\title{
Möglichkeiten des Steuerrechts zur Förderung des \\ ökologischen Landbaus - ein Ko-Instrument für eine effizientere Förderung
}

\author{
Dissertation \\ zur Erlangung des Doktorgrades \\ der Fakultät für Agrarwissenschaften \\ der Georg-August-Universität Göttingen
}

\author{
vorgelegt von \\ Jobst-Hendrik Held \\ geboren in Herford
}


D 7

1. Referent: Prof. Dr. E. Bahrs

2. Korreferent: Prof. Dr. L. Theuvsen

Tag der mündlichen Prüfung: 01.02.2007 


\section{VORWORT}

Die vorliegende Arbeit entstand während meiner Tätigkeit als Doktorand am Department für Agrarökonomie und Rurale Entwicklung der Georg-August-Universität Göttingen. Sie wurde gefördert von der Bundesanstalt für Landwirtschaft (BLE) im Rahmen des Bundesprogramms Ökologischer Landbau (BÖL). Für diese Unterstützung und den kontinuierlichen offenen Dialog möchte ich mich an dieser Stelle recht herzlich bedanken.

Mein ganz besonderer Dank gilt Herrn Prof. Dr. Enno Bahrs für die Überlassung des interessanten und innovativen Themas. Zusätzlich möchte ich mich bei ihm auch ganz speziell für das mir entgegengebrachte Vertrauen und die konstruktive und stets zielorientierte Betreuung bedanken. Seine methodischen und konzeptionellen Hinweise haben die Arbeit in grundlegender Weise geprägt und waren für die zügige Bearbeitung des Themas essentiell. Herrn Prof. Dr. Ludwig Theuvsen danke ich für die Übernahme des Korreferats. Danken möchte ich auch den Kolleginnen und Kollegen des Departments. Ihre Hilfsbereitschaft, der fachliche und private Gedankenaustausch sowie das äußerst angenehme Arbeitsklima haben großen Anteil am Gelingen dieser Arbeit gehabt. Ganz besonders bedanken möchte ich mich zudem bei meinen Göttinger Mitbewohnern, die mir während der Fertigstellung der Arbeit mit Rat, Verständnis und Rücksichtnahme zur Seite gestanden haben und die mit netten Ablenkungen stets dazu beigetragen haben, dass meine Promotionszeit mit viel Leben gefüllt war.

Schließlich möchte ich auch meinen Freunden und meiner Familie danken, die mir während der vergangenen Jahre soviel Verständnis entgegenbrachten und mir in jeder Phase der Arbeit den notwendigen Rückhalt gaben. 



\section{INHALTSVERZEICHNIS}

INHALTSVERZEICHNIS

TABELLENVERZEICHNIS

AbBildungsverzeichnis

VIII

ABKÜRZUNGSVERZEICHNIS

XI

1 Einleitung 1

$\begin{array}{lll}1.1 & \text { Problemstellung } & 1\end{array}$

1.2 ZieLSETZUNG 1

1.3 VORGEHENSWEISE 2

2 GEGENWÄRTIGE FÖRDERUNG DES ÖKOLOGISCHEN LANDBAUS IN DEUTSCHLAND 3

2.1 BiSHERIGE ZIELE UND RECHTFERTIGUNG EINER FöRDERUNG DES ÖKOLOGISCHEN LANDBAUS 3

2.1.1 DER BEITRAG DES ÖKOLOGISCHEN LANDBAUS ZU GESELLSCHAFTLICHEN ZIELEN 3

2.1.2 BEGRÜNDUNG STAATLICHER INTERVENTION

$\begin{array}{ll}\text { 2.1.3 EFFIZIENZ DER ZIELERREICHUNG } & 7\end{array}$

2.2 ÜBERBLICK ÜBER DIE DERZEITIGEN FÖRDERMAßNAHMEN IN DEUTSCHLAND 8

2.2.1 VON EU, BUND UND BUNDESLÄNDERN GEMEINSCHAFTLICH FINANZIERTE FÖRDERMAßNAHMEN 9

2.2.2 BUNDESFINANZIERTE FÖRDERMAßNAHMEN 11

2.2.3 LÄNDERFINANZIERTE FÖRDERMAßNAHMEN 12

2.2.4 BEDEUTUNG EINZELNER FÖRDERBEREICHE 14

\subsection{DIE FLÄCHENBEZOGENE ANGEBOTSFÖRDERUNG ALS SCHWERPUNKT DER} DERZEITIGEN FÖRDERPOLITIK 15

2.3.1 ENTWICKLUNG DER FLÄCHENBEZOGENEN FÖRDERUNG 15

2.3.2 RECHTLICHE RAHMENBEDINGUNGEN FÜR DIE BUNDESLÄNDER BEI DER AUSGESTALTUNG DER FLÄCHENBEZOGENEN FÖRDERUNG 17

2.3.3 AUSGESTALTUNG DER FLÄCHENBEZOGENEN FÖRDERUNG IN DEN BUNDESLÄNDERN 20 
2.3.4 DERZEITIGE UND ZUKÜNFTIGE FINANZIERUNG DER FLÄCHENBEZOGENEN FÖRDERUNG

2.3.5 SCHLUSSFOLGERUNG

2.4 WIRKUNGEN UND PROBLEMBEREICHE DER ÖKO-FLÄCHENPRÄMIEN

2.4.1 EFFEKTIVITÄT DER FÖRDERUNG

2.4.2 EFFIZIENZ DER FÖRDERUNG

2.5 SCHLUSSBETRACHTUNG UND WEITERE VORGEHENSWEISE

3 UNTERSUCHUNG STEUERLICHER FÖRDERALTERNATIVEN

3.1 GRUNDZÜGE DES STEUERRECHTS UND MÖGLICHKEITEN SEINER NUTZBARKEIT ALS FÖRDERINSTRUMENT

3.1.1 STEUERBEGRIFF UND STEUERARTEN

3.1.2 STEUERVERGÜNSTIGUNGEN

3.1.3 GRUNDSATZÜBERLEGUNGEN ZUR AUSWAHL STEUERLICHER FÖRDERINSTRUMENTE

3.2 KRITERIEN BEI DER BEURTEILUNG VON STEUERLICHEN FÖRDERALTERNATIVEN 50

3.2.1 WIRKUNG DER FÖRDERUNG 50

3.2.2 TRANSAKTIONSKOSTEN

3.2.3 GENEHMIGUNGS- UND KOFINANZIERUNGSFÄHIGKEIT GEMÄß DER EU-RAHMENGESETZGEBUNG

3.3 BISLANG DISKUTIERTE FÖRDERALTERNATIVEN IM RAHMEN DER STEUERGESETZE UND ABSCHÄTZUNG IHRER JEWEILIGEN ANWENDUNGSPROBLEME

3.3.1 DIFFERENZIERUNG DER BESTEHENDEN FLÄCHENPRÄMIEN NACH DEN ERTRAGSMESSZAHLEN (EMZ) DES STEUERLICHEN BEWERTUNGSGESETZES

3.3.2 FREISTELLUNG ÖKOLOGISCH BEWIRTSCHAFTETER FLÄCHEN VON DER GRUNDSTEUER

3.3.3 FreistelLUNG ÖKOLOGISCH ERZEUGTER PRODUKTE VON DER UMSATZSTEUER (UST)

\section{DiE FLEXIBLE FöRDERUNG - GEWINNERMITTLUNGSMETHODEN DES}

ERTRAGSTEUERRECHTS ALS VORBILD FÜR EINE FÖRDERUNG DES ÖKOLOGISCHEN LANDBAUS

4.1 ERHÖHTE BETRIEBSWIRTSCHAFTLICHE RISIKEN IM ÖKOLANDBAU ALS ANSATZ UND RECHTFERTIGUNG EINER FLEXIBLEN FÖRDERUNG

4.1.1 HINTERGRUND UND ZIELSETZUNG DER UNTERSUCHUNG

4.1.2 ANALYSE DER BETRIEBSWIRTSCHAFTLICHEN RISIKOSITUATION IM ÖKOLANDBAU 


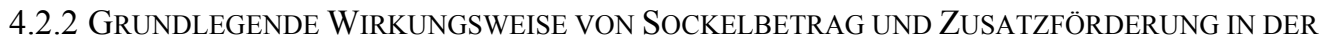
BASISKONSTELLATION 96

4.2.3 AUSWIRKUNGEN EINER VERÄNDERTEN BASISKONSTELLATION 102

4.2.4 ASPEKTE EINER ANGEMESSENEN BEMESSUNGSGRUNDLAGE FÜR DIE ZUSATZFÖRDERUNG 122

4.2.5 EXEMPLARISCHE DARSTELlung POTENZIELLER FehlanREIZE DURCh DIE FLEXIBLE FÖRDERUNG 123

4.2.6 FAZIT ZU DEN AUSWIRKUNGEN DER FLEXIBLEN FÖRDERUNG 132

\subsection{RECHTLICHE LEGITIMATION DER FLEXIBLEN FÖRDERUNG IM RAHMEN DER}

ELER-VERORDNUNG

4.4 ADMINISTRATIVE UMSETZUNG DER FLEXIBLEN FÖRDERUNG 136

4.4.1 Vorschlag FÜR DIE VERWALtUNGSTECHNISCHE HANDHABUNG DER FLEXIBLEN FöRdERUNG 136

4.4.2 VORSCHLAG FÜR EINE SACHGERECHTE SYSTEMUMSTELLUNG 140

4.4.3 SCHLUSSFOLGERUNGEN ZUR VERWALTUNGSTECHNISCHEN UMSETZUNG UND DEN ENTSTEHENDEN

$\begin{array}{ll}\text { TRANSAKTIONSKOSTEN } & 141\end{array}$

4.5 PotenZielle HeMmnisse Der IMPLEMENTIERUNG EINER FLEXIBLEN

FÖRDERUNG

4.5.1 WERTUNG DER ZEITLICHEN DIFFERENZ ZWISCHEN ERFASSUNG DER EINKOMMENSSCHWANKUNGEN UND GEWÄHRUNG DER FÖRDERUNG 141

4.5.2 GEWINNERMITTLUNG NACH DURCHSChNITTSSÄTZEN (§ 13A-BETRIEBE) 142

4.5.3 Personengesellschaften 143

$\begin{array}{ll}\text { 4.5.4 JURISTISCHE PERSONEN } & 144\end{array}$

4.5.5 TEILBETRIEBSUMSTELLUNG AUF ÖKOLOGISCHEN LANDBAU 146

4.5.6 RÜCKWIRKENDE ÄNDERUNG DER BEMESSUNGSGRUNDLAGE 146

$\begin{array}{ll}\text { 4.5.7 ANREIZ FÜR BETRIEBSTEILUNGEN } & 148\end{array}$

4.6 ÜBERLEGUNGEN ZU DEN AUSWIRKUNGEN UND ZUR UMSETZUNG IN FISKALISCHER

$\begin{array}{ll}\text { HINSICHT } & 149\end{array}$

4.6.1 FinANZIELLE AUSWIRKUNGEN EINER SYSTEMUMSTELlung AUS Sicht DES STAATES 149

4.6.2 PROBLEMATIK SCHWANKENDER FÖRDERBETRÄGE 152

\section{AKZEPTANZ FÜR DIE FLEXIBLE FÖRDERUNG IN DER LANDWIRTSCHAFT 155}

5.1 ZIELSETZUNG DER UNTERSUCHUNG

5.2 GESTALTUNG DER UNTERSUCHUNG 157

5.2.1 THEORIE DER EXPERIMENTELLEN WIRTSCHAFTSFORSCHUNG 157

$\begin{array}{ll}\text { 5.2.2 ORGANISATION, STRUKTUR UND ABLAUF DER UNTERSUCHUNG } & 159\end{array}$

5.3 ERGEBNISSE DER UNTERSUCHUNG

$\begin{array}{ll}\text { 5.3.1 CHARAKTERISIERUNG DER STICHPROBE } & 171\end{array}$

5.3.2 EINSCHÄTZUNGEN ZUR GEGENWÄRTIGEN FLÄCHENBEZOGENEN FÖRDERUNG 175 
5.3.3 UNTERSUCHUNG DER AKZEPTANZ FÜR DIE FLEXIBLE FÖRDERUNG

5.3.4 DIE PERSÖNLICHE RISIKOEINSTELLUNG ALS MÖGLICHE DETERMINANTE DER

ENTSCHEIDUNGSFINDUNG

\subsection{FAZIT}

6 ZUSAMMENFASSUNG

LITERATURVERZEICHNIS

VERZEICHNIS DER SONSTIGEN MITTEILUNGEN

GESPRÄCHSPARTNER IN DEN LÄNDERMINISTERIEN

XXVII

VERZEICHNIS DER RECHTSQUELLEN

XXIX

ANHANG

XXXI

A.1 ÖKO-FLÄCHENPRÄMIEN 2006 IN DEUTSCHLAND

XXXI

A.2 ERgebnisse Des Vergleichs DER BetriebsgewinNe

XXXVI

A.3 ÖKO-BETRIEBE NACH RECHTSFORMEN

XXXIX

A.4 Überblick Über die Programmierung in Z-Tree

XL

A.5 EXPERIMENTINSTRUKTIONEN FÜR DIE TEILNEHMER

XLVII

A.6 FRAgEbogen FÜR DIE TEILNEHMER AM EXPERIMENT

LX

A.7 ÜBerblick ÜBER DIE ERgeBNISSE DES PRÄMIENEXPERIMENTS 


\section{TABELLENVERZEICHNIS}

Tabelle 1: Höchstgrenzen für eine flächenbezogene Förderung im Rahmen der

Agrarumweltprogramme nach VO (EG) $1257 / 99 .$.

Tabelle 2: Vorgaben des GAK-Rahmenplanes 2006 zur Bemessung der Förderhöhen auf

Länderebene

Tabelle 3: Übersicht über die Öko-Fläche und den Einsatz von Fördermitteln auf

Landesebene in den Jahren 2001 bis 2004 23

Tabelle 4: Gliederung der Steuern 43

Tabelle 5: Jährliche Grundsteuerbelastung ldw. Betriebe in $€$

Tabelle 6: Schätzung des Steuerausfalls für die Kommunen bei Freistellung ökologisch bewirtschafteter Flächen in Deutschland

Tabelle 7: Umsatz an Öko-Lebensmitteln in Deutschland in Mrd. € (ohne Genussmittel und Außer-Haus-Verzehr)

Tabelle 8: Berechnung des USt-Volumens für die vergangenen Jahre. 73

Tabelle 9: Deskriptive Statistik zur mittleren einzelbetrieblichen Gewinnentwicklung (inkl. Prämienzahlungen) ökologischer und konventioneller Betriebe 85

Tabelle 10: Deskriptive Statistik zur mittleren einzelbetrieblichen Gewinnentwicklung (exkl. Prämienzahlungen) ökologischer und konventioneller Betriebe 86

Tabelle 11: Deskriptive Statistik zur mittleren einzelbetrieblichen Gewinnentwicklung (exkl. Prämienzahlungen der 2. Säule der GAP) ökologischer und konventioneller Betriebe

Tabelle 12: Risikoanalyse am Beispiel des Winterweizens auf Basis der einzelbetrieblichen

Daten des BMELV-TBN. .88

Tabelle 13: Risikoanalyse am Beispiel der Kartoffeln auf Basis der einzelbetrieblichen Daten des BMELV-TBN. 89

Tabelle 14: Beispielbetrachtungen zur Wirkung des ESt-Tarif 2006 94 
Tabelle 15: Beispielbetrachtung zur Wirkung einer dreijährigen Durchschnittsbesteuerung im Vergleich zur Wirkung der gegenwärtigen Besteuerung nach dem Jahresprinzip

Tabelle 16: Jährliche Zusatzförderung in $€ /$ Unternehmen (für die mittleren Variationskoeffizienten der ausgewerteten Öko-Betriebe aus dem BMELV-TBN)

Tabelle 17: Veränderungsempfehlungen für den Grenzsteuersatz

Tabelle 18: Ausgestaltung der Grenzsteuersatzmodifikationen

Tabelle 19: Annahmen für die untersuchten Beispielbetriebe

Tabelle 20: Vorteilhaftigkeit einer vorgezogenen Großreparatur (WJ 0/1 statt WJ 1/2) bei einem außerordentlich hohen Betriebsgewinn im Entscheidungsjahr

Tabelle 21: Vorteilhaftigkeit einer vorgezogenen Großreparatur (WJ 0/1 statt WJ 1/2) bei einem außerordentlich niedrigen Betriebsgewinn im Entscheidungsjahr ....

Tabelle 22: Übersicht über die Gewinnverteilung unter den Öko-Betrieben im BMELV-TBN

Tabelle 23: Übersicht über die mittleren Betriebsgewinne der gebildeten Betriebsgruppen. 150

Tabelle 24: Simulation der Gesamtausgaben für die Zusatzförderung

Tabelle 25: Ausgaben für die Zusatzförderung bei Annahme um $10 \%$ reduzierter Gewinnschwankungen im Vergleich zur Gewinnentwicklung im BMELV-TBN

Tabelle 26: Ausgaben für die Zusatzförderung bei Annahme um 10 \% erhöhter Gewinnschwankungen im Vergleich zur Gewinnentwicklung im BMELV-TBN 153

Tabelle 27: Chronologie der Einzelexperimente. 171

Tabelle 28: Charakterisierung der teilnehmenden Betriebe

Tabelle 29: Evaluierung der unternehmerischen Orientierung der Teilnehmer.

Tabelle 30: Stärken-Schwächen-Profil der Teilnehmer hinsichtlich ihrer landwirtschaftlichen Tätigkeit.

Tabelle 31: Relevanz der Förderung 176 
Tabelle 32: Beurteilung der Zukunft der Öko-Förderung 178

Tabelle 33: Darstellung der Plausibilitätsberechnung am Beispiel der

Entscheidungssituation aus Abbildung 42 181

Tabelle 34: Beweggründe zur Auswahl der Festbetragsförderung für den eigenen Betrieb . 186

Tabelle 35: Beweggründe zur Auswahl der Flexiblen Förderung für den eigenen Betrieb... 187

Tabelle 36: Nutzwertberechnung für die erhobenen Sicherheitsäquivalente der Teilnehmer 189

Tabelle 37: Übersicht über entscheidende Parameter der Risikoeinstellung 192

Tabelle 38: Einteilungsschema zur Klassifizierung der absoluten

Risikoaversionskoeffizienten 193

Tabelle 39: Häufigkeitstabelle zur Risikoeinstellung der Teilnehmer am Experiment 194 


\section{ABBILDUNGSVERZEICHNIS}

Abbildung 1: Fördermaßnahmen im Überblick ………………………………………….... 8

Abbildung 2: Finanzierungskonstellation bei der Finanzierung der Öko-Flächenprämien ..... 22

Abbildung 3: Wirkung einer Produktsubvention ohne Außenhandel ........................................ 29

Abbildung 4: Ökologisch bewirtschaftete Fläche, Staatsausgaben und

Einkommenswirkungen bei der Einheitsprämie.................................................... 36

Abbildung 5: Ökologisch bewirtschaftete Fläche, Staatsausgaben und Einkommenswirkungen bei einer gestaffelten Prämie ............................................................ 39

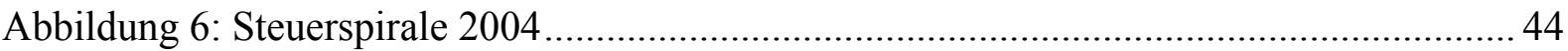

Abbildung 7: Auswahlprozess der für eine steuerliche Förderung infrage kommenden Steuerarten

Abbildung 8: Einkommensverluste, Fördersumme und Transaktionskosten von

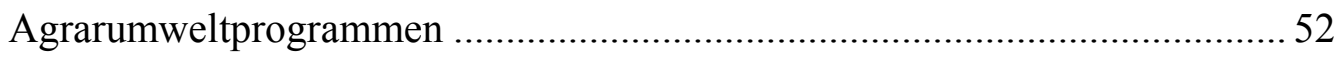

Abbildung 9: Angestrebtes Wirkungsszenario als Reaktion auf eine Freistellung ökologisch erzeugter Produkte von der Umsatzsteuer .............................................. 67

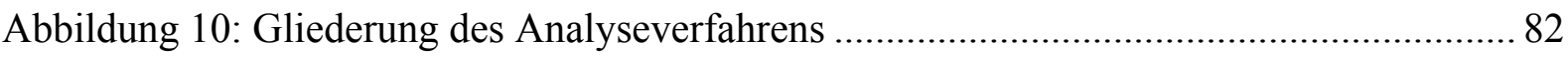

Abbildung 11: Beispielhafte Darstellung zweier Gewinnverteilungen..................................... 84

Abbildung 12: Aufbau der Flexiblen Förderung ................................................................... 91

Abbildung 13: Berechnungsschema für die Zusatzförderung.................................................. 92

Abbildung 14: Durchschnitts- und Grenzsteuersatz gemäß ESt-Tarif 2006 ............................ 93

Abbildung 15: Wirkungsweise der Zusatzförderung bei mittleren jährlichen Einkünften von 10.000 Euro und unterschiedlichen Einkunftsschwankungen

Abbildung 16: Wirkungsweise der Zusatzförderung bei mittleren jährlichen Einkünften von 50.000 Euro und unterschiedlichen Einkunftsschwankungen

Abbildung 17: Wirkungsweise der Zusatzförderung bei mittleren jährlichen Einkünften von 100.000 Euro und unterschiedlichen Einkunftsschwankungen.................. 98

Abbildung 18: Höhe der Zusatzförderung gemäß ESt-Tarif 2006 99 
Abbildung 19: Zusammenhang zwischen den Gewinnschwankungen und dem

Gewinnmittelwert bei Öko-Betrieben des BMELV-TBN 100

Abbildung 20: Einflussfaktoren auf die Flexible Förderung 102

Abbildung 21: Überblick über die Veränderungsmöglichkeiten 103

Abbildung 22: Zusatzförderung bei Anwendung eines fünfjährigen Bemessungszeitraums 104

Abbildung 23: Zusatzförderung bei Anwendung eines siebenjährigen Bemessungszeitraums

Abbildung 24: Vergleich der Förderwirkung unterschiedlicher Bemessungszeiträume bei

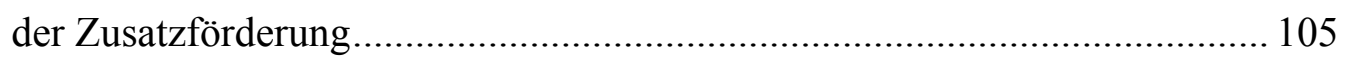

Abbildung 25: Auswirkungen einer Veränderung des Bemessungszeitraums ..... 107

Abbildung 26: Veränderung von Grenzsteuersatz und Förderwirkung infolge der

Änderung einzelner Stellgrößen 108

Abbildung 27: Grafische Darstellung der beiden Grenzsteuersatzmodifikationen...

Abbildung 28: Zusatzförderung bei Unterstellung der Grenzsteuersatzmodifikation 1 als Berechnungsgrundlage

Abbildung 29: Zusatzförderung bei Unterstellung der Grenzsteuersatzmodifikation 2 als Berechnungsgrundlage

Abbildung 30: Veränderung der Förderwirkung infolge der Grenzsteuersatzmodifikationen in $€ /$ Betrieb im Vergleich zur Basiskonstellation.

Abbildung 31: Auswirkungen einer Veränderung der Berechnungsgrundlage

Abbildung 32: Zusatzförderung bei Anwendung des Faktors 3

Abbildung 33: Zusatzförderung bei Anwendung des Faktors 5 116

Abbildung 34: Auswirkungen einer Einführung der Flexiblen Förderung für unterschiedlich flächenstarke Betriebe bei niedrigen Einkunftsschwankungen

Abbildung 35: Auswirkungen einer Einführung der Flexiblen Förderung für unterschiedlich flächenstarke Betriebe bei mittleren Einkunftsschwankungen. 
Abbildung 36: Auswirkungen einer Einführung der Flexiblen Förderung für unterschiedlich flächenstarke Betriebe bei hohen Einkunftsschwankungen .. 118

Abbildung 37: Wirkungsweise der Flexiblen Förderung bei geringen

Einkunftsschwankungen und der Multiplikation der Zusatzförderung mit unterschiedlichen Faktoren

Abbildung 38: Wirkungsweise der Flexiblen Förderung bei mittleren

Einkunftsschwankungen und der Multiplikation der Zusatzförderung mit verschiedenen Faktoren

Abbildung 39: Wirkungsweise der Flexiblen Förderung bei hohen

Einkunftsschwankungen und der Multiplikation der Zusatzförderung mit unterschiedlichen Faktoren....

Abbildung 40: Auswirkungen einer Veränderung der Gewichtung der Einzelkomponenten der Flexiblen Förderung

Abbildung 41: Schema zum technisch-organisatorischen Ablauf der Experimente 160

Abbildung 42: Entscheidungsbildschirm im Prämienexperiment.

Abbildung 43: Ergebnisbildschirm im Prämienexperiment.

Abbildung 44: Bildschirm zur Gebotsabgabe im Lotteriespiel.

Abbildung 45: Ergebnisbildschirm des Lotteriespiels

Abbildung 46: Analyse des Entscheidungsverhaltens hinsichtlich der Akzeptanz des

Probanden für die Flexible Förderung 180

Abbildung 47: Ergebnisse des Prämienexperiments zur Untersuchung der Akzeptanz für die Flexible Förderung. 183

Abbildung 48: Ermittlung der Risikonutzenfunktionen verschiedener Teilnehmer 190

Abbildung 49: Darstellung der Berechnung der ARAC's für die Fallbeispiele A bis C 193 


\section{ABKÜRZUNGSVERZEICHNIS}

\section{ABKÜRZUNGEN FÜR DIE BUNDESLÄNDER}

$\begin{array}{ll}\text { BB } & \text { Brandenburg } \\ \text { BE } & \text { Berlin } \\ \text { BW } & \text { Baden-Württemberg } \\ \text { BY } & \text { Bayern } \\ \text { HB } & \text { Bremen } \\ \text { HE } & \text { Hessen } \\ \text { HH } & \text { Hamburg } \\ \text { MV } & \text { Mecklenburg-Vorpommern } \\ \text { NI } & \text { Niedersachsen } \\ \text { NW } & \text { Nordrhein-Westfalen } \\ \text { RP } & \text { Rheinland-Pfalz } \\ \text { SH } & \text { Schleswig-Holstein } \\ \text { SL } & \text { Saarland } \\ \text { SN } & \text { Sachsen } \\ \text { ST } & \text { Sachsen-Anhalt } \\ \text { TH } & \text { Thüringen }\end{array}$

\section{Allgemeine AbKÜRZUngen}

Abl. Amtsblatt

AFP Agrarinvestitionsförderungsprogramm

AID Agrarinformationsdienst

AK Arbeitskraft

AO Abgabenordnung

BFH Bundesfinanzhof

BfN Bundesamt für Naturschutz

Bio biologisch/biologische/biologischer

BLE Bundesanstalt für Landwirtschaft und Ernährung

BMELV Bundesministerium für Ernährung, Landwirtschaft und Verbraucherschutz

BMF Bundesministerium für Finanzen 
BÖL Bundesprogramm Ökologischer Landbau

BNN Bundesverband Naturkost Naturwaren

bpb Bundeszentrale für politische Bildung

BSE Bovine Spongiforme Enzephalopathie (Rinderwahnsinn)

bspw beispielsweise

BVerfGE Bundesverfassungsgericht

BVL Bundesamt für Verbraucherschutz und Lebensmittelsicherheit

BWA Betriebswirtschaftliche Ausrichtung

bzw. beziehungsweise

DBV Deutscher Bauernverband

d.h. das heißt

dt Dezitonne

DVO Durchführungsverordnung

EDV Elektronische Datenverarbeitung

EG Europäische Gemeinschaft

EGE Europäische Größeneinheit

ELER Förderung der Entwicklung des ländlichen Raums durch den europäischen Landwirtschaftsfond zur Entwicklung des ländlichen Raums

EMZ Ertragsmesszahl

ESt Einkommensteuer

EU Europäische Union

EWG Europäische Wirtschaftsgemeinschaft

exkl. exklusive

FAL Bundesforschungsanstalt für Landwirtschaft

GAK Gemeinschaftsaufgabe „Verbesserung der Agrarstruktur und des Küstenschutzes“

GAP Gemeinsame Agrarpolitik der EU

GbR Gesellschaft bürgerlichen Rechts

GK Grenzkosten

GrSt Grundsteuer

ha Hektar

i.d.R. in der Regel

inkl. inklusive

INVEKOS Integriertes Verwaltungs- und Kontrollsystem 
JÜ Jahresüberschuss

KG Kommanditgesellschaft

KJ Kalenderjahr

KSt Körperschaftsteuer

LEH Lebensmitteleinzelhandel

LF landwirtschaftliche Fläche

LHO Landeshaushaltsordnung

LuF Land- und Forstwirtschaft

Mio. Million

Mrd. Milliarde

MSL Markt- und standortangepasste Landbewirtschaftung

MUNLV Ministerium für Umwelt und Naturschutz, Landwirtschaft und Verbraucherschutz des Landes Nordrhein Westfalen

OHG Offene Handelsgesellschaft

Öko ökologisch/ökologische/ökologischer

PLANAK Planungsausschuss „Agrarstruktur und Küstenschutz“

ARAC absoluter Risikoaversionskoeffizient

RdErl. Runderlass

RS Referenzszenario

SÖL Stiftung Ökologie \& Landbau

StAbw Standardabweichung

TBN Testbetriebsnetz

TK Transaktionskosten

US Untersuchungsszenario

USt Umsatzsteuer

u.U. unter Umständen

VC Variationskoeffizient

vgl. vergleiche

VO Verordnung

WJ Wirtschaftsjahr

ZMP Zentrale Markt- und Preisberichtsstelle

z.B. zum Beispiel

z.T. zum Teil

zvE $\quad$ zu versteuerndes Einkommen 


\section{EINLEITUNG}

\subsection{Problemstellung}

Der ökologische Landbau hat u. a. infolge des BSE-Skandals Ende des Jahres 2000 in Deutschland aber auch in der EU ein erhebliches Flächenwachstum vollzogen. Damit sind auch die Finanzmittelausgaben der öffentlichen Haushalte zur flächenbezogenen Förderung des ökologischen Landbaus erheblich angestiegen. Das hat in einigen Bundesländern in den Jahren 2004 und 2005 bereits dazu geführt, die Förderung für Neuumsteller bzw. für neu hinzukommende Flächen bereits bestehender Öko-Betriebe aus Finanzmittelknappheit einzuschränken oder sogar einzustellen. Dem übergeordneten Ziel dieser Förderung, die ökologisch bewirtschaftete Fläche in Deutschland stärker auszuweiten, ist diese Vorgehensweise abträglich. Die Situation wird sich aller Voraussicht nach gerade in den kommenden Jahren noch deutlich verschärfen. Denn die im Dezember 2005 von den Regierungschefs der EU-Staaten ausgehandelten Brüsseler Finanzbeschlüsse bedeuten für den kommenden Finanzplanungszeitraum 2007 bis 2013 deutliche Einschnitte in der Finanzierung der Agrarumweltmaßnahmen, wozu auch die flächenbezogene Förderung der ökologischen Bewirtschaftungsweise gehört. So haben einige Bundesländer als Reaktion darauf bereits einen teilweisen Förder-Stopp für 2006 sowie tief greifende Veränderungen bei der ÖkoFlächenprämie für die Zeit ab 2007 angekündigt.

\subsection{ZIELSETZUNG}

Ziel der vorliegenden Arbeit ist die Analyse zukünftiger Möglichkeiten der Angebotsförderung der ökologischen Landwirtschaft im Kontext des Steuerrechts. Vor dem Hintergrund der genannten Problemstellung sollte als grundlegende Handlungsmaxime dabei gelten: Mit den in Zukunft begrenzter zur Verfügung stehenden Finanzmitteln sollen maximale Förderergebnisse erzielt werden. Dazu werden die gegenwärtigen Flächenprämien hinsichtlich ihrer Effektivität und ihrer Effizienz überprüft und Verbesserungsmöglichkeiten diskutiert. Zu diesem Zweck werden ausgewählte steuerliche Förderinstrumente analysiert und hinsichtlich ihrer Eignung für eine Förderung des ökologischen Landbaus geprüft. Als Ergebnis soll eine ergänzende Förderalternative zur gegenwärtigen Angebotsförderung 
entwickelt werden, die Potentiale für einen wirksameren Einsatz der zur Verfügung stehenden Finanzmittel aufweist.

\subsection{VORGEHENSWEISE}

$\mathrm{Zu}$ Beginn der Arbeit wird in Kapitel 2 auf die gegenwärtige Förderung des ökologischen Landbaus eingegangen. Dazu wird neben grundlegenden Überlegungen zu den Zielen und zur Rechtfertigung einer Öko-Förderung ein Überblick über die derzeitig angebotenen Fördermaßnahmen präsentiert. Aufgrund der aus finanzieller Sicht außerordentlich hohen Bedeutung der flächenbezogenen Angebotsförderung sowie der Zielstellung dieser Arbeit wird in einem weiteren Unterkapitel auf die Konzeption und die Finanzierung des bislang eingesetzten Förderinstruments eingegangen. Eine allgemeine Evaluierung der flächenbezogenen Angebotsförderung in ihrer gegenwärtigen Ausgestaltung schließt das zweite Kapitel ab. Dabei werden speziell die Effektivität und die Effizienz dieses Förderinstruments vor dem Hintergrund zukünftig begrenzter finanzieller Mittel und dem politischen Ziel einer weiteren Ausdehnung des ökologischen Landbaus betrachtet.

Auf den Untersuchungsergebnissen aus Kapitel 2 aufbauend folgt in Kapitel 3 eine Untersuchung, inwieweit das Steuerrecht im Allgemeinen sowie einzelne Methoden des Steuerrechts im Speziellen geeignet sein können, eine Angebotsförderung des ökologischen Landbaus zu flankieren. In diesem Kontext werden drei bereits vielfach diskutierte steuerliche Förderinstrumente näher untersucht.

In Kapitel 4 erfolgt die Vorstellung der Flexiblen Förderung als konkreter Vorschlag für eine Systemergänzung. Dabei werden die Begründung für diese Art der Förderung, ihre Entwicklung sowie ihre konzeptionelle Ausgestaltung vorgestellt. Darüber hinaus wird die Flexible Förderung hinsichtlich ihrer europa- und bundesrechtlichen Eignung geprüft und es werden Vorschläge für eine administrative Umsetzung unterbreitet. Weiter werden potenzielle Hemmnisse für eine Implementierung der Flexiblen Förderung sowie Auswirkungen auf das Förderbudget diskutiert.

Für eine erfolgreiche Implementierung eines Förderinstruments ist auch die Akzeptanz der am Förderprozess Beteiligten notwendige Voraussetzung. Dazu wird in Kapitel 5 mittels des Instruments der experimentellen Wirtschaftsforschung die Akzeptanz für die Flexible Förderung durch Landwirte geprüft. Die Arbeit endet mit einer Zusammenfassung der Ergebnisse in Kapitel 6. 


\section{GEGENWÄRTIGE FÖRDERUNG DES ÖKOLOGISCHEN LANDBAUS IN DEUTSCHLAND}

\subsection{BISHERIGE ZIELE UND RECHTFERTIGUNG EINER FÖRDERUNG DES ÖKOLOGISCHEN LANDBAUS}

Während zu Beginn der gemeinsamen europäischen Agrarpolitik im Jahr 1957 die Versorgung der Bevölkerung $\mathrm{zu}$ angemessenen Preisen sowie die Steigerung der landwirtschaftlichen Produktivität im Vordergrund standen, sind es heute im Wesentlichen folgende Zielvorstellungen, die von der EU-Agrarpolitik vorrangig anvisiert werden (DABBERT et al., 2002: 60):

- Minimierung der negativen Umwelteinflüsse der landwirtschaftlichen Produktion.

- Sicherstellung der Versorgung der Bevölkerung mit qualitativ hochwertigen Lebensmitteln.

- Stabilisierung der Einkommen aus der Landwirtschaft und Förderung der Wettbewerbsfähigkeit der europäischen Agrarproduktion.

- Stärkung der Entwicklung der ländlichen Räume.

- Langfristige Senkung der Subventionierung der Landwirtschaft.

Einige dieser Grundziele der staatlichen Agrarförderung sind in den Grundsätzen des ökologischen Landbaus fest verankert und werden daher von ihm schon seit jeher direkt angesteuert. Es ist darum nicht verwunderlich, dass der ökologische Landbau in der Diskussion um die Erreichung dieser Ziele eine herausragende Rolle spielt.

\subsubsection{DER BEITRAg DES ÖKOLOGISCHEN LANDBAUS ZU GESELLSCHAFTLICHEN ZIELEN}

Insbesondere in den Bereichen Naturschutz und Lebensmittelqualität sehen Befürworter des ökologischen Landbaus die größten Vorzüge gegenüber dem konventionellen Landbau. Gleichzeitig liefern beide Bereiche für Konsumenten wichtige Kaufargumente für ÖkoProdukte (vgl. dazu HACCIUS, 2003: 47f). 
Ganz offensichtlich bringt der ökologische Landbau, wie jede andere Wirtschaftsweise auch, Auswirkungen auf die Natur mit sich. Dass die Umwelteffekte des ökologischen Landbaus weniger schädlich sind als die des konventionellen Landbaus belegt das Ergebnis einer vergleichenden wissenschaftlichen Untersuchung aus dem Jahr 2000. Die Studie (STOLZE et al., 2000) basiert auf einer Auswertung von wissenschaftlichen Arbeiten in 18 europäischen Ländern und untersucht die Effekte beider Produktionsverfahren auf Ökosystem, Boden, Grund- und Oberflächenwasser, Klima und Luft, Betriebsinput und -output sowie auf das Wohl und die Gesundheit der gehaltenen Tiere. Die Untersuchung kommt dabei zu dem Ergebnis, dass sich der ökologische Landbau in keiner der genannten Kategorien schlechter als der konventionelle Landbau auf die Umwelt auswirkt. In Bezug auf das Ökosystem trägt der ökologische Landbau sogar wesentlich zum Schutz der Biodiversität bei, da er einerseits keine synthetischen Dünge- und Pflanzenschutzmittel einsetzt und andererseits der Tierwelt vielseitigere Lebensräume anbietet. In der Untersuchungskategorie "Boden" fällt vor allem die deutlich höhere biologische Aktivität auf, die positive Auswirkungen auf die Fruchtbarkeit der Böden hat. Durch die stärker extensive ökologische Wirtschaftsweise werden auch Gewässer weitaus weniger belastet. Neben einer geringeren Nitratauswaschung existieren auch nicht die Risiken einer Kontamination von Gewässern mit synthetischen Pflanzenschutzmitteln. Insbesondere die größere Artenvielfalt bei Fauna und Flora (vgl. BrABAND et al., 2003: 155; PFIFFNER, 1997: 93ff oder WACHENDORF und TAUBE, 2001: 81) sowie ein niedrigeres Nitrat-N-Auswaschungsrisiko (vgl. BERG et al., 1999: 238 oder HANSEN et al., 2000: 65ff) konnten auch in einer Vielzahl anderer Untersuchungen nachgewiesen werden. Hinzu kommen Untersuchungen, die im Vergleich zum konventionellen Landbau auf eine höhere Energieeffizienz im ökologischen Landbau hinweisen (vgl. BoKISCH, 2000 oder HAAS et al., 1995: 387ff). Trotzdem gibt es auch Untersuchungen, die die positiven Umwelteigenschaften des ökologischen Landbaus relativieren. Neben SCHERINGER und ISSELSTEIN (2001: 284ff), die speziell ein niedrigeres Nitrat-N-Auswaschungsrisiko im ökologischen Landbau anzweifeln, gelangen TAUBE et al. (2005: 168) im Rahmen eines Vergleichs zwischen beiden Bewirtschaftungssystemen auf Hochertragsstandorten in Norddeutschland zu dem Ergebnis, dass der konventionelle Landbau trotz eines höheren Stickstoffeintrags sowie eines höheren Energieinputs aufgrund der standortbedingt höheren Erträge eine bessere Stickstoffeffizienz sowie eine vergleichbare Energieeffizienz aufweist.

Dennoch gelten die allgemein positiven Effekte des ökologischen Landbaus in Bezug auf biotische und abiotische Umweltaspekte in Fachkreisen mittlerweile als unbestritten. Es ist 
daher davon auszugehen, dass sich eine Zunahme der ökologisch bewirtschafteten Fläche in Bezug auf den Umwelt- und Ressourcenschutz positiv auf die aktuelle Situation im Agrarbereich auswirken würde.

Die Auswirkungen des ökologischen Landbaus auf die Lebensmittelqualität sind eingehend im Sommer 2003 von einer Arbeitsgruppe des Senats der Bundesforschungsanstalten untersucht worden. Dabei wurden vergleichende Untersuchungen von ökologisch und konventionell erzeugten Lebensmitteln durchgeführt. Die Ergebnisse dieser Studie (BMELV, 2003b: 34ff) zeigen, dass sich derzeit wissenschaftlich nicht eindeutig bestätigen lässt, dass Öko-Lebensmittel gesünder sind als konventionell produzierte Lebensmittel. Allerdings zeigen sich Tendenzen, dass dies u.U. so sein könnte. So seien in Öko-Produkten aufgrund der Nicht-Anwendung von chemischen Pflanzenschutzmitteln auch weniger Rückstände zu finden. Dieses Ergebnis bestätigen auch andere wissenschaftliche Untersuchungen. Nach Angaben des Bundesverbandes Naturkost Naturwaren (BNN) Herstellung und Handel war im Jahr 2004 im Bereich Bio-Obst und Bio-Gemüse lediglich bei 1,4\% der Proben eine Überschreitung der gesetzlichen Höchstmenge festzustellen (BNN, 2006: 8). Demgegenüber überschritten im konventionellen Landbau nach Angaben des Bundesamtes für Verbraucherschutz und Lebensmittelsicherheit (BVL) 7,4\% der Proben die gesetzliche Höchstmenge (BVL, 2005: 16ff oder AGRA-EUROPE, 2006e: 8)). Andere Untersuchungen verweisen auf höhere Anteile konjugierter Linolsäure in der Bio-Milch sowie auf höhere Anteile an Vitaminen, Mineralstoffen und sekundären Pflanzenstoffen in ökologisch erzeugtem Obst und Gemüse (vgl. dazu GORIS, 2006: 58). So sind auch hinsichtlich der Produktqualität ökologischer Erzeugnisse Vorzüge gegenüber konventionellen Erzeugnissen wahrscheinlich.

\subsubsection{BEGRÜNDUNG STAATLICHER INTERVENTION}

Nach Überlegungen zu den potentiellen Beiträgen des ökologischen Landbaus zu den gesellschaftlichen Zielen ist im Folgenden der Frage nachzugehen, ob die positiven Auswirkungen des ökologischen Landbaus ein politisches Eingreifen zugunsten des ökologischen Landbaus rechtfertigen. Die folgenden diesbezüglichen Ausarbeitungen orientieren sich eng an dem Beitrag von DABBERT und HÄRING (2003: 100ff).

Die spezifische Frage nach der Rechtfertigung eines politischen Eingriffs zugunsten des ökologischen Landbaus führt zur allgemeinen Frage nach der generellen Zulässigkeit von staatlichen Eingriffen in den laufenden Wirtschaftsprozess. Nach der Wohlfahrtstheorie kann 
ein unvollständiges Funktionieren oder ein vollständiges Versagen des Marktes staatliche Eingriffe rechtfertigen. Ein Marktversagen kann z.B. im Falle der "Öffentlichen Güter" vorliegen. Umweltgüter wie saubere Luft oder eine hohe Biodiversität sind beispielsweise solche öffentlichen Güter. Sie sind gekennzeichnet durch zwei wesentliche charakteristische Merkmale. Zum einen haben sie die Eigenschaft der Nichtausschließbarkeit, denn ein Nutzungsausschluss ist zumeist aus technischen Gründen nicht möglich, so dass kein Entgelt für eine Nutzung erhoben werden kann und jedes Mitglied einer Volkswirtschaft automatisch die Möglichkeit zur Nutzung dieses Gutes hat. Zum anderen gilt für öffentliche Güter im Allgemeinfall auch die Nichtrivalität, die besagt, dass der den Individuen aus dem Konsumakt zufließende Nutzen unabhängig von der Zahl der Konsumenten ist. So hat z.B. der "Konsum von Biodiversität" durch eine Person keinerlei Auswirkung auf den "Konsum von Biodiversität" einer anderen Person. Wenn also zwangsläufig jeder an der Leistung Biodiversität teilhaben kann, für die Nutzung bzw. den Genuss des Gutes aber nicht bezahlen muss, so wird in aller Regel auch niemand zu einer freiwilligen Gegenleistung bereit sein und damit seine marginale Zahlungsbereitschaft offen legen. Vielmehr werden die Nutzer als so genannte Trittbrettfahrer ("free rider") am Markt auftreten, d.h. sie nutzen das öffentliche Gut, ohne sich an den Kosten der Bereitstellung dieses Gutes entsprechend ihrer marginalen Zahlungsbereitschaft zu beteiligen. Das gesellschaftlich gewünschte Niveau an Biodiversität kann somit vom Markt nicht bereitgestellt werden. Um diesem Marktversagen entgegenzuwirken, kann der Staat in den Markt eingreifen, indem er für ein bestimmtes Angebotsniveau auf dem Markt für öffentliche Güter sorgt. In diesem Sinne wären damit staatliche Subventionen zugunsten des ökologischen Landbaus gerechtfertigt.

Auch OpPermanN (2001: 14) vertritt diese Position. Seiner Ansicht nach führt der Weg über den Markt eindeutig nicht dazu, eine ökologische Landwirtschaft zu etablieren, welche die gesellschaftlich notwendigen ökologischen Reproduktionsfunktionen erfüllen kann. Daher sei es für die zukünftige Entwicklung der Landwirtschaft notwendig, über eine Mischung aus marktbezogenen Entwicklungsformen und politischen Regelungen nachzudenken.

Speziell bei der Erzeugung von Umweltgütern, die wie aus Kapitel 2.1.1 ersichtlich ein Hauptargument für eine staatliche Förderung des ökologischen Landbaus darstellen, konkurriert dieser allerdings mit agrarumweltpolitischen Maßnahmen innerhalb der konventionellen Landwirtschaft. Bevor er daher für die Agrarpolitik als umweltpolitisches Leitbild etabliert wird, muss zuerst die Frage geklärt werden, ob es sich bei ihm um die 
Wirtschaftsform handelt, die die angestrebten Umweltziele effizient, d.h. mit dem geringsten Aufwand erreicht (VON AlvensLeBEn, 1998: 381).

\subsubsection{EFFIZIENZ DER ZIELERREICHUNG}

Die Effizienz der Zielerreichung misst sich an den Kosten, die verursacht werden, um ein bestimmtes Ziel zu erreichen. Zur Effizienzmessung ist es daher im Vorfeld unerlässlich, die anvisierten Umweltziele anhand von Umweltindikatoren $\mathrm{zu}$ definieren, um später die Kontrolle der Zielerreichung zu gewährleisten (BERG et al., 2003: 90). Um ihre Effizienz und damit letztlich auch ihre Förderwürdigkeit unter Beweis zu stellen, muss die ökologische Produktionsweise zu einem durch eine Reihe von Indikatoren definierten Umweltniveau führen und dabei im Vergleich zu allen anderen konkurrierenden Produktionsweisen die geringsten Kosten verursachen. In dieser Frage ist sich die Wissenschaft bisher uneins. So argumentieren einige Ökonomen, der Staat könne das angestrebte Umweltniveau mit einer Summe von Einzelmaßnahmen, von denen jede jeweils optimal auf ein Einzelziel zugeschnitten ist, im Rahmen des konventionellen Landbaus effizienter erreichen als mit der generellen Förderung des ökologischen Landbaus, bei der die Einzelziele nur ungenau anvisiert würden (vgl. VON ALVENSLEBEN, 1998: 382; vgl. KöSTER, 2001: 231). Andere Ökonomen (vgl. DABBERT und HÄRING, 2003: 102f) verweisen dagegen neben der kostenintensiven Messung einzelner Indikatoren und schwer bestimmbarer Interaktionen zwischen den Indikatoren vor allem auf die, mit einer Vielzahl verschiedener Politikinstrumente verbundenen, Transaktionskosten hin. Der ökologische Landbau wirke sich positiv auf eine große Bandbreite von Umweltindikatoren aus. Um eine große Breitenwirkung zu erreichen, sei er die kostengünstigere Variante.

Die Frage nach der Effizienz der Zielerreichung durch eine Förderung des ökologischen Landbaus kann aufgrund des derzeit noch unzureichenden Wissensstandes in Bezug auf die Administrierbarkeit und die damit verbundenen Verwaltungs- und Kontrollkosten von Einzelmaßnahmen noch nicht endgültig beantwortet werden. Es existieren aber bereits erste beispielhafte Untersuchungen zu dieser Fragestellung (vgl. u.a. HAGEDORN et al., 2004), die vermuten lassen, das die Förderung des ökologischen Landbaus bezüglich der entstehenden Transaktionskosten im Vorteil sein könnte. Die Ankündigung der neuen Bundesregierung, eine Ausweitung des ökologischen Landbaus auch in Zukunft zu fördern, kann daher nach dem heutigen Kenntnisstand als gerechtfertigt angesehen werden (vgl. dazu AGRA-EuROPE, 2006d: 33). Dies gilt umso mehr, da Öko-Betriebe nach NIEBERG und OfFERMANN (2006) 
trotz zukünftig voraussichtlich höherer Fördermittel aus der ersten Säule der GAP nach wie vor auf eine Sonderförderung angewiesen sind. Fraglich ist aber nach wie vor, mit welchen Fördermaßnahmen eine Ausweitung der ökologisch bewirtschafteten Fläche am effizientesten $\mathrm{zu}$ erreichen ist. Dazu soll im folgenden Kapitel zunächst auf das derzeitige Maßnahmenspektrum eingegangen werden, mit dem die angestrebte Ausweitung des ökologischen Landbaus erreicht werden soll.

\section{2 ÜBERBLICK ÜBER DIE DERZEITIGEN FÖRDERMAßNAHMEN IN DEUTSCHLAND}

Seit 1989 wird der ökologische Landbau in Deutschland finanziell gefördert. Angefangen mit einer ausschließlichen Förderung der ökologisch wirtschaftenden Betriebe über Flächenprämien im Rahmen des EU-Extensivierungsprogramms (VO (EWG) 4115/88), wird er heute durch viele, zum Teil sehr unterschiedliche, Maßnahmen politisch unterstützt. Gemäß Abbildung 1 können die Fördermaßnahmen in drei Gruppen eingeteilt werden.

Abbildung 1: Fördermaßnahmen im Überblick

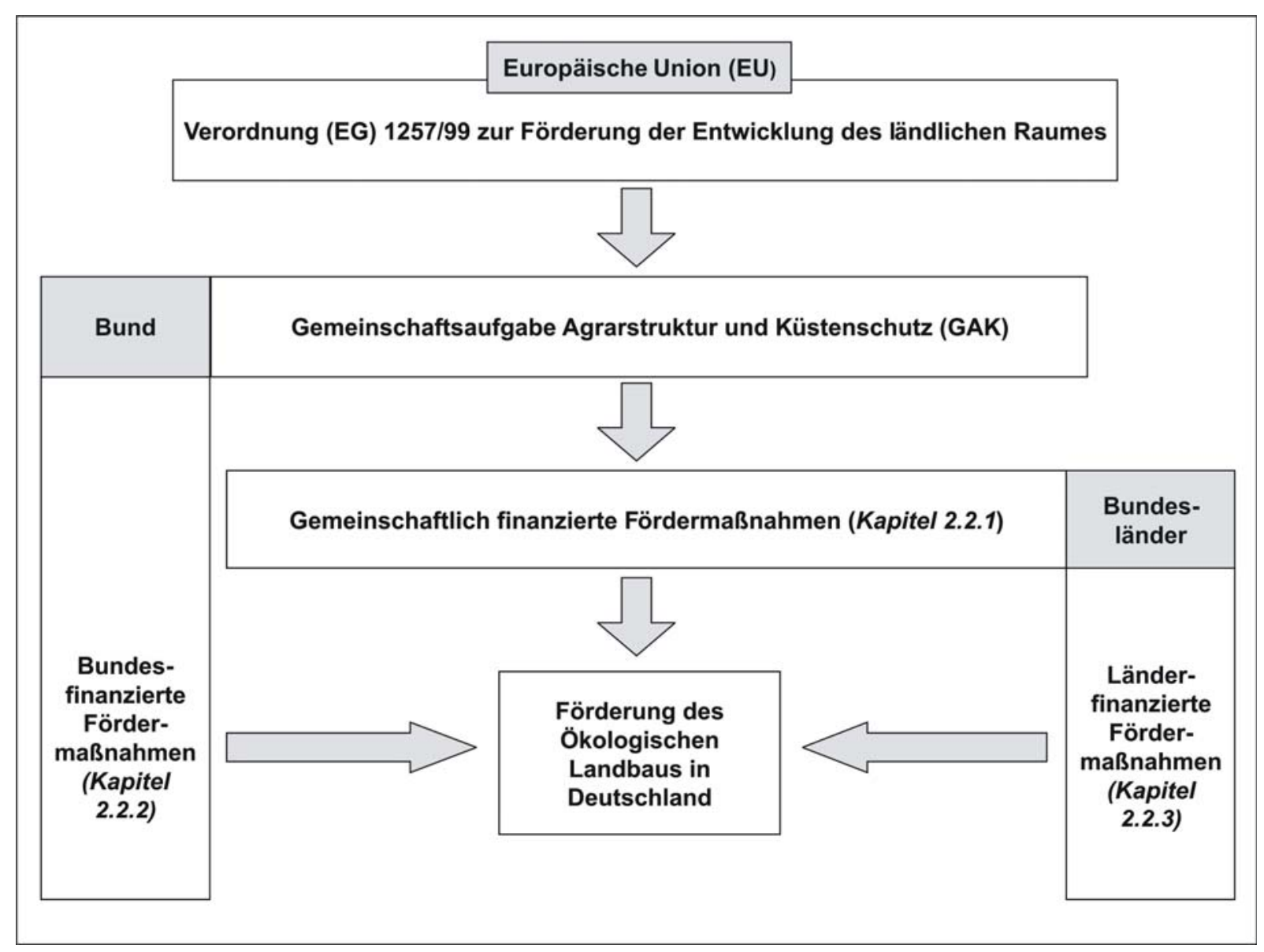

Quelle: Eigene Darstellung 
Die erste Gruppe bilden die gemeinschaftlich finanzierten Fördermaßnahmen. Weite Teile der Agrarstruktur- und Umweltpolitik fallen in den Kompetenzbereich der Bundesländer. Innerhalb der Gruppe der gemeinschaftlich finanzierten Maßnahmen bieten die EU und der Bund den Ländern jedoch eine finanzielle Beteiligung an, sofern die von ihnen diesbezüglich aufgestellten Rahmenvorgaben eingehalten werden. Daneben existieren Fördermaßnahmen, die ausschließlich aus dem Bundeshaushalt finanziert und auf Bundesebene konzipiert werden. Neben den gemeinschaftlich finanzierten Fördermaßnahmen werden auf Ebene der Bundesländer noch zusätzliche Maßnahmen angeboten, die für die Bundesländer nicht über die EU oder den Bund kofinanzierbar sind, sondern allein aus dem jeweiligen Landeshaushalt finanziert werden.

Im Folgenden sollen zunächst alle Maßnahmengruppen kurz vorgestellt werden, bevor auf deren finanzielle Bedeutung eingegangen wird.

\subsubsection{VON EU, BUND UND BUNDESLÄNDERN GEMEINSCHAFTLICH FINANZIERTE FÖRDERMAßNAHMEN}

Mit den Berliner Beschlüssen vom 26. März 1999 zur Agenda 2000 wurden eine Fortsetzung und vor allem eine Vertiefung der EU-Agrarreform von 1992 eingeleitet. Neben einer verstärkten Marktorientierung im Rahmen der ersten Säule der GAP zielte die Agenda 2000 auch auf eine zunehmende Berücksichtigung von Umweltzielen und eine stärkere Integration von Maßnahmen zur Förderung der Entwicklung des ländlichen Raumes ab (OSTERBURG und STRATMANN, 2002: 261f).

Diese Maßnahmen der zweiten Säule sind in der Verordnung (EG) 1257/99 zur Förderung der Entwicklung des ländlichen Raumes kodifiziert. Für die Förderung des ökologischen Landbaus sind insbesondere die Agrarumweltmaßnahmen relevant. Sie sind in den Artikeln 22 bis 24 der Verordnung (EG) 1257/99 geregelt und bieten den Bundesländern die Grundlage für flächenbezogene Fördermaßnahmen zugunsten des ökologischen Landbaus. Daneben weisen aber auch noch andere Maßnahmenbereiche Potentiale zur gezielten Förderung des ökologischen Landbaus auf, so etwa die Investitionsförderung in landwirtschaftlichen Betrieben im Rahmen der Artikel 4 bis 7, die Förderung von Weiterbildungsmaßnahmen gemäß Artikel 9 oder die Förderung einer Verbesserung der Verarbeitung und Vermarktung landwirtschaftlicher Erzeugnisse im Rahmen der Artikel 25 bis 28 . 
Die EU gibt mit der Verordnung (EG) 1257/99 aber lediglich einen Rahmen vor, innerhalb dessen sich die einzelnen Bundesländer mit ihren agrarpolitischen Maßnahmen zur Stärkung des ländlichen Raumes bewegen müssen, sofern sie eine Kofinanzierung der EU in Anspruch nehmen wollen. Die endgültigen Schwerpunkte der Förderpolitik können so, getreu dem Subsidiaritätsprinzip, auf Ebene der Bundesländer gesetzt werden. Weiterhin fungiert diese Verordnung auch als Rahmenverordnung für weitere Rahmenverordnungen auf Ebene der Nationalstaaten. Diese können sich über eigene Rahmenrichtlinien ebenfalls an der Finanzierung von Maßnahmen zugunsten des ländlichen Raumes beteiligen.

$\mathrm{Da}$ bundesgesetzliche Regelungen für die Agrarstrukturpolitik gänzlich fehlen, die Bundesländer also für die Agrarstrukturpolitik und deren Finanzierung allein verantwortlich sind, würden in Deutschland innerhalb kürzester Zeit große agrarstrukturelle Unterschiede und damit gravierende Unterschiede in der Wettbewerbsfähigkeit der landwirtschaftlichen Unternehmen entstehen. Um dem entgegenzuwirken, wurde die Verbesserung der Agrarstruktur und des Küstenschutzes im Jahr 1969 zu einer Gemeinschaftaufgabe von Bund und Ländern erklärt (BMELV, 2006c). Über diese Gemeinschaftsaufgabe Agrarstruktur und Küstenschutz (GAK) kann auf nationaler Ebene auf die Umsetzung der EUAgrarstrukturpolitik in den Bundesländern eingewirkt werden. Durch die Förderungsgrundsätze des GAK-Rahmenplanes werden die einzelnen Maßnahmen der Rahmenverordnung (EG) 1257/99 der EU auf Bundesebene weiter konkretisiert. Zusätzlich zur EU-Kofinanzierung können die Bundesländer dabei Bundesmittel in Höhe von 60 \% des nationalen Anteils in Anspruch nehmen, vorausgesetzt, sie halten sich bei der Ausgestaltung ihrer Fördermaßnahmen an die im Rahmenplan der GAK festgelegten Fördergrundsätze. Für die Förderung des ökologischen Landbaus sind analog zu den im Vorkapitel genannten Maßnahmen insbesondere folgende GAK-Grundsätze bedeutsam:

- Förderung einer markt- und standortangepassten Landbewirtschaftung (MSL):

Seit 1994 werden die Agrarumweltprogramme über den Förderungsgrundsatz Förderung einer markt- und standortangepassten Landbewirtschaftung (MSL) konkretisiert (vgl. dazu NiENDIEKER, 1998: 530). Die flächenbezogene Förderung des ökologischen Landbaus ist dabei nur ein Förderungsinstrument innerhalb der MSL. Wie sich speziell in Kapitel 2.2.4 noch zeigen wird, liegt der Schwerpunkt der Gesamtförderung des ökologischen Landbaus in der derzeitigen Ausgestaltung der Förderpolitik auf diesen Öko-Flächenprämien, auf die in Kapitel 2.3 detailliert eingegangen wird.

- Agrarinvestitionsprogramm (AFP): 
Die Aufgabe des AFP's liegt in der finanziellen Unterstützung landwirtschaftlicher Betriebe bei Investitionsvorhaben. Weil der ökologische Landbau im AFP als eine Produktionsform gilt, die zur Erfüllung besonderer Anforderungen an die Landwirtschaft beiträgt, können ökologisch wirtschaftende Landwirte bei allen förderfähigen Investitionen Zuschüsse in Anspruch nehmen.

- Grundsätze für die Förderung der Verarbeitung und Vermarktung ökologisch erzeugter landwirtschaftlicher Produkte :

Diese Fördergrundsätze umfassen vor allem Startbeihilfen u.a. zu Gründungs- und Organisationskosten, Investitionsbeihilfen sowie Beihilfen für die Erarbeitung von Vermarktungskonzepten. Als Förderungsempfänger kommen neben Unternehmen des Handels sowie der Be- und Verarbeitung vor allem Erzeugerzusammenschlüsse in Betracht.

Die Verfahrensweise der Bundesländer zielt generell darauf ab, landesbezogene Präferenzen und Ausgestaltungsanliegen unter gleichzeitiger maximaler Realisierung der Kofinanzierungsmöglichkeiten von Seiten der EU und des Bundes verwirklichen zu können (Mehl und PlankL, 2002). Einerseits ist das Verlangen, die bereitgestellten EU- und Bundesmittel anzunehmen, sehr groß. Andererseits sollen die Programme auch möglichst exakt den landespolitischen Anforderungen entsprechen.

\subsubsection{BUNDESFINANZIERTE FÖRDERMAßNAHMEN}

Nachdem im Vorkapitel auf Maßnahmen zugunsten des ökologischen Landbaus eingegangen wurde, die sich zwar auf Rahmenrichtlinien der EU und des Bundes stützen, letztendlich aber auf Ebene der Bundesländer entwickelt und angeboten werden, wird in diesem Kapitel ein Überblick über die Maßnahmen erstellt, die direkt von der Bundesregierung initiiert wurden und vollständig aus dem Bundeshaushalt finanziert werden.

Neben der Förderung des ökologischen Landbaus im Rahmen der GAK wurde Anfang 2002 das Bundesprogramm Ökologischer Landbau (BÖL) von der Bundesregierung aufgelegt, mit dem Ziel, die Rahmenbedingungen für eine weitere Ausdehnung des ökologischen Landbaus $\mathrm{zu}$ verbessern. Grundgedanke ist die Förderung eines nachhaltigen Wachstums durch eine ausgewogene Expansion von Angebot und Nachfrage (BMELV, 2003b: 68). Dabei fällt dem BÖL vor allem die Aufgabe zu, die Nachfrage nach Ökoprodukten zu steigern. Es umfasst insgesamt eine Vielzahl von Einzelmaßnahmen, die zentrale Wachstumshemmnisse auf dem 
Markt für ökologisch erzeugte Produkte beseitigen sollen und sich entlang der gesamten Wertschöpfungskette verteilen. Im Mittelpunkt stehen dabei Schulungs-, Aufklärungs- und allgemeine Informationsmaßnahmen sowie die Forschungsförderung und Maßnahmen zum Technologie- und Wissenstransfer (SÖL, 2004: 1ff). In den Jahren 2002 und 2003 war das Bundesprogramm mit jeweils 35 Mio. Euro ausgestattet. Bis 2007 soll es mit jährlich 20 Mio. Euro fortgeführt werden (BMELV, 2006a).

Neben den vielfältigen Maßnahmen des Bundesprogramms wird die weitere Ausdehnung des ökologischen Landbaus von der Bundesregierung derzeit noch mit einigen zusätzlichen Maßnahmen unterstützt. So werden Leistungen ökologisch wirtschaftender Betriebe in Bereichen, die besonders zur Verbesserung des ökologischen Landbaus, seiner Produktionstechnik sowie seiner Umwelt- und Verbraucherfreundlichkeit beitragen mit dem Förderpreis Ökologischer Landbau ausgezeichnet (BMELV, 2006a). Weiterhin existiert seit Mai 2001 auf Bundesebene zur Kennzeichnung von Produkten aus ökologischem Landbau ein staatliches Bio-Siegel. Dazu müssen die Erzeugnisse nach den Vorschriften der EG-ÖkoVerordnung produziert und kontrolliert worden sein. Zusätzlich müssen mindestens $95 \%$ der Zutaten landwirtschaftlichen Ursprungs aus dem ökologischen Landbau stammen. Der damit dem Bio-Siegel zugrunde liegende Standard erlaubt eine sehr breite Anwendung. So können auch Produkte aus anderen EU-Staaten und Drittländern mit dem Bio-Siegel gekennzeichnet werden (BMELV, 2006a). Zuletzt sei noch auf das im Dezember 2000 gegründete Institut für ökologischen Landbau mit Sitz in Trendhorst hingewiesen. Es soll zur Intensivierung der Forschung im ökologischen Landbau beitragen und untersteht deshalb der Bundesforschungsanstalt für Landwirtschaft (FAL) in Braunschweig-Völkenrode.

\subsubsection{LÄNDERFINANZIERTE FÖRDERMAßNAHMEN}

Neben den von EU, Bund und Ländern gemeinschaftlich finanzierten Fördermaßnahmen ist es den Ländern freigestellt, den ökologischen Landbau noch durch weitere ausschließlich länderfinanzierte Maßnahmen $\mathrm{zu}$ unterstützen. Viele dieser länderfinanzierten Fördermaßnahmen ähneln jedoch Einzelmaßnahmen aus dem Bundesprogramm Ökologischer Landbau (BÖL). Bevor das BÖL im Jahr 2002 eingerichtet wurde, waren die länderfinanzierten Maßnahmen die einzigen Fördermaßnahmen in den betreffenden Bereichen. Trotz des BÖL werden in den einzelnen Bundesländern aber auch weiterhin noch länderfinanzierte Maßnahmen angeboten. Es bestehen jedoch abhängig von der Finanzkraft, den Politikzielen und den landesspezifischen Gegebenheiten erhebliche Unterschiede 
zwischen den Bundesländern sowohl in Art und Anzahl der angebotenen Maßnahmen als auch im Fördervolumen.

Die von den Bundesländern regelmäßig angebotenen Maßnahmen lassen sich grob in die beiden Bereiche Bildung, Beratung und Forschung sowie Öffentlichkeitsarbeit und Absatzförderung einordnen. Bildungsleistungen im Bereich des ökologischen Landbaus verteilen sich über das Angebot von speziellen Studienrichtungen an Universitäten und Fachhochschulen, über spezielle Ausbildungsschwerpunkte an Fachschulen bis hin zu Fortbildungskostenzuschüssen. Daneben existieren in einigen Bundesländern staatliche Lehrund Versuchsgüter, die z.T. vollständig ökologisch bewirtschaftet werden. Hinsichtlich der Beratung reicht die Förderung der Bundesländer von Öko-Spezialberatern im Rahmen der staatlichen Offizialberatung oder der halbstaatlichen Beratung der Landwirtschaftskammern über Personal- und Sachkostenzuschüsse für private Öko-Beratungsdienste und ÖkoAnbauverbände bis hin zu Beratungskostenzuschüssen für ökologisch wirtschaftende Landwirte. Im Bereich der Forschungstätigkeit zum ökologischen Landbau sind neben den Universitäten und Fachhochschulen auch die in einigen Bundesländern bestehenden Landesforschungsanstalten $\mathrm{zu}$ nennen. Auch im Bereich Öffentlichkeitsarbeit und Absatzförderung werden von den meisten Bundesländern eigens finanzierte Maßnahmen angeboten. Der Maßnahmenkatalog der einzelnen Länder ist in diesem Bereich jedoch ganz besonders vielfältig. Er reicht von der Erstellung von Informationsbroschüren über die Durchführung von Öko-Aktionswochen bis hin zu eigenen regionalen Öko-Siegeln in Bayern und Baden-Württemberg. Öffentlichkeitswirksame und absatzfördernde Aktionen werden oftmals auch im Rahmen der Verbandstätigkeit von den Anbauverbänden durchgeführt, die bei diesen Vorhaben z.T. von den Landesregierungen finanziell unterstützt werden.

Die besprochenen ursprünglich ausschließlich länderfinanzierten Förderbereiche werden seit 2002 zunehmend durch Einzelmaßnahmen des Bundesprogramms Ökologischer Landbau (BÖL) abgedeckt. Über dieses bundesfinanzierte Förderprogramm wird der ökologische Landbau in allen Bundesländern gefördert. Das reduziert den Druck auf die Bundesländer, in den jeweiligen Bereichen eigene Maßnahmen anbieten zu müssen. Somit erfolgt durch die verstärkte bundesweite Förderung eine zunehmende, wenn auch nur indirekte, Standardisierung der Förderbedingungen in Deutschland. 


\subsubsection{BEDEUTUNG EINZELNER FÖRDERBEREICHE}

Die vorangegangenen Kapitel 2.2.1 bis 2.2.3 haben die Vielzahl von Fördermaßnahmen offenbart, mit denen in Deutschland der ökologische Landbau politisch unterstützt wird. Wurde er zu Beginn der neunziger Jahre nur über die Flächenprämien für Erzeuger gefördert, so hat sich seitdem eine Vielzahl ganz unterschiedlicher Maßnahmen herausgebildet, die den ökologischen Landbau in seiner Entwicklung stärken und voranbringen soll. Dabei drängt sich dem Außenstehenden der Eindruck einer umfangreichen und gleichgewichtigen Unterstützung in allen Förderbereichen auf.

Bei genauerer Analyse der finanziellen Bedeutung der angebotenen Maßnahmen wird jedoch deutlich, dass dieser Eindruck täuscht. Nach wie vor nimmt die flächenbezogene Angebotsförderung im Rahmen der Agrarumweltprogramme der VO (EG) 1257/99 eine dominierende Stellung innerhalb der Förderung des ökologischen Landbaus ein, obwohl sich seit Auflegung des Bundesprogramms Ökologischer Landbau (BÖL) die Akzentuierung schon deutlich verändert hat. Im Jahr 2002 wurde beispielsweise der ökologische Landbau in Bayern mit Gesamtmitteln von etwa 33 Mio. $€$ unterstützt (MILLER, 2003). Dieser Betrag umfasst die bereitgestellten Finanzmittel von EU, Bund und dem Freistaat Bayern. Davon wurden allerdings mit 25,1 Mio. € knapp 75\% der Gesamtmittel für die flächenbezogene Angebotsförderung ausgegeben (DRESSLER, 2005, vgl. VERZEICHNIS DER SONSTIGEN MitTEILUNGEN). In den anderen Bundesländern ist die Lage ähnlich. Für die flächenbezogene Förderung wurden im Förderjahr 2004 auf Bundesebene über 110 Mio. $€$ ausgegeben (vgl. Kapitel 2.3.4). Im Vergleich dazu beliefen sich die im Jahr 2004 im Rahmen des BÖL vergebenen Mittel lediglich auf 20 Mio. $€$. Für die Förderung der Verarbeitung und Vermarktung ökologisch erzeugter Produkte im Rahmen der gemeinschaftlich finanzierten Fördermaßnahmen wurden im Zeitraum 1993 bis 2004 sogar nur 18 Mio. $€$ aufgewendet (BMELV, 2006a). Wegen ihrer großen finanziellen Bedeutung und weil sich diese Arbeit in der Hauptsache mit der Untersuchung der Angebotsförderung zugunsten des ökologischen Landbaus beschäftigen soll, wird im folgenden Kapitel vertiefend auf die Entwicklung, die Ausgestaltung und die Finanzierung der flächenbezogenen Förderung eingegangen. 


\subsection{DIE FLÄCHENBEZOGENE ANGEBOTSFÖRDERUNG ALS SCHWERPUNKT DER DERZEITIGEN FÖRDERPOLITIK}

Neben den Direktzahlungen für landwirtschaftliche Betriebe, unabhängig von der ökologischen oder konventionellen Wirtschaftsweise, besteht für ökologisch wirtschaftende Betriebe seit 1989 die Möglichkeit, zusätzliche staatliche Zuwendungen speziell für die ökologische Wirtschaftsweise im Rahmen der Agrarumweltprogramme der Bundesländer zu erhalten. Bevor im Folgenden die Rahmenbedingungen, die länderspezifischen Ausgestaltungen und die Finanzierung dieser Fördermaßnahme dargestellt werden, wird zunächst auf die Entwicklung dieser Zahlungen und auf deren rechtliche Basis im Zeitablauf eingegangen.

\subsubsection{ENTWICKLUNG DER FLÄCHENBEZOGENEN FÖRDERUNG}

Die Entwicklung der flächenbezogenen Förderung lässt sich in drei Etappen skizzieren:

- $\quad$ EG-Extensivierungsverordnung (EWG) 4115/88

Grundlage der flächenbezogenen Förderung des ökologischen Landbaus in Deutschland war bis 1992 die EG-Extensivierungsverordnung VO (EWG) 4115/88. Als Voraussetzung für eine Förderung im Rahmen dieser Verordnung durften seinerzeit im gesamten Betrieb keine synthetischen Dünge- und Pflanzenschutzmittel ausgebracht werden. Weiterhin musste die Tierhaltung den Anforderungen ökologischer Wirtschaftsweisen entsprechen. Anlass dieses ersten Förderprogramms zugunsten des ökologischen Landbaus waren weniger umweltschutzorientierte Beweggründe als vielmehr die in den achtziger Jahren immer notwendiger werdende Marktentlastung im Bereich der Überschussprodukte (NIEBERG und STROHM-LÖMPKE, 2001: 413). Dabei wurde im Rahmen der Extensivierungsverordnung nur die Umstellung der Wirtschaftsweise innerhalb der ersten 5 Jahre bundesweit gefördert. Eine Förderung der Beibehaltung war in der damaligen Verordnung noch nicht vorgesehen. Die Höhe der Förderbeträge richtete sich nach der Nutzungsart der Fläche. So wurden Dauerkulturflächen bundesweit mit 1.416 DM je Hektar gefördert. Bei Ackerflächen wurde abermals unterschieden nach Flächen, auf denen zuvor Überschussprodukte wie Getreide, Ölsaaten, Körnerleguminosen oder Tabak angebaut worden waren und nach sonstigen Ackerflächen. Erstere wurden mit Flächenzahlungen von 425 bis 510 DM je Hektar gefördert, während die übrigen Ackerflächen wie auch die Grünlandflächen mit 300 bis $360 \mathrm{DM}$ je Hektar gefördert wurden (NIEBERG und STROHM-LÖMPKe, 2001: 413). An der Finanzierung 
beteiligte sich die EU mit $25 \%$. Der Bund richtete einen Sonderrahmenplan innerhalb der GAK ein und beteiligte sich darüber mit $70 \%$ am verbleibenden nationalen Anteil (Osterburg und Stratmann, 2002, 261).

- Verordnung (EWG) 2078/92 für umweltgerechte und den natürlichen Lebensraum schützende landwirtschaftliche Produktionsverfahren

Im Zuge der McSharry-Reform von 1992 wurden Agrarumweltprogramme als flankierende Maßnahme eingeführt. Grundlage dieser Agrarumweltprogramme war die Verordnung (EWG) 2078/92 zur Förderung umweltgerechter und den natürlichen Lebensraum schützender landwirtschaftlicher Produktionsverfahren. Sie ersetzte damit die vormals geltende EGExtensivierungsverordnung und sorgte neben einer Vielzahl anderer Extensivierungsvarianten erstmals für eine europaweite Förderung des ökologischen Landbaus. In Deutschland wird seitdem in den meisten Bundesländern im Rahmen der Agrarumweltprogramme neben der Einführung auch die Beibehaltung des ökologischen Landbaus finanziell honoriert. Im Zuge der Umstellung durch die Agrarreform 1992 wurden die Prämien zur Förderung des ökologischen Landbaus jedoch deutlich abgesenkt (NIEBERG und STROHM-LöMPKE, 2001: 413).

- Verordnung (EG) 1257/99 zur Förderung der Entwicklung des ländlichen Raumes Seit dem Jahr 2000 wird die flächenbezogene Förderung des ökologischen Landbaus von den Bundesländern im Rahmen der Agrarumweltprogramme der Verordnung (EG) 1257/99 zur Förderung der Entwicklung des ländlichen Raumes angeboten. Diese Umstellung hat in der Anfangsphase in den Bundesländern sowohl in Bezug auf die Fördervoraussetzungen als auch in Bezug auf die Förderhöhen zu keinen tief greifenden Änderungen geführt (NIEBERG und STROHM-LÖMPKE, 2001: 413). Erst mit der Anhebung der Prämiensätze im Rahmenplan der GAK im Jahr 2002 ist es auch zu Steigerungen bei den Förderhöhen in den Bundesländern gekommen. Bereits seit 2000 wird in vielen Bundesländern zusätzlich ein Kontrollkostenzuschuss gewährt.

Für den Förderzeitraum ab 2007 müssen von den Bundesländern neue Programme zur ländlichen Entwicklung auf Grundlage der dann gültigen Verordnung (EG) 1698/2005 über die Förderung der Entwicklung des ländlichen Raums durch den europäischen Landwirtschaftsfond zur Entwicklung des ländlichen Raums (ELER) verfasst werden. 


\subsubsection{RECHTLICHE RAHMENBEDINGUNGEN FÜR DIE BUNDESLÄNDER BEI DER Ausgestaltung DER FLÄCHENBEZOGENEN FÖRDERUNG}

Im vorliegenden Kapitel sollen zuerst die derzeit geltenden Regelungen für Agrarumweltprogramme auf EU-Ebene dargestellt werden. Anschließend wird auf die Regelungen im GAK-Rahmenplan bezüglich der MSL-Maßnahmen eingegangen, in deren Rahmen die flächenbezogene Förderung des ökologischen Landbaus in den meisten Bundesländern angeboten wird.

\subsubsection{RAHMENBEDINGUNGEN AUF EU-EBENE}

Die Artikel 22 bis 24 der Verordnung (EG) $1257 / 99$ beziehen sich auf die Agrarumweltförderung innerhalb der Maßnahmen zur Entwicklung des ländlichen Raumes. Konkretisiert werden diese Rahmenbedingungen durch die zugehörige Durchführungsverordnung (EG) 817/2004 der EU-Kommission. Die für die flächenbezogene Förderung des ökologischen Landbaus bedeutsamsten Regelungen sollen im Folgenden kurz angesprochen werden.

Als vorrangige Ziele der Agrarumweltmaßnahmen werden der Schutz der Natur und die Erhaltung des ländlichen Lebensraumes genannt. Zwingende Voraussetzung ist generell eine mindestens fünfjährige Verpflichtungsperiode. Die Verpflichtungen bezüglich der Agrarumweltmaßnahmen sollen insgesamt ausdrücklich über die Anwendung der guten landwirtschaftlichen Praxis hinausgehen. Bei der Gestaltung der Prämiensätze soll neben den Einkommensverlusten und den zusätzlichen Kosten, die infolge der eingegangenen Verpflichtung entstehen, auch eine Anreizkomponente für den Landwirt berücksichtigt werden. Nach VO (EG) 817/2004 darf dabei der Anreiz einen Anteil von $20 \%$ der Summe aus den Einkommensverlusten und den zusätzlichen Kosten nicht übersteigen; es sei denn, bei einzelnen Verpflichtungen wird ein höherer Anreiz als unerlässlich erachtet. Im Anhang der VO (EG) 1257/99 sind die bei einer Gemeinschaftsfinanzierung in Betracht kommenden Höchstgrenzen der Beihilfen aufgeführt. Sie sind in Tabelle 1 dargestellt: 
Tabelle 1: Höchstgrenzen für eine flächenbezogene Förderung im Rahmen der Agrarumweltprogramme nach VO (EG) 1257/99

\begin{tabular}{|c|c|}
\hline Flächennutzung & Förderhöchstbeträge \\
\hline Einjährige Kulturen & $600 €$ \\
\hline Mehrjährige Sonderkulturen & $900 €$ \\
\hline Sonstige Flächennutzung & $450 €$ \\
\hline
\end{tabular}

Quelle: VO (EG) 1257/99

Auch eine Kombination der flächenbezogenen Öko-Förderung mit anderen Agrarumweltmaßnahmen ist laut Durchführungsverordnung möglich. Allerdings sollten dazu die einzelnen Maßnahmen einander ergänzen und sich in ihrer Wirkung nicht entgegenstehen. Bei der Bemessung der Förderhöhen soll die Summe von Einkommensverlusten und zusätzlichen Kosten der kombinierten Verfahren für die Bundesländer maßgeblich sein.

\subsubsection{RAHMENBEDINGUNGEN AUF BUNDESEBENE}

Die Grundsätze für die Förderung einer markt- und standortangepassten Landbewirtschaftung (MSL) sind das zentrale Instrument der Agrarumweltförderung des Bundes im Rahmen der GAK. Im GAK-Rahmenplan 2006 werden den Bundesländern unter dem Förderungsgrundsatz MSL die folgenden fünf Fördermaßnahmen vorgeschlagen, innerhalb derer eine Kofinanzierung der Agrarumweltmaßnahmen möglich ist:

- Förderung extensiver Produktionsverfahren im Ackerbau oder bei Dauerkulturen

- Förderung extensiver Grünlandnutzung

- Förderung ökologischer Anbauverfahren

- Förderung mehrjähriger Stilllegung

- Förderung umwelt- und tiergerechter Haltungsverfahren

Unter der Fördermaßnahme $\mathrm{C}$ sind folglich die Rahmenbedingungen für eine Bundesbeteiligung an flächenbezogenen Fördermaßnahmen zugunsten des ökologischen Landbaus aufgeführt. Dabei richtet sich der Bund einerseits nach den Rahmenbedingungen für Agrarumweltprogramme auf EU-Ebene. Daneben werden aber auch weitere Beihilfebestimmungen speziell für die Förderung des ökologischen Landbaus benannt. Die folgenden Ausführungen zu den zusätzlichen Bestimmungen orientieren sich am GAKRahmenplan 2006 (vgl. BMELV, 2006b). 
Zwingende Voraussetzung für eine Förderung der Einführung oder der Beibehaltung eines ökologischen Anbauverfahrens im Rahmen der GAK ist die gesamtbetriebliche Umstellung. Unter der Anwendung eines ökologischen Anbauverfahrens wird dabei die Einhaltung der EG-Öko-Verordnung und des europäischen Folgerechts verstanden. Weitere Fördervoraussetzungen sind ein generelles Verbot des Grünlandumbruchs außer in speziell im Rahmenplan genannten Ausnahmefällen sowie die Verpflichtung, für mindestens $3 \%$ der landwirtschaftlichen Fläche ein weiteres Agrarumweltverfahren einzuführen, dessen Anforderungen allerdings die der EG-Öko-Verordnung noch übersteigen. Hinsichtlich der Prämienbemessung gibt es für die Bundesländer die Möglichkeit zur Flexibilisierung der Prämienstaffelung in der Einführungsphase. Danach können in den ersten beiden Jahren der Umstellung höhere Prämien gewährt werden, sofern danach direkt eine Absenkung auf das Beibehaltungsniveau erfolgt. Durch diese Option wird man der Problematik gerecht, dass Umstellungsbetriebe in den ersten Jahren der Umstellung ihre Produkte in der Regel weiterhin nur als konventionelle Produkte vermarkten können und daher an den Preisaufschlägen für ökologisch erzeugte Produkte nicht partizipieren können. Trotz der Anhebung der Prämiensätze im Jahr 2002 liegen die neuen Prämienvorgaben des Rahmenplanes immer noch deutlich unterhalb der Obergrenzen der VO (EG) 1257/99 auf Ebene der EU. Die aktuellen GAK-Vorgaben für die Fördersätze sind in Tabelle 2 aufgeführt. Den Ländern ist es bei der Festlegung der Prämiensätze jedoch ausdrücklich erlaubt, die GAK-Vorgaben um maximal $20 \%$ zu überschreiten bzw. um maximal $30 \%$ zu unterschreiten. Zusätzlich zu den Flächenprämien gibt der GAK-Rahmenplan den Ländern die Möglichkeit, ökologisch wirtschaftenden Betrieben einen Kontrollkostenzuschuss zu gewähren.

\section{Tabelle 2: Vorgaben des GAK-Rahmenplanes 2006 zur Bemessung der Förderhöhen auf} Länderebene

\begin{tabular}{|c|c|c|c|c|}
\hline \multirow{3}{*}{ Nutzungsart } & \multicolumn{3}{|c|}{ Prämienhöhe } & \multirow{3}{*}{$\begin{array}{c}\text { Kontrollkosten- } \\
\text { zuschuss }\end{array}$} \\
\hline & \multicolumn{2}{|c|}{$\begin{array}{l}\text { Einführung } \\
€ / \text { ha und Jahr }\end{array}$} & \multirow{2}{*}{$\begin{array}{c}\text { Beibehaltung } \\
€ / \text { ha und Jahr } \\
\text { ab 6.Jahr }\end{array}$} & \\
\hline & 1.-2.Jahr & 3.-5.Jahr & & \\
\hline \multirow{4}{*}{$\begin{array}{l}\text { Acker- und Grünland } \\
\text { Gemüsebau } \\
\text { Dauer- oder } \\
\text { Baumschulkulturen }\end{array}$} & $285^{\star}$ & 210 & 160 & \multirow{4}{*}{$\begin{array}{c}35 € \text { je ha, } \\
\text { maximal } 530 € \text { je } \\
\text { Unternehmen }\end{array}$} \\
\hline & $750^{*}$ & 480 & 300 & \\
\hline & & & & \\
\hline & $1220^{*}$ & 950 & 770 & \\
\hline
\end{tabular}

*Wird die Förderung in den ersten beiden Jahren auf diesen erhöhten Satz angehoben, so soll schon ab dem 3. Jahr die Beibehaltungsförderung gelten

Quelle: Eigene Aufstellung nach GAK Rahmenplan 2006 (BMELV, 2006b) 
Ende April 2006 erfolgte der PLANAK-Beschluss für den GAK-Rahmenplan 2007. Darin sind die in Tabelle 2 angegebenen Fördersätze um durchschnittlich $10 \%$ reduziert worden. Die Beibehaltungsprämie für Acker- und Grünland wurde sogar um knapp 15 \% gesenkt.

\subsubsection{AUSGESTALTUNG DER FLÄCHENBEZOGENEN FÖRDERUNG IN DEN BUNDESLÄNDERN}

Die folgenden Betrachtungen zur Ausgestaltung der flächenbezogenen Förderung auf Ebene der einzelnen Bundesländer basieren auf einer Übersicht von NIEBERG (2006), die in Anhang 1 dieser Arbeit einzusehen ist. Darin sind sowohl die Fördersätze zu den einzelnen Flächennutzungsarten als auch die Fördervoraussetzungen der Bundesländer aufgeführt. ${ }^{1}$

Aus der Übersicht von NiEBERG (2006) wird ersichtlich, dass sich die Angebote der einzelnen Bundesländer zur Förderung ökologischer Anbauverfahren über Flächenprämien insbesondere in Hinblick auf die Förderhöhen unterscheiden. Dieser Sachverhalt beruht zum einen auf den Handlungsspielräumen, die die Rahmenrichtlinien auf EU- und auf Bundesebene bieten und zum anderen auf der Tatsache, dass einige Bundesländer nach wie vor auf die Finanzierungsbeteiligung des Bundes im Rahmen der GAK verzichten.

Daneben bestehen, wie aus der Übersicht von NIEBERG (2006) in Anhang 1 ersichtlich, auch Unterschiede zwischen den Bundesländern in der Differenzierung der Prämienhöhe innerhalb der Einführungsphase bzw. zwischen Einführungsphase und Beibehaltungsphase. So bieten die Bundesländer Baden-Württemberg, Bayern und Hessen lediglich einen konstanten Fördersatz für die Einführungs- und Beibehaltungsphase an. Alle anderen Bundesländer bis auf Nordrhein-Westfalen variieren ihre Prämienhöhen entweder innerhalb der Einführungsphase oder zwischen Einführungs- und Beibehaltungsphase. NordrheinWestfalen und Rheinland-Pfalz bieten sogar drei verschiedene Prämiensätze an. Mit fortschreitender Laufzeit fallen in fast allen Bundesländern die Prämien vom höheren Einführungsniveau auf das niedrigere Beibehaltungsniveau ab.

Noch vor 4 Jahren war in Bundesländern wie Bayern und Rheinland-Pfalz die absolute Höhe der jährlichen Zahlungen pro Unternehmen durch einen Förderhöchstbetrag begrenzt. Dadurch wurden verstärkt kleinere und mittlere Betriebe gefördert. In den aktuellen Ausgestaltungen sind diese Beschränkungen ersatzlos gestrichen worden, so dass nun auch

\footnotetext{
${ }^{1}$ Zur detaillierten Ausgestaltung der Agrarumwelt- und Naturschutzprogramme in den einzelnen Bundesländern vgl. HARTMANN et al., 2006.
} 
größere Betriebe für ihre gesamte ökologisch bewirtschaftete Fläche den vollen Förderbetrag erhalten. Damit verbleibt Baden-Württemberg als letztes Bundesland mit einem Förderhöchstbetrag.

Mit Ausnahme der Bundesländer Berlin, Rheinland-Pfalz, Sachsen und Thüringen wird überall ein Kontrollkostenzuschuss angeboten. Dabei halten sich die meisten Bundesländer an die Vorgaben der GAK. Einzig Nordrhein-Westfalen bietet einen weit überdurchschnittlich hohen Zuschuss an.

Auch die Fördervoraussetzungen sind auf Länderebene unterschiedlich. Dies beruht auf der Freiheit der Bundesländer neben den Fördervoraussetzungen von EU und Bund den Landwirten noch weitere Verpflichtungen aufzuerlegen. Neben den in der Übersicht in Anhang 1 aufgeführten landesspezifischen Voraussetzungen ist der Nachweis über eine Kontrolle gemäß VO (EWG) 2092/91 in allen Bundesländern zwingende Voraussetzung zum Erhalt der Beihilfen. Ohne diese Voraussetzung würde die Kontrolle der Förderwürdigkeit für die Bundesländer erschwert. Auch in den Bundesländern, die ihre Fördermaßnahmen außerhalb der GAK anbieten, ist eine Gesamtbetriebsumstellung als Bedingung für den Erhalt von Fördergeldern zwingend vorgeschrieben.

Konsequenz der unterschiedlichen Förderbestimmungen auf Ebene der Bundesländer sind unterschiedliche Wettbewerbsbedingungen innerhalb des Bundesgebietes. Ein Problem, das durch die Existenz der GAK gemildert werden soll. Jedoch erscheint es gerade zum jetzigen Zeitpunkt äußerst fraglich, ob in Zukunft von den Bundesländern überhaupt noch flächendeckend in Deutschland eine Angebotsförderung des ökologischen Landbaus angeboten werden kann. Dazu soll im folgenden Kapitel zur Finanzierung der flächenbezogenen Förderung Stellung bezogen werden.

\subsubsection{DERZEITIGE UND ZUKÜNFTIGE FINANZIERUNG DER FLÄCHENBEZOGENEN FÖRDERUNG}

Die Finanzierung der gegenwärtigen flächenbezogenen Förderung erfolgt entweder als Gemeinschaftsfinanzierung von EU, Bund und Land oder ohne den Bund, sofern die Fördermaßnahmen außerhalb der GAK angeboten werden. Abbildung 2 zeigt die gegenwärtig geltende Finanzierungskonstellation. 


\section{Abbildung 2: Finanzierungskonstellation bei der Finanzierung der Öko-Flächenprämien}

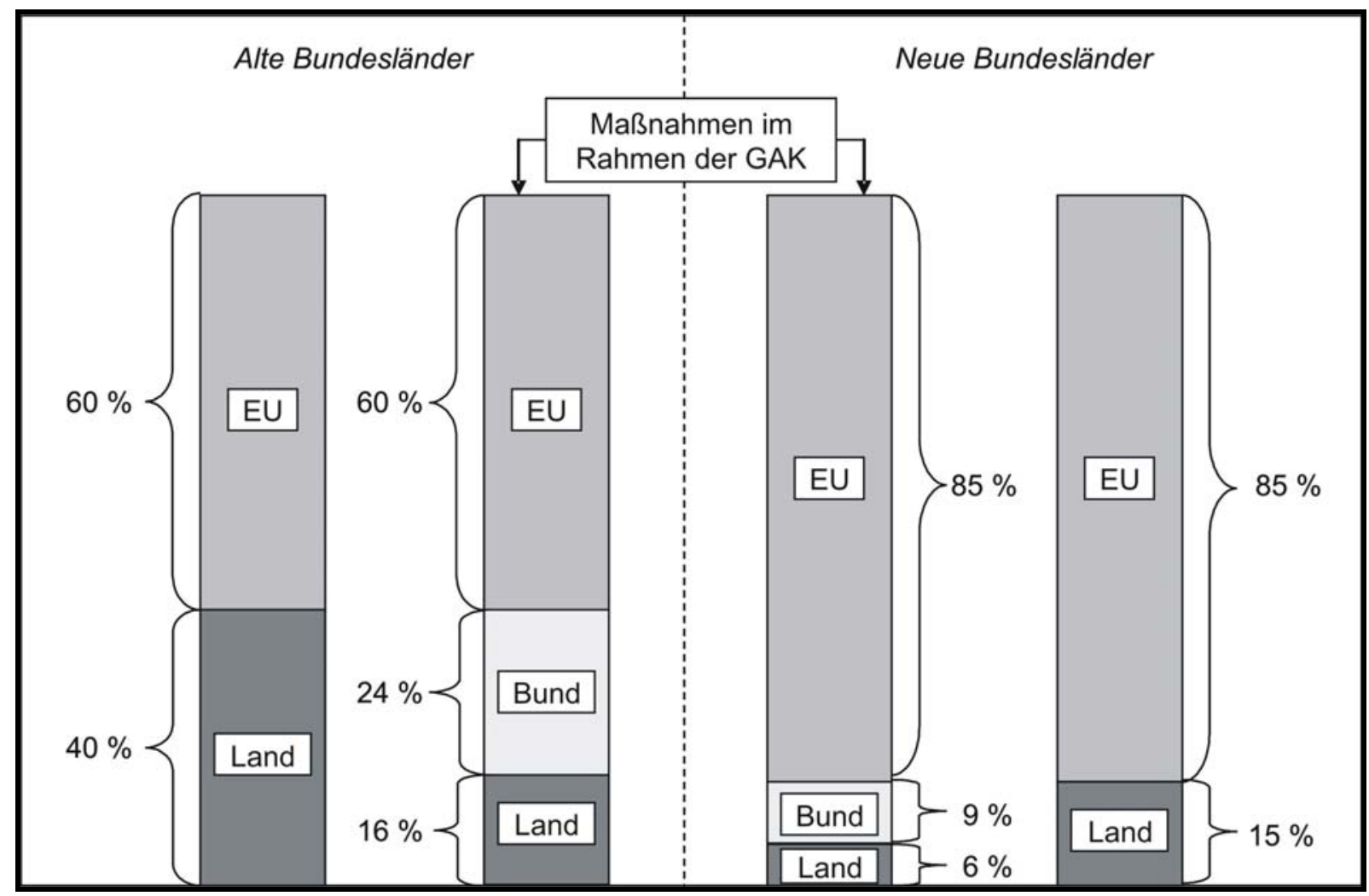

Quelle: Eigene Darstellung in Anlehnung an NIEBERG und StroHM-LÖMPKE (2001: 412)

Danach beträgt der maximale Kofinanzierungsanteil der EU in den alten Bundesländern derzeit $60 \%$. In den neuen Bundesländern übernimmt die EU sogar bis zu $85 \%$ der verausgabten Mittel. Der Grund dafür liegt in der Einstufung der neuen Bundesländer als Gebiete mit Entwicklungsrückstand (Ziel-1-Gebiete). Werden die Maßnahmen im Rahmen der GAK angeboten, so übernimmt der Bund weitere $60 \%$ vom verbleibenden nationalen Anteil.

Die folgende Tabelle 3 gibt eine Übersicht über den Einsatz von Fördergeldern in den Jahren 2001 bis 2004 sowohl auf Bundes- als auch auf Landesebene. Parallel dazu wird auch die Entwicklung der ökologisch bewirtschafteten Fläche (nach VO (EWG) 2092/91) auf Länderund Bundesebene dargestellt. Dabei zeigen sich höchst unterschiedliche Entwicklungen sowohl in Bezug auf die einzelnen Länder untereinander als auch in Bezug auf das Verhältnis der ökologisch bewirtschafteten Fläche und des Einsatzes von Fördermitteln zueinander. Bei den angeführten monetären Daten handelt es sich jeweils um die kumulierten Fördermittel von EU, Bund und Bundesländern. Die letzte Tabellenzeile zeigt den durchschnittlichen hektarbezogenen Förderbetrag in Deutschland. 
Tabelle 3: Übersicht über die Öko-Fläche und den Einsatz von Fördermitteln auf Landesebene in den Jahren 2001 bis 2004

\begin{tabular}{|c|c|c|c|c|c|c|c|c|c|c|}
\hline \multirow[b]{2}{*}{ Bundesland } & \multicolumn{2}{|c|}{2001} & \multicolumn{2}{|c|}{2002} & \multicolumn{2}{|c|}{2003} & \multicolumn{2}{|c|}{2004} & \multicolumn{2}{|c|}{$\begin{array}{l}\text { Veränderungen } 2001 \text { bis } \\
2004 \text { in } \%\end{array}$} \\
\hline & $\begin{array}{l}\text { Öko-Fläche } \\
\text { nach VO } \\
2092 / 91 \text { (ha) }\end{array}$ & $\begin{array}{c}\text { Finanzmittel } \\
\text { (in Mio. } € \text { ) }\end{array}$ & $\begin{array}{l}\text { Öko-Fläche } \\
\text { nach VO } \\
\text { 2092/91 (ha) }\end{array}$ & $\begin{array}{c}\text { Finanzmittel } \\
\text { (in Mio. €) }\end{array}$ & $\begin{array}{l}\text { Öko-Fläche } \\
\text { nach VO } \\
\text { 2092/91 (ha) }\end{array}$ & $\begin{array}{c}\text { Finanzmittel } \\
\text { (in Mio. } € \text { ) }\end{array}$ & $\begin{array}{l}\text { Öko-Fläche } \\
\text { nach VO } \\
\text { 2092/91 (ha) }{ }^{1}\end{array}$ & $\begin{array}{l}\text { Finanzmittel } \\
\text { (in Mio. } € \text { ) }\end{array}$ & $\begin{array}{l}\text { Öko-Fläche } \\
\text { nach VO } \\
\text { 2092/91 (ha) }\end{array}$ & $\begin{array}{l}\text { Finanz- } \\
\text { mittel }\end{array}$ \\
\hline BW & 77.380 & 9,92 & 82.418 & 10,50 & 85.825 & 11,20 & 86.416 & 11,10 & $+12 \%$ & $+12 \%$ \\
\hline BY & 106.614 & 21,30 & 116.163 & 25,10 & 127.151 & 26,70 & 132.044 & 28,90 & $+24 \%$ & $+36 \%$ \\
\hline BB & 104.539 & 9,87 & 111.170 & 11,87 & 119.270 & 12,88 & 129.745 & 13,96 & $+24 \%$ & $+41 \%$ \\
\hline $\mathrm{HE}$ & 55.616 & 8,41 & 58.014 & $8,30^{\star}$ & 56.578 & $8,32^{* *}$ & 55.971 & 6,41 & $+1 \%$ & $-24 \%$ \\
\hline MV & 97.226 & $3,89^{\star \star \star \star}$ & 103.803 & $4,73^{\star * *}$ & 107.412 & 7,74 & 105.532 & 11,65 & $+9 \%$ & $+199 \%$ \\
\hline NI & 43.175 & 4,86 & 51.989 & 5,93 & 55.959 & 9,81 & 61.172 & 8,63 & $+42 \%$ & $+78 \%$ \\
\hline NW & 38.656 & 3,87 & 45.039 & 8,45 & 47.579 & 10,67 & 51.084 & 10,34 & $+32 \%$ & $+167 \%$ \\
\hline RP & 15.136 & 2,05 & 16.978 & 2,57 & 18.604 & 2,80 & 18.957 & 2,71 & $+25 \%$ & $+32 \%$ \\
\hline SL & 2.738 & 0,45 & 3.465 & 0,60 & 4.691 & 0,81 & 5.006 & 0,96 & $+83 \%$ & $+112 \%$ \\
\hline SN & 17.441 & 2,22 & 19.062 & 3,17 & 20.342 & 3,30 & 22.548 & 3,76 & $+29 \%$ & $+69 \%$ \\
\hline ST & 28.318 & 2,48 & 32.997 & 3,14 & 34.855 & 3,82 & 40.825 & 5,23 & $+44 \%$ & $+111 \%$ \\
\hline SH & 19.949 & 1,62 & 22.877 & 1,77 & 27.765 & 3,23 & 29.915 & 3,70 & $+50 \%$ & $+129 \%$ \\
\hline $\mathrm{TH}$ & 27.130 & 2,14 & 31.590 & 2,73 & $26.659^{\star * * *}$ & 3,10 & $27.479^{* \star * \star}$ & 3,26 & $+1 \%$ & $+52 \%$ \\
\hline Bund $^{2}$ & 633.917 & 73,07 & 695.565 & 88,86 & 732.690 & 104,38 & 766.694 & 110,61 & $+21 \%$ & $+51 \%$ \\
\hline \begin{tabular}{|l|} 
Durchschnittl. \\
Förderbetrag \\
pro ha in $€:^{2}$
\end{tabular} & \multicolumn{2}{|c|}{115,27} & \multicolumn{2}{|c|}{127,75} & \multicolumn{2}{|c|}{142,46} & \multicolumn{2}{|c|}{144,26} & \multicolumn{2}{|c|}{$\begin{array}{c}\text { Veränderung des } \\
\text { durchschnittlichen Förder } \\
\text { betrags pro ha: }{ }^{2}+25 \%\end{array}$} \\
\hline
\end{tabular}

${ }^{1}$ Flächenmeldungen der Kontrollstellen jeweils zum 31.12. eines Jahres

${ }^{2}$ ohne Berücksichtigung der Stadtstaaten

* $80 \%$ des bewilligten Volumens wurden ausgeschüttet

* $120 \%$ des bewilligten Volumens wurden ausgeschüttet

*** geänderter Verpflichtungszeitraums; deshalb nur bedingte Vergleichbarkeit der Zahlen zu den verschiedenen Jahre

**** geänderte Flächenerfassung; deshalb nur bedingte Vergleichbarkeit der Zahlen zu den verschiedenen Jahre

Quelle: Eigene Darstellung nach den Ergebnissen einer Umfrage unter den zuständigen Länderministerien (vgl. VERZEICHNIS DER SONSTIGEN MITTEILUNGEN) 
Wie aus Tabelle 3 ersichtlich, hat sich der Fördermitteleinsatz auf Bundesebene im Zeitraum 2001 bis 2004 um 51 \% auf über 110 Mio. € erhöht. Im Vergleich zu dieser Entwicklung hat sich der Anteil der ökologisch bewirtschafteten Fläche an der gesamten landwirtschaftlich genutzten Fläche in Deutschland im gleichen Zeitraum nur um 21 \% erhöht. Geht man von einem konstanten ökologischen Grenznutzen der Umstellungsflächen aus, so können aus diesem Zusammenhang Rückschlüsse auf eine Ineffizienz der staatlichen Förderpolitik gezogen werden, denn die Budgetbelastung infolge jedes neu hinzugekommenen Hektars ökologisch bewirtschafteter Fläche ist im Zeitverlauf kontinuierlich angestiegen ${ }^{2}$. Dadurch hat sich auch der durchschnittliche Förderbetrag pro ha ökologisch bewirtschafteter Fläche im gleichen Zeitraum von etwa $115 €$ auf etwa $144 €$ erhöht. Ein wesentlicher Grund für die Verteuerung der Flächenförderung dürfte in der Anhebung der Fördersätze in vielen Bundesländern als Reaktion auf die Anhebung der GAK-Fördersätze im Rahmenplan 2002 liegen. Dadurch beinhalten die neu hinzukommenden Förderverpflichtungen jeweils höhere Fördersätze als die im Gegenzug auslaufenden Förderverpflichtungen. Als Konsequenz ergibt sich die angesprochene Erhöhung des Durchschnittsförderbetrags, die ein beträchtliches Potential an Mitnahmeeffekten erahnen lässt.

Die aufgezeigte historische Entwicklung der Förderung führt zum einen zwar zu der begründeten Annahme, dass die Anhebung der Förderung seit 2001 erheblich dazu beigetragen hat, den Anteil der Öko-Fläche an der gesamten landwirtschaftlich genutzten Fläche zu erhöhen. Daneben zeigen sich jedoch auch die in Relation zum Flächenwachstum hohen Kosten der Förderung. Deshalb ist davon auszugehen, dass ein weiteres Wachstum der Öko-Fläche mit weiteren erheblichen Budgeterhöhungen verbunden wäre. Vor einigen Jahren war das wenig problematisch. Einerseits war der Anteil der ökologisch bewirtschafteten Fläche und damit auch zwangsläufig das Gesamtvolumen der Förderung noch gering. Andererseits standen aber auch ausreichend finanzielle Mittel zur Verfügung. Es konnten alle umstellungswilligen Landwirte gefördert und damit eine Ausweitung des Ökolandbaus forciert werden. Gegenwärtig zeichnen sich jedoch die Grenzen dieses Fördersystems ab.

2 Der überproportionale Anstieg ist nicht auf eine überproportionale Zunahme an höher geförderten Sonderkulturflächen zurückzuführen. Im Gegenteil - der Anteil der Sonderkulturen an der gesamten ökologisch bewirtschafteten Fläche weist einen fallenden Trend auf. Demgegenüber wurde der Grün- und Ackerlandanteil, also der Anteil der weniger förderintensiven Flächen, bundesweit gesteigert (ZMP, 2004 sowie mündliche Bestätigung durch KASBOHM, ZMP, JULI 2004.). Auch der Anteil der geförderten ÖkoFlächen an den gesamten Öko-Flächen gemäß VO (EWG) 2092/91 ist stabil geblieben, so dass daraus keine relative Veränderung im Verhältnis von Förderfläche und Fördersumme resultiert. 
So wurden in 2004 erstmals Förderanträge von Betriebsleitern, die auf ökologischen Landbau umstellen wollten, in den Bundesländern Baden-Württemberg und MecklenburgVorpommern nicht mehr bewilligt (vgl. z.B. BIOLAND, 2004a). Auch Neuflächen bereits umgestellter Betriebe waren von diesem Förder-Stopp betroffen. Gemäß den zuständigen Landesregierungen standen dafür nicht mehr ausreichend Haushaltsmittel zur Verfügung. Die Zahl der Bundesländer mit derartigen Vorgehensweisen steigerte sich in 2005 mit BadenWürttemberg, Brandenburg, Hessen, Sachsen und Schleswig-Holstein sogar auf fünf (vgl. dazu Bioland, 2005: 3). Dem übergeordneten Ziel dieser Förderung, die ökologisch bewirtschaftete Fläche in Deutschland stärker auszuweiten, ist diese Vorgehensweise natürlich abträglich. Die Situation wird sich mit hoher Wahrscheinlichkeit in den kommenden Jahren noch deutlich verschärfen. Denn die im Dezember 2005 von den Regierungschefs der EU-Staaten ausgehandelten Brüsseler Finanzbeschlüsse für den kommenden EUFinanzplanungszeitraum 2007 bis 2013 bedeuten erhebliche Einschnitte für die Finanzierung der Agrarumweltmaßnahmen, und damit voraussichtlich auch für die Förderung der ökologischen Bewirtschaftungsweise (vgl. AGRA-EurOPE, 2005: 9). So werden für die zweite Säule der GAP in Zukunft nur noch 69,3 Mrd. € zur Verfügung stehen, anstatt der im ursprünglichen Kommissionsvorschlag anvisierten $87 \mathrm{Mrd} € €$, die angesichts der großen zukünftigen Herausforderungen, vor denen die EU-Agrarpolitik insbesondere im Hinblick auf die EU-Erweiterung steht, bereits als viel zu gering kritisiert worden waren (vgl. REIMER, 2005). Hinzu kommt, dass aus der zweiten Säule in Zukunft mit Umsetzung von Natura 2000 und der Wasserrahmenrichtlinie noch weitere neue Aufgaben finanziert werden müssen. So ergibt sich nach ersten Schätzungen aus der Kürzung des EU-Finanzbudgets für Deutschland gegenüber dem Status Quo eine deutliche Reduzierung der für Ausgaben im Rahmen der 2. Säule zur Verfügung stehenden Finanzmittel um $47 \%$ in den alten Bundesländern sowie um $30 \%$ in den neuen Bundesländern (WEHDE, 2006: 46). Als Reaktion darauf haben für 2006 bereits zehn Bundesländer einen Förder-Stopp für Neubewilligungen beschlossen (vgl. NIEBERG, 2006). Davon haben einige Bundesländer zusätzlich tief greifende Veränderungen bei der Öko-Flächenprämie für die Zeit ab 2007 angekündigt (vgl. KEMPKENS, 2006: 26 oder KETELHODT, 2005: 7).

Die Verringerung des zur Verfügung stehenden EU-Finanzbudgets ist jedoch nicht der einzige Anlass, neue Zusatzbelastungen befürchten zu müssen. ${ }^{3}$ Zum einen sind die Länderhaushalte

\footnotetext{
${ }^{3} \mathrm{Zu}$ möglichen zukünftigen Problembereichen der Angebotsförderung des ökologischen Landbaus vgl. auch BAHRS und HELD, 2004: 50.
} 
bereits finanziell angeschlagen. Zum anderen werden die jüngsten Sparvorschläge der Bundesregierung, die u.a. auch die Mittel für die Gemeinschaftsaufgabe Agrarstruktur und Küstenschutz (GAK) betreffen, Auswirkungen auf das Budget der flächenbezogenen Förderung des Ökolandbaus haben. Für 2006 ist derzeit eine Reduktion der für die GAK zur Verfügung stehenden Finanzmittel um 70 Mio. $€$ von 720 Mio. $€$ auf nur noch 650 Mio. $€$ geplant (AgrA-Europe, 2006b: 2). Höhere Belastungen für den Bundes- sowie die Länderhaushalte werden sich auch durch die geplante Absenkung des EUKofinanzierungssatzes um 5 Prozentpunkte von $85 \%$ auf $80 \%$ in den neuen Bundesländern bzw. von $60 \%$ auf $55 \%$ im übrigen Bundesgebiet mit Inkrafttreten der ELER-VO in 2007 ergeben (vgl. Art. 70 der VO (EG) 1698/2005). Hinzu kommt, dass im Rahmen der Reform der Kohäsionspolitik für die nächste EU-Förderperiode 2007 bis 2013 Teile der neuen Bundesländer aus der Ziel-1-Förderung fallen werden, weil verglichen mit dem durchschnittlichen Bruttoinlandsprodukt in der EU-25 ihr Bruttoinlandsprodukt mehr als 75 \% beträgt (vgl. dazu z.B. EU-KOMMISSION, 2004 oder AGRA-Europe, 2006f: 9), wodurch eine Rechtfertigung für die Einstufung als Ziel-1-Gebiete nicht mehr gegeben ist. In Anbetracht der geplanten langsamen degressiven Absenkung des Kofinanzierungssatzes resultiert daraus eine zukünftig stärkere Belastung der Landeshaushalte durch die bereits bestehenden Verpflichtungen der Öko-Förderung.

\subsubsection{SCHLUSSFOLGERUNG}

In Kapitel 2.3.4 konnte gezeigt werden, dass die ökologisch bewirtschaftete Fläche bundesweit in den Jahren 2001 bis 2004 um knapp $21 \%$ ausgedehnt werden konnte. Dazu war jedoch ein Anstieg der verausgabten Fördermittel um etwa $51 \%$ auf über 110 Mio. $€$ notwendig. Unter Berücksichtigung des Anteils der ökologisch bewirtschafteten Fläche an der landwirtschaftlichen Nutzfläche Ende des Jahres 2001 von ca. 3,7 \% im Vergleich zum Jahr 2004 mit etwa 4,5\% ${ }^{4}$, werden die hohen finanziellen Aufwendungen bei der derzeitigen Förderpolitik erkennbar, um das gesetzte Ziel einer weiteren Ausdehnung der ökologisch bewirtschafteten Fläche zu erreichen (vgl. dazu auch die Ausführungen in Kapitel 2.4.1.1.3.)

Gleichzeitig zeigen aber die vorherigen Ausführungen zur gegenwärtigen Situation der Förderpolitik sowie zu den in Zukunft zu erwartenden Veränderungen die Begrenztheit der

\footnotetext{
4 Zum Jahresende 2005 belief sich der Anteil der ökologisch bewirtschafteten Fläche an der gesamten landwirtschaftlichen Nutzfläche in Deutschland auf 4,7 \% (AGRA-EUROPE, 2006h)
} 
zur Verfügung stehenden Fördermittel auf. Daher stellt sich grundsätzlich die Frage, wie die Förderung der ökologischen Landwirtschaft auf der Angebotsseite in Zukunft organisiert sein könnte. Als Handlungsmaxime muss gelten: Mit den in Zukunft möglicherweise begrenzter zur Verfügung stehenden Finanzmitteln sollten maximale Ergebnisse erzielt werden (vgl. dazu auch WeHDE, 2005: 4 oder AgRA-Europe, 2006c: 14). In Anbetracht des politischen Zieles einer weiteren Ausdehnung des ökologischen Landbaus und vor dem Hintergrund der dafür möglicherweise zukünftig begrenzteren verfügbaren finanziellen Mittel soll deshalb im folgenden Kapitel eine Evaluierung der flächenbezogenen Förderung durchgeführt werden, bei der neben der Effektivität der Förderung vor allem die Effizienz des Mitteleinsatzes untersucht werden soll.

\subsection{WiRKUNGEN UND PROBLEMBEREICHE DER ÖKO- FLÄCHENPRÄMIEN}

Außer für die Marktteilnehmer ist die spezifische Wirkungsweise einer Fördermaßnahme auch für den Förderer von größter Bedeutung. Förderer bei der flächenbezogenen Förderung des ökologischen Landbaus ist der Staat und damit jeder einzelne Steuerzahler. Neben der generellen Rechtfertigung der staatlichen Zahlungen (vgl. Kapitel 2.1) ist für den Steuerzahler auch die Effizienz des Mitteleinsatzes von außerordentlicher Bedeutung. Will man nämlich bei zukünftig begrenzteren Fördermitteln auch weiterhin eine Zunahme der ökologisch bewirtschafteten Fläche erreichen, so muss der Frage nachgegangen werden, ob mit der derzeitigen Ausgestaltung der flächenbezogenen Förderung der ökologische Landbau effizient gefördert wird oder ob durch Veränderungen der Förderpraxis die Fördermitteleffizienz noch gesteigert werden kann und dadurch mit den begrenzten Mitteln eine stärkere Ausdehnung der ökologisch bewirtschafteten Fläche erreicht werden kann. In Kapitel 2.4.2 soll deshalb auf die aus Sicht der Steuerzahler fundamental wichtige Frage nach der Effizienz des Finanzmitteleinsatzes eingegangen werden. Zuvor soll jedoch in Kapitel 2.4.1 die Wirkungsweise der Flächenprämien in Bezug auf das politisch anvisierte Ziel einer Ausweitung des ökologischen Landbaus näher untersucht werden.

\subsubsection{EFFEKTIVITÄT DER FÖRDERUNG}

„Effizienz heißt, die Dinge richtig tun. Effektivität heißt, die richtigen Dinge tun“ (DRUCKER, 1954). Werden aber zur Förderung des ökologischen Landbaus wirklich die richtigen Dinge getan? Im Mittelpunkt steht also die Fragestellung, ob mit der flächenbezogenen Förderung in 
seiner derzeitigen Ausgestaltung das richtige Förderinstrument zur Erreichung des gesetzten Zieles angewandt wird.

Politisches Ziel der flächenbezogenen Förderung ist eine Ausweitung der ökologisch bewirtschafteten landwirtschaftlichen Fläche. Über eine solche Ausdehnung sollen, wie in Kapitel 2.1 dargestellt, letztendlich die angestrebten gesellschaftlichen Ziele erreicht werden. Um die Effektivität des betrachteten Förderinstrumentes im Hinblick auf die Erreichung dieser Ziele beurteilen zu können, müsste der individuelle Grenznutzen der neu hinzugewonnenen Flächen bekannt sein. Der Einfachheit halber soll deshalb im Folgenden in erster Linie die Effektivität dieses Instrumentes im Hinblick auf eine Ausweitung des ökologischen Landbaus betrachtet werden.

\subsubsection{WIRKUNG DER FÖRDERUNG}

Bevor die Auswirkungen der flächenbezogenen Förderung anhand eines einfachen Marktmodells erläutert werden, soll dieses Förderinstrument zunächst nach seiner Ausgestaltung näher spezifiziert werden. KÖSTER (1992: 299ff) unterteilt Subventionszahlungen nach der Basis ihrer Zurechnung in Produktsubventionen, faktorgebundene Subventionen und personenbezogene Subventionen. Bei den ÖkoFlächenprämien handelt es sich nach dieser Einteilung eindeutig um faktorgebundene Subventionen, da sie pro Hektar landwirtschaftlicher Fläche gewährt werden. Der Einfachheit halber wird jedoch bei den folgenden Betrachtungen vorerst davon ausgegangen, es handele sich um eine Produktsubvention. Auf die differenziertere Wirkung einer faktorgebundenen Subvention wird direkt im Anschluss daran verwiesen.

In Abbildung 3 wird die Wirkung einer konstanten Produktsubvention in einem Marktmodell nach HENRichSMEIER und WiTZKe (1994: 178) dargestellt. Dabei handelt es sich um ein, verglichen mit der realen Situation auf dem Öko-Markt, sehr vereinfachtes Grundlagenmodell mit einem national abgegrenzten Markt ohne Außenhandel und mit der Annahme einer konstanten Nachfrage. Auf die spezifischen Besonderheiten des Öko-Marktes und deren Einflüsse auf die Wirkung der Förderung wird im Anschluss Bezug genommen. 
Abbildung 3: Wirkung einer Produktsubvention ohne Außenhandel

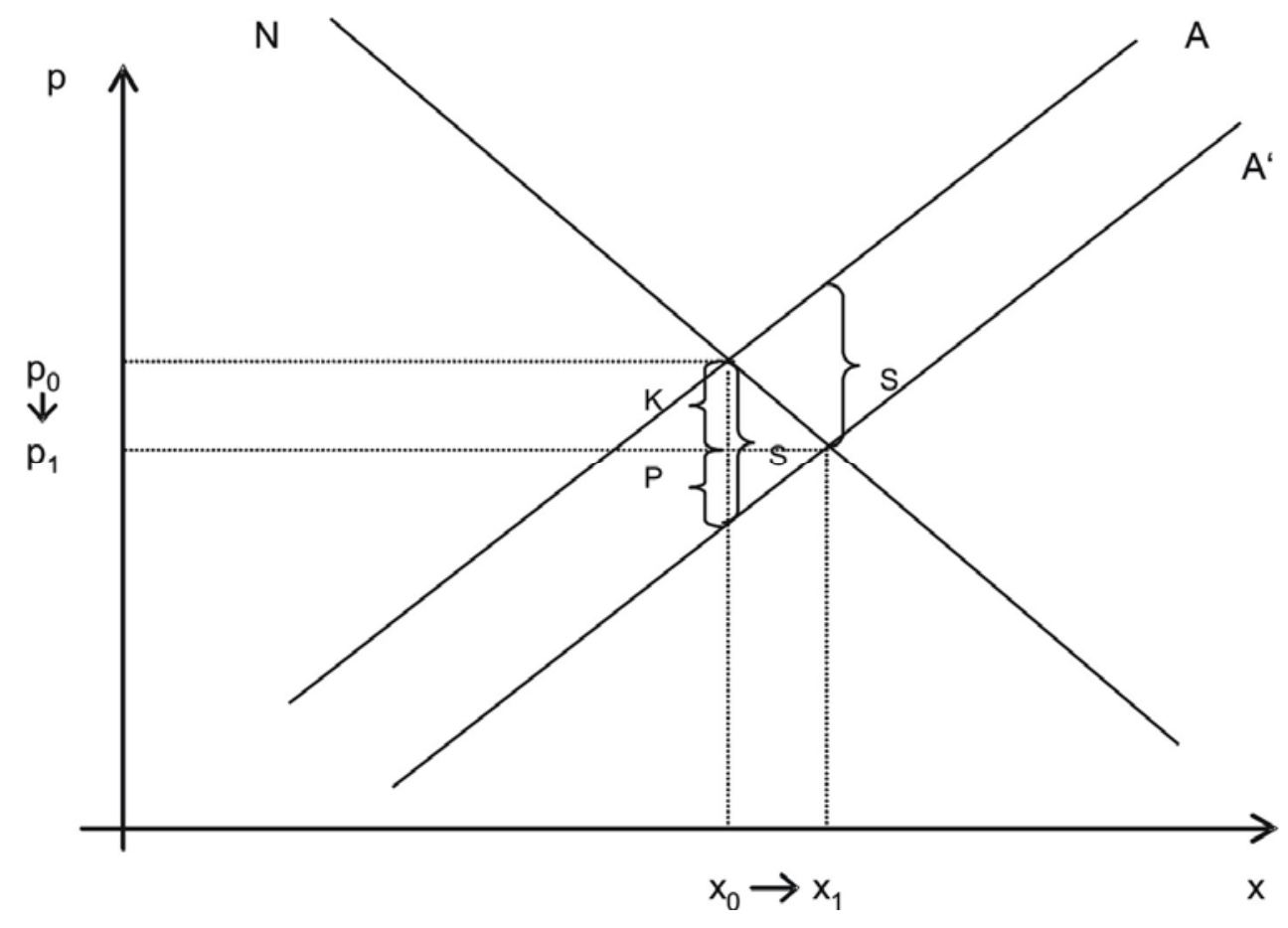

Quelle: Eigene Darstellung nach HENRICHSMEIER und WiTZKE (1994: 178)

Es wird unterstellt, dass ohne Subvention die Angebotskurve A und die Nachfragekurve N gelten. Als Folge der Subventionierung verschiebt sich die Angebotskurve um den Subventionsbetrag S je Mengeneinheit vertikal nach unten, so dass die Angebotskurve A' gilt. Der Grund für die Verschiebung liegt im veränderten Angebotsverhalten des Produzenten. Zur Maximierung seines Gewinns galt ursprünglich die Bedingung Grenzkosten = Marktpreis. Für den einzelnen Anbieter ist es jedoch unbedeutend, ob seine Grenzkosten über den Markt oder direkt durch die öffentliche Hand gedeckt werden. Seine Grenzkosten entsprechen folglich im Falle der Produktsubventionierung (Abbildung 3) genau der Summe aus Marktpreis und Stücksubvention (KÖSTER, 1992: 299ff).

Wie Abbildung 3 zeigt, hat sich durch die subventionsbedingte Verschiebung der Angebotskurve ein neues Marktgleichgewicht eingestellt. Dabei sind jedoch zwei gegenläufige Effekte zu beobachten:

(1) Die angebotene Menge steigt.

(2) Der Marktpreis sinkt.

Die Ausweitung der Ökoproduktion geht damit eindeutig zulasten des Marktpreises für Ökoprodukte. Für den Erhalt der Prämie haben die Produzenten Erlösverluste hinzunehmen. Subventionen, wie auch alle anderen staatlichen Zahlungen, belasten die Steuerzahler. Sie begünstigen in diesem Fall die Produzenten. Doch die Begünstigten sind nicht immer auch die 
letztendlichen Empfänger der Subvention. So verbleibt ein Teil der Prämie, in Abbildung 3 mit 'K' gekennzeichnet, nicht bei den Produzenten, sondern wird über den gesunkenen Marktpreis an die Konsumenten weitergereicht. Man spricht in diesem Zusammenhang auch von Überwälzungseffekten oder von einer Einpreisung der Subventionszahlungen. Abhängig von den Preiselastizitäten des Angebots und der Nachfrage wird die Subvention mehr zu einer Begünstigung der Produzenten oder der Konsumenten führen. Schon Mitte der neunziger Jahre wurde darauf verwiesen, dass die flächenbezogenen Zahlungen zu erhöhtem Preisdruck auf den Öko-Märkten führen (SchUlzE PALS, 1994). Mit zunehmendem zentralisiertem Handel im Öko-Food Sektor könnte ein derartiger Trend noch verstärkt werden (vgl. u. a. HAMM und WILD, 2004 oder RIPPIN, 2003).

Dadurch, dass es sich bei der flächenbezogenen Förderung des ökologischen Landbaus um eine faktorgebundene Subventionierung handelt, tritt dieser Effekt nur indirekt auf. Die Prämienzahlungen erfolgen pro Hektar. Deshalb lässt sich der gezahlte Subventionsbetrag nicht direkt auf den Produktpreis umlegen. Dies ändert jedoch nichts an der subventionsbedingten Angebotsausweitung und den aufgezeigten Verteilungseffekten.

Unterschiede zwischen einer produktgebundenen und einer faktorgebundenen Subventionierung liegen jedoch in der jeweiligen Wirkung auf qualitativ unterschiedliche Standorte. Während von einer produktgebundenen Subventionierung höher bonitierte Standorte überdurchschnittlich profitieren, spielt das jeweilige Ertragsniveau bei einer faktorgebundenen Subventionierung keine Rolle. Daher werden bei der flächenbezogenen Förderung gegenüber einer Produktsubventionierung extensive Standorte bevorzugt. Es kommt also lediglich $\mathrm{zu}$ standortabhängigen Unterschieden in den dargestellten Verteilungseffekten, auf die hier aber nicht weiter eingegangen werden soll (vgl. HENRICHSMEIER und WITZKE, 1994: 183).

Das steigende Angebot an ökologisch erzeugten Produkten als ein Resultat der Subventionierung impliziert eine Ausdehnung des ökologischen Landbaus. Da genau dies auch politisches Ziel der Förderung ist, kann für das untersuchte Förderinstrument insofern grundsätzlich von einer hohen Effektivität, d.h. von einem hohen Maß an Zielerreichung, ausgegangen werden. Es bleibt jedoch zu überlegen, ob diese Ausdehnung auch im Sinne aller Beteiligten erreicht wird. Mit Blick auf den zwischenzeitlichen Preisverfall auf einigen Märkten für ökologisch erzeugte Produkte wurde bereits auf ernste Zweifel an der derzeitigen Förderpolitik hingewiesen (vgl. dazu BIOLAND, 2004b: 4 oder GROß, 2004: 140). Dabei ist anzumerken, dass der aufgezeigte Preisdruck nicht in der speziellen Ausgestaltung der 
flächenbezogenen Förderung begründet liegt. Er ist vielmehr Konsequenz jeder rein angebotsinduzierenden Förderung und liegt dadurch im politischen Ziel einer flächenmäßigen Ausdehnung des ökologischen Landbaus begründet. Um ihn zu verhindern bzw. abzumildern, werden seit einigen Jahren verstärkt finanzielle Mittel für eine entsprechende Nachfragesteigerung bereitgestellt. Auf die verschiedenen diesbezüglichen Maßnahmen wurde in Kapitel 2.2 bereits eingegangen.

Insbesondere im vergangenen Jahr hat sich die Nachfrage nach Öko-Produkten sehr positiv entwickelt, was bei fast allen Produkten zu einer spürbaren Stabilisierung der Produktpreise geführt hat (vgl. dazu BRÜGGEMANN, 2006: 162ff oder RIPPIN, 2006: 64ff). Sollte sich die Absatzsituation jedoch wieder verschlechtern ${ }^{5}$, so stellt sich die Frage, welche Konsequenzen ein, sich durch eine Angebotsförderung tendenziell verstärkender, Preisrückgang für die einzelnen Beteiligten hat. Als Beteiligte gelten dabei neben den Konsumenten und Produzenten auch die Steuerzahler, die mit ihren Abgaben die Förderpolitik finanzieren. Im Folgenden sollen deshalb die unterschiedlichen Auswirkungen eines sukzessiven Abbaus der Preisaufschläge für ökologisch erzeugte Produkte auf Konsumenten, Produzenten sowie auf den Einsatz von Fördergeldern behandelt werden.

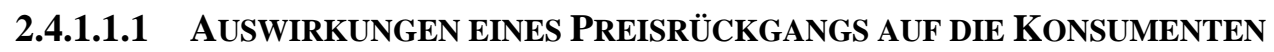

Für die Konsumenten kann ein Preisrückgang eine doppelt positive Wirkung haben. In einem ersten Schritt sinken die Verbraucherpreise durch den Erzeugerpreisverfall, vorausgesetzt, die erzielten Preisvorteile beim Einkauf werden vom Handel und von den Verarbeitern an die Verbraucher weitergegeben. Diese Senkung der Verbraucherpreise führt zu dem bereits angesprochenen erhöhten Absatz von Ökoprodukten. Infolge der zunehmenden Realisierung von Skaleneffekten durch den verstärkten Absatz von Ökoprodukten kommt es in einem zweiten Schritt zur Senkung der Transaktionskosten in Verarbeitung und Vermarktung und damit, vorausgesetzt auch diese Kostenvorteile werden an den Verbraucher weitergegeben, wiederum zur Senkung der Verbraucherpreise. Gerade die hohen Transaktionskosten in Verarbeitung und Vermarktung durch die $\mathrm{zu}$ geringen Umsatzvolumina wurden in der Vergangenheit von einer Reihe von Wissenschaftlern für den Ökomarkt als besonders wachstumshemmende Faktoren problematisiert (vgl. dazu HAMM, 2003: 15; NIEBERG und OFFERMANN, 2001: 427 oder auch SPILLER, 2001: 453). 
Aus Verbrauchersicht ist die Effektivität der flächenbezogenen Erzeugerförderung daher positiv zu bewerten.

\subsection{AUSWIRKUngen EINES PreISRÜCKgANGS AUf DIE ProdUZENTEN}

Aus Sicht der Landwirtschaft führt die Subventionierung zu einer veränderten Vorzüglichkeit des ökologischen Landbaus gegenüber dem konventionellen Landbau. Durch die daraus folgende Angebotsausdehnung kommt es, wie aus Abbildung 3 ersichtlich, zu dem bereits beschriebenen Abbau der Preisaufschläge für Ökoprodukte.

Nach Nieberg und OfFermann (2001: 424ff) haben jedoch die Preisaufschläge im Vergleich $\mathrm{zu}$ den Förderprämien eine weitaus größere Bedeutung für den ökonomischen Erfolg ökologisch wirtschaftender Betriebe. Die Aufschläge variieren zwischen den verschiedenen Produkten aber sehr stark. Sie reichen europaweit von durchschnittlich $+16 \%$ bei Milch bis zu durchschnittlich $+266 \%$ bei Kartoffeln im Vergleich zu den jeweiligen Notierungen für konventionelle Produkte (OffermanN, 2003: 101). Dieser Tatbestand führt dazu, dass es, infolge eines prämieninduzierten sukzessiven Abbaus der Preisaufschläge, auch unter den einzelnen ökologischen Produktionsverfahren zu veränderten Vorzüglichkeiten und damit zu erheblichen Änderungen in der Produktionsstruktur im ökologischen Landbau kommt (HOLlENBERG, 2001: 137). OfFERMANN (2003: 113ff) weist diesbezüglich z.B. auf eine drastisch eingeschränkte ökologische Veredelungsproduktion hin, da in diesem Bereich der Preisrückgang für die Verkaufsprodukte durch die Kostensenkungen im Futterbereich nicht kompensiert werden könne. Generell werden bei einem Wegfall oder einer starken Reduzierung der Preisaufschläge besonders die intensiv wirtschaftenden Betriebe auf eine Umstellung verzichten. Die Erlöseinbrüche können bei ihnen von den Subventionszahlungen nicht aufgefangen werden. Dadurch käme es nach OFFERMANN (2003: 113ff) im Falle erhöhter Subventionszahlungen und sinkender Preisaufschläge auch zu einer verstärkten regionalen Produktionsverlagerung auf extensivere Standorte. Dies ist auf die bereits angesprochene spezifische Wirkungsweise von Flächenprämien auf qualitativ unterschiedlichen Standorten zurückzuführen.

Es bleibt daher zu überlegen, ob die angesprochenen Effekte einer veränderten Produktionsstruktur und einer verstärkten regionalen Produktionsverlagerung mit den

\footnotetext{
${ }^{5}$ Trotz der aktuell positiven Marktentwicklung existieren nach wie vor Anzeichen, die langfristig auf einen Preisverfall hindeuten könnten (vgl. dazu AGRA-EUROPE, 2006g: 28)
} 
angestrebten gesellschaftspolitischen Zielen vereinbar sind und keine Schmälerung der positiven Effekte einer Ausweitung des ökologischen Landbaus bedeuten. Ansonsten wäre eine nicht nachfragegestützte weitere Ausdehnung des ökologischen Landbaus trotz der positiven Effekte eines Preisrückgangs für die Konsumenten wahrscheinlich insgesamt negativ zu beurteilen. Um diesbezüglich zu einem aussagekräftigen Ergebnis zu gelangen, müsste eine detaillierte Wohlfahrtsanalyse durchgeführt werden.

\subsection{AUSWIRKUngen EINES PREISRÜCKGANGS AUf DEN EINSATZ VON FÖRDERGELDERN}

OFFERMANN (2003: 120) führt Modellrechnungen zur Ausdehnung des ökologischen Landbaus in Europa durch. Neben einer subventionsinduzierten Ausdehnung auf $20 \%$ der landwirtschaftlichen Fläche in der EU unter der Annahme konstant hoher Preisaufschläge für Ökoprodukte, untersucht er dabei auch die Alternative einer solchen Ausdehnung bei vollständigem Abbau der Preisaufschläge. Er kommt dabei zu dem Ergebnis, dass im Falle konstanter Preisaufschläge den Erzeugern in der EU durchschnittliche Prämien von $342 €$ /ha gewährt werden müssten, um eine europaweite Ausdehnung des ökologischen Landbaus auf $20 \%$ der landwirtschaftlichen Fläche zu erreichen. Dies entspräche einem Gesamtfördervolumen von $8 \mathrm{Mrd} € €$ in der EU. Demgegenüber müssten bei einem kompletten Wegfall der Preisaufschläge sogar durchschnittliche Prämien von $526 € /$ ha gewährt werden, was einem Gesamtfördervolumen von $13 \mathrm{Mrd}$ € in der EU entspräche.

Dieses Extrembeispiel mit einer angenommenen Ausweitung des ökologischen Landbaus auf $20 \%$ der landwirtschaftlichen Fläche zeigt, dass eine Reduktion der Preisaufschläge eine deutliche Erhöhung der eingesetzten Fördergelder bedeutet, will man das gesetzte Ziel einer weiteren Ausdehnung des ökologischen Landbaus erreichen. Dies ist besonders unter dem Blickwinkel nachvollziehbar, dass durch den Abbau der Erzeugerpreisaufschläge die Prämiensätze nicht nur zur weiteren Ausdehnung der ökologisch bewirtschafteten Fläche, sondern auch zur Kompensation der Erlösverluste der schon ökologisch wirtschaftenden Betriebe angehoben werden müssen. Gelingt es also nicht, parallel zur Angebotsausdehnung auch die Nachfrage nach Ökoprodukten anzukurbeln, so müssen zur Zielerreichung unverhältnismäßig höhere Finanzmittel bereitgestellt werden. 


\subsubsection{FAZIT ZUR WIRKSAMKEIT DER FÖRDERUNG}

Wie zu Beginn des Kapitels gezeigt wurde, ist mit dem Instrument der flächenbezogenen Förderung das Ziel einer weiteren Ausdehnung des ökologischen Landbaus grundsätzlich zu erreichen.

Aus Sicht der Konsumenten gibt es keine Zweifel an der Effektivität des Förderinstrumentes. Auch im Falle einer nur schwachen Wirkung paralleler nachfrageinduzierender Maßnahmen wird der einsetzende Preisdruck zur Steigerung des Absatzes und damit zu einer politisch gewünschten verstärkten Versorgung der Bevölkerung mit Ökoprodukten führen.

Dagegen führt ein anhaltender Preisdruck im Bereich der Öko-Produktion im Falle einer stagnierenden Nachfrage und weiterer Prämienanhebungen zu tief greifenden Veränderungen, über deren politische Akzeptanz und letztendliche Konsequenzen weiterer Diskussions- und Forschungsbedarf besteht.

Neben den Produzenten und den Konsumenten als direkte bzw. indirekte Empfänger der Fördermittel sind jedoch auch die Steuerzahler als Geldgeber direkt an der Subventionierung beteiligt. Sie finanzieren die Förderung und sind dabei an einem möglichst effizienten Einsatz der zur Verfügung gestellten Gelder interessiert. Wie im vorangegangenen Kapitel jedoch dargelegt, führt ein Abschmelzen der Preisaufschläge für Ökoprodukte zu einem stärkeren Anstieg der zur Ausdehnung des ökologischen Landbaus benötigten Fördergelder bzw. bei einem begrenzten Förderbudget zu einem geringeren Ausmaß der ökologisch bewirtschafteten Fläche. Der Preisrückgang führt daher zu einer Minderung der Effizienz des Mitteleinsatzes.

Es stellt sich aber in diesem Zusammenhang auch die Frage, ob die derzeitige Ausgestaltung der flächenbezogenen Förderung im Falle konstanter Erzeugerpreisaufschläge überhaupt zu einem effizienten Einsatz der Fördergelder führt.

Im nächsten Kapitel soll daher mit der Effizienz die Wirtschaftlichkeit des Finanzmitteleinsatzes bei der flächenbezogenen Förderung näher untersucht werden. Dabei wird gemäß der aktuellen Marktlage davon ausgegangen, dass die angestoßenen nachfrageinduzierenden Maßnahmen Wirkungen zeigen und sich Angebot und Nachfrage gleichmäßig ausdehnen. Die Preise für ökologische Produkte werden also bei den folgenden Betrachtungen als konstant angenommen. 


\subsubsection{EFFIZIENZ DER FÖRDERUNG}

In Anbetracht der nur begrenzt zur Verfügung stehenden Fördermittel und der Vorgabe eines effizienten Einsatzes dieser Mittel seitens der Steuerzahler ist die Fördereffizienz von entscheidender Bedeutung, wenn es darum geht, mit einem festgelegten Budget eine möglichst großflächige Ausdehnung des ökologischen Landbaus zu erreichen.

Analog zur Betrachtung der Effektivität könnte bei einer Effizienzanalyse der eingesetzten Mittel bezogen auf das primäre Ziel einer Ausweitung des ökologischen Landbaus auch die Finanzmitteleffizienz im Hinblick auf das letztendliche Ziel einer Erreichung der angestrebten gesellschaftlichen Ziele betrachtet werden. So untersuchen beispielsweise AHRENS et al. (2000: 99ff) die flächenbezogene Förderung bezüglich ihrer Umwelteffizienz. Umwelteffizienz meint dabei das Verhältnis der eingesetzten Fördermittel zu den damit erreichten positiven Umwelteffekten. Wie schon bei der Betrachtung der Effektivität sollen aber auch die Untersuchungen zur Effizienz im vorliegenden Kapitel in erster Linie auf dem primären Förderziel einer Ausweitung des ökologischen Landbaus basieren.

\subsubsection{Problemstellung}

Wie in Kapitel 2.3.2.1 dargestellt wurde, ist es den Bundesländern von der EU auferlegt, die Prämienhöhen für Maßnahmen im Rahmen der Agrarumweltprogramme, wie in diesem Fall für die Förderung des ökologischen Landbaus über Flächenprämien, an den Kosten auszurichten, die dem Landwirt durch seine Teilnahme entstehen. NIEBERG und STROHMLÖMPKE (2001: 416) weisen in diesem Zusammenhang darauf hin, dass in verschiedenen landwirtschaftlichen Unternehmen diesbezüglich unterschiedlich hohe Kosten entstehen können. So bewirken vor allem unterschiedliche Standortbedingungen, wie z.B. ein unterschiedliches Ertragsniveau, die Verursachung sehr unterschiedlicher betriebsindividueller Kosten in Bezug auf die Teilnahme an der Fördermaßnahme. Da es dem Staat jedoch aufgrund der Komplexität der Umstellungswirkungen nicht möglich ist, die betriebsindividuellen Anpassungs- bzw. Beibehaltungskosten für eine größere Zahl von Betrieben abzuschätzen, wird auf der Grundlage von Durchschnittsdaten und relativ groben Kalkulationen und unter der Berücksichtigung der zur Verfügung stehenden finanziellen Mittel eine Einheitsprämie festgelegt. Diese Vorgehensweise und die mit ihr verbundenen Auswirkungen auf die Effizienz des Finanzmitteleinsatzes werden jedoch schon seit geraumer Zeit seitens der Agrarökonomie heftig kritisiert (vgl. OPPERMANN et al., 1997: 50f; vgl. AHRENS et al., 2000: 99ff; vgl. BAUDOUX, 2001: 260). 
Die Wirkungen der Einheitsprämie sind in Abbildung 4 schematisch dargestellt. Sowohl die schematische Darstellung als auch die nachfolgenden Erläuterungen sind dabei eng an die diesbezüglichen Ausführungen von NIEBERG und STROHM-LöMPKE (2001: 416) angelehnt.

\section{Abbildung 4: Ökologisch bewirtschaftete Fläche, Staatsausgaben und Einkommenswirkungen bei der Einheitsprämie}

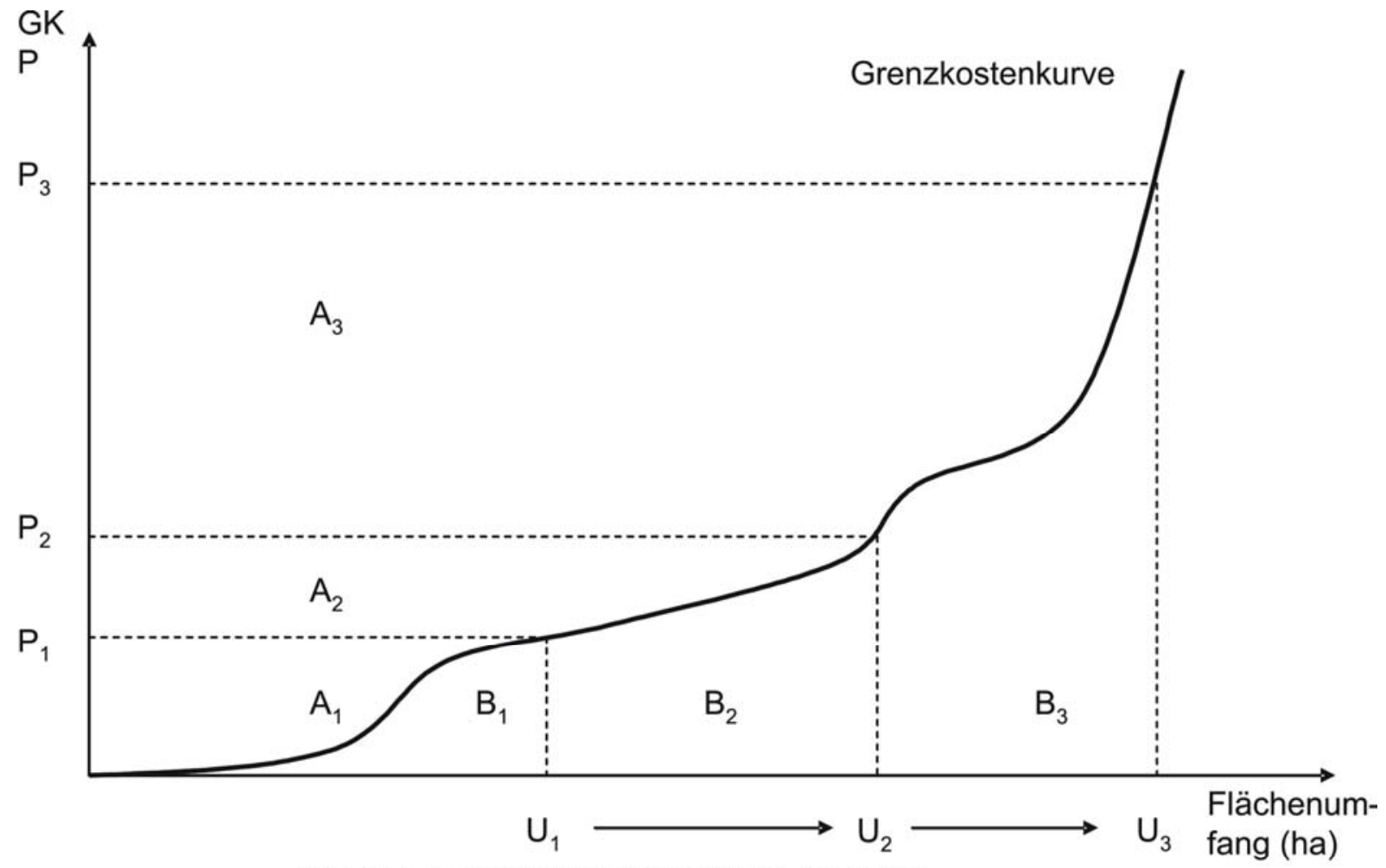

Flächen in aufsteigender Reihenfolge ihrer Umstellungskosten

$$
\begin{aligned}
& \text { GK: Grenzkosten } \\
& P \text { : Höhe der Prämie } \\
& \text { U : Flächenumfang }
\end{aligned}
$$

A : Einkommenseffekt

B : Umwelteffekt

$\mathrm{A}+\mathrm{B}$ : Summe der staatlichen Zahlungen

Quelle: NIEBERG und STROHM-LÖMPKE (2001: 416)

In Abbildung 4 sind auf der Abszisse die Flächen einer Region von links nach rechts geordnet gemäß der Höhe ihrer spezifischen Umstellungskosten. Weil nur eine konstante Einheitsprämie existiert, wird für alle Flächen einer Region für die Teilnahme am Programm jeweils die gleiche Prämie je Hektar gezahlt. Dabei werden drei verschiedene Szenarien mit jeweils unterschiedlichen Prämienhöhen (P1 bis P3) dargestellt. Die auf der Ordinate abgetragenen Grenzkosten entsprechen den Kosten aus der Programmteilnahme, d.h. der Summe aus Zusatzkosten und entgangenem Gewinn. Es wird vereinfachend angenommen, dass erstens alle Betriebsleiter ihre Umstellungskosten zutreffend abschätzen können und zweitens nur die Betriebe umstellen, für die sich dieser Schritt wirtschaftlich lohnt. Aus den Prämienhöhen (P1 bis P3) und den Flächenumfängen (U1 bis U3) ergibt sich der jeweilige 
Umfang der staatlichen Zahlungen. Sofern diese Zahlungen die Kosten aus der Programmteilnahme überkompensieren, entstehen Einkommenseffekte (A1 bis A3) für die Landwirte. Diese Einkommenseffekte werden von den Kritikern der derzeitigen Förderpraxis auch als Mitnahmeeffekte bezeichnet.

Es zeigt sich, dass auf einigen Flächen die Bewirtschaftung nach den Grundsätzen des ökologischen Landbaus keine oder nur verhältnismäßig geringe Kosten verursacht. Es handelt sich dabei um Flächen derjenigen Betriebe, deren Wirtschaftsweise schon immer nach sehr extensiven Grundsätzen erfolgte, d.h. bei denen vermutlich auch ohne die Zahlung einer Förderung keine Intensivierung der Produktion stattfinden würde. Durch die Zahlung der Prämie ändert sich folglich auf diesen Flächen an der Produktionsweise kaum etwas. Deshalb ist auch der positive Effekt der Förderung auf die Umwelt nur sehr gering oder sogar gleich null. Dementsprechend größer sind in diesen Fällen die Einkommenseffekte, die umso höher sind, je höher die Einheitsprämie ausfällt. Das Potential der Mitnahmeeffekte ist in diesen Fällen also ausgesprochen hoch.

Dem gegenüber gibt es, wie aus Abbildung 4 ersichtlich, andere Flächen, auf denen die Umstellung auf ökologischen Landbau hohe Kosten verursacht. Diese Kosten können durch die Einheitsprämie nicht abgedeckt werden. Eine Umstellung ist auf diesen Standorten deshalb unter den bestehenden Förderbedingungen nicht wirtschaftlich.

Es kann daher festgestellt werden, dass Standortunterschiede bei einer Förderung über eine Einheitsprämie zu erheblichen Produzentenrenten auf benachteiligten Standorten führen, während auf ertragreichen Standorten die Prämien häufig nicht ausreichen, um die Einkommensverluste der Landwirte abzudecken.

Bezüglich des mit der Förderung zu erreichenden Zieles einer Ausdehnung des ökologischen Landbaus führt bei einem flachen Verlauf der Grenzkostenkurve schon eine leichte Erhöhung der Prämie zu einer deutlichen Ausdehnung der Umstellungsfläche $(\mathrm{P} 1 \rightarrow \mathrm{P} 2$, U1 $\rightarrow \mathrm{U} 2)$. Dem gegenüber muss bei einem steileren Verlauf der Grenzkostenkurve die Prämie deutlich erhöht werden, um entsprechende Umstellungsraten $\mathrm{zu}$ induzieren $(\mathrm{P} 2 \rightarrow \mathrm{P} 3$, U2 $\rightarrow \mathrm{U} 3)$. Besonders auffällig ist dabei, dass sowohl die staatlichen Zahlungen als auch die Einkommenseffekte bei Betrieben mit niedrigeren Umstellungskosten mit voranschreitendem ökologisch bewirtschaftetem Flächenumfang überproportional zunehmen.

Dieser Zusammenhang offenbart, dass mit ansteigender Prämie auch die Effizienz der Zahlungen deutlich abnimmt. Jede weitere Anhebung der Förderprämie führt zwar zur 
Umstellung von ehemals konventionell wirtschaftenden Betrieben, deren Kosten nun durch die neue Prämie abgedeckt werden. Gleichzeitig werden aber auch die Prämien für jene Betriebe angehoben, deren Kosten bereits durch die zuvor gewährte Prämie abgedeckt wurden. Die zusätzlichen Zahlungen führen auf diesen Betrieben zu keinen weiteren ökologischen Produktionsausweitungen. Sie wirken also ausschließlich einkommenserhöhend. Mit zunehmender prämieninduzierter Ausdehnung der ökologisch bewirtschafteten Landfläche nimmt die Effizienz der staatlichen Zahlungen in Bezug auf die Ausweitung des ökologischen Landbaus daher sukzessive ab. Dementsprechend offenbarte der deutliche Anstieg des durchschnittlichen Förderbetrags pro ha in Tabelle 3 das vorhandene Potential an Mitnahmeeffekten. Das politische Ziel einer Ausweitung des ökologischen Landbaus - selbst bei ausreichend vorhandenen Finanzmitteln - kann daher mit einer einfachen Anhebung der Einheitsprämie (wie im GAK-Rahmenplan 2002 geschehen) nicht effizient erreicht werden.

\subsubsection{KONSEQUENZEN}

Den Schlüssel zur Lösung des aufgezeigten Effizienzproblems sehen viele Agrarökonomen in einer weiteren Differenzierung der Prämiensätze (vgl. OPPERMANN et al., 1997: 50f; vgl. AHRENS et al., 2000: 99ff). Dabei ist bezüglich der flächenbezogenen Förderung des ökologischen Landbaus auf die bereits bestehenden Prämiendifferenzierungen hinzuweisen (vgl. Kapitel 2.3.3). So werden die Prämiensätze in den Bundesländern bereits nach Flächennutzungsarten und dem Zeitpunkt der Umstellung differenziert. Einige Experten fordern jedoch noch weitergehende Differenzierungen z.B. nach der Standortqualität (vgl. KACHEL, 1999: 51ff).

Die effizienzsteigernde Wirkung von Prämiendifferenzierungen ist in Abbildung 5 dargestellt. Dabei wurde das schon in Kapitel 2.4.2.1 verwendete Diagramm entsprechend der neuen Prämienausgestaltung modifiziert. Statt einer konstanten Einheitsprämie, wie sie in Abbildung 4 angenommen wurde, sind die Flächen hier nach den Kosten ihrer Umstellung in drei Gruppen mit jeweils unterschiedlichen Prämienhöhen (P1 bis P3) unterteilt. 
Abbildung 5: Ökologisch bewirtschaftete Fläche, Staatsausgaben und Einkommenswirkungen bei einer gestaffelten Prämie

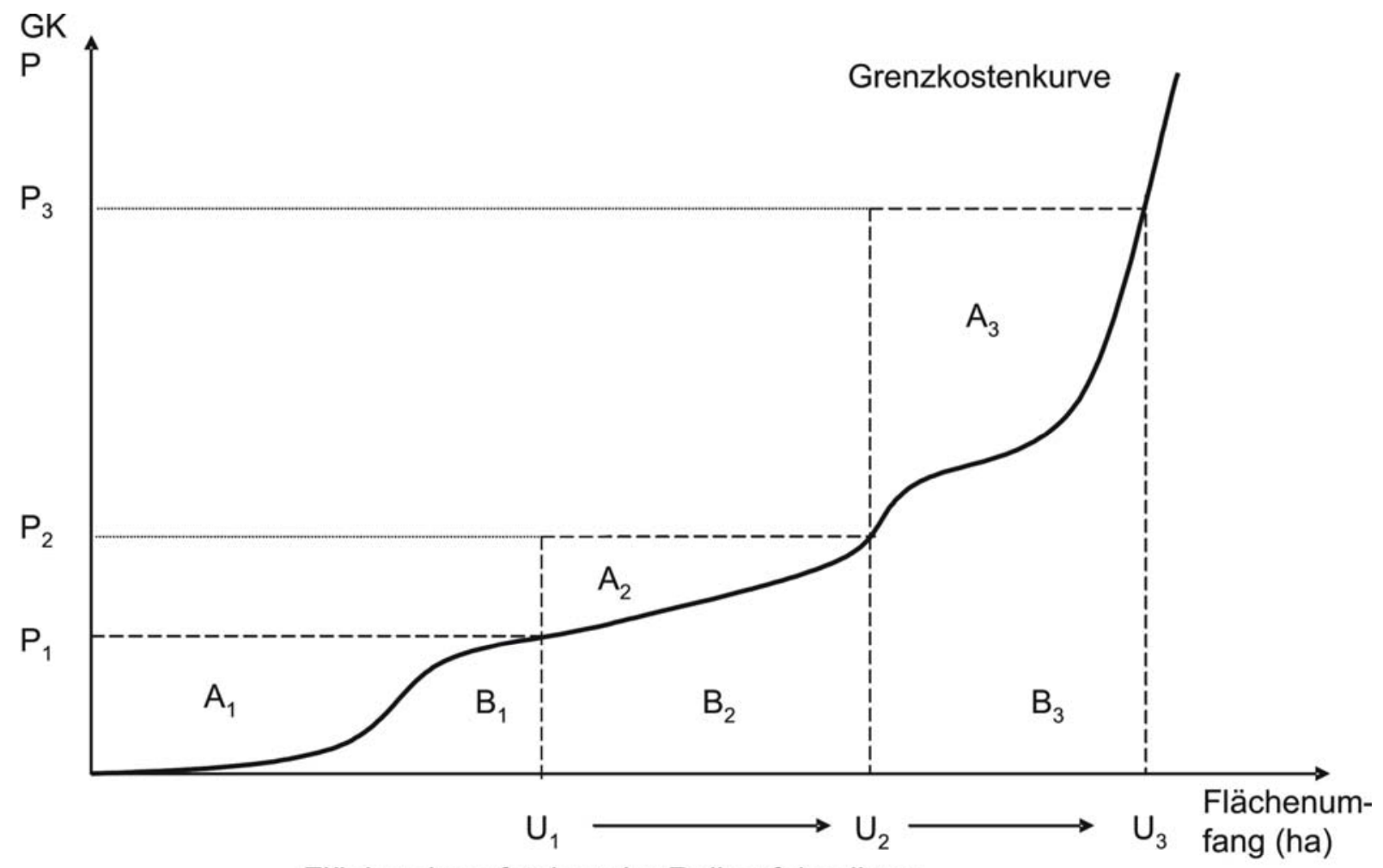

Flächen in aufsteigender Reihenfolge ihrer Umstellungskosten

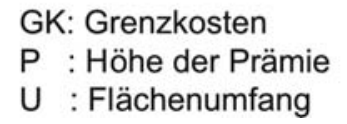

$U$ : Flächenumfang
A : Einkommenseffekt

B : Umwelteffekt

A+B: Summe der staatlichen Zahlungen

Quelle: NIEBERG und STROHM-LÖMPKE (2001: 417)

Es zeigt sich, dass z.B. ein angestrebter Flächenumfang U3 nun mit weitaus geringeren staatlichen Zahlungen erreicht werden kann. Der Grund dafür liegt in der drastischen Reduktion der Einkommenseffekte bei Betrieben mit niedrigeren Umstellungskosten. Sie erhalten nun nicht mehr dieselbe Prämie wie die Betriebe mit hohen Umstellungskosten (P3), sondern eine deutlich geringere (P2 bzw. P1). Durch die Differenzierung werden also die im Rahmen der Untersuchungen zur Einheitsprämie kritisierten Mitnahmeeffekte deutlich reduziert.

Würden die Prämien noch weiter differenziert, so könnten diese Mitnahmeeffekte sogar noch stärker reduziert werden. Im Extremfall könnte theoretisch eine exakte grenzkostenorientierte Förderung erreicht werden. Dabei ist allerdings zu bedenken, dass mit einer zunehmenden Prämiendifferenzierung im Regelfall auch die Kontroll- und Verwaltungskosten ansteigen (RUDLOFF und URFEI, 2000: 146). Bei der Überlegung, wie stark differenziert werden sollte, muss also neben der sich erhöhenden Fördereffizienz auch die gleichzeitig abnehmende 
Verwaltungseffizienz berücksichtigt werden. $\mathrm{Ob}$ daher über weitere Prämiendifferenzierungen eine Erhöhung der Effizienz des Finanzmitteleinsatzes erreicht werden kann, bleibt zunächst fraglich.

\subsection{SCHLUSSBETRACHTUNG UND WEITERE VORGEHENSWEISE}

Die bisherigen Betrachtungen offenbaren Defizite hinsichtlich der Eignung der gegenwärtigen flächenbezogenen Förderung des ökologischen Landbaus für eine zukünftig effiziente Förderpolitik. Auch die Überlegungen hinsichtlich einer weiteren Differenzierung der bisherigen Flächenprämien erlauben noch keine belastbaren Urteile.

Diese Defizite wirken gegenwärtig umso intensiver, weil trotz eines äußerst positiven Marktumfeldes kein deutliches Flächenwachstum im Inland zu verzeichnen ist. Während der Umsatz mit Öko-Produkten in Deutschland in 2005 um $15 \%$ angestiegen ist, stellte sich im gleichen Zeitraum lediglich ein Flächenwachstum von 5,2 \% ein. So scheint es, als könne der heimische Öko-Landbau am allgemeinen Marktwachstum nur unterdurchschnittlich partizipieren (AGRA-EUROPE, 2006h). Auch hier zeigt sich die Notwendigkeit einer Modifizierung der Angebotsförderung, um die Wettbewerbskraft der heimischen Unternehmen zu stärken und auf höhere Umstellungsraten und damit bei gleichzeitiger Reduktion von Mitnahmeeffekten auf eine höhere Effektivität der Förderung hinzuwirken.

In der Vergangenheit gab es immer wieder Vorschläge für eine Neukonzeption oder eine Modifizierung der Angebotsförderung zugunsten des ökologischen Landbaus, die jedoch nur teilweise in der Praxis auch Berücksichtigung fanden (vgl. u.a. NiEBERG und STROHMLÖMPKE (2001: 417). Zusätzlich gab es in Deutschland in den letzten Jahren wiederholt Vorschläge, die Teilnehmerauswahl bei Agrarumweltprogrammen und damit u.U. auch bei der flächenbezogenen Förderung des ökologischen Landbaus über Ausschreibungen zu organisieren (vgl. dazu LATACZ-LOHMANN und VAN DER HAMSVOORT, 1997 oder HOLMMÜLLER et al., 2002: 112ff). Die Grundidee dieser Vorschläge besteht darin, nicht wie bisher den Landwirten staatlich festgelegte Prämien anzubieten, sondern die Landwirte selbst im Rahmen einer Auktion zur Abgabe eines Gebotes und damit indirekt zur Bekanntgabe ihrer individuellen Grenzkosten $\mathrm{zu}$ bewegen. Die Informationen über die individuellen Grenzkosten der einzelnen Anbieter würden es den staatlichen Stellen erlauben, durch eine zielgerichtete Auswahl der Teilnehmer bei gegebenem Budget die positiven ökologischen Effekte zu maximieren. In der Theorie erscheint damit sogar eine exakt grenzkostenorientierte Förderung realisierbar. Erste Modellversuche sind in den letzten Jahren bereits mit positiven 
Ergebnissen zur Finanzmitteleffizienz abgeschlossen worden (vgl. HOLM-MüLLER und HILDEN, 2004 oder BERTKE et al., 2005: 41ff). Jedoch basierten diese Untersuchungen jeweils auf dem Agrarumweltprogramm Grünlandextensivierung. Ob Ausschreibungen sich auch für die flächenbezogene Öko-Förderung eignen und dort ebenfalls $\mathrm{zu}$ den angestrebten Effizienzsteigerungen führen, ist schon allein aufgrund des generell unterschiedlichen und vor allem deutlich kleineren potentiellen Teilnehmerkreises äußerst ungewiss. So könnten bspw. effizienzmindernde Absprachen hinsichtlich der Gebotshöhe unter den vergleichsweise wenigen Öko-Landwirten einer Region aufgrund einer durchaus denkbaren größeren Verbundenheit untereinander wahrscheinlicher sein und das Endergebnis belasten. Um diese Gefahr zu verringern, könnte von staatlicher Seite zwar mit einer Vergrößerung der Ausschreibungsregionen reagiert werden. Dadurch ginge jedoch die für eine effizienzsteigernde Wirkung von Ausschreibungen ebenfalls notwendige Symmetrie unter den Bietern zunehmend verloren.

Gemäß der Basisthematik dieser Arbeit sollen im folgenden Kapitel deshalb Möglichkeiten innerhalb des Steuerrechts aufgezeigt werden, die sich u.U. zur Förderung des ökologischen Landbaus eignen und Potentiale zur Verringerung der vieldiskutierten Mitnahmeeffekte und damit zur Erhöhung der Effizienz aufweisen. 


\section{UNTERSUCHUNG STEUERLICHER FÖRDERALTERNATIVEN}

In Kapitel 3.1 soll zunächst eine kurze Einführung in das Steuerrecht erfolgen. Im Anschluss daran werden in Kapitel 3.2 Kriterien für die Auswahl sinnvoller steuerlicher Förderalternativen ausgearbeitet, bevor in Kapitel 3.3 in Fachkreisen bereits diskutierte steuerliche Fördervorschläge hinsichtlich dieser Kriterien analysiert und beurteilt werden.

\subsection{GRUNDZÜGE DES STEUERRECHTS UND MÖGLICHKEITEN SEINER NUTZBARKEIT ALS FÖRDERINSTRUMENT}

\subsubsection{STEUERBEGRIFF UND STEUERARTEN}

Der verfassungsrechtliche Begriff der Steuer erhält seinen Inhalt im Wesentlichen aus $\S 3$ der Abgabenordnung (AO):

„Steuern sind Geldleistungen, die nicht eine Gegenleistung für eine besondere Leistung darstellen und von einem öffentlich-rechtlichen Gemeinwesen zur Erzielung von Einnahmen allen auferlegt werden, bei denen der Tatbestand zutrifft, an den das Gesetz die Leistungspflicht knüpft; die Erzielung von Einnahmen kann Nebenzweck sein. “

Anhand dieser Definition können die folgenden prägenden Merkmale des Steuerbegriffs abgeleitet werden:

- Die Steuer ist eine Geldleistung. Andere Leistungsarten werden von dem Begriff nicht erfasst.

- Für die Entrichtung der Steuer erhält der Steuerpflichtige keine andere Gegenleistung als die, dass sie im Rahmen der staatlichen Organisation für die öffentlichen Ausgaben verwendet wird.

- Die Geldleistung wird dem Steuerpflichtigen von einem öffentlich-rechtlichen Gemeinwesen auferlegt. Dies können in Deutschland der Bund, ein Land, eine Gemeinde oder die Kirche sein.

- Steuern werden allen denjenigen auferlegt, die den Tatbestand erfüllen, an den die Leistungspflicht knüpft. Damit wird die Gleichheit der Besteuerung unter den Steuerpflichtigen geregelt. 
- Steuern werden in der Hauptsache erhoben, um die Sachaufgaben des Staates zu finanzieren. Sie sind ein ökonomisches Instrument für die Deckung des allgemeinen Finanzbedarfs.

Trotzdem sind sie in der modernen Industriegesellschaft zwangsläufig auch zum zentralen Lenkungsinstrument aktiver staatlicher Wirtschafts- und Gesellschaftspolitik geworden (BVerfGE 67, 256 (282)).

Die Gesamtheit der erhobenen Steuern lässt sich wie in Tabelle 4 dargestellt nach einzelnen Steuerarten einteilen:

Tabelle 4: Gliederung der Steuern

\begin{tabular}{|c|c|c|c|}
\hline Substanzsteuern & Ertragsteuern & Verkehrsteuern & Sonstige Steuern \\
\hline Grundsteuer, & Einkommensteuer inkl. & Umsatzsteuer, & Verbrauchsteuern \\
Erbschaft- und & Lohn- und & Grunderwerbssteuer & (z.B. Stromsteuer, \\
Schenkungsteuer & Kapitalertragsteuer & & Mineralölsteuer, \\
& sowie & & Tabaksteuer, \\
& Solidaritätszuschlag, & & Branntweinabgaben), \\
& Körperschaftssteuer, & & weitere Steuern \\
& Gewerbesteuer & & (z.B. Kfz-Steuer, \\
& & & Kirchensteuer, \\
& & & Versicherungsteuer, \\
& & & Zölle) \\
\hline
\end{tabular}

Quelle: AID (2004: 7)

Abbildung 6 zeigt die Steuereinnahmen in Deutschland im Jahr 2004 und gibt damit einen Überblick über die fiskalische Bedeutung der einzelnen Steuerarten (vgl. für nähere Informationen zu den einzelnen Steuerarten ALtHOEFER et al., 2003 oder KUßMAUL, 2005) 
Abbildung 6: Steuerspirale 2004

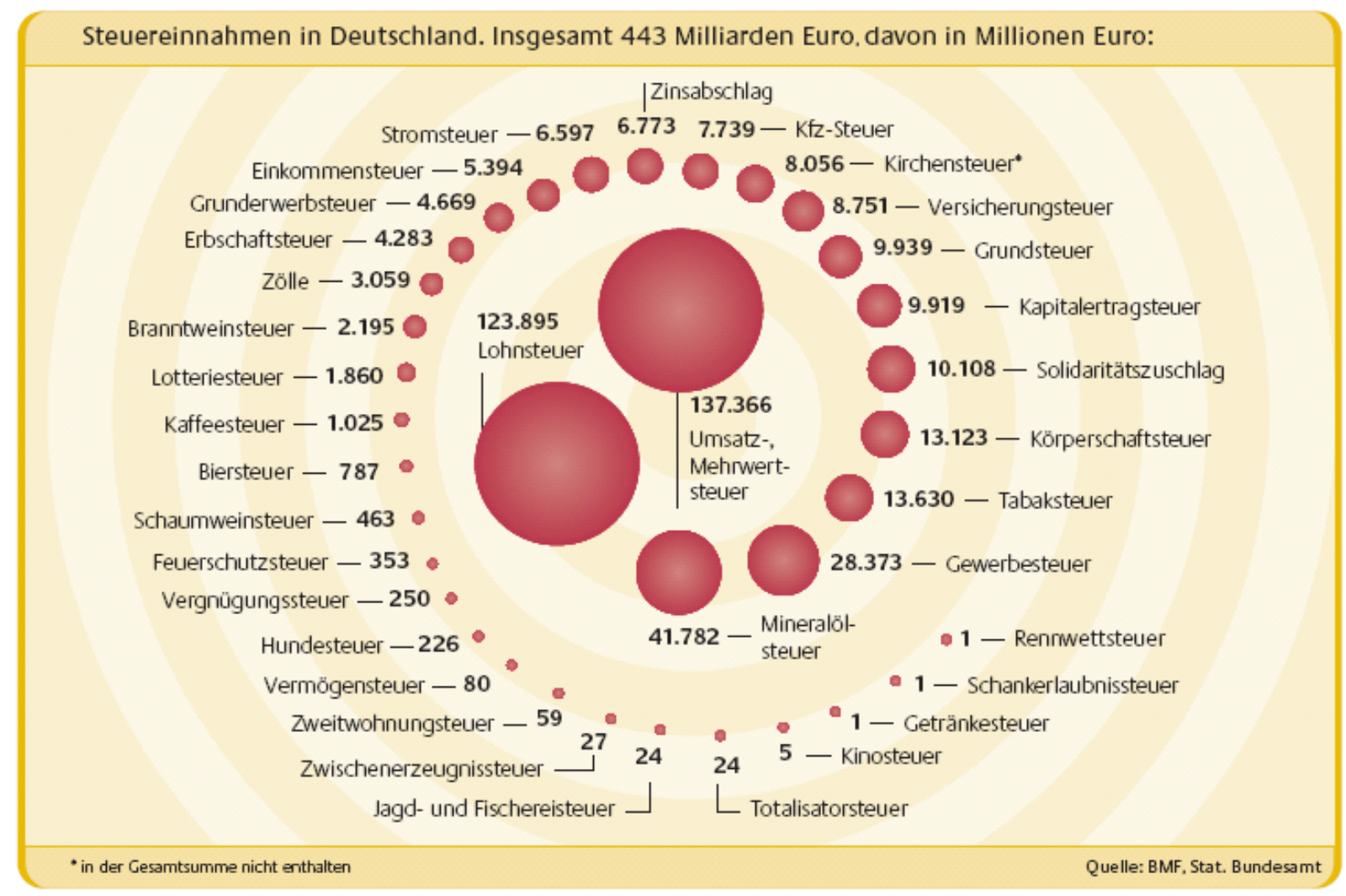

Quelle: Bundesministerium für Finanzen (2005)

\subsubsection{STEUERVERGÜNSTIGUNGEN}

\subsubsection{FISKALZWECKNORMEN VS. LENKUNGSNORMEN IM STEUERRECHT}

In Kapitel 3.1.1 wurde mit dem Hinweis auf $\S 3$ AO festgestellt, dass das vorrangige und ursprüngliche Ziel der Steuererhebung für den Staat die Erzielung von Einkünften ist, um seinen originären hoheitlichen Zielen nachzukommen. Dies bedeutet jedoch nicht, dass die Steuererhebung nicht auch anderen Zwecken dienen kann.

So kann und wird die Besteuerung vom Staat auch als Lenkungsmittel zur Verfolgung politischer Ziele eingesetzt. So genannte Lenkungs- oder auch Sozialzwecknormen haben die Aufgabe im Rahmen des Steuerrechts Verhaltensänderungen auf Seiten der Steuerzahler herbeizuführen. Die eigentliche Zielstellung, die Erzielung von Einkünften darf dabei in den Hintergrund treten. Damit unterscheiden sie sich von den so genannten Fiskalzwecknormen im Steuerrecht, die den ausschließlichen Zweck haben, dem Staat Einkommen zu verschaffen. Eventuelle Nebenzwecke haben dagegen nur eine untergeordnete bis gar keine Bedeutung. (TIPKE, 2000: 73ff) 
Lenkungsnormen schaffen im Steuerrecht in der Regel Steuerentlastungen. Sie können aber auch Steuersonderbelastungen oder sogar, wie im Falle der Ökosteuer, selbstständige Sondersteuern schaffen. Steuerentlastungen werden meist auch als Steuervergünstigungen oder auch als Steuervorteile bezeichnet. TIPKE (2000: 78) weist in diesem Zusammenhang darauf hin, dass Lenkungsnormen sachlich nicht zum Steuerrecht gehören. Soweit im Steuerrecht über Steuervergünstigungen Wirtschaftslenkung betrieben wird, handele es sich vielmehr um wirtschaftsrechtliche Normen, die lediglich steuergesetzlich eingekleidet seien, sich also lediglich der Technik der Steuergesetze bedienten. Wirtschaftslenkende Normen im Steuerrecht sind daher auch nicht anders zu qualifizieren als offene Subventionen. Aus diesem Grund sind Steuervergünstigungen genauso Bestandteil des jährlichen Subventionsberichts der Bundesregierung wie andere staatliche Transferleistungen und werden auch genauso von den Subventionsverboten des EU-Rechts (Art. 92 - 94 EGV) erfasst.

Dass Lenkungsnormen und damit Steuervergünstigungen als Nicht-Fiskalzwecknormen nicht dem originären Ziel der Besteuerung widersprechen, wird schon im besagten $\S 3 \mathrm{AO}$ festgelegt. Denn zum Steuerbegriff heißt es im 2. Halbsatz des 1. Absatzes: "Die Erzielung von Einnahmen kann Nebenzweck sein". Auch das Bundesverfassungsgericht hat durch mehrere Urteile und Beschlüsse die grundsätzliche Existenz von Lenkungsnormen im Steuerrecht anerkannt (vgl. bspw. BVerfGE 4, 7 oder 6, 55). Diese grundsätzliche Anerkennung von Lenkungsnormen im Steuerrecht erleichtert die verfassungsrechtliche Rechtfertigung von Lenkungsnormen als Instrumente bspw. der Umweltpolitik (SCHEMMEL, 1998: 21). Ein Verstoß gegen das dem Steuerrecht nach $\S 3$ AO zugrunde liegende Gebot gleichmäßiger Besteuerung nach der Leistungsfähigkeit, d.h. gegen die Verteilungsgerechtigkeit, kann aus Gründen der steuerlichen Lenkung also gerechtfertigt sein. Eine solche Rechtfertigung kann allerdings nach TIPKE (2000: 336ff) nur dann gelingen, wenn dem Lenkungszweck verfassungsrechtlich eine förderungswürdige Bedeutung zukommt.

Es stellt sich jedoch die Frage, welche Anforderungen an Steuervergünstigungen gestellt werden, damit eine verfassungsrechtliche Rechtfertigung als Lenkungsnorm ermöglicht wird. Für den Fortgang der Arbeit ergeben sich daraus folgende konkrete Fragestellungen:

- Zum einen ist es notwendig zu erörtern, welche Anforderungen im Allgemeinen an die Rechtfertigung von Lenkungsnormen gestellt werden und ob die mit dem ökologischen Landbau verfolgten Ziele grundsätzlich zur Rechtfertigung einer Lenkungsnorm im Steuerrecht genügen. 
- Zum zweiten stellt sich die Frage, ob die verschiedenen zur weiteren Beurteilung ausgewählten steuerlichen Förderinstrumente im Speziellen als Lenkungsnormen im Sinne der Steuerrechtslehre zu rechtfertigen wären.

Die Beantwortung dieser beiden Fragestellungen ist im Kontext der Überlegungen bezüglich einer steuerlichen Förderung von großer Wichtigkeit, da sie die rechtliche Legitimation eines solchen Förderinstrumentes sichern soll. Ohne eine steuerrechtliche Legitimation wären die Aussichten auf eine erfolgreiche Implementierung einer steuerlichen Förderung deutlich geschmälert.

Auf die erste Fragestellung soll im Folgenden eingegangen werden. Die zweite Fragestellung, die sich unmittelbar auf die spezifischen steuerlichen Förderinstrumente bezieht, ist im Rahmen der detaillierteren Untersuchung einzelner Förderinstrumente zu klären, sobald diese aufgrund ihrer sonstigen Spezifika in die engere Auswahl aufgenommen worden sind.

\subsubsection{LEGITIMIERUNG VON STEUERVERGÜNSTIGUNGEN}

Steuervergünstigungen in Form von Lenkungsnormen sind im Allgemeinen in die Steuergesetze eingebettet, ohne förmlich von den Fiskalzwecknormen getrennt zu sein. Im Unterschied zu diesen bezwecken sie aber keine gerechte, gleichmäßige Steuerverteilung. Die Rechtsqualität des Steuerrechts sowie seine Praktikabilität werden dadurch eingeschränkt. Es bedarf daher eines wichtigen Lenkungszwecks, der den Verstoß gegen die Verteilungsgerechtigkeit aufwiegt, um Lenkungsnormen im Steuerrecht zu implementieren. $\mathrm{Zu}$ der Frage, inwieweit Lenkungsnormen den verfassungsmäßigen Grundsatz einer gleichmäßigen Besteuerung verletzen, werden nach TIPKE (2000: 336ff) unter den Steuerrechtlern vier verschiedene Auffassungen vertreten:

- 1. Position: Steuervergünstigungen werden stets als ungerechtfertigt angesehen, weil sie eben jenes verfassungsmäßige Gebot der gleichmäßigen Besteuerung nach der Leistungsfähigkeit verletzen. Die Steuergerechtigkeit gilt danach kategorisch; Ausnahmen werden nicht toleriert. Dieser Auffassung zufolge dürfte damit auch nicht ökologisch gelenkt werden.

- 2. Position: Zwischen Lenkungsnormen und Sozialzwecknormen muss nicht getrennt werden: Das Steuerrecht wird als „legitimes Lenkungsinstrument“ der Wirtschafts- und Sozialpolitik angesehen. Durchbrechungen des Grundsatzes der Gleichmäßigkeit der Besteuerung sind gerechtfertigt, wenn diese Verletzungen einem besonderen 
wirtschaftlichen oder sozialen Zweck dienen, weil Willkür dann auszuschließen ist. Diese Position nimmt u.a. das Bundesverfassungsgericht in seinen bisherigen Urteilen ein. Der wirtschaftliche oder soziale Zweck wird aber nicht gewichtet. Ob die Verfolgung des wirtschaftlichen oder sozialen Zwecks gerechtfertigt, d.h. erforderlich, geeignet und verhältnismäßig ist, wird nicht überprüft.

- 3. Position: Zwischen Lenkungs- und Sozialzwecknormen muss strikt getrennt werden. Lenkungsnormen haben danach mit der eigentlichen Besteuerung nichts $\mathrm{zu}$ tun und bilden mit den eigentlichen Besteuerungsnormen, den Fiskalzwecknormen lediglich eine organisatorische Einheit. Steuervergünstigungen in Form von Lenkungsnormen müssten deshalb darauf überprüft werden, ob sie für ihren Zweck erforderlich, geeignet und verhältnismäßig sind.

- 4. Position: Zwischen Lenkungs- und Sozialzwecknormen ist zwar zu trennen. Jedoch ist abzuwägen, ob das Gemeinwohlinteresse an der Durchsetzung der Lenkungsmaßnahme den Einbruch in die Leistungsfähigkeitsbesteuerung rechtfertigt. Die Wirkungen, die mit der Lenkungsmaßnahme erzielt würden, sind folglich mit der Intensität der Verletzung des Leistungsfähigkeitsprinzips abzuwägen.

Die Darstellungen zeigen, dass schon in der Grundposition bezüglich der Rechtmäßigkeit der Existenz von Steuervergünstigungen unter den Steuerrechtlern große Differenzen bestehen. Wichtig erscheint dabei aber, dass das Bundesverfassungsgericht als oberster Hüter der Verfassung Steuervergünstigungen als durchaus legitim ansieht. Für die Rechtfertigung des wirtschaftlichen oder sozialen Zwecks schlägt TIPKE (2000, 340ff) vor, folgende Kriterien bei einer steuerrechtlichen Rechtfertigung von Steuervergünstigungen zu beachten:

1. Eine Steuervergünstigung muss im Interesse des Gemeinwohls ein Bedürfnis befriedigen oder ein bestimmtes Verhalten prämieren. Dem Gemeinwohl ist gedient, wenn die Steuervergünstigung $\mathrm{zu}$ einem gesamtgesellschaftlichen Nutzen führt, d.h. die Lebensgrundlagen des Gemeinwesens und seiner Mitglieder festigt, erhält oder verbessert.

2. Eine Steuervergünstigung muss zur Erreichung des angestrebten Gemeinwohlzwecks erforderlich, geeignet und verhältnismäßig sein. Neben der Frage nach der grundsätzlichen Notwendigkeit einer Subventionierung stellt sich damit auch die Frage nach der Wirkungsrichtung sowie der Wirkungsintensität der Steuervergünstigung. 
3. Bei einer Steuervergünstigung muss stets abgewogen werden zwischen dem Gemeinwohlgewicht der gleichen Steuerbelastung und dem Gemeinwohlgewicht der Steuervergünstigung. D.h. auch wenn feststeht, dass eine Steuervergünstigung dem Gemeinwohl dient und zu diesem Zweck auch erforderlich, geeignet und verhältnismäßig ist, ist noch $\mathrm{zu}$ prüfen, ob die Steuervergünstigung $\mathrm{zu}$ einem größeren gesamtgesellschaftlichen Nutzen führt als die gleichmäßige Besteuerung entsprechend der Leistungsfähigkeit.

Damit sind die Anforderungen, die im Allgemeinen an die Rechtfertigung von Lenkungsnormen gestellt werden, klar formuliert. Die Frage, ob im Sinne des 1. Kriteriums die mit dem ökologischen Landbau verfolgten Ziele grundsätzlich zur Rechtfertigung einer Lenkungsnorm im Steuerrecht genügen, kann nur unter Rückgriff auf Kapitel 2.1 beantwortet werden. Dort ist mit dem Hinweis auf die unbestritten positiven Umwelteffekte des ökologischen Landbaus versucht worden, den grundsätzlichen Eingriff des Staates gemäß der Wohlfahrtstheorie mit einem unvollständigen Funktionieren bzw. einem vollständigen Versagen des Marktes im Bereich der „Öffentlichen Güter“ zu rechtfertigen. Sollten grundsätzlich staatliche Maßnahmen zugunsten des ökologischen Landbaus im Rahmen der Erreichung gesellschaftlicher Ziele verantwortbar sein, so müssten auch Subventionen in Form von Steuervergünstigungen im Sinne des 1. Kriteriums zu rechtfertigen sein, denn bereits für die grundsätzliche Rechtfertigung von Fördermaßnahmen ist maßgeblich, dass sie zu einem gesamtgesellschaftlichen Nutzen führen, d.h. die Lebensgrundlagen des Gemeinwesens und seiner Mitglieder festigen, erhalten oder verbessern. In diesem Sinne wären also auch staatliche Subventionen in Form von Steuervergünstigungen zugunsten des ökologischen Landbaus gerechtfertigt.

Damit ist jedoch noch nicht geklärt, ob im Sinne des 2. sowie des 3. Kriteriums steuerliche Lenkungsnormen gerechtfertigt wären. Beide Kriterien beziehen sich jedoch im Unterschied zum 1. Kriterium auf die Wirkung der einzelnen spezifischen Steuervergünstigung und nicht mehr auf Steuervergünstigungen zugunsten des ökologischen Landbaus generell. Deshalb sind diese Kriterien unmittelbar in das in Kapitel 3.2.1 konzipierte Kriterium zur Beurteilung der Wirkung steuerlicher Förderalternativen eingegangen. Bevor darauf näher Bezug genommen wird, soll zunächst überlegt werden, im Rahmen welcher Steuerarten Steuervergünstigungen zugunsten des ökologischen Landbaus überhaupt sinnvoll erscheinen. 


\subsubsection{GrundSATZÜBERLEgUngen ZUR AUSWAHL STEUERLICHER FÖRDERINSTRUMENTE}

Bei der Auswahl steuerlicher Förderinstrumente stellt sich zu Beginn die Frage, welche Steuerarten in Hinblick auf ihre Charakteristika überhaupt zur Förderung des ökologischen Landbaus infrage kommen.

$\mathrm{Zu}$ diesem Zweck muss überlegt werden, welche grundsätzlichen Anforderungen eine steuerliche Förderung überhaupt erfüllen muss. Abbildung 7 zeigt dazu den angewandten Auswahlprozess bei der Eingrenzung der für eine steuerliche Förderung des ökologischen Landbaus in Betracht kommenden Steuerarten.

Abbildung 7: Auswahlprozess der für eine steuerliche Förderung infrage kommenden Steuerarten

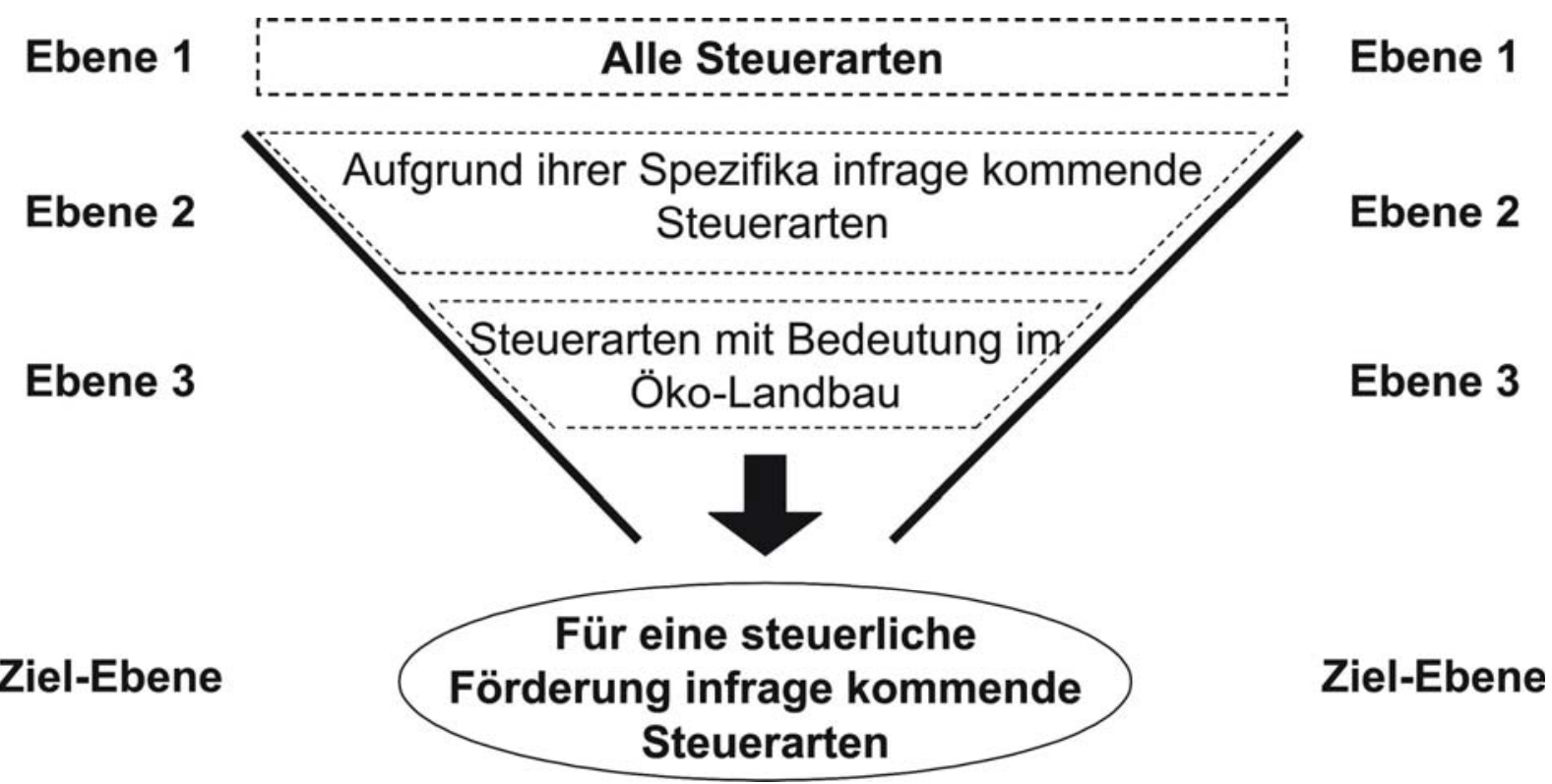

Quelle: Eigene Darstellung

Danach kommen für die zweite Ebene nur Steuerarten in Betracht, die aufgrund ihrer Spezifika für eine steuerliche Förderung geeignet erscheinen. Vorrangiges Kriterium ist dabei eine kontinuierliche regelmäßige Besteuerung. Daher sind die Grunderwerbssteuer sowie die Erbschaft- und Schenkungsteuer bei der Auswahl geeigneter steuerlicher Förderalternativen im Vorhinein auszuschließen, da sie für den ökologisch wirtschaftenden Landwirt nur unregelmäßig anfallen. Eine regelmäßige kontinuierliche Besteuerung ist ein notwendiges Kriterium für die Auswahl geeigneter Steuerarten, weil auch der Fördereffekt regelmäßig anfallen soll. Ein unregelmäßig anfallender Fördereffekt würde zu Ungerechtigkeiten führen 
und hätte keine Aussicht, als vollwertiges Alternativinstrument zur gegenwärtigen Angebotsförderung anerkannt zu werden.

Die verbleibenden Steuerarten müssen schließlich einen weiteren Auswahlschritt durchlaufen, um in die 3. Ebene Einzug zu halten. So ist ein weiteres notwendiges Kriterium, dass es sich um eine Steuerart handelt, die für möglichst viele ökologisch wirtschaftende Landwirte auch finanzielle Bedeutung hat. Eine Steuervergünstigung im Rahmen einer Steuerart, die für die Mehrzahl der ökologisch wirtschaftenden Landwirte gar nicht zur Zahlung in Betracht kommt, weil diese die Voraussetzungen für eine Besteuerung nicht erfüllen, könnte keinen wirksamen Beitrag zur Ausweitung des ökologischen Landbaus leisten und würde vermutlich lediglich Fehlallokationen von Fördermitteln nach sich ziehen. Aus diesem Grund muss auf dieser Auswahlebene die Gewerbesteuer aussortiert werden. Sie hat nur für wenige Landwirte praktische Bedeutung. Steuervergünstigungen innerhalb dieser Steuerarten würden daher nur einzelnen Betrieben zugute kommen. Die sich ergebende Auswahl der Förderungsempfänger wäre im Hinblick auf die gesellschaftlichen Leistungen der Betriebe nicht sachgemäß und deshalb auch nicht zu rechtfertigen.

Aus den ursprünglich auf Ebene 1 in Betracht kommenden Steuerarten der Tabelle 4 verbleiben damit für Überlegungen hinsichtlich einer steuerlichen Förderung des ökologischen Landbaus lediglich noch die Grundsteuer, die Ertragsteuern Einkommen- und Körperschaftsteuer, die Umsatzsteuer sowie sonstige Verbrauchssteuern.

\subsection{KRITERIEN BEI DER BEURTEILUNG VON STEUERLICHEN FÖRDERALTERNATIVEN}

Nach den Überlegungen im Vorkapitel hinsichtlich der für eine Förderung grundsätzlich infrage kommenden Steuerarten soll in diesem Kapitel aufgezeigt werden, welche Anforderungen an steuerliche Förderinstrumente $\mathrm{zu}$ stellen sind. Dazu sind wichtige Erfolgskriterien festgelegt worden, die neben der grundsätzlichen Durchführbarkeit vor allem auch die spezifischen Wirkungen umfassen.

\subsubsection{WIRKUNG DER FÖRDERUNG}

In Kapitel 2.4 ist die gegenwärtige flächenbezogene Angebotsförderung bezüglich ihrer Wirkungen untersucht worden. Dabei standen die Effektivität, d.h. der Zielerreichungsgrad, sowie die Finanzmitteleffizienz als elementare Erfolgskennziffern im Mittelpunkt der 
Betrachtungen. Wenn über alternative Förderinstrumente nachgedacht wird, so müssen diese ebenfalls hinsichtlich beider Kriterien beurteilt werden. Dieses ist umso wichtiger, da schon im Rahmen der Zielstellung dieser Arbeit eine Verbesserung der Finanzmitteleffizienz der Förderung des ökologischen Landbaus betont wurde.

Die Beurteilung steuerlicher Förderinstrumente hinsichtlich ihrer Auswirkungen soll dabei in drei Schritten erfolgen.

Zum einen soll der Beitrag des steuerlichen Förderinstrumentes zur Erreichung des mit der Förderung verfolgten Zieles untersucht werden. In Kapitel 2.1 war als vorrangiges Ziel der Förderung des ökologischen Landbaus dessen Ausweitung mit der Begründung benannt worden, dass sich daran die Erreichung verschiedener gesellschaftlicher Ziele anknüpft. Zur Beurteilung eines steuerlichen Förderinstruments hinsichtlich seiner Effektivität, d.h. seines Zielerreichungsgrades wäre deshalb eigentlich eine Simulation der weiteren Flächenentwicklung des Ökolandbaus und zwar mit und ohne Einsatz des untersuchten Instruments notwendig. Eine derartige Verfahrensweise wäre jedoch methodisch sehr aufwendig und zugleich der Zielstellung dieses Kapitels nicht angemessen. So sollen im Folgenden zunächst in Fachkreisen bereits diskutierte Instrumente untersucht werden. Bezüglich der Effektivität der Förderung sollen deshalb im Einzelnen jeweils die zu erwartenden betriebswirtschaftlichen Auswirkungen für den Antrag stellenden ökologisch wirtschaftenden Landwirt analysiert werden. Zur Beurteilung der Effizienz sollen in einem zweiten Schritt die bei Einführung des spezifischen steuerlichen Instruments zusätzlich zu erwartenden Finanzmittelausgaben des Staates untersucht werden. Zusammen mit den Ergebnissen des vorangegangenen Schrittes sollten sie in einem dritten Schritt eine erste Einschätzung der Finanzmitteleffizienz zulassen, um letztendlich ein Fazit zur Wirkung des steuerlichen Instruments formulieren zu können.

Hinsichtlich der letztendlichen Entscheidung für oder gegen das spezifische steuerliche Förderinstrument muss mit Bezug auf die Wirkungsanalyse schlussendlich folgende Handlungsmaxime gelten: Eine Änderung der Gestaltung der Angebotsförderung des ökologischen Landbaus kann nur sinnvoll sein, sofern sich dadurch deutliche Verbesserungen hinsichtlich der Effektivität bzw. der Effizienz der Förderung ergeben. Ein umfassender und zielgerichteter Fördereffekt ist auch deshalb eine notwendige Bedingung bei der Auswahl Erfolg versprechender Fördermaßnahmen, weil jede Änderung der Förderpraxis die Anstrengungen aller Beteiligten auch in administrativer Hinsicht erfordert. Diese im Falle 
einer Reform der Förderung zu erwartenden neu entstehenden Transaktionskosten sollen im folgenden Kapitel thematisiert werden.

\subsubsection{TRANSAKTIONSKOSTEN}

Transaktionskosten können definiert werden als Summe von Informations-, Administrations-, Kontroll- und Durchsetzungskosten. Sie können mitunter einen erheblichen Anteil an den Gesamtkosten von Förderprogrammen ausmachen und gelten daher als ein wichtiges Kriterium für die Vorteilhaftigkeit von Politikmaßnahmen (FALCONER und WhITBY, 1999; VATN et al., 2002). Abbildung 8 verdeutlicht dazu schematisch das Vorhandensein von Transaktionskosten sowohl beim teilnehmenden Landwirt als auch auf der Seite des Staates.

Abbildung 8: Einkommensverluste, Fördersumme und Transaktionskosten von Agrarumweltprogrammen

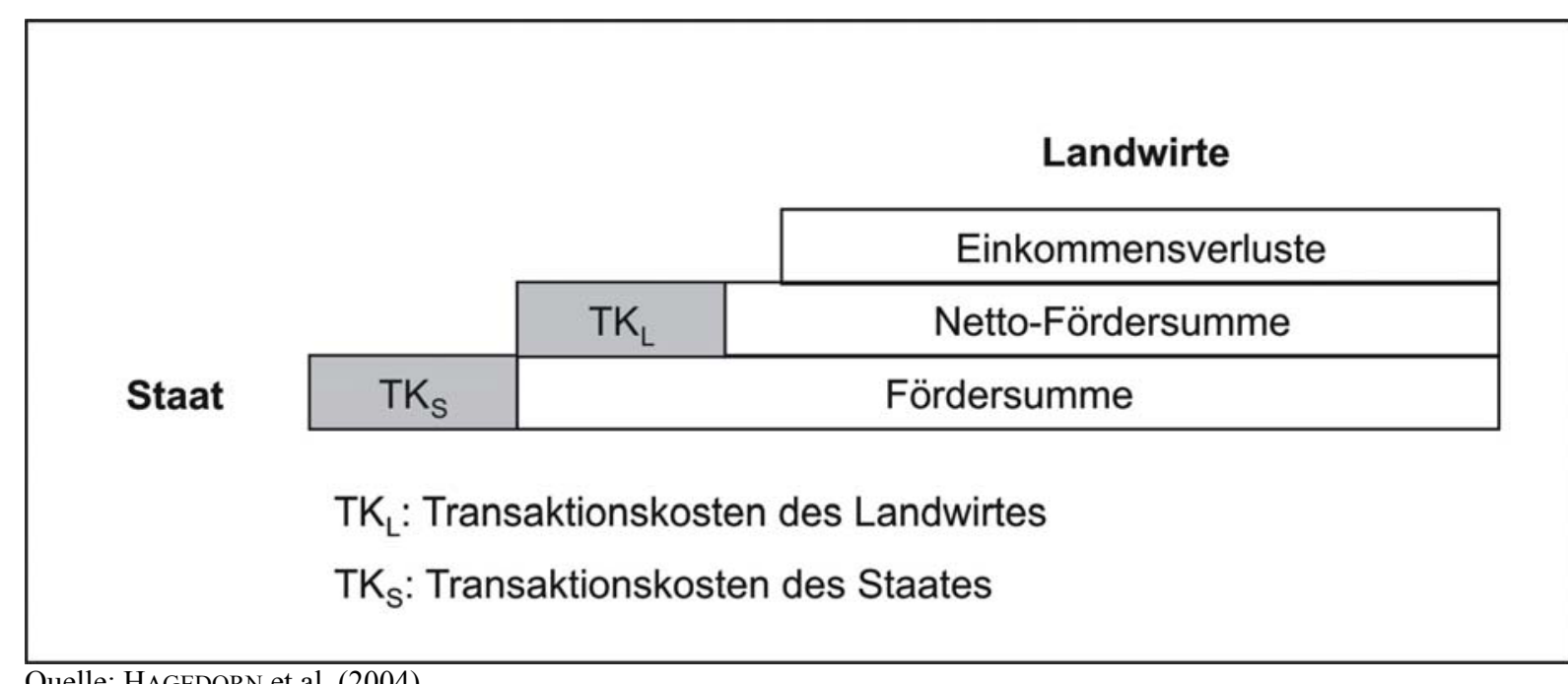

Quelle: HAGEDORN et al. (2004)

Auf Seiten des Staates erhöhen sich durch Verwaltungs- und Kontrollkosten die Gesamtausgaben für agrarumweltpolitische Maßnahmen. Auf Seiten der Landwirte verringern sich durch Informations- und Administrationsaufwendungen die Nettoförderung und der Anreiz zur Teilnahme an Fördermaßnahmen.

Wie bereits in Kapitel 3.2.1 angedeutet wurde, darf deshalb bei der Beurteilung von Förderinstrumenten nicht allein auf eine möglichst hohe Zielerreichung der Fördermaßnahme abgestellt werden („Umsetzungseffizienz“). Weil nämlich auch die Durchführungskosten berücksichtigt werden müssen, stellt eine möglichst einfache Administrierbarkeit der Förderung ein weiteres wichtiges Kriterium dar (,Verwaltungseffizienz“). Denn ein erhöhter Verwaltungsaufwand kann sonstige Effektivitäts- und Effizienzgewinne bei der Förderung schnell zunichte machen. Deshalb sollte auch bei der Beurteilung der zur Auswahl stehenden 
steuerlichen Förderinstrumente hinsichtlich der entstehenden Transaktionskosten folgender Grundsatz Geltung haben: Nur wenn die Verwaltungskosten für Landwirte und für die mit der Förderung betrauten staatlichen Organe im Vergleich zu den sonstigen Vorzügen einer geänderten Förderung verhältnismäßig und vertretbar bleiben, kann ein steuerliches Förderungsinstrument ernsthaft als Alternative zur derzeitigen Angebotsförderung in Erwägung gezogen werden.

Zur Untersuchung der entstehenden Transaktionskosten sollen zu den einzelnen steuerlichen Förderinstrumenten daher Überlegungen $\mathrm{zu}$ deren verwaltungstechnischer Umsetzung angestellt werden. Auf dieser Basis soll schließlich eine Beurteilung sowohl des bei der Einführung entstehenden sowie auch des nach Einführung laufenden Verwaltungsaufwands ermöglicht werden.

Unabhängig vom spezifischen steuerlichen Instrument lässt sich jedoch schon vorab sagen, dass speziell in Deutschland mit den fortgeschrittenen Möglichkeiten im Bereich der EDV sowie des rechtlich und administrativ ausgereiften Steuersystems grundsätzlich eine gute Basis für eine steuerliche Förderung des Ökolandbaus vorliegt. Hinzu kommt, dass im Zuge der ab 2007 geltenden EU-Rahmenbedingungen Transaktionskosten bei der Bemessung der Förderhöhe Berücksichtigung finden dürfen. Die EU beteiligt sich damit im Rahmen der Kofinanzierung an den entstehenden Transaktionskosten. Dies könnte u.U. entgegen der oben vorgeschlagenen Handlungsmaxime eine geringere Gewichtung der Transaktionskosten als Entscheidungskriterium bei der Auswahl infrage kommender steuerlicher Förderinstrumente rechtfertigen. Diesbezüglich sind jedoch zunächst die genauen Handlungsanweisungen in der Durchführungsverordnung zur ELER-Verordnung abzuwarten.

\subsubsection{GENEHMIGUNGS- UND KOFINANZIERUNGSFÄHIGKEIT GEMÄß DER EU- RAHMENGESETZGEBUNG}

Neben der grundsätzlichen Genehmigungsfähigkeit einer in Erwägung gezogenen steuerlichen Fördermaßnahme wird auch eine Kofinanzierung durch die EU unerlässlich sein. Ansonsten würden sich für Bund und Länder nicht akzeptable zusätzliche finanzielle Belastungen ergeben. Sollte sich im Rahmen der Untersuchungen die Kofinanzierung der EU bei einem diskutierten Förderinstrument daher als wenig wahrscheinlich herausstellen, so wäre dieses bereits ein hinreichender Grund für den Ausschluss des betreffenden Instruments von weitergehenden Überlegungen. Deshalb ist also zunächst im Einzelfall zu prüfen, ob mit einer Kofinanzierung der EU zu rechnen ist. Da mit der neuen Finanzplanungsperiode 2007 
bis 2013 die derzeitig gültige VO (EG) 1257/99 von der VO (EG) 1698/2005 (ELER-VO) abgelöst wird und die Bundesländer ihre Entwicklungsprogramme für den ländlichen Raum folglich ab 2007 auch an dieser Nachfolgeverordnung auszurichten haben, sollen sich diese Untersuchungen auch an dieser zukünftig gültigen Verordnung orientieren.

In Kapitel 2.3.2.1 wurden die derzeit gültigen Rahmenbedingungen für die flächenbezogene Förderung des ökologischen Landbaus auf Ebene der EU bereits erläutert. Für den weiteren Fortgang der flächenbezogenen Förderung sind nun die Änderungen interessant, die sich aus dem Wechsel der Grundlagenverordnung für die Programmplanung der Bundesländer ergeben. Dazu wird zunächst der für die Förderung des ökologischen Landbaus zukünftig relevante Verordnungstext aus der VO (EG) 1698/2005 an dieser Stelle zitiert:

\author{
VERORDNUNG (EG) Nr. 1698/2005 DES RATES \\ vom 20. September 2005 \\ über die Förderung der Entwicklung des ländlichen Raums durch den Europäischen \\ Landwirtschaftsfonds für die Entwicklung des ländlichen Raums (ELER)
}

Schwerpunkt 2: Verbesserung der Umwelt und der Landschaft

Artikel 39

Zahlungen für Agrarumweltmaßnahmen

(1) Die Mitgliedstaaten bieten die Beihilfen gemäß Artikel 36 Buchstabe a Ziffer IV in ihrem gesamten Hoheitsgebiet entsprechend den spezifischen Bedürfnissen an.

(2) Zahlungen für Agrarumweltmaßnahmen werden Landwirten gewährt, die freiwillig eine Agrarumweltverpflichtung eingehen. Soweit dies zur Erreichung der Umweltziele gerechtfertigt ist, können die Zahlungen für Agrarumweltmaßnahmen anderen Landbewirtschaftern gewährt werden.

(3) Die Zahlungen für Agrarumweltmaßnahmen betreffen nur die Verpflichtungen, die über die einschlägigen obligatorischen Grundanforderungen gemäß den Artikeln 4 und 5 und den Anhängen III und IV der Verordnung (EG) Nr. 1782/2003 sowie die Grundanforderungen für die Anwendung von

Düngemitteln und Pflanzenschutzmitteln und sonstige einschlägige verpflichtende Anforderungen hinausgehen, die im Rahmen von einzelstaatlichen Rechtsvorschriften festgelegt und in dem betreffenden Programm aufgeführt sind. Diese Verpflichtungen sind in der Regel für einen Zeitraum von fünf bis sieben Jahren einzugehen. Sofern erforderlich und begründet, wird für bestimmte Arten von Verpflichtungen nach dem in Artikel 90 Absatz 2 genannten Verfahren ein längerer Zeitraum festgelegt.

(4) Die Zahlungen werden jährlich gewährt und dienen zur Deckung der zusätzlichen Kosten und der Einkommensverluste infolge der eingegangenen Verpflichtungen. Gegebenenfalls können auch

Transaktionskosten gedeckt werden. Die Begünstigten können gegebenenfalls über eine Ausschreibung unter Anwendung von wirtschaftlichen und ökologischen Effizienzkriterien ausgewählt werden.

Beihilfehöchstbeträge:

Einjährige Kulturen: $600 €$ je ha

Mehrjährige Sonderkulturen: $900 €$ je ha

Sonstige Flächennutzung: $450 €$ je ha

Lokale Tierrassen: $200 €$ je GVE

In Ausnahmefällen können diese Höchstbeträge unter Berücksichtigung besonderer Umstände, die in den Entwicklungsprogrammen für den ländlichen Raum zu begründen sind, angehoben werden.

(5) Die Beihilfen können für nicht unter die Absätze 1 bis 4 fallende Maßnahmen zur Erhaltung genetischer Ressourcen in der Landwirtschaft gewährt werden. 
Artikel 70

Beteiligung des Fonds

(1) In der Entscheidung zur Genehmigung eines Entwicklungsprogramms für den ländlichen Raums wird die Höchstbeteiligung des ELER für jeden Schwerpunkt innerhalb einer Flexibilitätsobergrenze festgesetzt, die nach dem in Artikel 90 Absatz 2 genannten Verfahren festzulegen ist. Die etwaigen

Mittelzuweisungen für die im Rahmen des Konvergenzziels förderfähigen Regionen werden in der Entscheidung gesondert ausgewiesen.

(2) Die Beteiligung des ELER wird auf der Grundlage der zuschussfähigen öffentlichen Ausgaben berechnet.

(3) Der Beteiligungssatz des ELER wird auf die Schwerpunkte bezogen festgelegt.

a) (...).

b) Für den Schwerpunkt 2 „Verbesserung der Umwelt (...) gelten jeweils folgende Obergrenzen:

i) $80 \%$ der zuschussfähigen öffentlichen Ausgaben in den Regionen nach dem Konvergenzziel,

ii) $55 \%$ der zuschussfähigen öffentlichen Ausgaben in den übrigen Regionen.

Der Mindestsatz der Beteiligung des ELER auf Ebene eines Schwerpunkts wird auf $20 \%$ festgelegt.

Verglichen mit der derzeitig gültigen VO (EG) 1257/99 haben sich dabei für die zukünftige Förderung des ökologischen Landbaus über die Agrarumweltprogramme folgende Änderungen ergeben:

- Die bisher gültige $20 \%$ ige Anreizkomponente wurde ersatzlos gestrichen. Damit dürfen die Prämien für die Empfänger keine Einkommenswirkungen mehr aufweisen. Stattdessen ist es nun aber den Bundesländern erlaubt, bei der Bemessung der Prämien Transaktionskosten zu berücksichtigen. Allerdings steht eine konkretisierende Definition der Transaktionskosten noch aus. Die Streichung der bisherigen Anreizkomponente ist als Zugeständnis an die WTOVerhandlungen zu sehen, wonach die Agrarumweltprogramme möglichst wenig handelsverzerrend wirken sollen.

- Unter Einschränkungen werden zukünftig auch Ausschreibungsverfahren zur Auswahl der Begünstigten zugelassen. Unter der Annahme, dass viele potenzielle Teilnehmer zu erwarten und Preisabsprachen nicht wahrscheinlich sind, könnte damit im Vergleich zu festgelegten Beihilfebeträgen ein höheres Maß an Marktverhalten und damit ein effizienterer Mitteleinsatz zu erwarten sein. Auf eventuelle Probleme bei der Anwendung von Ausschreibungsverfahren im Zusammenhang mit der flächenbezogenen Förderung des ökologischen Landbaus ist in Kapitel 2.5 bereits hingewiesen worden.

- Die Höchstgrenzen für die EU-Kofinanzierungssätze sind mit der ELERVerordnung neu festgelegt worden. Gemäß der 1257/99 galten ursprünglich feste Kofinanzierungssätze von $75 \%$ für Ziel-1-Regionen und von $50 \%$ für die übrigen Regionen. Mit der zwischenzeitlichen Reform der VO (EG) 1257/99 im Rahmen der VO (EG) 1783/2003 sind sie auf die aktuell geltenden Sätze von 85 \% für Ziel- 
1-Regionen und von $60 \%$ für die übrigen Regionen angehoben worden, wobei es sich von nun an nicht mehr um feste Kofinanzierungssätze, sondern um Höchstgrenzen für eine EU-Kofinanzierung handelt. Diese Obergrenzen werden mit der ELER-VO wiederum gesenkt und zwar für Ziel-1-Regionen auf 80\% und für die übrigen Regionen auf $55 \%$. Darauf ist bereits im Zusammenhang mit der künftigen Finanzierung der flächenbezogenen Förderung in Kapitel 2.3.4 hingewiesen worden.

Unter Berücksichtigung dieser Veränderungen sind für die Beurteilung der Kofinanzierbarkeit von Fördermaßnahmen zugunsten des ökologischen Landbaus im Rahmen der VO (EG) 1698/2005 (ELER-VO) folglich drei Kriterien zu unterscheiden:

- Zur Inanspruchnahme der Kofinanzierung der EU ist die konkrete Nachvollziehbarkeit des absoluten jährlichen Fördervolumens notwendige Voraussetzung. Ansonsten kann der von der EU zu übernehmende Anteil an der Gesamtfinanzierung nicht ermittelt werden.

- In Artikel 39, Absatz 4 der ELER-VO wird die Kostenakzessorität bei der Bemessung der Förderprämien festgelegt, die besagt, dass sich die Förderprämie in ihrer Höhe an den individuellen Einkommensverlusten und Zusatzkosten bemessen soll, die dem Landwirt durch die Umstellung auf ökologischen Landbau bzw. durch die Beibehaltung des ökologischen Landbaus entstehen zuzüglich der entstehenden Transaktionskosten. Auch im Falle einer steuerlichen Förderung muss die Kostenakzessorität daher gewährleistet sein.

- Artikel 39, Absatz 4 der ELER-VO benennt zusätzlich Höchstgrenzen für die jährliche Förderung, die sich auf einen Hektar Fläche beziehen. Dieses könnte auf eine gewünschte Flächenakzessorität der Förderung hindeuten. Eine Kopplung der Förderung an die bewirtschaftete Fläche erscheint im Übrigen auch aus dem Grund sinnvoll, da die positiven externen Effekte des ökologischen Landbaus auch auf der Fläche auftreten. Gleichzeitig wird aber im Verordnungstext auch eindeutig darauf hingewiesen, dass in Ausnahmefällen unter Berücksichtigung besonderer Umstände diese Höchstgrenzen hinfällig sein können. Hier liegt die Vermutung nahe, dass in begründeten Ausnahmefällen, wie bspw. einem Förderinstrument, das $\mathrm{zu}$ einer effektiveren Förderung beiträgt, eine Flächenakzessorität entfallen kann. Daher sollte davon ausgegangen werden, dass 
betriebsbezogene oder personenbezogene Zahlungen dem Verordnungstext nicht grundsätzlich widersprechen.

So bleibt festzuhalten, dass bezüglich der Einschätzung der EU-Kofinanzierungsmöglichkeit eines Förderinstrumentes gemäß der zukünftig geltenden ELER-Verordnung zum einen das jährliche Fördervolumen eindeutig quantifizierbar sein muss und zum anderen die Kostenakzessorität hinsichtlicht des jährlichen Prämienniveaus gewährleistet sein muss. Eine Flächenakzessorität kann als wünschenswert erachtet werden. Sie wird aber aller Voraussicht nach einer sachgerechteren Förderung nicht im Weg stehen.

\subsection{BISLANG DISKUTIERTE FÖRDERALTERNATIVEN IM RAHMEN DER STEUERGESETZE UND ABSCHÄTZUNG IHRER JEWEILIGEN ANWENDUNGSPROBLEME}

Im vorangegangen Kapitel 3.2 wurden wichtige Erfolgskriterien für eine steuerliche Förderung des Ökolandbaus aufgezeigt. In diesem Kapitel sollen nun mögliche Förderalternativen dargestellt und in Hinblick auf diese Erfolgskriterien beurteilt werden. Dabei handelt es sich ausschließlich um steuerliche Förderinstrumente, die in der Öffentlichkeit als Alternativen zur Förderung des ökologischen Landbaus bereits mehrfach diskutiert wurden.

\subsubsection{DIFFERENZIERUNG DER BESTEHENDEN FLÄCHENPRÄMIEN NACH DEN ERTRAGSMESSZAHLEN (EMZ) DES STEUERLICHEN BEWERTUNGSGESETZES}

Als ersten Vorschlag für eine steuerliche Förderung soll im vorliegenden Kapitel die Differenzierung der bestehenden Flächenprämien nach den Ertragsmesszahlen (EMZ) des steuerlichen Bewertungsgesetzes untersucht werden. Die Ertragsmesszahl als Maßzahl für die natürlichen Standortbedingungen entstammt dem Bewertungsgesetz in Verbindung mit dem Bodenschätzungsgesetz und damit der steuerrechtlichen Gesetzgebung. Sie wurde ursprünglich zur Flächenbewertung im Steuerrecht eingeführt und ist somit auch ein steuerrechtliches Instrument. Eine zusätzliche Differenzierung der Öko-Flächenprämien nach der Ertragsfähigkeit des Standortes ist in den vergangenen Jahren bereits wiederholt vorgeschlagen und diskutiert worden (vgl. dazu KACHEL, 1999 oder BAUDOUX, 2001). 
- Wirkung der Förderung:

Als Auswirkung einer zusätzlichen Differenzierung der bestehenden Öko-Flächenprämien gemäß der Ertragsmesszahl (EMZ) wäre von einer gezielteren Förderung gemäß den einzelbetrieblichen standortabhängigen Einkommensverlusten auszugehen. Der Grund für diese Annahme liegt in der Tatsache, dass die Umstellung bzw. Beibehaltung des ökologischen Landbaus auf einem ertragsstarken Standort tendenziell mit höheren Einkommensverlusten verbunden ist als auf einem ertragsschwachen Standort. Dies würde einen höheren monetären Ausgleich auf dem ertragsstarken Standort rechtfertigen (vgl. dazu auch NiEBERG (2004: 7)).

Ein zweiter positiver Effekt einer zusätzlichen Differenzierung der bestehenden ÖkoFlächenprämien nach der EMZ, der allerdings unmittelbar aus dem Erstgenannten folgt, besteht in der Erhöhung der Maßnahmenakzeptanz speziell auf ertragsstärkeren Standorten. Derzeit verzeichnen wir vor allem in Ostdeutschland eine starke Konzentration des Ökolandbaus in landwirtschaftlich benachteiligten Regionen. Gemäß einer im letzten Jahr von der Bundesforschungsanstalt für Landwirtschaft (FAL) in Braunschweig vorgestellten Studie hat sich diese Konzentration in den vergangenen Jahren sogar noch deutlich verstärkt (OSTERBURG und ZANDER, 2004: 27). Danach besteht speziell in Ostdeutschland (abgeschwächt jedoch auch in Westdeutschland) eine hoch signifikante negative Korrelation zwischen der Ertragsfähigkeit der Böden und der Umstellungsbereitschaft der Landwirte (BICHLER et al., 2003). Der Grund dafür ist im Rahmen verschiedener Studien schon vor einiger Zeit ermittelt worden. Er liegt in der Pauschalität der derzeitigen Prämiengestaltung, die eine erhöhte Wirtschaftlichkeit des Ökolandbaus auf schwächeren Standorten bewirkt (vgl. Kapitel 2.4 oder auch KÖHNE und KÖHN, 1998: 347 oder HAAS, 2005: 125). Eine Differenzierung gemäß EMZ würde damit die Ausweitung des Ökolandbaus speziell in Regionen vorantreiben, in denen der Ökolandbau derzeit unterrepräsentiert ist. Daraus könnten positive Umwelteffekte resultieren. Denn mit einer gleichmäßigeren Verteilung des ökologischen Landbaus würde sich zwangsläufig auch eine gleichmäßigere Verteilung der positiven Umwelteffekte des ökologischen Landbaus ergeben. Gleichzeitig könnten analog zu den Erkenntnissen aus Kapitel 2.4.2.2 Mitnahmeeffekte erfolgreich reduziert werden, da auf ertragsschwächeren Standorten Einkommenseffekte reduziert würden. Abhängig vom im nächsten Schritt zu analysierenden zusätzlichen Verwaltungsaufwand einer zusätzlichen Prämiendifferenzierung nach EMZ könnte die Fördereffizienz somit gesteigert werden. 
- Einschätzung des administrativen Aufwands für Förderungsempfänger und die mit der Förderung betraute behördliche Verwaltung:

Aufgrund ihrer allgemein breiten Verwendungspraxis ist die EMZ als Maßzahl für die natürlichen Standortbedingungen in der Fachwelt anerkannt. Eine Differenzierung nach EMZ ist bereits gängige Verwaltungspraxis bei einer Vielzahl anderer angebotener Agrarumweltmaßnahmen. Als Beispiele sind die Förderung der zehnjährigen Stilllegung in Bayern und Hessen sowie die Förderung der Umwandlung von Acker- in Grünland in Rheinland-Pfalz und Nordrhein-Westfalen zu nennen. Und nicht zuletzt handelt es sich bei der Ertragsmesszahl auch um eine in hohem Maße rechtssichere Größe, da sie sich direkt aus der steuerlichen Gesetzgebung ergibt. Insofern dürften sich für die Verwaltung keine deutlichen Kostensteigerungen ergeben. Auch für die Landwirte als Förderungsempfänger dürfte der zusätzliche administrative Aufwand gering sein. Die Beantragung im Rahmen des INVEKOS-Verfahrens fordert den Landwirt bereits zur Abgabe eines detaillierten Flächenverzeichnisses auf, das sowohl für die Direktzahlungen als auch für die Agrarumweltprogramme und den Vertragsnaturschutz Gültigkeit hat. Infolge der zusätzlichen Prämiendifferenzierung nach der EMZ wäre vom Landwirt im Flächenverzeichnis lediglich die Angabe der EMZ der Einzelflächen, die den Katasterunterlagen zu entnehmen ist, einzufordern.

- Einschätzung der Kofinanzierungsfähigkeit gemäß der EU-Rahmenbedingungen

Im Vergleich zur Referenzsituation ist von keinen problematischen Veränderungen hinsichtlich der in Kapitel 3.2.3 vorgestellten Kriterien Nachvollziehbarkeit des absoluten Fördervolumens, Kostenakzessorität und Flächenakzessorität auszugehen. Im Gegenteil: Unter Berücksichtigung der zukünftig entfallenden $20 \%$ igen Anreizkomponente würde ein infolge der zusätzlichen Differenzierung gemäß der EMZ stärker an den konkreten einzelbetrieblichen Einkommensverlusten orientierter Ausgleich im Sinne der Kostenakzessorität die Wahrscheinlichkeit für eine EU-Kofinanzierung sogar erhöhen. Eine Gefährdung der EU-Kofinanzierung kann deshalb ausgeschlossen werden.

- Fazit zur Beurteilung:

Wie die Ausführungen zeigen, könnte eine zusätzliche Differenzierung der bestehenden ÖkoFlächenprämien nach der Ertragsmesszahl eine deutliche Steigerung der Effizienz des Finanzmitteleinsatzes bewirken. Gleichzeitig erscheint auch die administrative Umsetzung 
realisierbar. Dieses Instrument ist daher schon zum gegenwärtigen Zeitpunkt als sehr interessante Weiterentwicklung des derzeitigen Fördersystems vorzumerken.

Die Tatsache, dass dieses Instrument jedoch schon mehrfach als Ergebnis wissenschaftlicher Untersuchungen zur Implementierung vorgeschlagen wurde und infolge dessen auch schon mehrfach diskutiert wurde, war Anlass dafür, die zusätzliche Prämiendifferenzierung gemäß Ertragsmesszahl im Rahmen dieses Forschungsprojektes nicht in den Vordergrund zu stellen.

\subsubsection{FREISTELLUNG ÖKOLOGISCH BEWIRTSCHAFTETER FLÄCHEN VON DER GRUNDSTEUER}

Als weiterer Vorschlag für eine steuerliche Förderung soll im Folgenden die Freistellung ökologisch bewirtschafteter Flächen von der Grundsteuer untersucht werden, die sich thematisch direkt an die Prämiendifferenzierung gemäß EMZ anschließt. Über die Grundsteuer ist in den vergangenen Jahren eine intensiv geführte Reformdiskussion entbrannt. Neben einigen anderen Vorschlägen hat besonders der im Auftrag des Umweltbundesamtes erarbeitete Vorschlag von BIZER und LANG (2000) Bekanntheit erlangt, die gegenwärtige Grundsteuer in eine allgemeine Flächennutzungssteuer umzuwandeln. Vorgesehen ist dabei u.a. eine Einteilung der Flächennutzungsarten nach ökologischen Gesichtspunkten in Steuerklassen mit dem Ziel, ökologisch schonende Nutzungsarten steuerlich zu entlasten sowie schädliche Nutzungsarten stärker $\mathrm{zu}$ belasten. Gemäß dem offiziellen Änderungsvorschlag würde damit auch der ökologische Landbau im Vergleich zur gegenwärtigen Besteuerung steuerlich entlastet. Bedenkt man das vergleichsweise geringe Grundsteueraufkommen von etwa 10 Mrd. $€$ in 2005 insgesamt (BMF, 2005), könnte man sogar noch weiter gehen und die Forderung aufstellen, den ökologischen Landbau gänzlich von der Grundsteuer zu befreien, zumal das in 2005 auf die Grundsteuer A entfallende Aufkommen lediglich 342 Mio. $€$ betrug (ebenda). Diesem Vorschlag soll im weiteren Verlauf dieses Kapitels nachgegangen werden.

- Wirkung der Förderung:

Bei Überlegungen hinsichtlich einer vollständigen Befreiung ökologisch bewirtschafteter Flächen von der Grundsteuer ist zu bedenken, dass derzeit nur in den neuen Bundesländern auch der Bewirtschafter mit dem Steuerschuldner identisch ist. In Westdeutschland, wo die Grundsteuer in den meisten Fällen vom Grundstückseigentümer entrichtet wird, würden also von diesem Instrument in erster Linie die Verpächter von Flächen begünstigt, die ihr Land an 
einen Öko-Betrieb verpachtet haben. Es wäre allerdings überaus wahrscheinlich, dass eine Befreiung von der Grundsteuer automatisch in die Verhandlungen zwischen Pächter und Verpächter um den Pachtpreis einfließen würde. Sofern es sich bei den Mitbewerbern um konventionell wirtschaftende Landwirte handelt, hätte der ökologisch wirtschaftende Pächter vermutlich die bessere Verhandlungsposition, da nur die Bewirtschaftung durch ihn in seiner Eigenschaft als ökologisch wirtschaftender Landwirt für den Verpächter die Befreiung von der Grundsteuer zur Folge hätte. Somit wäre davon auszugehen, dass er voraussichtlich zumindest einen Teil der ersparten Grundsteuer über einen niedrigeren Pachtpreis für sich vereinnahmen könnte und die Überwälzungseffekte (vgl. dazu u.a. FUCHS, 2002, 396 ff.) dieser Förderung auf den Verpächter somit nur gering wären.

Um die finanzielle Belastung landwirtschaftlicher Betriebe durch die Grundsteuer A und damit auch die finanzielle Entlastung bei Wegfall der Grundsteuer zu vergegenwärtigen, ist in Tabelle 5 differenziert nach unterschiedlichen Einheitswerten bzw. Ersatzwirtschaftswerten beispielhaft die jährliche Steuerbelastung für unterschiedliche Hebesätze berechnet worden.

Tabelle 5: Jährliche Grundsteuerbelastung ldw. Betriebe in $€$

\begin{tabular}{|c|rrrrrrr|}
\hline $\begin{array}{c}\text { GrSt- } \\
\text { Hebesatz }\end{array}$ & $\mathbf{1 0 . 0 0 0}$ & $\mathbf{5 0 . 0 0 0}$ & $\mathbf{1 0 0 . 0 0 0}$ & $\mathbf{2 0 0 . 0 0 0}$ & $\mathbf{5 0 0 . 0 0 0}$ & $\mathbf{1 . 0 0 0 . 0 0 0}$ & $\mathbf{2 . 0 0 0 . 0 0 0}$ \\
\hline $\mathbf{1 0 0 \%}$ & 60 & 300 & 600 & 1.200 & 3.000 & 6.000 & 12.000 \\
$\mathbf{1 5 0} \%$ & 90 & 450 & 900 & 1.800 & 4.500 & 9.000 & 18.000 \\
$\mathbf{2 0 0} \%$ & 120 & 600 & 1.200 & 2.400 & 6.000 & 12.000 & 24.000 \\
$\mathbf{2 5 0} \%$ & 150 & 750 & 1.500 & 3.000 & 7.500 & 15.000 & 30.000 \\
$\mathbf{3 0 0} \%$ & 180 & 900 & 1.800 & 3.600 & 9.000 & 18.000 & 36.000 \\
$\mathbf{3 5 0} \%$ & 210 & 1.050 & 2.100 & 4.200 & 10.500 & 21.000 & 42.000 \\
$\mathbf{4 0 0} \%$ & 240 & 1.200 & 2.400 & 4.800 & 12.000 & 24.000 & 48.000 \\
\hline
\end{tabular}

Quelle: Eigene Berechnung gemäß § 13-31 GrStG

Die jährlichen Beträge für die derzeitige Grundsteuerbelastung bedeuten im Umkehrschluss die finanzielle Förderung des Ökolandbaus bei Freistellung ökologisch bewirtschafteter Flächen.

Dabei zeigt sich, dass bei dieser steuerlichen Förderung ökologisch wirtschaftende Betriebe mit hohen Einheitswerten proportional stärker gefördert würden. Der Einheitswert ergibt sich jedoch in erster Linie aus dem Umfang und der Standortqualität der Eigentumsflächen des jeweiligen Betriebes. Bei Betrieben mit umfangreichen Eigenflächen bzw. mit hoch bonitierten Böden sind die Einheitswerte entsprechend höher als bei Betrieben mit geringen Eigenflächen oder niedrigeren EMZ/ha-Werten. Bei einer Förderung des ökologischen 
Landbaus durch Freistellung ökologisch bewirtschafteter Flächen bemisst sich daher die Förderung vorrangig am Flächenumfang sowie an der Standortqualität gemäß der EMZ.

Daneben ist zu beachten, dass die Hebesätze, die auf die Grundsteuermessbeträge angewendet werden, von den Kommunen unterschiedlich festgesetzt werden. Öko-Betriebe in Kommunen mit hohen GrSt-Hebesätzen profitieren daher stärker von einer GrSt-Freistellung. Dies werden tendenziell Öko-Betriebe in strukturstarken Regionen sein, weil im Allgemeinen dort die GrSt-Hebesätze höher sein könnten als in strukturschwachen Regionen. Damit würden bspw. Betriebe in den ländlichen Regionen Brandenburgs gegenüber Betrieben im näheren Umkreis von Berlin tendenziell benachteiligt.

Bei der Betrachtung der Wirkung einer Freistellung ökologisch bewirtschafteter Flächen von der Grundsteuer dürfen aber auch die zu erwartenden Steuerausfälle für den Staat nicht außer Acht gelassen werden. Tabelle 6 gibt einen Überblick über das Grundsteueraufkommen in Deutschland und wagt eine Schätzung für den voraussichtlich zu erwartenden Steuerausfall auf der Basis historischer Daten.

\section{Tabelle 6: Schätzung des Steuerausfalls für die Kommunen bei Freistellung ökologisch} bewirtschafteter Flächen in Deutschland

\begin{tabular}{|lr|}
\hline $\begin{array}{l}\text { Grundsteueraufkommen in Deutschland im Jahr 2004 (Quelle: Statistisches } \\
\text { Bundesamt: Finanzen und Steuern) }\end{array}$ & $9.938 .200 .000 €$ \\
\hline \multicolumn{1}{|c}{ davon Grundsteuer A } & $347.400 .000 €$ \\
\hline $\begin{array}{l}\text { Landwirtschaftlich genutzte Fläche in Deutschland im WJ 2003/04 in ha } \\
\text { (Quelle: BMELV Statistisches Jahrbuch über Ernährung, Landwirtschaft und } \\
\text { Forsten 2004, Tabelle 6) }\end{array}$ & 17.000 .000 \\
\hline $\begin{array}{l}\text { Berechnung: Durchschnittliches Grundsteueraufkommen pro ha LF in } \\
\text { Deutschland }\end{array}$ & $20,44 €$ \\
\hline $\begin{array}{l}\text { Nach VO 2092/91 ökologisch bewirt. Fläche in zum 31.12.2004 in ha (Quelle: } \\
\text { Meldung der Kontrollstellen nach VO 2092/91 zum Stichtag 31.12.2004) }\end{array}$ \\
\hline $\begin{array}{l}\text { Berechnung: Grundsteueraufkommen aus der gesamten ökologisch } \\
\text { bewirtschafteten Fläche in Deutschland }\end{array}$ \\
\hline $\begin{array}{l}\text { Geschätzter Steuerausfall für die Kommunen in Deutschland bei } \\
\text { Freistellung ökologisch bewirtschafteter Flächen von der }\end{array}$ \\
\hline \begin{tabular}{l} 
Grundsteuer: \\
\hline
\end{tabular}
\end{tabular}

Quelle: Eigene Darstellung

Demnach wäre rückwirkend für 2004 bei einer Freistellung ökologisch bewirtschafteter Flächen von einem Grundsteuerausfall von etwa 15,7 Mio. $€$ auszugehen.

Sehr problematisch ist diesbezüglich die Verteilung der Grundsteuereinnahmen und damit zwangsläufig auch der Grundsteuerausfälle einzuschätzen. Da nämlich die Einnahmen des 
Staates aus der Grundsteuer ausschließlich den Kommunen zufließen, würde eine Freistellung ökologisch bewirtschafteter Flächen dazu führen, dass die Finanzierungslast dieser Förderung von den Kommunen zu tragen wäre. Damit würden speziell Kommunen mit einem hohen Anteil ökologisch bewirtschafteter Flächen stärker belastet. Um die Finanzierung der Förderung in der bisherigen Struktur zu erhalten, müsste daher ein finanzieller Ausgleich für die Kommunen initiiert werden, so dass dieses neue Förderinstrument auf Seiten der Kommunen letztendlich zu keinen finanziellen Belastungen führen würde. Das könnte beispielsweise über Veränderungen bei der Verteilung der Einkommensteuereinnahmen funktionieren.

Weiterhin ist hinsichtlich der $\mathrm{zu}$ erwartenden Steuerausfälle $\mathrm{zu}$ beachten, dass die Berechnungen in Tabelle 6 lediglich die finanziellen Konsequenzen einer Freistellung ökologisch bewirtschafteter Flächen auf Bundesebene abschätzen. Genauso wie die Wirkung einer derartigen steuerlichen Förderung für die landwirtschaftlichen Betriebe vom jeweiligen GrSt-Hebesatz der Kommune abhängig wäre, wäre der zu erwartende Steuerausfall der Kommune vom Anteil des ökologischen Landbaus in der betreffenden Kommune abhängig. Damit wären die voraussichtlichen Steuerausfälle im östlichen Mecklenburg-Vorpommern, im südlichen Baden-Württemberg oder in Hessen deutlich höher als in Niedersachsen oder Nordrhein-Westfalen. Weil die Finanzplanung der Agrarumweltprogramme aber ohnehin im Entscheidungsbereich der Bundesländer liegt und damit bereits jetzt Bundesländer mit einem hohen Anteil ökologisch bewirtschafteter Flächen eine höhere Finanzlast tragen, resultieren daraus keine Veränderungen ${ }^{6}$.

Hinsichtlich der Effektivität einer Grundsteuerbefreiung ökologisch bewirtschafteter Flächen ist zu hinterfragen, ob die angesprochenen Wirkungen dieses Instruments auch wirklich den mit der Förderung verfolgten Zielen zuträglich sind. Dabei ist stets ein Vergleich zur Wirksamkeit der derzeitigen Förderung zu ziehen. So ist einerseits festzustellen, dass dieses Instrument hinsichtlich seiner Wirkung auf die Landwirte deutliche Parallelen zu der im Vorkapitel behandelten zusätzlichen EMZ-Differenzierung aufweist, denn auch hier ist die Intensität der Wirkung von den betriebs- und flächenindividuellen EMZ abhängig. Parallel zu den Interpretationen in Kapitel 3.3.1 besäße diese Wirkungsweise deutliche Potentiale zur Steigerung der Effektivität der Förderung. Anders verhält es sich dagegen mit der zweiten

\footnotetext{
${ }^{6}$ Durch die EU-Kofinanzierung sowie durch die finanzielle Beteiligung des Bundes im Rahmen der GAK wird der Effekt einer höheren finanziellen Belastung der Länderhaushalte infolge eines höheren Anteils ökologisch bewirtschafteter Flächen in den jeweiligen Bundesländern allerdings abgeschwächt.
} 
angesprochenen Wirkungsweise. Die Tatsache, dass die Intensität der Wirkung dieser steuerlichen Förderung für die Öko-Betriebe zusätzlich vom GrSt-Hebesatz der Kommune abhängt, führt zu einer in hohem Maße willkürlichen Wirkung dieses Förderinstruments. Denn zusätzlich zu der Tatsache dass, wie bereits erwähnt, ein Zusammenhang zwischen den Hebesätzen und der Infrastruktur der Kommune bestehen könnte, ist auch eine Abhängigkeit der Hebesätze von der finanziellen Gesamtsituation der Kommune wahrscheinlich. Sowohl aufgrund dieser Erkenntnisse als auch aufgrund der Unsicherheiten bezüglich der Überwälzung der Förderung auf den Bodeneigentümer ist es daher äußerst fraglich, ob mit einer derartig ausgestalteten steuerlichen Förderung im Vergleich zur Referenzsituation die Effektivität und damit auch die Effizienz der Förderung angehoben werden könnte.

- Beurteilung des administrativen Aufwands für den Förderungsempfänger und für die mit der Förderung betraute behördliche Verwaltung:

Zunächst wäre zur Einführung einer Freistellung ökologisch bewirtschafteter Flächen von der Grundsteuer eine Änderung der gesetzlichen Grundlage der Grundsteuer notwendig. So müsste $\S 4$ GrStG („Sonstige Steuerbefreiungen“) um einen Ausnahmetatbestand für die ökologisch bewirtschaftete Fläche ergänzt werden. Hinzu käme der zusätzliche kontinuierliche jährliche Aufwand für die Finanzverwaltung. Dazu sei im Vorhinein kurz der gegenwärtige Ablauf der Grundsteuerfestsetzung in Westdeutschland erläutert ${ }^{7}$ :

Zunächst wird der Einheitswert des zu besteuernden Objekts durch das Finanzamt, in dessen Bezirk der Grundbesitz liegt, ermittelt. Der Einheitswert umfasst neben dem Wohnungswert auch den Wirtschaftswert des landwirtschaftlichen Betriebs und damit zwangsläufig auch die Eigentumsflächen. Er wird dem Eigentümer mit dem Einheitswertbescheid mitgeteilt und gilt in der Regel für mehrere Jahre. Die Ermittlung des Grundsteuermessbetrages erfolgt auf der Grundlage des festgestellten Einheitswerts. Auch der Grundsteuermessbetrag wird dem Eigentümer durch Grundsteuermessbescheid mitgeteilt und gilt ebenfalls in der Regel für mehrere Jahre. Da der Grundsteuermessbescheid Grundlage für die Grundsteuerberechnung und -festsetzung durch die Kommunalverwaltung ist, erhält diese eine Durchschrift des Messbescheides. Sie ist an den Inhalt des Messbescheides verbindlich gebunden und kann deshalb die mitgeteilten Daten nicht verändern. Anhand der Daten des Grundsteuermessbescheides nimmt sie die Steuerberechnung vor und setzt den 
Grundsteuerbetrag mit dem Grundsteuerbescheid fest (vgl. zur Systematik der Einheitswertermittlung AlthOEFER et al. (2003: 500ff) oder KUßMAUL (2005: 107ff)).

Die Ausführungen zum Ablauf der Grundsteuerfestsetzung lassen für eine Freistellung ökologisch bewirtschafteter Flächen deutliche Änderungen bei der bestehenden Verwaltungspraxis und damit grundsätzlich hohe Barrieren erahnen. So müsste eine Differenzierung der landwirtschaftlich genutzten Flächen bereits in die Einheitswertermittlung Einzug halten. Der Einheitswert selbst findet aber neben seiner Funktion als Bemessungsgrundlage für die Grundsteuer auch noch in mehreren anderen Bereichen Anwendung, so z.B. bei der Bemessung der Beiträge zur Landwirtschaftskammer, zu Berufsverbänden, zur Alterskasse oder zur landwirtschaftlichen Krankenkasse. Allein vor diesem Hintergrund wäre eine derart tiefgreifende Änderung der Einheitswerte sehr unwahrscheinlich. Hinzu kommt, dass insbesondere die Bewirtschaftung von Pachtflächen im Zeitverlauf durch häufige Pächterwechsel mancherorts häufigen Änderungen unterliegen kann. Die Finanzämter müssten infolge dessen regelmäßig die Bewirtschaftungsweise der Flächen überprüfen und bei Veränderungen neue Einheitswertbescheide erstellen. Damit würden zwangsläufig auch die Transaktionskosten des Landwirts bzw. des Bodeneigentümers steigen. Ein Verpächter in den alten Bundesländern müsste dem Finanzamt regelmäßig nachweisen, dass seine Flächen ökologisch bewirtschaftet werden, um eine Freistellung von der Besteuerung zu erreichen. Als Konsequenz dürfte sich im Vergleich zum bisherigen Verwaltungsablauf auch für den Landwirt ein Mehraufwand einstellen.

Äußerst problematisch dürfte sich auch die Initiierung eines finanziellen Ausgleichs für die Kommunen gestalten, da eine komplexe Finanzumverteilung zwischen Kommunen, Ländern und Bund mit einem schwierigen Kompromissfindungsprozess zu erwarten wäre.

- Einschätzung der Kofinanzierungsfähigkeit gemäß der EU-Rahmenbedingungen

Hinsichtlich der Nachvollziehbarkeit des absoluten Fördervolumens sind keine Probleme zu erwarten. Bezüglich der angestrebten Kostenakzessorität wäre das gegenwärtige Flächenprämienniveau um die Grundsteuerersparnis der Betriebe zu senken. Auch die Flächenakzessorität dürfte keine Probleme bereiten, da die Vergünstigung aus der Freistellung von der Grundsteuer zwangsläufig über der Fläche gewährt würde.

\footnotetext{
${ }^{7}$ In den neuen Bundesländern gilt die Einheitsbewertung nicht. Stattdessen werden vom zuständigen Finanzamt Ersatzwirtschaftswerte ermittelt, die im Rahmen der Grundsteuerfestsetzung die gleiche Funktion haben wie die Einheitswerte in den alten Bundesländern.
} 
Trotzdem ergibt sich in der Gesamtbetrachtung für eine Prämiendifferenzierung gemäß den EMZ im Hinblick auf die zu erwartenden Förderwirkungen sowie auf die voraussichtlich zusätzlich entstehenden Transaktionskosten ein deutlich erfolgversprechenderes Ergebnis.

\subsubsection{Freistellung ÖKOlOgisch ERZeUgter ProdukTe VON DER UMSATZSTEUER (UST)}

Aus dem Umsatzsteuerrecht ergibt sich mit der Umsatzsteuerfreistellung für ökologisch erzeugte Produkte eine weitere steuerliche Förderalternative. Im Gegensatz zu konventionell erzeugten Produkten würde dadurch für ökologisch erzeugte Produkte der allgemein geltende ermäßigte USt-Satz von $7 \%$ bzw. im Falle eines pauschalierenden Öko-Landwirts der Pauschalsteuersatz von 9\% (zukünftig 10,7\%) entfallen (vgl. zur Umsatzbesteuerung ALTHOEFER et al. (2003: 576ff) oder KuSSMAUL (2005: 397ff)).

- Ansatz und Wirkung der Förderung:

Der höhere Preis ökologisch erzeugter Produkte gilt allgemein als zentrales Hemmnis für eine stärkere Nachfrage nach ökologisch erzeugten Produkten (vgl. bspw. HAMM und WILD, 2004: 9). Der Preis, den der Endverbraucher für ein Produkt zu zahlen hat, besteht dabei zum einen aus dem vom Verkäufer festgelegten Produktpreis und zum zweiten aus der Umsatzsteuer, die abhängig davon, ob es sich bei dem Verkäufer um einen pauschalierenden Landwirt oder um einen sonstigen Verkäufer handelt, $9 \%$ bzw. im Regelfall $7 \%$ des Produktpreises beträgt. Eine Freistellung ökologisch erzeugter Produkte von der Umsatzsteuer würde also direkt am Preis, d.h. an einem zentralen Nachfragehemmnis ansetzen. Die allgemeine Senkung des Preisniveaus für ökologisch erzeugte Produkte würde gleichzeitig auch eine Senkung der Preisdifferenz zwischen ökologischen und konventionellen Produkten mit sich bringen. Der zentrale Vorteil einer Umsatzsteuersenkung wäre daher in einer Senkung der Verbraucherpreise und in einer Senkung des Preisaufschlags für Ökoprodukte zu sehen.

Dabei könnte dieses nachfrageinduzierende Instrument auch noch $\mathrm{zu}$ zwei weiteren als vorteilhaft erachteten Entwicklungen führen. Zum einen besteht ein immer wieder genannter Kritikpunkt der gegenwärtigen Flächenprämien in der einseitig angebotsfördernden Wirkung, was bei fehlender Nachfrage zu erhöhtem Preisdruck für die Produzenten und damit zu einer Senkung der Effektivität der Flächenförderung führen kann. Diese für Push-Maßnahmen spezifische problematische Förderwirkung besteht bei einer Senkung des Umsatzsteuertarifes nicht. Zum anderen wird ein wesentlicher Hemmfaktor für einen höheren Umsatz mit Öko- 
Produkten in den tendenziell höheren Transaktionskosten des Handels resultierend aus den bisher kleinen Verkaufsmengen gesehen (vgl. dazu HAMM, 2003: 15; NIEBERG und OfFERmANN, 2001: S. 427 oder SPILler, 2001: S. 453. Eine Ausdehnung des Handelsvolumens im Rahmen einer steigenden Nachfrage nach Öko-Produkten als Resultat der Preissenkungen könnte die Transaktionskosten des Handels senken. Würden diese Preisvorteile vom Handel an den Endkunden weitergegeben, so bestände die Chance auf einen Automatismus hinsichtlich sinkender Transaktionskosten, sinkender Produktpreise, einer steigenden Nachfrage und einem höheren Umsatz von ökologisch erzeugten Produkten.

Zunächst einmal ist aber $\mathrm{zu}$ analysieren, ob eine USt-Freistellung auch wirklich zu einem Nachfrageanstieg bei Öko-Produkten führt und ob dieser eventuelle Nachfrageanstieg im weiteren Verlauf seinerseits auch wirklich im Sinne des in Kapitel 2.1 formulierten Zieles der Öko-Förderung zu einer Ausdehnung der ökologisch bewirtschafteten Fläche in Deutschland führt. Im Rahmen einer theoretischen Betrachtung zur Entwicklung des ökologischen Landbaus wäre diesbezüglich von dem folgenden grundsätzlichen Wirkungsszenario auszugehen (Abbildung 9):

Abbildung 9: Angestrebtes Wirkungsszenario als Reaktion auf eine Freistellung ökologisch erzeugter Produkte von der Umsatzsteuer

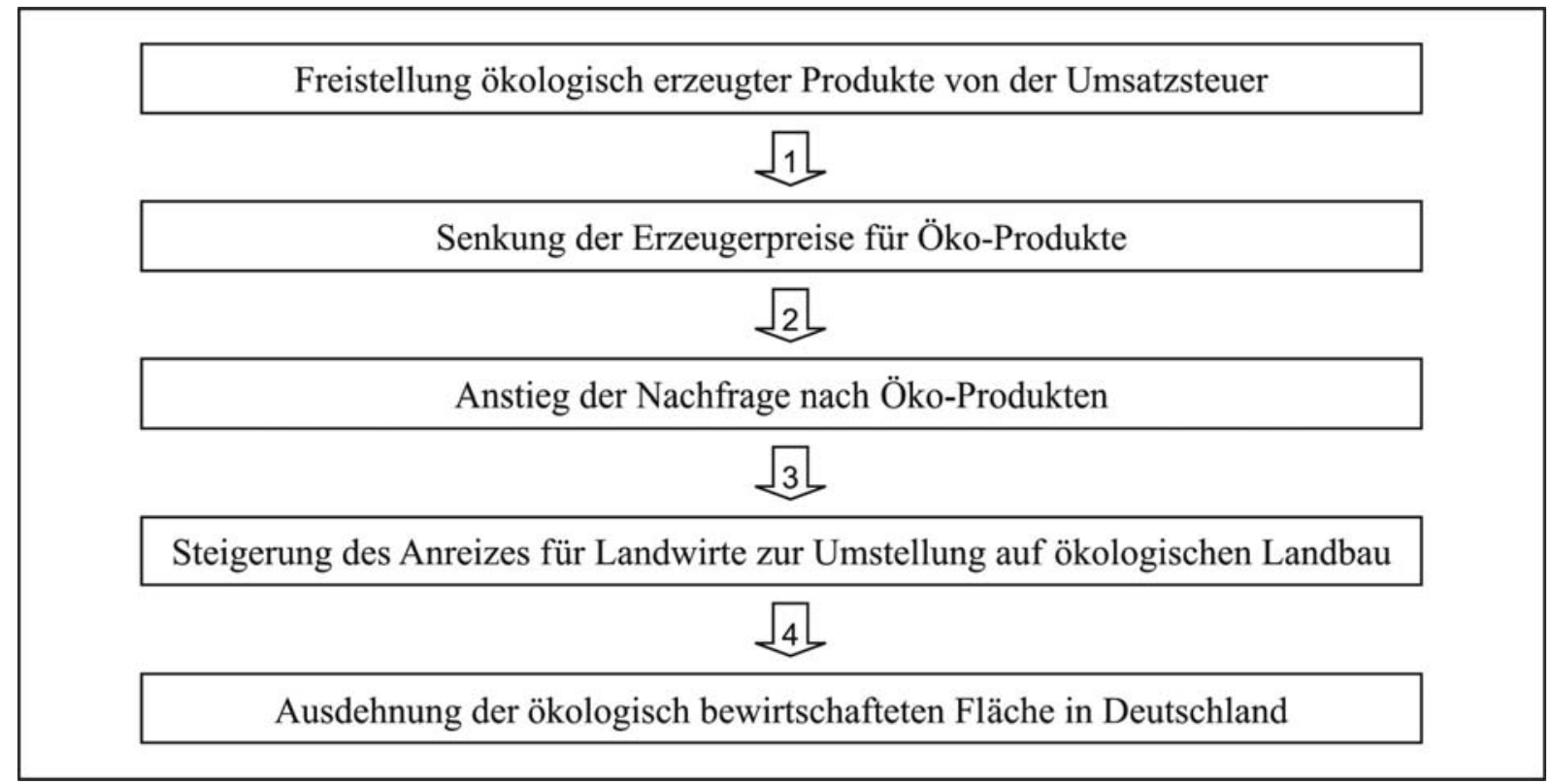

Quelle: Eigene Darstellung

Als Reaktion auf die Freistellung ökologisch erzeugter Produkte von der Umsatzsteuer wäre von einer Senkung der Erzeugerpreise um den Betrag der Umsatzsteuer auszugehen. Sofern die Preisvorteile auch an die Verbraucher weitergegeben werden, sollte sich ein Anstieg der Nachfrage nach Öko-Produkten einstellen. Als Folge des Nachfrageanstiegs wäre mit einem 
erhöhten Umstellungsanreiz und damit mit einer weiteren Ausdehnung der ökologisch bewirtschafteten Fläche in Deutschland zu rechnen.

Wichtig für den letztendlichen Erfolg einer Fördermaßnahme ist jedoch nicht nur deren grundsätzliche Wirkung, sondern vor allem deren tatsächliche Wirksamkeit. Dazu müssen die einzelnen Wirkungsschritte hinsichtlich ihrer Eintrittswahrscheinlichkeit überprüft werden:

Zunächst bleibt hinsichtlich des Wirkungsschrittes 1 fraglich, ob eine Freistellung von Ökoprodukten von der Umsatzsteuer auch wirklich zu einer deutlichen Senkung der Verbraucherpreise führt. Es besteht nämlich auch die Möglichkeit, dass die durch die Senkung der Umsatzsteuersätze entstandenen Preisvorteile von Landwirten (insbesondere von Direktvermarktern), der Industrie oder dem Handel nicht oder nur in geringem Maße an den Konsumenten weitergegeben werden. Insofern läge eine asymmetrische Preistransmission vor. Obwohl bei theoretischen Betrachtungen grundsätzlich immer von einem funktionierenden Wettbewerb ausgegangen werden sollte, liegt hier trotzdem eine latente Gefahr, die die Effektivität dieser steuerlichen Förderung deutlich reduzieren könnte.

Weiterhin wäre mit Bezug auf den Wirkungsschritt 2 nur mit einem geringen Nachfrageschub als Reaktion auf eine Senkung der Verbraucherpreise zu rechnen, sofern die Preiselastizitäten für Ökoprodukte nur sehr gering sind. Nachfrageelastizitäten für ökologisch erzeugte Produkte sind bisher nur vereinzelt für ausgewählte Produkte und Regionen ermittelt worden. Sie fielen dann aber im Vergleich zu den Nachfrageelastizitäten konventionell erzeugter Produkte relativ hoch aus (vgl. WIER und SMERD, 2000 oder GRINGS, 1991), wobei HAGNER (1994) bezüglich der Ergebnisse von GRINGS (1991) darauf hinweist, dass diese aufgrund der Datengrundlage und der verwendeten Modellannahmen äußerst vorsichtig zu interpretieren seien, da mit einer systematischen Verzerrung der Nachfrageelastizitäten zu rechnen sei. Doch auch bei einer höheren Preiselastizität der Nachfrage ist zumindest fraglich, ob die durch die Umsatzsteuerfreistellung ausgelöste Senkung der Verbraucherpreise ausreicht, um eine deutliche Nachfragesteigerung zu erreichen.

Aber selbst wenn sich ein deutlicher Nachfrageanstieg einstellen sollte, besteht hinsichtlich der Wirkungsschritte 3 und 4 zusätzlich die Gefahr, dass dieser Nachfrageanstieg nicht zur Ausweitung der ökologisch bewirtschafteten Fläche im Inland führt. Denn von einer UStFreistellung würden ebenfalls Importwaren profitieren. Im Falle erhöhter Importe würde eine Ausweitung der ökologisch bewirtschafteten Fläche im Ausland erreicht. Damit würden auch die positiven Umwelteffekte einer weiteren Ausdehnung des ökologischen Landbaus im 
Ausland anfallen. Insofern wäre eine unbefriedigende Effektivität der Fördermaßnahme aus deutscher Sicht die Folge.

Vor der Analyse der Auswirkungen auf ökologisch wirtschaftende Landwirte ist nochmals hervorzuheben, dass es sich bei der Umsatzsteuerfreistellung um ein Instrument handelt, das als so genannte Pull-Maßnahme direkt auf der Nachfrageseite ansetzt und damit auf die Angebotsseite nur indirekte Wirkungen hat. Dennoch können auch diese indirekten Wirkungen sehr bedeutsam sein. Das beweist insbesondere die Untersuchung der aus der UStFreistellung ökologischer Produkte resultierenden Auswirkungen auf den Vorsteuerabzug für regelbesteuerte Landwirte. Bisher gestaltet sich die Situation für einen regelbesteuerten ÖkoLandwirt wie folgt: Aus dem Verkauf landwirtschaftlich erzeugter Produkte ergeben sich für ihn Umsatzsteuereinnahmen. Gleichzeitig resultieren aus dem Einkauf von Vorleistungen für ihn Umsatzsteuerausgaben. Im Rahmen des Vorsteuerabzugs nach $§ 15$ UStG kann er nun diese Umsatzsteuerausgaben als Vorsteuer geltend machen und muss somit lediglich den Differenzbetrag aus seinen Umsatzsteuereinnahmen und seiner Vorsteuer an das Finanzamt abführen. Ist der Differenzbetrag negativ, so wird er vom Finanzamt erstattet. Nach LIPPROSS (2005: 745f) sind Vorsteuerbeträge aber nur abziehbar, wenn

- die positiven, anspruchsbegründenden Tatbestandsmerkmale des $\S 15$ Abs. 1 UStG erfüllt sind,

- die Ausschlusstatbestände nach $\S 15$ Abs. 1 a UStG nicht vorliegen und

- die negativen anspruchsausschließenden Tatbestandsmerkmale des $\S 15$ Abs.2 und 3 UStG nicht verwirklicht sind.

Diese negativen anspruchsausschließenden Tatbestandsmerkmale des $\S 15$ Abs. 2 UStG sind abhängig von der Verwendung der Eingangsumsätze (d.h. der bezogenen Leistungen) für Ausgangsumsätze. Vorsteuerschädlich in der Weise, dass die Vorsteuerausgaben vom Landwirt nicht von den Umsatzsteuereinnahmen abziehbar sind, ist die Verwendung u.a. für steuerfreie Umsätze ( $§ 15$ Abs. 2 Satz 1 Nr.1 UStG). Nach LipPross (2005: 748) ist es daher notwendig, die Ausgangsumsätze eines Unternehmers für den Vorsteuerabzug in Ausschlussund Abzugsumsätze $\mathrm{zu}$ untergliedern. Ausschlussumsätze sind folglich solche Ausgangsumsätze, die keinen Abzug der Vorsteuer erlauben. Dem gegenüber kann die Vorsteuer im Falle von Abzugsumsätzen geltend gemacht werden. Wird eine vom Unternehmer bezogene Leistung bzw. ein zugekaufter Gegenstand sowohl für die Ausführung von Ausschluss- als auch von Abzugsumsätzen verwendet, sind die angefallenen 
Vorsteuerbeträge gemäß $§ 15$ Abs. 4 UStG nach dem Maßstab der wirtschaftlichen Zurechnung aufzuteilen ( $\mathrm{Zu}$ der genauen Verfahrensweise bei der Aufteilung der Vorsteuerbeträge vgl. LIPPROSS (2005: 761ff)).

$\S 15$ Abs. 2 UStG macht den Ausschluss vom Vorsteuerabzug also daran fest, für welche Umsätze (d.h. Ausgangsumsätze) der Unternehmer die seinem Unternehmen zugeführten Gegenstände und Dienstleistungen verwendet. Verwendung bezeichnet dabei die wirtschaftliche Verbindung zwischen Eingangsbezügen und den Ausgangsumsätzen des Unternehmers. Der BFH spricht in diesem Zusammenhang auch von dem Prinzip der wirtschaftlichen Zuordnung (LIPPROSS (2005: 761ff)). Vom Ausschluss des Vorsteuerabzugs sind also nur die Eingangsbezüge betroffen, die wirtschaftlich mit USt-befreiten Ausgangsumsätzen verbunden sind. Für eine USt-Befreiung ökologisch erzeugter Produkte würde sich dadurch Folgendes ergeben:

- Eingangsbezüge (bezogene Leistungen oder Gegenstände), die wirtschaftlich allein mit der Herstellung von Öko-Produkten verbunden sind, wären nicht vorsteuerabzugsfähig.

- Eingangsbezüge (bezogene Leistungen oder Gegenstände), die sowohl mit der Herstellung von Öko-Produkten als auch mit nicht USt-befreiten Ausgangsumsätzen wirtschaftlich verbunden sind, wären gemäß $§ 15$ Abs. 4 UStG nach der wirtschaftlichen Zurechnung aufzuteilen. Der Anteil der Eingangsbezüge, die sich auf den Umsatz von Öko-Produkten beziehen, wäre dabei nicht vorsteuerabzugsfähig. Die gezahlte Umsatzsteuer, die sich auf Eingangsbezüge bezieht, die wirtschaftlich mit Ausgangsumsätzen verbunden sind, die nicht USt-befreit sind, könnte hingegen weiter als Vorsteuer geltend gemacht werden.

Die Ausführungen zeigen, dass sich aus der USt-Freistellung ökologischer Produkte und der daraus resultierenden Auswirkungen auf den Vorsteuerabzug für regelbesteuerte Landwirte deutliche Änderungen ergeben würden, die betriebswirtschaftlich zu einer teilweisen Angleichung der Ausgangssituation von pauschalierenden und regelbesteuerten Landwirten führen könnten. So käme es bei beiden Gruppen infolge der USt-Freistellung zu einem vollständigen Wegfall der Umsatzsteuereinnahmen aus dem Verkauf ökologisch erzeugter Produkte. Die gezahlte Vorsteuer auf die wirtschaftlich mit den USt-befreiten Ausgangsumsätzen verbundenen Eingangsbezüge ist beim pauschalierenden Landwirt schon heute in voller Höhe gewinnwirksam. Das würde nun auch für den regelbesteuerten Landwirt 
gelten. Da er zukünftig diese Vorsteuerausgaben auch nicht mehr vom Finanzamt einfordern könnte, würden sie bei ihm ebenfalls in voller Höhe gewinnwirksam. Diese Wirkungsweise hätte für die ökologisch wirtschaftenden Landwirte vor allem zwei Auswirkungen:

Zum einen würde für fast alle ökologisch wirtschaftenden Betriebe eine vollständige Neubetrachtung der Vor- und Nachteile von Pauschalierung und Regelbesteuerung sinnvoll werden, denn insbesondere auch für die pauschalierenden Betriebe, für die sich derzeitig Vorteile aus der USt-Pauschalierung nach $\S 24$ UStG ergeben, läge eine veränderte Situation vor. So würde sich die Entscheidung hinsichtlich der zukünftigen USt-Behandlung (Regelbesteuerung vs. Pauschalierung) im Falle einer USt-Freistellung ökologisch erzeugter Produkte im wesentlichen an dem Verhältnis der vereinnahmten USt aus den sonstigen nicht USt-befreiten Ausgangsumsätzen und der verausgabten USt für die mit diesen Umsätzen wirtschaftlich verbundenen Eingangsbezüge ausrichten. Generell wäre dazu analog zur jetzigen Entscheidung bezüglich der USt-Behandlung davon auszugehen, dass die Option zur Regelbesteuerung in den Fällen sinnvoll ist, in denen aufgrund betriebsbedingter Umstände damit zu rechnen ist, dass innerhalb der fünfjährigen Bindungsfrist bezogen auf die sonstigen nicht USt-befreiten Ausgangsumsätze ein Vorsteuerüberhang entstehen wird.

Zum anderen würden Öko-Landwirte durch den Wegfall der Abzugsmöglichkeit der Vorsteuer hinsichtlich der in ihren Betrieb eingeführten Gegenstände und Dienstleistungen zu Endverbrauchern, da sie die darauf entfallende Umsatzsteuer letztlich selbst zu tragen hätten.

Auch die Art der Vermarktung der Öko-Produkte auf den einzelnen Betrieben und die Intensität des Wettbewerbs hätten daher einen Einfluss auf die betriebswirtschaftlichen Auswirkungen einer USt-Freistellung. Zwar würde die Absenkung des Preisniveaus um den Umsatzsteuerbetrag verbunden mit der Nicht-Abzugsfähigkeit der Vorsteuer sowohl für pauschalierende als auch für regelbesteuerte Landwirte zu einer Verschlechterung der Ertragslage führen. Die Absenkung des Preisniveaus und damit die Definitiv-Belastung des Unternehmers aus der USt-Freistellung wären jedoch abhängig von der Intensität des Wettbewerbs. Bei einer sehr starken Wettbewerbsposition des einzelnen Betriebs, die insbesondere bei Direktvermarktern vielerorts vorliegen wird, wären durch die UStFreistellung keine oder nur geringe Erzeugerpreisveränderungen für die Landwirte zu erwarten. Damit wären die Auswirkungen auf die Verbraucherpreise aus der USt-Freistellung insgesamt reduziert und die Bedenken bezüglich des Wirkungsschrittes 1 aus Abbildung 9 würden sich bewahrheiten. Die Effektivität der steuerlichen Fördermaßnahme „Freistellung ökologisch erzeugter Produkte von der USt“" würde dadurch vermindert. 
Es gäbe allerdings auch einen Ausweg aus der Problematik der Nicht-Abzugsfähigkeit der Vorsteuer bei USt-befreiten Ausgangsumsätzen. Das wäre nämlich dann der Fall, wenn die Regelungen des $\S 15$ Abs. 3 auch auf die Umsätze von ökologisch erzeugten Produkten ausgeweitet würden. Die in $\S 15$ Abs. 2 UStG bezeichneten steuerfreien Umsätze führen nämlich nicht zum Ausschluss des Vorsteuerabzugs, wenn es sich um die besonderen Fälle des $§ 15$ Abs. 3 UStG handelt oder für Ökoprodukte ein Steuersatz von $0 \%$ erhoben wird (in diesem Fall würde keine Steuerbefreiung vorliegen, so dass § 15 II UStG nicht greift). Regelbesteuerte Öko-Landwirte könnten folglich auch zukünftig einen Vorsteuerabzug für Eingangsbezüge, die wirtschaftlich mit USt-befreiten Ausgangsumsätzen verbunden sind, geltend machen. Damit würde gleichzeitig die Vorzüglichkeit der Regelbesteuerung für die bisher pauschalierenden Öko-Landwirte zunehmen.

Infolge einer Sonderbehandlung der USt-freigestellten Öko-Produkte hinsichtlich der Abzugsfähigkeit der Vorsteuer würden sich die Gesamtwirkungen einer USt-Freistellung für die Öko-Landwirte verbessern. Dazu soll im Folgenden untersucht werden, welche Wirkung sich aus einer USt-Freistellung von Öko-Produkten für den Staat ergeben würde. Um den Steuerausfall für den Staat abzuschätzen, werden als Berechnungsbasis Daten zum Umsatz an Öko-Lebensmitteln für Deutschland benötigt. Dazu sei auf die folgende Tabelle 7 verwiesen.

Tabelle 7: Umsatz an Öko-Lebensmitteln in Deutschland in Mrd. $€$ (ohne Genussmittel und Außer-Haus-Verzehr)

\begin{tabular}{|c|c|c|c|c|c|c|c|c|c|}
\hline & 2000 & $\%$ & 2002 & $\%$ & 2003 & $\%$ & 2004 & $\%$ & ZMP-Schätzung \\
\hline Erzeuger ${ }^{1}$ & 0,35 & $17 \%$ & 0,52 & $17 \%$ & 0,52 & $17 \%$ & 0,56 & $16 \%$ & für den \\
\hline Handwerk ${ }^{2}$ & 0,14 & $7 \%$ & 0,22 & $7 \%$ & 0,23 & $7 \%$ & 0,24 & $7 \%$ & Gesamtumsatz \\
\hline Reformhäuser & 0,21 & $10 \%$ & 0,27 & $9 \%$ & 0,27 & $9 \%$ & 0,27 & $8 \%$ & an Öko- \\
\hline Naturkostfachgesch. & 0,57 & $28 \%$ & 0,78 & $26 \%$ & 0,81 & $26 \%$ & 0,9 & $26 \%$ & Lebensmitteln in \\
\hline LEH $^{3}$ & 0,68 & $33 \%$ & 1,05 & $35 \%$ & 1,09 & $35 \%$ & 1,28 & $37 \%$ & Deutschland \\
\hline Sonstige ${ }^{4}$ & 0,1 & $5 \%$ & 0,17 & $6 \%$ & 0,2 & $6 \%$ & 0,25 & $7 \%$ & 2005: \\
\hline Summe & 2,05 & $100 \%$ & 3,01 & $100 \%$ & 3,12 & $100 \%$ & 3,5 & $100 \%$ & 4,0 \\
\hline
\end{tabular}

${ }^{1}$ Landwirte einschließlich Wochenmärkte und Lieferdienste

${ }^{2}$ Bäckereien, Fleischereien

${ }^{3}$ Lebensmitteleinzelhandel einschließlich Feinkostgeschäfte und Lieferdienste

${ }^{4}$ Drogeriemärkte, Postversand und Lieferdienste von Verarbeitungsunternehmen

Quelle: ZMP, 2005: 26

Wie Tabelle 7 zeigt, hat der Umsatz an Öko-Lebensmitteln in den vergangenen Jahren stetig zugenommen. Und auch für die kommenden Jahre geht die ZMP von weiter deutlichen Steigerungsraten aus (ZMP, 2005: 26). Die angegebenen Zahlen zum Gesamtumsatz an Lebensmitteln verstehen sich inklusive der geltenden Umsatzsteuer (mündliche Mitteilung 
RIPPIN, 09.01.2006). Dabei kann insgesamt von einem Satz von $7 \%$ Umsatzsteuer ausgegangen werden. Hätte die Freistellung ökologisch erzeugter Produkte schon in der Vergangenheit Gültigkeit gehabt, so hätten sich folglich für den Staat die folgenden Umsatzsteuerausfälle ergeben (Tabelle 8).

Tabelle 8: Berechnung des USt-Volumens für die vergangenen Jahre

\begin{tabular}{|c|c|c|c|c|c|}
\hline Kalenderjahr & 2000 & 2002 & 2003 & 2004 & 2005 \\
\hline $\begin{array}{c}\text { Umsatz an Öko-Lebensmitteln in } \\
\text { Deutschland in Mrd. } €\end{array}$ & 2,05 & 3,01 & 3,12 & 3,50 & $4,00^{*}$ \\
\hline davon USt in Mrd. $€$ & 0,13 & 0,20 & 0,20 & 0,23 & 0,26 \\
\hline
\end{tabular}

*Schätzung ZMP

Quelle: Eigene Darstellung

Die Berechnungen zeigen, dass der Umsatzsteuerausfall für den Staat im Jahr 2005 auf bis zu 260 Mio. $€$ angestiegen wäre, wenn es in den vergangenen Jahren bereits eine Umsatzsteuerfreistellung ökologisch erzeugter Produkte gegeben hätte. Wenn man einerseits bedenkt, dass die Erwartungen an die zukünftige Umsatzentwicklung bei Öko-Produkten weiterhin sehr positiv sind und dass andererseits konventionelle Produkte im Falle einer UStFreistellung vermutlich noch stärker von ökologisch erzeugten Produkten substituiert werden könnten, liegt die Vermutung nahe, dass die Steuerausfälle in Zukunft sogar noch deutlich höher ausfallen könnten. Ein Einbruch der Steuereinnahmen und damit hohe Kosten für den Staat aus dieser Fördermaßnahme würden die anvisierte Steigerung der Fördereffizienz infrage stellen.

Die finanziellen Belastungen des Staates dürften sich erheblich reduzieren, wenn im Zuge der USt-Freistellung von Ökoprodukten auf eine Sonderbehandlung hinsichtlich der Abzugsfähigkeit der Vorsteuer verzichtet würde. Der USt-Ausfall des Staates infolge der UStFreistellung ökologisch erzeugter Produkte würde dann nämlich durch USt-Mehreinnahmen bei den Vorleistungen zur Herstellung von Öko-Produkten infolge der bereits diskutierten Definitivbelastung der Öko-Landwirte z.T. kompensiert werden. Inwieweit sich diese Mehrbelastungen durch eine ansteigende Nachfrage nach Öko-Produkten für die Landwirte ausgleichen würden, bleibt mit Verweis auf das in Abbildung 9 dargestellte Wirkungsszenario jedoch unklar.

Damit kann aus den verschiedenen Betrachtungen $\mathrm{zu}$ den Wirkungen einer Freistellung ökologisch erzeugter Produkte insgesamt geschlossen werden, dass die Effektivität wie auch die Effizienz einer derartigen steuerlichen Förderung aufgrund der weit verzweigten 
Wirkungszusammenhänge und der hohen Unsicherheiten hinsichtlich des grundsätzlichen Wirkungsszenarios äußerst fraglich sind. Sollte eine Sonderbehandlung hinsichtlich des Vorsteuerabzugs vereinbart werden, so wären die zu erwartenden hohen USt-Ausfälle für den Staat aller Voraussicht nach ein gewichtiges Argument gegen eine derart gestaltete steuerliche Förderung. Würde hingegen keine Sonderbehandlung von Ökoprodukten hinsichtlich des Vorsteuerabzugs anvisiert, so werden die absehbaren Zusatzbelastungen für die ökologisch wirtschaftenden Landwirte einer angestrebten verstärkten Ausdehnung der ökologisch bewirtschafteten Fläche entgegenstehen.

- Transaktionskosten:

Zunächst wäre zur Einführung einer Freistellung ökologisch erzeugter Produkte von der Umsatzsteuer eine Änderung der gesetzlichen Grundlage notwendig. So müsste $§ 4$ UStG („Steuerbefreiungen bei Lieferungen und sonstigen Leistungen“) um einen Steuerbefreiungstatbestand für ökologisch erzeugte Produkte ergänzt werden. Hinzu käme im Falle einer beabsichtigten Sonderbehandlung des Vorsteuerabzugs die Änderung von $\S 15$ Abs. 3 in der Weise, dass er zukünftig ebenfalls für die Umsätze von ökologisch erzeugten Produkten Geltung hat. Sollte dies nicht beabsichtigt sein, so dürfte allein die dann notwendige Differenzierung der Eingangsbezüge hinsichtlich ihrer wirtschaftlichen Verbundenheit mit USt-befreiten und Nicht-USt-befreiten Ausgangsumsätzen die Transaktionskosten dieser steuerlichen Förderung insbesondere für die Landwirtschaft in die Höhe treiben. Bei einem maßgeblichen USt-Tarif von $0 \%$ würde dies jedoch nicht gelten. Davon abgesehen wird der kontinuierliche Administrationsaufwand für die öffentliche Verwaltung allein dadurch erhöht, dass nun zwischen konventionellen und ökologischen Produkten bei Umsatzbesteuerung zu unterscheiden ist und jede weitere Differenzierung zusätzliche Verwaltungskosten mit sich bringt. So werden die zusätzlich entstehenden Transaktionskosten eine Entscheidung für eine Freistellung ökologisch erzeugter Produkte von der Umsatzsteuer eher erschweren.

- Genehmigungs- und Kofinanzierungsfähigkeit gemäß der EU-Rahmengesetzgebung:

Neben deutlichen Vorbehalten bezüglich der Effektivität und der Effizienz sowie voraussichtlich erhöhten Transaktionskosten sind aber auch noch weitere schwerwiegende Problembereiche hinsichtlich der rechtlichen Legitimierung $\mathrm{zu}$ beachten. So gilt eine Durchführung im Rahmen der EU-Gesetzgebung allgemein als fraglich. Dazu sei darauf hingewiesen, dass im Gegensatz bspw. zum Einkommensteuerrecht das Umsatzsteuerrecht 
innerhalb der EU bereits weitgehend harmonisiert ist. Einzelstaatliche Änderungen der Umsatzsteuersätze müssen mit der 6. EU-Umsatzsteuerrichtlinie (77/388/EWG) vereinbar sein, um nach dem EU-Recht genehmigungsfähig zu sein. In diesem Zusammenhang dürfte zu allererst die derzeitige Ausgestaltung des Artikels 13, der die Möglichkeiten von Steuerbefreiungen in den Mitgliedstaaten einschränkt, Probleme bereiten. Aller Voraussicht nach wäre hier zunächst ein neuer Befreiungstatbestand einzufügen, bevor ein Versuch zur Genehmigung einer Freistellung ökologisch erzeugter Produkte Aussicht auf Erfolg hätte. Damit es dazu käme, müsste aber vorher die Frage geklärt werden, ob eine unterschiedliche Besteuerung konventionell und ökologisch erzeugter Produkte dem allgemeinen Grundsatz der steuerlichen Neutralität widerspricht. Danach ist es unzulässig, gleichartige und deshalb miteinander im Wettbewerb stehende Waren steuerlich unterschiedlich zu behandeln. Obwohl es sicherlich Argumentationsmöglichkeiten gäbe, diese Fragestellung zu entkräften, sind Versuche, eine USt-Freistellung in den Niederlanden zu erreichen insbesondere an dieser Frage gescheitert (GARDEBROEK, 2005: schriftliche Mitteilung). Deshalb ist hinsichtlich der Genehmigungsfähigkeit dieses steuerlichen Förderinstruments im Rahmen der 6. EUUmsatzsteuerrichtlinie (77/388/EWG) festzustellen, dass dieses vermutlich wenn überhaupt nur mit erhöhten Kraftanstrengungen möglich wäre.

Noch erschwerender für eine Umsetzung der USt-Freistellung von Öko-Produkten wirken jedoch die Probleme hinsichtlich der Finanzierung des USt-Ausfalls. In Kapitel 3.2.3 wurde bereits darauf verwiesen, dass eine EU-Kofinanzierbarkeit fast eine Grundbedingung für eine Modifizierung des gegenwärtigen Fördersystems darstellt. Darum wirkt es umso schwerwiegender, dass bei dem voraussichtlich erheblichen Ausmaß der Steuerausfälle eine Kofinanzierung im Rahmen der ELER-VO nicht gegeben sein wird, weil im Sinne der Kriterien aus Kapitel 3.2.3 sowohl die Nachvollziehbarkeit des einzelbetrieblichen Fördervolumens als auch die Kosten- und Flächenakzessorität nicht gegeben sind. Die ohnehin derzeit ungewisse finanzielle Zukunft der Öko-Förderung würde dadurch noch zusätzlich belastet.

- Erfahrungen aus anderen EU-Staaten:

Die bisherigen Beurteilungen $\mathrm{zu}$ den verschiedenen Kriterien decken sich mit parallelen Untersuchungen in den Niederlanden und in Schweden. Insbesondere in den Niederlanden hat man sich in der Vergangenheit bereits ausgiebig mit dieser Thematik befasst. Hier wird der vergleichsweise höhere Preis ökologischer Produkte als zentrales Wachstumshemmnis für den Markt für ökologisch erzeugte Produkte und damit für den Ökolandbau insgesamt angesehen 
(JoAnKNeChT, 2004). Aus diesem Grund sollen bei der Politik zugunsten des ökologischen Landbaus Instrumente zur Reduzierung der Preisdifferenz zwischen ökologisch und konventionell erzeugten Produkten Priorität haben (MINISTRY OF AGRICULTURE, NATURE AND FoOD QUALITY NL, 2004: 11f). Schon in den neunziger Jahren gab es in den Niederlanden erste Überlegungen hinsichtlich einer Senkung der Umsatzsteuer für ökologische Erzeugnisse, die jedoch im Juni 2000 wieder verworfen wurden. Der Grund dafür lag in der Einschätzung, unterschiedliche USt-Sätze für ökologische und konventionelle Erzeugnisse widersprächen der EU-Umsatzsteuerharmonisierung im Rahmen der 6. EU-Umsatzsteuerrichtlinie (Ministry of Agriculture, NAture AND Food QuAlity NL, 2001: 12). Nachdem jedoch die EU-Kommission in ihrem „Green Paper on Integrated Product Policy“ eine Senkung der Umsatzsteuer für Ökoprodukte ausdrücklich als mögliche Option zur Förderung des ökologischen Landbaus aufgeführt hatte, wurde die Idee in den Niederlanden wieder aufgenommen (EU-Kommission, 2001: 11). Beim Centre for Agriculture and Environment (clm) wurde dazu eigens eine Studie initiiert, in der der Effekt einer Freistellung ökologisch erzeugter Produkte auf die Produktion und den Konsum von Öko-Produkten analysiert wurde (VerschuUR und VAN Well, 2001). Für die vier EU-Staaten Österreich, Dänemark, Schweden und die Niederlande wurden darin die Wirkungen auf den Umsatz mit Ökoprodukten und auf die Ausweitung des ökologischen Landbaus untersucht. Aufgrund der unterschiedlichen zumeist höheren Ausgangssteuersätze in den jeweiligen Ländern sind die Ergebnisse jedoch schwerlich auf Deutschland übertragbar. Lediglich die Niederlande mit einem derzeitigen USt-Satz von $6 \%$ kann bei einem USt-Satz von $7 \%$ in Deutschland auf die meisten ökologisch erzeugten Produkte als Vergleich herangezogen werden. Danach ergab sich durch den Wegfall der $6 \%$ igen Umsatzsteuer eine Steigerung der Nachfrage bei Brot, Milch, Joghurt und Butter um $5 \%$ sowie bei Kartoffeln um $10 \%$ (ebenda, 2001). Diesen Ergebnissen liegt jedoch die Annahme zugrunde, dass der Wegfall der Umsatzsteuer ausschließlich dem Verbraucher zugute kommt, d.h. dass sich die Verbraucherpreise um die volle Umsatzsteuer ermäßigen. Wie bereits in den Voruntersuchungen erwähnt, ist diese Annahme jedoch unter Beachtung der vielfältigen Wettbewerbsverhältnisse sehr fraglich. Die Berechnungen zur Auswirkung auf die ökologisch bewirtschaftete Fläche ergaben ebenfalls eine Flächenausweitung in den Niederlanden um $5 \%$, so dass hinsichtlich der Auswirkungen letztendlich ein positiver Schluss gezogen wurde und weitergehende Betrachtungen empfohlen wurden (ebenda, 2001). In der Folge gestartete weitergehende Untersuchungen ergaben jedoch aufgrund der hohen Steuerausfälle und damit der hohen Kosten einer derartigen Förderung negative Einschätzungen hinsichtlich der Effizienz (vgl. bspw. BUNTE, 
2004). Unsicherheiten hinsichtlich einer Genehmigungsfähigkeit seitens der EU sowie negative Einschätzungen hinsichtlich der Kosteneffizienz führten 2004 in den Niederlanden schließlich zu dem Entschluss, die Überlegungen vorerst nicht weiter zu verfolgen (GARDEBroeK, 2005: schriftliche Mitteilung). Auch in Schweden, wo man zwischenzeitlich intensiv über eine USt-Freistellung nachgedacht hatte, waren Bedenken bezüglich einer geringen Effizienz sowie der Vereinbarkeit mit dem geltenden EU-Recht die Gründe dafür, diese steuerliche Förderalternative bei der Suche nach neuen Förderinstrumenten zu vernachlässigen.

- Fazit zur Beurteilung:

Die Ausführungen haben gezeigt, dass der Grundgedanke, der hinter einer Umsatzsteuerfreistellung ökologisch erzeugter Produkte steht, durchaus im Sinne der Ziele der Förderung des ökologischen Landbaus ist. Es konnte aber auch gezeigt werden, dass sich durch die Auswirkungen einer so gestalteten steuerlichen Förderung sowohl für den Staat als auch für die ökologische Landwirtschaft deutliche Probleme ergeben können. Neben den zusätzlich zu erwartenden Problemen hinsichtlich einer Genehmigung im Rahmen des geltenden EU-Rechts liegt vielmehr auch die Vermutung nahe, dass es sich hierbei um einen sehr kostenintensiven Weg handeln könnte, um eine Ausweitung des ökologischen Landbaus zu erreichen, der dazu noch mit vielen Unwägbarkeiten versehen ist. Diese Einschätzung wird durch die Erfahrungen aus den Niederlanden und aus Schweden noch verstärkt. Insbesondere deshalb soll diese zunächst sinnvoll erscheinende steuerliche Förderalternative im weiteren Verlauf dieser Abhandlung nicht weiter beachtet werden.

Im vorliegenden Kapitel wurden bereits einige steuerliche Förderinstrumente auf ihre Eignung zur Förderung des ökologischen Landbaus hin überprüft. Dabei handelte es sich um Förderalternativen, die in der Vergangenheit bereits mehrfach zur Förderung des ökologischen Landbaus vorgeschlagen wurden. Dazu konnte festgestellt werden, dass lediglich eine weitere Differenzierung der gegenwärtigen Öko-Flächenprämien nach der EMZ Potentiale für eine Verbesserung der Effektivität sowie der Effizienz bietet. Die beiden anderen untersuchten Förderalternativen eignen sich dagegen nicht zur Substitution oder Modifikation der gegenwärtigen Angebotsförderung des ökologischen Landbaus. 


\section{DIE FLEXIBLE FöRDERUNG - GEWINNERMITTLUNGSMETHODEN DES ERTRAGSTEUERRECHTS ALS VORBILD FÜR EINE FÖRDERUNG DES ÖKOLOGISCHEN LANDBAUS}

Neben den drei im Vorkapitel untersuchten steuerlichen Förderalternativen sind noch eine Reihe weiterer Instrumente innerhalb der in Kapitel 3.1.3 ausgewählten Steuerarten untersucht worden. Die meisten von ihnen wurden jedoch bereits in frühen Untersuchungsstadien aufgrund ihrer spezifischen negativen Eigenschaften hinsichtlich der in Kapitel 3.2 definierten Erfolgskriterien ausgesondert. Bei anderen Instrumenten, bspw. bei jenen aus dem Bereich der Verbrauchssteuern, wurde im Vorfeld entschieden, aufgrund der vielen bereits vorhandenen diesbezüglichen Abhandlungen, sie im Rahmen dieser Arbeit nicht näher zu fokussieren.

Das vorliegende Kapitel ist deshalb allein einer Förderalternative gewidmet, die sich der Bemessungsgrundlage des Ertragssteuerrechts bedient und die in ganz besonderer Weise Potentiale zur Weiterentwicklung der Angebotsförderung des ökologischen Landbaus offenbaren könnte. Gemeint ist das System der Flexiblen Förderung. ${ }^{8}$ Die Vorgehensweise innerhalb dieses Kapitels orientiert sich dabei analog zur Vorgehensweise in den Kapiteln 3.3.1 bis 3.3.3 wiederum eng an den in Kapitel 3.2 definierten Erfolgskriterien für steuerliche Förderinstrumente, wobei insbesondere der Untersuchung der spezifischen Wirkungen dieses Förderinstrumentes mehr Raum gewährt wurde. Bevor nun aber näher auf die Konzeption und die spezifischen Eigenschaften der Flexiblen Förderung eingegangen wird, sollen in Kapitel 4.1 zunächst jene grundlegenden Überlegungen vorgestellt werden, die zur Idee der Flexiblen Förderung geführt haben.

\footnotetext{
${ }^{8}$ Vgl. zu den Überlegungen hinsichtlich einer ertragssteuerlichen Förderung des ökologischen Landbaus auch BAHRS, 2005: 148ff.
} 


\subsection{ERHÖHTE BETRIEBSWIRTSCHAFTLICHE RISIKEN IM ÖKOLANDBAU ALS ANSATZ UND RECHTFERTIGUNG EINER FLEXIBLEN FÖRDERUNG}

\subsubsection{HinTERgRUND UND ZielSETZUNG DER UNTERSUCHUNG}

In den vergangenen Wirtschaftsjahren haben ökologisch wirtschaftende Betriebe in Deutschland auf Basis der Agrarberichte in Relation zu vergleichbaren konventionell wirtschaftenden Betrieben vergleichbare oder höhere durchschnittliche Gewinne (absolut je Betrieb und je AK) erzielt (vgl. AgRARBERICHTE 2004, 2005 und 2006). Trotzdem ist der Umstellungswille konventionell wirtschaftender Landwirte nach wie vor nur schwach ausgeprägt (SCHRAMEK und SCHNAUT, 2004: 44ff). Als Erklärungsansatz für die Zurückhaltung der Landwirte werden neben Pfadabhängigkeiten (LATACZ-LOHMANN et. al., 2001: 434f), hohen Umstellungskosten (HADATSCH et al., 2000) sowie mehrdimensionalen Zielsetzungen der Landwirte (SchUlze-PALS, 1994: 102ff) auch wiederholt höhere mit der ökologischen Produktion einhergehende Einkommensrisiken angeführt (DARNHOFER, 2003, ODENING et al., 2004: 224ff).

Der Wechsel zwischen konventionellem und ökologischem Landbau weist vielfältige betriebswirtschaftliche Risiken auf. ${ }^{9}$ Da sind zum einen die mit der Umstellung verbundenen Kosten, die zumindest teilweise irreversibel sind, d.h. im Falle einer Rückumstellung versunken wären. Durch eine Umstellung verändern sich aber auch die Ein- und Auszahlungsströme. So sind nach der Umstellung infolge des Verbots synthetischer Pflanzenschutz- und Düngemittel im Allgemeinen nur geringere Naturalerträge zu erzielen. Um die Stickstoffversorgung trotzdem zu sichern, müssen nicht oder nur sehr eingeschränkt marktfähige Früchte ins Produktionsprogramm aufgenommen werden. KÖHNE und KÖHN (1998: 347) weisen in diesem Zusammenhang darauf hin, dass beispielsweise die Getreideerträge beträchtlichen Schwankungen in Abhängigkeit von den Witterungsverhältnissen unterliegen und dass es in ökologisch geführten Betrieben deutlich schwieriger ist, diesen Ertragschwankungen durch agrartechnische und phytosanitäre Maßnahmen zu begegnen. Im Gegenzug können Ökoprodukte aber nach einer Übergangszeit

\footnotetext{
${ }^{9} \mathrm{Zu}$ den potentiell höheren Produktionsrisiken im ökologischen Landbau als bedeutendes Hemmnis einer Ausweitung des ökologischen Landbaus in Deutschland vgl. u.a. KUHNERT et al, 2006: 22.
} 
von 2 Jahren i.d.R. zu deutlich höheren Preisen veräußert werden als konventionelle Produkte. Nach MuZIOL (1999: 20f) birgt der noch begrenzte Markt für Öko-Produkte im Vergleich mit dem konventionellen Lebensmittelmarkt die Gefahr höherer Schwankungen bei Angebot und Nachfrage und damit erhöhter Preisunsicherheiten. Diese generellen Preisunsicherheiten wurden in der Vergangenheit noch verstärkt durch die bei einigen Öko-Produkten bestehenden Absatzrisiken. So bestanden bei Milch und Fleisch in der Vergangenheit regelmäßig Verkaufsrisiken in der Weise, dass kein gesicherter Absatz zu den höheren Ökopreisen gewährleistet war und folglich die Öko-Ware z.T. nur zu konventionellen Preisen vermarktet werden konnte (vgl. AGRA-EUROPE, 2004 oder auch HAMM, 2003: 16). ${ }^{10}$ Auch ist eine höhere Sensibilität der Nachfrage nach Öko-Produkten gegenüber ÖkoLebensmittelskandalen zu erwarten, weil der Markt für Öko-Produkte, verglichen mit dem Markt für konventionelle Produkte, in höherem Maße auf Vertrauen basiert. Plötzliche deutliche Preiseinbrüche in Verbindung mit einem nachhaltigen Vertrauensverlust als Konsequenz von Lebensmittelskandalen könnten damit für den Öko-Landbau mit tendenziell höheren Einkommensrisiken verbunden sein.

$\mathrm{Zu}$ den spezifischen Motiven der (Nicht-) Umstellung auf ökologischen Landbau sind in der jüngeren Vergangenheit zahlreiche empirische Untersuchungen durchgeführt worden (vgl. z. B. SchneEberger et al. 2002: 24ff oder SchrameK und Schnaut 2004: 44ff). Als bedeutende Hemmnisse wurden dabei jeweils eben jene bei einer Umstellung zu erwartenden Naturalertrags- und Preisunsicherheiten ausgemacht. Weiterhin konnten sowohl GARDEBROEK (2002) als auch LIEN et al. (2004) aufzeigen, dass sich Öko-Landwirte im Vergleich zu ihren konventionell wirtschaftenden Berufskollegen im Allgemeinen durch eine schwächere Risikoaversion auszeichnen, sie also Risiken grundsätzlich aufgeschlossener gegenüberstehen.

Fraglich ist jedoch nach wie vor, ob die betriebswirtschaftlichen Risiken im ökologischen Landbau in der Tat höher sind, denn sowohl die Ergebnisse der Befragungen unter den Landwirten als auch die Ergebnisse zur Risikoaversion könnten auch auf „gefühlt“ höheren Risiken der Landwirte beruhen. Deshalb beschäftigt sich dieser Beitrag mit den tatsächlichen betriebswirtschaftlichen Risiken des ökologischen Landbaus, und zwar insbesondere im Vergleich zum konventionellen Landbau.

\footnotetext{
${ }^{10}$ Gegenwärtig zeigt sich jedoch ein Nachfrageüberhang nach diesen Produkten (vgl. dazu BRÜGGEMANN, 2006: 162ff oder RIPPIN, 2006: 64ff).
} 


\subsubsection{ANALYSE DER BETRIEBSWIRTSCHAFTLICHEN RISIKOSITUATION IM ÖKOLANDBAU}

Um Aussagen $\mathrm{zu}$ den betriebswirtschaftlichen Risiken im ökologischen Landbau im Vergleich $\mathrm{zu}$ denen des konventionellen Landbaus treffen $\mathrm{zu}$ können, wurde eine Risikoanalyse auf der Grundlage von Buchführungsergebnissen aus dem Testbetriebsnetz (TBN) des Bundesministeriums für Ernährung, Landwirtschaft und Verbraucherschutz (BMELV) initiiert. Bevor die Untersuchungsergebnisse präsentiert werden, wird zunächst auf den Ablauf und die Methodik der Untersuchung eingegangen.

\subsubsection{ABLAUf UND MethodiK}

\subsection{DATENGRUNDLAGE}

Für den Vergleich der betriebswirtschaftlichen Risiken von ökologischer und konventioneller Bewirtschaftungsweise auf der Basis historischer Buchführungsdaten stand ein Datensatz aus dem BMELV-Testbetriebsnetz der Wirtschaftsjahre 1996/97 bis 2003/04 zur Verfügung. Dieser umfasste zwar den Gesamtbestand aller Öko-Betriebe und aller konventionell wirtschaftenden Betriebe des Testbetriebsnetzes. Er enthielt jedoch nur die Daten zu ausgewählten Buchführungskennziffern, allen voran den Naturalerträgen und Verkaufspreisen ausgewählter Früchte sowie die Betriebsgewinne. 


\subsection{VORGEHENSWEISE}

Um die betriebswirtschaftlichen Risiken im konventionellen und im ökologischen Landbau zu analysieren und hinsichtlich ihrer Intensität miteinander vergleichen zu können, wurden zwei verschiedene Analysestrategien angewandt, die in Abbildung 10 dargestellt sind.

Abbildung 10: Gliederung des Analyseverfahrens

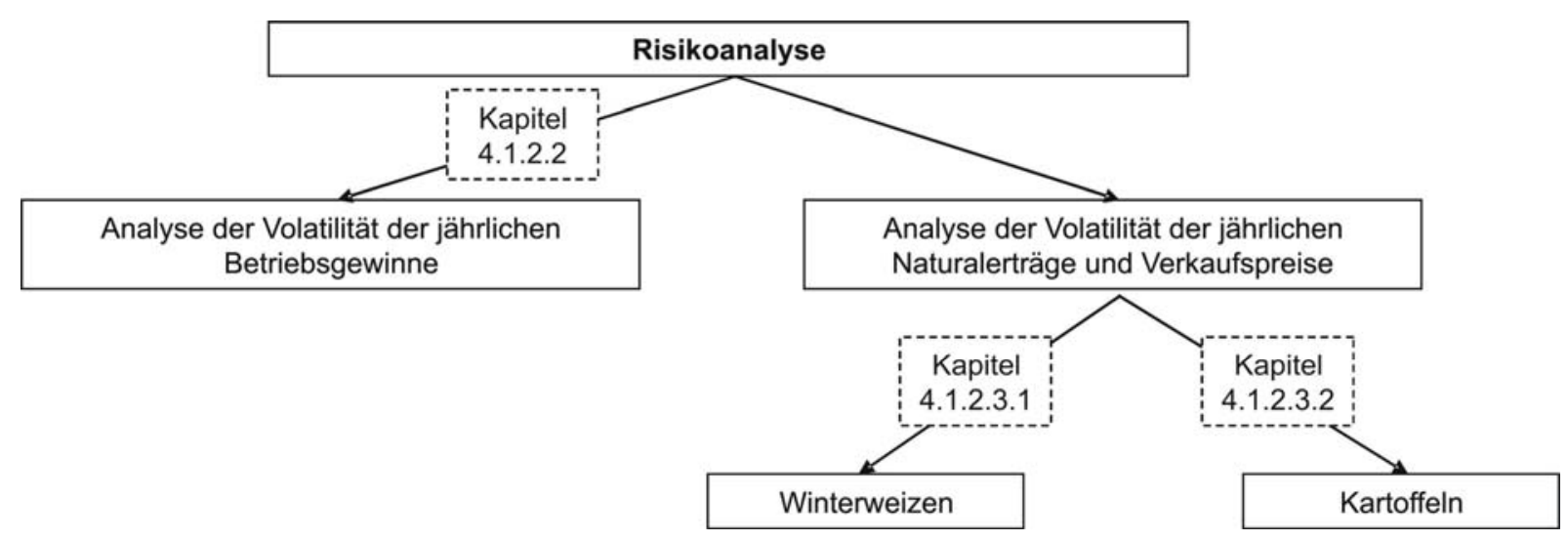

Quelle: Eigene Darstellung

In einer ersten Untersuchung wurden die Gewinne ökologisch und konventionell wirtschaftender Betriebe im Rahmen der deskriptiven Statistik analysiert und die Ergebnisse miteinander verglichen. Im Fall einer großen Streuung der Gewinne kann davon ausgegangen werden, dass im Rahmen des jeweils betrachteten Bewirtschaftungsverfahrens die Einkommenserzielung in hohem Maße mit Unsicherheit verbunden ist, weil der Gewinn z.T. stark vom durchschnittlich zu erwartenden Wert abweicht. Die Streuung der Gewinne kann deshalb als ein Maß für das Einkommensrisiko angenommen werden.

Um stärker das der Einkommenserzielung zugrunde liegende Ursprungsrisiko beider Bewirtschaftungsverfahren vergleichen zu können, wurden in einem zweiten Analyseschritt zunächst die Preis- und Ertragsverteilungen der beiden ausgesuchten Feldfrüchte Winterweizen und Kartoffeln im Rahmen der deskriptiven Statistik analysiert. Die Ergebnisse sollten über die Intensität der Ertrags- und Preisschwankungen sowohl für den konventionellen als auch für den ökologischen Landbau Aufschluss geben. Um die statistischen Abhängigkeiten zwischen beiden Verteilungen näher quantifizieren zu können, wurde anschließend auf die Berechnung der Korrelationskoeffizienten zurückgegriffen ${ }^{11}$.

\footnotetext{
${ }^{11}$ Zur Messung der Richtung und der Stärke des mathematischen Zusammenhangs zwischen den einzelbetrieblichen Naturalerträgen und Verkaufspreisen wurde betriebsindividuell der Korrelationskoeffizient nach BRAVAIS-PEARSON berechnet (für nähere Informationen zur Korrelationsanalyse bzw. zu den unterschiedlichen Korrelationskoeffizienten vgl. MOSLER und SCHMID, 2003: 165ff).
} 
Hinter dieser Vorgehensweise stand die Frage, ob sich eventuell höhere Einzelrisiken im ökologischen Landbau auf Ebene der Erlösverteilungen aufgrund negativer Korrelationskoeffizienten zwischen Preisen und Erträgen gegenseitig aufheben und sich damit ein so genannter „natural hedge“ ergibt. Wenn sich nämlich in Jahren mit niedrigeren Erträgen im Ökolandbau regelmäßig ein „Ausgleich“ über den Preis einstellt, so dass die Umsatzerlöse insgesamt stabil bleiben, werden eventuell höhere Einzelrisiken in Form höherer Verkaufspreis- und Naturalertragsschwankungen über den Markt abgefedert. Dann nämlich ergäben sich auch im Falle höherer Naturalertrags- und Verkaufspreisschwankungen im ökologischen Landbau keine erhöhten Einkommensrisiken. Insbesondere der Vergleich des Vorhandenseins bzw. der Intensität eines „,natural hedge“ bietet also die Möglichkeit, das Ausmaß der betriebswirtschaftlichen Risiken zwischen ökologischem und konventionellem Landbau miteinander vergleichen zu können, sofern von den Einkommensrisiko mindernden Direktzahlungen und sonstigen finanziellen Förderungen abstrahiert wird, die ihrerseits einen Einkommen stabilisierenden Effekt aufweisen.

\subsection{MODIFIZIERUNG DES TBN-DATENSATZES FÜR DIE UNTERSUCHUNGEN}

Für die Untersuchungen auf Basis der einzelbetrieblichen Daten des BMELV-TBN waren im Vorfeld einige Modifikationen am Datensatz notwendig. Für beide Analyseschritte war es notwendig, im Zeitablauf identische Betriebe zu untersuchen. Die Auswahl der im TBN vorhanden Betriebe unterliegt jedoch großen Fluktuationen. Daher war es notwendig, alle Betriebe aus dem Datensatz zu entfernen, von denen keine 8 Jahresdatensätze zur Verfügung standen. Hinsichtlich der ökologischen Betriebe ist in der Vergangenheit wiederholt von öffentlicher Seite auf die ungenügende Anzahl ökologisch wirtschaftender Betriebe im TBN hingewiesen worden (vgl. bspw. MEYER, 2005: 1). Dieses Manko gestaltete sich bei der einzelbetrieblichen Analyse als schwerwiegendes Hemmnis. Um eine genügend große Gruppe von Öko-Betrieben im Untersuchungsdatensatz zu belassen, verblieben deshalb für den ersten Analyseschritt, d.h. für die Analyse der Betriebsgewinne, auch jene Öko-Betriebe im Datensatz, von denen nur 6 oder 7 Jahresdaten im TBN vorlagen. Die Begründung dafür, warum dieses lediglich im Falle des ersten Analyseschrittes geschah, liegt darin, dass für diesen ersten Analyseschritt noch zwei weitere Modifikationen des Datensatzes notwendig waren. Um Verzerrungen beim Vergleich der Streuung der Betriebsgewinne weitestgehend auszuschließen, war es zum einen notwendig, die Gewinnverteilungen der einzelnen Betriebe auf einen signifikanten Trend hin zu untersuchen. Hierbei wurden die Datenreihen aber lediglich auf einen linearen Trend hin untersucht. Weil die einzelnen Datenreihen nur aus 
sechs bis acht Einzelwerten bestanden, wurde eine weitergehende Trenduntersuchung im Vorfeld der Untersuchungen als nicht sinnvoll erachtet. Abbildung 11 zeigt dazu exemplarisch zwei verschiedene Gewinnverteilungen.

\section{Abbildung 11: Beispielhafte Darstellung zweier Gewinnverteilungen}

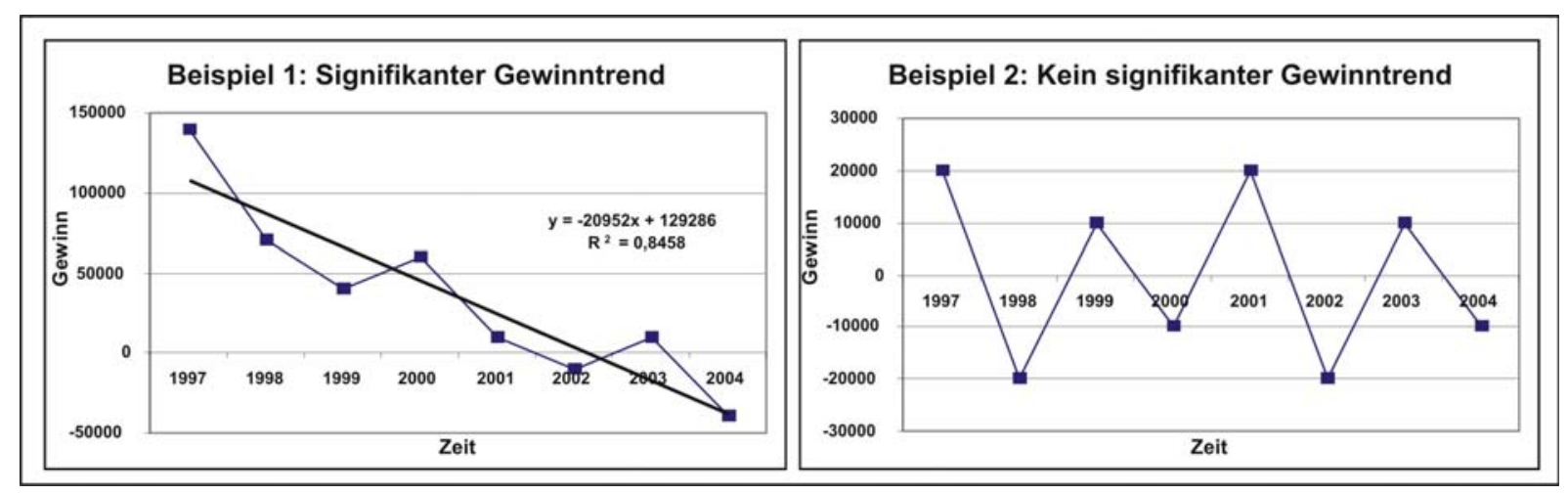

Quelle: Eigene Darstellung

Nur bei der ersten Gewinnverteilung kann im Rahmen einer linearen Einfachregression ein signifikanter Trend festgestellt werden. Bei der zweiten in der Abbildung dargestellten Gewinnverteilung liegt hingegen kein signifikanter Trend vor ${ }^{12}$. Alle Betriebe, bei denen ein signifikanter Trend festgestellt wurde, wurden ebenfalls dem Untersuchungsdatensatz entnommen. Grundsätzlich hätte auch bei diesen Betrieben die Intensität der Gewinnschwankungen analysiert und miteinander verglichen werden können. Dazu hätten die Standardfehler der einzelnen Gewinnverteilungen (d.h. die Abweichungen der Einzelgewinne vom jeweiligen Gewinntrend) ermittelt und analysiert werden müssen. Aufgrund der deutlich zu geringen Anzahl ökologisch wirtschaftender Betriebe im Datensatz mit einem signifikanten Trend wurde diese zunächst angedachte Vorgehensweise jedoch verworfen (siehe dazu auch Anhang 2).

Ein zweiter Grund für die Berücksichtigung von Öko-Betrieben im Datensatz, von denen nur sechs oder sieben Jahresdaten im TBN vorlagen, liegt darin, dass alle Betriebe für den ersten Analyseschritt gemäß ihrer betriebswirtschaftlichen Ausrichtung in Untergruppen eingeteilt wurden, um Aussagen zu den Gewinnschwankungen verschiedener Betriebstypen zu

\footnotetext{
${ }^{12}$ Für die Trenderkennung wurde für jeden Betrieb (d.h. für jede Gewinnverteilung) eine lineare Einfachregression mit der Zeit als erklärende Variable und dem Gewinn als zu erklärende Variable berechnet. Ergebnis war jeweils ein linearer mathematischer Zusammenhang, dessen Validität mit einem T-Test geprüft wurde. Konnte die Nullhypothese $\left(\mathrm{H}_{0}=\right.$ kein linearer Zusammenhang zwischen Zeit und Gewinn) auf einem 5\%igen Signifikanzniveau nicht abgelehnt werden, so war für die betreffende Gewinnverteilung kein signifikanter linearer Gewinntrend nachweisbar (für nähere Informationen zur Regressionsanalyse sowie zur Durchführung von Parametertests vgl. BLEYMÜLLER et al.; 2004: 107ff und 139ff).
} 
ermöglichen und ein Mindestmaß an Vergleichbarkeit zu gewährleisten. Sowohl die Gruppe der ökologisch wirtschaftenden als auch die der konventionell wirtschaftenden Betriebe wurden dazu in Ackerbau-, Futterbau- und sonstige Betriebe differenziert, von denen die sonstigen Betriebe in der Untersuchung aufgrund ihrer Heterogenität keine weitere Beachtung fanden. Eine weitere Differenzierung, bspw. nach der wirtschaftlichen Betriebsgröße wäre vor dem Hintergrund der generell zu geringen Anzahl der Öko-Betriebe im TBN nicht sinnvoll gewesen. Hätte man für den ersten Analyseschritt analog zur Vorgehensweise beim zweiten Analyseschritt lediglich auf die Gruppe der Öko-Betriebe mit acht aufeinander folgenden Jahresdaten zurückgegriffen, so wären bereits die Ergebnisse für den Acker- und den Futterbau aufgrund der zu geringen Anzahl an Betrieben wenig aussagekräftig gewesen.

\subsubsection{ANALYSE DER VOLATILITÄT DER BETRIEBSGEWINNE}

Die einzelbetrieblichen Gewinnschwankungen wurden für drei verschiedene Ergebnisgrößen analysiert, und zwar für die Gewinne inklusive aller Prämienzahlungen, für die Gewinne exklusive aller Prämienzahlungen und schließlich für die Gewinne exklusive der Prämienzahlungen aus der 2. Säule der Gemeinsamen Agrarpolitik (GAP). Die jeweiligen einzelbetrieblichen Gewinnverteilungen wurden dazu mittels deskriptiver Statistik analysiert. Anschließend wurden die Mittelwerte der einzelbetrieblichen Mittelwerte für den Betriebsgewinn sowie für den Variationskoeffizienten der Betriebsgewinne berechnet. Tabelle 9 bis Tabelle 11 zeigen die Ergebnisse. ${ }^{13}$ Zusätzlich ist wiederum jeweils die Anzahl der zugrunde liegenden Betriebe angegeben, die sich aus der Modifikation des Datensatzes ergeben hat.

Tabelle 9: Deskriptive Statistik zur mittleren einzelbetrieblichen Gewinnentwicklung (inkl. Prämienzahlungen) ökologischer und konventioneller Betriebe

\begin{tabular}{|c|c|c|c|c|}
\hline & \multicolumn{2}{|c|}{ ACKERBAU } & \multicolumn{2}{|c|}{ FUTTERBAU } \\
\hline & Öko-Betriebe* & $\begin{array}{c}\text { Konventionelle } \\
\text { Betriebe }^{*}\end{array}$ & Öko-Betriebe* & $\begin{array}{c}\text { Konventionelle } \\
\text { Betriebe }^{*}\end{array}$ \\
\hline Anzahl der Betriebe & 22 & 498 & 69 & 1095 \\
\hline $\begin{array}{l}\text { Mittelwert der mittleren Betriebsgewinne } \\
\text { (inkl. Prämienzahlungen) }\end{array}$ & $27.471 €$ & $49.629 €$ & $24.660 €$ & $30.534 €$ \\
\hline Mittelwert der Variationskoeffizienten & 1,33 & 0,83 & 0,89 & 0,75 \\
\hline
\end{tabular}

*Von insgesamt 143 Öko-Betrieben (3278 konventionellen Betrieben) verblieben 128 (2862) Betriebe in der Stichprobe, bei denen kein signifikanter Gewinntrend festgestellt wurde. Darunter waren 22 (498) Ackerbaubetriebe und 69 (1095) Futterbaubetriebe. Bei den restlichen 37 (1269) Betrieben handelte es sich um Betriebe, die keiner betriebswirtschaftlichen Ausrichtung eindeutig zugeordnet werden konnten. Sie fanden deshalb keine weitere Berücksichtigung.

Quelle: Eigene Darstellung auf Grundlage von TBN-Daten der WJ'e 1996/97 bis 2003/04

\footnotetext{
${ }^{13}$ Eine detailliertere Aufstellung der Ergebnisse ist in Anhang 2 einzusehen.
} 
Tabelle 10: Deskriptive Statistik zur mittleren einzelbetrieblichen Gewinnentwicklung (exkl. Prämienzahlungen) ökologischer und konventioneller Betriebe

\begin{tabular}{|c|c|c|c|c|}
\hline & \multicolumn{2}{|c|}{ ACKERBAU } & \multicolumn{2}{|c|}{ FUTTERBAU } \\
\hline & Öko-Betriebe* & $\begin{array}{c}\text { Konventionelle } \\
\text { Betriebe }^{*}\end{array}$ & Öko-Betriebe ${ }^{\star}$ & $\begin{array}{c}\text { Konventionelle } \\
\text { Betriebe }^{*}\end{array}$ \\
\hline Anzahl der Betriebe & 22 & 491 & 60 & 1088 \\
\hline $\begin{array}{l}\text { Mittelwert der mittleren Betriebsgewinne } \\
\text { (exkl. Prämienzahlungen) }\end{array}$ & $-34.370 €$ & $-16.844 €$ & $5.151 €$ & $4.737 €$ \\
\hline Mittelwert der Variationskoeffizienten & 1,69 & 1,44 & 1,59 & 1,46 \\
\hline
\end{tabular}

*Von insgesamt 143 Öko-Betrieben (3278 konventionellen Betrieben) verblieben 116 (2814) Betriebe in der Stichprobe, bei denen kein signifikanter Gewinntrend festgestellt wurde. Darunter waren 22 (491) Ackerbaubetriebe und 60 (1088) Futterbaubetriebe. Bei den restlichen 34 (1235) Betrieben handelte es sich um Betriebe, die keiner betriebswirtschaftlichen Ausrichtung eindeutig zugeordnet werden konnten. Sie fanden deshalb keine weitere Berücksichtigung.

Quelle: Eigene Darstellung auf Grundlage von TBN-Daten der WJ'e 1996/97 bis 2003/04

\section{Tabelle 11: Deskriptive Statistik zur mittleren einzelbetrieblichen Gewinnentwicklung (exkl. Prämienzahlungen der 2. Säule der GAP) ökologischer und konventioneller Betriebe}

\begin{tabular}{|c|c|c|c|c|}
\hline & \multicolumn{2}{|c|}{ ACKERBAU } & \multicolumn{2}{|c|}{ FUTTERBAU } \\
\hline & Öko-Betriebe* & $\begin{array}{c}\text { Konventionelle } \\
\text { Betriebe }^{*}\end{array}$ & Öko-Betriebe* & $\begin{array}{c}\text { Konventionelle } \\
\text { Betriebe* }^{*}\end{array}$ \\
\hline Anzahl der Betriebe & 19 & 502 & 64 & 1100 \\
\hline $\begin{array}{l}\text { Mittelwert der mittleren Betriebsgewinne } \\
\text { (exkl. Prämienzahlungen 2. Säule) }\end{array}$ & $21.215 €$ & $47.469 €$ & $17.305 €$ & $27.989 €$ \\
\hline Mittelwert der Variationskoeffizienten & 1,36 & 0,87 & 1,37 & 0,83 \\
\hline
\end{tabular}

*Von insgesamt 143 Öko-Betrieben (3278 konventionellen Betrieben) verblieben 121 (2878) Betriebe in der Stichprobe, bei denen kein signifikanter Gewinntrend festgestellt wurde. Darunter waren 19 (502) Ackerbaubetriebe und 64 (1100) Futterbaubetriebe. Bei den restlichen 38 (1276) Betrieben handelte es sich um Betriebe, die keiner betriebswirtschaftlichen Ausrichtung eindeutig zugeordnet werden konnten. Sie fanden deshalb keine weitere Berücksichtigung.

Quelle: Eigene Darstellung auf Grundlage von TBN-Daten der WJ'e 1996/97 bis 2003/04

Die Ergebnisse auf Ebene der Einzelbetriebe in Tabelle 9 (unter Berücksichtigung aller Prämienzahlungen) zeigen insbesondere bei den ökologisch wirtschaftenden Ackerbaubetrieben im Unterschied zu den Ergebnissen auf aggregierter Ebene höhere mittlere Gewinnschwankungen. Im Falle der konventionellen Ackerbaubetriebe betrug die mittlere Streuung der Gewinne im betrachteten 8-Jahres-Zeitraum nur $83 \%$ des mittleren Betriebsgewinns. Dem gegenüber betrug sie bei den ökologischen Ackerbaubetrieben im Mittel ganze $133 \%$ des mittleren Betriebsgewinns. Jedoch handelt es sich bei den untersuchten Öko-Betrieben, wie die Mittelwerte zur absoluten Höhe der Betriebsgewinne in den Tabellen zeigen, um Betriebe mit vergleichsweise geringeren absoluten Gewinnen. Um eine Vergleichbarkeit der Gewinnschwankungen zu ermöglichen, wurde daher wiederum der Variationskoeffizient (anstatt der Standardabweichung) als Vergleichsmaß für die Gewinnvariation gewählt. Zwar lässt sich aus der Analyse der einzelbetrieblichen Gewinne und ihrer Schwankungen insgesamt ein mit sinkender absoluter Gewinnhöhe steigender 
Variationskoeffizient vermuten (vgl. dazu auch Kapitel 4.2.2.2). Die absoluten Differenzen der Variationskoeffizienten machen dennoch höhere Einkommensrisiken im ökologischen Landbau wahrscheinlich. Die Risiko reduzierende Wirkung von Prämienzahlungen sowohl für die ökologisch wirtschaftenden als auch für die konventionell wirtschaftenden Betriebe zeigt sich in Tabelle 10. Infolge der potenziellen Reduzierung der flächenbezogenen ÖkoFörderung im Rahmen der beschlossenen Einsparungen in der 2. Säule der GAP werden sich, wie speziell Tabelle 11 offenbart, die Gewinnschwankungen im ökologischen Landbau vermutlich sogar noch weiter erhöhen.

\subsubsection{ANALYSE DER NATURALERTRÄGE UND VERKAUFSPREISE SOWIE DES „NATURAL HEDGE“}

Die Analyse der Naturalerträge und Verkaufspreise sowie des ,natural hedge“ beschränkt sich aufgrund des begrenzten zur Verfügung stehenden Datensatzes auf die beiden Feldfrüchte Winterweizen und Kartoffeln. Diese beiden Früchte wurden in erster Linie aufgrund ihrer großen Verbreitung bzw. erheblichen Bedeutung im Ökolandbau gewählt, die sich auch in der Breite der Datenbasis widerspiegelt.

\subsection{UNTERSUCHUNGSOBJEKT: WINTERWEIZEN}

Analog zur angekündigten Vorgehensweise wurden zunächst die Naturalerträge und die Verkaufspreise des Winterweizens analysiert. Tabelle 12 zeigt dazu die Ergebnisse aus der Analyse der einzelbetrieblichen Daten. 
Tabelle 12: Risikoanalyse am Beispiel des Winterweizens auf Basis der einzelbetrieblichen Daten des BMELV-TBN

\begin{tabular}{|c|c|c|c|c|}
\hline & \multicolumn{2}{|c|}{ ökologische Betriebe $^{1}$} & \multicolumn{2}{|c|}{ konventionelle Betriebe $^{1}$} \\
\hline & Naturalerträge & Betriebe & Naturalerträge & Betriebe \\
\hline Mittelwert in dt/ha & 37,29 & \multirow{7}{*}{44} & 68,48 & \multirow{7}{*}{1589} \\
\hline Standardabweichung & 9,06 & & 9,89 & \\
\hline Variationskoeffizient & 0,24 & & 0,14 & \\
\hline & Verkaufspreise & & Verkaufspreise & \\
\hline \multirow{4}{*}{$\begin{array}{l}\text { Mittelwert in } € / d t \\
\text { Standardabweichung } \\
\text { Variationskoeffizient }\end{array}$} & 43,56 & & 11,69 & \\
\hline & 11,37 & & 1,40 & \\
\hline & 0,26 & & 0,12 & \\
\hline & Natural Hedge & Betriebe & Natural Hedge & Betriebe \\
\hline Korrelationskoeffizient*: & $-0,04$ & 44 & $-0,01$ & 1589 \\
\hline Korrelationskoeffizient ${ }^{\star \star}$ : & 0,10 & 7 & $-0,06$ & 200 \\
\hline Korrelationskoeffizient ${ }^{\star \star \star}$ : & 0,14 & 5 & $-0,11$ & 113 \\
\hline
\end{tabular}

${ }^{1}$ TBN-Betriebe mit Buchführungsdaten zu den Wirtschaftsjahren 96/97 bis 03/04

*Alle Betriebe wurden berücksichtigt.

**Nur die Betriebe mit einem auf dem Signifikanzniveau von $p<0,1$ (2-seitig) signifikanten Korrelationskoeffizienten wurden berücksichtigt.

***Nur die Betriebe mit einem auf dem Signifikanzniveau von $p<0,05$ (2-seitig) signifikanten Korrelationskoeffizienten wurden berücksichtigt.

Quelle: Eigene Darstellung auf Grundlage von TBN-Daten der WJ'e 1996/97 bis 2003/04

Die Mittelwerte der Naturalerträge sowie der Erzeugerpreise zeigen das erwartete Bild. Während die Naturalerträge im ökologischen Landbau mit durchschnittlich 37,29 dt/ha im Vergleich zu 68,48 dt/ha geringer sind, sind die mittleren Verkaufspreise mit 43,56 €/dt im Vergleich zu 11,69 €/dt deutlich höher. Ein Blick auf die Schwankung beider Größen bestätigt wiederum die Einschätzungen vieler Beteiligter. Sowohl die Naturalerträge als auch die Erzeugerpreise zeigen über den betrachteten 8-Jahres-Zeitraum im ökologischen Landbau eine höhere Streuung. Dabei ist allerdings die relativ geringe Anzahl von Öko-Betrieben in der Untersuchung zu berücksichtigen. Das gilt insbesondere auch für die durchgeführte Korrelationsanalyse, für die deshalb die Korrelationskoeffizienten in der Tabelle auf verschiedenen Signifikanzebenen angegeben wurden. Trotzdem zeigt sich ein tendenziell geringer ausgeprägter „natural hedge“ bei den Winterweizen anbauenden Öko-Betrieben. Die höheren Einzelrisiken im ökologischen Weizenanbau gleichen sich somit nicht gegenseitig aus, was analog zur Untersuchung der Betriebsgewinne wiederum auf höhere betriebswirtschaftliche Risiken im Ökolandbau hindeutet.

\subsection{UNTERSUCHUNGSOBJEKT: KARTOFFELN}

Auch zum Kartoffelanbau wurden Naturalerträge, Verkaufspreise und der „natural hedge“ untersucht. Die Ergebnisse sind in Tabelle 13 dargestellt. 
Tabelle 13: Risikoanalyse am Beispiel der Kartoffeln auf Basis der einzelbetrieblichen Daten des BMELV-TBN

\begin{tabular}{|c|c|c|c|c|}
\hline & \multicolumn{2}{|c|}{ ökologische Betriebe ${ }^{1}$} & \multicolumn{2}{|c|}{ konventionelle Betriebe ${ }^{1}$} \\
\hline & Naturalerträge & Betriebe & Naturalerträge & Betriebe \\
\hline \multirow{4}{*}{$\begin{array}{l}\text { Mittelwert in dt/ha } \\
\text { Standardabweichung } \\
\text { Variationskoeffizient }\end{array}$} & 172,80 & \multirow{7}{*}{34} & 297,85 & \multirow{7}{*}{345} \\
\hline & 57,17 & & 68,64 & \\
\hline & 0,33 & & 0,23 & \\
\hline & Verkaufspreise & & Verkaufspreise & \\
\hline \multirow{4}{*}{$\begin{array}{l}\text { Mittelwert in } € / d t \\
\text { Standardabweichung } \\
\text { Variationskoeffizient }\end{array}$} & 39,48 & & 12,26 & \\
\hline & 10,09 & & 2,89 & \\
\hline & 0,26 & & 0,24 & \\
\hline & Natural Hedge & Betriebe & Natural Hedge & Betriebe \\
\hline Korrelationskoeffizient*: & $-0,18$ & 34 & $-0,26$ & 345 \\
\hline Korrelationskoeffizient ${ }^{\star \star}$ : & $-0,26$ & 8 & $-0,65$ & 84 \\
\hline Korrelationskoeffizient ${ }^{\star \star \star}$ : & $-0,52$ & 5 & $-0,70$ & 55 \\
\hline
\end{tabular}

${ }^{1}$ TBN-Betriebe mit Buchführungsdaten zu den Wirtschaftsjahren 96/97 bis 03/04

*Alle Betriebe wurden berücksichtigt.

**Nur die Betriebe mit einem auf dem Signifikanzniveau von $\mathrm{p}<0,1$ (2-seitig) signifikanten Korrelationskoeffizienten wurden berücksichtigt.

***Nur die Betriebe mit einem auf dem Signifikanzniveau von $\mathrm{p}<0,05$ (2-seitig) signifikanten Korrelationskoeffizienten wurden berücksichtigt.

Quelle: Eigene Darstellung auf Grundlage von TBN-Daten der WJ'e 1996/97 bis 2003/04

Hinsichtlich der mittleren absoluten Höhe der Naturalerträge und Verkaufspreise zeigt sich hier ein ähnliches Bild wie beim Winterweizen. Auch hier sind die Naturalerträge im Ökolandbau deutlich geringer, während die Verkaufspreise deutlich höher sind. Ebenso verhält es sich bei einem Blick auf die Streuungsmaße. Die Naturalerträge im ökologischen Landbau weisen wiederum eine höhere Streuung auf, was den Ergebnissen paralleler Untersuchungen genau entspricht (vgl. dazu DREYER, 2005: 30). Auch die Verkaufspreise zeigen bei den Kartoffeln im Ökolandbau wenn auch nur in schwächerem Umfang eine geringere Stabilität als im konventionellen Landbau. Hinsichtlich des statistischen Zusammenhangs von Naturalerträgen und Verkaufspreisen zeigt sich bei den Kartoffeln im Vergleich zum Winterweizen ebenfalls ein ähnliches Bild. In beiden Fällen liegen zwar negative Korrelationskoeffizienten und damit ein „natural hedge“ vor. Dieser ist im ökologischen Landbau aber schwächer ausgeprägt. Somit weisen auch hier die Korrelationskoeffizienten auf höhere der Einkommenserzielung im ökologischen Kartoffelanbau zugrunde liegende Risiken hin.

\subsubsection{FAZIT}

Die Ausführungen zur Datengrundlage haben bereits deutlich gemacht, dass ein wesentlicher Hemmschuh quantitativer Analysen zu betriebswirtschaftlichen Fragen im ökologischen Landbau nach wie vor die zu geringe Datenbasis zum ökologischen Landbau ist. Weil bereits 
bei den durchgeführten Analyseschritten die Anzahl der zugrunde liegenden Öko-Betriebe sehr gering war, wurden Überlegungen, die Untersuchung noch spezifischer auf verschiedene Betriebstypen oder direkt auf Deckungsbeitragsrechnungen auszudehnen verworfen. Zweifelsohne muss die geringe Anzahl ökologisch wirtschaftender Betriebe bei der Interpretation der Untersuchungsergebnisse berücksichtigt werden. Die Tatsache jedoch, dass, über den gesamten Verlauf der Analyse betrachtet, die nachgewiesenen Risiken bei den ÖkoBetrieben in allen Fällen höher waren, gibt Anlass zu der Vermutung, dass die Ergebnisse der empirischen Untersuchungen hinsichtlich etwaiger Umstellungshemmnisse nicht auf einem lediglich „gefühlt“ höheren Einkommensrisiko, sondern auf tatsächlich höheren Einkommensrisiken basieren. Insbesondere vor dem Hintergrund der zukünftig zurückgehenden Fördermittel aus der zweiten Säule und daraus möglicherweise resultierender geringerer Förderpotenziale für den ökologischen Landbau können die Erkenntnisse zu den höheren Einkommensrisiken im Ökolandbau von Bedeutung sein. Ist es nämlich der politische Wille, den ökologischen Landbau in Deutschland weiter auszuweiten, so sollten auch Politikmaßnahmen zur gezielten Reduktion der Einkommensrisiken in Betracht gezogen werden. Denn nur im Falle eines gezielten Abbaus der Umstellungshemmnisse ist auch eine effektive und vor allem effiziente Förderung des ökologischen Landbaus möglich.

\subsection{SYSTEMATIK UND AUSWIRKUNGEN DER FLEXIBLEN FÖRDERUNG}

Mit dem System der Flexiblen Förderung soll eine Alternative zum gegenwärtig geltenden Festbetragsprämiensystem vorgestellt werden, die genau den im Vorkapitel aufgezeigten potentiell höheren Einkommensrisiken im ökologischen Landbau entgegenwirken könnte. Dazu soll zunächst auf die Systematik dieses neuen Instruments eingegangen werden, bevor in den folgenden Kapiteln zu seinen Auswirkungen Stellung bezogen wird.

\subsubsection{SYSTEMATIK DER FLEXIBLEN FÖRDERUNG}

\subsubsection{GRUNDSÄTZLICHES}

Das Flexible Fördersystem setzt sich, wie Abbildung 12 verdeutlicht, aus zwei unterschiedlichen Komponenten zusammen. 
Abbildung 12: Aufbau der Flexiblen Förderung

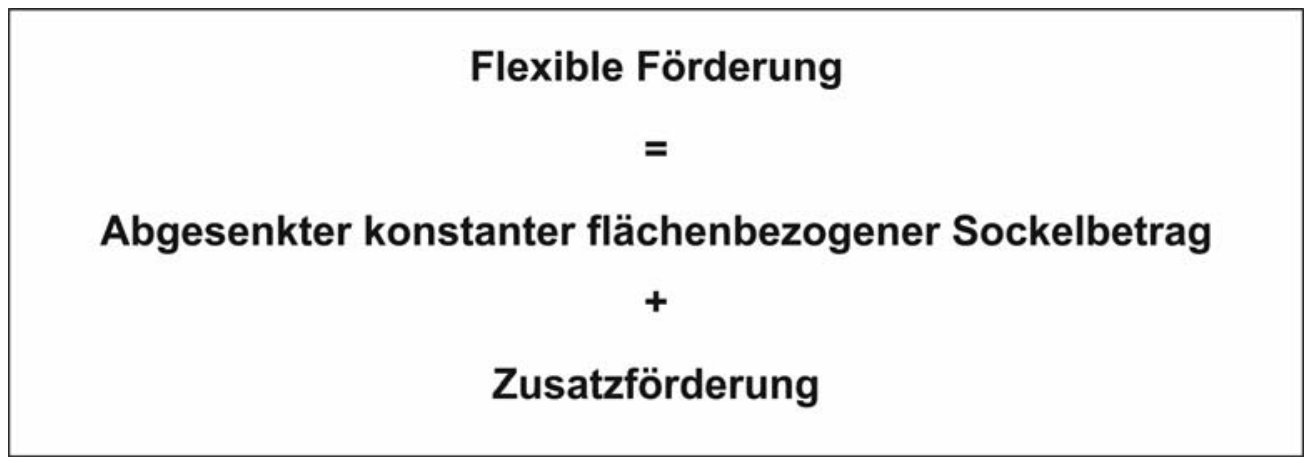

Quelle: Eigene Darstellung

Zum einen ist der reduzierte, konstante, flächenbezogene Sockelbetrag zu nennen. Er weist die gleichen Eigenschaften auf wie die derzeit geltende Festbetragsprämie. Der Hektarsatz ist jedoch geringer. Die Tatsache, dass er an die ökologisch bewirtschaftete Fläche gekoppelt ist und für eine vorgegebene Fläche folglich konstant ist, unterscheidet ihn von der Zusatzförderung, der zweiten Komponente der Flexiblen Förderung. Die Zusatzförderung, deren Konzeption in den folgenden Kapiteln näher erläutert wird, bemisst sich an den einzelbetrieblichen Schwankungen der ertragsteuerlichen Einkünfte aus Land- und Forstwirtschaft $(\mathrm{LuF})^{14}$ in einem festzulegenden Intervall mehrerer Wirtschaftsjahre. Sie ist damit in ihrer Höhe nicht standardisiert wie der Sockelbetrag, sondern wird jedes Jahr betriebsindividuell festgesetzt.

\subsubsection{DiE ZUSATZFördERUNG ALS BESTANDTEIL DER FLEXIBLEN FÖRDERUNG}

Abbildung 13 zeigt schematisch das Grundprinzip zur Berechung der Zusatzförderung, das im Rahmen der folgenden Unterkapitel Schritt für Schritt erklärt wird.

\footnotetext{
${ }^{14}$ Die Einkünfte aus Land- und Forstwirtschaft entsprechen gemäß $§ 2$ EStG einer von sieben Einkunftsarten. Sie sind nicht zu verwechseln mit dem ,zu versteuernden Einkommen' (zvE), das sich aus der Addition der Einkünfte aller Einkunftsarten ergibt.
} 
Abbildung 13: Berechnungsschema für die Zusatzförderung

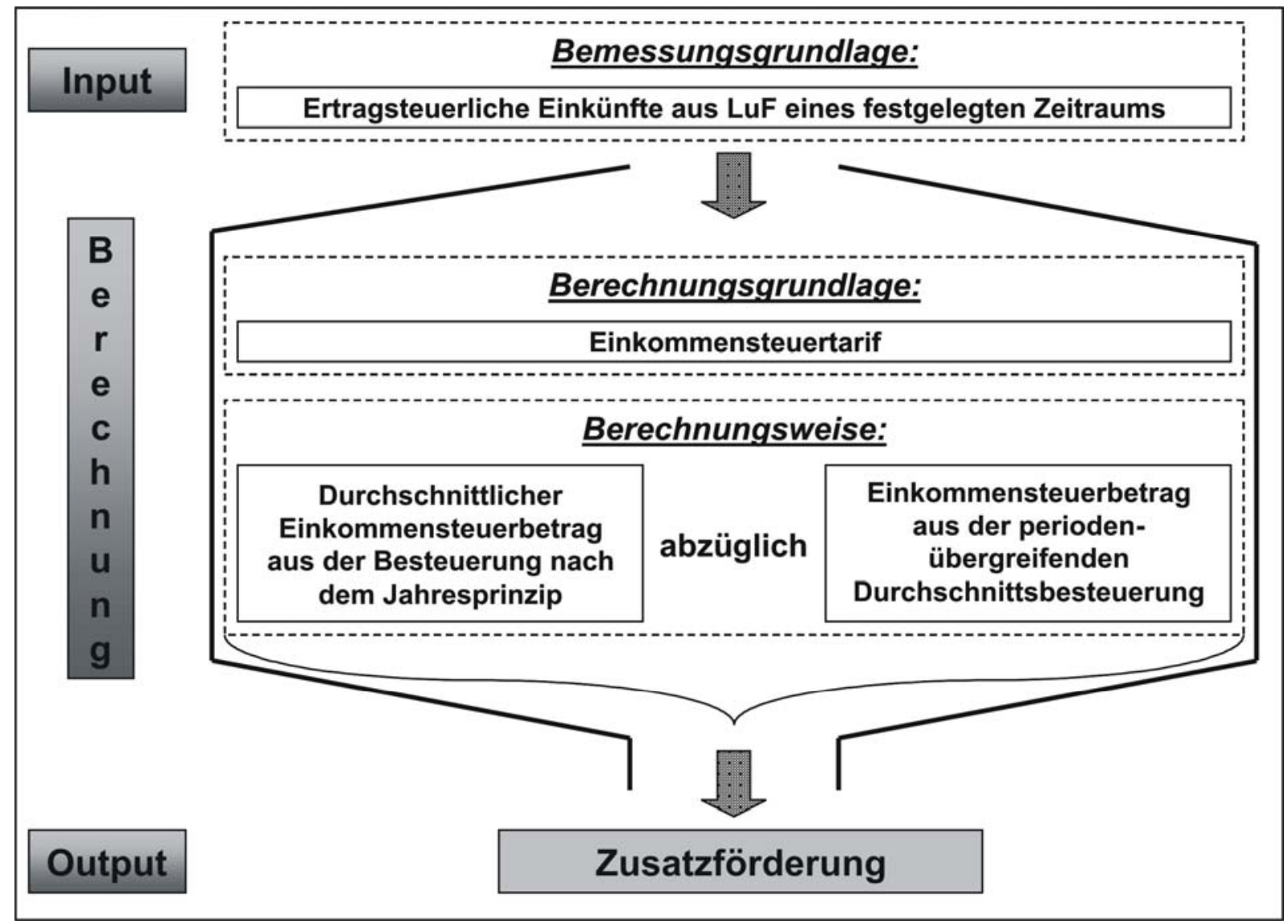

Quelle: Eigene Darstellung

\subsection{WIRKUNGSWEISE DES DEUTSCHEN EINKOMMENSTEUERTARIFS GEMÄß § 32A ESTG}

Der derzeit in Deutschland geltende Einkommensteuertarif ist ein progressiver Tarif: Je höher das zu versteuernde Einkommen (zvE) ist, desto höher ist auch der Durchschnittssteuersatz. Daraus ergibt sich der so genannte Progressionseffekt. Der Einkommensteuertarif wird in $\S 32 \mathrm{a}$ EStG definiert. Abbildung 14 zeigt die Verläufe von Durchschnitts- und Grenzsteuersatz für den Einkommensteuertarif 2006 sowohl für die getrennte Veranlagung als auch für die Zusammenveranlagung. 
Abbildung 14: Durchschnitts- und Grenzsteuersatz gemäß ESt-Tarif 2006

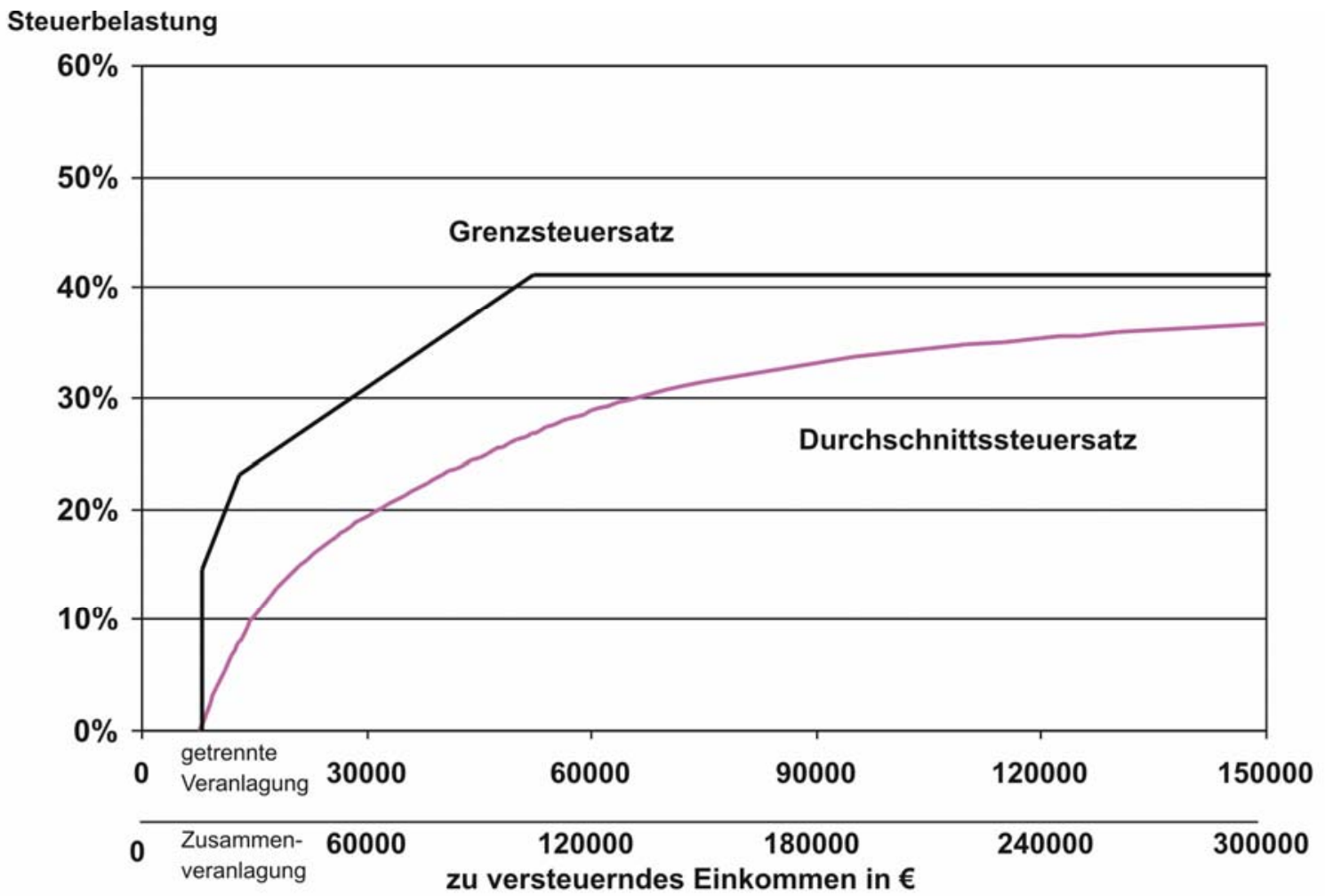

Quelle: Eigene Darstellung gemäß ESt-Tarif 2006

Der Einkommensteuertarif besteht aus drei Tarifzonen. Ist das zvE nicht höher als $7.664 €$, so fällt keine Einkommensteuer an. Dieser Betrag wird deshalb auch Grundfreibetrag genannt. Erst wenn das zvE $7.664 €$ übersteigt, fällt Einkommensteuer an. Der Eingangssteuersatz liegt bei $15 \%$. Die erste Progressionszone steigt bis zu einem ESt-Satz von $24 \%$ bei einem zvE von $12.740 €$ steil an. Danach beginnt die flacher ansteigende, zweite Progressionszone bis zu einem zvE von $51.151 €$ und einem Grenzsteuersatz von $42 \%$. Ab einem zvE von $52.152 €$ beginnt die Proportionalzone, in der der Grenzsteuersatz konstant bei $42 \%$ verbleibt. Jeder Euro, der das zvE in dieser Zone erhöht, wird mit einer Einkommensteuer von 0,42 € belegt.

Um die monetären Wirkungen des Einkommensteuertarifs $2006 \mathrm{zu}$ veranschaulichen, zeigt Tabelle 14 den Einkommensteuerbetrag, den Grenzsteuersatz sowie den Durchschnittssteuersatz für verschiedene beispielhafte zvE. 
Tabelle 14: Beispielbetrachtungen zur Wirkung des ESt-Tarif 2006

\begin{tabular}{|c|c|c|c|}
\hline Zu versteuerndes Einkommen (zvE) & Steuerbetrag & Durchschnittssteuersatz & Grenzsteuersatz \\
\hline 5000 & 0 & $0,00 \%$ & $0,00 \%$ \\
\hline $\mathbf{7 6 6 4}$ & $\mathbf{0}$ & $\mathbf{0 , 0 0} \%$ & $\mathbf{1 5 , 0 0 \%}$ \\
\hline 10000 & 398 & $3,98 \%$ & $20,00 \%$ \\
\hline $\mathbf{1 2 7 4 0}$ & $\mathbf{9 8 9}$ & $\mathbf{7 , 7 6} \%$ & $\mathbf{2 4 , 2 0 \%}$ \\
\hline 20000 & 2850 & $14,25 \%$ & $27,50 \%$ \\
\hline 30000 & 5807 & $19,36 \%$ & $32,10 \%$ \\
\hline 40000 & 9223 & $23,06 \%$ & $36,70 \%$ \\
\hline 50000 & 13096 & $26,19 \%$ & $41,20 \%$ \\
\hline $\mathbf{5 2 1 5 2}$ & $\mathbf{1 3 9 8 9}$ & $\mathbf{2 6 , 8 2} \%$ & $\mathbf{4 2 , 0 0} \%$ \\
\hline 75000 & 23586 & $31,45 \%$ & $42,00 \%$ \\
\hline 100000 & 34086 & $34,09 \%$ & $42,00 \%$ \\
\hline 150000 & 55086 & $36,72 \%$ & $42,00 \%$ \\
\hline
\end{tabular}

Quelle: Eigene Berechnung gemäß ESt-Tarif 2006

\subsection{BERECHNUNGSWEISE DER ZUSATZFÖRDERUNG}

Die geltende Einkommensteuergesetzgebung basiert auf dem Zeitabschnittsprinzip. Das Einkommen eines Kalenderjahres wird der Einkommensteuerfestsetzung zugrunde gelegt (= Jahresprinzip). Grundsätzlich wäre aber auch eine periodenübergreifende Durchschnittsbesteuerung denkbar, bei der das durchschnittliche $\mathrm{zvE}$ einer festgelegten Anzahl von Kalenderjahren der Einkommensteuerfestsetzung zugrunde gelegt wird. Tabelle 15 zeigt dazu mit einem Beispiel die Wirkung einer dreijährigen Durchschnittsbesteuerung im Vergleich zur Besteuerung gemäß dem Jahresprinzip. Die Berechnungen basieren auf den steuerlichen Einkünften aus Land- und Forstwirtschaft (LuF) als Teil des zvE.

Tabelle 15: Beispielbetrachtung zur Wirkung einer dreijährigen Durchschnittsbesteuerung im Vergleich zur Wirkung der gegenwärtigen Besteuerung nach dem Jahresprinzip

\begin{tabular}{|c|c|c|c|c|}
\hline \multicolumn{5}{|c|}{ Gegenwärtige Besteuerung nach dem Jahresprinzip } \\
\hline & \multirow[b]{2}{*}{$\begin{array}{c}\text { Einkünfte aus } \\
\text { LuF }\end{array}$} & \multicolumn{3}{|c|}{ Jährliche Besteuerung gemäß dem ESt-Tarif 2006} \\
\hline & & Steuerbetrag & $\begin{array}{c}\text { Durchschnittssteuersatz } \\
\text { (gerundet) }\end{array}$ & $\begin{array}{c}\text { Grenzsteuersatz } \\
\text { (gerundet) }\end{array}$ \\
\hline Jahr 1 & $32.604 €$ & $6.653 €$ & $20 \%$ & $33 \%$ \\
\hline Jahr 2 & $50.371 €$ & $13.248 €$ & $26 \%$ & $41 \%$ \\
\hline Jahr 3 & $5.186 €$ & $0 €$ & $0 \%$ & $0 \%$ \\
\hline \multicolumn{2}{|c|}{ Summe der Steuerbeträge: } & $19.901 €$ & & \\
\hline \multicolumn{2}{|c|}{ Durchschnittsbetrag pro Jahr: } & $6.634 €$ & & \\
\hline \multicolumn{5}{|c|}{ Wirkung einer Durchschnittsbesteuerung } \\
\hline \multirow{2}{*}{$\begin{array}{l}\text { Summe der } \\
\text { drei LuF- } \\
\text { Einkünfte }\end{array}$} & \multirow{2}{*}{$\begin{array}{l}\text { Durchschnittl. } \\
\text { LuF-Einkünfte } \\
\text { pro Jahr }\end{array}$} & \multicolumn{3}{|c|}{ Besteuerung gemäß dem ESt-Tarif 2006} \\
\hline & & Steuerbetrag & \multicolumn{2}{|c|}{$\begin{array}{l}\text { Durchschnittssteuersatz } \\
\text { (gerundet) }\end{array}$} \\
\hline $88.161 €$ & $29.387 €$ & $5.613 €$ & \multicolumn{2}{|l|}{$19 \%$} \\
\hline \multicolumn{2}{|c|}{$\begin{array}{l}\text { Jährliche Differenz der } \\
\text { Steuerbeträge: }\end{array}$} & $1.021 €$ & \multicolumn{2}{|c|}{$=$ jährliche Zusatzförderung } \\
\hline
\end{tabular}

Quelle: Eigene Berechnung gemäß ESt-Tarif 2006 
Das angegebene Beispiel offenbart, dass sich im Vergleich zur Durchschnittsbelastung der Besteuerung nach dem Jahresprinzip bei Anwendung der dreijährigen Durchschnittsbesteuerung für den Steuerpflichtigen eine Einkommensteuerminderbelastung von $1.021 €$ ergäbe. Genau diese Differenz entspricht der jährlichen Zusatzförderung. Im Folgenden ist die Berechnung der Zusatzförderung in allgemeiner Form dargestellt:

\section{Zusatzförderung}

$$
\begin{aligned}
& = \\
& \left(\frac{\operatorname{ESt}\left(\operatorname{EinkLu}_{\mathrm{T}}\right)+\operatorname{ESt}\left(\operatorname{EinkLu}_{\mathrm{T}-1}\right)+\operatorname{ESt}\left(\operatorname{EinkLu}_{\mathrm{T}-2}\right)+\ldots+\operatorname{ESt}\left(\operatorname{EinkLuF}_{\mathrm{T}-\mathrm{N}}\right)}{\mathrm{N}+1}\right. \\
& \left.\operatorname{ESt}\left(\frac{\operatorname{EinkLuF}_{\mathrm{T}}+\operatorname{EinkLuF}_{\mathrm{T}-1}+\operatorname{EinkLuF}_{\mathrm{T}-2}+\ldots+\operatorname{EinkLuF}_{\mathrm{T}-\mathrm{N}}}{N+1}\right)\right)
\end{aligned}
$$

ESt: $\quad$ Einkommensteuer

N: $\quad$ Anzahl der Jahre des Bemessungszeitraums

EinkLuF: $\quad$ Einkünfte aus Land- und Forstwirtschaft im Kalenderjahr

T: $\quad$ Das jüngste Kalenderjahr, für das zum Zeitpunkt der Beantragung der Förderung ein Einkommensteuerbescheid vorliegt.

Analog zur Beispielbetrachtung in Tabelle 15 könnten die Berechnungen auch mit deutlich unterschiedlichen Einkommensverteilungen und Periodenlängen durchgeführt werden. In keinem Fall ergäbe sich jedoch ein negativer Betrag für die Zusatzförderung. Ein Grund dafür liegt in dem geltenden Grundfreibetrag, der bei Anwendung der Durchschnittsbesteuerung effektiver ausgenutzt werden kann. Bei der Besteuerung nach dem Jahresprinzip führt er dazu, dass im Beispiel in Tabelle 15 im dritten Jahr bei Einkünften von lediglich $5.186 €$ keine Einkommensteuer anfällt. Hätte in diesem Jahr das Einkommen jedoch genau $7.664 €$ betragen, so wäre ebenfalls keine Steuer angefallen. Die Differenz zwischen beiden Beträgen kann bei Anwendung des Jahresprinzips nicht vor- oder zurückgetragen werden und verfällt somit, was bei Anwendung der Durchschnittsbesteuerung nicht passiert. Ein zweiter Grund für den stets geringeren Steuerbetrag aus der periodenübergreifenden Durchschnittsbesteuerung ergibt sich aus der Abschwächung des Progressionseffektes. Im Vorkapitel konnte gezeigt werden, dass infolge der in den beiden Tarifzonen ansteigenden Grenzsteuersätze hohe Einkommen einem höheren Durchschnittssteuersatz unterliegen als niedrigere. Dieser Effekt führt dazu, dass sich infolge der periodenübergreifenden 
Durchschnittsbesteuerung ein niedrigerer Durchschnittssteuersatz ergibt, woraus folglich auch eine niedrigere Einkommensteuerbelastung resultiert.

Beide Effekte führen aber nicht nur dazu, dass sich keine negative Zusatzförderung einstellt. Auf ihnen beruht auch die Wirkung dieses Instruments, auf die im folgenden Kapitel näher eingegangen werden soll.

Allerdings kann man sich bei dieser Methodik gleichermaßen eines vom Einkommensteuerrecht abgekoppelten, je nach Länderetat und Förderwillen individuell konstruierten Veranlagungstarifs bedienen. Das Einkommensteuerrecht bietet also lediglich die Methode, nicht aber die rechtlichen Voraussetzungen. Somit kann die Förderung im Ökolandbau unabhängig vom Steuerrecht variiert werden und ist nicht an das Steuereinzugsverfahren gebunden. Lediglich die Feststellung der rechtssicheren Bemessungsgrundlage „Einkünfte aus LuF“ wird dem Verwaltungsakt Steuerbescheid entnommen. Diese Vorgehensweise hat neben der unabhängigen Tarifgestaltung den Vorteil, dass die Förderung von der zuständigen Landesbehörde eigenständig konstruiert werden kann, ohne dass sich an den Steuereinnahmen und der Besteuerungspraxis des Staates gemäß Ertragsteuerrecht etwas ändert. Darin zeigt sich bereits die hohe Flexibilität bei der Umsetzung der Flexiblen Förderung.

\subsubsection{GRUNDLEGENDE WIRKUNGSWEISE VON SOCKELBETRAG UND ZUSATZFÖRDERUNG IN DER BASISKONSTELLATION}

Hinsichtlich der Auswirkungen der Flexiblen Förderung ist zu untersuchen, wodurch ihre grundlegende Wirkungsweise bestimmt ist und welcher Einfluss dabei den Unterkomponenten Sockelbetrag und Zusatzförderung zukommt. Für die Ausgestaltung der Flexiblen Förderung wurden dazu zunächst folgende Annahmen für eine Basiskonstellation getroffen:

- Sockelbetrag der Flexiblen Förderung pro ha landwirtschaftlich genutzter Fläche (LF): $100 € / J a h r$.

- Bemessungszeitraum der Zusatzförderung: 3 Jahre.

- Berechnungsgrundlage der Zusatzförderung: Einkommensteuertarif 2006. 


\section{- Die Zusatzförderung geht in einfacher Höhe in die Flexible Förderung ein. ${ }^{15}$}

Während sich der Sockelbetrag durch die einfache Multiplikation der bewirtschafteten Fläche mit dem jeweiligen Fördersatz errechnet, er also proportional zum Flächenumfang steigt, ist der monetäre Effekt aus der Zusatzförderung in Abhängigkeit seiner Bemessungsgrundlage differenzierter. Die spezifische Wirkungsweise der Zusatzförderung soll deshalb im Folgenden untersucht werden.

\subsubsection{DURCHFÜHRUNG VON PRAXISRECHNUNGEN ZUR SPEZIFISCHEN WIRKUNGSWEISE DER ZUSATZFÖRDERUNG}

Abbildung 15 bis Abbildung 17 zeigen die Höhe der Zusatzförderung in Abhängigkeit der mittleren Jahreseinkünfte aus Land- und Forstwirtschaft (LuF) sowie der jeweiligen Variationskoeffizienten ${ }^{16}$ als Maßzahlen für die Streuung der Jahreseinkünfte.

Abbildung 15: Wirkungsweise der Zusatzförderung bei mittleren jährlichen Einkünften von 10.000 Euro und unterschiedlichen Einkunftsschwankungen

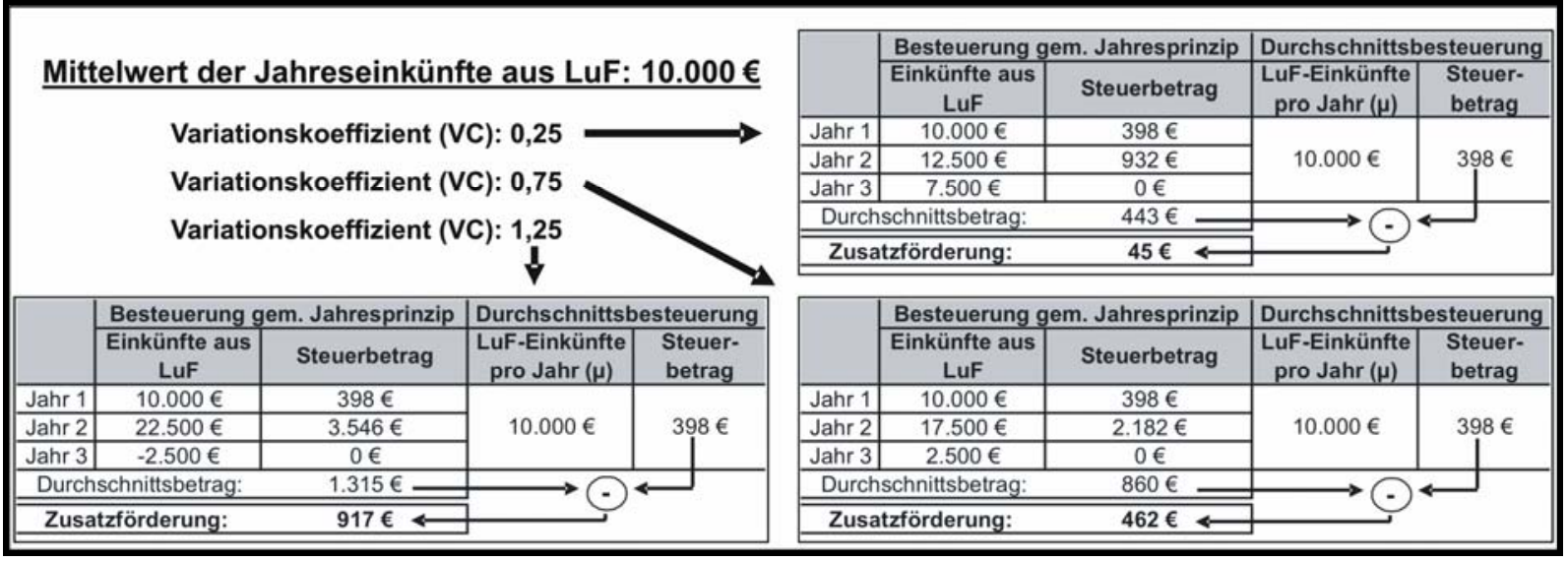

Quelle: Eigene Berechnungen gemäß ESt-Tarif 2006

\footnotetext{
${ }^{15}$ Wie speziell in Kapitel 4.2.3 deutlich werden wird, besteht bei der Ausgestaltung der Flexiblen Förderung die Möglichkeit, die Zusatzförderung vor Eingang in die Flexible Förderung mit einem Faktor zu multiplizieren, um ihr Gewicht innerhalb der Flexiblen Förderung zu verändern.

${ }^{16}$ Bei dem Variationskoeffizienten $(\mathrm{VC})$ handelt es sich um ein relatives Streuungsmaß $(\mathrm{VC}=\sigma / \mu)$, das den absoluten Streuungsmaßen Standardabweichung $(\sigma)$ und Varianz $\left(\sigma^{2}\right)$ vorgezogen wird, wenn Streuungen von Merkmalen verglichen werden sollen, die unterschiedliche Mittelwerte aufweisen (vgl. dazu auch MosLER und SCHMID, 2003).
} 
Abbildung 16: Wirkungsweise der Zusatzförderung bei mittleren jährlichen Einkünften von 50.000 Euro und unterschiedlichen Einkunftsschwankungen

\begin{tabular}{|c|c|c|c|c|c|c|c|c|c|}
\hline \multirow{2}{*}{\multicolumn{5}{|c|}{ Mittelwert der Jahreseinkünfte aus LuF: $50.000 €$}} & & \multicolumn{2}{|c|}{ Besteuerung gem. Jahresprinzip } & \multicolumn{2}{|c|}{ Durchschnittsbesteuerung } \\
\hline & & & & & & $\begin{array}{c}\text { Einkünfte aus } \\
\text { LuF }\end{array}$ & Steuerbetrag & $\begin{array}{c}\text { LuF-Einkünfte } \\
\text { pro Jahr }(\mu)\end{array}$ & $\begin{array}{l}\text { Steuer- } \\
\text { betrag }\end{array}$ \\
\hline \multirow{5}{*}{\multicolumn{5}{|c|}{$\begin{array}{l}\text { Variationskoeffizient (VC): } 0,25 \\
\text { Variationskoeffizient (VC): 0,75 } \\
\text { Variationskoeffizient (VC): } 1,25\end{array}$}} & \begin{tabular}{|l|l|} 
Jahr 1 \\
\end{tabular} & $50.000 €$ & $13.096 €$ & \multirow{3}{*}{$50.000 €$} & \multirow{3}{*}{$13.096 €$} \\
\hline & & & & & \begin{tabular}{|l|l|l|l|l}
$J a h r$ \\
\end{tabular} & $62.500 €$ & $18.336 \epsilon$ & & \\
\hline & & & & & Jahr 3 & $37.500 €$ & $8.326 €$ & & \\
\hline & & & & & \multicolumn{3}{|c|}{ Durchschnittsbetrag: } & \multirow{2}{*}{\multicolumn{2}{|c|}{$\square$}} \\
\hline & & & & & Zusa & atzförderung: & $157 € \ll$ & & \\
\hline & \multicolumn{2}{|c|}{ Besteuerung gem. Jahresprinzip } & \multicolumn{2}{|c|}{ Durchschnittsbesteuerung } & & \multicolumn{2}{|c|}{ Besteuerung gem. Jahresprinzip } & \multicolumn{2}{|c|}{ Durchschnittsbesteuerung } \\
\hline & \begin{tabular}{|c|} 
Einkünfte aus \\
LuF
\end{tabular} & Steuerbetrag & $\begin{array}{c}\text { LuF-Einkünfte } \\
\text { pro Jahr }(\mu)\end{array}$ & $\begin{array}{l}\text { Steuer- } \\
\text { betrag }\end{array}$ & & $\begin{array}{c}\text { Einkünfte aus } \\
\text { LuF }\end{array}$ & Steuerbetrag & $\begin{array}{c}\text { LuF-Einkünfte } \\
\text { pro Jahr }(\mu)\end{array}$ & $\begin{array}{l}\text { Steuer- } \\
\text { betrag }\end{array}$ \\
\hline Jahr 1 & $50.000 €$ & $13.096 \epsilon$ & \multirow{3}{*}{$50.000 €$} & \multirow{3}{*}{$13.096 \epsilon$} & \begin{tabular}{|l|l} 
Jahr 1 \\
\end{tabular} & $50.000 €$ & $13.096 \epsilon$ & \multirow{3}{*}{$50.000 €$} & \multirow{3}{*}{$13.096 €$} \\
\hline Jahr 2 & $112.500 €$ & $39.336 €$ & & & Jahr 2 & $87.500 €$ & $28.836 €$ & & \\
\hline Jahr 3 & $-12.500 €$ & $0 €$ & & & Jahr 3 & $12.500 €$ & $932 €$ & & \\
\hline \multicolumn{2}{|c|}{ Durchschnittsbetrag: } & $17.477 €=$ & & & \multicolumn{2}{|c|}{ Durchschnittsbetrag: } & $14.288 €$ & & \multirow[t]{2}{*}{$\longleftarrow$} \\
\hline \multicolumn{2}{|c|}{ Zusatzförderung: } & $4.381 € \ll$ & & & \multicolumn{2}{|c|}{ Zusatzförderung: } & $1.192 € \ll$ & & \\
\hline
\end{tabular}

Quelle: Eigene Berechnungen gemäß ESt-Tarif 2006

Abbildung 17: Wirkungsweise der Zusatzförderung bei mittleren jährlichen Einkünften von 100.000 Euro und unterschiedlichen Einkunftsschwankungen

\begin{tabular}{|c|c|c|c|c|c|c|c|c|c|}
\hline \multirow{2}{*}{\multicolumn{5}{|c|}{ Mittelwert der Jahreseinkünfte aus LuF: $100.000 €$}} & & \multicolumn{2}{|c|}{ Besteuerung gem. Jahresprinzip } & \multicolumn{2}{|c|}{ Durchschnittsbesteuerung } \\
\hline & & & & & & $\begin{array}{c}\text { Einkünfte aus } \\
\text { LuF }\end{array}$ & Steuerbetrag & \begin{tabular}{|c|} 
LuF-Einkünfte \\
pro Jahr $(\mu)$
\end{tabular} & $\begin{array}{l}\text { Steuer- } \\
\text { betrag }\end{array}$ \\
\hline \multirow{5}{*}{\multicolumn{5}{|c|}{$\begin{array}{l}\text { Variationskoeffizient (VC): } 0,25 \\
\text { Variationskoeffizient (VC): } 0,75 \\
\text { Variationskoeffizient (VC): } 1,25\end{array}$}} & Jahr 1 & $100.000 €$ & $34.086 €$ & \multirow{3}{*}{$100.000 €$} & \multirow{3}{*}{ 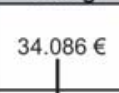 } \\
\hline & & & & & Jahr 2 & $125.000 €$ & $44.586 \epsilon$ & & \\
\hline & & & & & Jahr 3 & $75.000 €$ & $23.586 \epsilon$ & & \\
\hline & & & & & \multicolumn{3}{|c|}{ Durchschnittsbetrag: $\quad 34.086 €$} & \multirow{2}{*}{\multicolumn{2}{|c|}{ « }} \\
\hline & & & & & Zusa & tzförderung: & $\longleftrightarrow$ & & \\
\hline & \multicolumn{2}{|c|}{ Besteuerung gem. Jahresprinzip } & \multicolumn{2}{|c|}{ Durchschnittsbesteuerung } & & \multicolumn{2}{|c|}{ Besteuerung gem. Jahresprinzip } & \multicolumn{2}{|c|}{ Durchschnittsbesteuerung } \\
\hline & \begin{tabular}{|c|}
$\begin{array}{c}\text { Einkünfte aus } \\
\text { LuF }\end{array}$ \\
\end{tabular} & Steuerbetrag & $\begin{array}{c}\text { LuF-Einkünfte } \\
\text { pro Jahr }(\mu)\end{array}$ & $\begin{array}{l}\text { Steuer- } \\
\text { betrag }\end{array}$ & & $\begin{array}{c}\text { Einkünfte aus } \\
\text { LuF }\end{array}$ & Steuerbetrag & \begin{tabular}{|c|} 
LuF-Einkünfte \\
pro Jahr $(\mu)$ \\
\end{tabular} & $\begin{array}{l}\text { Steuer- } \\
\text { betrag }\end{array}$ \\
\hline Jahr 1 & $100.000 €$ & $34.086 \epsilon$ & \multirow{3}{*}{$100.000 €$} & \multirow{3}{*}{$34.086 \epsilon$} & Jahr 1 & $100.000 €$ & $34.086 \epsilon$ & \multirow{3}{*}{$100.000 €$} & \multirow{3}{*}{$34.086 €$} \\
\hline Jahr 2 & $225.000 €$ & $86.586 €$ & & & Jahr 2 & $175.000 €$ & $65.586 \epsilon$ & & \\
\hline Jahr 3 & $-25.000 €$ & $0 €$ & & & Jahr 3 & $25.000 €$ & $4.271 €$ & & \\
\hline \multicolumn{2}{|c|}{ Durchschnittsbetrag: } & $40.224 \epsilon \square$ & & & \multicolumn{2}{|c|}{ Durchschnittsbetrag: } & $34.648 \epsilon \_$ & \multicolumn{2}{|r|}{ 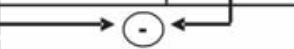 } \\
\hline \multicolumn{2}{|c|}{ Zusatzförderung: } & $6.138 € \ll$ & & & \multicolumn{3}{|c|}{ Zusatzförderung: $\quad 562 € \ll$} & & \\
\hline
\end{tabular}

Quelle: Eigene Berechnungen gemäß ESt-Tarif 2006

Die Berechnungen zeigen bereits eine deutliche Abhängigkeit der Zusatzförderung von den Einkunftsschwankungen sowie von der mittleren absoluten Einkunftshöhe. Um die Wirkungsrichtung noch stärker zu verdeutlichen, wurden mittels SIMETAR ${ }^{\circledR}$ (Simulation with Excel to analyze risk, (C) TAMU Texas, USA) Simulationsrechnungen durchgeführt, deren Ergebnisse Abbildung 18 zeigt. Simulationsgrundlage sind jeweils 1.000 Beobachtungen mittels der Latin Hypercube Methodik $^{17}$ (RICHARDSON, 2005a: Chapter 4). Als zugrunde liegende Verteilung der Einkünfte aus LuF wurde die Normalverteilung angenommen.

\footnotetext{
${ }^{17}$ Die Latin Hypercube Methodik stellt eine Weiterentwicklung der Monte Carlo Simulation dar und hat den Vorteil, dass die Anzahl der notwendigen Variantenrechnungen reduziert werden kann (HARDAKER ET AL., 2004: 158ff)
} 
Abbildung 18: Höhe der Zusatzförderung gemäß ESt-Tarif 2006

\begin{tabular}{|c|c|c|c|c|c|c|c|c|c|c|c|c|c|c|c|}
\hline \multicolumn{16}{|c|}{ Höhe der Zusatzförderung } \\
\hline \multicolumn{8}{|c|}{ Absolut in $€$ pro Betrieb: } & \multicolumn{8}{|c|}{ In Relation zu den mittleren LuF-Einkünften: } \\
\hline \multirow{2}{*}{$\begin{array}{c}\text { Jährl. Ein- } \\
\text { künfte aus } \\
\text { LuF in } €\end{array}$} & \multicolumn{7}{|c|}{ Variationskoeffizient } & \multirow{2}{*}{$\begin{array}{c}\text { Jährl. Ein- } \\
\text { künfte aus } \\
\text { LuF in } €\end{array}$} & \multicolumn{7}{|c|}{ Variationskoeffizient } \\
\hline & 0,1 & 0,25 & 0,5 & 0,75 & 1 & 1,25 & 1,5 & & 0,1 & 0,25 & 0,5 & 0,75 & 1 & 1,25 & 1,5 \\
\hline 1.000 & 0 & 0 & 0 & 0 & 0 & 0 & 0 & 1.000 & $0 \%$ & $0 \%$ & $0 \%$ & $0 \%$ & $0 \%$ & $0 \%$ & $0 \%$ \\
\hline 5.000 & 0 & 1 & 28 & 75 & 126 & 207 & 263 & 5.000 & $0 \%$ & $0 \%$ & $1 \%$ & $1 \%$ & $3 \%$ & $4 \%$ & $5 \%$ \\
\hline 10.000 & 6 & 62 & 190 & 302 & 410 & 628 & 744 & 10.000 & $0 \%$ & $1 \%$ & $2 \%$ & $3 \%$ & $4 \%$ & $6 \%$ & $7 \%$ \\
\hline 20.000 & 6 & 44 & 273 & 603 & 942 & 1.467 & 1.801 & 20.000 & $0 \%$ & $0 \%$ & $1 \%$ & $3 \%$ & $5 \%$ & $7 \%$ & $9 \%$ \\
\hline 30.000 & 14 & 88 & 434 & 958 & 1.487 & 2.289 & 2.781 & 30.000 & $0 \%$ & $0 \%$ & $1 \%$ & $3 \%$ & $5 \%$ & $8 \%$ & $9 \%$ \\
\hline 50.000 & 27 & 144 & 621 & 1.417 & 2.299 & 3.561 & 4.412 & 50.000 & $0 \%$ & $0 \%$ & $1 \%$ & $3 \%$ & $5 \%$ & $7 \%$ & $9 \%$ \\
\hline 75.000 & 0 & 35 & 576 & 1.739 & 3.106 & 4.898 & 6.269 & 75.000 & $0 \%$ & $0 \%$ & $1 \%$ & $2 \%$ & $4 \%$ & $7 \%$ & $8 \%$ \\
\hline 100.000 & 0 & 10 & 543 & 2.024 & 3.867 & 6.176 & 8.084 & 100.000 & $0 \%$ & $0 \%$ & $1 \%$ & $2 \%$ & $4 \%$ & $6 \%$ & $8 \%$ \\
\hline 200.000 & 0 & 1 & 609 & 3.205 & 6.855 & 11.233 & 15.258 & 200.000 & $0 \%$ & $0 \%$ & $0 \%$ & $2 \%$ & $3 \%$ & $6 \%$ & $8 \%$ \\
\hline
\end{tabular}

Quelle: Eigene Berechnungen gemäß ESt-Tarif 2006

Daraus ergibt sich die folgende grundsätzliche Wirkungsweise: Je stärker die steuerlichen Einkünfte aus LuF in einem bestimmten Intervall schwanken, umso höher ist die Zusatzförderung. Schwanken die Einkünfte im historischen Zeitraum überhaupt nicht $(\mathrm{VC}=0)$, so entfällt die Zusatzförderung für den jeweiligen Betrieb und es verbleibt folglich lediglich der Sockelbetrag. Im umgekehrten Fall ergibt sich bei sehr hohen Schwankungen eine vergleichsweise hohe Zusatzförderung, die zusammen mit dem konstanten lediglich flächenabhängigen Sockelbetrag zu einer vergleichsweise hohen Flexiblen Förderung führen kann. Daneben belegen die Ergebnisse für die Zusatzförderung eine Abhängigkeit von der Höhe der durchschnittlichen LuF-Einkünfte. So profitieren mittlere LuF-Einkünfte zwischen $20.000 €$ und $50.000 €$ bei gleichen Schwankungen in höherem Maße von der Zusatzförderung als geringere oder höhere LuF-Einkünfte. Für diesbezüglich noch spezifischere Aussagen zur Wirkungsweise der Zusatzförderung stellt sich die Frage nach einem möglichen Zusammenhang zwischen der absoluten Höhe der Jahreseinkünfte und deren Schwankung.

\subsubsection{DER ZUSAMMENHANG ZWISCHEN EINKUNFTSHÖHE UND}

\section{EINKUNFTSSCHWANKUNGEN IM BMELV-TESTBETRIEBSNETZ}

Auf der Basis real existierender, identischer Betriebe (Öko-Betriebe des BMELV- TBN) konnte ein Zusammenhang zwischen der Standardabweichung und dem Mittelwert der wirtschaftsjahrbezogenen Betriebsgewinne ${ }^{18}$ ermittelt werden. Dabei wurden mindestens sechs aufeinander folgende Jahresabschlüsse zwischen den Wirtschaftsjahren 1996/97 -

\footnotetext{
${ }^{18} \mathrm{Da}$ die Datengrundlage des BMELV-TBN hinsichtlich der kalenderjahrbezogenen Einkünfte aus LuF ungenügend war, wurde auf die wirtschaftsjahrbezogenen Betriebsgewinne zurückgegriffen.
} 
2003/04 analysiert. Die Ergebnisse belegen einen signifikanten linearen Zusammenhang zwischen der Standardabweichung und dem Mittelwert der wirtschaftsjahrbezogenen Betriebsgewinne $\left(B=0,28 ; R^{2}=0,31 ; \mathrm{T}-\right.$ Wert $\left.=7,14\right)$. Daraus resultiert der in Abbildung 19 ebenfalls dargestellte Zusammenhang zwischen dem Variationskoeffizienten und dem Mittelwert der Betriebsgewinne.

Abbildung 19: Zusammenhang zwischen den Gewinnschwankungen und dem Gewinnmittelwert bei Öko-Betrieben des BMELV-TBN

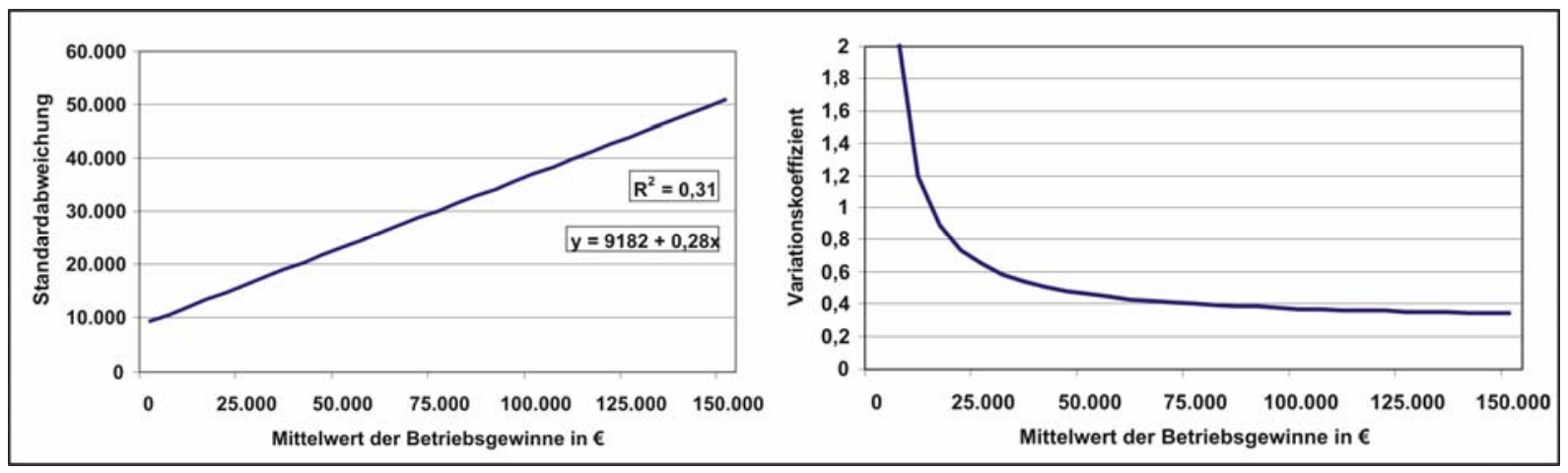

Quelle: Eigene Darstellung

Die Gewinnschwankungen in Betrieben mit niedrigen mittleren Gewinnen sind demnach deutlich höher als in Betrieben mit hohen mittleren Gewinnen. Mit zunehmend geringeren Mittelwerten der Betriebsgewinne steigt der Variationskoeffizient exponentiell an.

\subsubsection{ABSCHLUSSBETRACHTUNG ZUR WIRKUNGSWEISE DER FLEXIBLEN FÖRDERUNG}

Betrachtet man diesen Zusammenhang vor dem Hintergrund der in Abbildung 18 dargestellten Simulationsergebnisse, so lassen sich daraus weitergehende Erkenntnisse zur Wirkungsweise der Zusatzförderung ableiten. Unter der Annahme, der aufgezeigte Zusammenhang gelte für die kalenderjahrbezogenen Einkünfte aus LuF in gleichem Maße, wurden zunächst für ausgewählte beispielhafte Einkunftshöhen die entsprechenden Variationskoeffizienten gemäß Abbildung 19 berechnet. Für die sich daraus ergebenden Wertepaare wurde anschließend auf Basis der Simulationsrechungen aus Abbildung 18 wiederum die Zusatzförderung simuliert. Die Ergebnisse sind in Tabelle 16 dargestellt. 


\section{Tabelle 16: Jährliche Zusatzförderung in €/Unternehmen (für die mittleren} Variationskoeffizienten der ausgewerteten Öko-Betriebe aus dem BMELV-TBN)

\begin{tabular}{|c|c|c|c|c|c|c|c|c|c|}
\hline $\begin{array}{c}\text { Mittelwert der jährl. Einkünfte } \\
\text { aus LuF in Euro }\end{array}$ & 1000 & $\mathbf{5 0 0 0}$ & $\mathbf{1 0 0 0 0}$ & $\mathbf{2 0 0 0 0}$ & $\mathbf{3 0 0 0 0}$ & $\mathbf{5 0 0 0 0}$ & $\mathbf{7 0 0 0 0}$ & $\mathbf{1 0 0 0 0 0}$ & $\mathbf{2 0 0 0 0 0}$ \\
\hline $\begin{array}{c}\text { Landwirtschaftlich genutzte } \\
\text { Fläche (LF) in ha* }\end{array}$ & 18 & 30 & 45 & 75 & 105 & 165 & 225 & 315 & 615 \\
\hline $\begin{array}{c}\text { Variationskoeffizient der LuF } \\
\text { Einkünfte* }\end{array}$ & $\mathbf{9 , 4 6}$ & $\mathbf{2 , 1 1}$ & $\mathbf{1 , 2 0}$ & 0,74 & 0,58 & 0,46 & 0,41 & 0,37 & 0,32 \\
\hline Zusatzförderung in $€$ & $\mathbf{2 2 2}$ & $\mathbf{4 0 0}$ & $\mathbf{5 4 4}$ & $\mathbf{5 8 2}$ & $\mathbf{5 6 7}$ & $\mathbf{4 8 3}$ & $\mathbf{2 8 9}$ & $\mathbf{1 2 6}$ & $\mathbf{2 4}$ \\
\hline
\end{tabular}

*Ergebnisse auf der Basis einer Einfachregression ( $y=15,4+0,003 x)$ zwischen den Betriebsgewinnen (als unabhängige Variable) und der LF aller Öko-Betriebe im BMELV-TBN mit mindestens sechs aufeinander folgenden Jahresdatensätzen zwischen WJ 96/97 und WJ 03/04.

**Ergebnisse auf der Basis einer Einfachregression $(y=9182+0,28 x)$ zwischen den Betriebsgewinnen (als unabhängige Variable) und dem VC der Betriebsgewinne aller Öko-Betriebe im BMELV-TBN mit mindestens sechs aufeinander folgenden Jahresdatensätzen zwischen WJ 96/97 und WJ 03/04 (vgl. Vorkapitel).

Quelle: Eigene Berechnungen gemäß ESt-Tarif 2006

Die Berechnungen zeigen, dass der aufgezeigte Zusammenhang zwischen der Einkunftshöhe und den Einkunftsschwankungen die Wirkungsweise der Zusatzförderung hinsichtlich der mittleren Einkunftshöhe noch deutlich verstärkt. Weil Betriebe mit hohen Einkünften aus LuF tendenziell deutlich geringere relative Einkunftsschwankungen gemäß Variationskoeffizient aufweisen, wird ihr Profit aus der Zusatzförderung geringer sein als Abbildung 18 zunächst suggeriert. Eine entgegengesetzte Tendenz zeigt sich für Betriebe mit sehr geringen mittleren Einkünften. Aus Abbildung 18 ist eine vergleichsweise sehr niedrige Zusatzförderung für diese Betriebe ersichtlich.

Allerdings werden auch die insgesamt sehr geringen absoluten Förderbeträge für alle Betriebe deutlich. Durch die nachfolgend dargestellten Modifizierungsmöglichkeiten des Förderinstruments in Kapitel 4.2.3 können diese Förderbeträge jedoch erheblich erhöht oder reduziert werden.

Insgesamt wird erkennbar, dass mittlere jährliche Einkunftsniveaus zwischen $10.000 €$ und $50.000 €$ von der Zusatzförderung tendenziell am stärksten profitieren. Der Vorteil dieser Förderung liegt damit nicht allein im Einsparpotenzial und einer stärkeren Ausrichtung am Leistungsfähigkeitsprinzip, sondern auch darin, die Option zu nutzen, eine spezielle Auswahl der schwerpunktmäßig zu fördernden Betriebe treffen zu können.

Die aufgezeigten spezifischen Einflussfaktoren, die die letztliche Höhe der Flexiblen Förderung bestimmen, sind in Abbildung 20 nochmals zusammenfassend dargestellt. 
Abbildung 20: Einflussfaktoren auf die Flexible Förderung

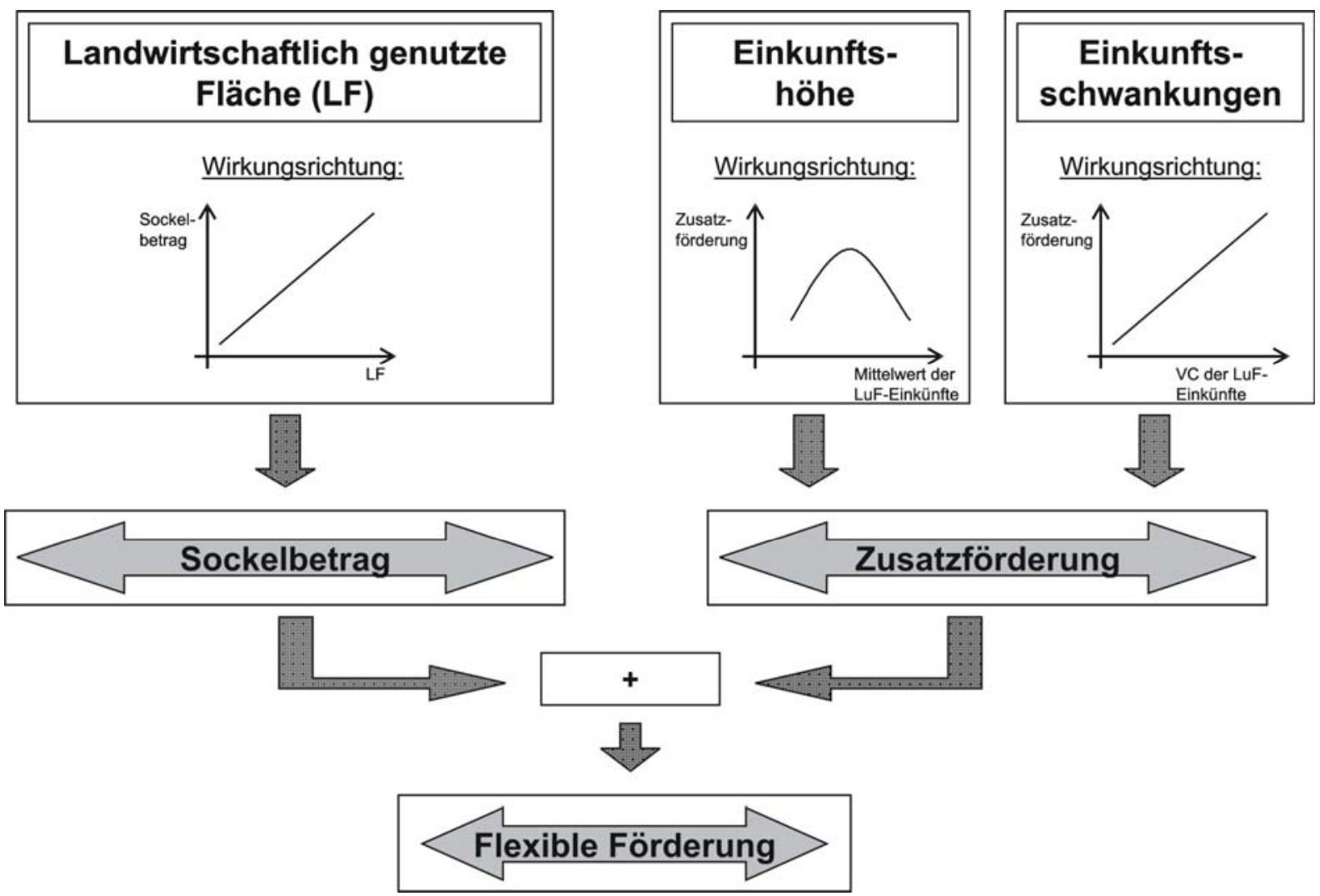

Quelle: Eigene Darstellung

Während die Flexible Förderung mit zunehmender landwirtschaftlich genutzter Fläche sowie mit zunehmenden Einkunftsschwankungen ansteigt, führen hinsichtlich der Einkunftshöhe als drittem Einflussfaktor mittlere jährliche Einkunftsniveaus im Vergleich zu niedrigen oder hohen Einkunftsniveaus zu einem Anstieg der Flexiblen Förderung.

\subsubsection{AUSWIRKUNGEN EINER VERÄNDERTEN BASISKONSTELLATION}

Für die bisherigen Betrachtungen zur betriebswirtschaftlichen Wirkung der Zusatzförderung ist hinsichtlich ihrer Ausgestaltung eine Basiskonstellation angenommen worden. Diese Annahmen sind jedoch nicht starr. Sie können bei Implementierung der Flexiblen Förderung vom jeweiligen Bundesland individuell an die Finanzausstattung, die strukturellen Gegebenheiten sowie an die politische Förderphilosophie angepasst werden. Abbildung 21 zeigt dazu in Anlehnung an Abbildung 13 Möglichkeiten für eine Veränderung der Basiskonstellation. 
Abbildung 21: Überblick über die Veränderungsmöglichkeiten

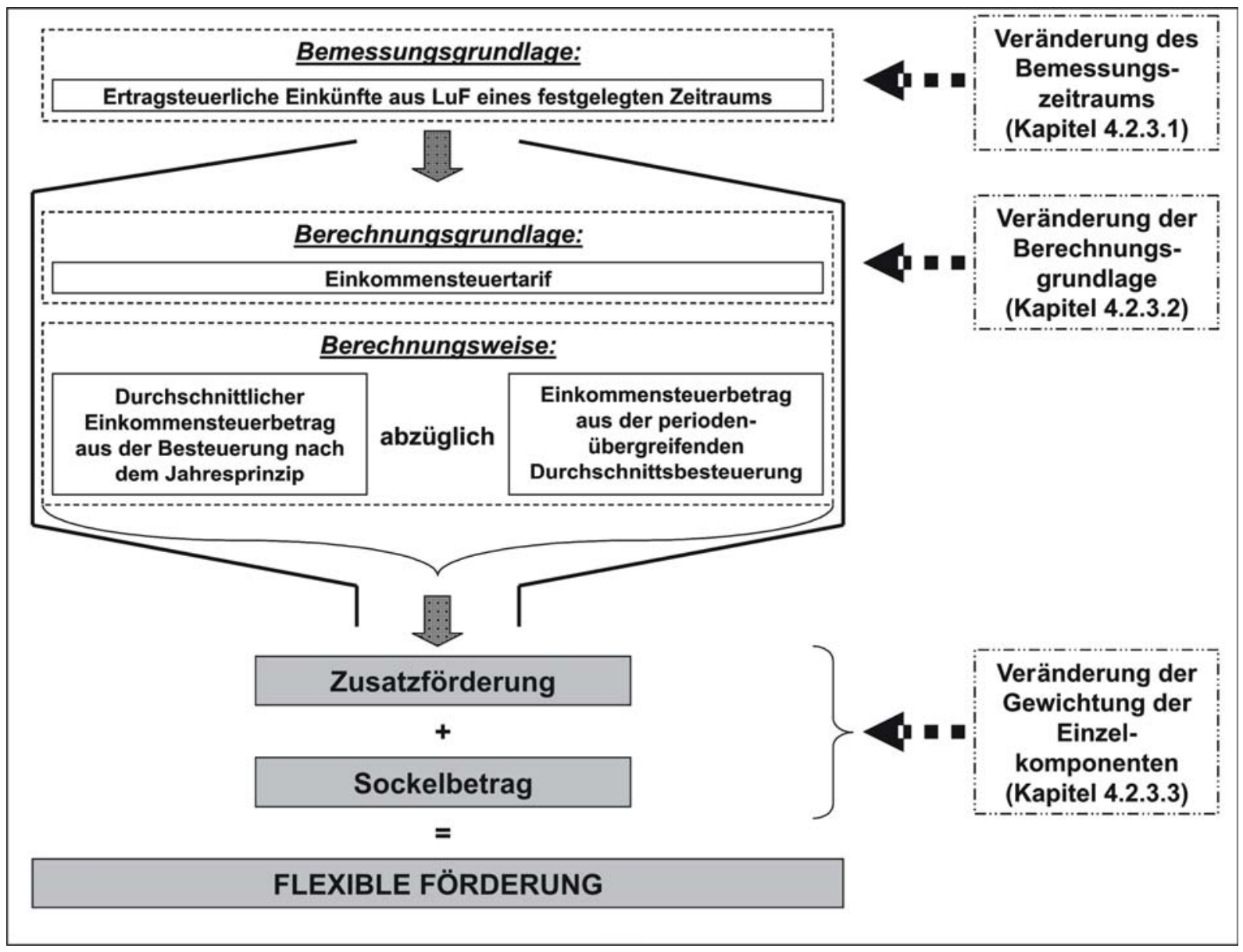

Quelle: Eigene Darstellung

Dabei stellt sich jedoch die Frage, welche Auswirkungen einzelne Veränderungen auf den monetären Effekt der Flexiblen Förderung haben. In den folgenden Unterkapiteln werden die einzelnen Veränderungsmöglichkeiten nacheinander separat untersucht.

\subsubsection{VERÄNDERUNG DES BEMESSUNGSZEITRAUMS DER ZUSATZFÖRDERUNG}

Eine erste Möglichkeit für eine Änderung der Basiskonstellation liegt in einer Veränderung des Zeitraums, dessen LuF-Einkünfte in die Berechnung der Zusatzförderung einfließen. Weil diese Änderung allein die Zusatzförderung betrifft und sich für den Sockelbetrag nichts ändert, soll dieser bei den folgenden Darstellungen außer Acht bleiben. Analog zu Abbildung 18 bilden Abbildung 22 und Abbildung 23 die Wirkungsweise der Zusatzförderung in absoluten jährlichen Förderbeträgen bei Anwendung eines 5-jährigen bzw. eines 7-jährigen Bemessungszeitraums ab. 


\section{Abbildung 22: Zusatzförderung bei Anwendung eines fünfjährigen Bemessungszeitraums}

\begin{tabular}{|c|c|c|c|c|c|c|c|c|c|c|c|c|c|c|c|}
\hline \multicolumn{16}{|c|}{ Höhe der Zusatzförderung bei einem 5-jährigen Bemessungszeitraum } \\
\hline \multicolumn{8}{|c|}{ Absolut in $€$ pro Betrieb: } & \multicolumn{8}{|c|}{ In Relation zu den mittleren LuF-Einkünften: } \\
\hline $\begin{array}{l}\text { Jährl. Ein- } \\
\text { künfte aus }\end{array}$ & \multicolumn{7}{|c|}{ Variationskoeffizient } & $\begin{array}{l}\text { Jährl. Ein-| } \\
\text { künfte aus }\end{array}$ & \multicolumn{7}{|c|}{ Variationskoeffizient } \\
\hline LuF in $\epsilon$ & 0,1 & 0,25 & 0,5 & 0,75 & 1 & 1,25 & 1,5 & LuF in $\epsilon$ & 0,1 & 0,25 & 0,5 & 0,75 & 1 & 1,25 & 1,5 \\
\hline 1.000 & 0 & 0 & 0 & 0 & 0 & 0 & 0 & 1.000 & $0 \%$ & $0 \%$ & $0 \%$ & $0 \%$ & $0 \%$ & $0 \%$ & $0 \%$ \\
\hline 5.000 & 0 & 1 & 30 & 90 & 161 & 245 & 314 & 5.000 & $0 \%$ & $0 \%$ & $1 \%$ & $2 \%$ & $3 \%$ & $5 \%$ & $6 \%$ \\
\hline 10.000 & 8 & 71 & 235 & 400 & 546 & 779 & 922 & 10.000 & $\%$ & $1 \%$ & $2 \%$ & $4 \%$ & $5 \%$ & $8 \%$ & $9 \%$ \\
\hline 20.000 & 7 & 51 & 309 & 748 & 1.248 & 1.760 & 2.210 & 20.000 & $0 \%$ & $0 \%$ & $2 \%$ & $4 \%$ & $6 \%$ & $9 \%$ & $11 \%$ \\
\hline 30.000 & 17 & 103 & 502 & 1.187 & 1.972 & 2.771 & 3.433 & 30.000 & $0 \%$ & $0 \%$ & $2 \%$ & $4 \%$ & $7 \%$ & $9 \%$ & $11 \%$ \\
\hline 50.000 & 33 & 168 & 723 & 1.745 & 3.000 & 4.337 & 5.430 & 50.000 & $\%$ & $0 \%$ & $1 \%$ & $3 \%$ & $6 \%$ & $9 \%$ & $11 \%$ \\
\hline 75.000 & 0 & 37 & 618 & 2.049 & 3.909 & 5.971 & 7.642 & 75.000 & $0 \%$ & $0 \%$ & $1 \%$ & $3 \%$ & $5 \%$ & $8 \%$ & $10 \%$ \\
\hline 100.000 & 0 & 10 & 559 & 2.314 & 4.741 & 7.518 & 9.776 & 100.000 & $0 \%$ & $0 \%$ & $1 \%$ & $2 \%$ & $5 \%$ & $8 \%$ & $10 \%$ \\
\hline 200.000 & 0 & 1 & 615 & 3.521 & 8.081 & 13.588 & 18.200 & 200.000 & $0 \%$ & $0 \%$ & $0 \%$ & $2 \%$ & $4 \%$ & $7 \%$ & $9 \%$ \\
\hline \multicolumn{16}{|c|}{ Differenz zur Zusatzförderung in der Basiskonstellation (3-jähriger Bemessungszeitraum) } \\
\hline \multicolumn{8}{|c|}{ Absolut in $€$ pro Betrieb: } & \multicolumn{8}{|c|}{ In Relation zu den mittleren LuF-Einkünften: } \\
\hline \begin{tabular}{|l|l} 
Jährl. Ein- \\
künfte aus
\end{tabular} & \multicolumn{7}{|c|}{ Variationskoeffizient } & \begin{tabular}{|c|} 
Jährl. Ein- \\
künfte aus
\end{tabular} & \multicolumn{7}{|c|}{ Variationskoeffizient } \\
\hline $\begin{array}{c}\text { Kunte aus } \\
\mathrm{LuF} \text { in } €\end{array}$ & 0,1 & 0,25 & 0,5 & 0,75 & 1 & 1,25 & 1,5 & $\begin{array}{c}\text { künfte aus } \\
\text { LuF in } €\end{array}$ & 0,1 & 0,25 & 0,5 & 0,75 & 1 & 1,25 & 1,5 \\
\hline 1.000 & 0 & 0 & 0 & 0 & 0 & 0 & 0 & 1.000 & $0 \%$ & $0 \%$ & $0 \%$ & $0 \%$ & $0 \%$ & $0 \%$ & $0 \%$ \\
\hline 5.000 & 0 & 0 & +3 & +15 & +36 & +38 & +51 & 5.000 & $0 \%$ & $0 \%$ & $+0,1 \%$ & $+0,3 \%$ & $+0,7 \%$ & \begin{tabular}{|l|}
$+0,8 \%$ \\
\end{tabular} & $+1,0 \%$ \\
\hline 10.000 & +1 & +9 & +45 & +98 & +136 & +151 & +178 & 10.000 & $0 \%$ & $+0,1 \%$ & $+0,5 \%$ & $+1,0 \%$ & $+1,4 \%$ & $\begin{array}{l}+5 \% \\
\end{array}$ & $+1,8 \%$ \\
\hline 20.000 & +1 & +6 & +37 & +145 & +306 & +293 & +409 & 20.000 & $0 \%$ & $0 \%$ & $+0,2 \%$ & $+0,7 \%$ & $+1,5 \%$ & $+1,5 \%$ & $+2,0 \%$ \\
\hline 30.000 & +3 & +14 & +68 & +229 & +485 & +482 & +652 & 30.000 & $0 \%$ & $0 \%$ & $+0,2 \%$ & $+0,8 \%$ & $+1,6 \%$ & \begin{tabular}{|l|}
$+1,6 \%$ \\
\end{tabular} & $+2,2 \%$ \\
\hline 50.000 & +6 & +24 & +102 & +328 & +701 & +775 & +1017 & 50.000 & $0 \%$ & $0 \%$ & $+0,2 \%$ & $+0,7 \%$ & $+1,4 \%$ & $+1,6 \%$ & $+2,0 \%$ \\
\hline 75.000 & 0 & +2 & +42 & +311 & +802 & +1072 & +1373 & 75.000 & $0 \%$ & $0 \%$ & $+0,1 \%$ & $+0,4 \%$ & $+1,1 \%$ & \begin{tabular}{|l|}
$+1,4 \%$ \\
\end{tabular} & $+1,8 \%$ \\
\hline 100.000 & 0 & 0 & +16 & +290 & +874 & +1343 & +1691 & 100.000 & $0 \%$ & $0 \%$ & $0 \%$ & $+0,3 \%$ & $+0,9 \%$ & \begin{tabular}{|l|}
$+1,3 \%$ \\
\end{tabular} & $+1,7 \%$ \\
\hline 200.000 & 0 & 0 & +6 & +316 & +1226 & +2355 & +2941 & 200.000 & $0 \%$ & $0 \%$ & $0 \%$ & $+0,2 \%$ & $+0,6 \%$ & $+1,2 \%$ & $+1,5 \%$ \\
\hline
\end{tabular}

Quelle: Eigene Berechnungen gemäß ESt-Tarif 2006

\section{Abbildung 23: Zusatzförderung bei Anwendung eines siebenjährigen Bemessungszeitraums}

\begin{tabular}{|c|c|c|c|c|c|c|c|c|c|c|c|c|c|c|c|}
\hline \multicolumn{16}{|c|}{ Höhe der Zusatzförderung bei einem 7-jährigen Bemessungszeitraum } \\
\hline \multicolumn{8}{|c|}{ Absolut in $€$ pro Betrieb: } & \multicolumn{8}{|c|}{ In Relation zu den mittleren LuF-Einkünften: } \\
\hline $\begin{array}{l}\text { Jährl. Ein- } \\
\text { künfte aus }\end{array}$ & \multicolumn{7}{|c|}{ Variationskoeffizient } & Jährl. Ein- & \multicolumn{7}{|c|}{ Variationskoeffizient } \\
\hline LuF in $€$ & 0,1 & 0,25 & 0,5 & 0,75 & 1 & 1,25 & 1,5 & LuF in $€$ & 0,1 & 0,25 & 0,5 & 0,75 & 1 & 1,25 & 1,5 \\
\hline 1.000 & 0 & 0 & 0 & 0 & 0 & 0 & 0 & 1.000 & $0 \%$ & $0 \%$ & $0 \%$ & $0 \%$ & $0 \%$ & $0 \%$ & $0 \%$ \\
\hline 5.000 & 0 & 1 & 31 & 93 & 167 & 257 & 341 & 5.000 & $0 \%$ & $0 \%$ & $1 \%$ & $2 \%$ & $3 \%$ & $5 \%$ & $7 \%$ \\
\hline 10.000 & 8 & 76 & 249 & 440 & 629 & 850 & 1.052 & 10.000 & $0 \%$ & $1 \%$ & $2 \%$ & $4 \%$ & $6 \%$ & $8 \%$ & $11 \%$ \\
\hline 20.000 & 8 & 55 & 321 & 790 & 1.327 & 1.929 & 2.485 & 20.000 & $0 \%$ & $0 \%$ & $2 \%$ & $4 \%$ & $7 \%$ & $10 \%$ & $12 \%$ \\
\hline 30.000 & 18 & 112 & 523 & 1.255 & 2.100 & 3.038 & 3.881 & 30.000 & $0 \%$ & $0 \%$ & $2 \%$ & $4 \%$ & $7 \%$ & $10 \%$ & $13 \%$ \\
\hline 50.000 & 36 & 185 & 758 & 1.848 & 3.189 & 4.713 & 6.122 & 50.000 & $0 \%$ & $0 \%$ & $2 \%$ & $4 \%$ & $6 \%$ & $9 \%$ & $12 \%$ \\
\hline 75.000 & 0 & 38 & 628 & 2.114 & 4.092 & 6.366 & 8.530 & 75.000 & $0 \%$ & $0 \%$ & $1 \%$ & $3 \%$ & $5 \%$ & $8 \%$ & $11 \%$ \\
\hline 100.000 & 0 & 12 & 564 & 2.354 & 4.926 & 7.927 & 10.833 & 100.000 & $0 \%$ & $0 \%$ & $1 \%$ & $2 \%$ & $5 \%$ & $8 \%$ & $11 \%$ \\
\hline 200.000 & 0 & 3 & 615 & 3.537 & 8.304 & 14.115 & 19.913 & 200.000 & $0 \%$ & $0 \%$ & $0 \%$ & $2 \%$ & $4 \%$ & $7 \%$ & $10 \%$ \\
\hline \multicolumn{16}{|c|}{ Differenz zur Zusatzförderung in der Basiskonstellation (3-jähriger Bemessungszeitraum) } \\
\hline \multicolumn{8}{|c|}{ Absolut in $€$ pro Betrieb: } & \multicolumn{8}{|c|}{ In Relation zu den mittleren LuF-Einkünften: } \\
\hline $\begin{array}{ll}\text { Jährl. Ein- } \\
\text { künfte aus }\end{array}$ & \multicolumn{7}{|c|}{ Variationskoeffizient } & $\begin{array}{ll}\text { Jährl. Ein- } \\
\text { künfte aus }\end{array}$ & \multicolumn{7}{|c|}{ Variationskoeffizient } \\
\hline $\mathrm{LuF}$ in $€$ & 0,1 & 0,25 & 0,5 & 0,75 & 1 & 1,25 & 1,5 & LuF in $€$ & 0,1 & 0,25 & 0,5 & 0,75 & 1 & 1,25 & 1,5 \\
\hline 1.000 & 0 & 0 & 0 & 0 & 0 & 0 & 0 & 1.000 & $0 \%$ & $0 \%$ & $0 \%$ & $0 \%$ & $0 \%$ & $0 \%$ & $0 \%$ \\
\hline 5.000 & 0 & 0 & +3 & +18 & +41 & +50 & +78 & 5.000 & $0 \%$ & $0 \%$ & $+0,1 \%$ & \begin{tabular}{|l|}
$+0,4 \%$ \\
\end{tabular} & $+0,8 \%$ & \begin{tabular}{|l|}
$+1,0 \%$ \\
\end{tabular} & $+1,6 \%$ \\
\hline 10.000 & +2 & +13 & +59 & +138 & +219 & +222 & +308 & 10.000 & $0 \%$ & $+0,1 \%$ & $+0,6 \%$ & \begin{tabular}{|l|}
$+1,4 \%$ \\
\end{tabular} & $+2,2 \%$ & $+2,2 \%$ & $+3,1 \%$ \\
\hline 20.000 & +2 & +11 & +48 & +187 & +385 & +462 & +684 & 20.000 & $0 \%$ & $+0,1 \%$ & $+0,2 \%$ & \begin{tabular}{|l|}
$+0,9 \%$ \\
\end{tabular} & $+1,9 \%$ & $+2,3 \%$ & $+3,4 \%$ \\
\hline 30.000 & +4 & +24 & +89 & +297 & +613 & +749 & +1100 & 30.000 & $0 \%$ & $+0,1 \%$ & $+0,3 \%$ & \begin{tabular}{|l|}
$+1,0 \%$ \\
\end{tabular} & $+2,0 \%$ & $+2,5 \%$ & $+3,7 \%$ \\
\hline 50.000 & +9 & +40 & +137 & +432 & +890 & +1152 & +1709 & 50.000 & $0 \%$ & $+0,1 \%$ & $+0,3 \%$ & \begin{tabular}{|l|}
$+0,9 \%$ \\
\end{tabular} & $+1,8 \%$ & $+2,3 \%$ & $+3,4 \%$ \\
\hline 75.000 & 0 & +3 & +52 & +376 & +985 & +1467 & +2261 & 75.000 & $0 \%$ & $0 \%$ & $+0,1 \%$ & \begin{tabular}{|l|}
$+0,5 \%$ \\
\end{tabular} & $+1,3 \%$ & $+2,0 \%$ & $+3,0 \%$ \\
\hline 100.000 & 0 & +2 & +21 & +330 & +1059 & +1752 & +2748 & 100.000 & $0 \%$ & $0 \%$ & $0 \%$ & $+0,3 \%$ & $+1,1 \%$ & $+1,8 \%$ & $+2,7 \%$ \\
\hline 200.000 & 0 & +3 & +6 & +332 & +1449 & +2882 & +4655 & 200.000 & $0 \%$ & $0 \%$ & $0 \%$ & \begin{tabular}{|l|}
$+0,2 \%$ \\
\end{tabular} & $+0,7 \%$ & $+1,4 \%$ & $+2,3 \%$ \\
\hline
\end{tabular}

Quelle: Eigene Berechnungen gemäß ESt-Tarif 2006 
Aus den Darstellungen wird ersichtlich, dass die absoluten Förderbeträge mit Ausdehnung des Bemessungszeitraums ansteigen. Damit würde die Zusatzförderung innerhalb der Flexiblen Förderung ein höheres Gewicht bekommen.

Wie auch die folgende Abbildung 24 zusätzlich bestätigt, ist der Anstieg der absoluten Förderbeträge umso größer, je höher die Schwankungen der LuF-Einkünfte sind. Weil die Zusatzförderung bei einem längeren Bemessungszeitraum elastischer auf Einkunftsschwankungen reagiert, würde eine Verlängerung des Bemessungszeitraums Betriebe mit hohen Einkunftsschwankungen stärker begünstigen als Betriebe mit geringen Einkunftsschwankungen. Der Fokus der Förderung auf die Schwankung der Einkünfte aus LuF würde damit verstärkt.

Abbildung 24: Vergleich der Förderwirkung unterschiedlicher Bemessungszeiträume bei der Zusatzförderung

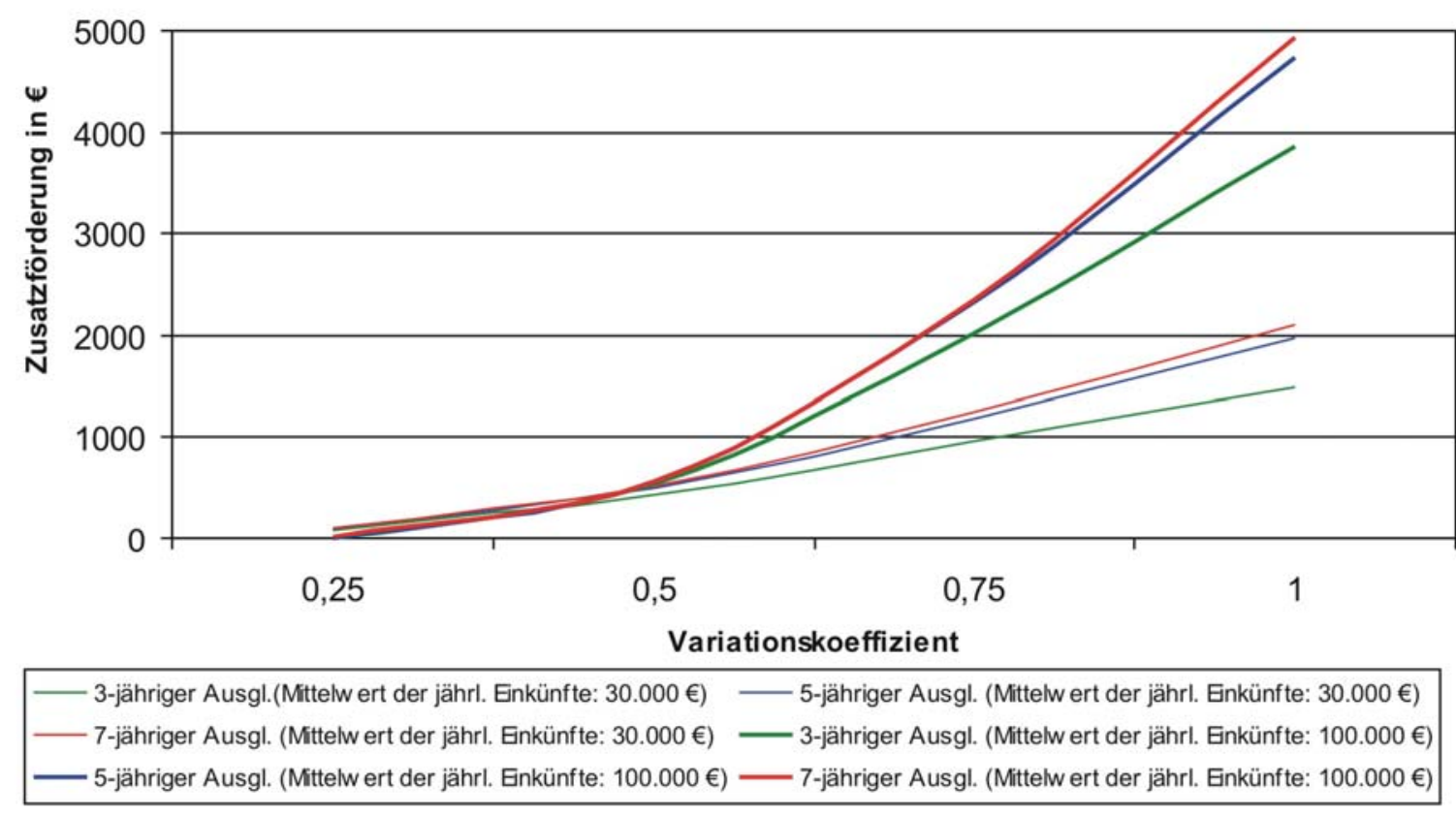

Quelle: Eigene Darstellung

Eine weitere Auswirkung eines verlängerten Bemessungszeitraums besteht, wie Abbildung 24 zeigt, darin, dass die Förderbeträge aus der Zusatzförderung deutlich variabler werden. Auf eventuelle Probleme variabler Förderbeträge für die Finanzplanung auf Landesebene wird in Kapitel 4.6.2 eingegangen.

Dadurch, dass sich die Zusatzförderung in Folge eines verlängerten Bemessungszeitraums stärker an den langfristigen Einkunftsschwankungen als an den kurzfristigen orientiert, ergäbe sich automatisch eine geringere Anfälligkeit für plötzliche, einmalige Gewinneinbrüche bzw. 
Gewinnanstiege, bspw. aufgrund außerordentlicher Ergebnisse in Form von z. B. erheblichen Veräußerungsgewinnen oder -verlusten. Das könnte auch ein geringeres Risiko für Fehlallokationen aufgrund steuerlicher Gestaltungsmöglichkeiten einschließen, weil der Betriebsgewinn einzelner Jahre automatisch geringeren Einfluss auf die Höhe der Zusatzförderung hätte.

Nachteilig dürfte sich eine Verlängerung des Bemessungszeitraums auf die administrative Umsetzung der Flexiblen Förderung auswirken. Bei Anwendung eines siebenjährigen Zeitraums müssen folglich auch die Einkünfte aus LuF der letzten sieben Kalenderjahre vorliegen. Daraus könnte zum einen ein erhöhter Verwaltungsaufwand sowohl für den Landwirt als auch für die Verwaltung resultieren. Daneben könnten sich auch ohnehin bestehende Problembereiche verstärken. So ergäben sich insbesondere bei Betrieben, die vor vier oder fünf Jahren noch der steuerlichen Gewinnermittlung nach Durchschnittssätzen gemäß $§ 13 \mathrm{a}$ EStG unterlagen oder die als Neuumsteller lediglich auf historische Einkünfte aus konventioneller Landwirtschaft zurückblicken können, Schwierigkeiten, diese in das System der Flexiblen Förderung zu integrieren. Auf die grundsätzlichen Überlegungen zur administrativen Einführung der Flexiblen Förderung und zur Behandlung der so genannten $\S 13 \mathrm{a}-$ Betriebe wird in den Kapiteln 4.4.2 und 4.5.2 eingegangen.

In Abbildung 25 ist die Möglichkeit, über eine Veränderung des Bemessungszeitraums eine stärkere Ausrichtung der Zusatzförderung auf die Einkunftsschwankungen zu erreichen, in Anlehnung an Abbildung 20 grafisch dargestellt. 
Abbildung 25: Auswirkungen einer Veränderung des Bemessungszeitraums

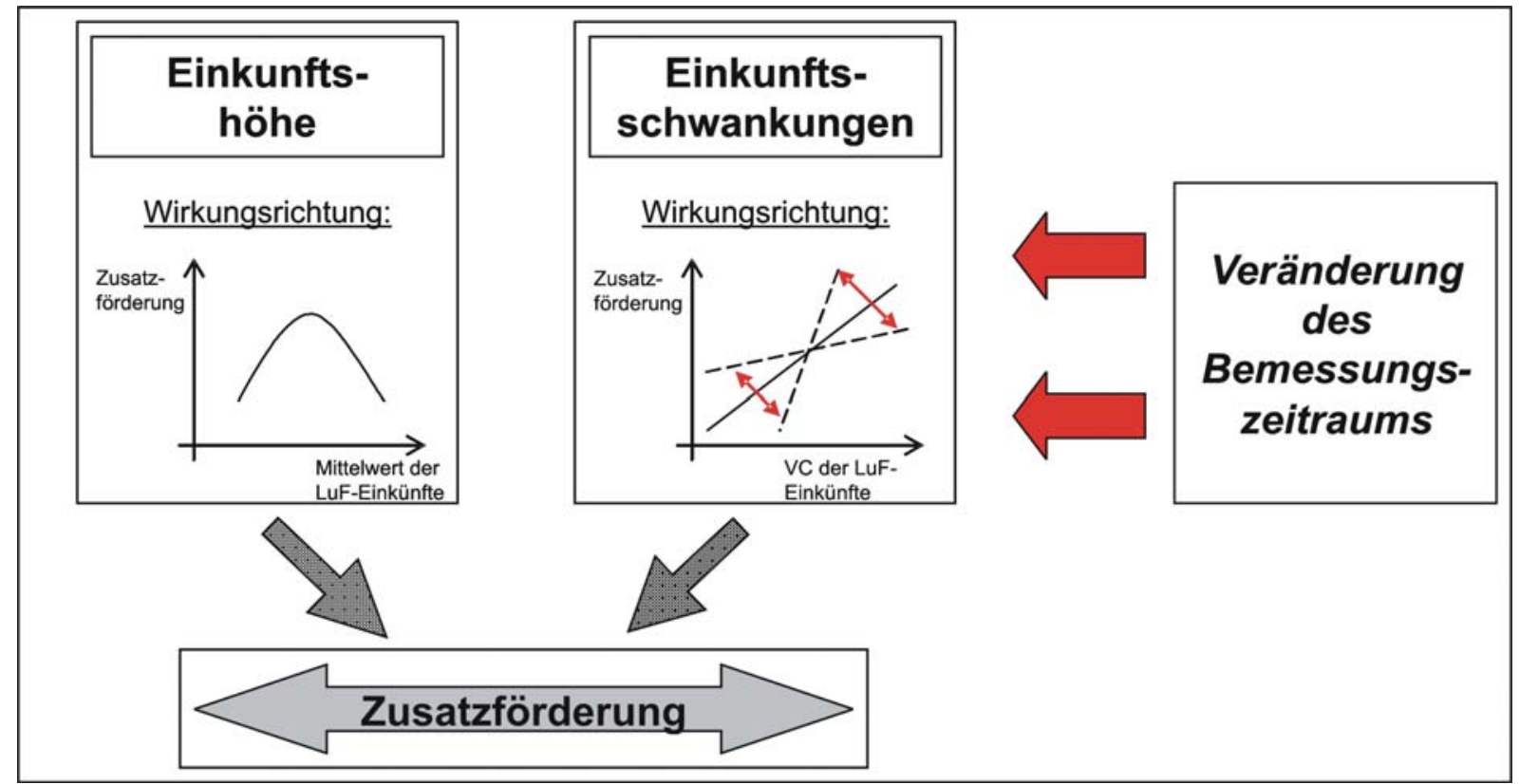

Quelle: Eigene Darstellung

Infolge einer Verlängerung des Bemessungszeitraums ergäbe sich ein steilerer Anstieg; im umgekehrten Fall ein flacherer Anstieg der dargestellten Gerade.

\subsubsection{VERÄNDERUNG DER BERECHNUNGSGRUNDLAGE DER ZUSATZFÖRDERUNG}

Für die Berechnung der Zusatzförderung wurden in der Basiskonstellation der EStGrundfreibetrag 2006 und der ESt-Tarif 2006 zugrunde gelegt, die beide gemeinsam den Grenzsteuersatz als Kernelement der Einkommensteuerveranlagung festlegen ${ }^{19}$. Für eine Veränderung des Verlaufes des Grenzsteuersatzes bestehen verschiedene Möglichkeiten:

A. Veränderung des Grundfreibetrags (ESt-Veranlagung 2006: $7.664 €$ )

B. Veränderung des Eingangssteuersatzes (ESt-Veranlagung 2006: $15 \%$ )

C. Verlegung des Endes der 1. Progressionszone (ESt-Veranlagung 2006: 12.739€)

D. Veränderung des Steuersatzes zu Beginn der 2. Progressionszone (EStVeranlagung 2006: 23,97\%)

E. Verlegung des Beginns der Proportionalzone (ESt-Veranlagung 2006: $52.152 €$ )

F. Veränderung des Spitzensteuersatzes (ESt-Veranlagung 2006: 42 \%). 
Für eine Modifizierung der Zusatzförderung auf Landesebene können gezielt einzelne Änderungen erfolgen. Oder aber es kann ein vollkommen neuer Grenzsteuersatz den Berechnungen zugrunde gelegt werden. Im Folgenden soll zunächst veranschaulicht werden, welche Auswirkungen auf die Zusatzförderung sich aus der Änderung einzelner Tarifbestandteile ergeben, um deren Wirkungsrichtung zu erkennen.

Veränderungen der Berechnungsgrundlage eignen sich insbesondere dazu, die Förderwirkung speziell auf eine basierend auf der mittleren Einkunftshöhe festgelegte Zielgruppe von Landwirten auszurichten. Deshalb wird die veränderte Förderwirkung mit dem einzelbetrieblichen Einkunftsniveau in Beziehung gesetzt.

\subsection{AUSWIRKUNGEN VON ÄNDERUNGEN EINZELNER STELLGRÖßEN AUF UNTERSCHIEDLICHE MITTLERE EINKUNFTSHÖHEN}

Welche Auswirkungen die Änderung einzelner Tarifbestandteile auf den der Berechnung zugrunde liegenden Grenzsteuersatz und in Abhängigkeit von der einzelbetrieblichen mittleren Einkunftshöhe auf die letztliche Höhe der Zusatzförderung in der Basiskonstellation haben, zeigt Abbildung 26. Für die Darstellung der Veränderung der Förderwirkung wurde ein konstanter Variationskoeffizient der Einkünfte aus LuF von 0,75 unterstellt.

Abbildung 26: Veränderung von Grenzsteuersatz und Förderwirkung infolge der Änderung einzelner Stellgrößen

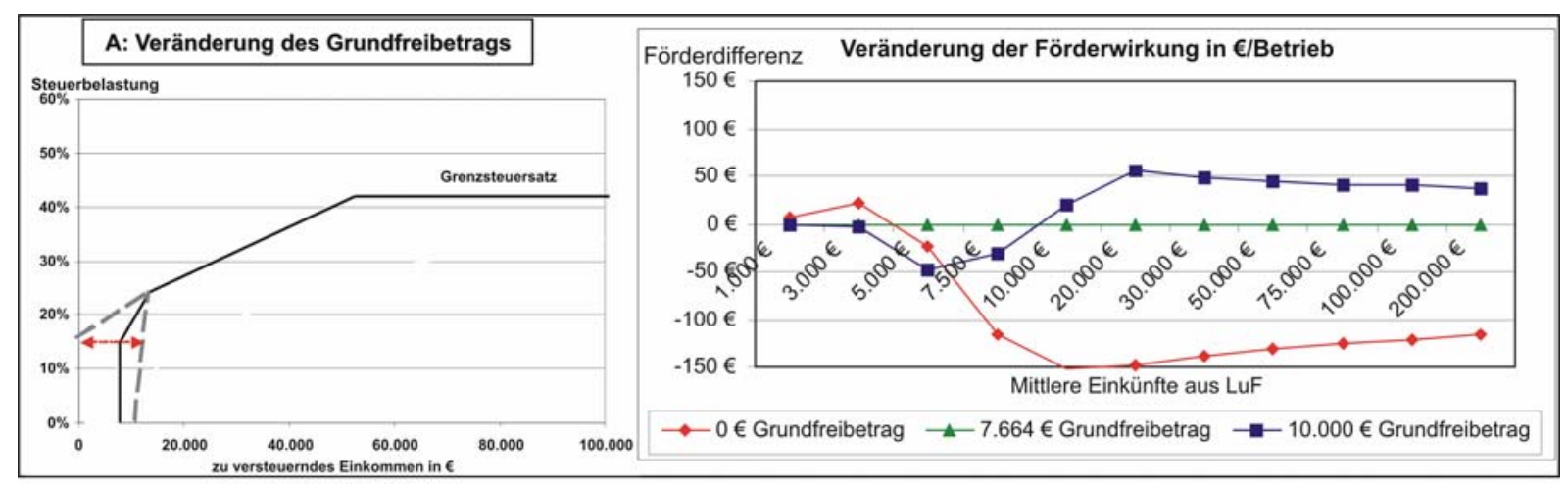

\footnotetext{
${ }^{19}$ Vgl. §32a Abs. 1 EStG zur Berechnungsweise der Einkommensteuer im Rahmen der ESt-Veranlagung 2006: Tarifformel: bis 7664€: 0 ; von $7665 €$ bis $12739 €$ : $(883,74 * \mathrm{y}+1500) * \mathrm{y}$; von $12740 €$ bis $52151 €$ : $(228,74 * z+2397) * z+989$; von $52152 €$ an: $0,42 * x-7914$
} 


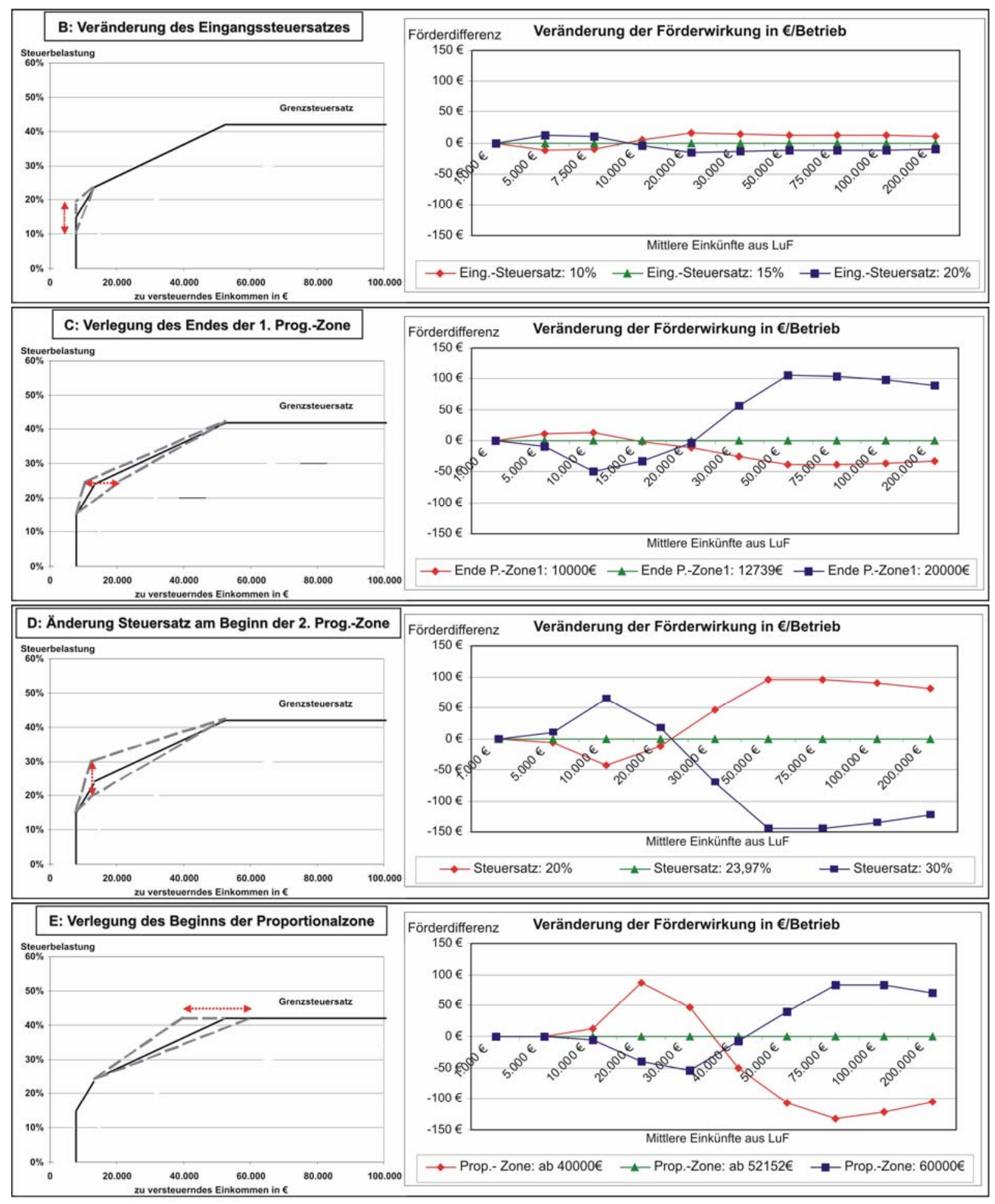




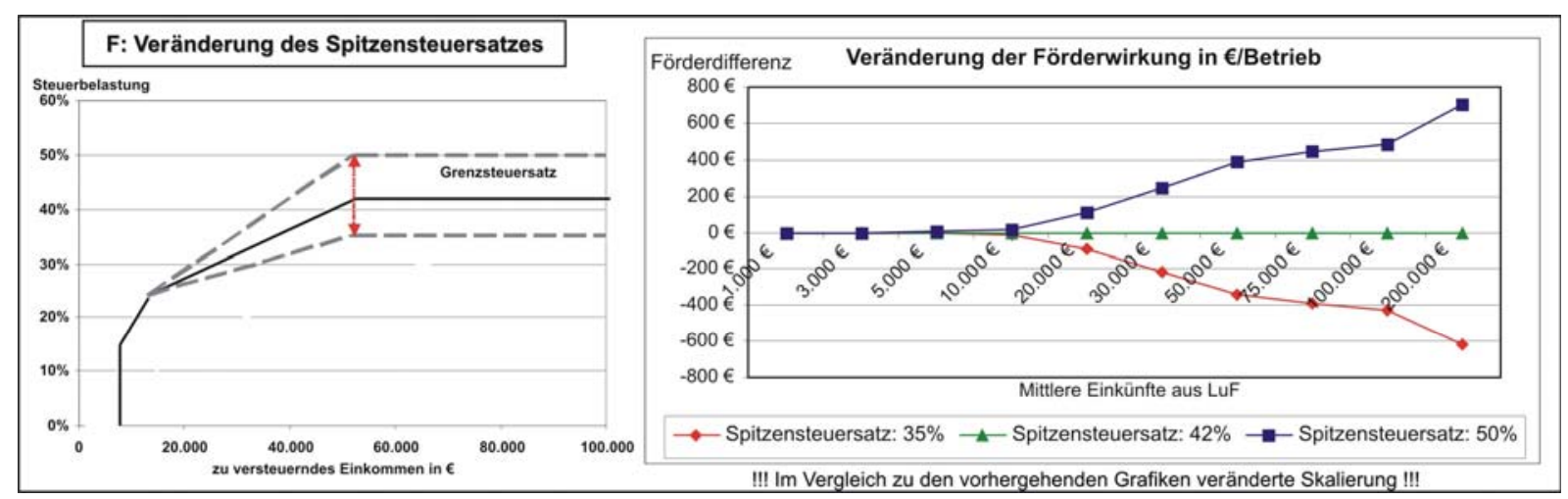

Quelle: Eigene Darstellung

Die Darstellungen zeigen die sehr unterschiedlichen Wirkungsrichtungen und Wirkungsintensitäten der einzelnen Veränderungsmöglichkeiten für unterschiedliche einzelbetriebliche Einkunftsniveaus. Während eine Rechtsverschiebung der Tarifzonen eine erhöhte Zusatzförderung für einkunftsstärkere Betriebe bedeutet, führt eine Linksverschiebung zu einer erhöhten Zusatzförderung für einkunftsschwächere Betriebe. Unter den Steuersätzen führt eine Anhebung des Eingangssteuersatzes zu einer erhöhten Förderwirkung bei einkunftsschwächeren Betrieben, während eine Anhebung des Steuersatzes $\mathrm{zu}$ Beginn der 2. Progressionszone sowie des Spitzensteuersatzes einkunftsstärkeren Betrieben zugute kommt. Gleichzeitig zeigt sich, dass bereits eine geringe Änderung des Spitzensteuersatzes vergleichsweise deutliche Auswirkungen auf die Zusatzförderung hat. Dem gegenüber ist die Wirkungsintensität bei einer Veränderung des Eingangssteuersatzes nur gering. Tabelle 17 veranschaulicht die Wirkungsintensität der einzelnen Stellgrößen und leitet aus den aufgezeigten Wirkungsrichtungen grundsätzliche Veränderungsempfehlungen ab.

Tabelle 17: Veränderungsempfehlungen für den Grenzsteuersatz

\begin{tabular}{|c|c|c|c|c|c|c|c|}
\hline \multirow{2}{*}{\multicolumn{2}{|c|}{ Stellgröße }} & \multirow{3}{*}{\begin{tabular}{|l}
$\begin{array}{l}\text { Wirkungs- } \\
\text { intensität }\end{array}$ \\
Mittel \\
\end{tabular}} & \multicolumn{5}{|c|}{$\begin{array}{c}\text { Veränderungsempfehlung für eine stärkere Fokussierung der } \\
\text { schwerpunktmäßigen Förderwirkung auf ... }\end{array}$} \\
\hline & & & \multicolumn{3}{|c|}{$\begin{array}{c}\text { einkommenschwächere } \\
\text { Betriebe }\end{array}$} & $\begin{array}{c}\text { einkommenstärkere } \\
\text { Betriebe }\end{array}$ & $\Rightarrow$ \\
\hline A & Veränderung des Grundfreibetrags & & $\ldots$ & Senkung & $\ldots$ & Anhebung & $\ldots$ \\
\hline B & $\begin{array}{l}\text { Veränderung des } \\
\text { Eingangssteuersatzes }\end{array}$ & Niedrig $\bigcirc$ & $\ldots$ & Anhebung & $\cdots$ & Senkung & $\cdots$ \\
\hline C & $\begin{array}{l}\text { Verlegung des Endes der } \\
\text { 1. Progressionszone }\end{array}$ & Mittel & $\cdots$ & Früh. Ende & $\cdots$ & Spät. Ende & $\cdots$ \\
\hline D & $\begin{array}{l}\text { Veränderung des Steuersatzes zu } \\
\text { Beginn der 2. Progressionszone }\end{array}$ & Mittel & $\cdots$ & Anhebung & $\cdots$ & Senkung & $\cdots$ \\
\hline E & $\begin{array}{l}\text { Verlegung des Beginns der } \\
\text { Proportionalzone }\end{array}$ & Mittel & $\cdots$ & Früh. Beginn & $\cdots$ & Spät. Beginn & $\cdots$ \\
\hline$F$ & $\begin{array}{l}\text { Veränderung des } \\
\text { Spitzensteuersatzes }\end{array}$ & Hoch & $\cdots$ & Senkung & $\cdots$ & Anhebung & $\cdots$ \\
\hline
\end{tabular}

Quelle: Eigene Darstellung 
Für die Konzeption der Zusatzförderung auf Landesebene ist es nahe liegend, auf Basis dieser Erkenntnisse $\mathrm{zu}$ den Einzelwirkungen einen speziellen Tarif zu konzipieren, um die Förderwirkung auf eine bestimmte Einkunftsgruppe besonders zielgerichtet zu konfigurieren.

Deshalb zeigt das nächste Kapitel zwei Beispiele für veränderte Grenzsteuersätze.

\subsection{KONZEPTION VERÄNDERTER GRENZSTEUERSÄTZE}

Um im Vergleich zur Basiskonstellation die Zusatzförderung noch stärker auf einkunftsschwächere (1) oder auf einkunftsstärkere Betriebe (2) zu fokussieren, sind analog $\mathrm{zu}$ den Handlungsempfehlungen aus Tabelle 17 die beiden entgegengesetzten Grenzsteuersatzmodifikationen 1 und 2 konstruiert worden. Tabelle 18 zeigt die Ausgestaltung beider Modifikationen im Vergleich zur derzeit geltenden ESt-Veranlagung 2006, die der Basiskonstellation zugrunde liegt.

Tabelle 18: Ausgestaltung der Grenzsteuersatzmodifikationen ${ }^{20}$

\begin{tabular}{|c|l|c|c|c|}
\hline \multicolumn{2}{|c|}{ Stellgröße } & $\begin{array}{c}\text { Grenzsteuersatz- } \\
\text { modifikation 1 }\end{array}$ & $\begin{array}{c}\text { ESt-Veranlagung } \\
\mathbf{2 0 0 6}\end{array}$ & $\begin{array}{c}\text { Grenzsteuersatz- } \\
\text { modifikation 2 }\end{array}$ \\
\hline A & Veränderung des Grundfreibetrags & $\mathbf{5 . 0 0 0 €}$ & $7.664 €$ & $\mathbf{1 0 . 0 0 0} €$ \\
\hline B & Veränderung des Eingangssteuersatzes & $\mathbf{2 0 \%}$ & $15 \%$ & $\mathbf{1 0 \%}$ \\
\hline C & $\begin{array}{l}\text { Verlegung des Endes der } \\
\text { 1. Progressionszone }\end{array}$ & $\mathbf{1 0 . 0 0 0 €}$ & $12.739 €$ & $\mathbf{2 0 . 0 0 0 €}$ \\
\hline D & $\begin{array}{l}\text { Veränderung des Steuersatzes zu Beginn } \\
\text { der 2. Progressionszone }\end{array}$ & $\mathbf{3 0 \%}$ & $24 \%$ & $\mathbf{2 0 \%}$ \\
\hline E & Verlegung des Beginns der Proportionalzone & $\mathbf{4 0 . 0 0 0 €}$ & $52.151 €$ & $\mathbf{6 0 . 0 0 0} €$ \\
\hline F & Veränderung des Spitzensteuersatzes & $\mathbf{3 5 \%}$ & $42 \%$ & $\mathbf{5 0 \%}$ \\
\hline
\end{tabular}

Quelle: Eigene Darstellung

Abbildung 27 veranschaulicht beide Modifikationen grafisch auf Grundlage der aus Kapitel 4.2.1.2.1 bekannten Grafik zum Grenzsteuersatz.

\footnotetext{
${ }^{20}$ Veränderte Tarifformel für Grenzsteuersatzmodifikation 1 analog zu §32a EStG: bis 5000€: 0; von 5001€ bis 10000€: $(1000 * \mathrm{y}+2000)^{*} \mathrm{y}$; von $10001 €$ bis $39999 €$ : $\left(83,34{ }^{*} \mathrm{z}+3000\right){ }^{*} \mathrm{z}+1250$; von $40000 €$ an: $0,35^{*} \mathrm{x}-3000$. Veränderte Tarifformel für Grenzsteuersatzmodifikation 2 analog zu §32a EStG: bis 10000€: 0; von 10001€ bis $20000 €:\left(500^{*} \mathrm{y}+1000\right) * \mathrm{y}$; von $20001 €$ bis $59999 €$ : $\left(375^{*} \mathrm{z}+3000\right)^{*} \mathrm{z}+1500$; von $60000 €$ an: $0,50 * \mathrm{x}-14499$.
} 
Abbildung 27: Grafische Darstellung der beiden Grenzsteuersatzmodifikationen

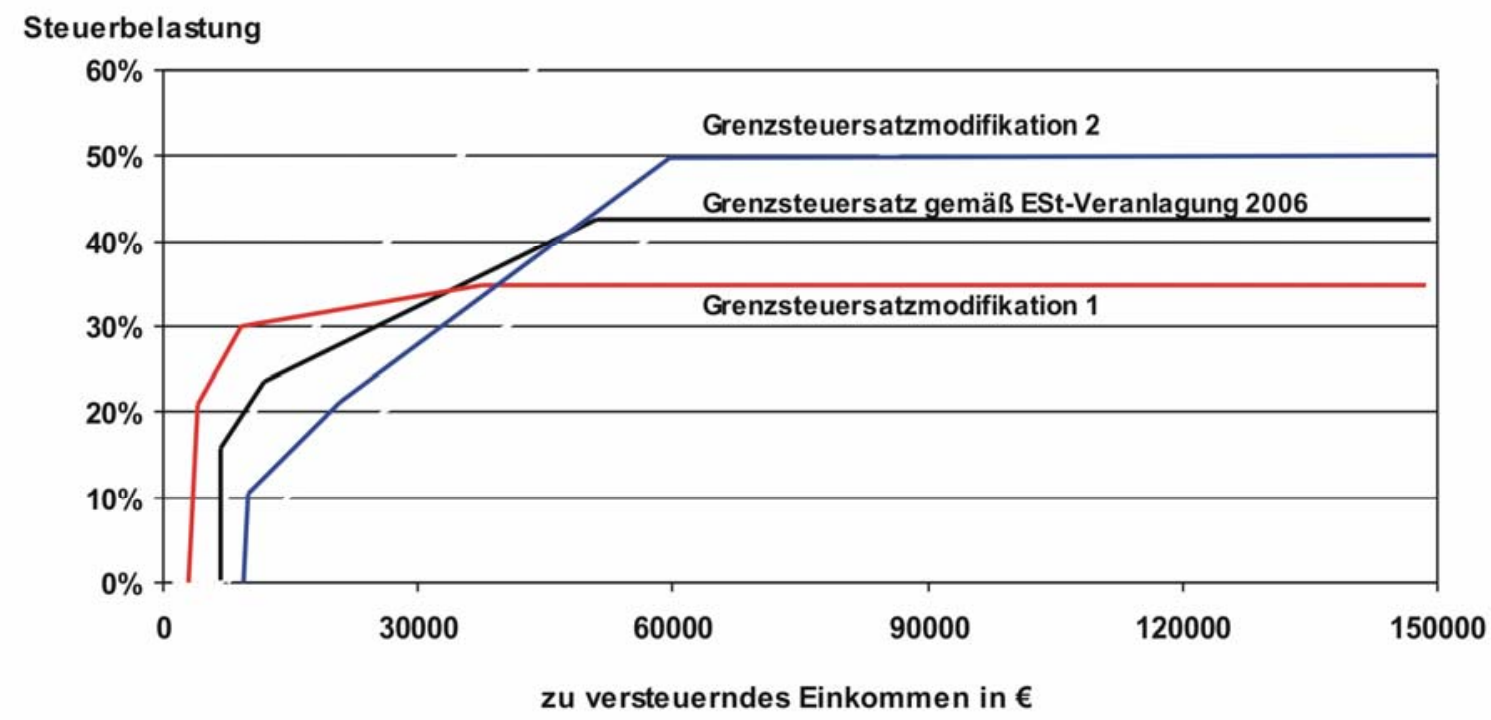

Quelle: Eigene Darstellung

Analog zu Abbildung 18 bilden Abbildung 28 und Abbildung 29 die Wirkungsweise der Zusatzförderung in absoluten jährlichen Förderbeträgen bei Anwendung der beiden Grenzsteuersatzmodifikationen ab.

Abbildung 28: Zusatzförderung bei Unterstellung der Grenzsteuersatzmodifikation 1 als Berechnungsgrundlage

\begin{tabular}{|c|c|c|c|c|c|c|c|c|c|c|c|c|c|c|c|}
\hline \multicolumn{16}{|c|}{ Höhe der Zusatzförderung bei der Grenzsteuersatzmodifikation 1} \\
\hline \multicolumn{8}{|c|}{ Absolut in $€$ pro Betrieb: } & \multicolumn{8}{|c|}{ In Relation zu den mittleren LuF-Einkünften: } \\
\hline \begin{tabular}{l|} 
Jährl. Ein- \\
künfte aus
\end{tabular} & \multicolumn{7}{|c|}{ Variationskoeffizient } & \begin{tabular}{|l|} 
Jährl. Ein- \\
künfte aus
\end{tabular} & \multicolumn{7}{|c|}{ Variationskoeffizient } \\
\hline $\mathrm{LuF}$ in $€$ & 0,1 & 0,25 & 0,5 & 0,75 & 1 & 1,25 & 1,5 & $\mathrm{LuF}$ in $€$ & 0,1 & 0,25 & 0,5 & 0,75 & 1 & 1,25 & 1,5 \\
\hline 1.000 & 0 & 0 & 0 & 0 & 0 & 0 & 0 & 1.000 & $0 \%$ & $0 \%$ & $0 \%$ & $0 \%$ & $0 \%$ & $0 \%$ & $0 \%$ \\
\hline 5.000 & 18 & 47 & 107 & 168 & 238 & 318 & 372 & 5.000 & $0 \%$ & $1 \%$ & $2 \%$ & $3 \%$ & $5 \%$ & $6 \%$ & $7 \%$ \\
\hline 10.000 & 4 & 26 & 147 & 286 & 403 & 630 & 757 & 10.000 & $0 \%$ & $0 \%$ & $1 \%$ & $3 \%$ & $4 \%$ & $6 \%$ & $8 \%$ \\
\hline 20.000 & 2 & 15 & 157 & 435 & 768 & 1.132 & 1.453 & 20.000 & $0 \%$ & $0 \%$ & $1 \%$ & $2 \%$ & $4 \%$ & $6 \%$ & $7 \%$ \\
\hline 30.000 & 5 & 30 & 199 & 584 & 1.081 & 1.611 & 2.093 & 30.000 & $0 \%$ & $0 \%$ & $1 \%$ & $2 \%$ & $4 \%$ & $5 \%$ & $7 \%$ \\
\hline 50.000 & 0 & 14 & 216 & 801 & 1.668 & 2.497 & 3.307 & 50.000 & $0 \%$ & $0 \%$ & $0 \%$ & $2 \%$ & $3 \%$ & $5 \%$ & $7 \%$ \\
\hline 75.000 & 0 & 3 & 227 & 1.049 & 2.317 & 3.573 & 4.797 & 75.000 & $0 \%$ & $0 \%$ & $0 \%$ & $1 \%$ & $3 \%$ & $5 \%$ & $6 \%$ \\
\hline 100.000 & 0 & 1 & 251 & 1.303 & 2.961 & 4.644 & 6.281 & 100.000 & $0 \%$ & $0 \%$ & $0 \%$ & $1 \%$ & $3 \%$ & $5 \%$ & $6 \%$ \\
\hline 200.000 & 0 & 0 & 382 & 2.341 & 5.538 & 8.917 & 12.203 & 200.000 & $0 \%$ & $0 \%$ & $0 \%$ & $1 \%$ & $3 \%$ & $4 \%$ & $6 \%$ \\
\hline \multicolumn{16}{|c|}{ Differenz zur Zusatzförderung in der Basiskonstellation (ESt-Veranlagung 2006) } \\
\hline \multicolumn{8}{|c|}{ Absolut in $€$ pro Betrieb: } & \multicolumn{8}{|c|}{ In Relation zu den mittleren LuF-Einkünften: } \\
\hline $\begin{array}{l}\text { Jährl. Ein- } \\
\text { künfte aus }\end{array}$ & \multicolumn{7}{|c|}{ Variationskoeffizient } & 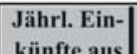 & \multicolumn{7}{|c|}{ Variationskoeffizient } \\
\hline $\operatorname{LuF}$ in $€$ & 0,1 & 0,25 & 0,5 & 0,75 & 1 & 1,25 & 1,5 & $\mathrm{LuF}$ in $€$ & 0,1 & 0,25 & 0,5 & 0,75 & 1 & 1,25 & 1,5 \\
\hline 1.000 & 0 & 0 & 0 & 0 & 0 & 0 & 0 & 1.000 & $0 \%$ & $0 \%$ & $0 \%$ & $0 \%$ & $0 \%$ & $0 \%$ & $0 \%$ \\
\hline 5.000 & +18 & +46 & +79 & +94 & +112 & +111 & +109 & 5.000 & $+0,4 \%$ & $+0,9 \%$ & $+1,6 \%$ & $+1,9 \%$ & $+2,2 \%$ & $+2,2 \%$ & $+2,2 \%$ \\
\hline 10.000 & -3 & -36 & -43 & -16 & -7 & +3 & +13 & 10.000 & $0 \%$ & $-0,4 \%$ & $-0,4 \%$ & $-0,2 \%$ & $-0,1 \%$ & $0,0 \%$ & $+0,1 \%$ \\
\hline 20.000 & -4 & -29 & -116 & -168 & -174 & -336 & -348 & 20.000 & $0 \%$ & $-0,1 \%$ & $-0,6 \%$ & $-0,8 \%$ & \begin{tabular}{|c|}
$-0,9 \%$ \\
\end{tabular} & $-1,7 \%$ & $-1,7 \%$ \\
\hline 30.000 & -9 & -58 & -235 & -374 & -406 & -678 & -688 & 30.000 & $0 \%$ & $-0,2 \%$ & $-0,8 \%$ & $-1,2 \%$ & $-1,4 \%$ & $-2,3 \%$ & $-2,3 \%$ \\
\hline 50.000 & -27 & -131 & -405 & -616 & -632 & -1065 & -1106 & 50.000 & $-0,1 \%$ & $-0,3 \%$ & $-0,8 \%$ & $-1,2 \%$ & $-1,3 \%$ & $-2,1 \%$ & $-2,2 \%$ \\
\hline 75.000 & 0 & -32 & -349 & -689 & -790 & -1325 & -1472 & 75.000 & $0 \%$ & $0 \%$ & $-0,5 \%$ & $-0,9 \%$ & $-1,1 \%$ & $-1,8 \%$ & $-2,0 \%$ \\
\hline 100.000 & 0 & -9 & -292 & -721 & -906 & -1532 & -1804 & 100.000 & $0 \%$ & $0 \%$ & $-0,3 \%$ & $-0,7 \%$ & $-0,9 \%$ & $-1,5 \%$ & $-1,8 \%$ \\
\hline 200.000 & 0 & 0 & -226 & -864 & -1317 & -2317 & -3056 & 200.000 & $0 \%$ & $0 \%$ & $-0,1 \%$ & $-0,4 \%$ & $-0,7 \%$ & $-1,2 \%$ & $-1,5 \%$ \\
\hline
\end{tabular}

Quelle: Eigene Berechnungen 
Abbildung 29: Zusatzförderung bei Unterstellung der Grenzsteuersatzmodifikation 2 als Berechnungsgrundlage

\begin{tabular}{|c|c|c|c|c|c|c|c|c|c|c|c|c|c|c|c|}
\hline \multicolumn{16}{|c|}{ Höhe der Zusatzförderung bei der Grenzsteuersatzmodifikation 2} \\
\hline \multicolumn{8}{|c|}{ Absolut in $€$ pro Betrieb: } & \multicolumn{8}{|c|}{ In Relation zu den mittleren LuF-Einkünften: } \\
\hline Jährl. Ein- & \multicolumn{7}{|c|}{ Variationskoeffizient } & \begin{tabular}{|l|} 
Jährl. Ein- \\
künfte aus
\end{tabular} & \multicolumn{7}{|c|}{ Variationskoeffizient } \\
\hline LuF in $€$ & 0,1 & 0,25 & 0,5 & 0,75 & 1 & 1,25 & 1,5 & $\mathrm{LuF}$ in $€$ & 0,1 & 0,25 & 0,5 & 0,75 & 1 & 1,25 & 1,5 \\
\hline 1.000 & 0 & 0 & 0 & 0 & 0 & 0 & 0 & 1.000 & $0 \%$ & $0 \%$ & $0 \%$ & $0 \%$ & $0 \%$ & $0 \%$ & $0 \%$ \\
\hline 5.000 & 0 & 0 & 2 & 17 & 46 & 82 & 126 & 5.000 & $0 \%$ & $0 \%$ & $0 \%$ & $0 \%$ & $1 \%$ & $2 \%$ & $3 \%$ \\
\hline 10.000 & 18 & 52 & 128 & 217 & 329 & 497 & 592 & 10.000 & $0 \%$ & $1 \%$ & $1 \%$ & $2 \%$ & $3 \%$ & $5 \%$ & $6 \%$ \\
\hline 20.000 & 12 & 76 & 333 & 659 & 1.088 & 1.619 & 1.955 & 20.000 & $0 \%$ & $0 \%$ & $2 \%$ & $3 \%$ & $5 \%$ & $8 \%$ & $10 \%$ \\
\hline 30.000 & 23 & 143 & 627 & 1.259 & 2.022 & $\begin{array}{l}2.867 \\
\end{array}$ & 3.371 & 30.000 & $0 \%$ & $0 \%$ & $2 \%$ & $4 \%$ & $7 \%$ & $10 \%$ & $11 \%$ \\
\hline 50.000 & 63 & 326 & 1.137 & 2.147 & 3.432 & \begin{tabular}{|l|}
4.698 \\
\end{tabular} & 5.627 & 50.000 & $0 \%$ & $1 \%$ & $2 \%$ & $4 \%$ & $7 \%$ & $9 \%$ & $11 \%$ \\
\hline 75.000 & 1 & 136 & 1.104 & 2.584 & 4.579 & 6.394 & 7.950 & 75.000 & $0 \%$ & $0 \%$ & $1 \%$ & $3 \%$ & $6 \%$ & $9 \%$ & $11 \%$ \\
\hline 100.000 & 0 & 41 & 994 & 2.896 & 5.541 & 8.152 & 10.136 & 100.000 & $0 \%$ & $0 \%$ & $1 \%$ & $3 \%$ & $6 \%$ & $8 \%$ & $10 \%$ \\
\hline 200.000 & 0 & 4 & 951 & 4.266 & 9.224 & 14.004 & 18.654 & 200.000 & $0 \%$ & $0 \%$ & $0 \%$ & $2 \%$ & $5 \%$ & $7 \%$ & $9 \%$ \\
\hline
\end{tabular}

Differenz zur Zusatzförderung in der Basiskonstellation (ESt-Veranlagung 2006)

Absolut in $€$ pro Betrieb:

\begin{tabular}{|c|c|c|c|c|c|c|c|}
\hline $\begin{array}{c}\text { Jährl. Ein- } \\
\text { künfte aus } \\
\text { LuF in } \boldsymbol{\epsilon}\end{array}$ & $\mathbf{0 , 1}$ & $\mathbf{0 , 2 5}$ & $\mathbf{0 , 5}$ & $\mathbf{0 , 7 5}$ & $\mathbf{1}$ & $\mathbf{1 , 2 5}$ & $\mathbf{1 , 5}$ \\
\hline $\mathbf{1 . 0 0 0}$ & 0 & 0 & 0 & 0 & 0 & 0 & 0 \\
\hline $\mathbf{5 . 0 0 0}$ & 0 & -1 & -26 & -57 & -80 & -125 & -137 \\
\hline $\mathbf{1 0 . 0 0 0}$ & +12 & -10 & -62 & -85 & -81 & -131 & -151 \\
\hline $\mathbf{2 0 . 0 0 0}$ & +6 & +32 & +60 & +56 & +146 & +151 & +154 \\
\hline $\mathbf{3 0 . 0 0 0}$ & +9 & +55 & +193 & +301 & +535 & +577 & +590 \\
\hline $\mathbf{5 0 . 0 0 0}$ & +36 & +182 & +516 & +731 & +1133 & +1137 & +1215 \\
\hline $\mathbf{7 5 . 0 0 0}$ & +1 & +101 & +528 & +846 & +1472 & +1496 & +1681 \\
\hline $\mathbf{1 0 0 . 0 0 0}$ & 0 & +31 & +451 & +871 & +1674 & +1976 & +2052 \\
\hline $\mathbf{2 0 0 . 0 0 0}$ & 0 & +4 & +342 & +1061 & +2369 & +2771 & +3396 \\
\hline
\end{tabular}

In Relation zu den mittleren LuF-Einkünften:

\begin{tabular}{|c|c|c|c|c|c|c|c|}
\hline $\begin{array}{c}\text { Jährl. Ein- } \\
\text { künfte aus } \\
\text { LuF in } \boldsymbol{€}\end{array}$ & \multicolumn{7}{|c|}{ Variationskoeffizient } \\
\hline $\mathbf{1 . 0 0 0}$ & $0 \%$ & $0 \%$ & $0 \%$ & $0 \%$ & $0 \%$ & $0 \%$ & $0 \%$ \\
\hline $\mathbf{5 . 0 0 0}$ & $0 \%$ & $0 \%$ & $-0,5 \%$ & $-1,1 \%$ & $-1,6 \%$ & $-2,5 \%$ & $-2,7 \%$ \\
\hline $\mathbf{1 0 . 0 0 0}$ & $+0,1 \%$ & $-0,1 \%$ & $-0,6 \%$ & $-0,8 \%$ & $-0,8 \%$ & $-1,3 \%$ & $-1,5 \%$ \\
\hline $\mathbf{2 0 . 0 0 0}$ & $0 \%$ & $+0,2 \%$ & $+0,3 \%$ & $+0,3 \%$ & $+0,7 \%$ & $+0,8 \%$ & $+0,8 \%$ \\
\hline $\mathbf{3 0 . 0 0 0}$ & $0 \%$ & $+0,2 \%$ & $+0,6 \%$ & $+1,0 \%$ & $+1,8 \%$ & $+1,9 \%$ & $+2,0 \%$ \\
\hline $\mathbf{5 0 . 0 0 0}$ & $+0,1 \%$ & $+0,4 \%$ & $+1,0 \%$ & $+1,5 \%$ & $+2,3 \%$ & $+2,3 \%$ & $+2,4 \%$ \\
\hline $\mathbf{7 5 . 0 0 0}$ & $0 \%$ & $+0,1 \%$ & $+0,7 \%$ & $+1,1 \%$ & $+2,0 \%$ & $+2,0 \%$ & $+2,2 \%$ \\
\hline $\mathbf{1 0 0 . 0 0 0}$ & $0 \%$ & $0 \%$ & $+0,5 \%$ & $+0,9 \%$ & $+1,7 \%$ & $+2,0 \%$ & $+2,1 \%$ \\
\hline $\mathbf{2 0 0 . 0 0 0}$ & $0 \%$ & $0 \%$ & $+0,2 \%$ & $+0,5 \%$ & $+1,2 \%$ & $+1,4 \%$ & $+1,7 \%$ \\
\hline
\end{tabular}

Quelle: Eigene Berechnungen

Für einen Variationskoeffizienten von 0,75 bzw. für mittlere jährliche Einkünfte aus LuF von $50.000 €$ veranschaulicht Abbildung 30 die Veränderung der Förderwirkung im Vergleich zur Basiskonstellation mit dem Grenzsteuersatz gemäß ESt-Veranlagung 2006.

Abbildung 30: Veränderung der Förderwirkung infolge der Grenzsteuersatzmodifikationen in $€ /$ Betrieb im Vergleich zur Basiskonstellation

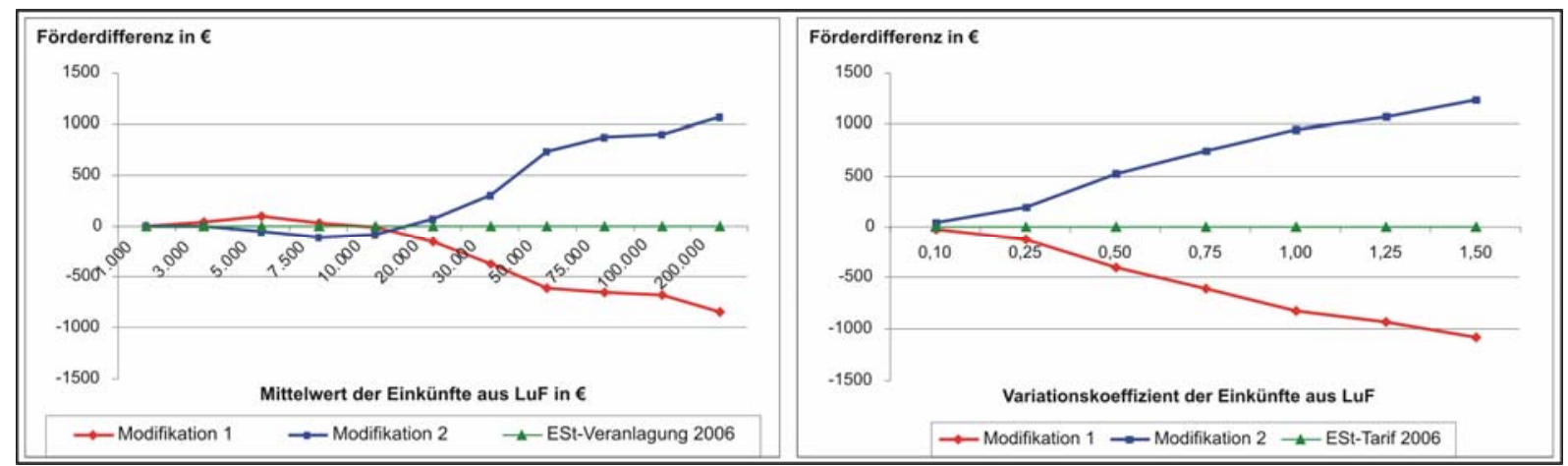

Quelle: Eigene Darstellung

Neben der verstärkten Fokussierung der Förderwirkung auf geringere bzw. höhere mittlere Einkünfte aus LuF wird dabei auch die gleichzeitig veränderte Förderwirkung für unterschiedlich intensive Einkunftsschwankungen deutlich. Mit zunehmenden 
Einkunftsschwankungen kommt es infolge der Modifikation 1 auch zu einer zunehmenden Verringerung der Förderwirkung. Demgegenüber erhöht sich die Zusatzförderung infolge der Anwendung der Modifikation 2 mit ansteigenden Einkunftsschwankungen in zunehmendem Maße. Weitergehende Betrachtungen haben bestätigt, dass sich infolge einer Modifikation des Grenzsteuersatzes die Zusatzförderung in €/Betrieb bei denjenigen Betrieben am stärksten verändert, die auch die stärksten Einkunftsschwankungen aufweisen.

Neben der Konzeption eines veränderten Grenzsteuersatzes ist es natürlich auch denkbar, als Berechnungsgrundlage für die Zusatzförderung einen vollständig vom Einkommensteuertarif abgekoppelten Tarif (z. B. logarithmisch) zu wählen.

\subsection{FAZIT ZUR WIRKUNGSWEISE EINER VERÄNDERUNG DER BERECHNUNGSGRUNDLAGE}

Mit einer Modifikation des der Berechnung der Zusatzförderung zugrunde liegenden Tarifs ist es möglich, die in Kapitel 4.2.2 dargestellte Wirkung der Zusatzförderung, wie Abbildung 31 veranschaulicht, auf der Skala der Einkunftshöhe zu verschieben. Das Schaubild zur Wirkungsrichtung ist dabei so zu verstehen, dass auf der Ordinate alle Öko-Betriebe bspw. eines Bundeslandes, geordnet nach dem Mittelwert ihrer Jahreseinkünfte, angeordnet sind. Eine Veränderung der Berechnungsgrundlage ermöglicht eine zielgerichtete Auswahl der zu fördernden Betriebe gemäß ihrer mittleren Einkunftshöhe.

Abbildung 31: Auswirkungen einer Veränderung der Berechnungsgrundlage

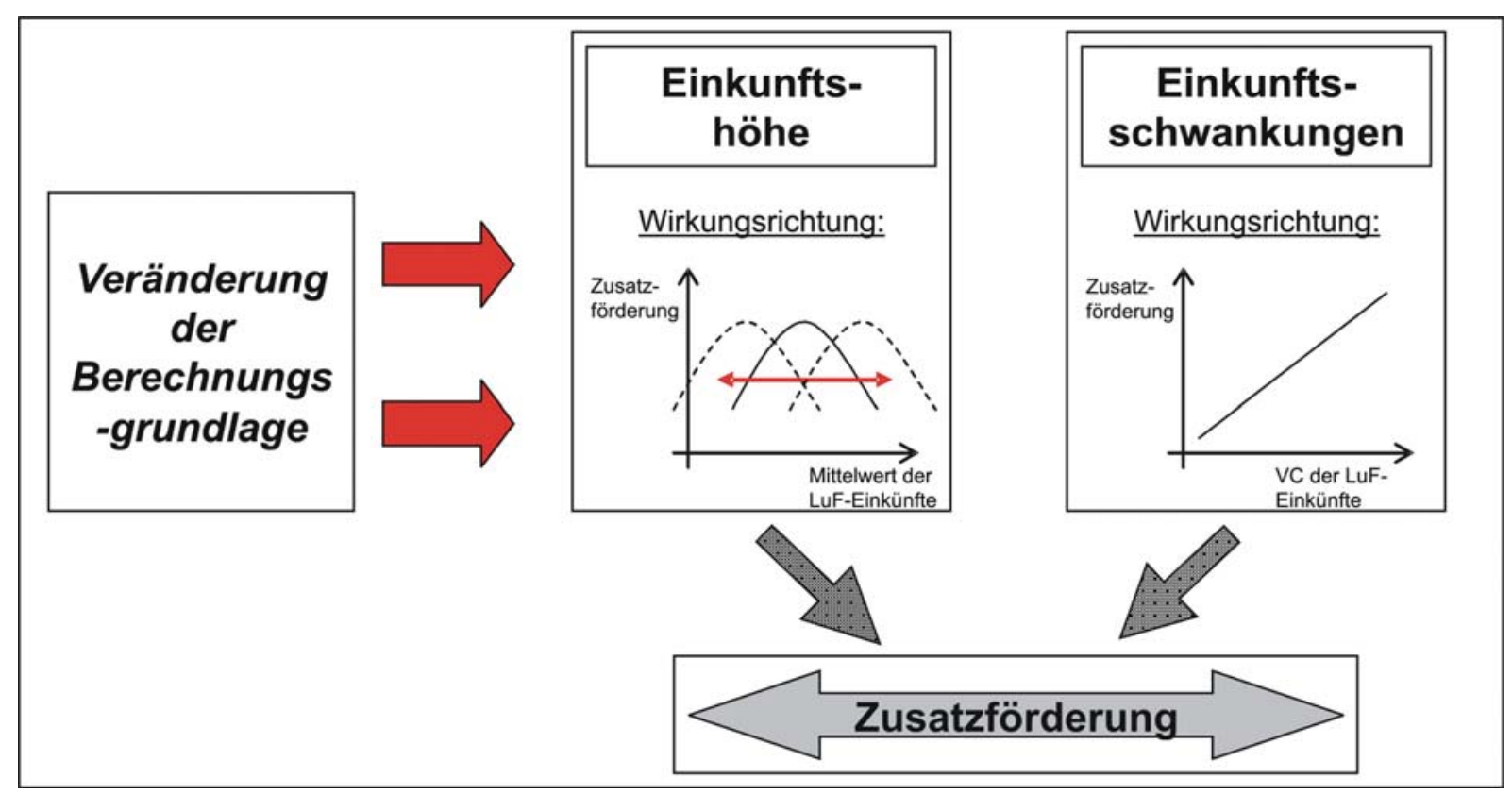

Quelle: Eigene Darstellung 
Damit kann die Förderwirkung passgenau den Zielvorstellungen für eine Förderung des ökologischen Landbaus auf Länderebene angepasst werden, was nochmals das hohe Maß an Flexibilität dieses Förderinstruments verdeutlich.

\subsubsection{VERÄNDERUNG DER GEWICHTUNG VON FLÄCHENBEZOGENEM SOCKELBETRAG UND ZUSATZFÖRDERUNG}

Besonders wichtig für die Wirkungsweise der Flexiblen Förderung insgesamt ist das Verhältnis der beiden Komponenten ,flächenbezogener Sockelbetrag' und ,Zusatzförderung’.

Die Gewichtung beider Bestandteile ergibt sich zum einen aus der Höhe des Hektarsatzes des flächenbezogenen Sockelbetrags, welcher innerhalb der Flexiblen Förderung variiert werden kann. Seine Relevanz, insbesondere im Zuge einer Systemumstellung von der gegenwärtigen Festbetragsförderung auf die Flexible Förderung, wird in Kapitel 4.2.3.3.1 näher untersucht werden.

Zum anderen ergibt sich die Gewichtung beider Einzelkomponenten aus dem Faktor, mit dem die Zusatzförderung multipliziert wird, bevor sie in die Flexible Förderung eingeht. Auf die spezifische Wirkung dieses Faktors wird im Folgenden zunächst isoliert vom flächenbezogenen Sockelbetrag eingegangen. Grundsätzlich gilt: Wird ein hoher Faktor gewählt, so hat die Zusatzförderung unter der Annahme eines unveränderten flächenbezogenen Sockelbetrags ein entsprechend höheres Gewicht innerhalb der Flexiblen Förderung als bei der Wahl eines niedrigeren Faktors. Mit Bezug auf Abbildung 18 in Kapitel 4.2.2.1, in der die Wirkung der Zusatzförderung ohne Anwendung eines Faktors aufgezeigt wurde, veranschaulichen Abbildung 32 und Abbildung 33 die Zusatzförderung bei Anwendung eines Faktors von 3 bzw. von 5.

Abbildung 32: Zusatzförderung bei Anwendung des Faktors 3

\begin{tabular}{|c|c|c|c|c|c|c|c|c|c|c|c|c|c|c|c|}
\hline \multicolumn{16}{|c|}{ Höhe der Zusatzförderung bei Multiplikation mit dem Faktor 3} \\
\hline \multicolumn{8}{|c|}{ Absolut in $€$ pro Betrieb: } & \multicolumn{8}{|c|}{ In Relation zu den mittleren LuF-Einkünften: } \\
\hline \multirow{2}{*}{$\begin{array}{c}\text { Jährl. Ein- } \\
\text { künfte aus } \\
\text { LuF in } €\end{array}$} & \multicolumn{7}{|c|}{ Variationskoeffizient } & \multirow{2}{*}{$\begin{array}{c}\text { Jährl. Ein- } \\
\text { künfte aus } \\
\text { LuF in } €\end{array}$} & \multicolumn{7}{|c|}{ Variationskoeffizient } \\
\hline & 0,1 & 0,25 & 0,5 & 0,75 & 1 & 1,25 & 1,5 & & 0,1 & 0,25 & 0,5 & 0,75 & 1 & 1,25 & 1,5 \\
\hline 1.000 & 0 & 0 & 0 & 0 & 0 & 0 & 0 & 1.000 & $0 \%$ & $0 \%$ & $0 \%$ & $0 \%$ & $0 \%$ & $0 \%$ & $0 \%$ \\
\hline 5.000 & 0 & 3 & 84 & 225 & 378 & 621 & 789 & 5.000 & $0 \%$ & $0 \%$ & $2 \%$ & $5 \%$ & $8 \%$ & $12 \%$ & $16 \%$ \\
\hline 10.000 & 18 & 186 & 570 & 906 & 1.230 & 1.884 & 2.232 & 10.000 & $0 \%$ & $2 \%$ & $6 \%$ & $9 \%$ & $12 \%$ & $19 \%$ & $22 \%$ \\
\hline 20.000 & 18 & 132 & 819 & 1.809 & 2.826 & 4.401 & 5.403 & 20.000 & $0 \%$ & $1 \%$ & $4 \%$ & $9 \%$ & $14 \%$ & $22 \%$ & $27 \%$ \\
\hline 30.000 & 42 & 264 & 1.302 & 2.874 & 4.461 & 6.867 & 8.343 & 30.000 & $0 \%$ & $1 \%$ & $4 \%$ & $10 \%$ & $15 \%$ & $23 \%$ & $28 \%$ \\
\hline 50.000 & 81 & 432 & 1.863 & 4.251 & 6.897 & 10.683 & 13.236 & 50.000 & $0 \%$ & $1 \%$ & $4 \%$ & $9 \%$ & $14 \%$ & $21 \%$ & $26 \%$ \\
\hline 75.000 & 0 & 105 & 1.728 & 5.217 & 9.318 & 14.694 & 18.807 & 75.000 & $0 \%$ & $0 \%$ & $2 \%$ & $7 \%$ & $12 \%$ & $20 \%$ & $25 \%$ \\
\hline 100.000 & 0 & 30 & 1.629 & 6.072 & 11.601 & 18.528 & 24.252 & 100.000 & $0 \%$ & $0 \%$ & $2 \%$ & $6 \%$ & $12 \%$ & $19 \%$ & $24 \%$ \\
\hline 200.000 & 0 & 3 & 1.827 & 9.615 & 20.565 & 33.699 & 45.774 & 200.000 & $0 \%$ & $0 \%$ & $1 \%$ & $5 \%$ & $10 \%$ & $17 \%$ & $23 \%$ \\
\hline
\end{tabular}

Quelle: Eigene Berechnungen gemäß ESt-Tarif 2006 
Abbildung 33: Zusatzförderung bei Anwendung des Faktors 5

\begin{tabular}{|c|c|c|c|c|c|c|c|c|c|c|c|c|c|c|c|}
\hline \multicolumn{16}{|c|}{ Höhe der Zusatzförderung bei Multiplikation mit dem Faktor 5} \\
\hline \multicolumn{8}{|c|}{ Absolut in $€$ pro Betrieb: } & \multicolumn{8}{|c|}{ In Relation zu den mittleren LuF-Einkünften: } \\
\hline \multirow{2}{*}{$\begin{array}{c}\text { Jährl. Ein- } \\
\text { künfte aus } \\
\text { LuF in } € \\
\end{array}$} & \multicolumn{7}{|c|}{ Variationskoeffizient } & \multirow{2}{*}{$\begin{array}{c}\text { Jährl. Ein- } \\
\text { künfte aus } \\
\text { LuF in } €\end{array}$} & \multicolumn{7}{|c|}{ Variationskoeffizient } \\
\hline & 0,1 & 0,25 & 0,5 & 0,75 & 1 & 1,25 & 1,5 & & 0,1 & 0,25 & 0,5 & 0,75 & 1 & 1,25 & 1,5 \\
\hline 1.000 & 0 & 0 & 0 & 0 & 0 & 0 & 0 & 1.000 & $0 \%$ & $0 \%$ & $0 \%$ & $0 \%$ & $0 \%$ & $0 \%$ & $0 \%$ \\
\hline 5.000 & 0 & 5 & 140 & 375 & 630 & 1.035 & 1.315 & 5.000 & $0 \%$ & $0 \%$ & $3 \%$ & $8 \%$ & $13 \%$ & $21 \%$ & $26 \%$ \\
\hline 10.000 & 30 & 310 & 950 & 1.510 & 2.050 & 3.140 & 3.720 & 10.000 & $0 \%$ & $3 \%$ & $10 \%$ & $15 \%$ & $21 \%$ & $31 \%$ & $37 \%$ \\
\hline 20.000 & 30 & 220 & 1.365 & 3.015 & 4.710 & 7.335 & 9.005 & 20.000 & $0 \%$ & $1 \%$ & $7 \%$ & $15 \%$ & $24 \%$ & $37 \%$ & $45 \%$ \\
\hline 30.000 & 70 & 440 & 2.170 & 4.790 & 7.435 & 11.445 & 13.905 & 30.000 & $0 \%$ & $1 \%$ & $7 \%$ & $16 \%$ & $25 \%$ & $38 \%$ & $46 \%$ \\
\hline 50.000 & 135 & 720 & 3.105 & 7.085 & 11.495 & 17.805 & 22.060 & 50.000 & $0 \%$ & $1 \%$ & $6 \%$ & $14 \%$ & $23 \%$ & $36 \%$ & $44 \%$ \\
\hline 75.000 & 0 & 175 & 2.880 & 8.695 & 15.530 & 24.490 & 31.345 & 75.000 & $0 \%$ & $0 \%$ & $4 \%$ & $12 \%$ & $21 \%$ & $33 \%$ & $42 \%$ \\
\hline 100.000 & 0 & 50 & 2.715 & 10.120 & 19.335 & 30.880 & 40.420 & 100.000 & $0 \%$ & $0 \%$ & $3 \%$ & $10 \%$ & $19 \%$ & $31 \%$ & $40 \%$ \\
\hline 200.000 & 0 & 5 & 3.045 & 16.025 & 34.275 & 56.165 & 76.290 & 200.000 & $0 \%$ & $0 \%$ & $2 \%$ & $8 \%$ & $17 \%$ & $28 \%$ & $38 \%$ \\
\hline
\end{tabular}

Quelle: Eigene Berechnungen gemäß ESt-Tarif 2006

Es zeigen sich die deutlich höheren monetären Effekte der Zusatzförderung sowohl absolut als auch in Relation zu den mittleren Einkünften aus LuF. Neben der Auswirkung des Faktors auf die isoliert betrachtete Zusatzförderung ist zusätzlich von Interesse, wie sich die Höhe des Faktors auf die Flexible Förderung insgesamt auswirkt. Dazu werden zunächst die grundsätzlichen Auswirkungen einer Systemumstellung von der Festbetragsförderung auf die Flexible Förderung analysiert.

\subsection{AUSWIRKUNGEN EINER UMSTELLUNG VON DER FESTBETRAGSFÖRDERUNG AUF DIE FLEXIBLE FÖRDERUNG}

Nicht allein die grundsätzliche Wirkungsweise der Flexiblen Förderung ist für Überlegungen zu ihrer Einführung maßgeblich. Auch die Veränderungen, die sich für die einzelnen ökologisch wirtschaftenden Betriebe aus einer Umstellung der Angebotsförderung von der gegenwärtigen Festbetragsförderung als Referenzsystem auf die Flexible Förderung ergeben, sind zu beachten. Für die diesbezüglichen Betrachtungen wird zusätzlich zu den Annahmen der Basiskonstellation eine Festbetragsprämie pro ha landwirtschaftlich genutzter Fläche (LF) von $160 € / \mathrm{Jahr}$ (Referenzsystem) unterstellt. Daneben wird angenommen, die Zusatzförderung gehe mit dem Faktor 3 multipliziert in die Flexible Förderung ein.

Durch diese zusätzlichen Annahmen wird erreicht, dass sich das Fördervolumen pro ha allein aus der Absenkung der Festbetragsprämie auf das Niveau des Sockelbetrages, d.h. ohne Betrachtung der Zusatzförderung, um 38 \% reduziert.

Abbildung 34 bis Abbildung 36 veranschaulichen die betriebswirtschaftlichen Auswirkungen einer Umstellung der Angebotsförderung für Öko-Betriebe mit unterschiedlichen Einkunftsschwankungen und unterschiedlichen Flächenumfängen. Für alle Fallbeispiele wurde ein Mittelwert der Jahreseinkünfte aus LuF von $50.000 €$ angenommen. Dieser Wert 
könnte auch beliebig höher oder niedriger sein, ohne dass sich der aufgezeigte Wirkungszusammenhang ändert. Aus Vereinfachungsgründen wurde an dieser Stelle aber auf eine weitere Differenzierung verzichtet.

Abbildung 34: Auswirkungen einer Einführung der Flexiblen Förderung für unterschiedlich flächenstarke Betriebe bei niedrigen Einkunftsschwankungen

\begin{tabular}{|c|c|c|c|c|c|c|c|c|c|c|c|}
\hline \multicolumn{6}{|c|}{ Mittelwert der Jahreseinkünfte aus LuF: $50.000 €$} & & $\begin{array}{c}\text { Einkünfte } \\
\text { aus LuF }\end{array}$ & $\begin{array}{l}\text { Sockel- } \\
\text { betrag }\end{array}$ & \begin{tabular}{|c|} 
Zusatz- \\
förderung
\end{tabular} & \begin{tabular}{|c|} 
Flexible \\
Förderung
\end{tabular} & $\begin{array}{c}\text { Festbetrags- } \\
\text { förderung }\end{array}$ \\
\hline \multicolumn{6}{|c|}{ Variationskoeffizient (VC): 0,25} & \begin{tabular}{|l|l|l|l|l}
$J a h r$ & 1 \\
\end{tabular} & $50.000 €$ & $\ldots$ & $\ldots$ & $\ldots$ & $\ldots$ \\
\hline \multirow{2}{*}{\multicolumn{6}{|c|}{ Landwirtschaftlich genutzte Fläche (LF): 50 ha }} & Jahr 2 & $62.500 €$ & ... & $\ldots$ & & \\
\hline & & & & & & Jahr 3 & $37.500 €$ & $5.000 €$ & $157 €$ & $5.470 €$ & $8.000 €$ \\
\hline \multicolumn{6}{|c|}{$\begin{array}{l}\text { Landwirtschaftlich genutzte Fläche (LF): } 100 \text { ha } \\
\text { Landwirtschaftlich genutzte Fläche (LF): } 500 \text { ha }\end{array}$} & \multicolumn{5}{|c|}{ Änderung der Fördersumme in Jahr 3 (absolut): } & Änderung der Fördersumme in Jahr 3 (relativ): \\
\hline & $\begin{array}{l}\text { Einkünfte } \\
\text { aus LuF }\end{array}$ & $\begin{array}{l}\text { Sockel- } \\
\text { betrag }\end{array}$ & \begin{tabular}{c|c|} 
Zusatz- \\
förderung
\end{tabular} & \begin{tabular}{c|c|} 
Flexible \\
Förderung
\end{tabular} & $\begin{array}{l}\text { Festbetrags- } \\
\text { förderung }\end{array}$ & & \begin{tabular}{c|} 
Einkünfte \\
aus LuF
\end{tabular} & $\begin{array}{l}\text { Sockel- } \\
\text { betrag }\end{array}$ & \begin{tabular}{|c|}
$\begin{array}{c}\text { Zusatz- } \\
\text { förderung }\end{array}$ \\
\end{tabular} & \begin{tabular}{|c|} 
Flexible \\
Förderung
\end{tabular} & $\begin{array}{c}\text { Festbetrags- } \\
\text { förderung }\end{array}$ \\
\hline Jahr 1 & $50.000 €$ & $\ldots$ & $\ldots$ & $\ldots$ & $\ldots$ & \begin{tabular}{|l|l|l|l}
$J a h r$ & 1 \\
\end{tabular} & $50.000 €$ & $\ldots$ & $\ldots$ & $\ldots$ & $\ldots$ \\
\hline Jahr 2 & $62.500 €$ & $\ldots$ & $\ldots$ & & & \begin{tabular}{|l|l}
$J a h r$ & 2 \\
\end{tabular} & $62.500 €$ & . & $\ldots$ & & \\
\hline Jahr 3 & $37.500 €$ & $50.000 \epsilon$ & $157 \epsilon$ & $50.470 €$ & $80.000 \epsilon$ & Jahr 3 & $37.500 €$ & $10.000 €$ & $157 \epsilon$ & $10.470 \epsilon$ & $16.000 \epsilon$ \\
\hline Änder & ng der För & ersumme & in Jahr 3 (a & bsolut): & $-29.530 €$ & Ändert & ung der Förc & dersumme & in Jahr 3 (a & bsolut): & $-5.530 €$ \\
\hline Änder & ng der För & ersumme & in Jahr 3 (r & elativ): & $-37 \%$ & Ändert & ung der Föro & dersumme & in Jahr 3 ( & elativ): & $-35 \%$ \\
\hline
\end{tabular}

Quelle: Eigene Berechnungen gemäß ESt-Tarif 2006

Abbildung 35: Auswirkungen einer Einführung der Flexiblen Förderung für unterschiedlich flächenstarke Betriebe bei mittleren Einkunftsschwankungen

\begin{tabular}{|c|c|c|c|c|c|c|c|c|c|c|c|}
\hline \multicolumn{6}{|c|}{ Mittelwert der Jahreseinkünfte aus LuF: $50.000 €$} & & $\begin{array}{l}\text { Einkünfte } \\
\text { aus LuF }\end{array}$ & $\begin{array}{l}\text { Sockel- } \\
\text { betrag }\end{array}$ & $\begin{array}{c}\text { Zusatz- } \\
\text { förderung }\end{array}$ & \begin{tabular}{|c|} 
Flexible \\
Förderung
\end{tabular} & $\begin{array}{l}\text { Festbetrags- } \\
\text { förderung }\end{array}$ \\
\hline \multicolumn{6}{|c|}{ Variationskoeffizient (VC): 0,75} & Jahr 1 & $50.000 €$ & \multirow{3}{*}{$\begin{array}{c}\ldots \\
\ldots \\
5.000 €\end{array}$} & \multirow{3}{*}{$\begin{array}{c}\ldots \\
\ldots \\
1.192 €\end{array}$} & & \\
\hline \multirow{3}{*}{\multicolumn{6}{|c|}{$\begin{array}{l}\text { Landwirtschaftlich genutzte Fläche (LF): } 50 \text { ha } \\
\text { Landwirtschaftlich genutzte Fläche (LF): } 100 \text { ha }\end{array}$}} & Jahr 2 & & & & & \\
\hline & & & & & & Jahr 3 & $12.500 €$ & & & $8.576 €$ & $8.000 €$ \\
\hline & & & & & & \multicolumn{6}{|c|}{ Änderung der Fördersumme in Jahr 3 (absolut): } \\
\hline & $\begin{array}{l}\text { Einkünfte } \\
\text { aus LuF }\end{array}$ & $\begin{array}{l}\text { Sockel- } \\
\text { betrag }\end{array}$ & \begin{tabular}{|c|}
$\begin{array}{c}\text { Zusatz- } \\
\text { förderung }\end{array}$ \\
\end{tabular} & \begin{tabular}{|c|}
$\begin{array}{c}\text { Flexible } \\
\text { Förderung }\end{array}$ \\
\end{tabular} & $\begin{array}{c}\text { Festbetrags- } \\
\text { förderung }\end{array}$ & & $\begin{array}{l}\text { Einkünfte } \\
\text { aus LuF }\end{array}$ & $\begin{array}{l}\text { Sockel- } \\
\text { betrag }\end{array}$ & \begin{tabular}{|c|}
$\begin{array}{c}\text { Zusatz- } \\
\text { förderung }\end{array}$ \\
\end{tabular} & \begin{tabular}{|c|}
$\begin{array}{c}\text { Flexible } \\
\text { Förderung }\end{array}$ \\
\end{tabular} & $\begin{array}{c}\text { Festbetrags- } \\
\text { förderung }\end{array}$ \\
\hline Jahr 1 & & \multirow{3}{*}{$\begin{array}{c}\ldots \\
\ldots \\
50.000 € \\
\end{array}$} & \multirow{3}{*}{$\begin{array}{c}\ldots \\
\ldots \\
1.192 € \\
\end{array}$} & & $\ldots$ & Jahr 1 & $50.000 €$ & \multirow{3}{*}{$\begin{array}{c}\ldots \\
\ldots \\
10.000 € \\
\end{array}$} & \multirow{3}{*}{$\begin{array}{c}\ldots \\
\ldots \\
1.192 € \\
\end{array}$} & $\ldots$ & 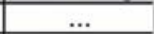 \\
\hline Jahr 2 & & & & & & Jahr 2 & $87.500 €$ & & & & \\
\hline Jahr 3 & $12.500 €$ & & & $53.576 €$ & $80.000 €$ & Jahr 3 & $12.500 €$ & & & $13.576 €$ & $16.000 €$ \\
\hline \multicolumn{6}{|c|}{ Änderung der Fördersumme in Jahr 3 (absolut): } & \multicolumn{5}{|c|}{\begin{tabular}{|l} 
Änderung der Fördersumme in Jahr 3 (absolut): \\
Änderung der Fördersumme in Jahr 3 (relativ)
\end{tabular}} & $-2.424 \epsilon$ \\
\hline Änder & Ing der För & ersumme & in Jahr 3 ( $\mathrm{r}$ & elativ): & $-33 \%$ & Ändert & ing der För & dersumme & in Jahr 3 ( & elativ): & $-15 \%$ \\
\hline
\end{tabular}

Quelle: Eigene Berechnungen gemäß ESt-Tarif 2006 
Abbildung 36: Auswirkungen einer Einführung der Flexiblen Förderung für unterschiedlich flächenstarke Betriebe bei hohen Einkunftsschwankungen

\begin{tabular}{|c|c|c|c|c|c|c|c|c|c|c|c|}
\hline \multicolumn{6}{|c|}{ Mittelwert der Jahreseinkünfte aus LuF: $50.000 €$} & & \begin{tabular}{|c|} 
Einkünfte \\
aus LuF
\end{tabular} & $\begin{array}{l}\text { Sockel- } \\
\text { betrag }\end{array}$ & \begin{tabular}{|c|} 
Zusatz- \\
förderung
\end{tabular} & \begin{tabular}{|c|}
$\begin{array}{c}\text { Flexible } \\
\text { Förderung }\end{array}$ \\
\end{tabular} & $\begin{array}{l}\text { Festbetrags- } \\
\text { förderung }\end{array}$ \\
\hline \multicolumn{6}{|c|}{ Variationskoeffizient (VC): 1,25} & \begin{tabular}{|l|} 
Jahr 1 \\
\end{tabular} & $50.000 €$ & & $\ldots$ & \begin{tabular}{|c|}
$\ldots$ \\
\end{tabular} & $\ldots$ \\
\hline \multirow{2}{*}{\multicolumn{6}{|c|}{$\begin{array}{l}\text { Landwirtschaftlich genutzte Fläche (LF): } 50 \text { ha } \\
\text { Landwirtschaftlich genutzte Fläche (LF): } 100 \text { ha }\end{array}$}} & \begin{tabular}{|l|} 
Jahr 2 \\
Jahr 3 \\
\end{tabular} & \begin{tabular}{|l|}
$112.500 €$ \\
$-12.500 €$ \\
\end{tabular} & $5.000 €$ & $4.381 €$ & $\begin{array}{cl}\ldots \\
18.144 \epsilon\end{array}$ & $8.000 €$ \\
\hline \multicolumn{2}{|c|}{ Landwirtschaftlich genutzte Fläche (LF): 500 ha } & & & & & \multicolumn{5}{|c|}{$\begin{array}{l}\text { Anderung der Fördersumme in Jahr } 3 \text { (absolut): } \\
\text { Änderung der Fördersumme in Jahr } 3 \text { (relativ): } \\
\end{array}$} & $\begin{array}{r}10.144 \epsilon \\
127 \% \\
\end{array}$ \\
\hline & $\begin{array}{l}\text { Einkünfte } \\
\text { aus LuF }\end{array}$ & $\begin{array}{c}\text { Sockel- } \\
\text { betrag }\end{array}$ & $\begin{array}{c}\text { Zusatz- } \\
\text { forderung }\end{array}$ & \begin{tabular}{|c|} 
Flexible \\
Förderung \\
\end{tabular} & \begin{tabular}{|c|}
$\begin{array}{c}\text { Festbetrags- } \\
\text { förderung }\end{array}$ \\
\end{tabular} & & \begin{tabular}{|c|}
$\begin{array}{c}\text { Einkünfte } \\
\text { aus LuF }\end{array}$ \\
\end{tabular} & $\begin{array}{c}\text { Sockel- } \\
\text { betrag }\end{array}$ & \begin{tabular}{|c|}
$\begin{array}{c}\text { Zusatz- } \\
\text { förderung }\end{array}$ \\
\end{tabular} & \begin{tabular}{|c|} 
Flexible \\
Förderung \\
\end{tabular} & $\begin{array}{c}\begin{array}{c}\text { Festbetrags } \\
\text { förderung }\end{array} \\
\end{array}$ \\
\hline Jahr 1 & $50.000 €$ & $\cdots$ & $\ldots$ & $\ldots$ & $\ldots$ & Jahr 1 & $50.000 €$ & $\ldots$ & $\cdots$ & $\ldots$ & $\ldots$ \\
\hline Jahr 2 & $112.500 €$ & $\cdots$ & $\cdots$ & & & Jahr 2 & $112.500 €$ & & $\ldots$ & & \\
\hline Jahr 3 & $-12.500 €$ & $50.000 €$ & $4.381 €$ & $63.144 €$ & $80.000 €$ & Jahr 3 & $-12.500 €$ & $10.000 €$ & $4.381 €$ & $23.144 €$ & $16.000 €$ \\
\hline Änder & ing der För & ersumme & in Jahr 3 (a & bsolut): & $-16.856 \epsilon$ & Änder & ung der För & ersumme & in Jahr 3 ( & bsolut): & $7.144 €$ \\
\hline Änder & ing der För & dersumme & in Jahr 3 ( $r$ & elativ): & $-21 \%$ & Änder & ung der För & dersumme & in Jahr 3 ( & elativ): & $45 \%$ \\
\hline
\end{tabular}

Quelle: Eigene Berechnungen gemäß ESt-Tarif 2006

Die Beispielrechungen zu den Auswirkungen einer Umstellung der Angebotsförderung auf die Flexible Förderung offenbaren wichtige Wirkungsmechanismen. Infolge der Reduktion der flächenbezogenen Komponente ergibt sich für die Öko-Betriebe eine Reduzierung der erhaltenen Fördermittel, die natürlich umso deutlicher ausfällt, je geringer der Sockelbetrag festgesetzt wird. Wie die Abbildungen zeigen, kann dieser Fördermittelverlust bei einer angenommenen LF von 500 ha auch bei hohen Einkunftsschwankungen nicht von der Zusatzförderung kompensiert werden. Dagegen führt die Umstellung auf die Flexible Förderung bei einer LF von nur 50 ha bereits bei mittleren Einkunftsschwankungen zu einer erhöhten Gesamtförderung. Damit zeigt sich, dass die Gesamtwirkung einer Systemumstellung hinsichtlich des einzelbetrieblichen Fördervolumens in hohem Maße vom Flächenumfang abhängig ist.

Diese Betrachtung setzt allerdings voraus, dass das gegenwärtige Festbetragsfördersystem bei Nichteinführung der Flexiblen Förderung noch unbegrenzt weiter Bestand hat. Zumindest eine Absenkung des flächenbezogenen Förderbetrags auf die abgesenkten Vorgaben des GAK-Rahmenplans 2007 wird aller Voraussicht nach in vielen Bundesländern aber ohnehin erfolgen.

Nichtsdestotrotz sind die Auswirkungen einer Absenkung der flächenbezogenen Förderkomponente für flächenstärkere Betriebe voraussichtlich erheblicher, als die Auswirkungen einer Einführung der Zusatzförderung. Mit Einführung der Flexiblen Förderung in der Basiskonstellation wäre deshalb zu erwarten, dass vor allem die potentiell flächenstärkeren Betriebe in Ostdeutschland auf sektoraler Basis verstärkt zu den Verlierern zählen könnten. In den Vorkapiteln ist aber bereits auf die Möglichkeiten zur individuellen 
Ausgestaltung der Flexiblen Förderung hingewiesen worden, mit denen die gezeigten Auswirkungen abgeschwächt oder gesteigert werden können. Eine weitere Möglichkeit dazu besteht in der Änderung des zuvor erläuterten Faktors, der auf die Zusatzförderung angewandt werden kann.

\subsection{AUSWIRKUNGEN EINER VERÄNDERUNG DES FAKTORS DER ZUSATZFÖRDERUNG}

Analog zur Darstellungsweise im Vorkapitel zeigen Abbildung 37 bis Abbildung 39 die Auswirkungen einer Veränderung des Faktors. Dabei wurden der Einfachheit halber jeweils mittlere Einkünfte von $50.000 €$ sowie eine landwirtschaftlich genutzte Fläche (LF) von 100 ha unterstellt. Für den Sockelbetrag, die Zusatzförderung und die Festbetragsförderung wurden wiederum die Annahmen aus dem Vorkapitel unterstellt.

Abbildung 37: Wirkungsweise der Flexiblen Förderung bei geringen Einkunftsschwankungen und der Multiplikation der Zusatzförderung mit unterschiedlichen Faktoren

\begin{tabular}{|c|c|c|c|c|c|c|c|c|c|c|c|}
\hline \multicolumn{6}{|c|}{ Mittelwert der Jahreseinkünfte aus LuF: $50.000 €$} & & $\begin{array}{c}\text { Einkünfte } \\
\text { aus LuF }\end{array}$ & $\begin{array}{l}\text { Sockel- } \\
\text { betrag }\end{array}$ & $\begin{array}{c}\text { Zusatz- } \\
\text { förderung }\end{array}$ & \begin{tabular}{|c|} 
Flexible \\
Förderung
\end{tabular} & $\begin{array}{c}\text { Festbetrags- } \\
\text { förderung }\end{array}$ \\
\hline \multicolumn{6}{|c|}{ Landw. genutzte Fläche: 100 ha } & \begin{tabular}{|l|} 
Jahr 1 \\
\end{tabular} & $50.000 €$ & & & $\ldots$ & $\ldots$ \\
\hline \multirow{2}{*}{\multicolumn{6}{|c|}{ Variationskoeffizient (VC): 0,25}} & \begin{tabular}{|l|} 
Jahr 2 \\
\end{tabular} & $62.500 €$ & & & & \\
\hline & & & & & & \begin{tabular}{|l|}
$J a h r$ \\
\end{tabular} & $37.500 €$ & $10.000 €$ & $157 €$ & $10.157 €$ & $16.000 €$ \\
\hline \multirow{2}{*}{\multicolumn{3}{|c|}{ Faktor: 5}} & \multirow{2}{*}{\multicolumn{3}{|c|}{ Faktor: 1 - }} & & $-5.843 \epsilon$ \\
\hline & & & & & & \multicolumn{5}{|c|}{ Änderung der Fördersumme in Jahr 3 (relativ): } & $-37 \%$ \\
\hline & $\begin{array}{l}\text { Einkünfte } \\
\text { aus LuF }\end{array}$ & $\begin{array}{l}\text { Sockel- } \\
\text { betrag }\end{array}$ & \begin{tabular}{|c|}
$\begin{array}{c}\text { Zusatz- } \\
\text { förderung }\end{array}$ \\
\end{tabular} & \begin{tabular}{c|} 
Flexible \\
Förderung
\end{tabular} & $\begin{array}{l}\begin{array}{l}\text { Festbetrags- } \\
\text { förderung }\end{array} \\
\end{array}$ & & $\begin{array}{c}\text { Einkünfte } \\
\text { aus LuF }\end{array}$ & $\begin{array}{l}\text { Sockel- } \\
\text { betrag }\end{array}$ & \begin{tabular}{|c|}
$\begin{array}{c}\text { Zusatz- } \\
\text { förderung }\end{array}$ \\
\end{tabular} & \begin{tabular}{|c|} 
Flexible \\
Förderung
\end{tabular} & $\begin{array}{c}\text { Festbetrags- } \\
\text { förderung }\end{array}$ \\
\hline \begin{tabular}{|l|} 
Jahr 1 \\
\end{tabular} & $50.000 €$ & & & $\ldots$ & $\ldots$ & \begin{tabular}{|l|} 
Jahr 1 \\
\end{tabular} & $50.000 €$ & & & $\ldots$ & $\ldots$ \\
\hline Jahr 2 & $62.500 €$ & & & & & \begin{tabular}{|l|} 
Jahr 2 \\
\end{tabular} & $62.500 €$ & & & & \\
\hline Jahr 3 & $37.500 €$ & $10.000 €$ & $157 €$ & $10.783 €$ & $16.000 €$ & \begin{tabular}{|l|l|} 
Jahr 3 \\
\end{tabular} & $37.500 €$ & $10.000 €$ & $157 €$ & $10.470 €$ & $16.000 €$ \\
\hline \multirow{2}{*}{\multicolumn{5}{|c|}{$\begin{array}{l}\text { Änderung der Fördersumme in Jahr } 3 \text { (absolut): } \\
\text { Änderung der Fördersumme in Jahr } 3 \text { (relativ): }\end{array}$}} & $-5.217 \epsilon$ & \multirow{2}{*}{\multicolumn{5}{|c|}{\begin{tabular}{|l} 
Ånderung der Fördersumme in Jahr 3 (absolut): \\
Änderung der Fördersumme in Jahr 3 (relativ):
\end{tabular}}} & $-5.530 \epsilon$ \\
\hline & & & & & $-33 \%$ & & & & & & $-35 \%$ \\
\hline
\end{tabular}

Quelle: Eigene Berechnungen gemäß ESt-Tarif 2006

Abbildung 38: Wirkungsweise der Flexiblen Förderung bei mittleren Einkunftsschwankungen und der Multiplikation der Zusatzförderung mit verschiedenen Faktoren

\begin{tabular}{|c|c|c|c|c|c|c|c|c|c|c|c|}
\hline \multirow{4}{*}{\multicolumn{6}{|c|}{$\begin{array}{l}\text { Mittelwert der Jahreseinkünfte aus LuF: } 50.000 € \\
\text { Landw. genutzte Fläche: } 100 \text { ha } \\
\text { Variationskoeffizient (VC): } \mathbf{0 , 7 5}\end{array}$}} & & $\begin{array}{l}\text { Einkünfte } \\
\text { aus LuF }\end{array}$ & $\begin{array}{l}\text { Sockel- } \\
\text { betrag }\end{array}$ & \begin{tabular}{|c|} 
Zusatz- \\
förderung
\end{tabular} & $\begin{array}{l}\text { Flexible } \\
\text { Förderung }\end{array}$ & $\begin{array}{l}\text { Festbetrags } \\
\text { förderung }\end{array}$ \\
\hline & & & & & & Jahr 1 & $50.000 €$ & $\ldots$ & \begin{tabular}{|c|}
$\ldots$ \\
\end{tabular} & $\ldots$ & $\ldots$ \\
\hline & & & & & & \begin{tabular}{|l|} 
Jahr 2 \\
\end{tabular} & $87.500 €$ & & & $\ldots$ & 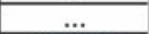 \\
\hline & & & & & & \begin{tabular}{|l|l|l} 
Jahr 3 \\
\end{tabular} & $12.500 €$ & $10.000 €$ & $1.192 €$ & $11.192 €$ & $16.000 €$ \\
\hline \multirow{2}{*}{\multicolumn{3}{|c|}{ Faktor: 5}} & \multirow[b]{2}{*}{ Faktor: 3} & \multirow{2}{*}{\multicolumn{2}{|c|}{ Faktor: 1 = }} & \multicolumn{5}{|c|}{ Ånderung der Fördersumme in Jahr 3 (absolut): } & $-4.808 €$ \\
\hline & & & & & & \multicolumn{5}{|c|}{ Änderung der Fördersumme in Jahr 3 (relativ): } & $-30 \%$ \\
\hline & $\begin{array}{l}\text { Einkünfte } \\
\text { aus LuF }\end{array}$ & $\begin{array}{l}\text { Sockel- } \\
\text { betrag }\end{array}$ & \begin{tabular}{|c|}
$\begin{array}{c}\text { Zusatz- } \\
\text { förderung }\end{array}$ \\
\end{tabular} & \begin{tabular}{|c|} 
Flexible \\
Förderung \\
\end{tabular} & \begin{tabular}{|c|}
$\begin{array}{c}\text { Festbetrags- } \\
\text { förderung }\end{array}$ \\
\end{tabular} & & $\begin{array}{l}\text { Einkünfte } \\
\text { aus LuF }\end{array}$ & $\begin{array}{l}\text { Sockel- } \\
\text { betrag }\end{array}$ & \begin{tabular}{|c|}
$\begin{array}{c}\text { Zusatz- } \\
\text { förderung }\end{array}$ \\
\end{tabular} & $\begin{array}{c}\text { Flexible } \\
\text { Förderung }\end{array}$ & \begin{tabular}{|c|}
$\begin{array}{c}\text { Festbetrags } \\
\text { förderung }\end{array}$ \\
\end{tabular} \\
\hline Jahr 1 & $50.000 €$ & $\ldots$ & $\ldots$ & \multirow{2}{*}{$\ldots$} & $\ldots$ & Jahr 1 & $50.000 €$ & $\ldots$ & $\ldots$ & $\ldots$ & $\ldots$ \\
\hline Jahr 2 & $87.500 €$ & & & & \multirow{2}{*}{$\frac{\ldots}{16.000 €}$} & \begin{tabular}{|l|} 
Jahr 2 \\
\end{tabular} & $87.500 €$ & 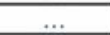 & $\ldots$ & $\ldots$ & $\ldots$ \\
\hline Jahr 3 & $12.500 €$ & $10.000 €$ & $1.192 €$ & $15.960 €$ & & \begin{tabular}{|l|l|l} 
Jahr 3 \\
\end{tabular} & $12.500 €$ & $10.000 €$ & $1.192 €$ & $13.576 €$ & $16.000 €$ \\
\hline \multicolumn{6}{|c|}{ Änderung der Fördersumme in Jahr 3 (absolut): } & \multicolumn{5}{|c|}{ Ånderung der Fördersumme in Jahr 3 (absolut): } & $-2.424 €$ \\
\hline \multicolumn{6}{|c|}{ Änderung der Fördersumme in Jahr 3 (relativ): } & \multicolumn{5}{|c|}{ Änderung der Fördersumme in Jahr 3 (relativ): } & $-15 \%$ \\
\hline
\end{tabular}

Quelle: Eigene Berechnungen gemäß ESt-Tarif 2006 
Abbildung 39: Wirkungsweise der Flexiblen Förderung bei hohen Einkunftsschwankungen und der Multiplikation der Zusatzförderung mit unterschiedlichen Faktoren

\begin{tabular}{|c|c|c|c|c|c|c|c|c|c|c|c|}
\hline \multirow{4}{*}{\multicolumn{6}{|c|}{$\begin{array}{c}\text { Mittelwert der Jahreseinkünfte aus LuF: } 50.000 € \\
\text { Landw. genutzte Fläche: } 100 \text { ha } \\
\text { Variationskoeffizient (VC): } 1,25\end{array}$}} & & \begin{tabular}{|l|} 
Einkünfte \\
aus LuF
\end{tabular} & $\begin{array}{l}\text { Sockel- } \\
\text { betrag }\end{array}$ & \begin{tabular}{|c|}
$\begin{array}{c}\text { Zusatz- } \\
\text { förderung }\end{array}$ \\
\end{tabular} & \begin{tabular}{|c} 
Flexible \\
Förderung
\end{tabular} & $\begin{array}{c}\text { Festbetrags } \\
\text { förderung }\end{array}$ \\
\hline & & & & & & Jahr 1 & $50.000 €$ & & & $\ldots$ & $\ldots$ \\
\hline & & & & & & Jahr 2 & $112.500 €$ & & & & \\
\hline & & & & & & Jahr 3 & $-12.500 €$ & $10.000 €$ & $4.381 \epsilon$ & $14.381 €$ & $16.000 €$ \\
\hline \multirow{2}{*}{\multicolumn{3}{|c|}{ Faktor: 5}} & \multirow{2}{*}{\multicolumn{3}{|c|}{ Faktor: 1 - }} & \multicolumn{5}{|c|}{ Änderung der Fördersumme in Jahr 3 (absolut): } & $-1.619 \epsilon$ \\
\hline & & & & & & \multicolumn{5}{|c|}{ Änderung der Fördersumme in Jahr 3 (relativ): } & $-10 \%$ \\
\hline & $\begin{array}{l}\text { Einkünfte } \\
\text { aus LuF }\end{array}$ & $\begin{array}{l}\text { Sockel- } \\
\text { betrag }\end{array}$ & $\begin{array}{c}\text { Zusatz- } \\
\text { förderung } \\
\end{array}$ & \begin{tabular}{|c|} 
Flexible \\
Förderung \\
\end{tabular} & \begin{tabular}{|l|}
$\begin{array}{c}\text { Festbetrags- } \\
\text { förderung }\end{array}$ \\
\end{tabular} & & \begin{tabular}{|l|}
$\begin{array}{l}\text { Einkünfte } \\
\text { aus LuF }\end{array}$ \\
\end{tabular} & $\begin{array}{l}\text { Sockel- } \\
\text { betrag }\end{array}$ & \begin{tabular}{|c|}
$\begin{array}{c}\text { Zusatz- } \\
\text { förderung }\end{array}$ \\
\end{tabular} & \begin{tabular}{|c|} 
Flexible \\
Förderung
\end{tabular} & \begin{tabular}{|c|}
$\begin{array}{c}\text { Festbetrags- } \\
\text { förderung }\end{array}$ \\
\end{tabular} \\
\hline Jahr 1 & $50.000 €$ & & & $\ldots$ & $\ldots$ & Jahr 1 & $50.000 €$ & & & $\ldots$ & $\ldots$ \\
\hline Jahr 2 & $112.500 €$ & & & & & Jahr 2 & $112.500 €$ & & & & \\
\hline Jahr 3 & $-12.500 €$ & $10.000 €$ & $4.381 €$ & $31.907 €$ & $16.000 €$ & Jahr 3 & $-12.500 €$ & $10.000 €$ & $4.381 €$ & $23.144 €$ & $16.000 €$ \\
\hline \multirow{2}{*}{\multicolumn{5}{|c|}{\begin{tabular}{|l} 
Änderung der Fördersumme in Jahr 3 (absolut): \\
Änderung der Fördersumme in Jahr 3 (relativ): \\
\end{tabular}}} & $15.907 \epsilon$ & \multirow{2}{*}{\multicolumn{5}{|c|}{$\begin{array}{l}\text { Änderung der Fördersumme in Jahr } 3 \text { (absolut): } \\
\text { Änderung der Fördersumme in Jahr } 3 \text { (relativ): } \\
\end{array}$}} & $7.144 \epsilon$ \\
\hline & & & & & $+99 \%$ & & & & & & $+45 \%$ \\
\hline
\end{tabular}

Quelle: Eigene Berechnungen gemäß ESt-Tarif 2006

Die Ergebnisse zeigen die hohe Relevanz des Faktors für Betriebe mit hohen Einkunftsschwankungen. Für Betriebe, die sehr stabile Einkünfte verzeichnen und daher generell auch nur eine sehr geringe Zusatzförderung erhalten, ergibt sich ein sehr geringer Nutzen aus einem erhöhten Multiplikator. Dem gegenüber kann im Falle hoher Einkunftsschwankungen, wie Abbildung 39 beweist, die Multiplikation der Zusatzförderung mit einem hohen Faktor zu einer deutlichen Steigerung der Gesamtförderung führen.

Als Konsequenz einer Erhöhung des Multiplikators der Zusatzförderung ergibt sich also eine verstärkte absolute Förderung von Betrieben mit erhöhten Einkunftsschwankungen. Daneben wird aber auch die aus dem Kapitel 4.2.2 bekannte Lenkungswirkung der Zusatzförderung gemäß der Einkunftshöhe verstärkt. Wenn bei einem begrenzten Förderbudget eine Anhebung des Faktors mit einer Senkung des flächenbezogenen Hektarsatzes einhergeht, führt das zusätzlich zu Fördermittelverlusten bei flächenstärkeren Betrieben. Der aus der Umstellung des Fördersystems von der bisherigen Festbetragsförderung auf die Flexible Förderung resultierende Umverteilungseffekt, der im Vorkapitel erkannt wurde, ist also umso größer, je höher der Faktor und damit das Gewicht der Zusatzförderung innerhalb der Flexiblen Förderung ist.

Damit ergeben sich insgesamt die in Abbildung 40 aufgezeigten Veränderungen infolge der Gewichtung von Sockelbetrag und Zusatzförderung. 
Abbildung 40: Auswirkungen einer Veränderung der Gewichtung der Einzelkomponenten der Flexiblen Förderung

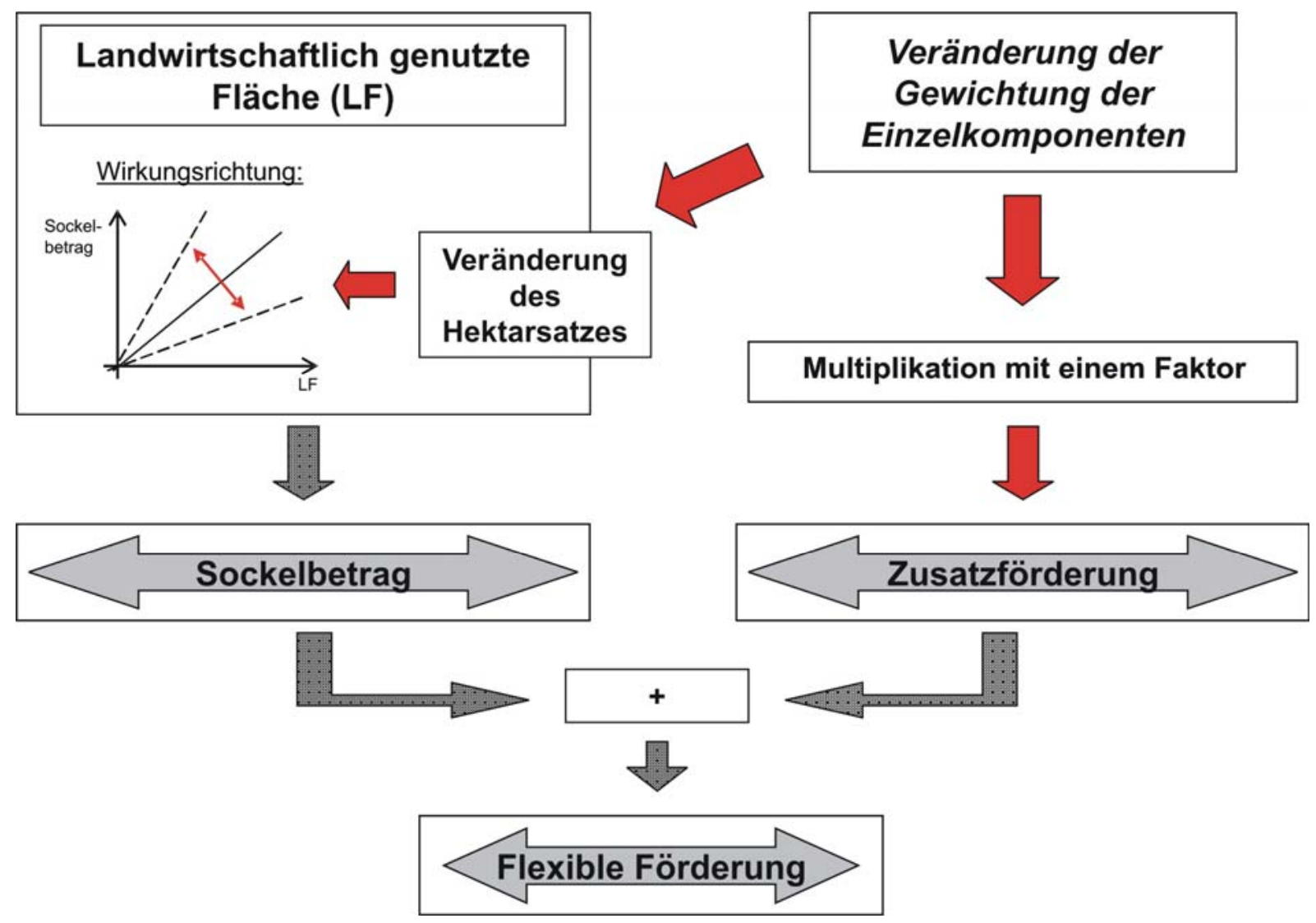

Quelle: Eigene Darstellung

Der Sockelbetrag als erste Komponente der Flexiblen Förderung bemisst sich an der ökologisch bewirtschafteten Fläche. Er ist daher umso höher, je mehr Fläche vom betreffenden Betrieb ökologisch bewirtschaftet wird oder je höher der Hektarsatz ist. Die Zusatzförderung als zweite Komponente der Flexiblen Förderung bemisst sich zum einen an der Schwankung der Einkünfte aus Land- und Forstwirtschaft. Sie ist daher umso höher, je stärker die Einkünfte des betreffenden Betriebs schwanken. Zum anderen ist sie in Abhängigkeit von der unterstellten Berechnungsgrundlage an die Höhe der einzelbetrieblichen Einkünfte geknüpft (=Lenkungswirkung). Wird die Gewichtung innerhalb der Flexiblen Förderung mit einem erhöhten Hektarsatz zugunsten des flächenbezogenen Sockebetrags verschoben, so gewinnt auch die bewirtschaftete Fläche als Bemessungsgrundlage an Gewicht. Wird im entgegen gesetzten Fall die Gewichtung zugunsten der Zusatzförderung verschoben, so gewinnen die Einkunftsschwankungen als Bemessungsgrundlage der Förderung sowie die Lenkungswirkung gemäß der Einkunftshöhe an Gewicht. Unabhängig von der variabel einzustellenden Lenkungswirkung ergeben sich daraus für die einzelnen Öko-Betriebe folgende Konsequenzen: Flächenstarke Betriebe mit 
geringen Einkunftsschwankungen profitieren von einer starken Gewichtung des flächenbezogenen Sockelbetrags, während flächenschwache Betriebe mit volatileren Einkünften von einer stärkeren Gewichtung der Zusatzförderung profitieren.

\subsubsection{ASPEKTE EINER ANGEMESSENEN BEMESSUNGSGRUNDLAGE FÜR DIE ZUSATZFÖRDERUNG}

Im Hinblick auf die Konzeption der Flexiblen Förderung stellt sich grundsätzlich die Frage, ob die Schwankung der steuerlichen Einkünfte aus Land- und Forstwirtschaft auf Basis des steuerlichen Gewinns als Maßzahl für das Einkommensrisiko als sachgerecht angesehen werden kann, oder anders ausgedrückt, ob die Schwankung der steuerlichen Einkünfte aus LuF eine geeignete Maßzahl für das real existierende Einkommensrisiko auf dem betrachteten landwirtschaftlichen Betrieb darstellt. Dazu ist zunächst festzustellen, dass sich erhöhte Risiken auf betriebswirtschaftlicher Ebene naturgemäß in höheren Schwankungen der jeweiligen betriebswirtschaftlichen Kennziffern ausdrücken. Ist die Einkommenserzielung aus einem landwirtschaftlichen Betrieb in hohem Maße mit Risiken verbunden, so wird auch das Einkommen über einen betrachteten Zeitraum hinweg stärkeren Schwankungen unterliegen. Die Anknüpfung des Einkommensrisikos an die Schwankung der LuF-Einkünfte kann also als sachgerecht angesehen werden. Jedoch bringt die Tatsache, dass es sich bei den zugrunde gelegten Einkünften um die steuerlichen Einkünfte handelt, einige $\mathrm{zu}$ beachtende Besonderheiten mit sich, die für eine Anknüpfung an das Einkommensrisiko u.U. nachteilig sein könnten. Hintergrund dieser Überlegungen ist, dass innerhalb des betriebswirtschaftlichen Rechnungswesens grundsätzlich zwischen dem steuerlichen und dem betriebswirtschaftlichen Gewinn unterschieden wird. Während bei der Berechnung des betriebswirtschaftlichen Gewinns das Abbilden der tatsächlichen wirtschaftlichen Leistungsfähigkeit des Betriebes im Vordergrund steht, soll der Gewinn im steuerlichen Abschluss zumindest im Schnitt der Jahre so gering wie möglich gehalten werden, um die Einkommensteuerlast zu drücken. Für eine Abbildung des Einkommensrisikos wäre daher der betriebswirtschaftliche Gewinn zunächst die sachgerechtere Bemessungsgrundlage. Es existieren jedoch zwei wesentliche Gründe, die gegen eine Verwendung des betriebswirtschaftlichen Gewinns als Bemessungsgrundlage der Zusatzförderung sprechen. Zum einen ist die Verbreitung der betriebswirtschaftlichen Buchführung in der Landwirtschaft bisher sehr gering. Obwohl ihr besonders im Hinblick auf eine vorausschauende Unternehmensführung eine stetig steigende Bedeutung zukommt, verfügen 
bisher nur wenige (ökologisch wirtschaftende) Betriebsleiter über ein derartig ausgereiftes Rechenwerk. Zum anderen gibt es bisher keine allgemeingültig festgelegten gesetzlichen Standards für die betriebswirtschaftliche Buchführung. Für die Bemessungsgrundlage von Prämienzahlungen ist eine abgesicherte rechtliche Basis jedoch unverzichtbar. Eine Anknüpfung der Zusatzförderung an den betriebswirtschaftlichen Gewinn scheidet damit aus. Deshalb soll im folgenden Kapitel analysiert werden, ob die Besonderheiten der steuerlichen Gewinnermittlung die Sachgerechtigkeit der steuerlichen Einkünfte als Bemessungsgrundlage der Zusatzförderung in Frage stellen. Dieses wäre der Fall, wenn es für den Betriebsleiter erhebliche Anreize einer Beeinflussung der Bemessungsgrundlage gäbe, um erhebliche Einkunftsschwankungen zu induzieren. Damit würde er möglicherweise steuerliche Nachteile in Kauf nehmen, die aber durch Fördervorteile überkompensiert werden könnten. In diesem Fall wäre die Flexible Förderung kontraproduktiv und aus volkswirtschaftlicher Sicht wenig zielführend und systemgerecht.

\subsubsection{EXEMPLARISCHE DARSTELLUNG POTENZIELLER FEHLANREIZE DURCH DIE FLEXIBLE FÖRDERUNG}

Fehlanreize bzw. Fehlallokationen wären gegeben, wenn es dem Betriebsleiter einerseits technisch und rechtlich möglich wäre und andererseits auch ökonomisch sinnvoll erschiene, die Höhe der Zusatzförderung aus Gründen, die nicht dem ökologischen Landbau nahe stehen, zu modifizieren. Da deren Bemessung auf den steuerlichen Einkünften aus LuF gründet, hätte eine Veränderung dieser Größe direkten Einfluss auf die Höhe der Zusatzförderung. In der Tat gibt es für den Betriebsleiter verschiedene Möglichkeiten, den steuerlichen Gewinn des Wirtschaftsjahres als Grundlage der Einkünfte aus Land- und Forstwirtschaft zu beeinflussen bzw. die LuF-Einkünfte gezielt schwanken zu lassen. Dazu zählen z.B.

- die Möglichkeit, durch Rückstellungen, Großreparaturen oder Teilwertabschreibungen Aufwand vorzuziehen bzw. kurzfristig erhöhten Aufwand zu verursachen, dem wirtschaftlich ein längerer Zeitraum gegenübersteht, oder auch

- die Möglichkeit, über Ansparrücklagen/Sonderabschreibungen nach §7g EStG die Abschreibungen für bewegliche Anlagegegenstände unabhängig von der realen Nutzung vorzuziehen oder zurückzuschieben.

Allerdings werden diese Instrumente in der Praxis und in der Regel dazu genutzt, steuerlichen Aufwand so früh wie möglich zu induzieren, damit durch entsprechende Steuerersparnisse 
zum einen ein Zins- und Liquiditätseffekt (durch Steueraufschub) und zum anderen evtl. ein Progressionseffekt erzielt wird. Bewusst Einkunftsschwankungen im Sinne der Flexiblen Förderung herbeizuführen, würde bedeuten, auf diese steuerlichen Zins-, Liquiditäts- und Progressionseffekte zumindest partiell zu verzichten. Darauf würde sich ein Betriebsleiter lediglich einlassen, wenn der Effekt der Flexiblen Förderung größer wäre.

Die Wirkung eines kurzfristig erhöhten Aufwands in Form einer Großreparatur soll im Kontext der Flexiblen Förderung im Folgenden simuliert und dargestellt werden. ${ }^{21} \mathrm{Im}$ Vordergrund steht dabei die Frage, ob die Existenz der Flexiblen Förderung anstelle der Festbetragsförderung zu Verhaltensänderungen seitens des Landwirts hinsichtlich des Durchführungszeitpunkts der Großreparatur führt, die eine Erhöhung des Fördermittelbudgets des Landwirts zur Folge hätten. Wäre es nämlich für den Landwirt möglich, über eine zeitliche Verschiebung der Reparaturmaßnahme seine zukünftigen Fördermitteleinnahmen deutlich zu erhöhen, so könnten sich dadurch deutliche Nachteile für eine Systemumstellung von der Festbetragsförderung auf die Flexible Förderung ergeben.

Mit dem unterstellten Beispiel soll versucht werden, genau die Situation zu konzipieren, in der die Entscheidung über den betriebswirtschaftlich optimalen Zeitpunkt einer anstehenden Großreparatur in der Praxis regelmäßig ansteht. So wurde für die Untersuchung angenommen, ein Landwirt befinde sich im Mai des laufenden Wirtschaftsjahres, d.h. zu einem Zeitpunkt, an dem der Gewinn des laufenden Wirtschaftsjahres (Ende des Wirtschaftsjahres: 30. Juni) für ihn bereits abzusehen ist, und überlege nun, ob er die ursprünglich für den Herbst geplante Großreparatur aus betriebswirtschaftlichen Gründen doch noch im laufenden Wirtschaftsjahr durchführen lassen soll. Bei der Simulation wurde daher jeweils zwischen einem Referenzszenario (RS), in dem sich der Landwirt für eine Durchführung der Großreparatur im kommenden Wirtschaftsjahr (WJ 1/2) entscheidet und einem Untersuchungsszenario (US), in dem der Landwirt die geplante Großreparatur in das laufende Wirtschaftsjahr (WJ 0/1) vorzieht, unterschieden.

Hinsichtlich der Ausgestaltung der Zusatzförderung wurde in der Untersuchung die Basiskonstellation aus Kapitel 4.2.2 unterstellt, mit dem Unterschied, dass wiederum angenommen wurde, dass die Zusatzförderung mit dem Faktor 3 multipliziert wird. Für die Simulationsrechnungen wurde wiederum auf $\operatorname{SIMETAR}^{\circledR}$ (Simulation with Excel to analyze

21 Die Durchführung einer Großreparatur steht synonym für andere Maßnahmen zur Beeinflussung der steuerlichen Bemessungsgrundlage, wie z.B. der Inanspruchnahme einer Ansparrücklage/Sonderabschreibung gemäß $§ 7 \mathrm{~g}$ EStG. 
risk, (C) TAMU Texas, USA) zurückgegriffen. Als zugrunde liegende Verteilung der Einkünfte aus LuF wurde parallel zur Vorgehensweise in Kapitel 4.2.2 die Normalverteilung angenommen. Um Ergebnisse zu verschiedenen Einkommensgruppen zu erhalten, wurden für die Untersuchung drei Beispielbetriebe mit verschiedenen Mittelwerten der Betriebsgewinne gebildet. Aus einer Analyse der Betriebsgewinne des BMELV-Testbetriebsnetzes ergaben sich mittlere Standardabweichungen, die für die gebildeten Beispielbetriebe in dieser Untersuchung angenommen wurden. Der Umfang einer Großreparatur ist von der Betriebsgröße abhängig. Für die drei Beispielbetriebe wurde daher auch ein jeweils unterschiedliches finanzielles Volumen der Großreparatur angenommen. Die nachfolgende Tabelle zeigt nochmals die getroffenen Annahmen hinsichtlich der Untersuchungsbetriebe.

Tabelle 19: Annahmen für die untersuchten Beispielbetriebe

\begin{tabular}{|l|r|r|r|}
\hline & $\begin{array}{c}\text { Mittelwert des } \\
\text { Betriebsgewinns }\end{array}$ & $\begin{array}{c}\text { Standard- } \\
\text { abweichung* }\end{array}$ & $\begin{array}{c}\text { Aufwand für die } \\
\text { Großreparatur }\end{array}$ \\
\hline Betrieb 1 (untere Einkommensgruppe) & $15.000 €$ & $13.359 €$ & $20.000 €$ \\
\hline Betrieb 2 (mittlere Einkommensgruppe) & $40.000 €$ & $23.103 €$ & $40.000 €$ \\
\hline Betrieb 3 (obere Einkommensgruppe) & $100.000 €$ & $37.022 €$ & $80.000 €$ \\
\hline
\end{tabular}

*Ergebnisse auf der Basis einer Einfachregression $(\mathrm{y}=9182+0,28 \mathrm{x})$ zwischen den Betriebsgewinnen (als unabhängige Variable) und VC der Betriebsgewinne aller Öko-Betriebe im BMELV-TBN mit mindestens sechs aufeinander folgenden Jahresdatensätzen zwischen WJ 96/97 und WJ 03/04 (vgl. Kapitel 4.2.2.2).

Quelle: Eigene Darstellung

Ziel der Simulationsrechnungen war es, zu zeigen, wie sich ein Vorziehen der Großreparatur für den Landwirt betriebswirtschaftlich auswirkt. Neben der angenommenen Gewinnverteilung (Mittelwert und Standardabweichung der Betriebsgewinne sowie Verteilung) und der angenommenen Stärke des Einmaleffektes (Finanzielles Volumen der Großreparatur) existiert mit der zum Entscheidungszeitpunkt vorherrschenden Gewinnsituation des Betriebes eine dritte Größe, die maßgeblich zur betriebswirtschaftlichen Vorteilhaftigkeit einer vorgezogenen Großreparatur beiträgt. Deshalb wurden für den Landwirt zwei unterschiedliche Ausgangssituationen angenommen.

Bei der Ausgangssituation A handelt es sich um die typische Situation, in der ein Unternehmer aus ertragssteuerlichen Gründen über ein Vorziehen einer ohnehin anstehenden Großreparatur nachdenkt. Nach konstanten Betriebsgewinnen in den Vorjahren fällt der Betriebsgewinn im Wirtschaftsjahr der Entscheidung (WJ 0/1) außerordentlich hoch aus. Folglich wäre gemäß der ESt-Veranlagung 2006 mit einer hohen Einkommensteuerlast zu rechnen. Da der Landwirt für die kommenden Jahre wieder mit deutlich niedrigeren Betriebsgewinnen rechnet, könnte durch ein Vorziehen der Großreparatur der Gewinn im 
Entscheidungsjahr gedrückt werden und infolge der Gewinnglättung die Einkommensteuerlast reduziert werden. Tabelle 20 stellt die sich für die Betriebe 1 bis 3 ergebende betriebswirtschaftliche Situation zum Zeitpunkt der Entscheidung dar. Da sich sowohl die Ertragsbesteuerung als auch die Bemessung der Zusatzförderung an den kalenderjahrbezogenen steuerlichen Einkünften aus LuF anstatt der wirtschaftsjahrbezogenen Steuergewinne ausrichtet, war dazu eine Umrechung der Gewinne in die Einkünfte aus LuF notwendig. Zur Begrenzung der Komplexität der Darstellungen wurden Zins- und Liquiditätseffekte bei den Berechnungen außer Acht gelassen.

Tabelle 20: Vorteilhaftigkeit einer vorgezogenen Großreparatur (WJ 0/1 statt WJ 1/2) bei einem außerordentlich hohen Betriebsgewinn im Entscheidungsjahr

\begin{tabular}{|c|c|c|c|c|c|c|c|c|}
\hline \multirow{2}{*}{\begin{tabular}{|c|} 
Betrieb 1 \\
WJ
\end{tabular}} & \multicolumn{2}{|c|}{ Gewinn } & \multirow[b]{2}{*}{ KJ } & \multicolumn{2}{|c|}{ LuF-Einkünfte } & \multirow{2}{*}{$\begin{array}{l}\text { Steuervorteil = } \\
\text { ESt-Last (RS) } \\
\text { abzgl. ESt-Last } \\
\text { (US) })^{\star \star \star \star \star}\end{array}$} & \multirow{2}{*}{$\begin{array}{l}\text { Fördervorteil = } \\
\text { Zusatzförderung } \\
\text { (US) abzgl. Zusatz- } \\
\text { förderung (RS) })^{\star \star \star \star \star} \\
\end{array}$} & \multirow{2}{*}{$\begin{array}{l}\text { Gesamtvorteil } \\
\text { einer vorge- } \\
\text { zogenen Groß- } \\
\text { reparatur (GR) }\end{array}$} \\
\hline & $\begin{array}{c}\text { GR in WJ } 0 / 1 \\
(=U S)\end{array}$ & $\begin{array}{l}\text { GR in WJ } \\
1 / 2(=R S)\end{array}$ & & $\begin{array}{c}\text { GR in } \\
\text { WJ } 0 / 1 \\
(=U S)\end{array}$ & $\begin{array}{c}\text { GR in } \\
\text { WJ } 1 / 2 \\
(=R S)\end{array}$ & & & \\
\hline WJ $-3 /-2$ & 15000 & 15000 & $\mathrm{KJ}-2$ & 15000 & 15000 & 0 & - & - \\
\hline WJ $-2 /-1$ & 15000 & 15000 & $\mathrm{KJ}-1$ & 15000 & 15000 & 0 & - & 0 \\
\hline WJ $-1 / 0$ & 15000 & 15000 & KJ 0 & 18359 & 28359 & 2883 & -255 & 2628 \\
\hline WJ $0 / 1$ & $41718-20000$ & 41718 & $\mathrm{KJ} 1$ & $\ldots{ }^{\star \star \star}$ & $\ldots{ }^{\star \star \star}$ & -164 & -205 & \\
\hline WJ $1 / 2$ & $z^{*}$ & $z^{* *}-20000$ & $\mathrm{KJ} 2$ & $\ldots{ }^{* \star *}$ & $\ldots * * *$ & -1463 & -1416 & -2879 \\
\hline WJ $2 / 3$ & $z^{*}$ & $z^{* * k}$ & KJ 3 &..$^{* \star \star}$ &..$^{* \star *}$ & -27 & -598 & -625 \\
\hline WJ $3 / 4$ & $z^{*}$ & $z^{* *}$ & $\mathrm{KJ} 4$ & $\ldots{ }^{* \star \star *}$ & $\ldots{ }^{* \star \star}$ & -16 & -468 & -484 \\
\hline \multicolumn{6}{|c|}{ Summe } & 1212 & -2942 & -1730 \\
\hline \multicolumn{9}{|c|}{$\begin{array}{l}\text { "Zufallszahlen (Normalverteilung, Mittelwert }=15.000 € \text {, Standardabweichung }=13.359 € \text { ) } \\
\text { "ZZufallszahlen des Referenzszenarios } \\
\text { "**Berechnung der LuF-Einkünfte aus den hälftigen Gewinnen der anteiligen Wirtschaftsjahre } \\
\text { ***Simulierte Mittelwerte (Simulationsgrundlage: } 1.000 \text { Beobachtungen mittels der Latin Hypercube Methodik) }\end{array}$} \\
\hline \multicolumn{9}{|c|}{$\mathrm{GR}=\mathrm{Großreparatur;} \mathrm{RS}=$ Referenzszenario; US = Untersuchungsszenario; $\mathrm{WJ}=$ Wirtschaftsjahr; $\mathrm{KJ}=$ Kalenderjah } \\
\hline
\end{tabular}

\begin{tabular}{|c|c|c|c|c|c|c|c|c|}
\hline \multirow{2}{*}{$\begin{array}{c}\text { Betrieb } 2 \\
\text { WJ }\end{array}$} & \multicolumn{2}{|c|}{$\overline{\text { Gewinn }}$} & \multirow[b]{2}{*}{ KJ } & \multicolumn{2}{|c|}{ LuF-Einkünfte } & \multirow{2}{*}{$\begin{array}{l}\text { Steuervorteil = } \\
\text { ESt-Last (RS) } \\
\text { abzgl. ESt-Last } \\
\text { (US) } \\
\end{array}$} & \multirow{2}{*}{$\begin{array}{l}\text { Fördervorteil = } \\
\text { Zusatzförderung } \\
\text { (US) abzgl. Zusatz- } \\
\text { förderung (RS) }{ }^{\star \star \star \star *}\end{array}$} & \multirow{2}{*}{$\begin{array}{l}\text { Gesamtvorteil } \\
\text { einer vorge- } \\
\text { zogenen Groß- } \\
\text { reparatur (GR) }\end{array}$} \\
\hline & $\begin{array}{c}\text { GR in WJ } 0 / 1 \\
(=U S)\end{array}$ & $\begin{array}{l}\text { GR in WJ } \\
1 / 2 \text { (=RS) }\end{array}$ & & $\begin{array}{c}\text { GR in } \\
\text { WJ } 0 / 1 \\
\text { (=US) }\end{array}$ & $\begin{array}{c}\text { GR in } \\
\text { WJ } 1 / 2 \\
(=R S)\end{array}$ & & & \\
\hline WJ $-3 /-2$ & 40000 & 40000 & $\mathrm{KJ}-2$ & 40000 & 40000 & 0 & - & - \\
\hline WJ $-2 /-1$ & 40000 & 40000 & $\mathrm{KJ}-1$ & 40000 & 40000 & 0 & - & 0 \\
\hline $\mathrm{WJ}-1 / 0$ & 40000 & 40000 & \begin{tabular}{|l|}
$\mathrm{KJ} 0$ \\
\end{tabular} & 43103 & 63103 & 8213 & -524 & 7689 \\
\hline WJ 0/1 & $83206-40000$ & 86206 & KJ 1 & $\ldots{ }^{* \star \star}$ & $\ldots^{* \star \star}$ & -230 & -440 & -670 \\
\hline WJ $1 / 2$ & $\mathrm{z}^{*}$ & $z^{* * *}-40000$ & KJ 2 & $\ldots{ }^{* \star \star *}$ & $\ldots^{* * *}$ & -5858 & -2345 & -8203 \\
\hline WJ $2 / 3$ & $z^{*}$ & $z^{k * k}$ & KJ 3 & $\ldots{ }^{* \star \star}$ & $\ldots{ }^{\star \star \star \star}$ & -17 & -1127 & -1145 \\
\hline WJ $3 / 4$ & $z^{*}$ & $z^{* * *}$ & \begin{tabular}{|l|}
$\mathrm{KJ} 4$ \\
\end{tabular} & $\ldots{ }^{* \star \star}$ & $\ldots{ }^{* \star \star}$ & -6 & -1031 & -1037 \\
\hline \multicolumn{6}{|c|}{ Summe } & 2103 & -5468 & -3365 \\
\hline \multicolumn{9}{|c|}{$\begin{array}{l}\text { "Zufallszahlen (Normalverteilung, Mittelwert }=40.000 € \text {, Standardabweichung }=23.103 € \text { ) } \\
\text { "ZZufallszahlen des Referenzszenarios } \\
* * * \text { Berechnung der LuF-Einkünfte aus den hälftigen Gewinnen der anteiligen Wirtschaftsjahre } \\
* * * \text { Simulierte Mittelwerte (Simulationsgrundlage: } 1.000 \text { Beobachtungen mittels der Latin Hypercube Methodik) }\end{array}$} \\
\hline
\end{tabular}




\begin{tabular}{|c|c|c|c|c|c|c|c|c|}
\hline \multirow{2}{*}{$\begin{array}{c}\text { Betrieb } 3 \\
\text { WJ }\end{array}$} & \multicolumn{2}{|c|}{$\overline{\text { Gewinn }}$} & \multirow[b]{2}{*}{ KJ } & \multicolumn{2}{|c|}{ LuF-Einkünfte } & \multirow{2}{*}{$\begin{array}{c}\text { Steuervorteil = } \\
\text { ESt-Last (RS) } \\
\text { abzgl. ESt-Last } \\
\text { (US) }\end{array}$} & \multirow{2}{*}{\begin{tabular}{|c|} 
Fördervorteil = \\
Zusatzförderung \\
(US) abzgl. Zusatz- \\
förderung (RS)
\end{tabular}} & \multirow{2}{*}{$\begin{array}{c}\text { Gesamtvorteil } \\
\text { einer vorge- } \\
\text { zogenen Groß- } \\
\text { reparatur (GR) }\end{array}$} \\
\hline & $\mid \begin{array}{c}\text { GR } \operatorname{in~WJ~} 0 / 1 \\
(=\mathrm{US})\end{array}$ & $\begin{array}{l}\text { GR in WJ } \\
1 / 2(=R S)\end{array}$ & & $\begin{array}{c}\text { GR in } \\
\text { WJ } 0 / 1 \\
(=U S)\end{array}$ & $\begin{array}{c}\text { GR in } \\
\text { WJ } 1 / 2 \\
(=R S)\end{array}$ & & & \\
\hline WJ $-3 /-2$ & 100000 & 100000 & $\mathrm{KJ}-2$ & 100000 & 100000 & \begin{tabular}{|c|}
0 \\
\end{tabular} & \begin{tabular}{|c|}
- \\
\end{tabular} & - \\
\hline$W J-2 /-1$ & 100000 & 100000 & $\mathrm{KJ}-1$ & 100000 & 100000 & 0 & - & 0 \\
\hline WJ $-1 / 0$ & 100000 & 100000 & KJ 0 & 97022 & 137022 & 16800 & 0 & 16800 \\
\hline WJ $0 / 1$ & $174044-80000$ & 174044 & KJ 1 & $\ldots{ }^{* \star \star}$ & $\ldots{ }^{\star \star \star}$ & -46 & 0 & -46 \\
\hline WJ $1 / 2$ & $z^{*}$ & $\mathrm{Z}^{* *}-80000$ & KJ 2 &..$^{\star \star \star \star}$ & $\ldots{ }^{* * *}$ & -16250 & -550 & -16800 \\
\hline WJ $2 / 3$ & $z^{*}$ & $z^{* * *}$ & KJ 3 & $\ldots{ }^{* \star \star}$ & $\ldots{ }^{\star \star \star *}$ & 0 & -529 & -529 \\
\hline WJ $3 / 4$ & $z^{*}$ & $z^{* \star}$ & $\mathrm{KJ} 4$ & $\ldots{ }^{\star \star \star \star}$ & $\ldots{ }^{\star \star \star}$ & 0 & -529 & -529 \\
\hline \multicolumn{6}{|c|}{ Summe } & 504 & -1607 & -1103 \\
\hline $\begin{array}{l}\text { "Zufallszahlen } \\
\text { **Zufallszahler } \\
\text { ***Berechnung } \\
* * * \text { Simulierte }\end{array}$ & $\begin{array}{l}\text { (Normalverteilung, } \\
\text { n des Referenzszen } \\
\text { g der LuF-Einkünfte } \\
\text { Mittelwerte (Simulat }\end{array}$ & $\begin{array}{l}\text { Mittelwert }=100 \\
\text { aarios } \\
\text { aus den hälftige } \\
\text { tionsgrundlage: }\end{array}$ & $\begin{array}{l}\text { n Gewin } \\
1.000 \mathrm{Be}\end{array}$ & $\begin{array}{l}\text { nnen der ante } \\
\text { eobachtunge }\end{array}$ & $\begin{array}{l}\text { eiligen Wirts } \\
\text { en mittels de }\end{array}$ & $\begin{array}{l}\text { schaftsjahre } \\
\text { er Latin Hypercube Me }\end{array}$ & thodik) & \\
\hline \multicolumn{9}{|c|}{ GR = Großreparatur; RS = Referenzszenario; US = Untersuchungsszenario; WJ = Wirtschaftsjahr; KJ = Kalender } \\
\hline
\end{tabular}

Quelle: Eigene Berechnungen gemäß ESt-Tarif 2006

Die Tabellen zeigen für alle drei Beispielbetriebe zum einen den Steuervorteil. Er entspricht jeweils dem Unterschied in der Einkommensteuerbelastung zwischen einer vorgezogenen Durchführung der Großreparatur im WJ 0/1 und einer Durchführung im WJ 1/2 und ist vollkommen unabhängig von der Einführung der Zusatzförderung. Ohne Einführung der Zusatzförderung würde sich die Entscheidung des Landwirts lediglich am Steuervorteil zzgl. des Zins- und Liquiditätseffekts ausrichten. Mit Einführung der Zusatzförderung erhält mit dem in den Tabellen ebenfalls dargestellten Fördervorteil ein weiterer Effekt Einzug in die Entscheidungssituation des Landwirts. Zusammen mit dem Steuervorteil ergibt er den Gesamtvorteil einer vorgezogenen Großreparatur und damit die Entscheidungsgrundlage für den Landwirt.

Zusätzlich kann in den Tabellen zwischen einem kurzfristigen und einem langfristigen Effekt unterschieden werden. Der kurzfristige Effekt ist für den Landwirt zum Zeitpunkt seiner Entscheidung bereits vollständig einsehbar. Er entspricht dem Gesamtvorteil im KJ 0. Im Falle des Betriebs 1 ist es für den Landwirt schon sicher, dass er bei einer vorgezogenen Durchführung der Großreparatur im KJ 0 einen Gesamtvorteil von $2.628 €$ verzeichnen kann. Dem gegenüber ist der langfristige Effekt in Form des kumulierten Gesamtvorteils für den Landwirt mit hoher Unsicherheit verbunden, da er auf der Erwartung des Landwirts hinsichtlich seiner zukünftigen Betriebsgewinne basiert.

Die Ergebnisse zeigen, dass der kurzfristige Gesamtvorteil in allen Fällen aufgrund des positiven Steuereffektes positiv ist. Insofern ändert sich am Entscheidungsverhalten des Landwirts mit Einführung der Zusatzförderung aus kurzfristiger Sicht also nichts. Anders zeigt sich die Situation bei der langfristigen Betrachtung. Dadurch dass der außerordentlich 
hohe Gewinn mit Durchführung der Großreparatur geglättet wird, fällt die Zusatzförderung im Vergleich zum Referenzszenario in den Folgejahren deutlich geringer aus. Die Einbußen bei der Zusatzförderung führen bei allen drei Betrieben auf Basis der zukünftigen Gewinnprognosen der Landwirte jeweils zu einer negativen Vorteilhaftigkeit einer vorgezogenen Großreparatur. Die Existenz der Flexiblen Förderung anstelle der Festbetragsförderung führt damit zu einer abnehmenden Attraktivität einer Gewinnglättung in der Ausgangssituation A, in der sie in der Praxis regelmäßig Anwendung findet. Besonders deutlich zeigt sich die abnehmende Attraktivität im Falle des Betriebs 2, da hier der negative Fördervorteil den finanziellen Gesamteffekt einer vorzeitigen Großreparatur am deutlichsten belastet. Dem gegenüber wird der Fördereffekt für Betrieb 3 bei Überlegungen hinsichtlich der Großreparatur vermutlich keine Berücksichtigung finden, da er in Relation zum Durchschnittsgewinn nur gering ist und durch die Zins- und Liquiditätseffekte der hohen kurzfristigen Vorteilhaftigkeit wahrscheinlich aufgezehrt wird. Es ergibt sich daher die folgende Wirkungsbeziehung: Infolge einer vorgezogenen Großreparatur spart der Staat bei der Zusatzförderung Fördermittel ein. Wird die Großreparatur nicht vorgezogen, so erhöhen sich die Steuereinnahmen des Staates.

Bei der Konzeption der Ausgangssituation B stand die Kernfrage dieses Kapitels im Vordergrund: Kann es für einen Landwirt unter bestimmten Umständen lohnend sein, durch ein Vorziehen einer Großreparatur bereits vorhandene Gewinnschwankungen künstlich zu verstärken, um damit die Fördermitteleinnahmen zu erhöhen? Dazu wurde konträr zur Ausgangssituation A angenommen, dass nach konstanten Betriebsgewinnen in den Vorjahren der Betriebsgewinn im Wirtschaftsjahr der Entscheidung (WJ 0/1) außerordentlich niedrig ausfällt. Da der Landwirt für die kommenden Jahre wieder mit deutlich höheren Betriebsgewinnen rechnet, könnte es für ihn lohnend sein, durch ein Vorziehen der Großreparatur die ohnehin hohen Gewinnschwankungen weiter zu erhöhen, um die Zusatzförderung zu optimieren. Analog zu Tabelle 20 stellt Tabelle 21 die sich für die Betriebe 1 bis 3 ergebende betriebswirtschaftliche Situation zum Zeitpunkt der Entscheidung dar. 
Tabelle 21: Vorteilhaftigkeit einer vorgezogenen Großreparatur (WJ 0/1 statt WJ 1/2) bei einem außerordentlich niedrigen Betriebsgewinn im Entscheidungsjahr

\begin{tabular}{|c|c|c|c|c|c|c|c|c|}
\hline \multirow{2}{*}{$\begin{array}{c}\text { Betrieb } 1 \\
\text { WJ }\end{array}$} & \multicolumn{2}{|c|}{ Gewinn } & \multirow[b]{2}{*}{ KJ } & \multicolumn{2}{|c|}{ LuF-Einkünfte } & \multirow{2}{*}{$\begin{array}{c}\text { Steuervorteil = } \\
\text { ESt-Last (RS) } \\
\text { abzgl. ESt-Last } \\
\text { (US) }{ }^{\star \star \star *}\end{array}$} & \multirow{2}{*}{\begin{tabular}{|c|} 
Fördervorteil = \\
Zusatzförderung \\
(US) abzgl. Zusatz- \\
förderung (RS)
\end{tabular}} & \multirow{2}{*}{$\begin{array}{l}\text { Gesamtvorteil } \\
\text { einer vorge- } \\
\text { zogenen Groß- } \\
\text { reparatur (GR) }\end{array}$} \\
\hline & $\mid \begin{array}{c}\text { GR in WJ } 0 / 1 \\
(=U S)\end{array}$ & $\begin{array}{l}\text { GR in WJ } \\
1 / 2 \text { (=RS) }\end{array}$ & & $\begin{array}{c}\text { GR in } \\
\text { WJ } 0 / 1 \\
(=U S)\end{array}$ & $\begin{array}{c}\text { GR in } \\
\text { WJ } 1 / 2 \\
\text { (=RS) }\end{array}$ & & & \\
\hline WJ $-3 /-2$ & 15000 & 15000 & $\mathrm{KJ}-2$ & 15000 & 15000 & 0 & $-\left.\right|^{-}$ & - \\
\hline WJ $-2 /-1$ & 15000 & 15000 & $\mathrm{KJ}-1$ & $6641^{\mathrm{a}}$ & 15000 & 1542 & - & 1542 \\
\hline WJ $-1 / 0$ & 15000 & 15000 & $\mathrm{KJ} 0$ & 0 & 1641 & 0 & 1515 & 1515 \\
\hline WJ $0 / 1$ & $-11718-20000$ & -11718 & KJ 1 &.$^{* \star *}$ & $\ldots{ }^{* \star \star}$ & 0 & 5 & 5 \\
\hline WJ $1 / 2$ & $z^{*}$ & $z^{* k}-20000$ & KJ 2 & $\ldots{ }^{\star \star \star *}$ & $\ldots{ }^{* \star \star}$ & -840 & 1467 & 627 \\
\hline WJ $2 / 3$ & $z^{*}$ & $z^{* *}$ & $\mathrm{KJ} 3$ &..$^{\star \star \star}$ & $\ldots{ }^{* \star \star}$ & -305 & 624 & 319 \\
\hline WJ $3 / 4$ & $z^{*}$ & $z^{* *}$ & KJ 4 &..$^{\star \star \star}$ & $\ldots{ }^{\star \star \star}$ & -133 & -468 & -601 \\
\hline \multicolumn{6}{|c|}{ Summe } & 264 & 3143 & 3407 \\
\hline \multicolumn{9}{|c|}{ 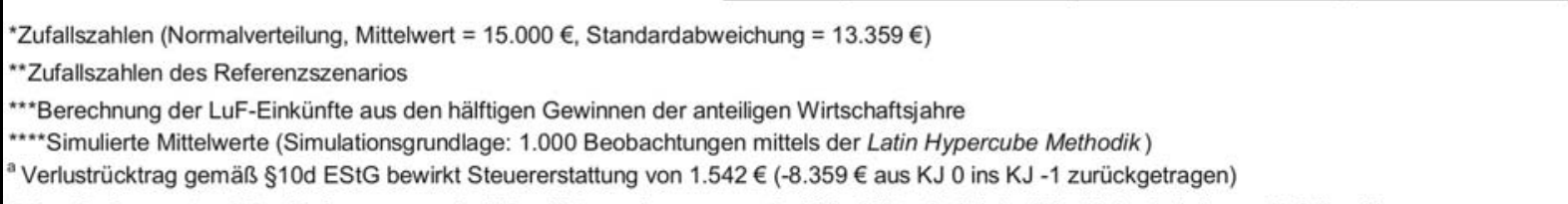 } \\
\hline \multicolumn{9}{|c|}{ GR = Großreparatur; RS = Referenzszenario; US = Untersuchungsszenario; WJ = Wirtschaftsjahr; $\mathrm{KJ}=$ Kalenderjahr; $\mathrm{z}=$ Zufallszahl } \\
\hline
\end{tabular}

\begin{tabular}{|c|c|c|c|c|c|c|c|c|}
\hline \multirow{2}{*}{$\begin{array}{c}\text { Betrieb } 2 \\
\text { WJ }\end{array}$} & \multicolumn{2}{|c|}{ Gewinn } & \multirow[b]{2}{*}{ KJ } & \multicolumn{2}{|c|}{ LuF-Einkünfte } & \multirow{2}{*}{$\begin{array}{c}\text { Steuervorteil = } \\
\text { ESt-Last (RS) } \\
\text { abzgl. ESt-Last } \\
\text { (US) })^{\star \star * *}\end{array}$} & \multirow{2}{*}{\begin{tabular}{|c|} 
Fördervorteil = \\
Zusatzförderung \\
(US) abzgl. Zusatz- \\
förderung (RS)
\end{tabular}} & \multirow{2}{*}{$\begin{array}{l}\text { Gesamtvorteil } \\
\text { einer vorge- } \\
\text { zogenen Groß- } \\
\text { reparatur (GR) }\end{array}$} \\
\hline & $\begin{array}{c}\text { GR in WJ } 0 / 1 \\
(=\mathrm{US})\end{array}$ & $\begin{array}{l}\text { GR in WJ } \\
1 / 2 \text { (=RS) }\end{array}$ & & $\begin{array}{c}\text { GR in } \\
\text { WJ } 0 / 1 \\
\text { (=US) }\end{array}$ & $\begin{array}{c}\text { GR in } \\
\text { WJ } 1 / 2 \\
\text { (=RS) }\end{array}$ & & & \\
\hline WJ $-3 /-2$ & 40000 & 40000 & $\mathrm{KJ}-2$ & 40000 & 40000 & 0 & - & - \\
\hline WJ $-2 /-1$ & 40000 & 40000 & $\mathrm{KJ}-1$ & $36897^{\mathrm{a}}$ & 40000 & 1109 & - & 1109 \\
\hline $\mathrm{WJ}-1 / 0$ & 40000 & 40000 & KJ 0 & 0 & 16897 & 1088 & 4254 & 42 \\
\hline WJ 0/1 & $-6206-40000$ & -6206 & $\mathrm{KJ} 1$ &.$* \star \star$ & $\ldots^{* \star \star}$ & 0 & 2564 & 2564 \\
\hline WJ $1 / 2$ & $z^{*}$ & $z^{* k}-40000$ & KJ 2 & $\ldots^{\star \star * \star}$ & $\ldots{ }^{\star \star \star}$ & -4498 & 3843 & -655 \\
\hline WJ $2 / 3$ & $z^{*}$ & $z^{* *}$ & $\mathrm{KJ} 3$ &..$^{* * *}$ & $\ldots{ }^{* * *}$ & -101 & 732 & 631 \\
\hline WJ $3 / 4$ & $z^{*}$ & $z^{* *}$ & $\mathrm{KJ} 4$ &.$^{\star \star \star \star}$ & $\ldots{ }^{\star \star \star \star}$ & -26 & -1031 & -1058 \\
\hline \multicolumn{6}{|c|}{ Summe } & -2428 & 10361 & 7933 \\
\hline \multicolumn{9}{|c|}{ 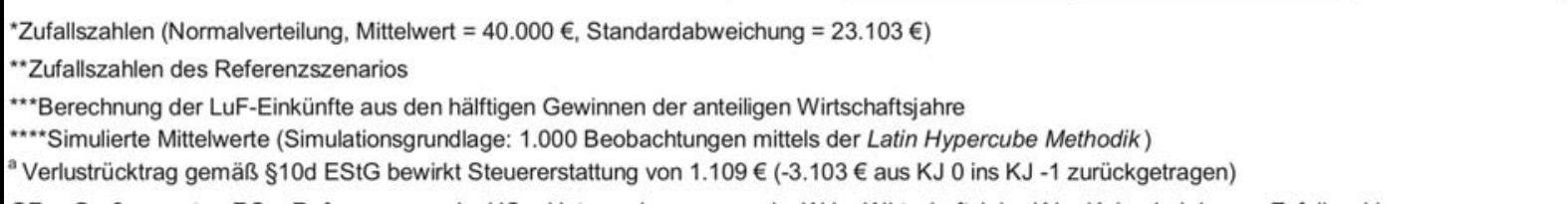 } \\
\hline
\end{tabular}

\begin{tabular}{|c|c|c|c|c|c|c|c|c|}
\hline \multirow{2}{*}{$\begin{array}{c}\text { Betrieb } 3 \\
\text { WJ }\end{array}$} & \multicolumn{2}{|c|}{ Gewinn } & \multirow[b]{2}{*}{ KJ } & \multicolumn{2}{|c|}{ LuF-Einkünfte } & \multirow{2}{*}{$\begin{array}{l}\text { Steuervorteil = } \\
\text { ESt-Last (RS) } \\
\text { abzgl. ESt-Last } \\
\text { (US) })^{\star \star \star \star *}\end{array}$} & \multirow{2}{*}{\begin{tabular}{|c|} 
Fördervorteil = \\
Zusatzförderung \\
(US) abzgl. Zusatz- \\
förderung (RS) $)^{\star \star \star \star \star}$
\end{tabular}} & \multirow{2}{*}{$\begin{array}{l}\text { Gesamtvorteil } \\
\text { einer vorge- } \\
\text { zogenen Groß- } \\
\text { reparatur (GR) }\end{array}$} \\
\hline & $\begin{array}{c}\text { GR in WJ } 0 / 1 \\
(=U S)\end{array}$ & $\begin{array}{l}\text { GR in WJ } \\
1 / 2 \text { (=RS) }\end{array}$ & & $\begin{array}{c}\text { GR in } \\
\text { WJ } 0 / 1 \\
\text { (=US) }\end{array}$ & $\begin{array}{c}\text { GR in } \\
\text { WJ } 1 / 2 \\
(=R S)\end{array}$ & & & \\
\hline WJ $-3 /-2$ & 100000 & 100000 & $\mathrm{KJ}-2$ & 100000 & 100000 & 0 & - & - \\
\hline WJ $-2 /-1$ & 100000 & 100000 & $\mathrm{KJ}-1$ & \begin{tabular}{|l|}
100000 \\
\end{tabular} & 100000 & 0 & - & 0 \\
\hline WJ $-1 / 0$ & 100000 & 100000 & $\mathrm{KJ} 0$ & 22978 & 62978 & 14670 & 1947 & 16617 \\
\hline WJ 0/1 & $25956-80000$ & 25956 & KJ 1 & $\ldots^{* \star \star}$ & $\ldots{ }^{* \star \star}$ & -3 & 1655 & 1652 \\
\hline WJ $1 / 2$ & $z^{*}$ & $z^{* * *}-80000$ & KJ 2 & $\ldots{ }^{\star \star \star \star}$ & $\ldots{ }^{* \star \star *}$ & -16238 & 1397 & -14841 \\
\hline WJ $2 / 3$ & $z^{*}$ & $z^{* *}$ & $\mathrm{KJ} 3$ & $\ldots{ }^{\star \star \star}$ & $\ldots{ }^{\star \star \star}$ & -35 & -104 & -140 \\
\hline WJ $3 / 4$ & $z^{*}$ & $z^{* *}$ & KJ 4 & $\ldots{ }^{* \star \star *}$ & $\ldots{ }^{* \star \star}$ & 0 & -529 & -529 \\
\hline \multicolumn{6}{|c|}{ Summe } & -1606 & 4366 & 2760 \\
\hline \multicolumn{9}{|c|}{$\begin{array}{l}\text { "Zufallszahlen (Normalverteilung, Mittelwert }=100.000 €, \text { Standardabweichung }=37.022 € \text { ) } \\
\text { "Zufallszahlen des Referenzszenarios } \\
* * * \text { Berechnung der LuF-Einkünfte aus den hälftigen Gewinnen der anteiligen Wirtschaftsjahre } \\
* * * \text { Simulierte Mittelwerte (Simulationsgrundlage: } 1.000 \text { Beobachtungen mittels der Latin Hypercube Methodik) }\end{array}$} \\
\hline \multicolumn{9}{|c|}{ GR = Großreparatur; RS = Referenzszenario; US = Untersuchungsszenario; WJ = Wirtschaftsjahr; KJ = Kalenderjahr; z = Zufallszahl } \\
\hline
\end{tabular}

Quelle: Eigene Berechnungen gemäß ESt-Tarif 2006 
Die Darstellungen ergeben für Betrieb 1 einen positiven Steuervorteil sowohl bei der kurzfristigen als auch bei der langfristigen Betrachtung. Folglich würde sich der Betriebsleiter im Regelfall auch ohne Vorhandensein der Zusatzförderung für ein Vorziehen der Großreparatur entscheiden. Damit ergibt sich aus der Einführung der Zusatzförderung keine Verhaltensänderung. Für diesen Fall sind Fehlanreize daher ausgeschlossen.

Etwas anders verhält es sich bei den Betrieben 2 und 3. Aus den Simulationsrechnungen resultiert für beide Betriebe zwar ebenfalls kurzfristig ein positiver Steuervorteil. Bei der langfristigen Betrachtung ergibt sich jedoch ein negativer Steuervorteil. Die Betriebsleiter würden sich daher ohne Vorhandensein der Zusatzförderung im Regelfall nur bei hohen Zinsund Liquiditätseffekten für ein Vorziehen der Großreparatur entscheiden. Anders gestaltet es sich, wenn die Zusatzförderung für die Entscheidungssituation unterstellt wird. Denn der Fördervorteil ist in beiden Fällen deutlich positiv und überragt den steuerlichen Nachteil um ein Vielfaches. Das deutet darauf hin, dass in beiden Fällen eine Verhaltensänderung des Landwirts in der Weise erfolgen könnte, dass er die Großreparatur zeitlich vorzieht, um seine Fördermitteleinnahmen zu maximieren. Damit deuten sich für die Ausgangssituation B aus dem Vorhandensein der Zusatzförderung Fehlanreize für den Landwirt an.

Allerdings werden gleichermaßen verschiedene andere Umstände dazu führen, dass diesen Überlegungen in der Praxis nur relativ wenig Gewicht zukommt.

Erstens ist $\mathrm{zu}$ bedenken, dass mit betrieblichen Anpassungsmaßnahmen vielfach Transaktionskosten verbunden sind, die unverhältnismäßig zur Zusatzförderung sein können.

Zweitens ist für das Entscheidungsverhalten des Landwirts wiederum zwischen der kurzfristigen und der langfristigen Vorteilhaftigkeit einer vorgezogenen Großreparatur zu unterscheiden. Zum Entscheidungszeitpunkt bietet lediglich der Gesamtvorteil des Kalenderjahres 0 zuzüglich einer eventuellen Steuerrückerstattung aus dem Kalenderjahr -1 (siehe bspw. Betrieb 2) dem Landwirt eine sichere Planungsgrundlage. Die weiteren Fördereffekte sind als Simulationsergebnisse von den Zukunftserwartungen des Landwirts abhängig, die naturgemäß einer Vielzahl unsicherer Einflussfaktoren unterliegen. Sollten die zukünftigen Gewinne in den beiden Betrieben entgegen der bisherigen Erwartungen des Landwirts deutlich niedriger ausfallen, so werden auch die Fördereffekte aus der Zusatzförderung aufgrund der dann geringeren Einkunftsschwankungen deutlich geringer sein. Der positive Fördereffekt einer vorgezogenen Großreparatur würde dadurch im Nachhinein abnehmen. 
Drittens handelt es sich bei der Ausgangssituation B um ein Extremszenario, in dem zum einen nach konstanten historischen Gewinnen für die Betriebe für das Jahr der Entscheidung ein deutlicher Ergebniseinbruch angenommen wurde. Zum anderen wurde in Relation zum Mittelwert der Unternehmensgewinne jeweils eine von ihrem finanziellen Volumen her immense Großinvestition angenommen. Ob allerdings ein Landwirt, der im laufenden Wirtschaftsjahr ein für seine Verhältnisse außerordentlich schlechtes Ergebnis zu verzeichnen hat, die Intention und die Finanzkraft besitzt, eine für seine Verhältnisse sehr große Reparatur sogar noch vorzuziehen, erscheint zumindest fraglich. Insofern werden die Beispiele der Tabelle 21 vermutlich nur Ausnahmecharakter besitzen.

Zuletzt wird insbesondere beim Vergleich der Ergebnisse zu den beiden Ausgangssituationen für die Wirkung der Zusatzförderung auf die Entscheidungssituation eine zentrale Tendenz erkennbar: Die vormals bestehende deutliche steuerliche Attraktivität der Gewinnglättung nimmt ab (Ausgangssituation A) und im Gegenzug nimmt die Attraktivität einer Erzeugung bzw. Verstärkung von Einkunftsschwankungen zu (Ausgangssituation B). Aus staatlicher Sicht wirkt sich das so aus: Dadurch, dass der Landwirt in der Ausgangssituation B die Großreparatur vorzieht, erhöhen sich für den Staat die Fördermittelausgaben und es verringern sich die Steuereinnahmen. Im Gegenzug erhöhen sich für den Staat aber die Steuereinnahmen, weil der Landwirt in der Ausgangssituation A die Großreparatur aufgrund der negativen langfristigen Vorzüglichkeit nicht vorzieht. Die veränderten Vorzüglichkeiten in der Ausgangssituation A bedeuten damit ebenfalls eine Relativierung der veränderten Attraktivitäten aus der Ausgangssituation B.

Eine Gefahr für Fehlallokationen von Fördermitteln ist daher insgesamt nicht festzustellen. Wichtig ist dabei auch, dass für den Landwirt im Rahmen von Steuergestaltungsmöglichkeiten lediglich eine Beeinflussung der wirtschaftsjahrbezogenen Betriebsgewinne möglich ist. Da sich die steuerlichen Einkünfte aus LuF als Bemessungsgrundlage für die Zusatzförderung aber aus den hälftigen Anteilen zweier Wirtschaftsjahrgewinne zusammensetzen, sind diese für den Landwirt über die Betriebsgewinne nur mittelbar zu beeinflussen. Die Umrechnung der Wirtschaftsjahrgewinne in die LuF-Einkünfte führt zu einer Glättung der Ergebnisentwicklung, die die Wirkung einmalig außerordentlich hoher Gewinne bspw. aufgrund von Veräußerungsgewinnen in ihrer 
Wirkung auf die Zusatzförderung deutlich abmildert. Der Einfluss der genannten Steuergestaltungsmöglichkeiten auf die Zusatzförderung nimmt dadurch erheblich ab. ${ }^{22}$

Sofern im Rahmen der Flexiblen Förderung eine stärkere Gewichtung der Zusatzförderung erfolgen sollte, ist mit einer zunehmenden Gefahr von Fehlanreizen zu rechnen. Damit sind dem Einfluss der Zusatzförderung innerhalb der Flexiblen Förderung eindeutig Grenzen gesetzt. $^{23}$

Die Ausführungen verdeutlichen, dass die in Kapitel 4.2.4 aufgeworfene Frage, ob es sich bei den steuerlichen Einkünften aus Land- und Forstwirtschaft auch um eine sachgerechte Bemessungsgrundlage für die Zusatzförderung im Rahmen der Flexiblen Förderung handelt, insgesamt positiv beantwortet werden kann.

\subsubsection{FAZIT ZU DEN AUSWIRKUNGEN DER FLEXIBLEN FöRDERUNG}

Die aufgezeigten betriebswirtschaftlichen Wirkungsweisen der Flexiblen Förderung lassen positive Auswirkungen auf die Effektivität der Förderung vermuten. So ist die monetäre Wirkung aus der Zusatzförderung im Falle durchgängig sehr niedriger Einkünfte aus LuF deutlich abgeschwächt. Das betrifft insbesondere Kleinstbetriebe, die evtl. nicht langfristig im System bleiben und z.T. lediglich Mitnahmeeffekte im Sinne eines nicht nachhaltigen Ökolandbaus nutzen. In diesen Fällen stellt sich daher grundsätzlich die Frage, ob eine erhöhte Förderung über die Zusatzförderung zweckmäßig wäre. Vielmehr sollten solche Betriebe mit einer erhöhten Förderung versehen werden, die möglichst effektiv und mit einem für die eigene Familie bzw. die eigenen Mitarbeiter ausreichenden Haupterwerbseinkommen in der Lage sind, nachhaltig ökologischen Landbau $\mathrm{zu}$ betreiben. ${ }^{24}$ Denn nur diejenigen Betriebe, die ökonomisch nachhaltig ökologischen Landbau betreiben können, sind auch imstande, nachhaltig die mit der ökologischen Wirtschaftsweise verbundenen positiven

\footnotetext{
${ }^{22}$ Allerdings wäre auch eine direkte Anknüpfung der Zusatzförderung an die wirtschaftsjahrbezogenen Betriebsgewinne im Rahmen der Möglichkeiten zur Ausgestaltung der Flexiblen Förderung prinzipiell denkbar.

${ }^{23}$ In Kapitel 4.6 wird auf den finanziell entlastenden Effekt der Systemumstellung für die öffentlichen Haushalte eingegangen.

${ }^{24}$ Damit ist allerdings auch der Effekt verbunden, dass Nebenerwerbsbetriebe und ihre Betriebsleiter, die andere Haupterwerbsquellen außerhalb des LuF-Einkommens aufweisen, eine geringere Förderung erhalten, obgleich sie sehr effizient und effektiv einen nachhaltigen ökologischen Landbau betreiben. Aufgrund Ihres nicht aus der Landwirtschaft stammenden Haupteinkommens sowie vielfach ihrer besonderen Passion zum ökologischen Landbau kann aber auch hier die ökologische Bewirtschaftungsweise nachhaltig gewährleistet sein.
} 
externen Effekte zu generieren und somit die gesellschaftlichen Leistungen zu erbringen, auf die sich die Rechtfertigung der Förderung des ökologischen Landbaus stützt (vgl. dazu Kapitel 2.1). Dabei ist allerdings zu beachten, dass auch jenen Betrieben, die von der Zusatzförderung nicht profitieren, in Form des Sockelbetrages eine flächenbezogene Grundförderung erhalten bleibt, die die Umstellungs- bzw. Beibehaltungskosten zumindest teilweise ausgleichen soll. Die Flexible Förderung ermöglicht also eine gezielte Fokussierung der Förderung auf zukunftsfähige Betriebe und damit im Sinne einer weiteren Ausdehnung des ökologischen Landbaus eine Stärkung der Effektivität und der Effizienz der Förderung.

Weiter zeigen die betriebswirtschaftlichen Wirkungen der Flexiblen Förderung, dass auch bei durchgängig sehr hohen Einkünften aus LuF ein verringerter Fördereffekt aus der Zusatzförderung zu erwarten ist. In diesem Zusammenhang wäre zu hinterfragen, ob diese Betriebe einer erhöhten Förderung über die Zusatzförderung noch bedürfen. Diese Betriebe weisen offensichtlich derart niedrige Grenzkosten bzw. derart hohe Grenzleistungen auf, dass die Zusatzförderung für sie als verzichtbar angesehen werden kann. Gleiches gilt für den Effekt einer vergleichsweise stärker reduzierten Förderung infolge der Systemumstellung bei flächenstarken Betrieben. Diese Betriebe erzielen aus der derzeitigen flächenbezogenen Festbetragsförderung bereits ein derart hohes absolutes Fördervolumen, dass in der Vergangenheit aus Politik und Praxis wiederholt die Forderung nach degressiven Fördersätzen erhoben wurde (vgl. dazu AGRA-EUROPE, 2006a: 30 oder NIEBERG, 2004: 7). Auch an dieser Stelle ist wiederum darauf hinzuweisen, dass den angesprochenen Betrieben in Form des festen Sockelbetrags eine flächenbezogene Grundförderung erhalten bleibt, die auch weiterhin Anreize zur Umstellung bieten bzw. der Gefahr einer Rückumstellung entgegenwirken und gleichzeitig die ökologischen Leistungen entlohnen soll.

Die Überlegungen zu den Auswirkungen einer Umstellung der Förderung auf das System der Flexiblen Förderung zeigen, dass sich dadurch Umverteilungseffekte ergeben würden. Diese eröffnen jedoch die Möglichkeit, in der Vergangenheit wiederholt kritisierte Mitnahmeeffekte der derzeitigen Förderausgestaltung (vgl. dazu Kapitel 2.4.2) ganz offensichtlich zu reduzieren. Der damit verbundene Gewinn an Effektivität und Effizienz wäre ganz im Sinne der zu Beginn der Arbeit formulierten Zielsetzung für eine modifizierte Förderung des ökologischen Landbaus. Durch die Kopplung der Prämienbemessung an die Einkommensrisiken im ökologischen Landbau könnte dieser Effekt sogar noch verstärkt werden, weil dadurch zusätzliche neue Umstellungsanreize für konventionell wirtschaftende Landwirte geschaffen bzw. alte Umstellungshemmnisse abgebaut werden. 


\subsection{RECHTLICHE LEGITIMATION DER FLEXIBLEN FÖRDERUNG IM RAHMEN DER ELER-VERORDNUNG}

In Kapitel 3.2.3 wurden hinsichtlich der Beurteilung der Kofinanzierbarkeit steuerlicher Förderalternativen die Kriterien Nachvollziehbarkeit des jährlichen absoluten Fördervolumens, Kostenakzessorität und Flächenakzessorität eingeführt. Diese Kriterien sollen auch bei der Beurteilung der Flexiblen Förderung als ein Förderinstrument, das an die ertragsteuerliche Bemessungsgrundlage gebunden ist, Verwendung finden. Dazu sollen analog zur Vorgehensweise in Kapitel 3.3 die Veränderungen im Vergleich zur Referenzsituation der gegenwärtig geltenden Angebotsförderung untersucht werden.

Bezüglich des ersten Kriteriums, der Nachvollziehbarkeit des jährlichen absoluten Fördervolumens, bestehen keine Bedenken für eine Kofinanzierbarkeit, da der Förderbetrag jährlich von den dafür verantwortlichen Behörden quantifiziert und direkt an den Empfänger ausbezahlt wird.

Bei der Beurteilung der Kofinanzierungsfähigkeit anhand des wichtigen Kriteriums der Kostenakzessorität der Förderung kann auf die diesbezügliche Beurteilung der Differenzierung gemäß der EMZ (Kapitel 3.3.1) verwiesen werden. Dort ist bereits darauf hingewiesen worden, dass ein stärker an den konkreten einzelbetrieblichen Einkommensverlusten und Zusatzkosten der ökologischen Bewirtschaftung orientierter Ausgleich im Sinne der Kostenakzessorität in Zukunft zunehmend wichtiger sein wird, weil im Zuge der Umstellung von der VO (EG) 1257/99 auf die ELER-VO bei den Agrarumweltprogrammen die bisherige 20 \%ige Anreizkomponente entfallen wird. Mit Blick auf die bereits aufgezeigten Wirkungen der Flexiblen Förderung würde eine so ausgestaltete Angebotsförderung den Veränderungen der EU-Rahmenverordnung daher sogar entgegenkommen. Der Grund dafür liegt in der Anknüpfung der Zusatzförderung als Bestandteil der Flexiblen Förderung an den Schwankungen der einzelbetrieblichen Einkünfte und damit am betriebsindividuellen Einkommensrisiko. Durch ein höheres betriebliches Einkommensrisiko entstehen für einen Landwirt im Vergleich zu Berufskollegen mit einem geringeren betrieblichen Einkommensrisiko zusätzliche Kosten. Er hat im Durchschnitt der Jahre eine höhere Einkommensteuerlast $\mathrm{zu}$ tragen und muss größere Anstrengungen unternehmen, um bspw. durch eine verstärkte Diversifizierung sein jährliches Einkommen gegen existenzbedrohende Schwankungen abzusichern. Da es, wie in Kapitel 4.1 gezeigt wurde, sehr wahrscheinlich ist, dass ökologisch wirtschaftende Betriebe im direkten 
Vergleich zu konventionell wirtschaftenden Betrieben durch ein tendenziell höheres Einkommensrisiko gekennzeichnet sind, sind auch die risikobedingten Zusatzkosten für die ökologisch wirtschaftenden Betriebe höher. Die Ursachen dieser Zusatzkosten liegen damit nicht allein in der persönlichen betrieblichen Situation des Einzelnen, sondern knüpfen direkt an die Form der Bewirtschaftung an. Das hat zur Folge, dass auch diese Zusatzkosten bei der Bemessung der Förderung Berücksichtigung finden müssen, denn sie hat sich gemäß der derzeitigen und der zukünftigen EU-Rahmengesetzgebung an den einzelbetrieblichen Einkommensverlusten und Zusatzkosten zu orientieren, die infolge der Bewirtschaftung nach den Regeln der EG-Öko-Verordnung entstehen. Eine Systemumstellung auf die Flexible Förderung könnte damit der zukünftig geforderten verstärkten Kostenakzessorität eher gerecht werden, so dass hinsichtlich dieses Kriteriums von ausschließlich positiven Einflüssen auf die Kofinanzierungsfähigkeit der Flexiblen Förderung auszugehen ist. Der Forderung, dass eine positive Einkommenswirksamkeit der Förderung zukünftig nicht mehr gewährleistet sein soll, könnte der Tatbestand, dass die Flexible Förderung insbesondere bei einkommensstarken Betriebsleitern die Förderung zurückführt, sogar noch in besonderer Weise Rechnung tragen. Die Flexible Förderung wird den neuen rechtlichen Anforderungen damit stärker gerecht, als das bislang bekannte System der pauschalen Festbetragsförderung.

Geringfügig anders stellt sich die Situation bezüglich einer in der EU-Rahmenverordnung eventuell geforderten Flächenakzessorität der Förderung dar. Zwar ist der Sockelbetrag, der gemäß den Ergebnissen zur Wirkung in Kapitel 4.2.2 im Regelfall die gewichtigere Komponente innerhalb der Flexiblen Förderung darstellen wird, nach wie vor ausschließlich flächenbezogen. Dies trifft jedoch nicht auf die Zusatzförderung zu. Allerdings ist, wie schon in Kapitel 3.2.3 angemerkt, in der EU-Rahmenverordnung eine Flächenbezogenheit der Förderung auch nicht explizit gefordert. Lediglich die in Artikel 39, Absatz 4 der ELER-VO benannten Höchstgrenzen für die jährliche Förderung sind als flächenbezogene Größen angegeben. Laut Verordnungstext können in Ausnahmefällen, unter Berücksichtigung besonderer Umstände, diese Höchstgrenzen aber hinfällig sein. Deshalb und weil der Sockelbetrag als im Regelfall gewichtigere Komponente innerhalb der Flexiblen Förderung weiterhin flächenbezogen ist, sollte hinsichtlich der Flexiblen Förderung davon ausgegangen werden können, dass sie dem Verordnungstext auch in diesem Punkt nicht widerspricht. Diese Sichtweise wird noch dadurch verstärkt, dass die Flexible Förderung - würde man sie als Förderung je ha auszahlen - im Regelfall die Höchstgrenzen des Förderbetrags gemäß ELERVO nicht überschreiten würde. Sollte sie aber dennoch als betriebsbezogene Förderung der 
Rahmengesetzgebung aus Sicht der EU entgegenstehen, so dürfte eine Umlage auf die beantragte Fläche keine Probleme bereiten. Der errechnete Zusatzförderbetrag pro Betrieb wäre dabei lediglich durch die beantragte Fläche zu dividieren und zum Hektarsatz der Sockelförderung zu addieren. Die von ihrer Konzeption her eigentlich betriebsbezogene Zusatzförderung könnte so in eine formal flächenbezogene Zusatzförderung umgewandelt werden.

Gemäß dem zukünftig geltenden Verordnungstext der VO (EG) 1698/2005 (ELER-VO) ist daher für die Flexible Förderung hinsichtlich der EU-Kofinanzierbarkeit von keinen Veränderungen im Vergleich zum gegenwärtigen Fördersystem auszugehen. Eine Gefährdung der EU-Kofinanzierung infolge einer Systemumstellung kann deshalb ausgeschlossen werden.

\subsection{ADMINISTRATIVE UMSETZUNG DER FLEXIBLEN FÖRDERUNG}

Wichtig bei einer Einführung der Flexiblen Förderung ist auch ihre administrative Umsetzung. Das betrifft zum einen die konkrete Systemumstellung. Zum anderen ist damit aber auch der kontinuierliche Verwaltungsablauf nach Einführung der Flexiblen Förderung gemeint, auf den im Folgenden zunächst eingegangen werden soll.

\subsubsection{VORSCHLAG FÜR DIE VERWALTUNGSTECHNISCHE HANDHABUNG DER FLEXIBLEN FÖRDERUNG}

Bevor auf die im Zuge einer Systemumstellung notwendigen Anpassungen eingegangen wird, soll zunächst die derzeitige Verwaltungspraxis erläutert werden. Da sich die Vorgehensweise in den einzelnen Bundesländern geringfügig unterscheidet, wurde für die folgenden beispielhaften Betrachtungen das Bundesland Nordrhein-Westfalen (NRW) und das Beantragungsjahr 2006 ausgewählt.

\subsubsection{STATUS QUO}

In Nordrhein-Westfalen wird der ökologische Landbau auf Grundlage der Richtlinien über die Gewährung von Zuwendungen für die Förderung einer markt- und standortangepassten Landbewirtschaftung (RdErl. d. MUNLV v. 01.09.2005) gefördert. Weil in NRW zusätzlich zur EU-Kofinanzierung auch noch die Kofinanzierung des Bundes in Anspruch genommen wird, basieren diese Richtlinien zum einen auf der EU-Rahmengesetzgebung (VO (EG) 1257/99 und DVO (EG) 817/2004) und zum anderen auf den Grundsätzen zur Förderung 
einer markt- und standortangepassten Landbewirtschaftung im Rahmen der GAK auf Bundesebene. Als Rechtsgrundlage für den verwaltungstechnischen Ablauf der Förderung fungieren die Verwaltungsvorschriften zu $\S 44$ LHO (Landeshaushaltsordnung). Danach ist der verwaltungstechnische Ablauf für die flächenbezogene Öko-Förderung in NRW in drei Stufen unterteilt:

Zunächst ist vom Landwirt der Grundantrag auf Förderung ökologischer Anbauverfahren im Rahmen der Förderung einer markt- und standortangepassten Landbewirtschaftung (MSL) bei der Bewilligungsbehörde einzureichen. In dem Grundantrag sind der Umfang der Betriebsflächen, aufgeteilt nach den Flächennutzungsarten Ackerland, Dauergrünland, Gemüseanbau, Dauerkulturen und Unterglasfläche, und die zuständige amtliche ÖkoKontrollstelle anzugeben. Zusätzlich kann mit dem Grundantrag auch der Kontrollkostenzuschuss beantragt werden. Das Flächenverzeichnis und die dazugehörigen Luftbildkarten, auf denen die Betriebsflächen vom Landwirt eindeutig zu kennzeichnen sind, müssen nicht gesondert eingereicht werden. Sie liegen der Bewilligungsbehörde bereits aus der parallelen Beantragung der Direktzahlungen vor. Bewilligungsbehörde ist in NordrheinWestfalen der Direktor der Landwirtschaftskammern als Landesbevollmächtigter. Der jährliche Verpflichtungszeitraum beginnt in Nordrhein-Westfalen am 01.07.2006 und endet am 30.06.2007. Er ist damit wirtschaftsjahrbezogen und nicht kalenderjahrbezogen wie bspw. in Niedersachsen. Letzter Termin für die Einreichung des Grundantrags ist in NRW der 30.06.2006.

Auf den vom Landwirt eingereichten Grundantrag folgt im positiven Fall im Herbst der behördliche Zuwendungsbescheid. Er bewilligt die vom Landwirt beantragte Zuwendung für die Dauer von 5 Jahren. Der Landwirt hat sich damit verpflichtet, die Bestimmungen der VO (EWG) 2092/91 (EG-Öko-Verordnung) in der Pflanzenproduktion und die Bestimmungen der VO (EG) 1804/1999 in der Tierproduktion mindestens bis zum 30.06.2011 gesamtbetrieblich einzuhalten.

Die Förderung wird jedes Jahr nach Beendigung des jährlichen Verpflichtungszeitraums ausbezahlt. Dazu muss der Landwirt jährlich zum 15.05. einen Auszahlungsantrag bei der Bewilligungsbehörde stellen. Im gegebenen Fall hat dieses folglich erstmals bis zum 15.05.2007 zu geschehen. Mit der ersten Prämienzahlung ist im Spätherbst 2007, d.h. etwa 1,5 Jahre nach Stellung des Grundantrags, zu rechnen. 


\subsubsection{NOTWENDIGE ANPASSUNGEN}

Bei einer Systemumstellung von der gegenwärtigen flächenbezogenen Festbetragsförderung auf die Flexible Förderung werden geringfügige Anpassungen des bisherigen verwaltungstechnischen Ablaufs notwendig, die in der Hauptsache den jährlichen Auszahlungsantrag betreffen. Dazu wird für die folgenden Betrachtungen davon ausgegangen, dass die Flexible Förderung in der vorgeschlagenen Basiskonstellation eingeführt wird.

Um die Zusatzförderung zu berechnen, entstehen für die Bewilligungsbehörde lediglich zwei Neuerungen:

1. Zum einen müssen die steuerlichen Einkünfte, die aus dem betreffenden landwirtschaftlichen Betrieb erzielt wurden, für einen rückwärtigen Zeitraum von z.B. 3 Jahren vorliegen. Verglichen mit dem terminlichen Ablauf der bisherigen Beantragung der Öko-Flächenprämien, stellt sich der terminliche Ablauf der Einkommensbesteuerung wie folgt dar: Ausgehend von einem landwirtschaftlichen Wirtschaftsjahr vom 01.07. bis 30.06. wird der steuerliche Gewinn im Regelfall im Laufe des darauf folgenden Wirtschaftsjahres festgestellt. Der steuerliche Gewinn des Wirtschaftsjahres 2005/2006 wird folglich innerhalb des Wirtschaftsjahres 2006/2007 festgestellt. Die Festsetzung der Einkommenssteuer erfolgt jedoch nicht wirtschaftsjahrbezogen, sondern kalenderjahrbezogen. Daher sind für die Einkommensteuererklärung 2005 die hälftigen steuerlichen Gewinne der Wirtschaftsjahre 2004/05 sowie 2005/06 maßgeblich. Damit kann der Einkommensteuerbescheid für den Veranlagungszeitraum 2005 auch erst nach Erstellung des steuerlichen Buchführungsabschlusses für das Wirtschaftsjahr 2005/06 erfolgen. Aus dem Vorkapitel ist bekannt, dass jährlich zum 15.05. der Auszahlungsantrag für die Öko-Förderung zu stellen ist. Zum 15.05.2007 wird der Einkommensteuerbescheid 2005 im Regelfall vorliegen. Daher ist der jährliche Auszahlungsantrag um die zusätzliche Eingabemöglichkeit dreier steuerlicher LuFEinkünfte zu erweitern. Im Beispielfall hätte der Landwirt folglich neben den bisherigen Angaben zur Beantragung der Zusatzförderung auch noch die steuerlichen Einkünfte aus LuF der Veranlagungszeiträume 2003 bis 2005 auf dem Auszahlungsantrag für den Verpflichtungszeitraum WJ 2006/07 anzugeben.

Sollte der Einkommensteuerbescheid 2005 zum 15.05.2007 nicht vorliegen, so kommen dafür im Wesentlichen zwei Gründe infrage. Zum einen kann es sein, dass der Betriebsleiter den steuerlichen Jahresabschluss nicht früh genug fertiggestellt hat 
bzw. nicht früh genug hat fertigstellen lassen. Die Zeitverzögerung ist damit vom antragstellenden Landwirt verschuldet, so dass in diesem Fall von der Bewilligungsbehörde zunächst nur der Sockelbetrag gewährt werden könnte. Bei einer späteren Nachreichung des Steuerbescheids könnte die Zusatzförderung nachgezahlt werden. Wenn dies nicht gewollt ist, bleiben zwei Möglichkeiten. Entweder es wird in diesen Fällen definitiv nur den Sockelbetrag ausgezahlt oder zur Ermittlung der Bemessungsgrundlage wird noch ein weiteres Jahr auf 2002 zurückgegangen.

2. Neben den Angaben des Landwirts zu den steuerlichen Einkünften aus LuF benötigt die Bewilligungsbehörde zusätzlich eine spezielle Software zur Berechnung der Zusatzförderung aus den drei Betragsangaben. Die Berechnungsformel ist analog zu der Darstellung in Kapitel 4.2 im Folgenden für die Beispielbetrachtung dargestellt:

\section{Zusatzförderung WJ 2006/07}

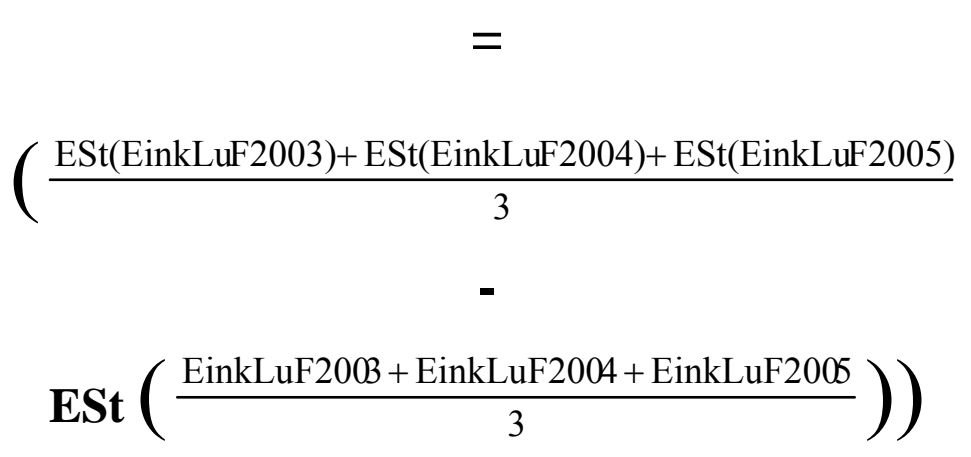

ESt: Einkommensteuer

EinkLuF2003: Einkünfte aus Land- und Forstwirtschaft im Kalenderjahr 2003

Weil die Berechnungsweise sehr einfach ist, sollte sie problemlos in der Weise in die INVEKOS-Software integrierbar sein, dass der Sachbearbeiter lediglich die Einkünfte aus Land- und Forstwirtschaft der drei Kalenderjahre in den Computer eingibt und dieser dann unter Rückgriff auf die Berechnungsformel sowie auf die Flächendaten den Förderbetrag für die komplette Flexible Förderung errechnet und dem Sachbearbeiter anzeigt.

Sollte zukünftig in den Bewilligungsbehörden sogar ein automatisches Einlesesystem für die INVEKOS-Anträge zum Einsatz kommen, so dürften die Zusatzkosten für die Administration der Flexiblen Förderung noch geringer sein. 


\subsubsection{VORSCHLAG FÜR EINE SACHGERECHTE SYSTEMUMSTELLUNG}

Vor dem Hintergrund einer 5-jährigen vertraglichen Verpflichtung zwischen Landwirt und Bewilligungsbehörde sollten bestehende Verträge von einer Systemumstellung unberührt bleiben. Nur für neu abzuschließende 5-jährige Verpflichtungen käme die Flexible Förderung in Betracht. Diese teilen sich in die Umstellungsförderung, sofern es sich bei dem Landwirt um einen Neuumsteller handelt, und in die Beibehaltungsförderung, sofern bereits vorher eine 5-jährige Verpflichtung bestand. Für den Fall einer neu abzuschließenden 5-jährigen Beibehaltungsverpflichtung gestaltet sich die Systemumstellung von der ausschließlich flächenbezogenen Festbetragsförderung auf die Flexible Förderung problemlos. Es kann mit der neuen Verpflichtung direkt auf die Flexible Förderung übergegangen werden. Bei der Ausgestaltung der Förderung für Neuumsteller sollte darauf geachtet werden, dass sie ihre ökologisch erzeugten Produkte in den ersten beiden Jahren noch nicht zu den höheren Preisen ökologischer Produkte verkaufen dürfen. Weil sie aber bereits die Bestimmungen der EGÖko-Verordnung einhalten müssen, sinken die erzielten Naturalerträge auf das üblicherweise niedrigere Niveau im ökologischen Landbau deutlich ab, woraus sich für Neuumsteller erhebliche außerordentliche Belastungen ergeben können (NIEBERG, 2001: 7). Daher könnte die Flexible Förderung für Neuumsteller z.B. mit einem höheren Multiplikator für die Zusatzförderung ausgestattet sein (oder - wie bislang - mit einem höheren Sockelbetrag für die Flächenförderung). Eine erhöhte Neuumstellerförderung könnte bei Einführung der Flexiblen Förderung beibehalten werden. In diesem Zusammenhang wäre bspw. auch in Erwägung zu ziehen, die Zusatzförderung nur für Neuumsteller zu gewähren. Die Beibehaltungsprämie ergäbe sich dann lediglich aus dem flächenbezogenen Sockelbetrag. Dadurch würden speziell die erwartungsgemäß höheren Einkunftsschwankungen während der Umstellungszeit berücksichtigt, was dem Umstellungsbetrieb helfen könnte, die betriebswirtschaftlichen Auswirkungen einer möglicherweise turbulenten Umstellungsphase abzumildern. ${ }^{25}$ Die Vielfalt an Kombinationsmöglichkeiten der Flexiblen Förderung ist in jedem Fall groß und kann sich den individuellen Anforderungsprofilen anpassen.

\footnotetext{
${ }^{25}$ Problematisch wäre in diesem Fall die Tatsache, dass lediglich Einkünfte aus konventioneller Landwirtschaft vorliegen, an die die Zusatzförderung anknüpfen würde. Ist dies nicht gewollt, kann man zunächst mit Abschlagsbeträgen fördern, die mit den tatsächlichen Förderbeträgen zu späteren Zeitpunkten verrechnet werden.
} 


\subsubsection{SCHLUSSFOLGERUNGEN ZUR VERWALTUNGSTECHNISCHEN UMSETZUNG UND DEN ENTSTEHENDEN TRANSAKTIONSKOSTEN}

Die Ausführungen zeigen, dass auch hinsichtlich der Transaktionskosten, die die Vorteile einer erhöhten Fördereffizienz schnell wieder nivellieren können, bei Einführung der Flexiblen Förderung nur mit sehr geringem Mehraufwand sowohl für die Landwirte als auch für die behördliche Verwaltung zu rechnen ist. Der Grund dafür liegt vor allem darin, dass die Einkünfte aus Land- und Forstwirtschaft direkt aus den jährlichen ESt-Bescheiden ablesbar sind und als Verwaltungsakt ohne zusätzliche Mehrarbeit einer wünschenswerten Rechtssicherheit entsprechen. Ein weiterer wichtiger Grund besteht in dem hohen EDVtechnischen Effizienzgrad des bestehenden Fördersystems, dass sich relativ problemlos um zusätzliche Dateneingaben und Rechenprozeduren erweitern lässt. Auch hinsichtlich der Systemumstellung sind, wie die Ausführungen gezeigt haben, bei Anwendung der vorgeschlagenen Verfahrensweise aufgrund der geringen Komplexität der Veränderungen nur geringe Zusatzkosten zu erwarten.

\subsection{POTENZIELLE HEMMNISSE DER IMPLEMENTIERUNG EINER FLEXIBLEN FÖRDERUNG}

Im Folgenden sollen einige etwaige Problembereiche diskutiert werden, die im Vorfeld der Gesamtüberlegungen zur Flexiblen Förderung hinsichtlich einer Implementierung als bedeutsam angenommen wurden.

\subsubsection{WERTUNG DER ZEITLICHEN DIFFERENZ ZWISCHEN ERFASSUNG DER EINKOMMENSSCHWANKUNGEN UND GEWÄHRUNG DER FÖRDERUNG}

Im Vorkapitel wurde im Zusammenhang mit der grundsätzlichen verwaltungstechnischen Umsetzung der Flexiblen Förderung auch auf deren zeitliche Umsetzung eingegangen. Danach könnten der Zusatzförderung, bei einer Beantragung im Frühjahr 2007 und bei Annahme eines fünfjährigen Bemessungszeitraums, die Einkünfte aus LuF der Kalenderjahre 2001 bis 2005 zugrunde gelegt werden. Weiter ist angeführt worden, dass der Zeitraum zwischen Beantragung und Gewährung der Förderung im Falle des Bundeslandes NRW in der Regel etwa 18 Monate beträgt. Damit vergehen zwischen der Erfassung der betriebsindividuellen Einkommensschwankungen und der Auszahlung der Förderung ungefähr drei Jahre. Es ist daher schnell ersichtlich, dass sich die Zusatzförderung nicht für 
einen direkten monetären Ausgleich höherer Einkommensrisiken eignet. Eine Liquiditätssicherung ist aber auch gar nicht das Ziel, welches mit der Einführung des Systems der Flexiblen Förderung verfolgt werden soll. Ziel der Zusatzförderung soll vielmehr sein, die zusätzlichen Kosten auszugleichen, die aus den potentiell höheren Einkommensrisiken im ökologischen Landbau resultieren ${ }^{26}$. Denn höhere betriebswirtschaftliche Risiken haben bspw. höhere Kapitalkosten zur Folge, dadurch, dass zur Sicherung der Liquidität bspw. höhere Geldbeträge auf kurzfristigen Konten vorgehalten oder kurzfristig ungünstige Finanzierungen eingegangen werden müssen. Insofern entstehen durch die zeitliche Differenz zwischen der Erfassung der einzelbetrieblichen Einkommensschwankungen und der Gewährung der Zusatzförderung keinerlei Nachteile für eine Rechtfertigung der Flexiblen Förderung. Das Erfordernis zeitnaher Einkommensdaten für die Berechnung der Zusatzförderung besteht lediglich im Sinne des Erfordernisses einer möglichst zeitnahen Erfassung der individuellen betriebswirtschaftlichen Risikosituation.

\subsubsection{GEWINNERMITTLUNG NACH DURCHSCHNITTSSÄTZEN (§ 13A-BETRIEBE)}

Neben der Überschussrechnung ( $§ 4$ Abs. 3 EStG) und der Buchführung (§ 4 Abs. 1 EStG) besteht in der Land- und Forstwirtschaft mit der Gewinnermittlung nach Durchschnittssätzen (§ 13a EStG) noch eine weitere Gewinnermittlungsmethode. Der Durchschnittssatzgewinn wird auf der Grundlage der landwirtschaftlichen Vergleichswerte ( $\$ 40$ BewG) ermittelt und ist aus betriebswirtschaftlicher Sicht eine grobe und pauschale Gewinnschätzung, die das tatsächliche Gewinnpotential nur unzureichend wiedergibt. Durch die flächenbezogene pauschale Gewinnschätzung sind die LuF-Einkünfte bei fehlender Flächenveränderung und fehlenden sonstigen LuF-Einkünften (z.B. Dienstleistungen) stabil und nicht schwankend. Somit würde auch keine Zusatzförderung anfallen.

Es ist allerdings $\mathrm{zu}$ überlegen, ob sich dieser Zusammenhang für eine mögliche Implementierung der Flexiblen Förderung, wie zunächst zu vermuten, wirklich nachteilig auswirkt. Zum einen handelt es sich bei den $\S 13 \mathrm{a}$-Betrieben zumeist um sehr kleine Betriebe mit z.T. ungewissen Zukunftsperspektiven. Insbesondere in diesen Fällen ist zu hinterfragen, ob diese Betriebe im Sinne der gesellschaftlichen Ziele der Öko-Förderung einer Zusatzförderung bedürfen. Bei diesen Betrieben ist die Wahrscheinlichkeit groß, dass sie nicht mehr über einen längeren Zeitraum hinaus Ökolandbau betreiben, weil sie nicht die

\footnotetext{
${ }^{26}$ Vgl. Kapitel 4.1
} 
kritische Größe erreichen, um langfristig als Haupterwerbsbetriebe zu bestehen. Ist dies nicht der Fall, so können die Betriebsleiter zu Gunsten der Förderung jeder Zeit ihre Gewinnermittlung auf die Überschussrechnung oder die Buchführung umstellen. Verzichtet daher ein $\S 13 \mathrm{a}$-Betrieb auf eine Umstellung seiner Gewinnermittlungsmethode, so scheinen entweder die für ihn aus der Gewinnermittlung nach § 13a ESt resultierenden Vorteile so groß zu sein, dass er auf die Zusatzförderung verzichten kann oder er scheut den zusätzlichen Verwaltungsaufwand aus der Überschussrechnung bzw. der Buchführung, weil keine Wachstumsmaßnahmen geplant sind oder ohnehin in den kommenden Jahren die Betriebsaufgabe geplant ist. Darüber hinaus ist die Gruppe der ,passionierten Nebenerwerbslandwirte“ zu nennen, die zwar effizient und effektiv ökologischen Landbau betreiben, daneben aber noch andere Haupterwerbsquellen aufweisen, aufgrund derer die Fortführung des ökologische Landbaus auch mit einer ausschließlichen Gewährung des Sockelbetrags nachhaltig gewährleistet werden kann.

Die Ausführungen offenbaren, dass der Zusammenhang zwischen der Gewinnermittlung nach $\S 13 \mathrm{a}$ EStG und der Anwendbarkeit der Flexiblen Förderung sogar Potential zur Verringerung von Mitnahmeeffekten und ,ineffektiven Förderungen“ bietet und somit kein Hemmnis darstellen muss.

\subsubsection{Personengesellschaften}

Bei den bisherigen Betrachtungen wurde immer davon ausgegangen, dass es sich bei den untersuchten ökologisch wirtschaftenden Betrieben um Betriebe in der Rechtsform einer Einzelunternehmung handelt. In der Tat ist der Anteil der Einzelunternehmungen mit fast 94,3\% aller landwirtschaftlichen Betriebe nach wie vor sehr hoch (STATISTISCHES BundeSAMT, 2004: 33). Mit 4,5\% stellen die Personengesellschaften aber die zweitgrößte Gruppe dar. An Personengesellschaften, wie bspw. einer GbR, einer OHG oder einer KG, um nur die wichtigsten zu nennen, sind mehrere Gesellschafter beteiligt. Das bedeutet, dass sich die steuerpflichtigen Einkünfte aus einer Personengesellschaft auch auf alle diese Gesellschafter verteilen. Daher müssten zur Ermittlung der gesamten steuerlichen Einkünfte eigentlich die Steuerbescheide aller Gesellschafter vorliegen und deren Einkünfte aus dem betreffenden Betrieb addiert werden. Dies könnte jedoch speziell bei großen Personengesellschaften mit vielen Gesellschaftern mitunter zu erhöhten Transaktionskosten führen. Hinzu kommt die Tatsache, dass Gesellschafter auch an zwei unterschiedlichen landwirtschaftlichen Betrieben beteiligt sein können. Weil die Aufteilung dem Steuerbescheid 
nicht zu entnehmen ist, würden auch daraus erhebliche Umsetzungsprobleme resultieren. Um dem $\mathrm{zu}$ entgehen, bietet sich für Personengesellschaften eine einfachere administrative Vorgehensweise an. Bei Personengesellschaften werden vom Finanzamt stets so genannte Gewinnfeststellungsbescheide als Grundlagenbescheide für die Steuerbescheide (=Folgebescheide) der einzelnen Gesellschafter erstellt (vgl. dazu $§ \S 179$ AO ff). Bei dem Gewinnfeststellungsbescheid handelt es sich um einen Feststellungsbescheid und nicht wie bei dem Steuerbescheid um einen Festsetzungsbescheid. Mit dem Gewinnfeststellungsbescheid werden die ertragsteuerlichen Einkünfte aus dem betreffenden Betrieb auf der Basis der beiden entsprechenden wirtschaftsjahrbezogenen Steuergewinne festgestellt. Er liefert damit direkt die Bemessungsgrundlage für die Zusatzförderung. Dementsprechend müsste den Bearbeitungshinweisen zum Auszahlungsantrag ein Vermerk hinzugefügt werden, dass im Falle einer Personengesellschaft die steuerlichen Einkünfte dem Gewinnfeststellungsbescheid $\mathrm{zu}$ entnehmen sind. Auch in zeitlicher Hinsicht sind keine Probleme zu erwarten, weil der Gewinnfeststellungsbescheid als Grundlagenbescheid i.d.R. noch vor den Steuerbescheiden vom Finanzamt ausgestellt wird. Insofern ergeben sich bei der Flexiblen Förderung sowohl beim Verwaltungsablauf als auch bei der Wirkung keine Unterschiede zwischen Personengesellschaften und Einzelunternehmen.

\subsubsection{Juristische Personen}

Juristische Personen erwirtschaften keinen Gewinn sondern einen Jahresüberschuss (JÜ), dessen jährliche Schwankung als Bemessungsgrundlage für die Zusatzförderung verwendet werden kann. Jedoch unterliegen juristische Personen nicht der Einkommensteuer (ESt) sondern der Körperschaftsteuer (KSt), was dazu führen könnte, dass Anreize entstehen, künstlich Schwankungen des Jahresüberschusses zu erzeugen, die man im Fall eines progressiv wirkenden ESt-Tarifs nicht induziert hätte.

Während der Anreiz bei Personenunternehmen darin besteht, Aufwendungen vorzuziehen, um sowohl Zins- und Liquiditätsgewinne zu maximieren als evtl. auch den Progressionseffekt zu reduzieren, sind Geschäftsführer von juristischen Personen lediglich daran interessiert, durch die Nutzung von Sonderabschreibungen etc. die Zins- und Liquiditätseffekte zu maximieren. Damit kann sich insgesamt ein stärkerer Anreiz ergeben, zur Maximierung der Flexiblen Förderung die Jahresüberschüsse mit Hilfe von Sondermaßnahmen, wie Sonderabschreibungen oder Rückstellungen etc., stärker schwanken zu lassen. Hierbei ist jedoch zu bedenken, dass mit Anpassungsmaßnahmen vielfach Transaktionskosten verbunden 
sind, die unverhältnismäßig zur Zusatzförderung sein können. Daneben kann die Induzierung künstlicher JÜ-Schwankungen bei juristischen Personen auch zu weiteren Nachteilen, bspw. hinsichtlich der Ausschüttungsmöglichkeiten, führen.

Es besteht aber noch ein weiteres Problem, das eine genauere Betrachtung juristischer Personen notwendig macht. Zwar können die Jahresüberschüsse als Bemessungsgrundlage der Zusatzförderung problemlos dem Körperschaftssteuerbescheid der juristischen Person entnommen werden. Der Jahresüberschuss selbst setzt sich aber nicht zwangsläufig ausschließlich aus Erträgen aus landwirtschaftlicher Tätigkeit zusammen. Diese Heterogenität des Jahresüberschusses könnte in der Weise zu Fehlallokationen von Fördermitteln führen, dass auch Unternehmenserträge in die Bemessungsgrundlage der Zusatzförderung Einzug halten, die nicht der ökologischen Landbewirtschaftung entstammen, sondern bspw. aus dem Vertrieb sonstiger Handelswaren, aus Lohnunternehmertätigkeit oder aus Energieerzeugung resultieren. Dies würde zum einen die Rechtfertigung der Zusatzförderung als Instrument zum Ausgleich potentiell erhöhter Einkunftsschwankungen im Ökolandbau erschweren. Zum anderen könnte dies im Widerspruch zu den maßgeblichen europäischen und nationalen Rahmenverordnungen stehen. Auch um einen sachgerechten Einsatz der Fördermittel zu gewährleisten, ist daher im Falle juristischer Personen eine Einzelfallbetrachtung unabdingbar. Dazu bietet es sich an, die Gewährung der Zusatzförderung daran zu binden, dass ein festgelegter Mindestanteil der betrieblichen Umsatzerlöse der Landwirtschaft entstammen muss. Im GAK-Rahmenplan ist für das Agrarinvestitionsförderprogramm (AFP) bereits eine derartige Fördervoraussetzung vorgeschrieben. So sind im Rahmen des AFP nur Unternehmen förderfähig, deren Umsatzerlöse zu mindestens $25 \%$ aus landwirtschaftlichen Tätigkeiten stammen. Dieser Mindestsatz müsste als Fördervoraussetzung für die Zusatzförderung deutlich erhöht werden, um sicherzustellen, dass der eindeutige Schwerpunkt der Geschäftstätigkeit auf landwirtschaftlichen Tätigkeiten liegt. Die Verwaltungspraxis zur Bestimmung dieses Umsatzanteils wäre für die Zusatzförderung vollständig zu übernehmen, da sie als praxiserprobte rechtssichere Verfahrensweise bereits vorliegt. Nach Eingang des Grundantrags zur Öko-Förderung wäre der Umsatzanteil von der Bewilligungsbehörde einmalig $\mathrm{zu}$ berechnen. Das Ergebnis sollte der Entscheidung über einen Zuwendungsbescheid zugrunde gelegt werden. Insofern wäre aus der teilweisen Sonderbehandlung juristischer Personen zwar eine Erhöhung der Transaktionskosten für Verwaltung und Landwirte zu erwarten, deren Höhe in Anbetracht des gesamten zu erwartenden Nutzens jedoch tragbar erscheint. 
Obwohl die juristischen Personen an der Gesamtzahl der ökologisch wirtschaftenden landwirtschaftlichen Betriebe in Deutschland nur $4 \%$ ausmachen, bewirtschaften sie in Ostdeutschland etwa $40 \%$ der gesamten ökologisch bewirtschafteten Fläche (vgl. dazu Anhang 3). Insoweit dürfen die zuvor dargestellten Problemfelder zumindest für die Neuen Bundesländer nicht außer Acht gelassen werden.

\subsubsection{TeILBETRIEBSUMSTELLUNG AUf ÖKOLOGISCHEN LANDBAU}

Das zuvor bei den juristischen Personen erörterte Problem stellt sich in ähnlicher Form auch bei Teilbetriebsumstellungen. Nach der EG-Öko-Verordnung sind Teilbetriebsumstellungen grundsätzlich möglich. Nach den Fördervoraussetzungen für die Öko-Flächenprämien ist allerdings für eine Beantragung der Prämien nach GAK eine Gesamtbetriebsumstellung zwingend erforderlich. Das bedeutet, dass zumindest in den Bundesländern, in denen eine Kofinanzierung des Bundes im Rahmen der GAK in Anspruch genommen wird, Teilumstellungen bei der Förderung ohnehin keine Beachtung finden. Lediglich in den Bundesländern, die sich bei der Konzeption ihrer Fördermaßnahmen nicht an den Fördergrundsätzen des GAK-Rahmenplanes orientieren, kommt prinzipiell auch für Teilumstellungen eine Förderung in Betracht.

Bei Teilumstellungen besteht hinsichtlich der Flexiblen Förderung die Problematik, dass die Einkünfte aus Land- und Forstwirtschaft immer eine gemischte Größe aus Einkünften aus der konventionellen und der ökologischen Erzeugung darstellen. Die Rechtfertigung der Zusatzförderung, dass dadurch ein Ausgleich für die potentiell höheren Einkommensrisiken im ökologischen Landbau gewährt wird, wäre daher in Frage zu stellen. Die Gewährung der Zusatzförderung im Rahmen der Flexiblen Förderung wäre somit kaum möglich. Da jedoch Teilumstellungen in Deutschland faktisch keine Bedeutung haben, resultiert daraus wahrscheinlich kein wesentlicher Nachteil für eine Implementierung der Flexiblen Förderung. Im Gegenteil: Die Existenz der Zusatzförderung könnte für einen Landwirt, der nur einen Teil seines Betriebes ökologisch bewirtschaftet, ein Anreiz für eine Gesamtbetriebsumstellung sein.

\subsubsection{RÜCKWIRKENDE ÄNDERUNG DER BEMESSUNGSGRUNDLAGE}

Eine rückwirkende Änderung der Bemessungsgrundlage ist in zweierlei Hinsicht denkbar. Erstens kommt es bei Flächenförderungen immer wieder $\mathrm{zu}$ nachträglichen Flächenaberkennungen. Insofern käme es zu einer Veränderung der Bemessungsgrundlage 
des Sockelbetrags und somit zu einer rückwirkenden Rückforderung anteiliger Fördermittel. Zweitens kann sich infolge einer steuerlichen Betriebsprüfung herausstellen, dass die historischen Betriebsgewinne und damit die steuerlichen Einkünfte aus LuF als Bemessungsgrundlage der Zusatzförderung nicht korrekt ermittelt wurden.

\subsubsection{RÜCKFORDERUNGEN BEI FEHLERHAFTEN FLÄCHENANGABEN}

Bei der Rückforderung anteiliger Fördermittel infolge der nachträglichen Aberkennung beantragter Flächen stellt sich grundsätzlich die Frage, ob sich der Rückforderungsanspruch lediglich auf den flächenbezogenen Sockelbetrag oder auch auf die Zusatzförderung und damit auf die gesamte Flexible Förderung erstrecken soll. Wie im Regelfall verfahren werden soll, entscheidet sich mit Bezug auf Kapitel 4.3 letztlich an der Frage, ob die Zusatzförderung im Rahmen der ELER-VO betriebsbezogen gewährt werden darf oder ob ein Flächenbezug unverzichtbar ist. Wäre eine betriebsbezogene Zusatzförderung zulässig, so bezöge sich der Rückforderungsanspruch lediglich auf den dann einzig flächenbezogenen Sockelbetrag. Dies kann im Zweifelsfall auch als die sachgerechtere Methode angesehen werden, da sich die Zusatzförderung nicht an den Flächenangaben bemisst, sie also unabhängig von ihnen ist und daher Flächenänderungen keine Auswirkung auf die Höhe der Zusatzförderung haben (siehe dazu Kapitel 4.2.1.2). Sofern jedoch eine betriebsbezogene Zusatzförderung der EURahmengesetzgebung entgegensteht, müsste auch die dann flächenbezogene Zusatzprämie vom Landwirt bei nachträglicher Flächenaberkennung anteilig zurückbezahlt werden. Beide Verfahrensweisen würden aus administrativer Sicht voraussichtlich keine zusätzlichen Belastungen zur Folge haben, da die gegenwärtige Verwaltungspraxis beibehalten werden könnte.

\subsubsection{WIRKUNGEN VON STEUERLICHEN BETRIEBSPRÜFUNGEN}

Im Rahmen einer Betriebsprüfung bzw. Außenprüfung überprüfen die Finanzbehörden die steuerlichen Angaben des Steuerpflichtigen. Sie kann eine oder mehrere Steuerarten und einen oder mehrere Besteuerungszeiträume umfassen oder sich auf bestimmte Sachverhalte beschränken. Wie oft ein Betrieb geprüft wird, hängt insbesondere von seiner wirtschaftlichen Größe ab. Das Bundesministerium für Finanzen (BMF) veröffentlicht diesbezüglich alle zwei Jahre die aktualisierten Merkmale für Groß-, Mittel- und Kleinbetriebe. Grundsätzlich gilt dabei, dass mit zunehmender Betriebsgröße auch häufiger eine Betriebsprüfung vorgenommen wird. So werden, statistisch gesehen, Kleinbetriebe nur alle 25 Jahre und mittlere Betriebe nur alle 12 Jahre einer Betriebsprüfung unterzogen. (BDP, 2005: 34ff). 
Als Konsequenz einer Betriebsprüfung kann sich eine rückwirkende Änderung der steuerlichen Bemessungsgrundlage ergeben. Damit würde sich auch die Bemessungsgrundlage für die Zusatzförderung nachträglich ändern.

Hinsichtlich einer Implementierung der Flexiblen Förderung könnte dieser Problematik in zweierlei Weise begegnet werden. Entweder es erfolgt eine nachträgliche Änderung der bereits gewährten Zusatzförderbeträge für vergangene Verpflichtungszeiträume. Infolgedessen wäre voraussichtlich mit einem zusätzlichen Verwaltungsaufwand für die Bewilligungsbehörden zu rechnen. Eine zweite Möglichkeit besteht in der Hinnahme nachträglicher Änderungen bei der Bemessungsgrundlage ohne nachträgliche Anpassungen bei der Zusatzförderung. Angesichts der strafrechtlichen Behandlung absichtlicher Falschangaben bei der steuerlichen Gewinnermittlung in Verbindung mit der vorbeugenden Wirkung von Betriebsprüfungen und des gleichzeitig geringen Prüfungsturnus insbesondere bei Klein- und Mittelbetrieben (vgl. dazu auch BMF, 2006) sollte auch vor dem Hintergrund der Erkenntnisse zum absoluten monetären Wirkungspotential der Zusatzförderung in der vorgestellten Basiskonstellation diese zweite Möglichkeit in Betracht gezogen werden.

\subsubsection{ANREIZ FÜR BETRIEBSTEILUNGEN}

Aufgrund ihrer spezifischen Wirkungsweise könnte auf die Einführung der Flexiblen Förderung von einkunftsstarken Betrieben mit einer Betriebsteilung reagiert werden. Dabei könnte vornehmlich versucht werden, speziell risikoreiche Betriebsteile in einem insgesamt einkunftsschwächeren Betrieb zusammenzufassen, um so das gesamte Prämienvolumen über alle Betriebe zu maximieren.

Dabei sind jedoch die Transaktionskosten einer solchen Betriebsteilung nicht zu vergessen, die mitunter erhebliches Ausmaß annehmen können. Bei der Frage nach der betriebswirtschaftlichen Vorzüglichkeit einer Betriebsteilung wäre also zu untersuchen, ob die $\mathrm{zu}$ erwartenden positiven Fördereffekte einer Betriebsteilung diese Transaktionskosten rechtfertigen. Mit Bezug auf den vergleichsweise geringen Fördereffekt aus der Zusatzförderung in der Basiskonstellation wird dies voraussichtlich nur in Ausnahmefällen der Fall sein. Es ist daher nicht zu erwarten, dass es allein infolge der Einführung der Flexiblen Förderung zu Betriebsteilungen kommen wird. 


\section{6 ÜBERLEGUNGEN ZU DEN AUSWIRKUNGEN UND ZUR}

\section{UMSETZUNG IN FISKALISCHER HINSICHT}

\subsubsection{FinANZIELLE AUSWIRKUNGEN EINER SySTEMUMSTELLUNG AUS SiCHT DES STAATES}

Für quantitative Betrachtungen liefern die vorliegenden Daten des BMELVTestbetriebsnetzes nur eine begrenzte Grundlage. Im Zusammenhang mit den Risikountersuchungen in Kapitel 4.1 ist bereits darauf verwiesen worden, dass die derzeit im Testbetriebsnetz vorhandenen Öko-Betriebe die Gesamtheit der ökologischen Betriebe in Deutschland nicht genügend repräsentieren. Das erschwert auch eindeutige Voraussagen hinsichtlich der fiskalischen Auswirkungen einer Systemumstellung. Die deutliche Senkung der Fördermittelauszahlungen in den Beispielbetrachtungen in Kapitel 4.2.3.3.1 deutet jedoch für die Flexible Förderung in der Ausgestaltung der Basiskonstellation auf deutliche Ersparnisse für die öffentlichen Haushalte hin. $\mathrm{Zu}$ diesem Ergebnis kommen auch die folgenden, speziell zur fiskalischen Wirkung einer Systemumstellung durchgeführten Simulationen.

So wurde in einer ersten Analyse versucht, speziell das voraussichtliche Finanzvolumen der Zusatzförderung abzuschätzen. Dazu wurden zunächst für die drei Wirtschaftsjahre 2001/02 bis 2003/04 alle Öko-Betriebe im BMELV-TBN auf ihren absoluten Gewinn hin untersucht und, wie Tabelle 22 und Tabelle 23 veranschaulichen, hinsichtlich dieser Größe in verschiedene Betriebsgruppen eingeteilt.

Tabelle 22: Übersicht über die Gewinnverteilung unter den Öko-Betrieben im BMELV-TBN

\begin{tabular}{|c|c|c|c|c|}
\hline \multirow{2}{*}{ Gewinn in $€$ im WJ } & \multicolumn{3}{|c|}{ Anteile der Öko-Betriebsgruppen im TBN in \% } & \multirow{3}{*}{$\begin{array}{c}\text { mittlere Anteile der } \\
\text { Gruppen in } \%\end{array}$} \\
\hline & WJ 03/04 & WJ $02 / 03$ & WJ 01/02 & \\
\hline Gruppe 1: & $16 \%$ & $19 \%$ & $15 \%$ & \\
\hline Gruppe 2: $\quad>3.000$ und $<7.500$ & $8 \%$ & $8 \%$ & $7 \%$ & $8 \%$ \\
\hline Gruppe 3: $>7.500$ und $<15.000$ & $14 \%$ & $12 \%$ & $13 \%$ & $13 \%$ \\
\hline Gruppe 4: $>15.000$ und $<25.000$ & $17 \%$ & $16 \%$ & $21 \%$ & $18 \%$ \\
\hline Gruppe 5: $>25.000$ und $<40.000$ & $19 \%$ & $19 \%$ & $19 \%$ & $19 \%$ \\
\hline Gruppe 6: $>40.000$ und $<67.500$ & $14 \%$ & $15 \%$ & $13 \%$ & $14 \%$ \\
\hline Gruppe 7: $>67.500$ und $<87.500$ & $5 \%$ & $5 \%$ & $5 \%$ & $5 \%$ \\
\hline Gruppe 8: $>87.500$ und $<150.000$ & $6 \%$ & $5 \%$ & $6 \%$ & $6 \%$ \\
\hline Gruppe 9: & $1 \%$ & $1 \%$ & $1 \%$ & $1 \%$ \\
\hline Mittelwerte: & $100 \%$ & $100 \%$ & $100 \%$ & $100 \%$ \\
\hline
\end{tabular}

Quelle: Eigene Darstellung auf der Basis der Daten des BMELV-TBN der WJ 01/02 bis 03/04 
Tabelle 23: Übersicht über die mittleren Betriebsgewinne der gebildeten Betriebsgruppen

\begin{tabular}{|c|c|c|c|c|}
\hline \multirow{2}{*}{ Gewinn in $€$ im WJ } & \multicolumn{3}{|c|}{ Mittlerer Gewinn der Öko-Betriebe im TBN in $€$} & \multirow{2}{*}{$\begin{array}{c}\text { Mittelwerte der } \\
\text { Gruppen in } € \\
\end{array}$} \\
\hline & WJ 03/04 & WJ 02/03 & WJ 01/02 & \\
\hline Gruppe 1: & -10.777 & -8.917 & -13.158 & -10.951 \\
\hline Gruppe 2: $\quad>3.000$ und $<7.500$ & 5.271 & 5.270 & 4.942 & 5.161 \\
\hline Gruppe 3: $>7.500$ und $<15.000$ & 11.486 & 11.397 & 11.233 & 11.372 \\
\hline Gruppe 4: $>15.000$ und $<25.000$ & 20.553 & 20.195 & 19.997 & 20.248 \\
\hline Gruppe 5: $>25.000$ und $<40.000$ & 31.769 & 31.566 & 31.632 & 31.655 \\
\hline Gruppe 6: $>40.000$ und $<67.500$ & 50.357 & 50.757 & 51.177 & 50.764 \\
\hline Gruppe 7: $>67.500$ und $<87.500$ & 76.710 & 80.114 & 75.325 & 77.383 \\
\hline Gruppe 8: $>87.500$ und $<150.000$ & 114.364 & 112.742 & 111.313 & 112.806 \\
\hline Gruppe 9: & 262.554 & 256.214 & 189.871 & 236.213 \\
\hline Mittelwerte: & 31.451 & 29.519 & 29.972 & 30.314 \\
\hline
\end{tabular}

Quelle: Eigene Darstellung auf der Basis der Daten des BMELV-TBN der WJ 01/02 bis 03/04

Auf der Basis der Daten dieser Tabellen und der Erkenntnisse aus Kapitel 4.2.2.2 bezüglich des Zusammenhangs zwischen der absoluten Höhe und der Schwankung der Betriebsgewinne von Öko-Betrieben im BMELV-TBN entstand schließlich auf Basis der Daten des BMELVTBN die folgende Schätzung für das für die Zusatzförderung notwendige Finanzvolumen (Tabelle 24). Dabei wurde gemäß BMELV-Agrarbericht (2006: 26) von einer Gesamtzahl ökologisch wirtschaftender Betriebe in Deutschland zum 31.12.2004 von 16.591 Betrieben ausgegangen. Für die Simulationsrechnungen wurde wiederum auf $\operatorname{SIMETAR}^{\circledR}$ (Simulation with Excel to analyze risk, (C) TAMU Texas, USA) zurückgegriffen (vgl. Kapitel 4.2.2). Für die Ausgestaltung der Zusatzförderung wurde die Basiskonstellation unterstellt, mit dem Unterschied, dass erneut angenommen wurde, dass die Zusatzförderung vor Eingang in die Flexible Förderung mit dem Faktor 3 multipliziert wird.

Tabelle 24: Simulation der Gesamtausgaben für die Zusatzförderung

\begin{tabular}{|c|c|c|c|c|c|c|c|c|c|}
\hline & $\begin{array}{c}\text { Betriebs- } \\
\text { gruppe 1 }\end{array}$ & $\begin{array}{c}\text { Betriebs- } \\
\text { gruppe 2 }\end{array}$ & $\begin{array}{c}\text { Betriebs- } \\
\text { gruppe 3 }\end{array}$ & $\begin{array}{c}\text { Betriebs- } \\
\text { gruppe 4 }\end{array}$ & $\begin{array}{c}\text { Betriebs- } \\
\text { gruppe 5 }\end{array}$ & $\begin{array}{c}\text { Betriebs- } \\
\text { gruppe 6 }\end{array}$ & $\begin{array}{c}\text { Betriebs- } \\
\text { gruppe 7 }\end{array}$ & $\begin{array}{c}\text { Betriebs- } \\
\text { gruppe 8 }\end{array}$ & $\begin{array}{c}\text { Betriebs- } \\
\text { gruppe 9 }\end{array}$ \\
\hline $\begin{array}{c}\text { Mittelwert der } \\
\text { Betriebsgewinne in } €\end{array}$ & -10.951 & 5.161 & 11.372 & 20.248 & 31.655 & 50.764 & 77.383 & 112.806 & 236.213 \\
\hline $\begin{array}{c}\text { Mittlere Variations- } \\
\text { koeffizienten im TBN }\end{array}$ & $-0,56$ & 2,06 & 1,09 & 0,73 & 0,57 & 0,46 & 0,40 & 0,36 & 0,32 \\
\hline $\begin{array}{c}\text { Simulierte jährliche } \\
\text { Zusatzförderung pro Öko- } \\
\text { Betrieb in € }\end{array}$ & 0 & 1.257 & 1.731 & 1.806 & 1.824 & 1.500 & 753 & 285 & 63 \\
\hline $\begin{array}{c}\text { \%ualer Anteil der } \\
\text { Betriebsgruppen an der } \\
\text { Gesamtheit der Öko-Betriebe } \\
\text { im TBN }\end{array}$ & $17 \%$ & $8 \%$ & $13 \%$ & $18 \%$ & $19 \%$ & $14 \%$ & $5 \%$ & $6 \%$ & $1 \%$ \\
\hline \hline $\begin{array}{c}\text { Ausgaben für } \\
\text { die Zusatz- } \begin{array}{c}\text { für einzelne } \\
\text { Betriebs- } \\
\text { gruppen }\end{array}\end{array}$ & 0 & 1.620 .263 & 3.720 .152 & 5.311 .593 & 5.657 .625 & 3.519 .251 & 612.792 & 265.776 & 15.197 \\
\hline förderung in $€$ insgesamt & & & & & & & & & \\
\hline
\end{tabular}

Quelle: Eigene Darstellung auf der Basis der Daten des BMELV-TBN sowie des ESt-Tarif 2006

Wie Tabelle 24 zeigt, wäre im Zuge der Einführung der Zusatzförderung als Teil der Flexiblen Förderung in Deutschland mit Mehrkosten in Höhe von voraussichtlich etwa 20 Mio. Euro zu rechnen, sofern der bisherige pauschale Flächenbetrag unverändert bleiben 
würde (komparativ-statische Analyse). Im Vergleich zu den gegenwärtigen Ausgaben für die flächenbezogene Öko-Förderung von etwa 110 Mio. Euro (vgl. Kapitel 2.3.4) ist für eine Gesamtbeurteilung von Interesse, welche finanziellen Gesamtauswirkungen sich durch eine Systemumstellung von der ausschließlich flächenbezogenen Festbetragsförderung auf die Flexible Förderung ergeben.

Dazu wurden in einer zweiten Betrachtung die diesbezüglichen Auswirkungen für die 77 Öko-TBN-Betriebe mit einem vollständigen Datensatz zu den Wirtschaftsjahren 96/97 bis 03/04 untersucht. Diese Betriebe weisen insgesamt einen durchschnittlichen wirtschaftsjahrbezogenen Gewinn von etwa $20.000 €$ auf. Die Umstellung des Fördersystems von der Festbetragsprämie mit dem angenommenen Fördersatz von $160 € /$ ha auf die Flexible Förderung in der Basiskonstellation inkl. eines Faktors für die Zusatzförderung von 3 ergibt eine Senkung des staatlichen Fördervolumens um mehr als 30\%. Damit wird der Kürzungseffekt aus der Basiskonstellation heraus erkennbar. Würde man jedoch eine dynamische Analyse durchführen, wäre gemäß den Angaben in Abbildung 19, Kapitel 4.2.2.2 damit zu rechnen, dass die Zusatzförderung aufgrund der abgesenkten Pauschalförderung zunehmen müsste (gemäß der dann höheren Variationskoeffizienten). Dem steht jedoch entgegen, dass die durchschnittlich bewirtschaftete Acker- und Grünlandfläche bei den betrachteten Betrieben mit 45 ha unter dem Durchschnitt aller ökologisch wirtschaftenden Betriebe in Deutschland lag. Da infolge der Systemumstellung durch den abgesenkten flächenbezogenen Betrag flächenstarke Betriebe umso deutlicher an Fördervolumen verlieren und diese in der Stichprobe unterrepräsentiert sind, wären bei einer Implementierung aus dieser Sicht größere Einsparungen wahrscheinlich.

Hinzu kommt, dass mit Bezug auf die Erkenntnisse aus Kapitel 4.2.5 zu dem mitunter abgeschwächten Anreiz zur Inanspruchnahme steuerlicher Gestaltungsmöglichkeiten infolge der Einführung der Flexiblen Förderung auch Veränderungen bei den Steuereinnahmen des Staates denkbar wären.

Hinsichtlich der fiskalischen Auswirkungen einer Umstellung der Förderung von der ausschließlich flächenbezogenen Festbetragsförderung auf die Flexible Förderung darf jedoch nicht vergessen werden, dass neben der Fördereffizienz auch die Verwaltungseffizienz entscheidenden Einfluss auf die Gesamteffizienz des Finanzmitteleinsatzes hat. Auf den voraussichtlichen administrativen Aufwand für Verwaltung und Landwirte ist im Rahmen der Ausführungen im Vorkapitel detailliert eingegangen worden. Dort konnte ein positives Fazit hinsichtlich der zu erwartenden Transaktionskosten gezogen worden. 
Insgesamt lassen Fördereffizienz und Verwaltungseffizienz deshalb im Hinblick auf eine Systemumstellung auf die Flexible Förderung positive Auswirkungen auf die Gesamteffizienz des Finanzmitteleinsatzes erwarten.

\subsubsection{PROBLEMATIK SCHWANKENDER FÖRDERBETRÄGE}

Bisher war das finanzielle Gesamtvolumen aus jeder einzelnen 5-jährigen Verpflichtung zum Zeitpunkt der Ausstellung des Zuwendungsbescheids für die Bewilligungsbehörde eindeutig bestimmbar. Damit war für die Bundesländer das zur Förderung jährlich notwendige Finanzvolumen gut prognostizierbar und in die mittelfristige Haushaltsfinanzplanung gut integrierbar, wenn auch bei Festlegung der Finanzplanungen die letztlich beantragten Gelder nie vollständig antizipierbar waren. Aus der Flexiblen Förderung mit ihren jährlich variablen einzelbetrieblichen Förderbeträgen resultieren für die öffentlichen Haushalte hingegen jährlich schwankende Fördervolumina. Daraus ergibt sich ein zusätzlicher Unsicherheitsfaktor, selbst wenn die förderfähige Fläche vorab bekannt sein sollte. Inwieweit daraus für die öffentlichen Haushalte Budgetprobleme entstehen, dürfte in erster Linie daran festzumachen sein, welches Ausmaß die Schwankungen annehmen.

Auf Basis der Simulationsrechnungen zum voraussichtlichen Finanzvolumen der Zusatzförderung im Vorkapitel wurden deshalb in der Weise Variationsrechnungen angestellt, dass die den Berechungen bisher zugrunde liegenden Variationskoeffizienten der mittleren Betriebsgewinne in den neun angenommenen Betriebsgruppen verändert wurden. So wurden für eine erste Betrachtung die Variationskoeffizienten jeweils um $10 \%$ reduziert (vgl. Tabelle 25) und für eine zweite Betrachtung um $10 \%$ erhöht (vgl. Tabelle 26). 
Tabelle 25: Ausgaben für die Zusatzförderung bei Annahme um $10 \%$ reduzierter Gewinnschwankungen im Vergleich zur Gewinnentwicklung im BMELV-TBN

\begin{tabular}{|c|c|c|c|c|c|c|c|c|c|c|}
\hline & & \begin{tabular}{|l} 
Betriebs- \\
gruppe 1
\end{tabular} & \begin{tabular}{|l} 
Betriebs- \\
gruppe 2
\end{tabular} & \begin{tabular}{|l} 
Betriebs- \\
gruppe 3
\end{tabular} & \begin{tabular}{|l|} 
Betriebs- \\
gruppe 4
\end{tabular} & \begin{tabular}{|l} 
Betriebs- \\
gruppe 5
\end{tabular} & \begin{tabular}{|l} 
Betriebs- \\
gruppe 6
\end{tabular} & \begin{tabular}{|l} 
Betriebs- \\
gruppe 7
\end{tabular} & \begin{tabular}{|l} 
Betriebs- \\
gruppe 8
\end{tabular} & $\begin{array}{l}\text { Betriebs } \\
\text { gruppe } 9\end{array}$ \\
\hline \multicolumn{2}{|c|}{$\begin{array}{c}\text { Mittelwert der } \\
\text { Betriebsgewinne in } €\end{array}$} & -10.951 & 5.161 & 11.372 & 20.248 & 31.655 & 50.764 & 77.383 & 112.806 & 236.213 \\
\hline \multicolumn{2}{|c|}{$\begin{array}{c}\text { Mittlere Variations- } \\
\text { koeffizienten im TBN }\end{array}$} & $-0,50$ & 1,85 & 0,98 & 0,66 & 0,51 & 0,41 & 0,36 & 0,32 & 0,29 \\
\hline \multicolumn{2}{|c|}{$\begin{array}{c}\text { Simulierte jährliche } \\
\text { Zusatzförderung pro Öko- } \\
\text { Betrieb in } €\end{array}$} & 0 & 1.103 & 1.510 & 1.492 & 1.449 & 1.129 & 482 & 137 & 26 \\
\hline \multicolumn{2}{|c|}{$\begin{array}{c}\text { \%ualer Anteil der } \\
\text { Betriebsgruppen an der } \\
\text { Gesamtheit der Öko-Betriebe } \\
\text { im TBN } \\
\end{array}$} & $17 \%$ & $8 \%$ & $13 \%$ & $18 \%$ & $19 \%$ & $14 \%$ & $5 \%$ & $6 \%$ & $1 \%$ \\
\hline \multirow{2}{*}{$\begin{array}{l}\text { Ausgaben für } \\
\text { die Zusatz- } \\
\text { förderung in } €\end{array}$} & $\begin{array}{l}\text { für einzelne } \\
\text { Betriebs- } \\
\text { gruppen }\end{array}$ & 0 & 1.422 .005 & 3.244 .705 & 4.387 .240 & 4.494.071 & 2.648.314 & 392.473 & 127.989 & 6.171 \\
\hline & insgesamt & \multicolumn{9}{|c|}{16.722 .970} \\
\hline
\end{tabular}

Quelle: Eigene Darstellung auf der Basis der Daten des BMELV-TBN

\section{Tabelle 26: Ausgaben für die Zusatzförderung bei Annahme um $10 \%$ erhöhter} Gewinnschwankungen im Vergleich zur Gewinnentwicklung im BMELV-TBN

\begin{tabular}{|c|c|c|c|c|c|c|c|c|c|c|}
\hline & & \begin{tabular}{|l} 
Betriebs- \\
gruppe 1
\end{tabular} & \begin{tabular}{|l|} 
Betriebs- \\
gruppe 2
\end{tabular} & \begin{tabular}{|l} 
Betriebs- \\
gruppe 3
\end{tabular} & \begin{tabular}{|l|} 
Betriebs- \\
gruppe 4
\end{tabular} & \begin{tabular}{|l} 
Betriebs- \\
gruppe 5
\end{tabular} & \begin{tabular}{|l} 
Betriebs- \\
gruppe 6
\end{tabular} & \begin{tabular}{|l|} 
Betriebs- \\
gruppe 7
\end{tabular} & \begin{tabular}{|l|} 
Betriebs- \\
gruppe 8
\end{tabular} & $\begin{array}{l}\text { Betriebs } \\
\text { gruppe } 9\end{array}$ \\
\hline \multicolumn{2}{|c|}{$\begin{array}{c}\text { Mittelwert der } \\
\text { Betriebsgewinne in } €\end{array}$} & -10.951 & 5.161 & 11.372 & 20.248 & 31.655 & 50.764 & 77.383 & 112.806 & 236.213 \\
\hline \multicolumn{2}{|c|}{$\begin{array}{c}\text { Mittlere Variations- } \\
\text { koeffizienten im TBN }\end{array}$} & $-0,62$ & 2,26 & 1,19 & 0,81 & 0,63 & 0,51 & 0,44 & 0,40 & 0,35 \\
\hline \multicolumn{2}{|c|}{$\begin{array}{c}\text { Simulierte jährliche } \\
\text { Zusatzförderung pro Öko- } \\
\text { Betrieb in } €\end{array}$} & 3 & 1.422 & 1.928 & 2.135 & 2.203 & 1.922 & 1.094 & 562 & 195 \\
\hline \multicolumn{2}{|c|}{$\begin{array}{c}\text { \%ualer Anteil der } \\
\text { Betriebsgruppen an der } \\
\text { Gesamtheit der Öko-Betriebe } \\
\text { im TBN } \\
\end{array}$} & $17 \%$ & $8 \%$ & $13 \%$ & $18 \%$ & $19 \%$ & $14 \%$ & $5 \%$ & $6 \%$ & $1 \%$ \\
\hline \multirow{2}{*}{$\begin{array}{l}\text { Ausgaben für } \\
\text { die Zusatz- } \\
\text { förderung in } €\end{array}$} & $\begin{array}{l}\text { für einzelne } \\
\text { Betriebs- } \\
\text { gruppen }\end{array}$ & 9.081 & 1.833 .169 & 4.143 .136 & 6.278 .488 & 6.833 .698 & 4.508 .628 & 890.023 & 523.716 & 46.959 \\
\hline & insgesamt & \multicolumn{9}{|c|}{25.066 .898} \\
\hline
\end{tabular}

Quelle: Eigene Darstellung auf der Basis der Daten des BMELV-TBN

Die Ergebnisse zeigen, dass sich die Gesamtausgaben für die Zusatzförderung im Falle reduzierter Gewinnschwankungen von ursprünglich etwa 20,7 Mio. Euro ${ }^{27}$ auf etwa 16,7 Mio. Euro reduzieren. Im Gegenzug erhöhen sich die Ausgaben bei erhöhten Gewinnschwankungen auf etwa 25 Mio. Euro. Damit ergibt sich auf gesamtdeutscher Ebene ein Schwankungsbereich von etwa 8 Mio. Euro für die jährliche Vorausplanung der Zusatzförderung. ${ }^{28}$ Dabei ist jedoch zu bedenken, dass die angegebenen Finanzvolumina die

\footnotetext{
${ }^{27}$ Vgl. Tabelle 24 mit den Simulationsergebnissen zum voraussichtlichen Finanzvolumen der Zusatzförderung auf der Basis der historischen Gewinnschwankungen der Öko-Betriebe im BMELV-TBN

${ }^{28}$ Bei einer Extrembetrachtung mit um 50\% reduzierten bzw. um 50\% erhöhten Variationskoeffizienten ergeben sich auf Bundesebene jährliche Budgetschwankungen von 40 Mio. $€$. Dabei wird aber unterstellt, dass entweder alle ökologischen Betriebe im angenommenen 3-Jahres-Zeitraum außerordentlich gering
} 
Budgetsituation auf Bundesebene anzeigen. Die Finanzplanung zur Angebotsförderung des ökologischen Landbaus erfolgt aber auf Landesebene. Dort werden die Schwankungen in ihrem absoluten Ausmaß folglich deutlich geringer sein.

schwankende oder außerordentlich stark schwankende Gewinne aufweisen. Diese Situation wird sich jedoch in der Praxis selbst bei Einmaleffekten immensen Ausmaßes, wie bspw. großflächigen Dürrekatastrophen oder Überschwemmungen, kaum ergeben, da es immer auch Betriebe geben wird, die von diesen Einmaleffekten nicht oder nur in geringem Ausmaß betroffen sind und sich deshalb in ihrer Gewinnentwicklung von der Masse der betroffenen Betriebe unterscheiden. Daher werden die „wahren“ Ausgabenschwankungen bei der Zusatzförderung dieses Ausmaß aller Voraussicht nach nie erreichen. 


\section{AKZEPTANZ FÜR DIE FLEXIBLE FÖRDERUNG IN DER LANDWIRTSCHAFT}

Nachdem im vorherigen Kapitel zunächst auf die positiven Förderwirkungen der Flexiblen Förderung verwiesen wurde, hat sich im weiteren Verlauf gezeigt, dass auch hinsichtlich der rechtlichen Legitimierung, der administrativen Umsetzung sowie der Behandlung eventueller spezifischer Problemkomplexe keine schwerwiegenden Probleme erkennbar sind. Gleiches gilt auch für die Einführung der Flexiblen Förderung aus der Perspektive der Transaktionskosten. Insbesondere hier haben sich Möglichkeiten zur Senkung der Gesamtkosten und gleichzeitig zur stärkeren Fokussierung der Fördermittel gezeigt. Bei den Überlegungen zur Konzeption der Flexiblen Förderung seitens der fördernden Institution dürfen auch die Ansprüche und Belange der Landwirte nicht außer Acht gelassen werden. Sie entscheiden letztlich mit ihren Anträgen darüber, inwieweit eine veränderte Systematik akzeptiert wird. In diesem Kontext ist die Verständlichkeit der Flexiblen Förderung für die Betroffenen von Bedeutung. Komplexe und schwer verständliche Förderinstrumente werden von der Landwirtschaft nicht in Anspruch genommen und von Verwaltung und Politik nicht unterstützt. Aus diesem Grund ist im Rahmen dieser Arbeit eine Untersuchung durchgeführt worden, mit der die Akzeptanz und damit auch das Verständnis der Flexiblen Förderung unter ökologisch wirtschaftenden Landwirten untersucht werden sollte. Im folgenden Kapitel soll zunächst auf die genaue Zielrichtung der Untersuchung eingegangen werden.

\subsection{ZIELSETZUNG DER UNTERSUCHUNG}

Die Betrachtungen im Vorkapitel haben gezeigt, dass sich das System der Flexiblen Förderung vom bestehenden System der Festbetragsprämie in zwei wesentlichen Punkten unterscheidet. Zum einen ist die Tatsache zu nennen, dass die Förderbeträge in den einzelnen Jahren variabel und zum Zeitpunkt der Entscheidung für die Flexible Förderung dem Landwirt nicht bekannt sind. Zum zweiten ist es die Tatsache, dass sich diese variablen Förderbeträge an den einzelbetrieblichen Schwankungen der Einkünfte aus LuF bemessen. Will man überprüfen, wie Landwirte diesem neuen Fördermodell gegenüberstehen, so müssen folglich auch diese zwei Kernelemente im Fokus der Betrachtung stehen. Hinter der Untersuchung der Akzeptanz stehen also grundsätzlich die beiden folgenden Fragen:

1. Besteht seitens der Landwirte Akzeptanz für ein Fördersystem, dessen jährliche Förderbeträge variabel und schwer vorhersehbar sind? 
2. Besteht seitens der Landwirte Akzeptanz für eine teilweise Bemessung der Förderung an Einkunftsschwankungen aus LuF?

Um diese Kernfragen zunächst möglichst allgemeingültig und unverfälscht untersuchen zu können, galt es bei der Konzeption dieser Untersuchung einen möglichst standardisierten Untersuchungsgang $\mathrm{zu}$ entwickeln und dabei verzerrende Einflüsse bestmöglich auszublenden. Dazu gehören alle Einflüsse, die zu einem individuell unterschiedlichen Verhalten der Landwirte aufgrund ihrer persönlichen Situation führen könnten.

In Kapitel 4 offenbarten sich verschiedene Elemente, an denen sich die Wirkung der Flexiblen Förderung ausrichtet. So ist gezeigt worden, dass es infolge einer Umstellung der Förderung des ökologischen Landbaus von der derzeitigen Festbetragsprämie auf die Flexible Förderung unter den Landwirten, abhängig von der Höhe ihrer LuF-Einkünfte, „Gewinner“ und „Verlierer“ geben wird. Würde man nun die Akzeptanz dieser Flexiblen Förderung bei den Landwirten untersuchen und die Landwirte auf die genaue Wirkungsweise dieses Fördersystems hinsichtlich der mittleren Einkunftshöhe hinweisen, so würde diese Information die Landwirte in ihrer Entscheidung für oder gegen die Flexible Förderung maßgeblich beeinflussen. Obgleich sie sich in der Befragung bzw. im Experiment aus rationalen Gründen für eine Flexible Förderung entscheiden müssten, würden sie mit der Perspektive, dass sie in der Realität mit der Flexiblen Förderung zu den „Verlierern“ zählen würden, keine schlüssige und im Sinne der Analyse verwertbare Antwort geben bzw. sie würden das Ergebnis bewusst verfälschen. Diese Effekte sollen deshalb bei der Akzeptanzbetrachtung ausgeblendet werden, um die oben formulierten Fragestellungen möglichst unverfälscht untersuchen zu können. Daher wird den Landwirten die Berechnungsweise der Zusatzförderung grob vereinfacht dargestellt, indem sie lediglich erfahren, dass sich die Zusatzförderung an der Schwankung der Einkünfte aus LuF der letzten fünf Jahre bemisst. Eine detaillierte Erläuterung der Berechnung der Zusatzförderung unterbleibt somit. Die Landwirte gehen deshalb davon aus, dass sich die Wirkungsweise lediglich an den Schwankungen der Einkünfte orientiert und nicht an der Höhe der mittleren Einkünfte oder des Flächenumfangs. Mit dieser vereinfachten Darstellung gelingt eine ceteris paribus Analyse, ob und inwieweit eine Fördermethodik, die sich grundsätzlich an Einkunftsschwankungen orientiert, verstanden und akzeptiert wird. 


\subsection{GESTALTUNG DER UNTERSUCHUNG}

Um Verständnis und Akzeptanz der Landwirte, d.h. der potentiell Begünstigten der Förderung, für die Flexible Förderung zu ergründen, wurde eine empirische Erhebung in Form einer Kombination aus zwei hintereinander angeordneten Feldexperimenten und einem Fragebogen ausgewählt. Der Grund für die Wahl dieser Kombination lag vor allem in der Natur der Flexiblen Förderung mit ihrer Anknüpfung an die Volatilität der steuerlichen Einkünfte aus Land- und Forstwirtschaft, wodurch sich, verglichen mit der Festbetragsförderung, eine höhere Komplexität der Wirkungen ergibt. Um den Landwirten die Funktionsweise und die Wirkungsweise anschaulicher und damit transparenter darzustellen, wurden computergestützte Feldexperimente einer ausschließlichen Befragung via Fragebogen vorgezogen. Jedoch sollte der Fragebogen nicht vollständig entfallen. Vielmehr sollten mit ihm zusätzliche Informationen zur Akzeptanz im Anschluss an das Feldexperiment eingeholt werden. Doch zunächst sollen kurz die theoretischen Grundlagen zu computergestützten Feldexperimenten erläutert werden.

\subsubsection{THEORIE DER EXPERIMENTELLEN WIRTSCHAFTSFORSCHUNG}

Die experimentelle Wirtschaftsforschung bezeichnet eine Teildisziplin der Wirtschaftswissenschaften und beschäftigt sich mit der experimentellen Bewertung ökonomischer Theorien sowie mit der Entwicklung neuer ökonomischer Verhaltensmodelle (HELD, 2003: 21ff). Dazu wird das Entscheidungsverhalten von Versuchspersonen in ökonomisch relevanten Situationen beobachtet, wobei in Abhängigkeit vom Ergebnis des Experiments den Versuchspersonen Geld ausbezahlt wird, so dass infolge der Versuchsanordnung reale monetäre Anreize entstehen. Auf diese Weise ist es möglich, Rückschlüsse auf reales Entscheidungsverhalten zu ziehen. Experimente stellen aber kein eigenständiges Datengewinnungsverfahren dar. Sie sind eine bestimmte Form der Befragung bzw. der Beobachtung unter bestimmten festgelegten Bedingungen. Die Zielsetzung von Experimenten ist es zu prüfen, ob ein Kausalzusammenhang zwischen verschiedenen Faktoren vorliegt. Charakteristisch für Experimente ist die Veränderung eines Faktors (hier: Bemessung der Förderung) und seine Auswirkung auf einen anderen Faktor (hier: Auswahlverhalten der Förderungsempfänger) unter kontrollierten Bedingungen. „Kontrollierte Bedingungen“ bedeutet dabei, dass störende Umweltfaktoren so weit wie möglich ausgeschlossen werden. 
Die experimentelle Wirtschaftsforschung nahm in den 1980er und 1990er Jahren einen Aufsehen erregenden Aufschwung. Von einer eher am Rande betrachteten Disziplin fand sie den Weg in den Mainstream der Ökonomik (RuBInsteIn, 2001: 625). Ihre Erfolge, die sich materiell in einem beachtlichen Publikationsboom in den führenden Zeitschriften des Fachs manifestierten, wurden zuletzt mit den Nobelpreisen für Vernon Smith und Daniel Kahnemann für die Erforschung begrenzter Rationalität sowie für Robert Aumann und Thomas Schelling für die Erforschung spieltheoretischer Fragestellungen gewürdigt.

Experimente lassen sich generell in Labor- und Feldexperimente einteilen. Laborexperimente finden unter speziellen, für das Experiment geschaffenen, künstlichen, möglichst abstrakten Bedingungen statt. Feldexperimente werden dem gegenüber unter „natürlichen“ Bedingungen durchgeführt. Sie haben den Vorteil der größeren Realitätsnähe und damit verbunden eine höhere externe Validität als Laborexperimente. Eine vergleichsweise höhere externe Validität bedeutet, dass Schlussfolgerungen aus dem Experiment besser in die Realität übertragbar sind. Nachteilig bei Feldexperimenten ist die geringere interne Validität gegenüber der von Laborexperimenten. Damit ist gemeint, dass Veränderungen der abhängigen Faktoren weniger eindeutig auf die Variation der im Experiment variierenden unabhängigen Faktoren zurückzuführen sind. Daraus folgt, dass die Wiederholbarkeit eingeschränkt sein kann, weil im Vergleich zu Laborexperimenten die externen Bedingungen schlechter zu kontrollieren sind. Damit diese Nachteile keine Auswirkungen auf die Qualität der Ergebnisse haben und die interne Validität erhalten bleibt, müssen bei Feldexperimenten die externen Bedingungen möglichst standardisiert werden.

HARRISON und LIST (2004: 1012f) geben 6 Unterscheidungskriterien an, die benutzt werden können, um den Feld-Kontext eines Experimentes zu bestimmen:

- Beschaffenheit des Teilnehmerkreises,

- Beschaffenheit des Basiswissens der Teilnehmer,

- Beschaffenheit/Art des Untersuchungsfeldes/Untersuchungsgegenstandes,

- Art der Aufgabenstellung bzw. der Experimentinstruktionen,

- Art des Anreizes,

- Beschaffenheit des Umfeldes, in dem das Experiment durchgeführt wird.

Danach kann das in dieser Untersuchung durchgeführte Experiment vor allem deshalb den Feldexperimenten zugeordnet werden, weil es sich beim Teilnehmerkreis um Öko-Landwirte 
mit einem spezifischen Basiswissen zur Förderung des Ökolandbaus handelte und dementsprechend auch der Untersuchungsgegenstand, die flächenbezogene Öko-Förderung, direkt ihrem Arbeitsumfeld entstammte. Für Laborexperimente gelten häufig Studenten als Standardteilnehmer. Studenten werden dabei als lern- und anpassungsfähig eingestuft. Ihnen gegenüber steht die Gruppe von Experten auf dem untersuchten Gebiet. Sie werden als weniger lern- und anpassungsfähig beschrieben. Sie haben die Tendenz, sich so zu verhalten, wie sie es im Rahmen ihrer Tätigkeit tun würden (FRIEDMANN und SUNDER, 1994: 40ff). Anders ausgedrückt: Man kann unterstellen, dass sich Landwirte, also die Expertengruppe, im Experiment so verhalten, wie sie es von ihrer betrieblichen Situation gewohnt sind. Da aus der Verhaltensweise im Experiment Rückschlüsse auf die Verhaltensweise in der Realität gezogen werden sollen, ist dieses Verhalten ganz im Sinne der Zielsetzung der Untersuchung. So war es auch ein vorrangiges Ziel im Vorfeld der Untersuchung, das Experiment möglichst praxisnah zu gestalten, damit sich der Proband schnell in seiner Umgebung zurechtfindet und somit möglichst realitätsnah entscheiden kann. Ein abstraktes Modell wäre schwerer verständlich gewesen und hätte dementsprechend stärker zu Verzerrungen beim Versuchsergebnis geführt.

\subsubsection{ORganisation, STRUKTUR UND ABLAUf DER UNTERSUChUNG}

Die Konzeption der Untersuchung unterteilt sich in 3 Schritte. Begonnen wurde mit dem eigentlichen Experiment zur Beurteilung der Akzeptanz der Flexiblen Förderung in der Landwirtschaft. Danach folgte ein Lotteriespiel zur Messung der individuellen Risikoeinstellung der Probanden. Dadurch sollte ermöglicht werden, die individuelle Risikoeinstellung der Probanden den Ergebnissen zur Akzeptanz gegenüberzustellen. Dies erschien im Vorfeld sehr interessant, um herauszufinden, ob sich eine mangelnde Akzeptanz auf einer generell fehlenden Risikobereitschaft oder auf einer wirklichen Abneigung gegenüber dem Instrument begründet.

Abschließend wurde von den Teilnehmern ein Fragebogen ausgefüllt, der die Hintergründe der Entscheidungen im Prämienexperiment, die Grundeinstellung des Teilnehmers zu wichtigen Fragen rund um die Förderung des ökologischen Landbaus sowie Daten zur Person und zum Betrieb des Probanden offen legen sollte.

Hinsichtlich des technischen Ablaufs konnte bei der Untersuchung auf ein mobiles PC-Labor mit 22 Laptops nebst Funknetzwerk zurückgegriffen werden. Die softwaretechnische Umsetzung sowohl des Prämienexperiments als auch des Lotteriespiels erfolgte im Rahmen 
der Experimentalsoftware ,z-Tree“ (FISCHBACHER, 1998) ${ }^{29}$. z-Tree ist eine Basissoftware, auf deren Grundlage computerbasierte Experimente programmiert werden können. Abbildung 41 zeigt schematisch den technischen Ablauf der Feldexperimente.

Abbildung 41: Schema zum technisch-organisatorischen Ablauf der Experimente

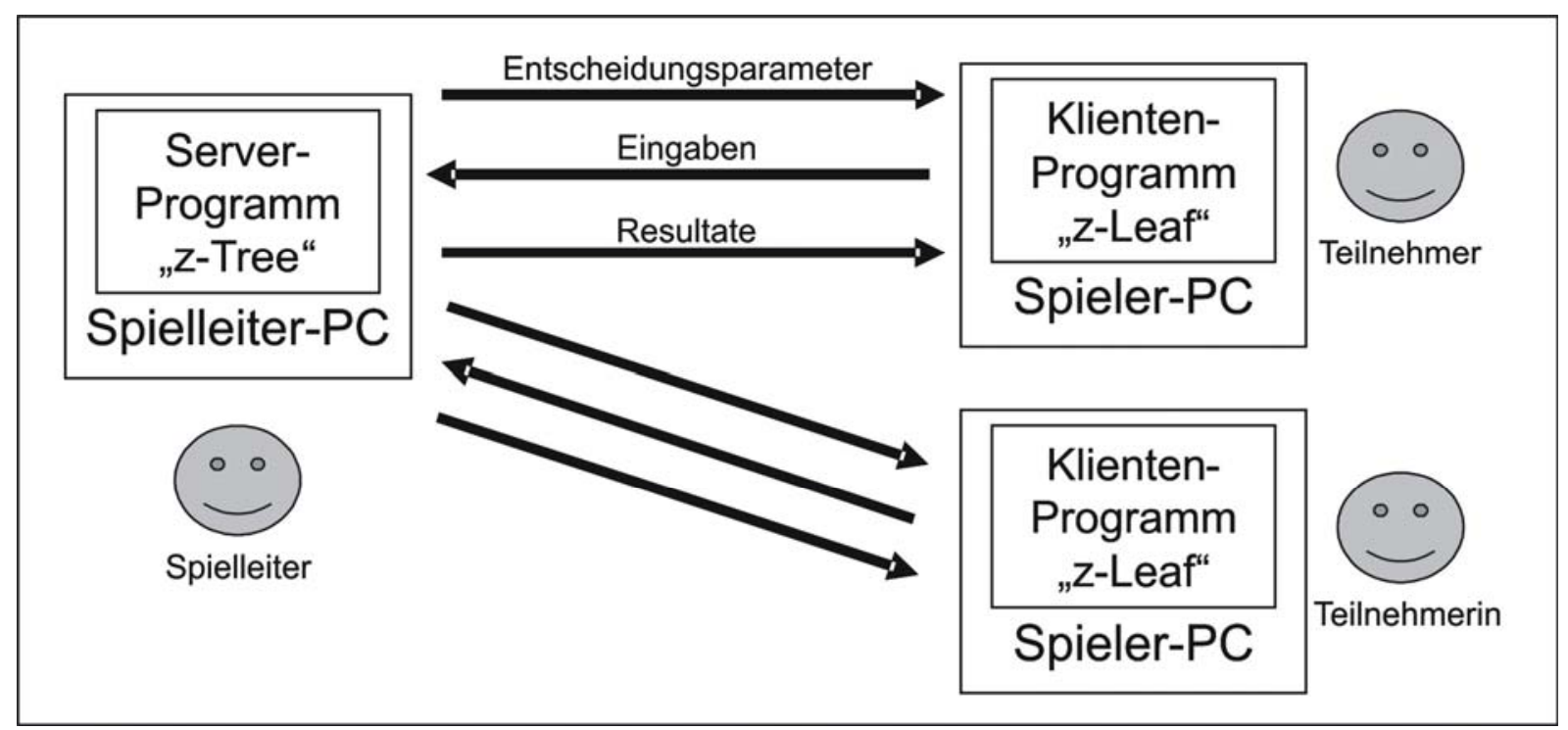

Quelle: Eigene Darstellung in Anlehnung an FISCHBACHER (1998: 6)

Der Spielleiter bedient den Spielleiter-PC. Von hieraus wird des Prämienexperiment zentral gestartet. Die Probanden bedienen jeweils einen der Spieler-PC's, die über ein Funknetzwerk mit dem Spielleiter-PC verbunden sind. Sobald das Prämienexperiment vom Spielleiter gestartet wurde, erscheint der erste Programmbildschirm auf den Spieler-PC's. Die Probanden haben nun die Möglichkeit sich mit der angezeigten individuellen Situation vertraut zu machen und ihre Eingaben $\mathrm{zu}$ machen. Sowohl die vom Probanden vorgefundene Ausgangskonstellation als auch die Eingaben des Probanden werden auf dem Spielleiter-PC gespeichert und stehen damit dem Spielleiter für die Auswertung zur Verfügung.

Die durchgeführte Gesamtuntersuchung teilt sich in sechs Einzeluntersuchungen auf, die in sechs verschiedenen Bundesländern an unterschiedlichen Terminen durchgeführt wurden. Als Veranstaltungsorte dienten jeweils Schulungsräume von Lehranstalten oder Veranstaltungsräume in Gastwirtschaften oder auf größeren landwirtschaftlichen Betrieben. $\mathrm{Zu}$ Beginn jeder Veranstaltung erfolgte eine einführende Beamer-Präsentation. Damit sollten die Teilnehmer einerseits in die Basisthematik eingeführt werden. Zum anderen sollte die Flexible Förderung vorgestellt und der Ablauf der Veranstaltung erläutert werden. Gemäß den Anforderungen an Feldexperimente aus der Theorie der experimentellen

\footnotetext{
${ }^{29}$ Die Programmierung in z-Tree ist in Anhang 4 detailliert einzusehen.
} 
Wirtschaftsforschung und zur Sicherung der internen Validität waren identische Ausgangsbedingungen und Instruktionen für die Probanden in den einzelnen Experimenten sowie ein möglichst identischer Ablauf der einzelnen Experimente unabdingbar. Deshalb folgte die Einführungsveranstaltung immer einer detailliert festgelegten Vorgehensweise, die in Anhang 5 dieser Arbeit in Form der „Experimentinstruktionen“ einzusehen ist (am Beispiel Niedersachsens). Für den Leser dieser Arbeit legen diese Experimentinstruktionen den genauen Gang der Untersuchung und die Ausgangsbedingungen für die Probanden offen.

Mit Bezug auf die in der Theorie der experimentellen Wirtschaftsforschung für die Durchführung von Experimenten geforderten „kontrollierten Bedingungen“ galt es auch zu gewährleisten, dass jeder Teilnehmer seine Entscheidung allein treffen konnte und keinen äußeren Beeinflussungen unterlag. Dazu wurden den Teilnehmern im Vorfeld genaue Verhaltensregeln auferlegt, die ebenfalls aus den Experimentinstruktionen zu ersehen sind. Zusätzlich wurde darauf geachtet, dass sich für die einzelnen Teilnehmer keine Möglichkeiten boten, auf die Bildschirme anderer Mitspieler zu schauen. Lagen bei einem Experiment keine optimalen räumlichen Verhältnisse vor, so wurden zusätzlich Trennwände zwischen den einzelnen Teilnehmern aufgebaut.

Die bisherigen Ausführungen zeigen, dass Planung, Konzeption und Durchführung von Untersuchungen in Form von Feldexperimenten hohe organisatorische Anforderungen stellen. Wie bereits erwähnt kann der Ablauf der Einzelveranstaltungen anhand der Experimentinstruktionen in Anhang 5 der Arbeit genau nachvollzogen werden. Deshalb soll bei den folgenden Betrachtungen die Konzeption der einzelnen Untersuchungsteile im Vordergrund stehen und der praktische Ablauf nur der Vollständigkeit halber verkürzt erläutert werden.

\subsubsection{PRÄMIENEXPERIMENT}

Den ersten Teil der Untersuchung bildete das so genannte Prämienexperiment, in dem sich jeder Teilnehmer auf Basis eines standardisierten Beispielbetriebes mit 50 ha Ackerland zwischen der gegenwärtigen Festbetragsprämie und der neuen Flexiblen Prämie zu entscheiden hatte. Dazu wurde analog zur Basiskonstellation aus Kapitel 4.2 für die Flexible Prämie ein fester Sockelbetrag von $100 € /$ ha festgesetzt. Für die Festbetragsprämie wurde mit $160 € /$ ha der derzeitige Fördersatz des GAK-Rahmenplanes angenommen. Die Zusatzprämie als zweiter Bestandteil der Flexiblen Prämie wurde gemäß den Vorgaben zur Funktionsweise aus Kapitel 4.2.1 an die betriebsindividuellen Schwankungen der LuF-Einkünfte des Beispielbetriebes geknüpft. Für die Verteilung der Einkünfte aus LuF wurde eine 
Dreiecksverteilung mit einem Mittelwert von 23.000€ und einer maximalen Abweichung von $15.000 €$ herangezogen. Diese Werte ergaben sich näherungsweise aus einer Analyse der Gewinnverteilungen der ökologisch wirtschaftenden Betriebe des BMELV-Testbetriebsnetzes auf Basis der Öko-Betriebe mit mehr als sechs Jahresdaten aus den vergangenen acht Wirtschaftsjahren $^{30}$. Entgegen den Annahmen zur Basiskonstellation wurde jedoch ein 5jähriger anstatt des 3-jährigen Bemessungszeitraums für die Zusatzförderung angenommen. Jeder Proband sollte sich für einen folgenden 5-Jahres-Zeitraum zwischen der Festbetragsprämie und der Flexiblen Prämie entscheiden. Dazu erhielt er die steuerlichen Einkünfte aus LuF der vergangenen sieben Jahre. Über einen internen Förderfaktor wurde der Erwartungswert der fünfjährigen Auszahlung, der im Falle der Festbetragsförderung automatisch bei $40.000 €$ lag, für die Flexible Prämie auf $42.000 €$ festgesetzt, so dass ein Risikoaufschlag von $5 \%$ gewährt wurde. Allerdings war dieser Erwartungswert der Flexiblen Förderung den Landwirten nicht bekannt. Letztere mussten sich allein auf die Analyse der ihnen vorliegenden vergangenen sieben Jahre verlassen, in der Hoffnung oder Vermutung, dass sich daraus ein Trend für die zukünftigen fünf Jahre ableiten ließe $\mathrm{e}^{31}$.

Die Entscheidung wurde insgesamt siebenmal wiederholt, d.h. jeder Proband musste sich achtmal für den folgenden 5-Jahres-Zeitraum für eines der beiden zur Auswahl stehenden Fördersysteme entscheiden. Am Ende jeder Runde wurden jedem Teilnehmer die Auswirkungen seiner getroffenen Entscheidung offenbart.

Das Prämienexperiment repräsentiert auch gleichzeitig den Hauptteil der Untersuchung. Hinter der Entscheidung des Probanden für eines der beiden Prämiensysteme steht die grundsätzliche Frage aus Kapitel 5.1 nach der Akzeptanz der Flexiblen Prämie. Der einzelne Proband sollte sich bei seiner Entscheidung soweit wie möglich in die Situation des Beispielbetriebs hineinversetzen. Um eine möglichst rationale Entscheidung anzuregen, wurde den Teilnehmern gemäß der Theorie der experimentellen Wirtschaftsforschung ein monetärer Anreiz von mindestens $10 €$ und durchschnittlich $15 €$ geboten, für den das Gesamtprämienvolumen der einzelnen Teilnehmer am Ende der acht Spielrunden die Bemessungsgrundlage darstellte. Abbildung 42 zeigt den so genannten Entscheidungsbildschirm, d.h. den Bildschirm, mit dem jede Runde des Experiments begann und auf dessen Grundlage die Teilnehmer ihre Entscheidung fällten.

\footnotetext{
${ }^{30}$ Dabei handelt es sich um dieselbe Stichprobe, die auch schon für die Risikoanalyse in Kapitel 4.1.2.2 verwendet wurde.

${ }^{31}$ Vgl. Anhang 5 für detaillierte Informationen zur Entscheidungssituation.
} 
Abbildung 42: Entscheidungsbildschirm im Prämienexperiment ${ }^{32}$

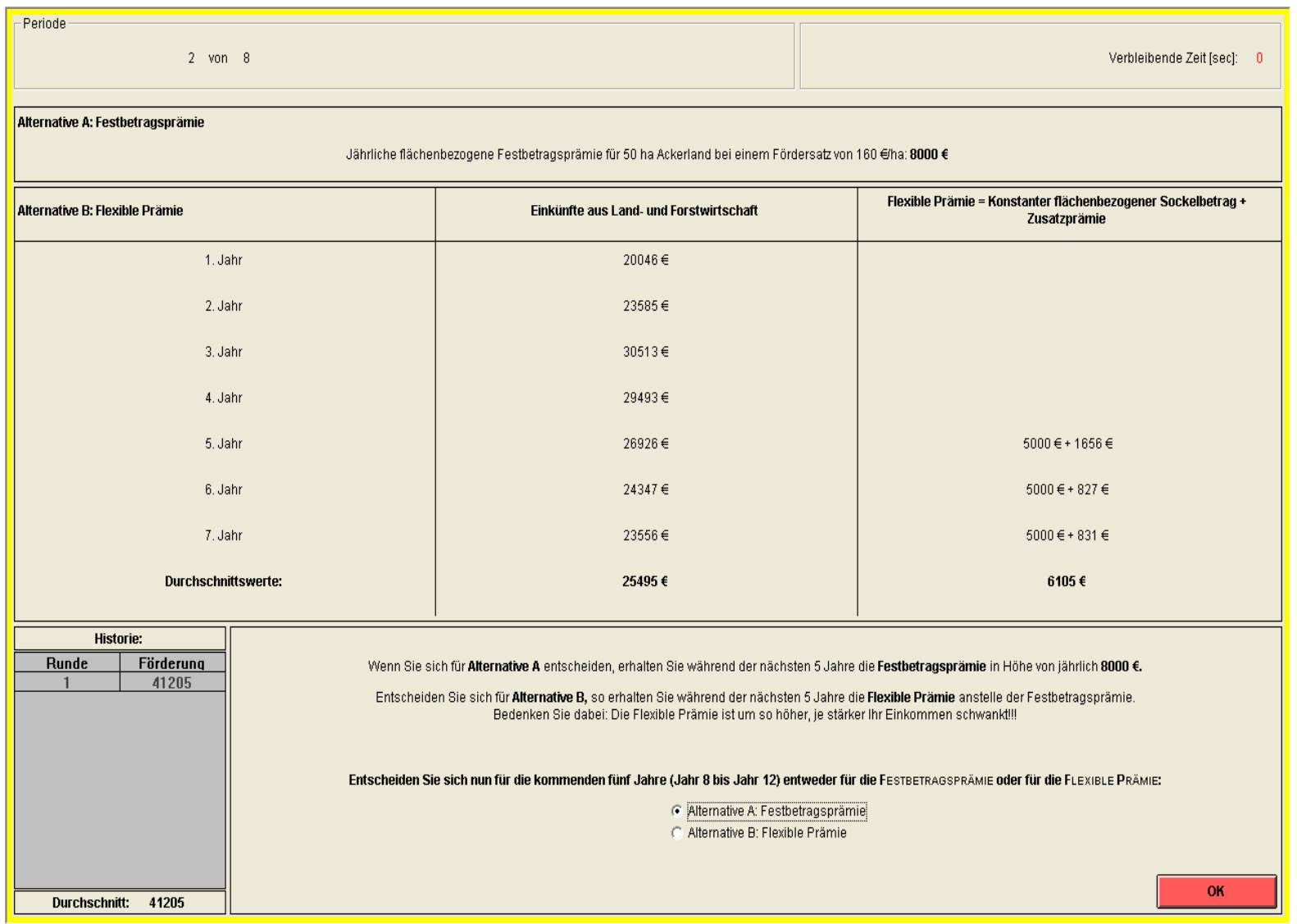

Quelle: Eigene Darstellung

Die historischen Einkünfte des fiktiven Betriebes - der mit dem realen Betrieb des Probanden nichts gemeinsam hatte - wurden dem Entscheider auf diesem Bildschirm angezeigt. Daran konnte er die Schwankung der Einkünfte einschätzen. Im unteren Bereich des Bildschirms konnte die Prämienart für die folgenden 5 Jahre vom Teilnehmer individuell festgelegt werden. Nach der getroffenen Entscheidung, wurde der Ergebnisbildschirm (Abbildung 43) eingeblendet. Der 5-Jahres-Zeitraum, für den sich der Teilnehmer für eine Prämienart festgelegt hatte, war nun vergangen und die Einkünfte aus LuF ersichtlich. Weiterhin wurden jetzt die Zusatzförderungen der einzelnen Jahre angezeigt. Mit den jährlichen Sockelbeträgen ergaben sie aufsummiert die Gesamtförderung für den 5-Jahreszeitraum, der mit der Gesamtförderung von $40.000 €$ der Festbetragsförderung verglichen werden konnte. Nun zeigte es sich, ob der Teilnehmer mit seiner Wahl die im Nachhinein richtige oder falsche Entscheidung getroffen hatte.

\footnotetext{
${ }^{32}$ Der abgebildete Entscheidungsbildschirm zeigt eine Ausgangssituation, in der die Festbetragsförderung für die vergangenen Jahre deutlich vorteilhafter gewesen wäre (Durchschnittlicher Jahresbetrag: $6.105 €$ ) und die LuF-Einkünfte relativ geringen Schwankungen unterliegen. Genauso gut kann die Ausgangssituation aber auch ganz anders aussehen.
} 
Abbildung 43: Ergebnisbildschirm im Prämienexperiment

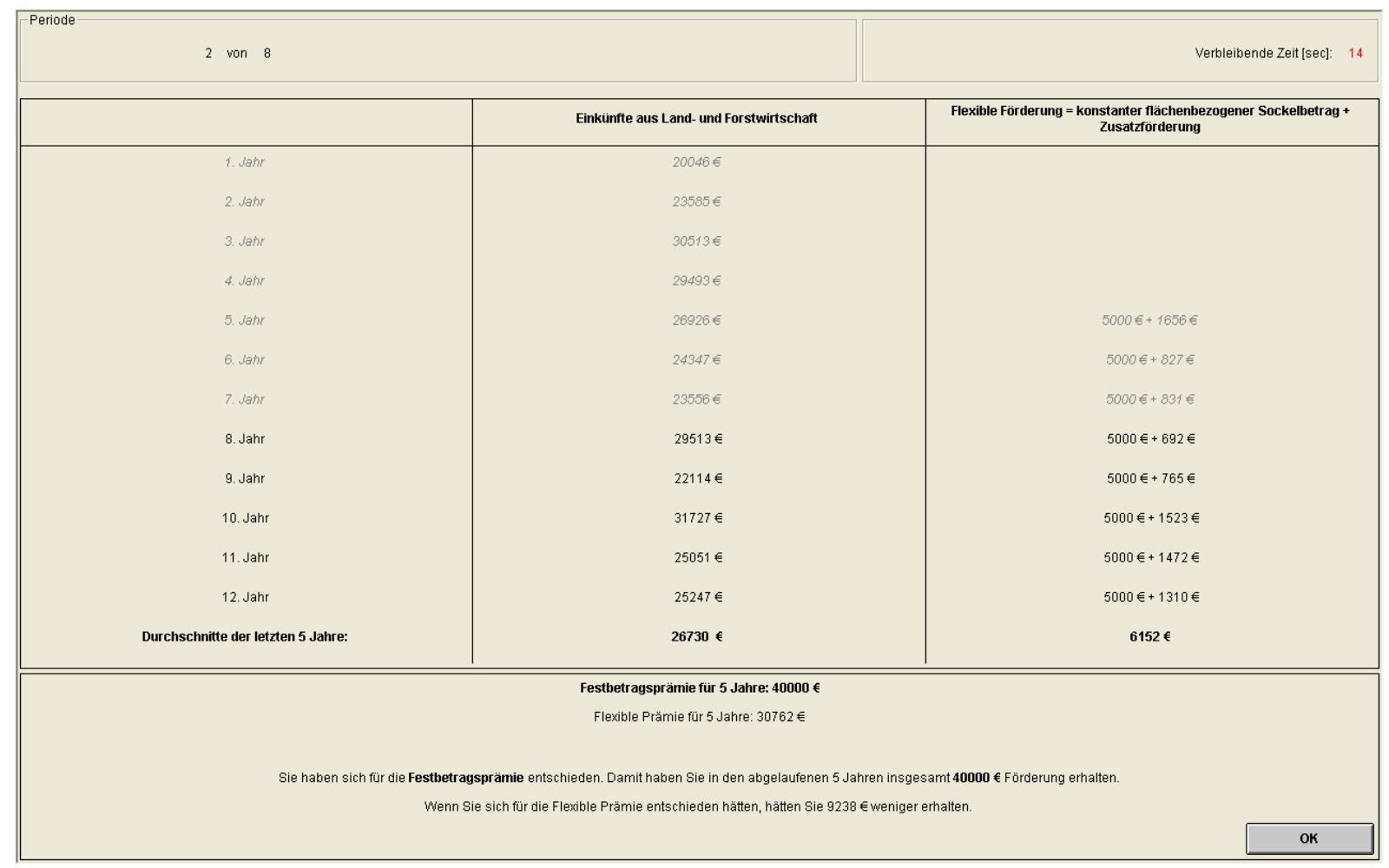

Quelle: Eigene Darstellung

Nach acht gespielten Runden hatten die Teilnehmer in Abhängigkeit ihres persönlichen Erfolgs einen bestimmten Geldbetrag eingespielt. Dieser Geldbetrag wurde an dieser Stelle den Teilnehmern jedoch noch nicht genannt, sondern bildete den finanziellen Grundstock der Teilnehmer für das Lotteriespiel, in dem dieses Geld von den Teilnehmern nochmals ins Spiel gebracht werden sollte, um die persönliche Auszahlung am Ende des Spieles zu optimieren.

\subsubsection{LOTTERIESPIEL}

Bei der Konzeption des Lotteriespiels, das den zweiten Teil der Untersuchung ausmacht, ging es um die Frage, welche Möglichkeiten es generell gibt, um die persönliche Risikoeinstellung eines Probanden zu ergründen, denn die Risikoeinstellung eines Probanden beeinflusst die Wahl des risikoreicheren Förderinstruments „Flexible Förderung“, während bislang die „risikolose“ Pauschalförderung in Anspruch genommen werden konnte.

Auch dieser zweite Teil der Untersuchung wurde in Form eines Feldexperiments durchgeführt. Zwischen beiden Untersuchungsteilen besteht trotzdem ein gravierender Unterschied. Während das Prämienexperiment im vollen Umfang eigenständig entwickelt wurde, geht das Lotteriespiel in seiner Konzeption auf die theoretischen Überlegungen von ANDERSON et al. (1977) zurück. Aus diesem Grund sollen im Folgenden zunächst die 
theoretischen Überlegungen dargelegt werden, die zur Konzeption des Lotteriespiels geführt haben, bevor seine spezielle Ausgestaltung im Rahmen eines Feldexperimentes erläutert wird.

Im Rahmen der Experimentellen Wirtschaftsforschung wird seit langer Zeit versucht, individuelle Risikoeinstellungen zu messen (vgl. bspw. Mosteller und NogeE, 1951 oder KAHNEMANN und TVERSKY, 1979). Der große Vorteil liegt darin, dass der Proband reale Entscheidungen zu treffen hat, die für ihn auch monetäre Konsequenzen haben. Denn auch der Erfolg des Spielers in diesem Teil der Untersuchung beeinflusste den letztendlichen Auszahlungsbetrag des Spielers in seiner Höhe. Bei äquivalenten Methoden zur Ermittlung der persönlichen Risikoeinstellung, allen voran den Selbsteinschätzungen über einen Fragebogen, wird die Tatsache, dass die Probanden oftmals direkt auf die Untersuchungsabsicht schließen können, als sehr negativ beurteilt. Dieses Kriterium, welches prinzipiell auch auf dieses Experiment zutreffen könnte, ist bei Experimenten im Rahmen der Experimentellen Wirtschaftsforschung jedoch weit weniger entscheidend als bei Befragungen, weil das Experiment nicht nur hypothetisch vorgenommen wird, sondern die von den Probanden gewählten Alternativen auch tatsächlich auszahlungsrelevant sind und somit ein finanzieller Anreiz besteht, die wahren Präferenzen offen zu legen.

Der Sinn dieser Anreizkompatibilität ist, eventuell vorhandene Motivationen der Probanden in den Hintergrund treten zu lassen, die nicht der zu untersuchenden Entscheidungssituation entsprechen (näheres dazu in SMITH, 1976: 274ff). Eine Schwierigkeit besteht allerdings in Experimenten darin, anreizkompatible Mechanismen zu implementieren, bei denen die Versuchspersonen einen Anreiz haben, ihre wahren Präferenzen zu offenbaren und sich nicht in Form falscher Angaben strategisch verhalten.

Eine weit verbreitete Methode, die Risikoeinstellung eines Individuums aus ökonomischer Sicht zu messen, besteht darin, die subjektiven Sicherheitsäquivalente zu verschiedenen Lotterien zu ermitteln (vgl. ANDERSON et al., 1977 oder auch Mosteller und NogEe, 1951). Prinzipiell lässt sich dieses Verfahren darauf zurückführen, dass eine Kombination aus einer Lotterie und einer sicheren Auszahlung ermittelt wird, zwischen denen der Proband indifferent ist. Dazu wurde im Experiment ein einfacher Münzwurf mit zwei möglichen unterschiedlichen Auszahlungen verwendet, bei dem naturgemäß gilt, dass die beiden Auszahlungen jeweils mit der gleichen Wahrscheinlichkeit, d.h. mit $p=0,5$ eintreffen. Die Probanden waren nun aufgefordert, Gebote für die Teilnahme an der Lotterie abzugeben. Das individuelle Gebot eines jeden Spielers entsprach dem sicheren Wert bzw. Geldbetrag, bei 
dem der Spieler indifferent ist, diesen zu behalten oder an der Lotterie teilzunehmen, d.h. also seinem persönlichen Sicherheitsäquivalent.

Wenn die Probanden, wie im vorliegenden Fall, aufgefordert werden, eine vorgegebene Lotterie mit einem Festbetrag auf ihre Präferenz hin zu vergleichen, und die Lotterie dabei auszahlungsrelevant ist, so besteht kein Grund zu der Befürchtung, die Probanden könnten aus strategischen Gründen die Unwahrheit sagen. Das Problem der Anreizkompatibilität ist hierbei also durch die Wahl der Messmethode gelöst.

Diese so genannte Sicherheitsäquivalentmethode, bei der die Lotterie vorgegeben ist und das Sicherheitsäquivalent ermittelt wird, gehört nach SCHOEMAKER und HERSHEY (1992: 398) zu den am weitesten verbreiteten Methoden zur Messung der persönlichen Risikoeinstellung. Die Methode hat aber auch einige Nachteile, die u.U. wichtigen Einfluss auf die Aussagefähigkeit der Ergebnisse haben können:

- Die Risikoeinstellung eines Individuums kann nach ANDERSEN et al. (1977) bei dieser Methode nicht durch einen einzigen derartigen Messwert charakterisiert werden. Die Probanden müssen daher mehrere Entscheidungen für verschiedene Ausgangskonstellationen vornehmen, die sich teilweise nur geringfügig unterscheiden. Dies kann zu Motivationsproblemen führen. Daher muss diese Messung für verschiedene Ausgangskonstellationen wiederholt werden. Aus diesem Grund waren im vorliegenden Experiment die Probanden aufgefordert, nacheinander vier Gebote für verschiedene Ausgangskonstellationen, d.h. für jeweils verschiedene Auszahlungsbeträge abzugeben. Die Bemessung der unterschiedlichen Auszahlungsbeträge lehnten sich dabei an die allgemein anerkannte Vorgehensweise von ANDERSEN et al. (1977) an.

- Weil das Gesamtbudget begrenzt ist, kann jede einzelne Entscheidung nur eine geringe Auszahlung zur Folge haben, so dass es fraglich wird, ob die Geldauszahlung noch den gewünschten Anreizcharakter behält. Untersuchungen, die in China (KACHELMEIER und SHeHATA, 1992: 1120ff) und in Indien (BINSWANGER, 1980: 395ff), also in Ländern mit deutlich geringeren Einkommensniveaus, durchgeführt wurden, weisen jedoch auf keine grundsätzlich anderen Verhaltensweisen hin als Untersuchungen mit relativ geringen Auszahlungsniveaus. Trotzdem wurde versucht, diese Problematik abzumildern, indem für das Prämienspiel und das Lotteriespiel insgesamt nur ein Geldbetrag ausgezahlt wurde, der allerdings von beiden Spielen beeinflusst wurde. Die letztendliche Berechnung wurde dem Spieler jedoch nicht offen 
gelegt. Der in Aussicht gestellte Auszahlungsbetrag von mindestens $10 €$ konnte daher für beide Spiele gleichermaßen als Anreizfaktor verwendet werden.

- Durch die Vielzahl von Einzellotterien entsteht eine neue, zusammengesetzte Lotterie mit eigenem Charakter. Gemäß der Vorgehensweise von ANDERSEN et al. (1977) ist es daher wichtig, die Ergebnisse der einzelnen Runden erst am Ende des Experimentes dem Spieler anzuzeigen, um sein Gebotsverhalten nicht zu beeinflussen. So wurde auch im Lotteriespiel verfahren. Erst nach den 4 Runden wurden den Probanden die Ergebnisse der Lotterien angezeigt.

Eine weitere Problematik dieser Methode besteht noch in dem folgenden Sachverhalt: Würde man einfach nach dem Gebot fragen und anschließend die Lotterie spielen, so hätte der Proband einen Anreiz, ein zu niedriges Gebot zu nennen, da dann der Erwartungswert seines Gewinns und damit seiner Auszahlung entsprechend höher wäre. Als Ausweg bietet sich hier die von VICKREY 1961 vorgeschlagene Second-price-sealed-bid-Auction an, ein Mechanismus, der die wahre Zahlungsbereitschaft offenbart.

Bei diesem Auktionstyp wird ein gegebenes Gut (hier die Teilnahme an der Lotterie) an denjenigen Bieter verkauft, der das höchste Gebot abgegeben hat, allerdings muss er lediglich den Preis des zweithöchsten Gebots zahlen. Bei mindestens zwei Probanden gleichzeitig ist dieses daher ein Verfahren, bei dem es für die Probanden optimal ist, für die Teilnahme an der Lotterie das wirkliche individuelle Sicherheitsäquivalent $\mathrm{zu}$ bieten, d.h. die maximale Zahlungsbereitschaft abzufragen, um denjenigen Betrag zu erhalten, bei dem sie indifferent zwischen der Lotterie und dem gebotenen Betrag sind (vgl. VICKREY, 1961).

Um dieses Verfahren für eine größere Gruppe von Teilnehmern zu öffnen, wurde eigens für diese Untersuchung festgelegt, dass nur die $50 \%$ der Teilnehmer der Veranstaltung mit den höchsten Geboten zur Lotterie zugelassen wurden. Es herrschte also Konkurrenz unter den Probanden um die Teilnahme an der Lotterie und damit um die Chance auf einen Geldgewinn. Die Strategie der Teilnehmer bei der Gebotsabgabe im Lotteriespiel sah also wie folgt aus: Bei einem zu niedrigen Gebot bestand für den Spieler die Gefahr, von der Lotterie und damit von der Chance auf einen Geldgewinn ausgeschlossen zu werden. Bei einem zu hohen Gebot hingegen bestand für den Spieler die Gefahr, nur die geringere der beiden Auszahlungen $\mathrm{a}_{1}$ und $\mathrm{a}_{2} \mathrm{zu}$ erhalten und damit Geld zu verlieren.

Diese Methode wurde in leicht abgewandelter Form auch von GÜTH et al. (1997) angewandt, um individuelle Sicherheitsäquivalente zu erheben. Die Autoren weisen in diesem Zusammenhang auf ein grundlegendes Problem hin. Danach bietet dieser Auktionstyp 
Anreize zur Kartellbildung seitens der Bieter (vgl. dazu auch RoтнKOPF et al., 1990). Diese Problematik wurde bei der Konzeption des Lotteriespiels dadurch entschärft, dass gemäß den Anforderungen an Feldexperimente die einzelnen Teilnehmer strikt voneinander getrennt die Entscheidungen trafen.

Um zu verhindern, dass die Probanden die Auswirkungen ihrer Entscheidung nicht nachvollziehen können und sich daher unkontrolliert verhalten, sind vor Durchführung der Experimente Instruktionen notwendig. Der Ablauf der Einführungsveranstaltung für das Lotteriespiel ist ebenfalls aus den im Zusammenhang mit dem Ablauf des Prämienexperiments bereits erwähnten „Experimentinstruktionen“, die in Anhang 5 dargestellt sind, ersichtlich. Deshalb soll darauf an dieser Stelle nur verkürzt eingegangen werden.

Im Anschluss an die Instruktionen konnte jeder Spieler seinen individuellen Bildschirm zur Gebotsabgabe einsehen, der in Abbildung 44 beispielhaft dargestellt ist.

Abbildung 44: Bildschirm zur Gebotsabgabe im Lotteriespiel

\begin{tabular}{|c|c|c|c|}
\hline Runde 2 von 4 & & & Verbleibende Zeit [sec]: $\quad 19$ \\
\hline \multicolumn{4}{|c|}{ Glücksspiel } \\
\hline \multicolumn{4}{|c|}{ Achtung: Bei diesem Spiel werden die Ergebnisse erst $N A C H$ der vierten Runde angezeigtt!! } \\
\hline Gewinn bei "Kopr" & Gewinn bei "Zahl" & \multicolumn{2}{|l|}{ Ihr Einsatz } \\
\hline $50 €$ & $100 €$ & \multicolumn{2}{|l|}{$65 €$} \\
\hline \multirow[t]{3}{*}{$50 €$} & $65 €$ & \multicolumn{2}{|l|}{1} \\
\hline & \multicolumn{3}{|c|}{ Drücken Sie "OK, wenn Sie inren Einsatz gemacht haben. } \\
\hline & & & ок \\
\hline
\end{tabular}

Quelle: Eigene Darstellung

Der einzelne Teilnehmer war nun aufgefordert, für vier Lotteriesituationen, bei denen die Gewinnbeträge jeweils variierten, sein Gebot für die Teilnahme an der Lotterie abzugeben. Um die angesprochenen theoretischen Anforderungen an die Ermittlung der persönlichen Risikoeinstellung einzuhalten, durften die Ergebnisse der vier Spielrunden erst am Spielende 
offen gelegt werden. Für den nach Abschluss der 4. und letzten Gebotsrunde folgenden Ergebnisbildschirm ist in Abbildung 45 ein Beispiel dargestellt.

Abbildung 45: Ergebnisbildschirm des Lotteriespiels

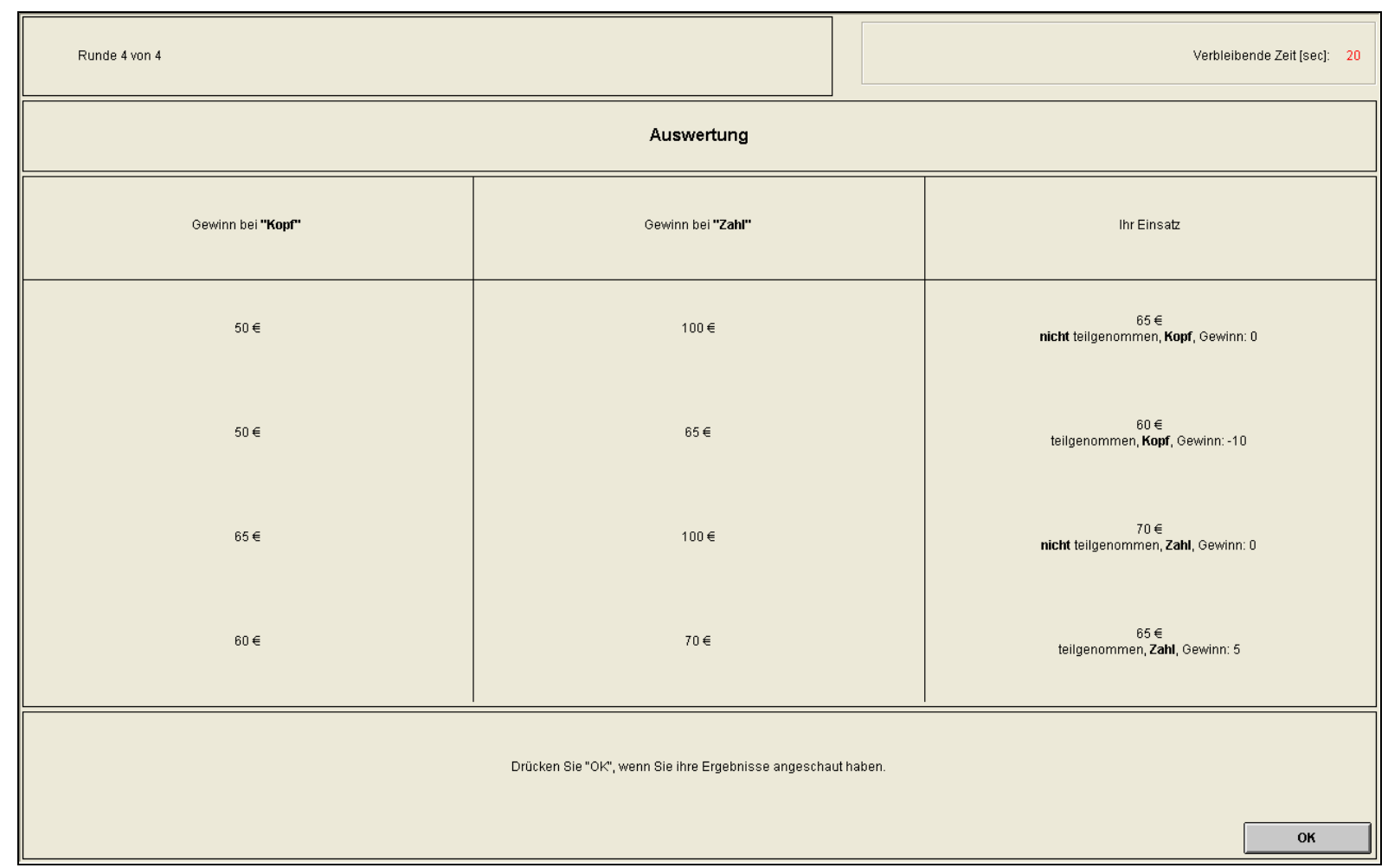

Quelle: Eigene Darstellung

Allerdings kann man mit dieser Methodik zur Ermittlung der individuellen Risikoeinstellung einer Person nicht allen Anforderungen gerecht werden. KACHELMEIER et al. (1992) verweisen darauf, dass bei mehreren aneinander gereihten Lotterien wie im vorliegenden Fall, die Risikoeinstellung durch den Eindruck einer „großen Lotterie“, bei der alle Spielrunden zusammenhängen, beeinflusst werden kann. Die Risikoaversion kann dadurch vermindert werden. Dieser Effekt ist nicht als Problem der Anreizkompatibilität zu sehen, sondern entsteht, weil die verschiedenen Lotterien zusammengenommen ein Lotterieportfolio mit eigener Risikostruktur ergeben. Dieser Sachverhalt wäre nur auszuräumen, indem man die Komplexität des Experimentes erhöhen würde, etwa indem nicht alle vier Entscheidungen auszahlungsrelevant sind, sondern bspw. nur zwei, die am Schluss ausgelost werden. Da diese zunehmende Komplexität aber die Verständlichkeit und vermutlich auch die Akzeptanz des Experimentes unter den Probanden einschränken würde, fand eine solche Strategie bei dieser Untersuchung keine Anwendung. 


\subsubsection{FRAGEBOGEN}

Im Anschluss an die beiden computerbasierten Teile des Experiments wurde an die Probanden ein Frageboden ausgeteilt. Mit ihm sollten Informationen zu folgenden Bereichen eingeholt werden (vgl. Anhang 6):

- Informationen zum Betrieb des Probanden,

- Informationen zur persönlichen Einstellung des Probanden hinsichtlich der Förderung des ökologischen Landbaus,

- Informationen zum Entscheidungsverhalten des Probanden im Prämienexperiment,

- Informationen zur Akzeptanz der Flexiblen Förderung für den eigenen Betrieb,

- Informationen zur persönlichen Einstellung gegenüber der zugrunde liegenden Idee einer Ausrichtung der Förderung an den betriebsindividuellen Einkunftsschwankungen und der Anreizwirkung der Flexiblen Förderung,

- Informationen zur Person der Probanden.

Bei der Konzeption des Fragebogens stand die Intention im Vordergrund, die Aussagekraft der Ergebnisse des Prämienexperimentes zu erhöhen. Dazu wurden schwerpunktmäßig geschlossene Fragen mit vorgegebenen Antwortmöglichkeiten verwendet. Sie haben im Vergleich zu offenen Fragen den Vorteil einer einfacheren statistischen Auswertung. Ergänzend wurden aber auch offene Fragen gewählt. Dies war vornehmlich bei Fragestellungen der Fall, bei denen im Vorfeld viele verschiedene Antwortmöglichkeiten denkbar waren, der Proband in seinem Antwortverhalten aber nicht durch vorgegebene Antwortmöglichkeiten eingeschränkt werden sollte. Offene Fragestellungen bieten zusätzlich den Vorteil, die Kreativität des Probanden zu fordern. Dies sollte bspw. bei der Frage nach interessanten eigenen Vorschlägen für eine Ausgestaltung der Förderung genutzt werden. Zusätzlich zu den offenen Fragen wurden z.T. auch bei den geschlossenen Fragen für den Probanden Möglichkeiten eingebunden, unter „Sonstiges“ außerhalb der vorgegebenen Antwortmöglichkeiten eigene Ansätze und Vorschläge $\mathrm{zu}$ nennen. Obwohl diese Vorgehensweise eine statistische Auswertung erschwert, wurde sie trotzdem bei dieser Untersuchung als notwendig erachtet, da die unterschiedlichen Positionen der Probanden zum Modell der Flexiblen Förderung aufgrund seiner Neuheit im Vorhinein nur schwer ausgemacht werden konnten.

Zur Bewertung von Statements dienen überwiegend diskrete, fünfstufige Rating-Skalen, die sog. Likert-Skalen (vgl. DiEKMANN, 2002: 183 und 209). In diesem Zusammenhang muss 
darauf hingewiesen werden, dass Rating-Skalen streng genommen ordinal skaliert sind, so dass Messverfahren, die ein metrisches Messniveau verlangen, wie bspw. die Berechnung des arithmetischen Mittels oder der Standardabweichung, nicht angewendet werden dürfen (BEREKOVEN et al. 2004: 76). Da jedoch von gleichgroßen Skalenabschnitten ausgegangen wird, nimmt man eine Intervallskalierung an (BACKHAUS et al. 2003: 5), wodurch diese Methoden genutzt werden können.

\subsection{ERGEBNISSE DER UNTERSUCHUNG}

Im Rahmen der Gesamtuntersuchung wurden die Ergebnisse von insgesamt 76 Probanden erhoben, von denen allerdings zur Auswertung vier aus der Stichprobe entfernt wurden, da es sich bei Ihnen um konventionell wirtschaftende Landwirte ohne Umstellungsinteresse handelte. Die Erhebung erfolgte in Form von sechs einzelnen Feldexperimenten im Rahmen von Tagungsveranstaltungen landwirtschaftlicher Beratungseinrichtungen zu betriebswirtschaftlichen Themenstellungen. Die Untersuchung wurde in den Monaten Januar bis März 2006 durchgeführt. Tabelle 27 zeigt die Chronologie sowie bereits einige erste Informationen zu den einzelnen Experimenten.

Tabelle 27: Chronologie der Einzelexperimente

\begin{tabular}{|c|c|c|c|}
\hline Nr. des Experiments & Anzahl der Teilnehmer & Art der Teilnehmer & Experiment wurde durchgeführt in: \\
\hline 1 & 12 & Landwirte & Schleswig-Holstein \\
\hline 2 & 10 & Landwirte & Hessen \\
\hline 3 & 13 & Landwirte & Nordrhein-Westfalen \\
\hline 4 & 15 & Landwirte & Mecklenburg-Vorpommern \\
\hline 5 & 10 & Landwirte & Rheinland-Pfalz \\
\hline 6 & 12 & Landwirte & Baden-Württemberg \\
\hline
\end{tabular}

Quelle: Eigene Darstellung

\subsubsection{CHARAKTERISIERUNG DER STICHPROBE}

Voraussetzung für die Teilnahme an der Untersuchung war, dass es sich bei jedem Probanden um einen Leiter eines landwirtschaftlichen Betriebes handelte. Dieses war besonders deshalb von größter Wichtigkeit, da dieser Personengruppe auch in der Realität die Beantragung der flächenbezogenen Öko-Prämie obliegt. Der zu beantwortende Fragebogen zeigt zunächst die wesentlichen Charakteristika der Stichprobe auf, die im Folgenden vorgestellt werden.

Von den 72 Betriebsleitern bewirtschafteten 70 einen bereits auf ökologischen Landbau umgestellten Betrieb. Zwei Betriebsleiter gaben an, ihren derzeit noch konventionell bewirtschafteten Betrieb in nächster Zeit umstellen zu wollen. Die Betriebsleiter der bereits 
ökologisch wirtschaftenden Betriebe wurden nach dem historischen Umstellungszeitpunkt befragt. Dabei stellte sich das Jahr 1993 als durchschnittlicher historischer Umstellungszeitpunkt heraus. Es fiel jedoch auf, dass insbesondere an der Veranstaltung in NRW Betriebsleiter teilnahmen, deren Betriebe erst in der jüngeren Vergangenheit auf Ökolandbau umgestellt worden waren.

Die 70 bereits ökologisch wirtschaftenden Landwirte wurden in einer offenen Frage nach den Gründen gefragt, die zur Umstellung ihres Betriebes geführt hatten. Von 63 Betriebsleitern, die diese Frage beantworteten, nannten $63 \%$ ausschließlich Gründe wie bspw. die eigene Weltanschauung, den Naturschutz, die Produktion gesünderer Nahrungsmittel oder ein Wirtschaften ohne Einsatz chemischer Betriebsmittel. Dem gegenüber stellten nur $21 \%$ der Befragten ihren Betrieb ausschließlich aus betriebswirtschaftlichen Gründen um. In diesem Zusammenhang wurden Argumente wie etwa die profitablere Nutzung schwächerer Standorte genannt, die im Kontext höherer Fördermittel oder von Vorteilen bei der Vermarktung zu sehen sind. Die restlichen $16 \%$ der Befragten gaben jeweils sowohl betriebswirtschaftliche als auch ideelle Gründe für ihre Betriebsumstellung an. Daraus ergibt sich eine generell stark ausgeprägte ideologische Orientierung der Probanden in der Stichprobe.

Hinsichtlich der bewirtschafteten Betriebe ergab sich durch die sehr unterschiedlichen und räumlich weit auseinander liegenden Durchführungsorte ein sehr heterogenes Bild. Während die Betriebe in den Experimenten in Schleswig-Holstein und Mecklenburg-Vorpommern flächenmäßig relativ groß waren, bewirtschafteten die Probanden der übrigen Experimente deutlich kleinflächigere Betriebe. Daher ist auch der Durchschnitt der bewirtschafteten Fläche von 298 ha aufgrund der hohen Standardabweichung von 652 ha kaum aussagekräftig. Tabelle 28 zeigt deshalb Mittelwerte und Standardabweichungen der bewirtschafteten Fläche der Betriebe unterteilt nach den einzelnen Experimenten.

Tabelle 28: Charakterisierung der teilnehmenden Betriebe

\begin{tabular}{|l|r|r|r|}
\hline \multirow{2}{*}{ Ort des Experiments } & \multirow{2}{*}{ Anzahl der Teilnehmer } & \multicolumn{2}{|c|}{ Bewirtschaftete Fläche in ha } \\
\cline { 3 - 4 } & & Mittelwert & Standardabweichung \\
\hline Schleswig-Holstein & 12 & 299,33 & 372,96 \\
\hline Hessen & 10 & 75,20 & 37,86 \\
\hline Nordrhein-Westfalen & 13 & 52,85 & 32,86 \\
\hline Mecklenburg-Vorpommern & 15 & 1003,53 & 1153,89 \\
\hline Rheinland-Pfalz & 10 & 65,10 & 47,65 \\
\hline Baden-Württemberg & 12 & 60,83 & 25,27 \\
\hline
\end{tabular}

Quelle: Eigene Darstellung 
Die große Heterogenität der Stichprobe beruht auf der real existierenden nicht minder großen Heterogenität der ökologischen Betriebe in Deutschland. Trotzdem ist darauf hinzuweisen, dass die Stichprobe keinesfalls als repräsentativ hinsichtlich des ökologischen Landbaus in Deutschland angesehen werden kann. Ziel bei der Auswahl der Stichprobe war vielmehr, die gesamte Bandbreite der vorherrschenden Betriebsgrößen in die Stichprobe aufzunehmen, um mögliche unterschiedliche Verhaltensmuster hinsichtlich des Prämienexperiments untersuchen zu können. Ähnlich groß wie die Unterschiede bezüglich der bewirtschafteten Fläche waren auch die Unterschiede hinsichtlich der Tierbestände. Zur grundsätzlichen Einordnung des Betriebes wurde im Fragebogen nach der betriebswirtschaftlichen Ausrichtung (BWA) gefragt. Zum besseren Verständnis wurde den Probanden dabei das Einteilungsschema gemäß der Zusammensetzung des gesamtbetrieblichen Standarddeckungsbeitrags erläutert (vgl. dazu SAUER et al., 2004: 8ff). Danach handelte es sich bei 45,8\% der Betriebe um Futterbaubetriebe, bei 23,6\% der Betriebe um Ackerbaubetriebe und bei $29,2 \%$ der Betriebe um Verbundbetriebe. Lediglich ein Veredelungsbetrieb befand sich unter den teilnehmenden Betrieben. Der geringe Anteil der Veredelung in der Stichprobe entspricht dem geringen Anteil der Veredelungsbetriebe innerhalb des gesamten Ökolandbaus in Deutschland. Diese Aufteilung in Futterbau- und Ackerbaubetriebe ergibt sich insbesondere aus dem hohen Anteil an Futterbaubetrieben in Mecklenburg-Vorpommern sowie in den Mittelgebirgslagen Hessens und NordrheinWestfalens. Dem gegenüber ist in Schleswig-Holstein der Anteil der Ackerbaubetriebe relativ hoch.

Die Experimente in Hessen und in Nordrhein-Westfalen wurden in landwirtschaftlich tendenziell benachteiligten Regionen durchgeführt. Daraus resultiert ein Anteil von knapp $28 \%$ an Nebenerwerbsbetrieben in der Stichprobe. $42 \%$ der Probanden bewirtschafteten ihren Betrieb im Haupterwerb. Weitere $15 \%$ leiteten eine GbR. Bei den restlichen Betriebsleitern handelte es sich zumeist um solche von Genossenschaften und Kapitalgesellschaften. Unter allen Rechtsformen betrug der Anteil der Direktvermarkter in der Stichprobe $30 \%$.

Das durchschnittliche Alter der Befragten betrug 45 Jahre. Bezüglich des Ausbildungsstands wurde jeweils der höchste Abschluss berücksichtigt. Er erscheint in der Stichprobe generell recht hoch. Bei $18 \%$ der Probanden handelte es sich um staatlich geprüfte Landwirte bzw. staatlich geprüfte Agrarbetriebswirte. Weitere $18 \%$ haben die landwirtschaftliche Meisterprüfung abgelegt und $33 \%$ haben ein landwirtschaftliches Studium absolviert. Lediglich $18 \%$ der Probanden gaben an, überhaupt keine landwirtschaftliche Ausbildung 
absolviert zu haben. Dabei handelte es sich zur einen Hälfte um Nebenerwerbslandwirte und zur anderen Hälfte um im Vergleich zum Durchschnitt ältere Landwirte.

Die Zufriedenheit der Landwirte mit der Bewirtschaftungsweise „Ökologischer Landbau“ war unter den Teilnehmern insgesamt sehr hoch. Knapp 85 \% der Probanden gaben an, wieder auf ökologischen Landbau umzustellen, wenn sie die Möglichkeit hätten, die Zeit zurückzudrehen und sich nochmals entscheiden müssten. Nur $7 \%$ waren unzufrieden mit ihrer beruflichen Situation. Sie gaben an, in der gegebenen Situation auf keinen Fall wieder auf Ökolandbau umzustellen. Bezüglich der Frage nach einer persönlichen Einschätzung der Zukunft des ökologischen Landbaus ergab sich ein ähnliches jedoch leicht abgeschwächt positives Bild. Knapp 60 \% der Befragten gaben sich hinsichtlich der Zukunft des ökologischen Landbaus in Deutschland optimistisch. $22 \%$ wagten keine genaue Prognose und standen dieser Frage folglich unentschlossen gegenüber. Nur etwa $18 \%$ sahen die Zukunft des ökologischen Landbaus pessimistisch.

Tabelle 29: Evaluierung der unternehmerischen Orientierung der Teilnehmer

\begin{tabular}{|c|c|c|c|c|c|c|c|}
\hline \multirow[t]{2}{*}{$n=72$} & \multirow{2}{*}{$\begin{array}{l}\text { Mittel- } \\
\text { wert }\end{array}$} & \multirow[t]{2}{*}{ StAbw } & \multicolumn{3}{|c|}{$\begin{array}{l}\text { Lehne voll- } \\
\text { ständig ab }\end{array}$} & & \multirow{2}{*}{$\begin{array}{r}\begin{array}{r}\text { Stimme voll- } \\
\text { ständig zu }\end{array} \\
2\end{array}$} \\
\hline & & & -2 & -1 & 0 & & \\
\hline $\begin{array}{l}\text { Mir ist die Zeit am Schreibtisch } \\
\text { genauso wichtig wie die tägliche } \\
\text { Arbeit im Betrieb. }\end{array}$ & 0,10 & 1,30 & $\begin{array}{c}1 \\
1 \\
1 \\
1\end{array}$ & i & & & $\begin{array}{c}T_{1} \\
1 \\
1 \\
1\end{array}$ \\
\hline $\begin{array}{l}\text { Ich riskiere auch mal Dinge, die } \\
\text { eventuell scheitern können. }\end{array}$ & 0,76 & 0,76 & $\begin{array}{l}1 \\
1 \\
1\end{array}$ & i & I & & $\begin{array}{l}1 \\
1 \\
1\end{array}$ \\
\hline $\begin{array}{l}\text { Ich bin an technologischen } \\
\text { Neuerungen interessiert. }\end{array}$ & 1,08 & 0,71 & i & i & i & & i \\
\hline $\begin{array}{l}\text { Bei der Umsetzung geplanter } \\
\text { gesetzlicher Bestimmungen sehe } \\
\text { ich mich als Vorreiter. }\end{array}$ & $-0,25$ & 0,83 & $\begin{array}{l}1 \\
1 \\
1 \\
1 \\
1\end{array}$ & $\begin{array}{c}1 \\
1 \\
1 \\
1 \\
1\end{array}$ & & & $\begin{array}{l}1 \\
1 \\
1 \\
1 \\
1\end{array}$ \\
\hline $\begin{array}{l}\text { Der Betriebsleiter sollte seine } \\
\text { betriebswirtschaftlichen } \\
\text { Kennzahlen aus dem Stehgreif } \\
\text { kennen. }\end{array}$ & 0,90 & 0,73 & $\begin{array}{c}1 \\
1 \\
1 \\
1 \\
1 \\
1\end{array}$ & $\begin{array}{l}1 \\
1 \\
1 \\
1 \\
1 \\
1\end{array}$ & $\begin{array}{c}1 \\
1 \\
1 \\
1 \\
1\end{array}$ & & $\begin{array}{c}1 \\
1 \\
1 \\
1 \\
1 \\
1\end{array}$ \\
\hline $\begin{array}{l}\text { Um meine Zukunft mache ich mir } \\
\text { keine Sorgen, da sich gute } \\
\text { Unternehmen durchsetzen werden. }\end{array}$ & 0,44 & 0,96 & 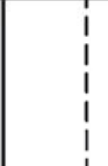 & $\begin{array}{l}1 \\
1 \\
1 \\
1\end{array}$ & $\begin{array}{l}1 \\
1 \\
1 \\
1 \\
1\end{array}$ & 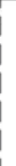 & $\begin{array}{c}1 \\
1 \\
1 \\
1 \\
1\end{array}$ \\
\hline $\begin{array}{l}\text { Ich verbringe sehr viel Zeit an der } \\
\text { Planung meines Betriebes. }\end{array}$ & 0,63 & 0,74 & i & i & i & & i \\
\hline $\begin{array}{l}\text { Ich beobachte den Markt sehr } \\
\text { genau und passe mich frühzeitig } \\
\text { an sich verändernde } \\
\text { Marktbedingungen an. }\end{array}$ & 0,64 & 0,77 & i & $\begin{array}{l}i_{1} \\
1 \\
1 \\
i\end{array}$ & $\begin{array}{l}1 \\
1 \\
1 \\
1 \\
1\end{array}$ & 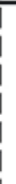 & i \\
\hline
\end{tabular}

Quelle: Eigene Darstellung 
In einem abschließenden Block wurde einerseits die unternehmerische Orientierung der Betriebsleiter beleuchtet (vgl. Tabelle 29). Dabei ergab sich gegenüber technologischen Neuerungen eine verbreitete Aufgeschlossenheit. Die Relevanz der Betriebswirtschaft wurde innerhalb der Gruppe sehr heterogen beurteilt.

Andererseits sollte jeder Betriebsleiter seinen Betrieb hinsichtlich seiner Stärken und Schwächen gewichten (vgl. Tabelle 30).

Tabelle 30: Stärken-Schwächen-Profil der Teilnehmer hinsichtlich ihrer landwirtschaftlichen Tätigkeit

\begin{tabular}{|c|c|c|c|c|c|c|c|}
\hline \multirow[t]{2}{*}{ Wir ... } & \multirow{2}{*}{$\begin{array}{l}\text { Mittel- } \\
\text { wert }\end{array}$} & \multirow[t]{2}{*}{ StAbw } & \multicolumn{4}{|c|}{$\begin{array}{l}\text { Lehne voll- } \\
\text { ständig ab }\end{array}$} & \multirow{2}{*}{$\begin{array}{l}\begin{array}{l}\text { Stimme voll- } \\
\text { ständig zu }\end{array} \\
2\end{array}$} \\
\hline & & & -2 & -1 & 0 & 1 & \\
\hline $\begin{array}{l}\text { sind besonders gut in der } \\
\text { Produktion (hohe Erträge...). }\end{array}$ & 0,54 & 0,77 & i & $i_{1}$ & $i_{1}$ & 1 & 1 \\
\hline $\begin{array}{l}\text { produzieren besonders gute } \\
\text { Qualität. }\end{array}$ & 0,85 & 0,73 & i & $i_{1}^{1}$ & i & i & i \\
\hline $\begin{array}{l}\text { produzieren zu besonders } \\
\text { niedrigen Kosten. }\end{array}$ & 0,38 & 0,88 & 1 & $i_{1}^{1}$ & $i_{1}$ & i & 1 \\
\hline $\begin{array}{l}\text { erzielen besonders gute Preise am } \\
\text { Markt. }\end{array}$ & 0,58 & 0,76 & I & $i_{1}$ & i & 1 & 1 \\
\hline $\begin{array}{l}\text { nutzen besonders intensiv } \\
\text { Fördergelder. }\end{array}$ & 0,47 & 0,89 & i & i & $i_{1}^{1}$ & 1 & i \\
\hline $\begin{array}{l}\text { haben besonders lukrative } \\
\text { Marktnischen besetzt. }\end{array}$ & 0,17 & 0,87 & i & i & & 1 & i \\
\hline $\begin{array}{l}\text { verfügen über eine optimale } \\
\text { Betriebsorganisation } \\
\text { (Arbeitsverteilung, } \\
\text { Maschinenauslastung ....). }\end{array}$ & 0,51 & 0,89 & i & $i_{i}$ & i & $\begin{array}{c}1 \\
1 \\
1 \\
1 \\
1 \\
1\end{array}$ & i \\
\hline
\end{tabular}

Quelle: Eigene Darstellung

Beim Stärken-Schwächen-Profil zeigt sich insbesondere der Qualitätsaspekt der produzierten Güter, gekoppelt mit entsprechenden Preisansprüchen als bedeutend. Die relativ geringe Ausrichtung auf Marktnischen ergibt sich vermutlich aus dem vergleichsweise geringen Anteil an Direktvermarktern in der Stichprobe.

\subsubsection{EINSCHÄTZUNGEN ZUR GEGENWÄRTIGEN FLÄCHENBEZOGENEN} FÖRDERUNG

Interessant erschien die Frage, welche Bedeutung die gegenwärtige flächenbezogene Förderung für die Wirtschaftlichkeit der Betriebe und für die Betriebsleiter insgesamt hat. Dazu konnten mit Hilfe einer Faktorenanalyse die Fragekonstrukte, die zur Bedeutung der Förderung führten, verdichtet werden. Die Faktorenanalyse dient der Komplexitätsreduktion 
und fasst Fragen, die zu gleichem Antwortverhalten geführt haben, zusammen (BACKHAUS et al. 2003: 260 ff.). Es wird von der Annahme ausgegangen, dass hinter einer Anzahl von manifesten Variablen ein latenter Faktor steht, der den zentralen Sachverhalt abbildet und damit die komplexe Struktur der Ausgangsdaten verdichtet (HÜTTNER und SCHWARTING 1999: 383). Als Maß zur Beurteilung der Eignung einer Stichprobe für eine Faktorenanalyse dient das KMO-Kriterium (nach Kaiser-Meyer-Olkin). Es gibt an, inwieweit die Ausgangsvariablen zusammengehören (BACKHAUS et al. 2003: 276f.). Die KMO-Werte können zwischen 0 und 1 liegen. Die Auswahl der zur Beurteilung der Relevanz der Förderung sinnvoll erscheinenden Fragekonstrukte ergab einen KMO-Wert von 0,715, was als ,ziemlich gut““ (middeling) zu bezeichnen ist (BACKHAUS et al. 2003: 276). Die erklärte Gesamtvarianz beträgt 72,167 \%. Um die Zuverlässigkeit des gebildeten Faktors beurteilen $\mathrm{zu}$ können, wurde eine Reliabilitätsanalyse durchgeführt. Die Reliabilitätsanalyse ist ein Verfahren, bei dem überprüft wird, inwieweit die Zusammenfassung einer Anzahl manifester Merkmale dazu geeignet ist, das latente Merkmal zuverlässig (reliabel) abzubilden. Ein Verfahren zur Berechnung der Reliabilität ist das Cronbach'sche Alpha. Der Wertebereich für Cronbach's Alpha liegt ebenfalls zwischen 0 und 1. Für den gebildeten Faktor „Relevanz der Förderung“ ergab sich ein Wert von 0,801. Als Faustregel wird angenommen, dass ein Wert größer 0,7 auf eine zuverlässige Abbildung hindeutet. Tabelle 31 zeigt Mittelwerte, Standardabweichungen und die Faktorladungen der einzelnen Fragekonstrukte:

Tabelle 31: Relevanz der Förderung

\begin{tabular}{|c|c|c|c|}
\hline $\mathbf{n}=\mathbf{7 0}$ & Mittelwert & StAbw & Faktorladung \\
\hline${ }^{1}$ Wir nutzen besonders intensiv Fördergelder. & $\mathbf{0 , 4 7}$ & $\mathbf{0 , 8 9}$ & $\mathbf{0 , 8 5 0}$ \\
\hline $\begin{array}{l}{ }^{1} \text { Bei der zukünftigen Ausrichtung meines Betriebes } \\
\text { spielen Fördermittel eine entscheidende Rolle. }\end{array}$ & 0,92 & 1,08 & 0,849 \\
\hline $\begin{array}{l}{ }^{2} \text { Welche Bedeutung messen Sie den gegenwärtigen } \\
\text { flächenbezogenen Öko-Prämien für die Wirtschaftlichkeit } \\
\text { Ihres Betriebes zu? }\end{array}$ & 0,97 & $\mathbf{0 , 8 8}$ & $\mathbf{0 , 8 5 0}$ \\
\hline \multicolumn{4}{|c|}{$\begin{array}{l}{ }^{1} \text { Skala }=+2: \text { Stimme vollständig zu, }+1: \text { Stimme zu, 0: weder noch, }-1: \text { Lehne ab, }-2: \text { Lehne vollständig ab } \\
{ }^{2} \mathrm{Skala}=+2 \text { : elementar wichtig, }+1 \text { : hohe Bedeutung, } 0 \text { : durchschnittliche Bedeutung, }-1 \text { : geringe Bedeutung, } \\
\text { 2: völlig unwichtig } \\
\text { Faktorenanalyse: KMO }=0,715 \text {; Erklärte Varianz }=72,167 \% \text {; Cronbach's Alpha }=0,801\end{array}$} \\
\hline
\end{tabular}

Quelle: Eigene Darstellung

Die Bedeutung der flächenbezogenen Förderprämien für die Wirtschaftlichkeit der Betriebe wird von den Betriebsleitern als hoch angesehen. Die Beobachtung einer hohen Abhängigkeit der Wirtschaftlichkeit von den Fördermitteln deckt sich mit vergleichbaren Untersuchungen aus der Vergangenheit (vgl. dazu NieBerg, 2004: 6). Die hohe Bedeutung der Prämien für die 
Wirtschaftlichkeit wird vermutlich auch der Grund dafür sein, warum sich eine deutliche Mehrzahl der Teilnehmer trotz der vorrangig ideologischen Prägung intensiv mit den Fördermöglichkeiten auseinandersetzt und auch die Ausrichtung des Betriebs von den Fördermöglichkeiten abhängig macht.

Weiter wurde gefragt, wie nach Meinung der Landwirte mit der Förderung des Ökolandbaus in der Zukunft weiter verfahren werden sollte. Zur Auswahl standen die drei Antwortmöglichkeiten:

- Die Förderung sollte abgebaut werden.

- Die Förderung sollte verändert werden.

- Die Förderung sollte so beibehalten werden.

Dabei entschieden sich mit mehr als $47 \%$ der Teilnehmer die meisten für die Beibehaltung der gegenwärtigen Förderung. Etwa $40 \%$ gaben an, die Förderung solle verändert werden. Der Meinung, dass die Förderung abgebaut werden sollte, waren nur etwa 8 \%. Eine Person konnte sich für keine der drei Alternativen entscheiden. Dieses Entscheidungsverhalten zeigt, dass etwa die Hälfte der Teilnehmer mit der gegenwärtigen Situation zufrieden ist. Speziell im Falle der letzten beiden Antwortmöglichkeiten wurde weitergehend nach den Hintergründen der Entscheidung gefragt. Die Forderung nach einem Abbau der Förderung wird vielfach begründet mit der Erwartung steigender Preise, einer geringeren Abhängigkeit von der Politik sowie einem erwarteten Abbau der Bürokratie infolge des Abbaus der Förderung. Hinsichtlich der zweiten Antwortmöglichkeit wurde speziell nach Vorschlägen für eine Modifikation der Förderung gefragt. Von 29 Landwirten, die für eine Veränderung der Förderung votiert hatten, sprachen sich sechs Landwirte und damit die größte Einzelgruppe für eine Kopplung der Förderung an die im Betrieb beschäftigten Arbeitskräfte aus. Weitere wiederholt genannte Vorschläge waren eine stärkere Kopplung der Förderung an die tatsächlichen positiven Umwelteffekte der ökologischen Wirtschaftsweise (ergebnisorientierter Ansatz anstatt des gegenwärtigen handlungsbezogenen Ansatzes) sowie eine Degression der Fördersätze für besonders flächenstarke Betriebe. Vier Landwirte votierten für eine im Vergleich zum gegenwärtigen Niveau höhere Förderung. Aus der Frage nach Vorschlägen für eine zukünftige Modifikation der Förderung ergibt sich die grundsätzliche Frage, wie die Landwirte die Zukunft der flächenbezogenen Förderung einschätzen. Die folgende Tabelle 32 zeigt diesbezüglich das Antwortverhalten der Teilnehmer. 
Tabelle 32: Beurteilung der Zukunft der Öko-Förderung

\begin{tabular}{|c|c|c|c|c|c|c|c|}
\hline \multirow[t]{2}{*}{$n=72$} & \multirow{2}{*}{$\begin{array}{c}\text { Mittel- } \\
\text { wert }\end{array}$} & \multirow[t]{2}{*}{ StAbw } & \multicolumn{4}{|c|}{$\begin{array}{l}\text { Lehne voll- } \\
\text { ständig ab }\end{array}$} & \multirow{2}{*}{$\begin{array}{c}\text { Stimme voll- } \\
\text { ständig zu }\end{array}$} \\
\hline & & & -2 & -1 & 0 & 1 & \\
\hline $\begin{array}{l}\text { Ich bin davon überzeugt, dass die } \\
\text { derzeitigen Öko-Flächenprämien } \\
\text { zukünftig auch bei knapperen } \\
\text { öffentlichen Haushalten nicht in } \\
\text { Gefahr sind. }\end{array}$ & $-0,18$ & 1,23 & i & i & 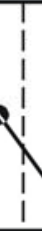 & $\begin{array}{l}1 \\
1 \\
1 \\
1\end{array}$ & i \\
\hline $\begin{array}{l}\text { Das gegenwärtige Fördersystem } \\
\text { muss reformiert werden. } \\
\text { Ansonsten wird die Förderung in } \\
\text { Zukunft in Gefahr sein. }\end{array}$ & 0,85 & 0,97 & i & i & $\begin{array}{l}\mathrm{i} \\
\mathrm{i} \\
\mathrm{i} \\
\mathrm{i}\end{array}$ & i & i \\
\hline
\end{tabular}

Quelle: Eigene Darstellung

Mit einem Mittelwert von 0,85 waren die Landwirte davon überzeugt, dass eine Reform des gegenwärtigen Fördersystems notwendig ist, um die Zukunft der Förderung zu sichern. Allerdings gab es auch Teilnehmer, die entgegen aller Verlautbarungen in der Öffentlichkeit, in ihrem Glauben an einen ungefährdeten Fortbestand der Flächenprämien auf dem gegenwärtigen Niveau weiterhin unbeirrt festhalten.

\subsubsection{UNTERSUCHUNG DER AKZEPTANZ FÜR DIE FLEXIBLE FöRDERUNG}

Mit Hilfe des Prämienexperiments wurde die Akzeptanz der Teilnehmer der Untersuchung für die Flexible Förderung untersucht. Dazu sollen zunächst Vorüberlegungen zum zu erwartenden Entscheidungsverhalten der Teilnehmer angestellt werden, um danach die Ergebnisse der Untersuchung detailliert vorzustellen.

\subsubsection{VORÜBERLEGUNGEN ZUM ENTSCHEIDUNGSVERHALTEN DES LANDWIRTS IM PRÄMIENEXPERIMENT}

Bei der Entscheidung für ein Prämiensystem gilt es für die Landwirte folgendes zu bedenken: Bei der bestehenden Festbetragsprämie weiß der Landwirt sehr genau, wie viel Prämie er erhalten wird. Sie ergibt sich durch einfache Multiplikation des Prämiensatzes mit der bewirtschafteten Fläche und ist damit lediglich von dieser abhängig. Die Flexible Förderung gründet sich auf der Schwankung der Einkünfte aus LuF. Diese Einkünfte sind aber nicht im Voraus bekannt. Deshalb ist die Förderung für den Landwirt nur schwer abzuschätzen. Der Landwirt hat deshalb die Auswahl zwischen einer sicheren und einer unsicheren Alternative.

Zur Möglichkeit der Einschätzung der unsicheren Alternative, d.h. der Flexiblen Prämie, sei nochmals folgendes in Erinnerung gerufen: Gemäß der in Anhang 5 einzusehenden 
Experimentinstruktionen verpflichtet sich der Landwirt, wie bei Agrarumweltmaßnahmen derzeitig in der Praxis üblich, für die nächsten fünf Jahre für eine der beiden Förderalternativen. Zum Zeitpunkt der Entscheidung im Experiment sind dem Landwirt die Einkünfte aus LuF der vergangenen vier Jahre bekannt. Da sich die Flexible Förderung des kommenden Jahres an den vier Einkünften aus LuF der vergangenen vier Jahre zuzüglich der Einkünfte aus LuF des kommenden Jahres orientiert (also eine Fünfjahresdurchschnittsbetrachtung unterstellt wird), kann sie für das folgende Jahr in gewissen Grenzen vorausgesagt werden. Für das darauf folgende Jahr wird eine Prognose zum Zeitpunkt der Antragstellung schon schwieriger, weil zum Zeitpunkt der Entscheidung nun nur noch drei Jahre bekannt sind, die in die Berechnung eingehen. Für die Flexible Förderung des letzten Förderjahrs des 5-jährigen Verpflichtungszeitraums kann er zum Zeitpunkt der Antragstellung keine Aussage mehr treffen.

Der Landwirt muss anhand seiner historischen Einkünfte aus LuF sowie seiner grundsätzlichen Einstellung zur Flexiblen Förderung abwägen, ob er sich für die folgenden fünf Jahre für die sichere Festbetragsprämie und damit für die absolute Planungssicherheit entscheidet oder die unsichere Flexible Förderung wählt, die jedoch die Möglichkeit bietet insbesondere in Jahren mit hohen Einkunftsschwankungen die Förderung zu optimieren, d.h. dem Einkommensrisiko entgegen zu wirken.

\subsubsection{Methodik Der AusWertung Des PrÄMIENEXPERIMENTS}

Bei der Auswertung des Prämienexperiments wurde ein zweistufiges Auswertungsschema angewandt. Die folgenden Fragen standen dabei grundsätzlich im Mittelpunkt:

- Hat der Spieler die Funktionsweise der Flexiblen Förderung verstanden? $(\rightarrow$ Verständnis)

- Ist die Flexible Förderung für den Spieler eine wirkliche Alternative zum gegenwärtigen Festbetragssystem? ( $\rightarrow$ Akzeptanz)

Als Ergebnis des Prämienexperiments liegen von jedem Teilnehmer acht Entscheidungen für oder gegen die Flexible Förderung vor dem Hintergrund unterschiedlicher historischer Gewinnverteilungen vor. Ziel der Auswertung ist es nun, aus diesem Datensatz Aussagen zur Akzeptanz der Teilnehmer abzuleiten. Abbildung 46 zeigt die Vorgehensweise bei der Analyse einer Einzelentscheidung innerhalb des Datensatzes, die im Folgenden ausführlich erläutert wird. 
Abbildung 46: Analyse des Entscheidungsverhaltens hinsichtlich der Akzeptanz des Probanden für die Flexible Förderung

Hat sich der Proband bei mindestens 5 seiner Entscheidungen im Sinne der Funktionsweise der Flexiblen Förderung plausibel entschieden?

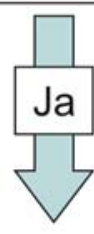

Anzahl der Entscheidungen für die Festbetragsprämie im Rahmen der „unplausiblen“ Entscheidungen:

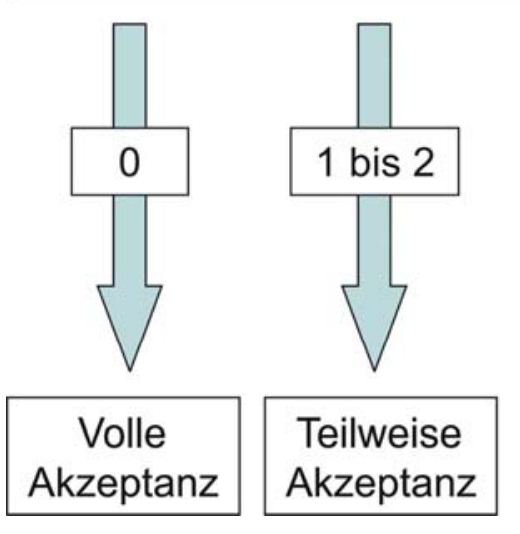

Quelle: Eigene Darstellung

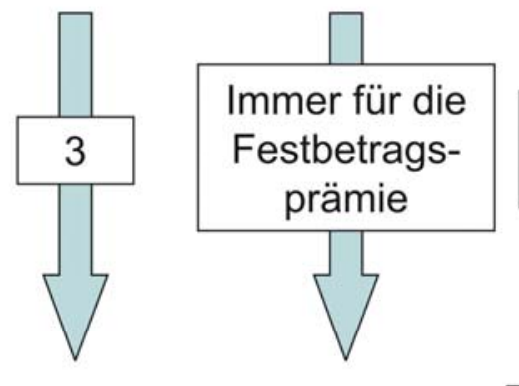

Keine Akzeptanz
Wofür hat sich der Proband im Rahmen der "unplausiblen“ Entscheidungen entschieden?

Dem Spieler liegt zu seiner Entscheidung die historische Gewinnverteilung seines 50 ha Ackerbaubetriebes vor, von der er resultierend aus dem Einführungsvortrag weiß, das sie neben den noch unbekannten zukünftigen Einkünften einen Teil die Bemessungsgrundlage für die zukünftige Flexible Förderung bildet. Handelt der Teilnehmer rational, so wird er sich bei seiner Entscheidung an der Schwankung der LuF-Einkünfte der letzten vier Jahre orientieren und somit plausibel im Sinne der Funktionsweise der Flexiblen Förderung agieren. Es gibt jedoch auch Gründe, warum das nicht unbedingt bei jedem Teilnehmer der Fall sein muss:

- Der Spieler hat die Funktionsweise der Flexiblen Förderung mit ihrer Anknüpfung an die historischen Einkünfte aus LuF nicht verstanden. In diesem Fall orientiert sich der Spieler entweder überhaupt nicht an den Angaben auf dem Bildschirm oder er orientiert sich ausschließlich an Angaben, die keinen direkten Einfluss auf die Flexible Förderung der folgenden Jahre haben, wie etwa die auf dem Entscheidungsbildschirm angegebene imaginäre historische Flexible Förderung oder die Historie der eigenen Entscheidungen.

- Der Spieler hat sich bereits während des Einführungsvortrags fest für eine der beiden Förderalternativen entschieden. Hat der Spieler bspw. generell eine tiefe Abneigung 
gegen die Konzeption oder die Funktionsweise der Flexiblen Prämie, so hat er sich bereits vor Beginn des eigentlichen Experiments für die Festbetragsprämie entschieden. Im umgekehrten Fall wäre es denkbar, dass der Teilnehmer von der Flexiblen Förderung so überzeugt ist, dass er sich schon vor Beginn des Experiments für die Flexible Förderung entschieden hat. In beiden Fällen ist also die Entscheidung ohne Orientierung an den historischen Einkünften, d.h. an der eigentlichen Bemessungsgrundlage der Flexiblen Förderung, getroffen worden.

In einem ersten Analyseschritt ist der Datensatz deshalb daraufhin untersucht worden, wie viele Teilnehmer unter Berücksichtigung ihrer Ausgangsbedingungen eine plausible Entscheidung im Sinne der Funktionsweise der Flexiblen Förderung getroffen haben. Dazu wurde zu jeder Entscheidung der Variationskoeffizient ${ }^{33}$ der vier dem Spieler zum Zeitpunkt der Entscheidung bekannten historischen Einkünfte gebildet. Bei der Prüfung auf Plausibilität der Entscheidung wurde nun überprüft, ob die vom Teilnehmer getroffene Entscheidung der im Sinne des Variationskoeffizienten der historischen Einkünfte plausiblen Entscheidung entspricht. Die genaue Vorgehensweise dazu ist in Tabelle 33 beispielhaft dargestellt.

\section{Tabelle 33: Darstellung der Plausibilitätsberechnung am Beispiel der Entscheidungssituation aus Abbildung 42}

\begin{tabular}{|c|c|c|c|c|}
\hline & $\begin{array}{l}\text { Einkünfte aus Land- } \\
\text { und Forstwirtschaft }\end{array}$ & Mittelwert & $\begin{array}{l}\text { Standard- } \\
\text { abweichung }\end{array}$ & $\begin{array}{l}\text { Variations- } \\
\text { koeffizient }\end{array}$ \\
\hline 4. Jahr & $29.493 €$ & \multirow{4}{*}{$26.081 €$} & \multirow{4}{*}{2.692} & \multirow{4}{*}{0,10} \\
\hline 5. Jahr & $26.926 €$ & & & \\
\hline 6. Jahr & $24.347 €$ & & & \\
\hline 7. Jahr & $23.556 €$ & & & \\
\hline \multicolumn{4}{|c|}{ Mittlerer empirischer Variationskoeffizient*: } & 0,255 \\
\hline \multicolumn{5}{|c|}{$\begin{array}{c}\text { Bewertung der Entscheidungssituation: } \\
\text { Die Einkünfte unterliegen nur vergleichsweise geringen Schwankungen. } \\
\text { Daher wäre die Wahl der Festbetragsförderung plausibel. }\end{array}$} \\
\hline \multicolumn{5}{|c|}{ Entscheidung des Teilnehmers: Festbetragsförderung } \\
\hline \multicolumn{5}{|c|}{$\begin{array}{l}\text { Bewertung der Entscheidung des Spielers: } \\
\text { hmer hat sich in dieser Spielrunde plausibel entschieden. }\end{array}$} \\
\hline
\end{tabular}

*bei der unterstellten Dreiecksverteilung der Einkünfte aus LuF

(Mittelwert: $23.000 €$; max. Abweichung: $15.000 €$ )

Quelle: Eigene Darstellung

\footnotetext{
${ }^{33}$ Bei dem Variationskoeffizient $(\mathrm{VC})$ handelt es sich um ein relatives Streuungsmaß $(\mathrm{VC}=\sigma / \mu)$, das den absoluten Streuungsmaßen Standardabweichung $(\sigma)$ und Varianz $\left(\sigma^{2}\right)$ vorgezogen wird, wenn Streuungen von Merkmalen verglichen werden sollen, die unterschiedliche Mittelwerte aufweisen (vgl. dazu auch MosLER und SCHMID, 2003: 42ff und 91ff)
} 
Für die acht Runden des Experiments wurde eine plausible Entscheidungsfindung des Teilnehmers angenommen, sofern er sich in fünf der acht Runden plausibel entschieden hat.

In einem zweiten Analyseschritt sollte nun versucht werden, die beiden gebildeten Gruppen weiter zu unterteilen und sie hinsichtlich ihrer Akzeptanz für die Flexible Förderung einzuordnen.

Dazu wurden im Falle der Landwirte, die sich in der Mehrzahl der Runden nicht plausibel verhalten haben, diese unplausiblen Entscheidungen zugrunde gelegt. Hinsichtlich der Bewertung der Akzeptanz wurden gemäß der bereits beschriebenen möglichen Hintergründe dieses Verhaltens wiederum drei Gruppen gebildet:

- Hat sich der Teilnehmer im Falle aller unplausiblen Entscheidungen für die Festbetragsprämie entschieden, so kann davon ausgegangen werden, dass bei ihm die Flexible Förderung keine Akzeptanz findet.

- Hat sich der Teilnehmer im Falle aller unplausiblen Entscheidungen für die Flexible Förderung entschieden, so kann davon ausgegangen werden, dass bei ihm die Flexible Förderung volle Akzeptanz findet.

- Hat sich der Teilnehmer bei seinen unplausiblen Entscheidungen in einigen Fällen für die Festbetragsprämie und in anderen Fällen für die Flexible Prämie entschieden, so kann davon ausgegangen werden, dass der Spieler das System der Flexiblen Förderung nicht verstanden hat. In diesen Fällen ist auf Basis des Prämienexperiments keine Aussage zur Akzeptanz der Flexiblen Förderung möglich.

Im Falle der Landwirte, die sich in der Mehrzahl der Entscheidungen unter Berücksichtigung ihrer Ausgangsbedingungen plausibel im Sinne der Funktionsweise der Flexiblen Förderung entschieden haben, kann schon im Vorfeld weiterer Untersuchungen angenommen werden, dass eine grundsätzliche Akzeptanz für die Flexible Förderung besteht. Im Rahmen des zweiten Analyseschrittes sollte diese Gruppe aber hinsichtlich der Intensität der Akzeptanz weiter untergliedert werden. Dazu wurde gemäß Abbildung 46 die Anzahl der Entscheidungen für die Festbetragsförderung im Rahmen der „unplausiblen“ Entscheidungen zugrunde gelegt. Hinsichtlich der Akzeptanz der Teilnehmer ergab sich die folgende Einteilung:

- Hat sich der Teilnehmer entweder in allen acht Entscheidungen plausibel im Sinne der Funktionsweise der Flexiblen Förderung entschieden oder hat er sich in seinen „unplausiblen“ Entscheidungen immer für die Flexible Förderung entschieden, so 
kann von einer vollen Akzeptanz des Teilnehmers für die Flexible Förderung ausgegangen werden.

- Hat sich der Teilnehmer in seinen ,unplausiblen“ Entscheidungen in maximal zwei Fällen für die Festbetragsförderung entschieden, so kann von einer teilweisen Akzeptanz des Teilnehmers für die Flexible Förderung ausgegangen werden.

- Hat sich der Teilnehmer in seinen „unplausiblen“ Entscheidungen in drei Fällen für die Festbetragsförderung entschieden, so kann davon ausgegangen werden, dass der Teilnehmer der Flexiblen Förderung ablehnend gegenübersteht, d.h. keine Akzeptanz zeigt.

Nachdem das Einteilungsschema zur Auswertung der Ergebnisse nun beschrieben worden ist, sollen im folgenden Kapitel die Ergebnisse dargestellt werden.

\subsubsection{ERGEBNISSE DES PRÄMIENEXPERIMENTS}

In die Auswertung des Prämienexperiments flossen die Entscheidungen aller 72 Teilnehmer ein. Abbildung 47 zeigt die Verteilung der Ergebnisse ${ }^{34}$ :

Abbildung 47: Ergebnisse des Prämienexperiments zur Untersuchung der Akzeptanz für die Flexible Förderung

\begin{tabular}{|cr|}
\hline \multicolumn{2}{|c|}{ Ergebnisse gemäß Einteilungsschema: } \\
Anzahl der Teilnehmer mit < 5 plausiblen Entscheidungen: & 13 \\
davon Teilnehmer mit allen unplausiblen Entscheidungen für die Festbetragsförderung: & 3 \\
davon Teilnehmer mit allen unplausiblen Entscheidungen für die Flexible Förderung: & 1 \\
davon Teilnehmer mit Entscheidungen sowohl für die Flexible als auch für die Festbetragsförderung: & 9 \\
& \\
Anzahl der Teilnehmer mit > 4 plausiblen Entscheidungen: \\
davon Teilnehmer mit keinen unplausiblen Entscheidungen für die Festbetragsförderung: \\
davon Teilnehmer mit 1 oder 2 unplausiblen Entscheidungen für die Festbetragsförderung: \\
davon Teilnehmer mit $\underline{3}$ unplausiblen Entscheidungen für die Festbetragsförderung: \\
\\
Auswertung der Ergebnisse: \\
Volle Akzeptanz: & 39 \\
Teilweise Akzeptanz: & $\mathbf{3 8}$ \\
Keine Akzeptanz: & $\mathbf{2 2}$ \\
Keine Aussage möglich: & $\mathbf{9}$ \\
Teilnehmer gesamt: & $\mathbf{7 2}$ \\
\hline
\end{tabular}

Quelle: Eigene Darstellung

\footnotetext{
${ }^{34}$ Für eine detaillierte Übersicht über die Ergebnisse sei an dieser Stelle auf Anhang 7 verwiesen.
} 
Die Ergebnisse zeigen, dass sich mit 38 von insgesamt 72 Teilnehmern am Prämienexperiment über 52 \% eindeutig für die Flexible Förderung entschieden haben. Hinzu kommen weitere 22 Teilnehmer, bei denen infolge ihrer Entscheidungen ebenfalls Akzeptanz zu vermuten ist, diese aber nicht so eindeutig ausfällt wie im Falle der erstgenannten Gruppe. Drei Teilnehmer haben sich im Experiment eindeutig für die gegenwärtige Festbetragsprämie entschieden. Bei neun Teilnehmern ist auf der Grundlage ihrer Entscheidungen im Experiment keine Aussage zur Akzeptanz möglich. Hier kann vermutet werden, dass Konzeption und Funktionsweise der Flexiblen Förderung vom Teilnehmer nicht verstanden worden sind oder bei ihm grundsätzlich kein Interesse an der Gesamtthematik ,Förderung des ökologischen Landbaus' bestand.

Bei dieser Auswertungsmethodik ist der Einfluss des historischen Erfolges bei der Wahl einer Förderalternative nicht berücksichtigt worden. Ein Teilnehmer, bei dem nach bspw. fünf gespielten Runden trotz sehr unterschiedlicher Ausgangssituationen im Nachhinein jedes Mal die Flexible Förderung vorteilhaft gewesen wäre, könnte sich in der sechsten Runde vermutlich auch für die Flexible Prämie entscheiden, wenn die Einkunftsschwankungen der vergangenen vier Jahre vergleichsweise gering sind. Diese Verhaltensweise dürfte vermutlich dem Verhalten vieler Landwirte in der Praxis entsprechen. Wer mit einem System lange Zeit die richtige Wahl getroffen hat, zeigt naturgemäß eine geringe Wechselbereitschaft. In gleicher Weise ist diese Situation auch für die Festbetragsförderung denkbar, wobei hier die Konsequenz erwartungsgemäß noch deutlicher ausfallen könnte. Der Grund dafür ist, dass es sich bei der Festbetragsprämie um das etablierte vertraute Fördersystem handelt. Eine in der Landwirtschaft tendenziell ausgeprägte Pfadabhängigkeit wird vermutlich dazu führen, dass sich bei Enttäuschungen mit der Flexiblen Prämie vergleichsweise schneller eine grundsätzliche Präferenz für die Festbetragsprämie ausbildet. Im Gegenzug ist allerdings zu bedenken, dass gemäß der Konzeption des Experiments der Erwartungswert der Flexiblen Förderung um $5 \%$ über der Festbetragsförderung liegt, was dazu führt, dass die Wahrscheinlichkeit, sich mit der Wahl der Flexiblen Förderung im Nachhinein richtig entschieden zu haben, höher ausfallen lässt. Zusammenfassend könnte man zu der Vermutung gelangen, dass die Anreize für die jeweiligen Förderinstrumente bei der 8jährigen Betrachtung relativ ausgeglichen sein könnten.

Ein anderer Faktor kann jedoch dazu führen, dass die Akzeptanz der Flexiblen Förderung überschätzt wird. Es ist bereits auf den monetären Anreiz für die Spieler eingegangen worden. Er ist gekoppelt an sein Prämienvolumen zum Ende der acht Spielrunden. Im Mittel wurden je Spieler $15 €$ ausbezahlt. Die Auszahlung wurde den Teilnehmern zu Beginn der Veranstaltung 
mit mindestens $10 €$ angekündigt. Gegenüber diesem Auszahlungsbetrag hätte die Entscheidung des Spielers in der Praxis für ihn deutlich höhere monetäre Konsequenzen. Bei dieser Diskrepanz handelt es sich um ein grundsätzliches Problem aller Experimente. Es wird zwar ein monetärer Anreiz geboten, der rationales Verhalten gewährleisten soll. Trotzdem besteht aber ein deutlicher Unterschied zur Entscheidung in der Praxis, der u.U. zur Konsequenz haben könnte, dass die Ernsthaftigkeit bei der Entscheidung verringert ist. Für das hier dargestellte Experiment könnte das bedeuten, dass der Spieler z.B. aus reiner Neugierde die Flexible Förderung ausprobieren möchte, was er bei einem höheren monetären Anreiz nicht so einfach machen würde. Dieser Effekt könnte im Gegensatz zum oben angesprochenen Effekt dazu führen, dass die Akzeptanz der Landwirte für die Flexible Förderung in der Auswertung überschätzt wird. Um diesen Effekt allerdings möglichst gering zu halten, wurden die Teilnehmer, wie auch aus den Experimentinstruktionen deutlich wird, direkt vor Beginn des Experiments nochmals gezielt darauf hingewiesen, sich bei ihrer Entscheidung soweit wie möglich in die vorgegebene Ausgangssituation hineinzuversetzen und genau so zu entscheiden, wie sie entscheiden würden, wenn sie wirklich in der gegebenen Situation wären.

Weitergehend erschien es interessant, einmal zu untersuchen, inwiefern sich die Teilnehmer mit voller Akzeptanz von den Teilnehmern ohne Akzeptanz hinsichtlich ihres Antwortverhaltens im Fragebogen unterscheiden. Da allerdings die Gruppe der Teilnehmer ohne Akzeptanz nur drei Landwirte umfasst, sind signifikante Zusammenhänge nicht nachweisbar. Es zeigt sich aber die klare Tendenz, dass die Teilnehmer mit Akzeptanz die Zukunft der flächenbezogenen Förderung realistischer einschätzen. Bei dieser Gruppe ist also die Erkenntnis, dass eine Modifikation der gegenwärtigen Förderung unerlässlich ist, um die Förderung insgesamt $\mathrm{zu}$ sichern, sehr viel weiter fortgeschritten. Das zeigt auch die Untersuchung der Frage nach der persönlichen Einstellung zur flächenbezogenen Förderung. Während die Teilnehmer ohne Akzeptanz zu $100 \%$ die Beibehaltung des gegenwärtigen Systems befürworten, sprechen sich die Teilnehmer mit Akzeptanz mehrheitlich für eine Veränderung des gegenwärtigen Systems aus.

\subsubsection{4 ÜBERTRAGBARKEIT DER ERGEBNISSE AUF DIE REALE SITUATION DER LANDWIRTE}

Die Landwirte haben sich im Prämienexperiment für einen Beispielbetrieb zwischen der Festbetragsförderung und der Flexiblen Förderung entscheiden müssen. Dadurch wurde die persönliche Situation der Probanden ausgeblendet und die Entscheidungssituation weitgehend standardisiert. Hinsichtlich der Beurteilung der Flexiblen Förderung ist aber auch die 
Entscheidung des Landwirts für seinen eigenen Betrieb interessant. Aus diesem Grund wurde eine entsprechende Frage in den Fragebogen aufgenommen:

- „Angenommen die Flexible Förderung stände für Ihren eigenen Betrieb im nächsten Jahr wirklich zur Auswahl. Für welche Prämienart würden Sie sich dann entscheiden?“"

Zur Auswahl standen äquivalent zum Prämienexperiment die gegenwärtige Festbetragsförderung sowie die Flexible Förderung, und zwar in der Ausgestaltung wie sie den Teilnehmern aus dem Prämienexperiment bekannt war. Die Auswertung dieser Frage ergab, dass sich von den 72 Teilnehmern 39 für die Festbetragsförderung und 33 für die Flexible Förderung entschieden. Damit zeigte sich im Gegensatz zu den Ergebnissen des Prämienexperiments insgesamt eine leichte Tendenz bei den Landwirten zur Festbetragsförderung. Dabei ist jedoch zu berücksichtigen, dass diese Entscheidung rational betrachtet richtig sein kann, weil die Einkünfte im eigenen Betrieb weniger stark schwanken. Bei der Untersuchung der statistischen Beziehung zwischen der Akzeptanz der Teilnehmer im Experiment und dem Antwortverhalten zur besagten Frage konnte eine erwartungsgemäß positive, jedoch nicht signifikante Korrelation zwischen beiden Faktoren ermittelt werden.

Tabelle 34: Beweggründe zur Auswahl der Festbetragsförderung für den eigenen Betrieb

\begin{tabular}{|c|c|c|c|c|c|c|}
\hline \multirow[t]{2}{*}{$\mathrm{n}=\mathbf{3 9}$} & \multirow{2}{*}{$\begin{array}{l}\text { Mittel- } \\
\text { wert }\end{array}$} & \multirow[t]{2}{*}{ StAbw } & \multicolumn{3}{|c|}{$\begin{array}{l}\text { Lehne voll- } \\
\text { ständig ab }\end{array}$} & \multirow{2}{*}{$\begin{array}{r}\begin{array}{r}\text { Stimme voll- } \\
\text { ständig zu }\end{array} \\
2\end{array}$} \\
\hline & & & -2 & -1 & 0 & \\
\hline $\begin{array}{l}\text { Die Flexible Prämie habe ich nicht } \\
\text { verstanden. }\end{array}$ & $-1,28$ & 0,94 & i & 1 & i & 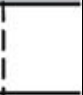 \\
\hline $\begin{array}{l}\text { Bei der Festbetragsprämie weiß } \\
\text { ich was ich habe. }\end{array}$ & 1,08 & 1,09 & i & 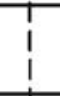 & i & 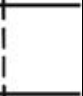 \\
\hline $\begin{array}{l}\text { Die Gewinne auf meinem Betrieb } \\
\text { unterlagen in den vergangenen } \\
\text { Jahren nur geringen } \\
\text { Schwankungen. }\end{array}$ & 1,28 & 0,83 & i & i & $i_{i}^{1}$ & 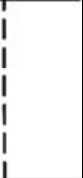 \\
\hline $\begin{array}{l}\text { Die Höhe der Auszahlung bei der } \\
\text { Flexiblen Prämie ist mir zu } \\
\text { unsicher. }\end{array}$ & 0,77 & 1,09 & i & i & i & 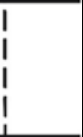 \\
\hline $\begin{array}{l}\text { Die Idee einer Kopplung der } \\
\text { Förderung an die } \\
\text { Einkommensschwankungen gefällt } \\
\text { mir nicht. }\end{array}$ & 0,67 & 1,18 & i & i & i & i \\
\hline
\end{tabular}

Quelle: Eigene Darstellung

Die Landwirte wurden weiter nach den Beweggründen für ihre Entscheidung gefragt. Dazu standen für beide Gruppen jeweils verschiedene Statements zur Bewertung. Tabelle 34 zeigt 
dazu die Befragungsergebnisse der Landwirte, die sich für ihren eigenen Betrieb für die Festbetragsförderung entschieden hatten:

Es zeigt sich, dass nicht die Verständlichkeit des neuen Fördersystems, sondern in erster Linie das Wissen über geringe Schwankungen im eigenen Betrieb zur Ablehnung der Flexiblen Förderung geführt haben könnte. Das erklärt auch die Diskrepanz im Entscheidungsverhalten zwischen dem Prämienexperiment und der Entscheidung für den eigenen Betrieb. Allerdings spielen auch die Vertrautheit mit dem gegenwärtigen System (Pfadabhängigkeit) und damit eng verbunden die Unsicherheit der Prämienhöhe bei der Flexiblen Förderung eine entscheidende Rolle. Dagegen hat die grundsätzliche Einstellung zu einer Förderung, die sich an den Einkunftsschwankungen orientiert, insgesamt weiniger Einfluss. Die hohe Standardabweichung zeigt jedoch, dass bei diesem Faktor die Ansichten am stärksten auseinander liegen.

Die nachfolgende Tabelle 35 zeigt die Befragungsergebnisse der Landwirte, die sich für ihren eigenen Betrieb für die Flexible Förderung entschieden hatten:

Tabelle 35: Beweggründe zur Auswahl der Flexiblen Förderung für den eigenen Betrieb

\begin{tabular}{|c|c|c|c|c|c|c|c|}
\hline \multirow[t]{2}{*}{$\mathrm{n}=\mathbf{3 3}$} & \multirow{2}{*}{$\begin{array}{c}\text { Mittel- } \\
\text { wert }\end{array}$} & \multirow[t]{2}{*}{ StAbw } & \multicolumn{3}{|c|}{$\begin{array}{l}\text { Lehne voll- } \\
\text { ständig ab }\end{array}$} & & \multirow{2}{*}{$\begin{array}{l}\begin{array}{r}\text { Stimme voll- } \\
\text { ständig zu }\end{array} \\
2\end{array}$} \\
\hline & & & -2 & -1 & 0 & 1 & \\
\hline $\begin{array}{l}\text { Bei der Flexiblen Förderung habe } \\
\text { ich die Chance auf eine höhere } \\
\text { Auszahlung. }\end{array}$ & 1,12 & 0,70 & 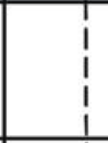 & $i_{i}^{1}$ & $\begin{array}{r}1 \\
1 \\
1 \\
1 \\
\end{array}$ & |ه & $\begin{array}{c}1 \\
1 \\
1 \\
1 \\
\end{array}$ \\
\hline $\begin{array}{l}\text { Die Gewinne auf meinem Betrieb } \\
\text { unterlagen in der Vergangenheit } \\
\text { starken Schwankung. }\end{array}$ & 1,09 & 0,72 & i & i & i & & $\begin{array}{c}1 \\
1 \\
1 \\
1 \\
1\end{array}$ \\
\hline $\begin{array}{l}\text { Ich stehe neuen Sachen } \\
\text { grundsätzlich immer } \\
\text { aufgeschlossen gegenüber. }\end{array}$ & 0,67 & 0,92 & i & $i_{1}^{1}$ & i & I & $i$ \\
\hline $\begin{array}{l}\text { Es wäre für mich einfach, meine } \\
\text { jährlichen Gewinne stärker } \\
\text { schwanken zu lassen, um so } \\
\text { höhere Prämienzahlungen zu } \\
\text { realisieren. }\end{array}$ & $-0,03$ & 1,02 & i & i & $\hat{\sigma}_{1}^{1}$ & $\begin{array}{l}1 \\
1 \\
1\end{array}$ & i \\
\hline
\end{tabular}

Quelle: Eigene Darstellung

Wie bei der Betrachtung der Beweggründe zur Auswahl der Festbetragsförderung spielt auch bei den Landwirten, die sich für die Flexible Förderung entschieden haben die eigene betriebliche Situation eine entscheidende Rolle bei der Wahl der Förderung. Allerdings wird auch die Chance auf eine höhere Auszahlung bei der Flexiblen Förderung als entscheidendes Argument angeführt. Demgegenüber wird die Möglichkeit, gezielt Einfluss auf die 
Schwankung der jährlichen Gewinne auszuüben, um die Förderung zu optimieren, gering eingeschätzt. Dies könnte jedoch auch auf eine teilweise Unkenntnis der Möglichkeiten im Rechnungswesen zurückzuführen sein.

\subsubsection{DIE PERSÖNLICHE RISIKOEINSTELLUNG ALS MÖGLICHE DETERMINANTE DER ENTSCHEIDUNGSFINDUNG}

Die in Kapitel 5.3.3.4 dargestellten Ergebnisse zu den Beweggründen der Teilnehmer bei der Wahl der Festbetrags- bzw. der Flexiblen Förderung für den eigenen Betrieb legen nahe, dass diesbezüglich die persönliche Risikoeinstellung des Landwirts ein wichtiger Entscheidungsfaktor sein könnte. Weil dieses aufgrund der Konzeption der Flexiblen Förderung bereits vor der Durchführung der Feldexperimente zu vermuten war, wurde das hinsichtlich seiner Methodik in Kapitel 5.2.2.2 beschriebene Lotteriespiel in den Untersuchungsablauf integriert. Im Folgenden soll nun zunächst die Methodik zur Auswertung des Lotteriespiels aufgezeigt werden, bevor die Ergebnisse ausgewertet und zu den Ergebnissen des Prämienexperiments ins Verhältnis gesetzt werden.

\subsubsection{VORÜBERLEGUNGEN ZUR AUSWERTUNG DES LOTTERIESPIELS}

In Kapitel 5.2.2.2 wurde bereits auf die Konzeption des Lotteriespiels hingewiesen. Die Ausführungen zur Auswertung des Lotteriespiels in diesem Kapitel orientieren sich an einer von HARDAKER et al. (2004: 93ff) auf der Basis der Abhandlungen von ANDERSEN et al. (1977) eigens zur Auswertung dieses Lotteriespiels entwickelten Methodik, die von RICHARDSON (2005b: 36) in einer MICROSOFT-EXCEL ${ }^{\odot}$-Programmierung automatisiert wurde.

Um auf der Grundlage der Eingaben der Teilnehmer, d.h. der vier jeweiligen persönlichen Sicherheitsäquivalente, Aussagen über deren Risikoeinstellung treffen zu können, war es zunächst notwendig, aus diesen Sicherheitsäquivalenten die persönlichen Risikonutzenfunktionen der Teilnehmer zu generieren. Wie in Kapitel 5.2.2.2 dargelegt, betrugen die möglichen Auszahlungen beim ersten Münzwurf $50 €$ bei ,Kopf' und $100 €$ bei ,Zahl'. Damit kamen für den Teilnehmer als Einsatz lediglich Zahlen zwischen $50 €$ und $100 €$ infrage. Da sich die Auszahlungen der folgenden Runden an die Eingaben des Teilnehmers sowie die Auszahlungen der ersten Runde knüpften, lagen alle Eingaben des Teilnehmers im Lotteriespiel zwangsläufig in den Grenzen von $50 €$ und $100 €$. Das ermöglichte es, die Risikonutzenfunktion in der Weise $\mathrm{zu}$ normieren, dass dem 
Auszahlungsbetrag $50 €$ ein Nutzen (U) von 0 und dem Auszahlungsbetrag $100 €$ ein Nutzen (U) von 1 zugeordnet wurde. Durch diese Festlegungen ließ sich nun auch der Nutzen der vom Teilnehmer erhobenen Sicherheitsäquivalente angeben. Die zugrunde liegenden Berechnungsschritte werden in Tabelle 36 offen gelegt. ${ }^{35}$

Tabelle 36: Nutzwertberechnung für die erhobenen Sicherheitsäquivalente der Teilnehmer

\begin{tabular}{|c|c|c|}
\hline Lotterierunde & Eingetragene Sicherheitsäquivalente (CE) des Teilnehmers & Berechnung des Nutzens (U) \\
\hline vor Spielbeginn: & $\begin{array}{l}\text { Definition der Gewinnbeträge a und } f \text { der ersten Lotterierunde } \\
\text { (hier: } a=50 \text { und } f=100 \text { ) }\end{array}$ & $U(a)=U(50)=0 ; \quad U(f)=U(100)=1$ \\
\hline 1 & $(\mathbf{b} ; 1,0) \sim(a, f ; 0,5 ; 0,5)$ & $U(b)=0,5^{*} U(a)+0,5^{*} U(f)=0,5$ \\
\hline 2 & $(\mathbf{c} ; 1,0) \sim(a, b ; 0,5 ; 0,5)$ & $U(c)=0,5^{*} U(a)+0,5 * U(b)=0,25$ \\
\hline 3 & $(\mathbf{d} ; 1,0) \sim(b, f ; 0,5 ; 0,5)$ & $U(\mathrm{~d})=0,5^{*} \mathrm{U}(\mathrm{b})+0,5^{*} \mathrm{U}(\mathrm{f})=\mathbf{0 , 7 5}$ \\
\hline 4 & $(\mathbf{e} ; 1,0) \sim(c, d ; 0,5 ; 0,5)$ & $U(e)=0,5^{*} U(c)+0,5^{*} U(d)=0,5$ \\
\hline
\end{tabular}

Quelle: Eigene Darstellung nach HARDAKER (2004: 97)

Die sich ergebende Wertetabelle von Sicherheitsäquivalenten (CE) und zugehörigen Nutzwerten (U) ermöglichte im nächsten Schritt das Abschätzen der persönlichen Risikonutzenfunktionen der Teilnehmer. Dazu wurde analog zur Vorgehensweise von RICHARDSON (2005b) eine multivariate Regression mit dem Nutzen (U) als 'zu erklärende Variable' und dem Sicherheitsäquivalent (CE), dem quadrierten Sicherheitsäquivalent $\left(\mathrm{CE}^{2}\right)$ und dem mit der Zahl drei potenzierten Sicherheitsäquivalent $\left(\mathrm{CE}^{3}\right)$ als 'erklärende Variablen' berechnet. Als Ergebnis erhält man die Funktionsgleichung der Risikonutzenfunktion des jeweiligen Teilnehmers in der folgenden Grundform:

$$
U=\beta_{0}+\beta_{1} C E+\beta_{2} C E^{2}+\beta_{3} C E^{3}
$$

Abbildung 48 zeigt exemplarisch für drei Teilnehmer mit deutlich unterschiedlichen Risikoeinstellungen deren Eingaben sowie die dazugehörigen Risikonutzenfunktionen.

\footnotetext{
${ }^{35}$ Zur Erklärung der Schreibweise: $(\mathrm{c} ; 1,0) \sim(\mathrm{a}, \mathrm{b} ; 0,5 ; 0,5)$ bedeutet, dass der Teilnehmer bei der Auswahl zwischen einer sicheren $(\mathrm{p}=1,0)$ Auszahlung von $\mathrm{c} €$ und einer Lotterie, bei der er mit einer Wahrscheinlichkeit von $\mathrm{p}=0,5$ eine Auszahlung von a $€$ und mit der gleichen Wahrscheinlichkeit eine Auszahlung von $b €$ erhält, indifferent ist.
} 
Abbildung 48: Ermittlung der Risikonutzenfunktionen verschiedener Teilnehmer

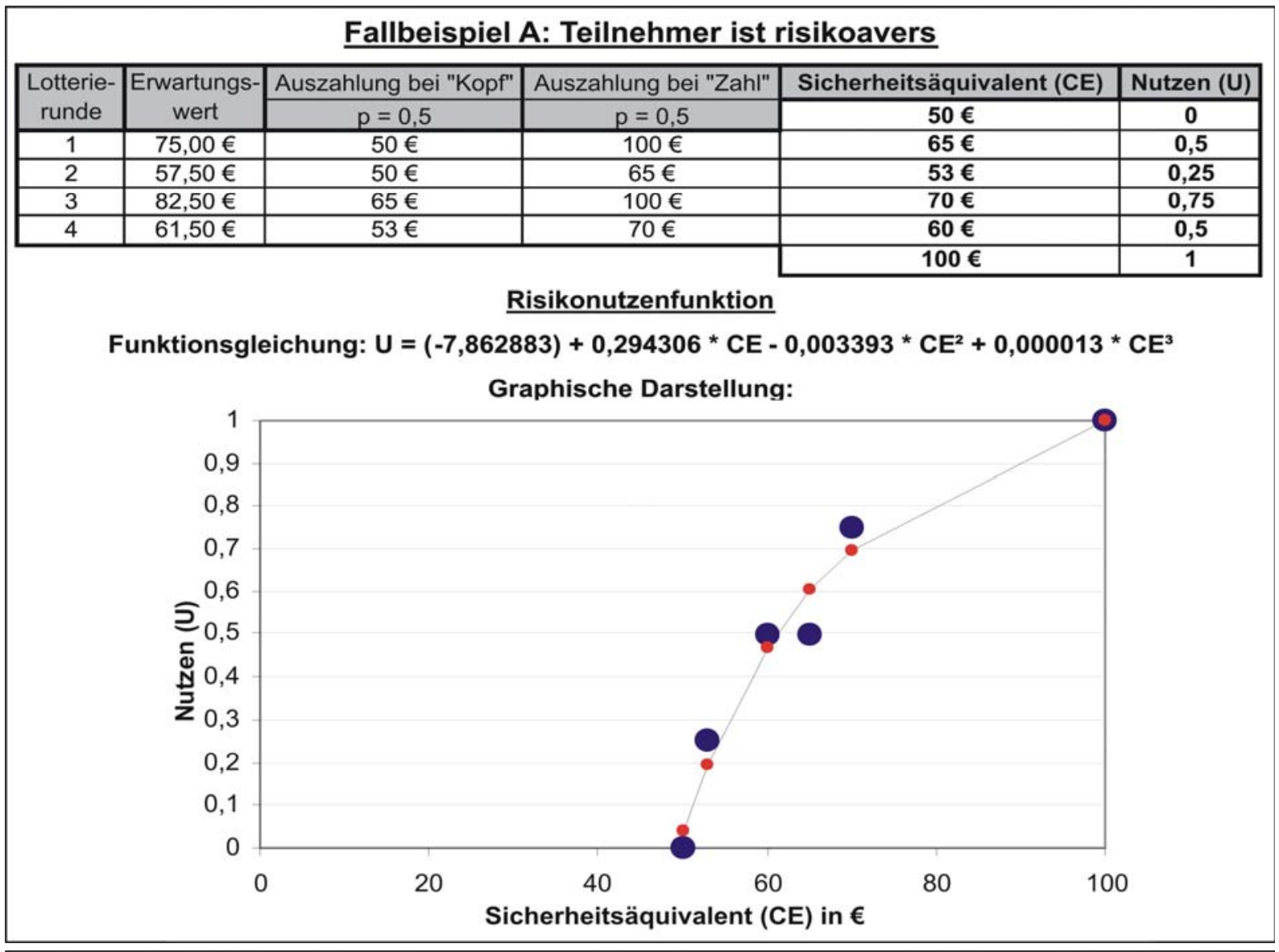

\begin{tabular}{|c|c|c|c|c|c|}
\hline \multicolumn{6}{|c|}{ Fallbeispiel B: Teilnehmer ist risikoneutral } \\
\hline \multirow{2}{*}{$\begin{array}{c}\text { Lotterie- } \\
\text { runde }\end{array}$} & \multirow{2}{*}{$\begin{array}{c}\text { Erwartungs- } \\
\text { wert }\end{array}$} & Auszahlung bei "Kopf" & Auszahlung bei "Zahl" & Sicherheitsäquivalent (CE) & Nutzen (U) \\
\hline & & $p=0,5$ & $p=0,5$ & $50 €$ & 0 \\
\hline 1 & $75,00 €$ & $50 €$ & $100 €$ & $75 €$ & 0,5 \\
\hline 2 & $62,50 €$ & $50 €$ & $75 €$ & $62,50 €$ & 0,25 \\
\hline 3 & $87,50 €$ & $75 €$ & $100 €$ & $87,50 €$ & 0,75 \\
\hline 4 & $75,00 €$ & $62,50 €$ & $87,50 €$ & $75 €$ & 0,5 \\
\hline & & & & $100 €$ & 1 \\
\hline
\end{tabular}

Risikonutzenfunktion

Funktionsgleichung: $U=(-1)+0,02$ * $C E$

Graphische Darstellung:

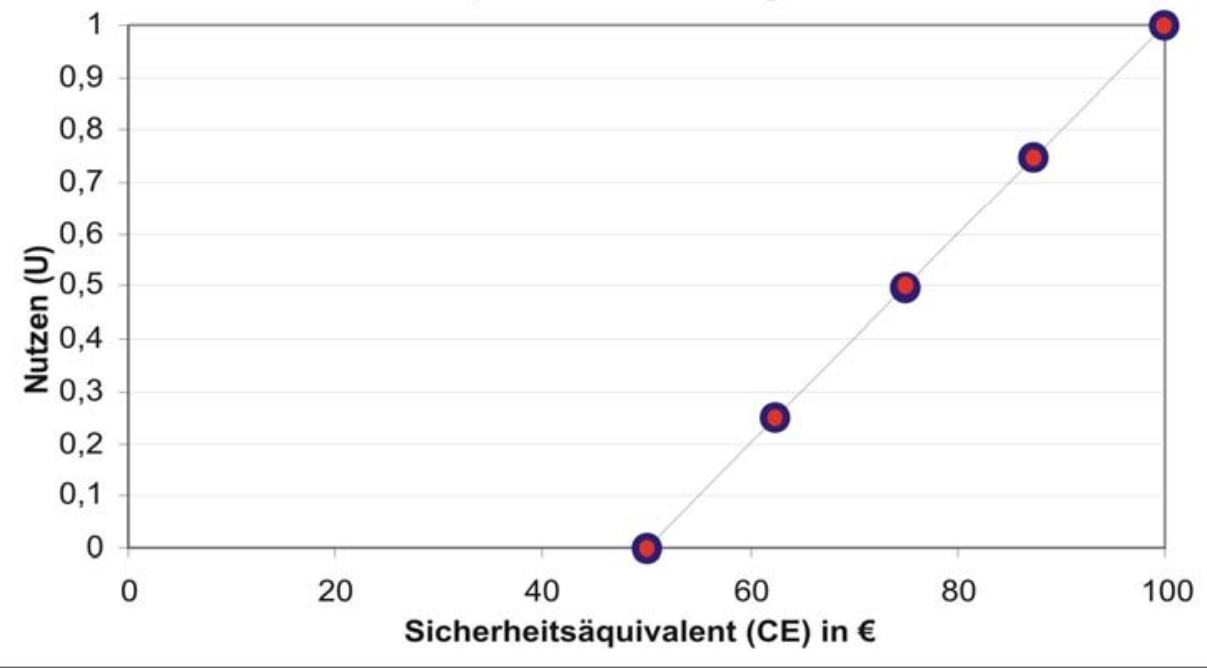




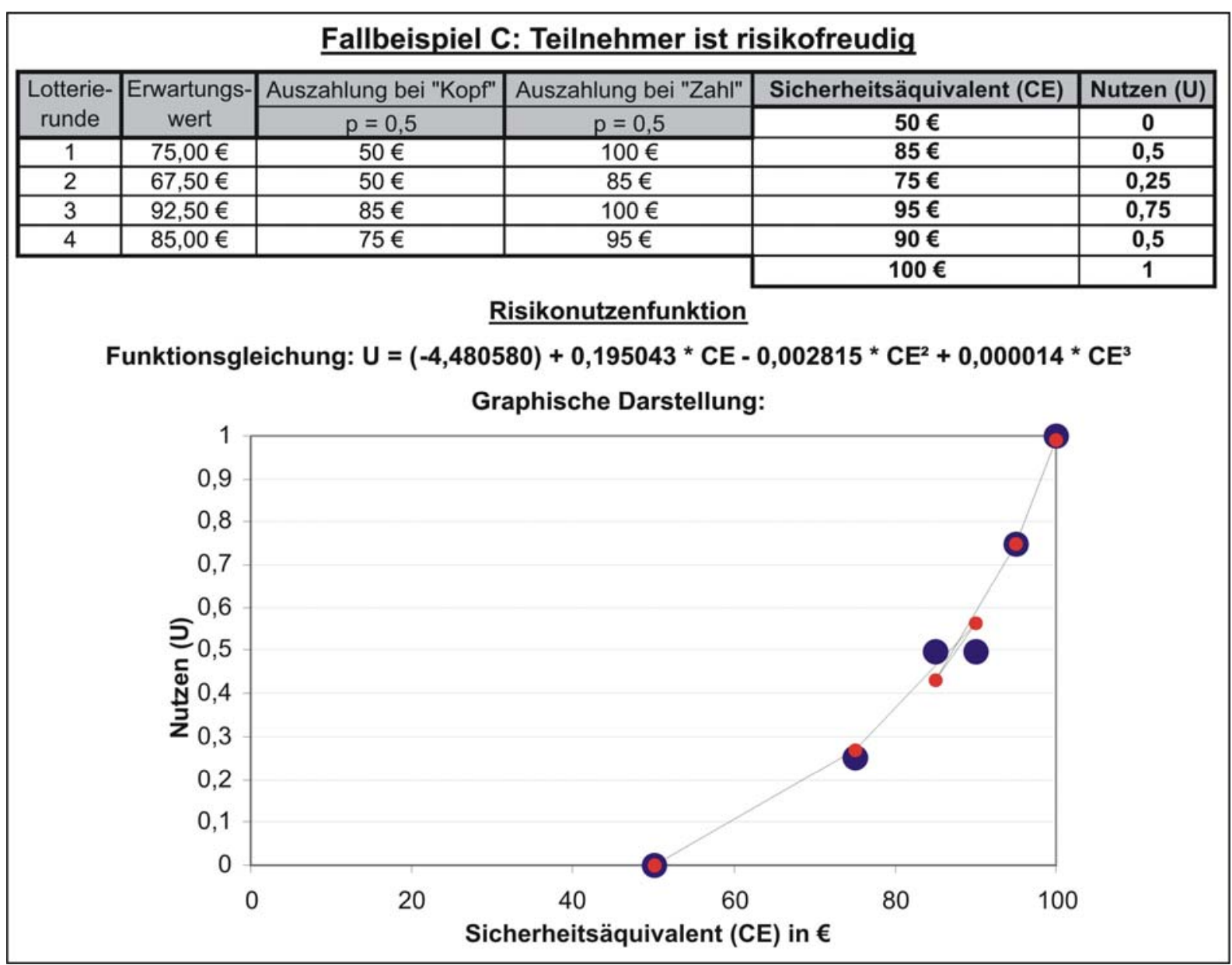

Quelle: Eigene Darstellung nach RiCHARDSON (2005b)

Dabei kennzeichnen die größeren blauen Punkte in den graphischen Darstellungen die Nutzenwerte aus der anfänglichen Datentabelle, während die kleineren roten Punkte die Funktionswerte der empirisch erhobenen Sicherheitsäquivalente entlang des Funktionsgraphen markieren.

Allein die grafische Darstellung des Funktionsverlaufs lässt bereits auf die Risikoeinstellung des Teilnehmers schließen (vgl. dazu auch ROMMELFANGER und EICKEMEIER, 2002: 84ff). Eine Person, deren Präferenzen durch eine konkave Risikonutzenfunktion abgebildet werden, verhält sich risikoavers bei ihren Entscheidungen (Fallbeispiel 1). Sind Sicherheitsäquivalent und Erwartungswert der Lotterie wie im Fallbeispiel 2 genau identisch, so stellt sich eine lineare Risikonutzenfunktion ein. Ein linearer Verlauf drückt daher risikoneutrales Entscheidungsverhalten des Entscheidenden aus. Verläuft die Risikonutzenfunktion dagegen konvex zum Ursprung ist dieses ein Indiz für Risikofreude (Fallbeispiel 3).

Für einen detaillierteren Überblick über die Risikoeinstellungen der einzelnen Teilnehmer ist diese Einteilung jedoch sehr grob. Um den Grad der Risikoaversion des Entscheiders deshalb durch eine Maßzahl zu quantifizieren, wurde im nächsten Berechnungsschritt auf das ARROW- 
PRATT-Maß ${ }^{36}$ zurückgegriffen, für das auch die Bezeichnung ,absoluter Risikoaversionskoeffizient (ARAC)“ verwendet wird (vgl. z.B. BRANDES und ODENING, 1992; 199):

$$
A R A C=\frac{-U^{\prime \prime}}{U^{\prime}}
$$

Wie die Berechnungsformel offenbart, muss zunächst für jeden Teilnehmer die erste sowie die zweite Ableitung seiner Risikonutzenfunktionen gebildet werden. Anschließend kann für den Mittelwert der Sicherheitsäquivalente der absolute Risikoaversionskoeffizient des jeweiligen Teilnehmers berechnet werden. Wie Tabelle 37 zeigt, nimmt er im Falle eines risikoaversen (risikofreudigen) Entscheidungsverhaltens positive (negative) Werte an.

Tabelle 37: Übersicht über entscheidende Parameter der Risikoeinstellung

\begin{tabular}{|c|c|c|c|}
\hline $\begin{array}{c}\text { Verlauf der } \\
\text { Risikonutzenfunktion }\end{array}$ & $\begin{array}{c}\text { Realtion zwischen dem Erwartungswert } \\
\text { der Lotterie und dem persönlichen } \\
\text { Sicherheitsäquivalent des Teilnehmers }\end{array}$ & $\begin{array}{c}\text { Einstellung des } \\
\text { Teilnehmers zum } \\
\text { Risiko }\end{array}$ & $\begin{array}{c}\text { Absoluter } \\
\text { Risikoaversions- } \\
\text { koeffizient (ARAC) }\end{array}$ \\
\hline streng konkav & Erwartungswert > Sicherheitsäquivalent & risikoavers & ARAC $>0$ \\
\hline linear & Erwartungswert = Sicherheitsäquivalent & risikoneutral & ARAC $=0$ \\
\hline streng konvex & Erwartungswert < Sicherheitsäquivalent & risikofreudig & ARAC $<0$ \\
\hline
\end{tabular}

Quelle: Eigene Darstellung nach BAMBERG und COENENBERG (2004)

Die Berechung der absoluten Risikoaversionskoeffizienten für die drei Entscheidungsträger aus den Fallbespielen A bis C aus Abbildung 48 ist in Abbildung 49 dargestellt.

\footnotetext{
${ }^{36}$ Für detaillierte Informationen zum ARROW-PRATT-Maß vgl. PRATT, 1964 und ARROW, 1970
} 
Abbildung 49: Darstellung der Berechnung der ARAC's für die Fallbeispiele A bis C

\begin{tabular}{|c|}
\hline Fallbeispiel A: \\
\hline Funktionsgleichung: $U=(-7,862883)+0,294306 * \mathrm{CE}-0,003393{ }^{*} \mathrm{CE}^{2}+0,000013 * \mathrm{CE}^{3}$ \\
\hline 1. Ableitung: $U^{\prime}=0,294306-2 * 0,003393 * C E+3 * 0,000013 * C^{2}$ \\
\hline 2. Ableitung: $U^{\prime \prime}=2 * 0,003393+6 * 0,000013 * \mathrm{CE}$ \\
\hline Mittelwert der Sicherheitsäquivalente: $66 €$ \\
\hline ARAC = -U“/U“ \\
\hline 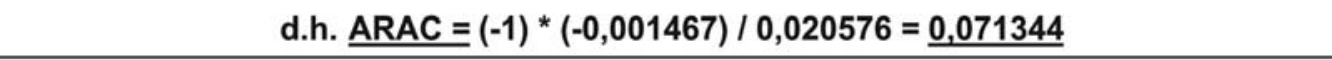 \\
\hline Fallbeispiel B: \\
\hline Funktionsgleichung: $U=(-1)+0,02 * \mathrm{CE}$ \\
\hline 1. Ableitung: $U^{\prime}=0,02$ \\
\hline 2. Ableitung: $U "=0$ \\
\hline Mittelwert der Sicherheitsäquivalente: $75 €$ \\
\hline ARAC = -U“/U“ \\
\hline d.h. $\underline{A R A C}=(-1) * 0 / 0,02=\underline{0}$ \\
\hline Fallbeispiel C: \\
\hline Funktionsgleichung: $U=(-4,480580)+0,195043 * \mathrm{CE}-0,002815 * \mathrm{CE}^{2}+0,000014{ }^{*} \mathrm{CE}^{3}$ \\
\hline 1. Ableitung: $U^{\prime}=0,195043-2 * 0,002815 * \mathrm{CE}+3 * 0,000014 * \mathrm{CE}^{2}$ \\
\hline 2. Ableitung: U" $=2 * 0,002815+6 * 0,000014$ * CE \\
\hline Mittelwert der Sicherheitsäquivalente: $83 €$ \\
\hline ARAC $=-U “ / U \times$ \\
\hline d.h. $\underline{A R A C}=(-1) * 0,001357 / 0,018822=\underline{-0,072129}$ \\
\hline
\end{tabular}

Quelle: Eigene Darstellung nach RICHARDSON (2005b)

Um auf der Grundlage der berechneten absoluten Risikoaversionskoeffizienten konkretere Aussagen zur Risikoeinstellung der Landwirte treffen zu können, wurden alle Teilnehmer gemäß ihrer ARAC's in Risikogruppen eingeteilt. Das dazu verwendete Einteilungsschema zeigt Tabelle 38 .

Tabelle 38: Einteilungsschema zur Klassifizierung der absoluten Risikoaversionskoeffizienten

\begin{tabular}{|c|l|}
\hline Absoluter Risikoaversionskoeffizient (ARAC) & Risikoeinstellung des Teilnehmers \\
\hline ARAC $<0,05$ & Teilnehmer ist: sehr risikofreudig \\
\hline $0,05<$ ARAC $<0,005$ & Teilnehmer ist: risikofreudig \\
\hline $0,005<$ ARAC $<0,002$ & Teilnehmer ist: leicht risikofreudig \\
\hline $0,002<$ ARAC $<0,002$ & Teilnehmer ist: risikoneutral \\
\hline $0,002<$ ARAC $<0,005$ & Teilnehmer ist: leicht risikoavers \\
\hline $0,005<$ ARAC $<0,05$ & Teilnehmer ist: risikoavers \\
\hline ARAC $>0,05$ & Teilnehmer ist: sehr risikoavers \\
\hline
\end{tabular}

Quelle: Eigene Darstellung 


\subsubsection{ERGEBNISSE DES LOTTERIESPIELS}

Tabelle 39 zeigt die Häufigkeitsverteilung der Risikoeinstellung der Teilnehmer am Experiment. $^{37}$

Tabelle 39: Häufigkeitstabelle zur Risikoeinstellung der Teilnehmer am Experiment

\begin{tabular}{|l|c|c|c|}
\hline ARAC-Einteilung & Häufigkeit & Prozent & Kumulierte Prozente \\
\hline sehr risikofreudig & 3 & 4,17 & 4,17 \\
\hline risikofreudig & 18 & 25,00 & 29,17 \\
\hline leicht risikofreudig & 4 & 5,56 & 34,72 \\
\hline risikoneutral & 12 & 16,67 & 51,39 \\
\hline leicht risikoavers & 6 & 8,33 & 59,72 \\
\hline risikoavers & 16 & 22,22 & 81,94 \\
\hline sehr risikoavers & 13 & 18,06 & 100,00 \\
\hline Gesamt & 72 & 100,00 & \\
\hline
\end{tabular}

Quelle: Eigene Darstellung

Es zeigt sich, dass die Mehrzahl der Teilnehmer in ihrer Grundeinstellung nicht risikofreudig ist. Fast $50 \%$ der Teilnehmer sind risikoavers; über $15 \%$ sind risikoneutral und nur weniger als $35 \%$ sind risikofreudig. Damit ergibt sich eine mittlere Risikoeinstellung im Teilnehmerkreis von ,leicht risikoavers', die allerdings durch eine hohe Standardabweichung in ihrer Aussagekraft eingegrenzt wird.

Die Motivation, das Lotteriespiel in das Feldexperiment zu integrieren, wurde von der Vermutung getragen, es könnte ein eindeutiger Zusammenhang zwischen der Risikoeinstellung des Landwirts und seiner Akzeptanz für die Flexible Förderung bestehen. Deshalb wurde die ARAC-Verteilung in einer Korrelationsanalyse den beiden Verteilungen ,Akzeptanz im Prämienexperiment' und ,Akzeptanz für den eigenen Betrieb' gegenübergestellt. Heraus kamen mit $-0,084$ sowie $-0,111$ jeweils schwach negative Korrelationskoeffizienten $^{38}$, für die allerdings keine Signifikanz ermittelt werden konnte. Damit kann in der Tendenz die Vermutung bestätigt werden, dass eine stark ausgeprägte Risikoaversion der Akzeptanz der Flexiblen Förderung eher abträglich ist. Jedoch lassen diese Ergebnisse vermuten, dass der Zusammenhang deutlich geringer sein könnte als zunächst angenommen.

\footnotetext{
${ }^{37}$ Eine detaillierte Aufstellung der Ergebnisse ist in Anhang 8 einzusehen.

${ }^{38} \mathrm{Da}$ es sich bei den Verteilungen ,Akzeptanz im Prämienexperiment' und ,Akzeptanz für den eigenen Betrieb' um ordinalskalierte Daten handelt, wurde der Korrelationskoeffizient nach Spearman gewählt. Er ist im Übrigen weniger stark durch Ausreißer beeinflussbar (für nähere Informationen zur Korrelationsanalyse bzw. zu den unterschiedlichen Korrelationskoeffizienten vgl. MOSLER und SCHMID, 2003: 165ff).
} 


\subsection{FAZIT}

Die Untersuchung der Akzeptanz für die Flexible Förderung in der Landwirtschaft hat vielversprechende Ergebnisse hervorgebracht. Es konnte im Prämienexperiment gezeigt werden, dass die grundsätzliche Akzeptanz für die Flexible Förderung sehr hoch ist. Bei der Entscheidung für den eigenen Betrieb zeigt sich eine geringere aber immer noch hohe Akzeptanz, was primär auf die eigenen einzelbetrieblichen historischen Gewinnschwankungen zurückzuführen sein wird.

Mit Blick auf die Zielstellung der Flexiblen Förderung, die Effizienz und die Effektivität der Förderung zu erhöhen, ist die Anreizwirkung der Förderung auf Öko-Landwirte und Umstellungsinteressierte interessant. Eine wichtige Frage im Fragebogen lautete daher:

- „Sehen Sie im Falle einer Einführung der Flexiblen Prämie für sich selbst einen zusätzlichen Anreiz zur Betriebsumstellung auf ökologischen Landbau bzw. einen zusätzlichen Anreiz zur Beibehaltung des ökologischen Landbaus?“‘

Dabei ergab sich ein sehr unterschiedliches Antwortverhalten, je nach dem, welche Förderung zuvor vom Landwirt für seinen eigenen Betrieb gewählt worden war. Auf einer Skala von -2 (nein) bis +2 (ja) bildete sich in der Gruppe der Landwirte, die die Flexible Förderung für ihren Betrieb gewählt hatten, ein Mittelwert von 0,3 bei einer Standardabweichung von 1,4 heraus, was einem neutralen bis eher bejahenden Votum der Teilnehmer zu dieser Frage gleichkommt. Diesbezüglich ist wiederum auf die vielfältige Flexibilität des Förderers bei der letztendlichen Ausgestaltung der Förderung, auf die bereits im Verlauf des Kapitels 4 mehrfach eingegangen wurde, hinzuweisen. Unterschiedliche Ausgestaltungen werden auch unterschiedliche Anreizwirkungen entfalten. Dabei wird die Anreizwirkung bei einer Veränderung der Ausgestaltung nicht für alle potentiell Umstellungsinteressierten identisch sein. Abhängig von der einzelbetrieblichen Situation wird die jeweilige Anreizwirkung sogar sehr unterschiedlich sein. So wird bspw. hinsichtlich der Gewichtung der Einzelkomponenten der Flexiblen Förderung (vgl. Kapitel 4.2.3.3) für einen flächenstarken Betrieb mit geringen Einkommensschwankungen eine stärkere Gewichtung des flächenbezogenen Sockelbetrags einen hohen Anreiz bieten. Demgegenüber wird der Anreiz zur Umstellung auf ökologischen Landbau bzw. zur Beibehaltung des ökologischen Landbaus für einen flächenschwächeren Betrieb mit hohen Einkommensschwankungen bei einer stärkeren Gewichtung der Zusatzförderung höher sein. Auch eine Veränderung des Bemessungszeitraums (vgl. Kapitel 4.2.3.1) oder der Berechnungsweise (vgl. Kapitel 4.2.3.2) der Zusatzförderung wird sich in unterschiedlicher Weise auf den Anreiz auswirken, den die Förderung auf einzelne Gruppen 
von Öko-Landwirten und Umstellungsinteressierten bietet. Die Möglichkeit zur flexiblen Ausgestaltung der Förderung bietet für den Förderer deshalb auch die Möglichkeit, speziell die Anreizwirkung der Förderung zielgenau auszurichten. 


\section{ZUSAMMENFASSUNG}

Ziel dieser Arbeit war es, sich ausführlich mit den Anforderungen an eine zukünftige Förderung der ökologischen Landwirtschaft auf der Angebotsseite auseinanderzusetzen und dabei für die gegenwärtige Angebotsförderung Alternativen mit Hilfe des Steuerrechts zu entwickeln, die Potentiale für einen wirksameren Einsatz der zur Verfügung stehenden Finanzmittel aufweisen.

Nach einem Überblick über die spezifischen Ziele und die derzeit angebotenen Maßnahmen zur Förderung des ökologischen Landbaus zeigte sich in Kapitel 2, dass der finanzielle Schwerpunkt der Förderpolitik weiterhin auf der flächenbezogenen Angebotsförderung liegt. Im Anschluss an eine detaillierte Darstellung der Konzeption dieser finanziell bedeutsamsten Fördermaßnahme in ihrer aktuellen Ausgestaltung wurde auf die derzeitig unsichere Situation der zukünftigen Finanzierung hingewiesen. Die zunehmende Begrenztheit der zur Ausdehnung des ökologischen Landbaus zur Verfügung stehenden Finanzmittel wurde zum Anlass genommen, die Öko-Flächenprämien hinsichtlich des Verhältnisses der Flächenentwicklung zur Fördermittelentwicklung zu evaluieren. Neben der Effektivität der Förderung war vor allem die Effizienz des Mitteleinsatzes von besonderem Interesse. Die Ergebnisse deckten sich mit denen vieler anderer Untersuchungen, wonach die flächenbezogenen Festbetragszahlungen zu z.T. erheblichen Mitnahmeeffekten führen können und sich damit Anzeichen für eine derzeitig nicht effiziente Angebotsförderung ergeben.

In der Mehrzahl der Bundesländer besteht ein finanziell bedingter Förder-Stopp für Neuumsteller. Zukünftig ist im Rahmen der ELER-Verordnung nicht auszuschließen, mit noch geringeren Finanzmittelbudgets rechnen zu müssen. Vor dem Hintergrund dieser Rahmenbedingungen sowie der gegenwärtig nicht effizienten Angebotsförderung wurden in Kapitel 3 Förderalternativen des Steuerrechts evaluiert. Nach einem kurzen Einstieg wurde zunächst speziell die grundsätzliche Eignung des Steuerrechts als Förderinstrument diskutiert. Dabei wurde darauf hingewiesen, dass sich für eine Angebotsförderung lediglich Steuerarten eignen, die für ökologisch wirtschaftende Landwirte einerseits überhaupt eine finanzielle Bedeutung haben und andererseits auch in möglichst regelmäßigen Abständen zur Zahlung anstehen. So verblieben lediglich die Ertragsteuern sowie die Grundsteuer, die Umsatzsteuer und andere Verbrauchsteuern für weitergehende Betrachtungen. Daneben wurden drei Erfolgskriterien definiert, denen im Vorfeld der Überlegungen eine große Bedeutung für die Eignung steuerlicher Förderinstrumente für eine effizientere Öko-Angebotsförderung 
beigemessen wurde. Neben der Wirkung der Förderung sind dies die voraussichtlich entstehenden Transaktionskosten sowie die Genehmigungs- und Kofinanzierungsfähigkeit gemäß der EU-Rahmengesetzgebung. Anhand dieser drei Kriterien wurden in der Folge mit der Prämiendifferenzierung gemäß der EMZ, der Freistellung ökologisch bewirtschafteter Flächen von der Grundsteuer und der Freistellung ökologisch erzeugter Produkte von der Umsatzsteuer zunächst drei in Fachkreisen bereits vieldiskutierte steuerliche Förderinstrumente hinsichtlich ihrer spezifischen Eigenschaften analysiert. Dabei zeigten sich lediglich im Falle der Prämiendifferenzierung gemäß der EMZ Anzeichen für eine effizienzsteigernde Wirkung. Die beiden anderen untersuchten Förderalternativen eignen sich dagegen nicht zur Substitution oder Modifikation der gegenwärtigen Angebotsförderung des ökologischen Landbaus.

Kapitel 4 bildet mit der Vorstellung der Flexiblen Förderung als Förderalternative, die ihren Ursprung im Ertragsteuerrecht findet, den Schwerpunkt der Untersuchung. Ausgangspunkt ihrer Eignung sind Überlegungen im Kontext einer vergleichenden Risikoanalyse auf Basis der Buchführungsergebnisse aus dem BMELV-Testbetriebsnetz, aus der sich Hinweise für potentiell höhere Einkommensrisiken im ökologischen Landbau im Vergleich zum konventionellen Landbau ergeben. Mit Bezug auf eine gewünschte Effektivitäts- und Effizienzsteigerung der Förderung wurde daher mit der Zusatzförderung im Rahmen der Flexiblen Förderung ein Instrument ausgewählt, mit dem ein gezielter Abbau dieses spezifischen Umstellungshemmnisses möglich erschien, bei gleichzeitiger Beachtung der durch die ELER-Verordnung vorauszusetzenden Kostenakzessorität der Förderung .

Die weitere Vorgehensweise innerhalb des Kapitels 4 orientierte sich wiederum eng an den im Vorkapitel definierten Erfolgskriterien für steuerliche Förderinstrumente. Vorher wurde jedoch die Konzeption der Flexiblen Förderung dargestellt. Sie besteht aus einem reduzierten, aber weiterhin flächenbezogenen Sockelbetrag und der neu entwickelten Zusatzförderung. Diese wiederum resultiert aus einer fiktiven Ersparnis, die sich ergeben würde, wenn schwankende Einkünfte anstatt einer jährlichen Veranlagung mit einem progressiven Einkommensteuertarif mit dem jährlichen Durchschnitt der Summe mehrjähriger (schwankender) Einkünfte veranlagt werden würden.

Dieses Instrument ist kostenakzessorisch, da es die höheren Kosten stärker schwankender Einkünfte in Form höherer Steuern und sonstiger Absicherungen kompensiert, ohne Einkommensanreize bieten zu müssen. Beide Förderkomponenten (Sockelbetrag und Zusatzförderung) können auf Ebene der Bundesländer individuell miteinander kombiniert 
werden, so dass sie den individuellen Finanzmittelbudgets und den zu fördernden Zielgruppen angepasst werden können. Darüber hinaus ist diese Förderung durch geringstmögliche Transaktionskosten realisierbar. Aufgrund des Verwaltungsaktes Einkommensteuerbescheid kann die Agrarverwaltung auf bereits verfügbare Informationen rechtssicher und ohne erhebliche Zusatzarbeit zurückgreifen. Dies darf allerdings nicht verwechselt werden mit einer möglicherweise suggerierten Förderung innerhalb des Steuerrechts. Die Finanzverwaltung hat mit diesem Förderinstrument weder zusätzliche Arbeit noch wird der Förderbetrag mit Steuern verrechnet. Das System löst sich weitgehend vom Steuerrecht und nutzt lediglich deren Informationen bzw. Methoden.

Für die Analyse der Wirkungen der Flexiblen Förderung wurde zunächst eine Basiskonstellation definiert, die neben dem Bemessungszeitraum und der Berechnungsweise der Zusatzförderung auch die Höhe des flächenbezogenen Sockelbetrags festlegte. Dabei offenbarte sich zum einen die beabsichtigte Abhängigkeit des Auszahlungsbetrags von der Intensität der Einkunftsschwankungen. Es zeigte sich aber auch, dass mit einer Anknüpfung der Zusatzförderung an gegenwärtig bestehende Freibeträge und ESt-Tarife, mittlere jährliche LUF-Einkünfte zwischen $10.000 €$ und $50.000 €$ von der Zusatzförderung tendenziell stärker profitieren als niedrigere oder höhere Einkunftsniveaus. Allerdings muss man sich bei der Zusatzförderung nicht zwangsläufig an bestehenden Tarifen (z. B. an dem Einkommensteuertarif gemäß $§ 32 \mathrm{a} E \mathrm{EStG})$ orientieren. Die verantwortlichen Institutionen können sich völlig vom Einkommensteuergesetz lösen und die Zusatzförderung durch die Umgestaltung des Einkommensteuertarifs (individuellere Freibeträge und Tarifgestaltung) oder durch ganz neue Tarifverläufe (z. B. abschnittsweise logarithmierter Funktionsverlauf) auch für andere Einkommensgruppen attraktiver werden lassen. Dies ist auch eine Stärke der Zusatzförderung, da man sie gemäß des föderalen Prinzips gezielt auf die individuellen Verhältnisse in den Bundesländern (Betriebsgrößen, Betriebsausrichtungen, Finanzmittelbudgets) abstellen oder gemäß GAK einheitlich koordinieren kann. Wesentlicher Eckpfeiler der Flexiblen Förderung ist die Reduktion der bisher zu verzeichnenden Mitnahmeeffekte, da sich dieses Förderinstrument direkt an den individuellen Einkünften aus Land- und Forstwirtschaft orientiert. Somit liegt ein weiterer Vorteil dieser Förderung in der Option, eine spezielle Auswahl der schwerpunktmäßig zu fördernden Betriebe treffen zu können. Allerdings ist bei einer Umstellung der gegenwärtig ausschließlich flächenbezogenen Festbetragsförderung auf die Flexible Förderung davon auszugehen, dass neben den zu erwartenden allgemein eintretenden Kürzungseffekten auch von Umverteilungseffekten auszugehen sein wird. 
Verschiedene Faktoren beeinflussen die Wirksamkeit der Flexiblen Förderung: Dazu zählen die Änderung des Bemessungszeitraums der Zusatzförderung (z. B. dreijährige oder fünfjährige Durchschnittsbetrachtung), die Änderung der Berechnungsweise in Form unterschiedlicher Tarifgestaltungen oder die Gewichtung des flächenbezogenen Sockelbetrags im Vergleich zur Zusatzförderung. Flächenstarke Betriebe mit geringen Einkunftsschwankungen profitieren von einer starken Gewichtung des flächenbezogenen Sockelbetrags, während flächenschwache Betriebe mit volatileren Einkünften von einer stärkeren Gewichtung der Zusatzförderung profitieren. So kann über die Gewichtung der Einzelkomponenten speziell die Zielrichtung der Förderung hinsichtlich der Förderungsempfänger variiert werden. Vor dem Hintergrund der unterschiedlichen durchschnittlichen Flächenausstattung der Ökobetriebe in den einzelnen Bundesländern (insbesondere zwischen West- und Ostdeutschland) kann damit eine unterschiedliche Gewichtung der Einzelkomponenten angezeigt sein. Allerdings spielt dabei auch das jeweilige Budget der einzelnen Bundesländer eine maßgebliche Rolle.

Nachdem ausführlich auf die Frage nach der Sachgerechtigkeit der Bemessungsgrundlage der Zusatzförderung eingegangen worden war, wurde im weiteren Verlauf der Untersuchung zur Gefahr von Fehlallokationen bzw. Fehlanreizen Stellung bezogen. Derartige Bedenken wären berechtigt, sofern es dem Betriebsleiter einerseits technisch und rechtlich möglich wäre und es ihm andererseits auch ökonomisch sinnvoll erschiene, die Höhe der Zusatzförderung aus Gründen, die nicht mit der ökologischen Wirtschaftsweise in Verbindung stehen, zu modifizieren. Betriebswirtschaftliche Analysen am Beispiel der optimalen zeitlichen Terminierung einer Großreparatur zeigen jedoch, dass in der Praxis die Gefahren von Fehlallokationen relativ gering sind. Insoweit sind kaum negative Auswirkungen auf die Vorzüglichkeit des Instruments zu vermuten.

Auch aus den Untersuchungen zur Genehmigungs- und Kofinanzierungsfähigkeit der Flexiblen Förderung gemäß der EU-Rahmengesetzgebung ergaben sich durchweg positive Erkenntnisse hinsichtlich einer Eignung der Flexiblen Förderung zur Weiterentwicklung der bisherigen Angebotsförderung.

Hinsichtlich der administrativen Umsetzung zeigte sich, dass zusätzliche Transaktionskosten für Verwaltung und Landwirte nur in einem geringen Umfang $\mathrm{zu}$ erwarten sind. Die erforderlichen Einkünfte aus Land- und Forstwirtschaft sind direkt aus dem jährlichen EStBescheid ablesbar, der als bereits vorhandener rechtsverbindlicher Verwaltungsakt keine zusätzliche Mehrarbeit mit sich bringt. Ein weiterer wichtiger Grund besteht in dem hohen 
EDV-technischen Effizienzgrad des bestehenden Fördersystems, dass sich vergleichsweise problemlos um die wenigen zusätzlichen Dateneingaben und Rechenprozeduren erweitern lässt. Auch hinsichtlich der konkreten verwaltungstechnischen Systemumstellung sind somit aufgrund der geringen Komplexität der Veränderungen lediglich niedrige Zusatzkosten zu erwarten. Eine erhöhte Fördereffizienz der Flexiblen Förderung wird daher nicht in Frage gestellt.

Von größter Bedeutung für eine Steigerung der Effizienz der Förderung sind schließlich die Auswirkungen einer Systemumstellung auf das Förderbudget. Im Vergleich zu den gegenwärtigen Ausgaben für die flächenbezogene Öko-Förderung könnten diese Ausgaben auf der Basis der bereits geförderten Flächen erheblich reduziert werden. Dies hängt aber von der gewählten Ausgestaltung der Flexiblen Förderung ab. Je stärker die flächenbezogene Komponente reduziert wird, umso deutlicher können die Einsparungen bei den Fördermitteln sein.

Die Flexible Förderung weist aber auch Problempotenziale auf. Sie reichen von weniger gut planbaren (variablen) Finanzmittelbudgets bis zu höheren Transaktionskosten bei der Förderung juristischer Personen. Bezüglich Ersterem zeigen die in diesem Zusammenhang durchgeführten Simulationsrechnungen, dass die Intensität der Ausgabenschwankungen für die öffentlichen Haushalte voraussichtlich gering sein wird. Im Falle juristischer Personen wird eine detailliertere Einzelfallbetrachtung notwendig sein, um eventuelle Fehlanreize und Fehlallokationen im Vorhinein auszuschließen.

Zusammengefasst lassen Förder- bzw. Verwaltungseffizienz im Hinblick auf eine Systemumstellung auf die Flexible Förderung sehr positive Auswirkungen auf die Gesamteffizienz des Finanzmitteleinsatzes erwarten.

In Kapitel 5 werden die Erfahrungen zusammengefasst, die mit der Flexiblen Förderung im Rahmen von Feldexperimenten bei verschiedenen Gruppen von Landwirten gemacht wurden. Dabei wurden das Verständnis und die Akzeptanz dieses Förderinstruments geprüft. Auch daraus ergaben sich positive Erkenntnisse, so dass als Resultat aller Untersuchungen eine Implementierung der Flexiblen Förderung empfohlen werden kann, insbesondere um zukünftigen Neuumstellern den Einstieg in den Ökolandbau zu ermöglichen. 


\section{LITERATURVERZEICHNIS}

AID (2004): Besteuerung der Land- und Forstwirtschaft. AID-Heft Nr. 1247, Bonn.

AgrA-EuROPE (2004): Der Markt für Ökoprodukte steht vor großen Herausforderungen. Agra-Europe 30/04, Sonderbeilage.

Agra-Europe (2005): Zweite Säule oder zweites Säulchen? Agra-Europe 52/05, EuropaNachrichten 9.

Agra-EuROPE (2006a): Hauk unterstützt betriebliche Beihilfeobergrenze. Agra-Europe 1/2/06, Kurzmeldungen 30.

Agra-Europe (2006b): Seehofer muss 200 Millionen Euro einsparen. Agra-Europe 9/06, Länderberichte 1-3.

AgrA-EuROPE (2006c): Grüne fordern ökologische Schwerpunkte in der ländlichen Entwicklung. Agra-Europe 11/06, Kurzmeldungen 14-15.

Agra-EuROPE (2006d): Agrarminister bekennen sich zur Honorierung ökologischer Leistungen. Agra-Europe 11/06, Kurzmeldungen 33.

Agra-EuroPe (2006e): Weniger Lebensmittel ohne Pestizidrückstände. Agra-Europe 12/06, Länderberichte 8 .

AgRA-EuROPE (2006f): Brüsseler Ministerrat vereinbart Förderregeln für EU-Strukturpolitik. Agra-Europe 20/06, Europa-Nachrichten 9.

Agra-Europe (2006g): Minister Tillich warnt vor Preisverfall bei Biolebensmitteln. AgraEurope 20/06, Kurzmeldungen 28.

AgrA-EuROPE (2006h): Öko-Branche in Deutschland weiter gewachsen. Agra-Europe 27/06, Länderberichte 19.

AHrens, H.; LiPPERT, C.; RitTERShOFER, M. (2000): Überlegungen $\quad$ zu Umwelt- und Einkommenswirkungen von Agrarumweltprogrammen nach VO (EWG) Nr. 2078/92 in der Landwirtschaft. In: Agrarwirtschaft, Band 49 , S. 99-115.

Althoefer, K.; Bauer, K.; Eisele, D.; Fichtelmann, H.; Walter, H. (2003): Besteuerung der Land- und Forstwirtschaft. 4. Auflage, Herne/Berlin. 
ALVENSLEBEN, R. VON (1998): Ökologischer Landbau: ein umweltpolitisches Leitbild? In: Agrarwirtschaft, Band 47, S. 381/382.

Anderson, J.R.; Dillon, J.L.; Hardaker, B. (1977): Agricultural Decision Analysis, Ames, Iowa.

ARROW, K.J. (1970): Essays in the theory of risk-bearing. Amsterdam/London.

Backhaus, K.; Erichson, B.; Plinke, W.; Weiber, R. (2003): Multivariate Analysemethoden, 10. Auflage, Berlin, Heidelberg, New York.

BAHRS, E. (2005): Proposal for a more efficient subsidy system for organic farming Potential use of the tax system within the European Union. In: Journal of Renewable Agriculture and Food Systems, 3/2005 (20), S. 148-154.

BAHRS, E.; HelD, J.-H. (2004): Die Förderung des Öko-Landbaus muss modifiziert werden. In: Ökologie \& Landbau, Ausgabe 4/2004, S. 50.

Bamberg, G.; Coenenberg, A.G. (2004): Betriebswirtschaftliche Entscheidungslehre. 12. Auflage, München.

BAudoux, P. (2001): Beurteilung von Agrarumweltprogrammen - eine einzel-betriebliche Analyse in Baden-Württemberg und Nordbrandenburg. In: Agrarwirtschaft, Band 50, S. 249-261.

Berekoven, L.; EcKert,W.; Ellenrieder, P. (2004): Marktforschung - Methodische Grundlagen und praktische Anwendung, 10. Auflage, Wiesbaden.

BerG, M.; HAAS, G.; KÖPKE, U. (1999): Nitrataustrag im Systemvergleich: Produkt- und Flächenbezug. In: Mitt. Ges. Pflanzanbauwiss. 12, S. 237-238.

Berg, M.; Eisele, J.-A.; Schulze Pals, L. (2003): Umweltindikatoren als Element agrarpolitischer Maßnahmen - Möglichkeiten und Grenzen aus Sicht der Administration. In: Schriftenreihe agrarspectrum, Band 36: Umweltindikatoren Schlüssel für eine umweltverträgliche Land- und Forstwirtschaft, S. 85-96.

BertKe, E.; Groth, M.; Richter, A (2005): Die Honorierung von Umweltleistungen per Ausschreibung - Ergebnisse der modellhaften Umsetzung eines marktanalogen Agrarumweltprogramms. In: Ländlicher Raum, Agrarsoziale Gesellschaft e.V. (Hrsg.), Ausgabe Nov./Dez. 2005, S. 41-44. 
BiChleR, B.; HÄrING, A. M.; LIPPERT, C. (2003): Die Bestimmungsgründe für die räumliche Struktur des ökologischen Landbaus in Deutschland. Ökologischer Landbau der Zukunft, Wien.

BIOLAND (2004a):Ökolandbauförderung zwischen Anspruch und Wirklichkeit - Bioverbände kritisieren Einsparmaßnahmen beim MEKA. Pressemitteilung vom 23.03.04, Internet: http://www.bioland-bw.de/presse/pm_2004_03_23.html, Abrufdatum: 27.02.2005.

Bioland (2004b): Keine Visionen mehr? In: Bioland, Heft 07/2004, S. 4-5.

BIOLAND (2005): Länderwillkür bremst Öko-Landbau, In: Bioland, Heft 03/2005, S.3-4.

Binswanger, H. P. (1980): Attitudes toward Risk: Experimental Mesurement In Rural India. In: American Journal of Agricultural Economics 62, S. 395-407.

BIZER, K.; LANG, J. (2000): Ansätze für ökonomische Anreize zum sparsamen und schonenden Umgang mit Bodenflächen. Forschungsbericht im Auftrag des Umweltbundesamtes, Berlin.

BlEYMÜLlER, J.; GEHLERT, G.; GÜLICHER, H. (2004): Statistik für Wirtschaftswissenschaftler. WiSt Studienkurs, 14. Auflage, München.

BMF (2005): Kassenmäßige Steuereinnahmen nach Steuerarten in den Kalenderjahren 2002 2005. In: BMF-Finanzbericht 2005, Tabelle 11, Berlin.

BMF (2006): Ergebnisse der steuerlichen Betriebsprüfung 2005. Internet: http://www.bundesfinanzministerium.de/cln_06/nn_3790/DE/Steuern/Veroeffentlichu ngen_zu_Steuerarten/Betriebspruefung/003.html; Abrufdatum: 12.05.2006.

BMELV (1998 bis 2006): Agrarberichte der Bundesregierung 1998 bis 2006, Berlin.

BMELV (2003a): Bewertung von Lebensmitteln verschiedener Produktionsverfahren Statusbericht 2003. Senat der Bundesforschungsanstalten, Berlin.

BMELV (2003b): Bundesprogramm Ökologischer Landbau. Informationsbroschüre des Referates für Öffentlichkeitsarbeit, Berlin.

BMELV (2006a): Ökologischer Landbau in Deutschland. Stand: März 2006, Internet: http://www.bmelv.de $>$ Startseite $>$ Landwirtschaft $>$ Ökologischer Landbau, Abrufdatum: 06.04.2006. 
BMELV (2006b): Rahmenplan der Gemeinschaftsaufgabe „Verbesserung der Agrarstruktur und des Küstenschutzes“ für den Zeitraum 2006 bis 2009. Internet: http://www.bmelv.de>Startseite> Landwirtschaft $>$ Förderung $>$ GAK, Abrufdatum: 21.04.2006.

BMELV (2006c): Gemeinschaftsaufgabe „Verbesserung der Agrarstruktur und des Küstenschutzes“; Erläuterungen zu Rechtsgrundlagen und zur Funktionsweise. Internet: http://www.bmelv.de $>$ Startseite $>$ Landwirtschaft $>$ Förderung $>$ GAK, Abrufdatum: 21.04.2006.

BNN (2006): Bilanz im Pestizid-Monitoring für Obst und Gemüse: 1000 Mal beprobt, selten nur gedopt. In: BNN-Nachrichten Naturkosthandel, Ausgabe März 2006, S. 8.

BocKISCH, F.-J. (2000): Bewertung von Verfahren der ökologischen und konventionellen landwirtschaftlichen Produktion im Hinblick auf den Energieeinsatz und bestimmte Schadgasemissionen. Wissenschaftliche Mitteilungen der FAL, Sonderheft 211. Bundesforschungsanstalt für Landwirtschaft (FAL), Braunschweig.

BDP (2005): Steuern und Finanzen. Bundeszentrale für politische Bildung (bpb) (Hrsg.), Informationen für politische Bildung, Ausgabe 288.

Braband, D.; V. Elsen, T.; OpPeRMAnN, H.; HAACK, S. (2003): Ökologisch bewirtschaftete Ackerflächen - eine ökologische Leistung? Ein ergebnisorientierter Ansatz für die Praxis. In: Freyer, B. (Hrsg.): Beiträge zur 7. Wissenschaftstagung zum Ökologischen Landbau - Ökologischer Landbau der Zukunft. Wien, S. 153-156.

BRANDES, W.; OdEnING, M. (1992): Investition, Finanzierung und Wachstum in der Landwirtschaft. Stuttgart.

BRÜGgemanN, C. (2006): Biofleisch verlässt die Nische. In: top agrar, Ausgabe 3/2006, S. 162-164.

BuNTE, F. (2004): In de markt geprijsd. Rapport 6.04.16, LEI, Den Haag.

BVL (2005): Lebensmittel-Monitoring 2004 - Ergebnisse eines bundesweiten LebensmittelMonitorings. Gemeinsamer Bericht des Bundes und der Länder, Berlin.

DABBert, S.; HÄRING, A.; ZANOLI, R. (2002): Politik für den Öko-Landbau. Bonn.

DABBERT, S.; HÄRING, A. (2003): Vom Aschenputtel zum Lieblingskind - Zur politischen Förderung des Ökolandbaus. In: GAIA, Ausgabe 02/2003, S. 100-106. 
DARNHOFER, I (2003): Umstellung auf biologischen Landbau aus Sicht der Landwirte. Vortrag am 01. Juli 2003 in Wien im Rahmen der Aktion Österreich-Tschechien: Vergleich umweltpolitischer Maßnahmen in der landwirtschaftlichen Produktion der Tschechischen Republik und Österreichs.

Diekmann, A. (2002): Empirische Sozialforschung. Grundlagen, Methoden, Anwendung. 9. Auflage, Reinbek bei Hamburg.

DREYER, W. (2005): Durchschnittlich reicht nicht. In: Bioland, 11/2005, S. 30.

DRUCKER, P.F. (1956): Praxis des Management: Ein Leitfaden für die Führungs-Aufgaben in der modernen Wirtschaft. Düsseldorf.

EUROPÄISCHE KOMMISSION (2001): Green Paper on Integrated Product Policy, Brüssel.

EUROPÄISCHE KOMMISSION (2004): Eine Partnerschaft für die Kohäsion - Dritter Bericht über den wirtschaftlichen und sozialen Zusammenhalt. Internet: http://europa.eu.int/comm/regional_policy/sources/docoffic/official/reports/ cohesion3/cohesion3_de.htm.

Falconer, K.; Whitby, N. (1999): The Invisible Costs of Scheme Implementation and Administration. In: Van Huylenbroek, G. And Whiby, M (eds.) Countryside Stewardship: Farmers, Policies and Markets. Oxford, S. 67-68.

FISCHBACHER, U. (1998): z-Tree 1.0 Instruktionen für Experimentatoren, Internet: http://www.iew.unizh.ch/ztree/ztreemand.pdf, Abrufdatum: 15.09.2005.

FRIEDMAN, D.; SUNDER, S. (1994): Experimental methods - Aprimer for economists. Cambridge.

Fuchs, C. (2002): The Influence of Per-hectare Premiums on Prices for Rented Agricultural Area and on Agricultural Land Prices. In: Agrarwirtschaft 51, S. 396-403.

GARDEBROEK, K. (2002): Are organic farmers less risk averse than non-organic farmers? Vortrag präsentiert auf dem 10. Kongress der European Association of Agricultural Economists (EAAE) in Saragossa, Spanien, 28.-31. August 2002.

GoRIS, E. (2006): Wie gut ist Bio-Kost aus dem Supermarkt? In: Bild am Sonntag, Ausgabe vom 7. Mai 2006, S. 56-59. 
GRINGS, M. (1991): Verbrauchselastizitäten für Produkte aus ökologischem Landbau. In: Thimm, C. (Hrsg.): Neue Absatzwege und Elastizität für Produkte aus ökologischem Landbau in Nordeuropa. Studie im Auftrag der EG-Kommission, Fallingbostel.

GrOß, D. (2004): Mit Bauern sind „20 Prozent Bio“ so nicht zu machen. In: Der kritische Agrarbericht 2004, S. 138-142.

GÜTH, W.; KRAHNEN, J.P.; RIECK, C. (1997): Financial markets with asymmetric information: A pilot study focusing on insider advantages. In: Journal of Economic Psychology 18, S. $235-257$.

HAAs, G.; GeIER, U.; Schulz, D.G.; KÖPKE, U. (1995): Klimarelevanz des Agrarsektors der Bundesrepublik Deutschland: Reduzierung der Emissionen von Kohlendioxid. In: Berichte über Landwirtschaft 73, S. 387-400.

HAAS, G. (2005): Ökologischer Landbau in Nordrhein-Westfalen: Empirische Analyse und Bestimmungsgründe der heterogenen räumlichen Verteilung. In: Agrarwirtschaft, Band 54, S. 119- 127.

HACCIUS, M (2003): Ökologischer Landbau - Grundlagen und Praxis. AID-Heft Nr. 1070, Bonn.

Hadatsch, S.; Kratochvil, R.; Vabitsch, A.; Freyer, B.; Götz, B. (2000): Biologische Landwirtschaft im Marchfeld. Potentiale zur Entlastung des Natur- und Landschaftshaushaltes. Umweltbundesamt, Serie: Monographien, Band 127, Wien.

Hagedorn, K.; Beckmann, V.; Tiemann, S.; Reuter, K. (2004): Kosten der Erreichung von Umweltqualitätszielen in ausgewählten Regionen durch Umstellung auf Ökologischen Landbau im Vergleich $\mathrm{zu}$ anderen Agrarumweltmaßnahmen unter besonderer Berücksichtigung von Administrations- und Kontrollkosten, Bericht Nr. 02OE227, Bundesprogramm Ökologischer Landbau. Unveröffentlicht.

Hagner, C. (1994): Nachfrage nach Produkten des ökologischen Landbaus. Stand der Literatur und Ergebnisse einer ökonometrischen Nachfrageanalyse für Müsliprodukte. Agrarökonomische Diskussionsbeiträge 23, Institut für Agrarpolitik und Marktforschung, Universität Gießen.

HAMM, U. (2003): Förderung des ökologischen Landbaus - aber wie? In: B\&B Agrar, Ausgabe 1/03, S. 14-19. 
Hamm, U.; WiLD, S. (2004): Der Preis bestimmt den Absatz. In: bioHandel, Ausgabe 1/2004, S. 8-13.

Hansen, B.; Kristensen, E. S.; Grant, R.; Högh-Jensen, H.; SimmelsgaArd, S. E.; OLESEN, J. E. (2000): Nitrogen Leaching from conventional versus organic farming systems - a system modelling approach. In: European Journal of Agronomy 13, S. 6582.

Hardaker, J. B.; Huirne, R. B. M.; Andersen, J. R.; Lien, G. (2004): Coping with Risk in Agriculture, 2. Auflage, Wallingford.

HARRISON, G. W.; List; J. A. (2004): Field Experiments. In: Journal of Economic Literature, Vol. XLII, S. 1009-1055.

Hartmann, E.; ScheKahn, A.; Luick, R.; Thomas, F. (2006): Kurzfassung der Agrarumwelt- und Naturschutzprogramme - Darstellung und Analyse von Maßnahmen der Agrarumwelt- und Naturschutzprogramme in der Bundesrepublik Deutschland. BfN-Skripten 161. Internet: www.bfn.de/fileadmin/MDB/documents/skript161.pdf, Abrufdatum: 30.06.2006.

Held, M.; KUBON-GILKE, G.; STURN, R. (2003): Experimente in der Ökonomik. In: Jahrbuch Normative und institutionelle Grundfragen der Ökonomik. Band 2, Marburg, S. 7-38.

HenrichsmeieR, W.; Witzke, H.-P. (1994): Agrarpolitik Band 2 - Bewertung und Willensbildung. Stuttgart.

Hollenberg, K. (2001): Auswirkungen einer Umstellung der Landwirtschaft auf ökologischen Landbau. Landwirtschaft und Umwelt, Schriften zur Umweltökonomik, Band 18, Kiel.

Holm-MÜlleR, K.; RADKe, V.; WeIS, J. (2002): Umweltfördermaßnahmen in der Landwirtschaft - Teilnehmerauswahl durch Ausschreibungen? In: Agrarwirtschaft, Band 51, S. 112-120.

Holm-MülleR, K.; Hilden, R. (2004): Modellprojekt „Erprobung der Ausschreibung von Agrarumweltprogrammen am Beispiel der Grünlandextensivierung“. Vortrag anlässlich der 44. GeWiSoLa-Tagung vom 27. bis 29. September 2004 in Berlin.

HÜTTNer, M.; Schwarting, U. (1999): Exploratorische Faktorenanalyse. In: Homburg, C.; Herrmann, A. (Hrsg.): Marktforschung, Wiesbaden, S. $381-412$. 
JOANKNECHT, N. (2004): Aktionsplan Ökologische Landwirtschaft in den Niederlanden. Vortrag auf der BMELV-Tagung „Ökologische Lebensmittel in Deutschland Perspektiven zur Weiterentwicklung des Ökologischen Landbaus“ am 7. und 8.12.2004 in Berlin.

KACHEL, K.-U. (1999): Wirkungen der Förderung umweltgerechter landwirtschaftlicher Nutzung von Ackerflächen; betriebswirtschaftliche Untersuchungen zu ausgewählten Förderprogrammen in Landwirtschaftsbetrieben im Biosphärenreservat SchorfheideChorin. Landwirtschaft und Umwelt, Schriften zur Umweltökonomik, Band 16, Kiel.

Kachelmeier, S.J.; Shehata, M. (1992): Examing Risk Preferences Under High Monetary Incentives: Experimental Evidence from the People's Republic of China. In: The American Economic Review 82, S. 1120-1141.

Kahnemann, D.; TVERsky, A. (1979): Prospect theory: An analysis of decision under risk. In: Econometrica 47, S. 263-291.

KEMPKENS, K. (2006): Ökolandbau nicht vergessen. In: Landwirtschaftliches Wochenblatt Westfalen-Lippe, Ausgabe 6/2006; S. 26.

Ketelhodt, C. (2005): Klare Signale sind gefordert! In: Bioland, Ausgabe 05/2005, S. 7.

KÖHNE, M.; KÖHN, O. (1998): Betriebsumstellung auf ökologischen Landbau - Auswirkung der EU-Förderung in den neuen Bundesländern. In: Berichte über Landwirtschaft, Band 76, S. 329-365.

KÖSTER, U. (1992): Grundzüge der landwirtschaftlichen Marktlehre. 2. Auflage, München.

KÖSTER, U. (2001): Grundsätze für eine Neuausrichtung der Agrarpolitik. In: Agrarwirtschaft, Band 50, S. 229-231.

Kuhnert, H.; FeIndT, P. H.; Beusmann, V. (2006): Ausweitung des Ökologischen Landbaus in Deutschland. In: ASG Ländlicher Raum, Ausgabe März/April, S. 21-23.

KußMaul, H. (2005): Betriebswirtschaftliche Steuerlehre. 4. Auflage, München.

Latacz-Lohmann, U.; Hamsvoort, C. P. C. M. van DeR (1997): Auktioning Conservation Contracts: A Theoretical Analysis and an Application. In: American Journal of Agricultural Economics, Ausgabe 79, S. 407-418. 
Latacz-Lohmann, U.; Recke, G.; WolfF, H. (2001): Die Wettbewerbsfähigkeit des ökologischen Landbaus: Eine Analyse mit dem Konzept der Pfadabhängigkeit. In: Agrarwirtschaft, Band 50, S. 433-438.

Lien, G.; Flaten, M.; Ebbesvik, M.; Koesling, M.; Valle, P.S. (2004): Risk and risk management in organic and conventional dairy farming: empirical results from Norway. In: Farm Management, Band 11, S. 665-675.

LIPPROSS, O.-G. (2005): Umsatzsteuer. Grüne Reihe, Band 11, 21. Auflage, Achim.

Miller, J. (2003): Öko-Erlebnistage 2003 - Situation des Öko-Landbaus in Bayern. Rede vom Staatminister Miller anlässlich einer Pressekonferenz am 04.09.2003 in München. Internet: http://www.stmlf.bayern.de/publikationen/ministerreden/2003/re091-03.html, Abrufdatum: 09.01.2004.

Ministry of Agriculture, NATURe AND Food QuAlity NL (2000): An organic market to conquer. Policy document on Organic Agriculture 2001-2004, Den Haag.

Ministry of Agriculture, NAture AND Food Quality NL (2004): Dutch Policy Document on Organic Agriculture 2005-2007, Den Haag.

Mehl, P.; PlankL, R. (2002): Regionale Agrarpolitik in Deutschland - Handlungsspielräume in einem verflochtenen Politikfeld. In: Jahrbuch des Föderalismus - Föderalismus, Subsidiarität und Regionen in Europa, Europäisches Zentrum für Föderalismusforschung, S. 199-210.

MEYER, R. (2005): Darstellung der Ertragslage der Öko-Betriebe in Deutschland. In: ZMPÖKOMARKT-Forum, Nr. 8, S. 1-10.

Mosler, K.; Schmid, F. (2003): Beschreibende Statistik und Wirtschaftsstatistik. 1. Auflage, Berlin und Heidelberg.

Mosteller, F.; Nogee, P. (1951): An Experimental Mesurement of Utility. In: Journal of Political Economy 59, S. 371-404.

MuZiol, O. (1999): Voraussetzungen, Chancen und Risiken des ökologischen Landbaus aus Sicht der Unternehmensberatung. In: HLBS-Report 5/99, S. 15-22.

NiEBERG, H. (2001): Umstellung auf ökologischen Landbau: Wer profitiert? In: Ökologie\&Landbau, Ausgabe 02/2001, S. 6-9.

NiEBERG, H. (2004): Wie wichtig sind die Öko-Prämien? In: bioland 08/2004, S. 6/7. 
NieBERG, H. (2006): Flächenbezogene Förderung bei Einführung und Beibehaltung ökologischer Wirtschaftsweisen und Kontrollkostenzuschuss nach Bundesländern (2006). Internet: http://www.oekolandbau.de/fileadmin/redaktion/dokumente/erzeuger/ flaechenpraemien_060419.pdf, Abrufdatum: 06.05.2006.

NieBerg, H.; OfFERMANN, F. (2001): Wirtschaftliche Situation ökologischer Betriebe in ausgewählten Ländern Europas: Stand, Entwicklung und wichtige Einflussfaktoren. In: Agrarwirtschaft, Band 50, S. 421-427.

NiEBERG, H.; OFFERMANN, F. (2006): Einkommensvergleich zwischen ökologischen und konventionellen Betrieben. In: Agra-Europe, 18/06, Sonderbeilage.

NieBERG, H.; STROHM-LÖMPKE, R. (2001): Förderung des ökologischen Landbaus in Deutschland: Entwicklung und Zukunftsaussichten. In: Agrarwirtschaft, Band 50, S. $410-420$.

NiENDIEKER, V. (1998): Die Ratsverordnung (EWG) Nr. 2078/92 als Instrument der europäischen und nationalen Agrarumwelt- und Agrarstrukturpolitik. In: Berichte über Landwirtschaft, Band 76 (1998), S. 520-539.

Odening,M.; MußhOFF, O.; Utesch, V. (2004): Der Wechsel vom konventionellen zum ökologischen Landbau: Eine investitionstheoretische Betrachtung. In: Agrarwirtschaft, Band 53, S. 223-232.

OfFERMANN, F. (2003): Quantitative Analyse der sektoralen Auswirkungen einer Ausdehnung des ökologischen Landbaus in der EU. Berliner Schriften zur Agrar- und Umweltökonomik, Band 5, Aachen.

Oppermann, B.; Lutz, F.; Kaule, G. (1997): Der „Runde Tisch“ als Mittel zur Umsetzung der Landschaftsplanung. In: Angewandte Landschaftsökologie, Schriftenreihe des Bundesamtes für Naturschutz, Band 11, S. 50-51.

OPPERMANN, R. (2001): Ökologischer Landbau am Scheideweg: Chancen und Restriktionen für eine ökologische Kehrtwende in der Agrarwirtschaft. ASG - Kleine Reihe Nr. 62, Göttingen.

OsterburG, B. (2002): Analyse der Bedeutung von naturschutzorientierten Maßnahmen in der Landwirtschaft im Rahmen der Verordnung (EG) 1257/1999 über die Förderung der Entwicklung des ländlichen Raumes. Materialien zur Umweltforschung, Stuttgart. 
Osterburg, B.; Stratmann, U. (2002): Die regionale Agrarumweltpolitik in Deutschland unter dem Einfluss der Förderangebote der Europäischen Union. In: Agrarwirtschaft, Band 51, S. 259-279.

OsterburG, B.; ZANDER, K. (2004): Regionale Strukturen des ökologischen Landbaus in Deutschland. Arbeitsberichte des Bereiches Agrarökonomie, FAL, Braunschweig.

PFIFFNER, L. (1997): Welchen Beitrag leistet der ökologische Landbau zur Förderung der Kleintierfauna? In: Weiger, H.; Willer, H. (Hrsg.): Naturschutz durch Ökologischen Landbau. Bad Dürkheim, S. 93-120.

PRATT, J.W. (1964): Risk aversion in the small and in the large. In: Econometrica Nr. 32, S. 122-136.

ReIMER, W. (2005): Zukunft der 2. Säule der Agrarpolitik - Chancen, Bedeutung und Unwägbarkeiten. In: ASG (Hrsg.): Landwirtschaft in einer beschleunigten Globalisierung. Schriftenreihe für ländliche Sozialfragen Nr. 146, Göttingen.

Richardson, J.W. (2005a): Simulation for Applied Risk Management. Department of Agricultural Economics, Texas A\&M University.

Richardson, J.W. (2005b): Developing and Using Simulation Models for Risk Analysis. Begleitheft zum Simulations-Workshop in College Station (Texas) vom 17.-19. Januar 2005; unveröffentlicht.

RIPPIN, M. (2003): Wachstum durch niedrige Erzeugerpreise erkauft? In: top agrar, Ausgabe 3/2003, S. 138-142.

RIPPIN, M. (2006): Wachstum ohne die Deutschen. In: DLG-Mitteilungen, Ausgabe 5/2006, S. 64-69.

Rommelfanger, H.J.; EICKEMEIER; S.H. (2002): Entscheidungstheorie - Klassische Konzepte und Fuzzy-Erweiterungen. Berlin/Heidelberg.

Rothkopf, M.H.; Teisberg, T.; Kahn, E.P. (1990): Why Are Vickrey Auctions Rare? In: Journal of Political Economy 98, S.94-109.

Rubinstein, A. (2001): A theorists view of experiments. In: European Economic Review 45, S. $615-628$. 
RUDLOFF, B.; URFEI, G. (2000): Agrarumweltpolitik nach dem Subsidiaritätsprinzip: Kategorisierung von Umwelteffekten und Evaluierung geltender Politikmaßnahmen. Schriften zur Agrarforschung und Agrarpolitik, Band 3, Berlin.

SAUER, N.; WeIERSHÄUSER, L.; HARDEWEG, B. (2004): Standarddeckungsbeiträge （SDB) 2000/01, 2001/02, 2002/03. KTBL (Hrsg.), Münster.

SCHEMMEL, L. (1998): Ökosteuern - kein geeigneter Weg aus der Beschäftigungs- und Umweltmisere. Wiesbaden.

SCHERINGER, J.; ISSELSTEIN, J. (2001): Nitrogen budgets of organic and conventional dairy farms in North-West Germany. In: Isselstein, J.; Spatz, G.; Hofmann, M.: Organic Grassland Farming. Proc. Int. Occ. Symp. Europ. Grassland Fed., Witzenhausen, 10. bis 12. Juli 2001. Grassland Science in Europe, Vol. 6, S. 284-287.

SCHNEEBERGER, W.; DARNHOFER, I.; EDER, M. (2002): Barriers to the adoption of organic farming by cash crop producers in Austria. In: American Journal of Alternative Agriculture, Band 17, S. 24-31.

SchrameK, J.; SchNAUT, G. (2004): Motive der (Nicht-)Umstellung auf Öko-Landbau. In: Ökologie\&Landbau, Ausgabe 3/2004, S. 44-46.

SCHUlZE-PALS, L. (1994): Ökonomische Analyse der Umstellung auf ökologischen Landbau. Münster.

SmitH, V.-L. (1976): Experimental Economics: Induced Value Theory. In: American Economic Review 66, S. 274-279.

SÖL (2004): Bundesprogramm Ökologischer Landbau - Rückblick und Perspektiven. In: Ökologie\&Landbau, Ausgabe 1/2004, Spezialbeilage.

SPILLER, A. (2001): Preispolitik für ökologische Lebensmittel: Eine neo-institutionalistische Analyse. In: Agrarwirtschaft, Band 50, S. 451-461.

STATISTISCHES BundESAMT (2004): Statistisches Jahrbuch über Ernährung, Landwirtschaft und Forsten 2004. Münster-Hiltrup.

StATISTISCHES BundeSAMT (2005): Betriebe mit ökologischem Landbau, Agrarstrukturerhebung 2005, Fachserie 3, Reihe 2.2.1. 
Stolze, M.; Piorr, A.; HÄring, A.; DabBert, S. (2000): The environmental impacts of organic farming in Europe. Schriftenreihe: Organic farming in Europe: Economies and Policy Vol. 6, Stuttgart-Hohenheim.

Taube, F.; Loges, R.; Kelm, M.; LAtaCZ-Lohmann, U. (2004): Vergleich des ökologischen und konventionellen Ackerbaus im Hinblick auf Leistungen und ökologische Effekte auf Hochertragsstandorten Norddeutschlands. In: Berichte über Landwirtschaft, Band 83 (2005), S. 165-176.

TIPKE, K. (2000): Die Steuerrechtsordnung - Band 1: Wissenschaftsorganisatorische, systematische und grundrechtlich rechtsstaatliche Grundlagen. 2. Auflage, Köln.

Vatn, A.; Kvakkestad, V.; Rorstad, P.-K. (2002): Policies for Multifunctional Agriculture - The Trade-off between Transaction Costs and Precision. Department of Economics \& Resource Management. As, Norway.

VerschuUr, G.; VAN Well, E. (2001): Stimulating organic farming in the EU - With economic and fiscal instruments. Centre for Agriculture and Environment (clm), Utrecht.

ViCKREY, W. (1961): Counterspeculation, Auctions and Competitive Sealed Tenders. In: Journal of Finance 16, S. 8-37.

WACHENDORF, M.; TAUBE, F. (2001): Artenvielfalt, Leistungsmerkmale und bodenchemische Kennwerte der Dauergrünlands im konventionellen und ökologischen Landbau in Nordwestdeutschland. In: Pflanzenbauwissenschaften 2, S. 75-86.

WEHDE, G. (2005): Priorität für den Bio-Landbau! In: bioland, Ausgabe 04/2005, S. 3-4.

WEHDE, G. (2006): EU-Finanzbeschlüsse stehen nachhaltiger Landwirtschaft entgegen. In: Ökologie\&Landbau, Ausgabe 2/2006, S. 46-48.

WIER, M.; SMERD, S. (2000): Forbrug af okologiske fodevarer. Del 2: Modellering og eftersporgsel. Faglig rapport fra DMU 319. Danmarks Miljoundersogelser.

ZMP (2004): Ökomarkt-Jahrbuch 2004 - Verkaufspreise im ökologischen Landbau. Materialen zur Marktberichterstattung, Band 51, Bonn.

ZMP (2005): ZMP-Jahresbericht 2005/2006, Rückblick und Vorschau auf die Agrarmärkte. Bonn. 


\section{VERZEICHNIS DER SONSTIGEN MITTEILUNGEN}

DRESSLER (2005): Bayerisches Staatsministerium für Landwirtschaft und Forsten, Referat L 2, mündliche Mitteilung im Juni 2005.

Gardebroek, K. (2004): Universität Wageningen, Agricultural Economics and Rural Policy Group, Department of Social Sciences, schriftliche Mitteilung im November 2004.

Kasbohm, A. (2004): ZMP, Zuständigkeitsbereich Obst und Gemüse, mündliche Mitteilung im Juli 2004.

RIPPIN, M. (2006): ZMP, Zuständigkeitsbereich Öko-Markt, mündliche Mitteilung im Januar 2006.

\section{GESPRÄCHSPARTNER IN DEN LÄNDERMINISTERIEN}

Baden Württemberg: Herr Dr. Norbert Haber, Ministerium für Ernährung und ländlichen Raum Baden-Württemberg, Referat Agrarpolitik.

Bayern: Frau Ingeborg Bauer, Bayerisches Staatsministerium für Landwirtschaft und Forsten, Referat L 2.

Brandenburg: Frau Dr. Karen Krüger, Ministerium für Landwirtschaft, Umweltschutz und Raumordnung des Landes Brandenburg, Referat 22.

Hessen: Herr Martin Fuchs, Hessisches Ministerium für Umwelt, Landwirtschaft und Forsten, Abteilung VII.

Mecklenburg-Vorpommern: Herr Peter Ditz, Ministerium für Ernährung, Landwirtschaft, Forsten und Fischerei Mecklenburg-Vorpommern, Referat 330.

Niedersachsen: Herr Dr. Stefan Dreesmann, Niedersächsisches Ministerium für Ernährung, Landwirtschaft und Forsten, Referat 107.

Nordrhein-Westfalen: Herr Wolfgang Neuerburg, Ministerium für Umwelt und Naturschutz, Landwirtschaft und Verbraucherschutz des Landes NRW, Referat II-6.

Rheinland-Pfalz: Frau Sabine Hohn-Braun, Ministerium für Wirtschaft, Verkehr, Landwirtschaft und Weinbau Rheinland-Pfalz, Referat 8602.

Saarland: Herr Dr. Ferdinand Ecker, Ministerium für Umwelt Saarland, Referat B2. 
Sachsen: Herr Herwig Vopel, Sächsisches Ministerium für Umwelt und Landwirtschaft, Referat 32.

Sachsen-Anhalt: Herr Hagen, Ministerium für Landwirtschaft und Umwelt Sachsen-Anhalt, Referat 55.

Schleswig-Holstein: Herr Andreas Koppe, Ministerium für ländliche Räume, Landesplanung, Landwirtschaft und Tourismus Schleswig-Holstein, Referat 24.

Thüringen: Frau Ilka Bergner, Thüringer Ministerium für Landwirtschaft, Naturschutz und Umwelt, Referat 31. 


\section{VERZEICHNIS DER RECHTSQUELLEN}

Verordnung (EWG) Nr. 4115/88 der Kommission vom 21. Dezember 1988 mit Durchführungsbestimmungen zur Beihilferegelung für die Extensivierung der Erzeugung. Abl. EG Nr. L 361 vom 29.12.1988.

Verordnung (EWG) Nr. 2092/91 des Rates vom 24. Juni 1991 über den ökologischen Landbau / die biologische Landwirtschaft und die entsprechende Kennzeichnung der landwirtschaftlichen Erzeugnisse und Lebensmittel. Abl. EG Nr. L 198 vom 22.07.1991.

Verordnung (EWG) Nr. 2078/92 des Rates vom 30. Juni 1992 für umweltgerechte und den natürlichen Lebensraum schützende landwirtschaftliche Produktionsverfahren. Abl. EG Nr. L 215 vom 30.07.1992.

Verordnung (EG) Nr. 1257/99 des Rates vom 17. Mai 1999 über die Förderung der Entwicklung des ländlichen Raumes durch den Europäischen Ausrichtungs- und Garantiefonds für die Landwirtschaft (EAGFL) und zur Änderung bzw. Aufhebung bestimmter Verordnungen. Abl. EG Nr. L 160/80 vom 26.06.1999.

Verordnung (EG) Nr. 1804/99 des Rates vom 19. Juli 1999 zur Einbeziehung der tierischen Erzeugung in den Geltungsbereich der Verordnung (EWG) Nr. 2092/91 über den ökologischen Landbau und die entsprechende Kennzeichnung der landwirtschaftlichen Erzeugnisse und Lebensmittel. Abl. EG Nr. L 222/1 vom 24.08.1999.

Verordnung (EG) Nr. 817/2004 der Kommission vom 29. April 2004 mit Durchführungsvorschriften zur Verordnung (EG) Nr. 1257/1999 des Rates über die Förderung der Entwicklung des ländlichen Raumes durch den Europäischen Ausrichtungs- und Garantiefonds für die Landwirtschaft (EAGFL). Abl. EG Nr. L 231 vom 30.06.2004.

Verordnung (EG) Nr. 1698/2005 des Rates vom 20. September 2005 über die Förderung der Entwicklung des ländlichen Raumes durch den Europäischen Landwirtschaftsfonds für die Entwicklung des ländlichen Raums (ELER). Abl. EG Nr. L 277 vom 21.10.2005. 
Richtlinien über die Gewährung von Zuwendungen für die Förderung einer markt- und standortangepassten Landbewirtschaftung. RdErl. d. Ministeriums für Umwelt und Naturschutz, Landwirtschaft und Verbraucherschutz II-6 - 72.40.32 v. 01.09.2005 (MB1. NRW. 2005 S. 1149); Internet: http://sgv.im.nrw.de/mbl/2003/Ausg4/MBL41.htm.

Verwaltungsvorschrift (VV) zu § 44 Abs. 1 LHO (Landeshaushaltsordnung) NRW 


\title{
ANHANG
}

\section{A.1 ÖKO-FLÄCHENPRÄMIEN 2006 IN DEUTSCHLAND}

\author{
Flächenbezogene Förderung bei Einführung und Beibehaltung ökologischer \\ Wirtschaftsweisen und Kontrollkostenzuschuss nach Bundesländern im Jahr 2006
}

(Stand April 2006)

Zusammengestellt von

Dr. Hiltrud Nieberg, Institut für Betriebswirtschaft, Bundesforschungsanstalt für Landwirtschaft (FAL) Braunschweig

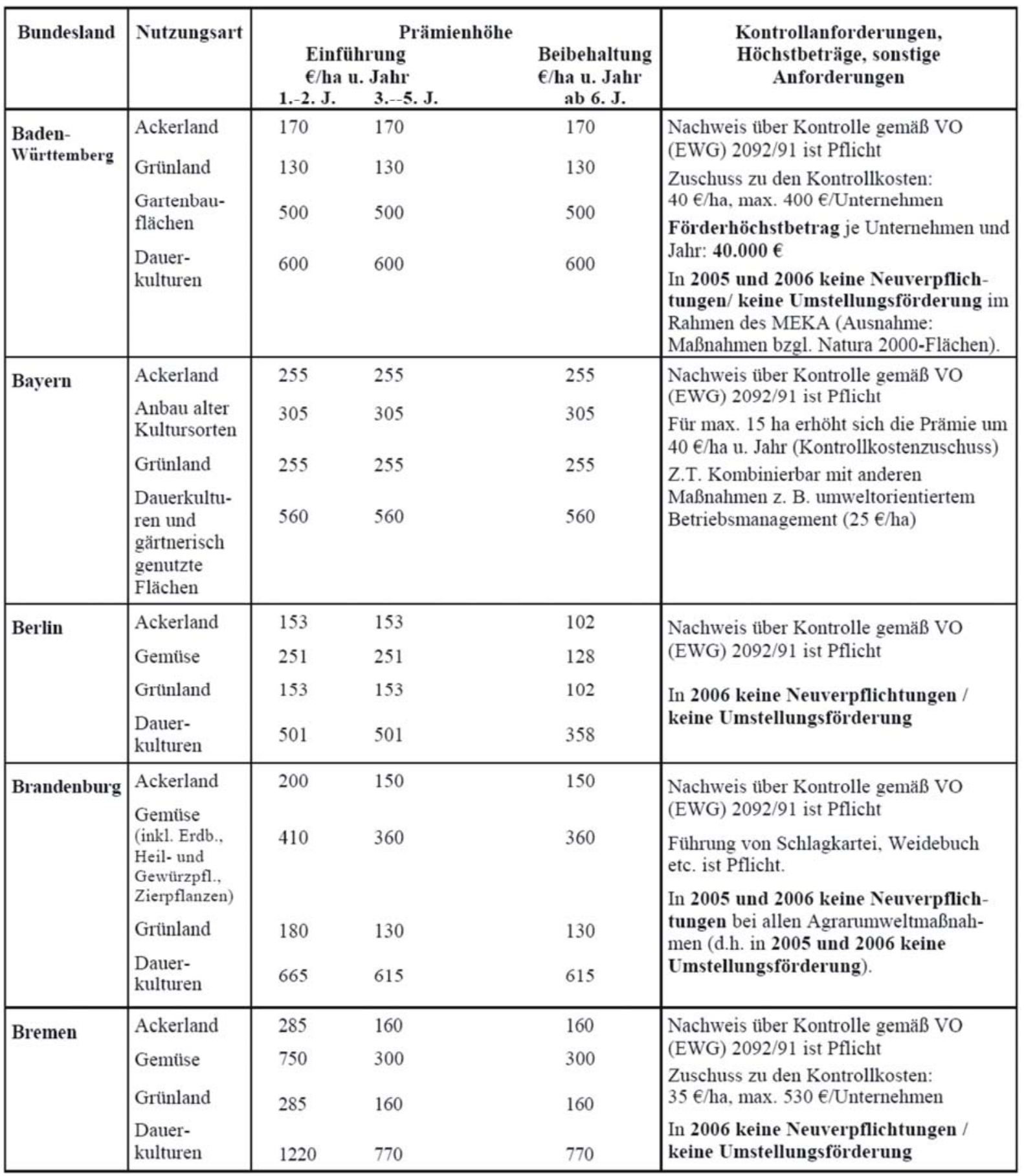




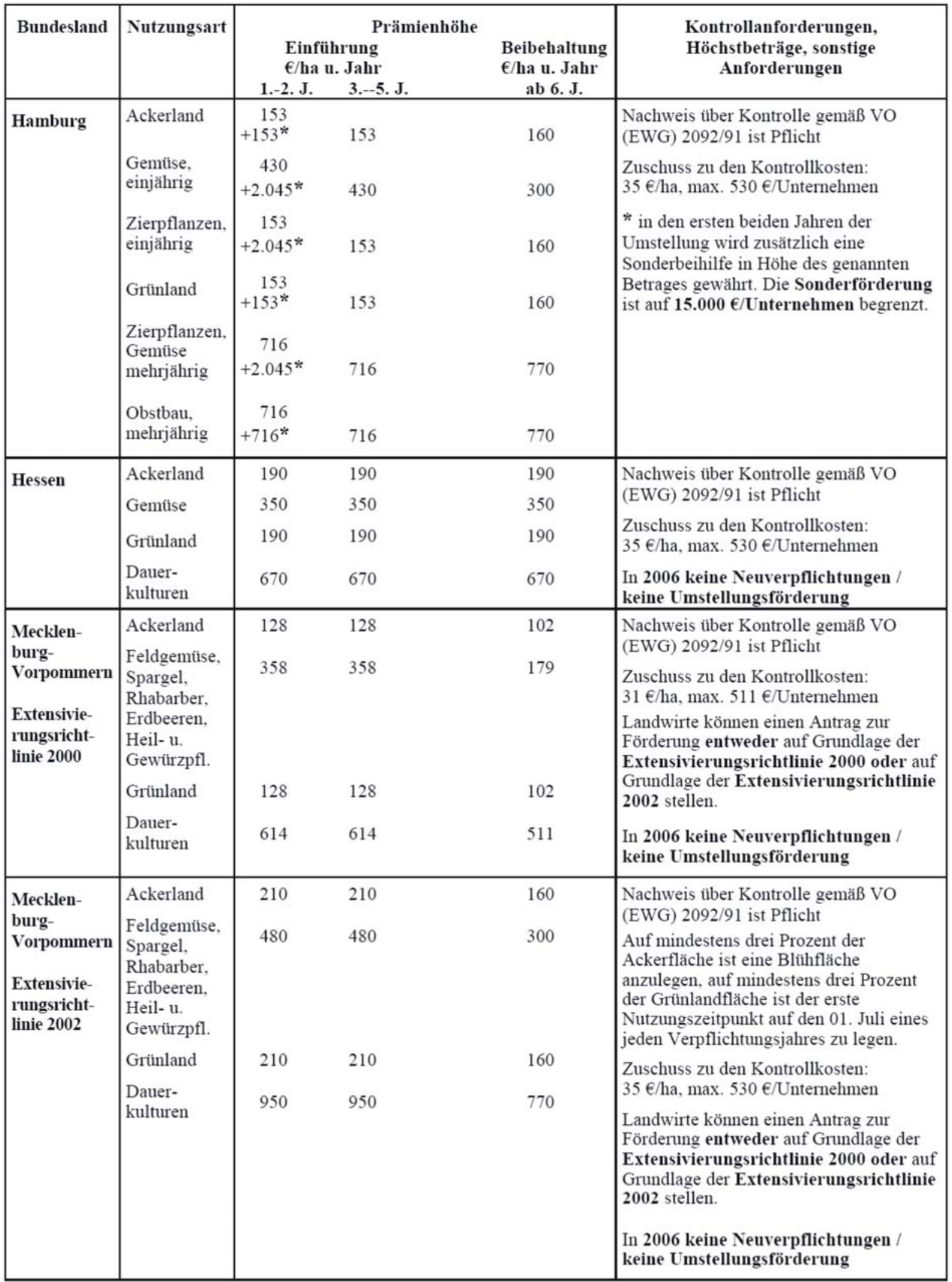




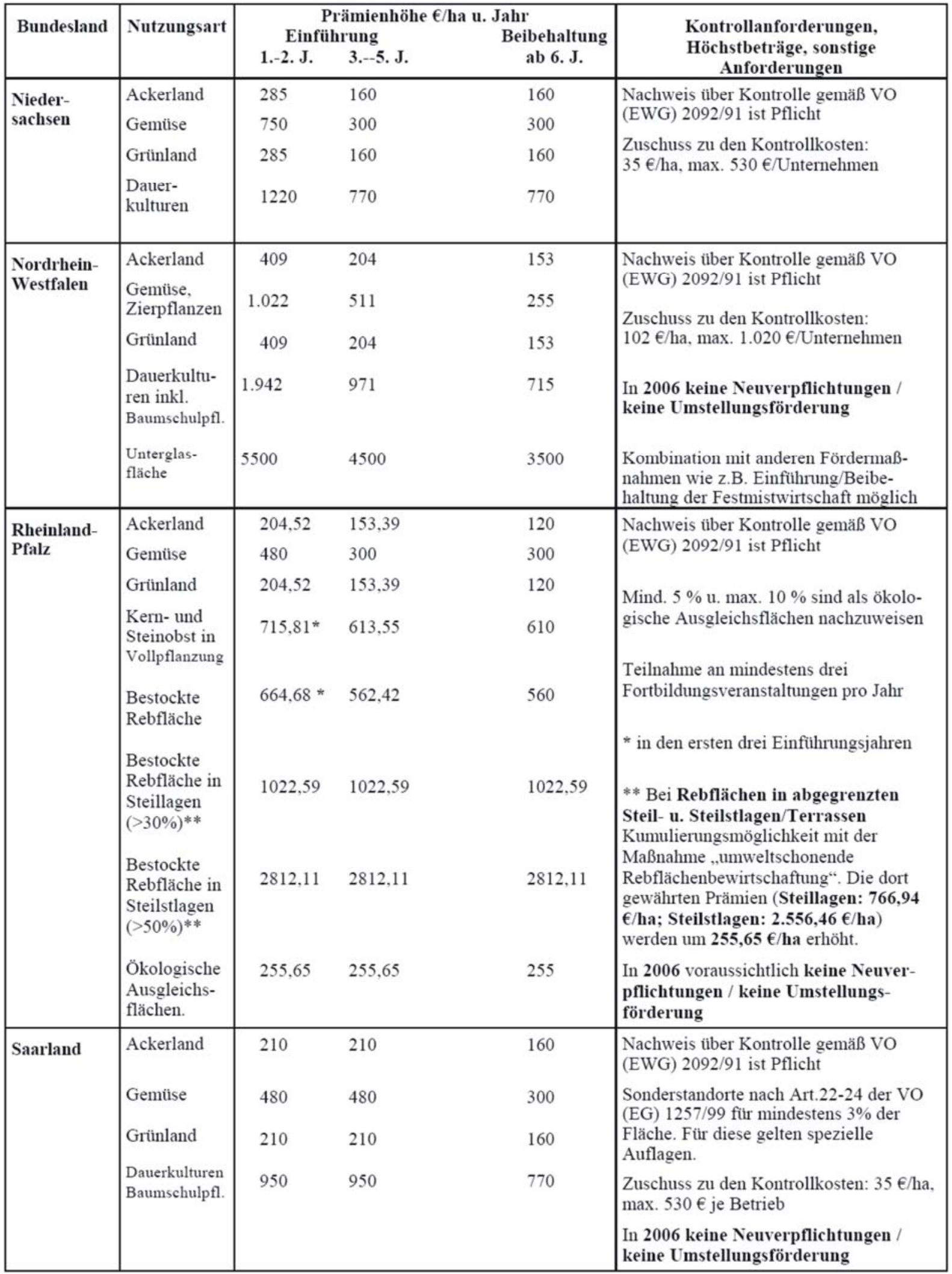




\begin{tabular}{|c|c|c|c|c|c|}
\hline \multirow{2}{*}{ Bundesland } & \multirow{2}{*}{ Nutzungsart } & \multicolumn{3}{|c|}{ Prämienhöhe $€ /$ ha u. Jahr } & \multirow[b]{2}{*}{$\begin{array}{c}\text { Kontrollanforderungen, } \\
\text { Höchstbeträge, sonstige } \\
\text { Anforderungen }\end{array}$} \\
\hline & & $\begin{array}{l}\text { Einf } \\
\text { 1.-2. J. }\end{array}$ & 3.--5. J. & $\begin{array}{l}\text { Beibehaltung } \\
\text { ab } 6 . \mathrm{J} .\end{array}$ & \\
\hline \multirow[t]{8}{*}{ Sachsen } & Ackerland & 337 & 230 & 230 & \multirow{5}{*}{$\begin{array}{l}\text { Nachweis über Kontrolle gemäß VO } \\
\text { (EWG) 2092/91 ist Pflicht } \\
\text { Führung schlag- bzw. anlagenbezogener } \\
\text { Aufzeichnungen. } \\
\text { Durchführung von Bodenuntersuchungen. }\end{array}$} \\
\hline & Gemüse & & & & \\
\hline & $\begin{array}{l}\text { (inkl. Heil- u. } \\
\text { Gewürzpfl.) }\end{array}$ & 490 & 351 & 357 & \\
\hline & Grünland & 244 & 244 & 244 & \\
\hline & $\begin{array}{l}\text { Obstbau u. } \\
\text { Baumschulpfl. }\end{array}$ & $914 *$ & 664 & 664 & \\
\hline & Weinbau & $914^{*}$ & 664 & 664 & \multirow{3}{*}{$\begin{array}{l}\text { *in den ersten drei Einführungsjahren } \\
\text { In } 2005 \text { und } 2006 \text { keine Neuverpflich- } \\
\text { tungen (d.h. in } 2005 \text { und } 2006 \text { keine } \\
\text { Umstellungsförderung) }\end{array}$} \\
\hline & $\begin{array}{l}\text { Weinbau } \\
\text { (Steillage) }\end{array}$ & $1043^{\circ}$ & 766 & 766 & \\
\hline & $\begin{array}{l}\text { Gemüse } \\
\text { (inkl. Heil- u. } \\
\text { Gewürzpfl.) } \\
\text { unter } \\
\text { Glas/Folie }\end{array}$ & 4294 & 3068 & 3068 & \\
\hline \multirow{9}{*}{$\begin{array}{l}\text { Sachsen- } \\
\text { Anhalt } \\
\text { Programm } \\
\text { MSL* }\end{array}$} & \multirow{9}{*}{$\begin{array}{l}\text { Ackerland } \\
\text { Gemüse } \\
\text { Grünland } \\
\text { Dauer- } \\
\text { kulturen }\end{array}$} & 252 & 252 & 192 & \multirow{9}{*}{$\begin{array}{l}\text { * Programm ,markt- und standortange- } \\
\text { passte Landbewirtschaftung"- relevant } \\
\text { vor allem für landwirtschaftliche } \\
\text { Betriebe - nicht kombinierbar mit dem } \\
\text { Programm UA } \\
\text { Nachweis über Kontrolle gemäß VO } \\
\text { (EWG) } 2092 / 91 \text { ist Pflicht } \\
\text { Zuschuss zu den Kontrollkosten: } \\
35 € / \text { ha, max.530 } € / \text { Unternehmen } \\
\text { Führung schlag- bzw. anlagenbezogener } \\
\text { Aufzeichnungen. } \\
\text { Durchführung von Bodenuntersuchungen } \\
\text { In } 2006 \text { keine Umstellungsförderung. }\end{array}$} \\
\hline & & 576 & 576 & 360 & \\
\hline & & 252 & 252 & 192 & \\
\hline & & 1140 & 1140 & 924 & \\
\hline & & & & & \\
\hline & & & & & \\
\hline & & & & & \\
\hline & & & & & \\
\hline & & & & & \\
\hline \multirow[t]{7}{*}{$\begin{array}{l}\text { Sachsen- } \\
\text { Anhalt } \\
\text { Programm } \\
\text { UA }^{*}\end{array}$} & $\begin{array}{l}\text { Gemüse, } \\
\text { Heil- u. Ge- } \\
\text { würzpfl. }\end{array}$ & 409 & 409 & 409 & $\begin{array}{l}\text { * Programm „umweltschonender Anbau“ } \\
\text { - relevant vor allem für spezialisierte } \\
\text { Gemüse-, Obst-, Wein-, } \\
\text { Gartenbaubetriebe - nicht kombinierbar } \\
\text { mit dem Programm MSL }\end{array}$ \\
\hline & Gemüse & 4090 & 4090 & 4090 & $\begin{array}{l}\text { Nachweis über Kontrolle gemäß VO } \\
\text { (EWG) 2092/91 ist Pflicht }\end{array}$ \\
\hline & & & & & $\begin{array}{l}\text { Zuschuss zu den Kontrollkosten: } \\
409 € / \text { Unternehmen }\end{array}$ \\
\hline & $\begin{array}{l}\text { Kern- u. } \\
\text { Steinobst }\end{array}$ & 716 & 716 & 716 & $\begin{array}{l}\text { Führung schlag- bzw. anlagenbezogener } \\
\text { Aufzeichnungen. }\end{array}$ \\
\hline & Wein & 716 & 716 & 716 & \multirow{3}{*}{$\begin{array}{l}\text { Durchfuhrung von Bodenuntersuchungen. } \\
\text { In } 2006 \text { keine Umstellungsförderung. }\end{array}$} \\
\hline & $\begin{array}{l}\text { Wein } \\
\text { (Steillage) }\end{array}$ & 1023 & 1023 & 1023 & \\
\hline & Hopfen & 511 & 511 & 511 & \\
\hline
\end{tabular}




\begin{tabular}{|c|c|c|c|c|c|}
\hline \multirow{2}{*}{ Bundesland } & \multirow{2}{*}{ Nutzungsart } & \multicolumn{3}{|c|}{ Prämienhöhe $€ /$ ha u. Jahr } & \multirow[b]{2}{*}{$\begin{array}{c}\text { Kontrollanforderungen, } \\
\text { Höchstbeträge, sonstige } \\
\text { Anforderungen }\end{array}$} \\
\hline & & $\begin{array}{r}\text { Ein } \\
\text { 1.-2. J. }\end{array}$ & rung & $\begin{array}{c}\text { Beibehaltung } \\
\text { ab } 6 . \mathrm{J} .\end{array}$ & \\
\hline \multirow{5}{*}{$\begin{array}{l}\text { Schleswig- } \\
\text { Holstein }\end{array}$} & \multirow{5}{*}{$\begin{array}{l}\text { Ackerland } \\
\text { Gemüse } \\
\text { Grünland } \\
\text { Dauerkulturen }\end{array}$} & 285 & 160 & 160 & \multirow{5}{*}{$\begin{array}{l}\text { Nachweis über Kontrolle gemäß VO } \\
\text { (EWG) } 2092 / 91 \text { ist Pflicht } \\
\text { Zuschuss zu den Kontrollkosten: } 35 € / \text { ha, } \\
\text { max. } 530 € \text { je Betrieb } \\
\text { In } 2005 \text { keine Neuverpflichtungen / } \\
\text { keine Umstellungsförderung (ob dies } \\
\text { auch für das Jahr } 2006 \text { gilt, ist noch nicht } \\
\text { geklärt) }\end{array}$} \\
\hline & & 750 & 300 & 300 & \\
\hline & & 285 & 160 & 160 & \\
\hline & & 1220 & 770 & 770 & \\
\hline & & & & & \\
\hline \multirow[t]{4}{*}{ Thüringen } & Ackerland & 180 & 180 & 155 & \multirow{4}{*}{$\begin{array}{l}\text { Nachweis der Kontrolle gemäß VO } \\
\text { (EWG) 2092/91 ist Pflicht } \\
\text { Landwirte müssen Schlagkartei führen }\end{array}$} \\
\hline & $\begin{array}{l}\text { Gemüse, } \\
\text { Duft-, Heil-, } \\
\text { Gewürzpfl. }\end{array}$ & 600 & 600 & 450 & \\
\hline & $\begin{array}{l}\text { Griinland u. } \\
\text { Streuobstw. }\end{array}$ & 230 & 230 & 205 & \\
\hline & Dauerkulturen & 900 & 900 & 900 & \\
\hline
\end{tabular}

Details zur Förderung sind den aktuellen Richtlinien der Länder zu entnehmen.

Nieberg, H. (2006): Flächenbezogene Förderung bei Einführung und Beibehaltung ökologischer Wirtschaftsweisen und Kontrollkostenzuschuss nach Bundesländern im Jahr 2006. FAL-Erhebung in den zuständigen Länderministerien. Institut für Betriebswirtschaft der FAL Braunschweig. 


\section{A.2 ERGEBNISSE DES VERGLEICHS DER BETRIEBSGEWINNE}

\section{Deskriptive Statistik zur mittleren einzelbetrieblichen Gewinnentwicklung ökologischer und konventioneller Betriebe des BMELV- Testbetriebsnetzes}

\section{Betrachtung des Gewinns inklusive aller Prämienzahlungen}

\begin{tabular}{|c|c|c|c|c|}
\hline \multirow[b]{2}{*}{ Datengrundlage } & \multirow{2}{*}{$\begin{array}{l}\text { Konventionell } \\
\text { wirtschaftende } \\
\text { Betriebe }\end{array}$} & \multicolumn{3}{|c|}{ Ökologisch wirtschaftende Betriebe } \\
\hline & & 8 Jahresdaten & 7 Jahresdaten & 6 Jahresdaten \\
\hline Betriebe insgesamt & 3278 & 77 & 31 & 35 \\
\hline $\begin{array}{l}\text { Mittelwert der jährlichen } \\
\text { Betriebsgewinne in } €\end{array}$ & 32673 & 20300 & 24025 & 27607 \\
\hline Betriebstyp & \multicolumn{4}{|c|}{ ACKERBAU } \\
\hline Gewinntrend? & \multicolumn{4}{|c|}{ ja } \\
\hline Anzahl der Betriebe & 74 & 2 & 0 & 0 \\
\hline $\begin{array}{l}\text { Mittelwert der jährlichen } \\
\text { Betriebsgewinne in } €\end{array}$ & 39101 & 74335 & - & - \\
\hline $\begin{array}{c}\text { Mittlerer Quotient aus } \\
\text { Standardfehler und Mittelwert }\end{array}$ & 0,18 & 0,11 & - & - \\
\hline Gewinntrend? & \multicolumn{4}{|c|}{ nein } \\
\hline Anzahl der Betriebe & 498 & 8 & 8 & 6 \\
\hline $\begin{array}{l}\text { Mittelwert der jährlichen } \\
\text { Betriebsgewinne in } €\end{array}$ & 49629 & 22578 & 12523 & 53926 \\
\hline Mittlerer Variationskoeffizient & 0,83 & 1,72 & 1,25 & 0,92 \\
\hline Betriebstyp & \multicolumn{4}{|c|}{ FUTTERBAU } \\
\hline Gewinntrend? & \multicolumn{4}{|c|}{ ja } \\
\hline Anzahl der Betriebe & 169 & 3 & 3 & 0 \\
\hline $\begin{array}{l}\text { Mittelwert der jährlichen } \\
\text { Betriebsgewinne in } €\end{array}$ & 29108 & 17451 & 28725 & - \\
\hline $\begin{array}{c}\text { Mittlerer Quotient aus } \\
\text { Standardfehler und Mittelwert }\end{array}$ & 0,16 & 0,05 & 0,09 & - \\
\hline Gewinntrend? & \multicolumn{4}{|c|}{ nein } \\
\hline Anzahl der Betriebe & 1095 & 42 & 11 & 16 \\
\hline $\begin{array}{l}\text { Mittelwert der jährlichen } \\
\text { Betriebsgewinne in } €\end{array}$ & 30534 & 21021 & 36232 & 26256 \\
\hline Mittlerer Variationskoeffizient & 0,75 & 1,02 & 0,39 & 0,91 \\
\hline Betriebstyp & \multicolumn{4}{|c|}{ SONSTIGE } \\
\hline Gewinntrend? & \multicolumn{4}{|c|}{ ja } \\
\hline Anzahl der Betriebe & 173 & 3 & 2 & 2 \\
\hline $\begin{array}{l}\text { Mittelwert der jährlichen } \\
\text { Betriebsgewinne in } €\end{array}$ & 27496 & 18288 & 41315 & 29618 \\
\hline $\begin{array}{c}\text { Mittlerer Quotient aus } \\
\text { Standardfehler und Mittelwert }\end{array}$ & 0,22 & 0,19 & 0,08 & 0,19 \\
\hline Gewinntrend? & \multicolumn{4}{|c|}{ nein } \\
\hline Anzahl der Betriebe & 1269 & 19 & 7 & 11 \\
\hline $\begin{array}{c}\text { Mittelwert der jährlichen } \\
\text { Betriebsgewinne in } €\end{array}$ & 28671 & 12829 & 11934 & 14849 \\
\hline Mittlerer Variationskoeffizient & 1,16 & 1,25 & 1,68 & 0,70 \\
\hline
\end{tabular}


Betrachtung des Gewinns exklusive aller Prämienzahlungen

\begin{tabular}{|c|c|c|c|c|}
\hline \multirow[b]{2}{*}{ Datengrundlage } & \multirow{2}{*}{$\begin{array}{c}\text { Konventionell } \\
\text { wirtschaftende } \\
\text { Betriebe }\end{array}$} & \multicolumn{3}{|c|}{ Ökologisch wirtschaftende Betriebe } \\
\hline & & 8 Jahresdaten & 7 Jahresdaten & 6 Jahresdaten \\
\hline Betriebe insgesamt & 3278 & 77 & 31 & 35 \\
\hline $\begin{array}{l}\text { Mittelwert der jährlichen } \\
\text { Betriebsgewinne in } €\end{array}$ & -13955 & -676 & -32529 & -25563 \\
\hline Betriebstyp & \multicolumn{4}{|c|}{ ACKERBAU } \\
\hline Gewinntrend? & \multicolumn{4}{|c|}{ ja } \\
\hline Anzahl der Betriebe & 81 & 1 & 0 & 1 \\
\hline $\begin{array}{l}\text { Mittelwert der jährlichen } \\
\text { Betriebsgewinne in } €\end{array}$ & -17305 & -4595 & - & 4925 \\
\hline $\begin{array}{c}\text { Mittlerer Quotient aus } \\
\text { Standardfehler und Mittelwert }\end{array}$ & 0,31 & 1,11 & - & 0,17 \\
\hline Gewinntrend? & \multicolumn{4}{|c|}{ nein } \\
\hline Anzahl der Betriebe & 491 & 9 & 8 & 5 \\
\hline $\begin{array}{l}\text { Mittelwert der jährlichen } \\
\text { Betriebsgewinne in } €\end{array}$ & -16844 & -4293 & -13596 & -121748 \\
\hline Mittlerer Variationskoeffizient & 1,44 & 2,40 & 1,53 & 0,66 \\
\hline Betriebstyp & \multicolumn{4}{|c|}{ FUTTERBAU } \\
\hline Gewinntrend? & \multicolumn{4}{|c|}{ ja } \\
\hline Anzahl der Betriebe & 176 & 9 & 5 & 1 \\
\hline $\begin{array}{c}\text { Mittelwert der jährlichen } \\
\text { Betriebsgewinne in } €\end{array}$ & 3639 & 1101 & 8239 & -7417 \\
\hline $\begin{array}{c}\text { Mittlerer Quotient aus } \\
\text { Standardfehler und Mittelwert }\end{array}$ & 1,19 & 0,42 & 0,87 & 0,18 \\
\hline Gewinntrend? & \multicolumn{4}{|c|}{ nein } \\
\hline Anzahl der Betriebe & 1088 & 36 & 9 & 15 \\
\hline $\begin{array}{l}\text { Mittelwert der jährlichen } \\
\text { Betriebsgewinne in } €\end{array}$ & 4737 & 2708 & 12305 & 6720 \\
\hline Mittlerer Variationskoeffizient & 1,46 & 1,32 & 0,78 & 2,73 \\
\hline Betriebstyp & \multicolumn{4}{|c|}{ SONSTIGE } \\
\hline Gewinntrend? & \multicolumn{4}{|c|}{ ja } \\
\hline Anzahl der Betriebe & 207 & 6 & 2 & 2 \\
\hline $\begin{array}{l}\text { Mittelwert der jährlichen } \\
\text { Betriebsgewinne in } €\end{array}$ & -18453 & -8447 & -489839 & -64544 \\
\hline $\begin{array}{c}\text { Mittlerer Quotient aus } \\
\text { Standardfehler und Mittelwert }\end{array}$ & 3,38 & 0,11 & 0,02 & 0,03 \\
\hline Gewinntrend? & \multicolumn{4}{|c|}{ nein } \\
\hline Anzahl der Betriebe & 1235 & 16 & 7 & 11 \\
\hline $\begin{array}{c}\text { Mittelwert der jährlichen } \\
\text { Betriebsgewinne in } €\end{array}$ & -30808 & -4098 & -10274 & -23198 \\
\hline Mittlerer Variationskoeffizient & 1,88 & 1,17 & 2,52 & 1,35 \\
\hline
\end{tabular}


Betrachtung des Gewinns inklusive der Prämienzahlungen der 1. Säule, aber exklusive der Prämienzahlungen der 2. Säule

\begin{tabular}{|c|c|c|c|c|}
\hline \multirow[b]{2}{*}{ Datengrundlage } & \multirow{2}{*}{$\begin{array}{c}\text { Konventionell } \\
\text { wirtschaftende } \\
\text { Betriebe }\end{array}$} & \multicolumn{3}{|c|}{ Ökologisch wirtschaftende Betriebe } \\
\hline & & 8 Jahresdaten & 7 Jahresdaten & 6 Jahresdaten \\
\hline Betriebe insgesamt & 3278 & 77 & 31 & 35 \\
\hline $\begin{array}{l}\text { Mittelwert der jährlichen } \\
\text { Betriebsgewinne in } €\end{array}$ & 30048 & 12432 & 10542 & 13081 \\
\hline Betriebstyp & \multicolumn{4}{|c|}{ ACKERBAU } \\
\hline Gewinntrend? & \multicolumn{4}{|c|}{ ja } \\
\hline Anzahl der Betriebe & 70 & 1 & 1 & 3 \\
\hline $\begin{array}{l}\text { Mittelwert der jährlichen } \\
\text { Betriebsgewinne in } €\end{array}$ & 36731 & 25419 & 130 & -22593 \\
\hline $\begin{array}{c}\text { Mittlerer Quotient aus } \\
\text { Standardfehler und Mittelwert }\end{array}$ & 0,35 & 0,20 & 8,81 & 0,17 \\
\hline Gewinntrend? & \multicolumn{4}{|c|}{ nein } \\
\hline Anzahl der Betriebe & 502 & 9 & 7 & 3 \\
\hline $\begin{array}{l}\text { Mittelwert der jährlichen } \\
\text { Betriebsgewinne in } €\end{array}$ & 47469 & 21032 & 6346 & 56457 \\
\hline Mittlerer Variationskoeffizient & 0,87 & 1,67 & 1,09 & 1,06 \\
\hline Betriebstyp & \multicolumn{4}{|c|}{ FUTTERBAU } \\
\hline Gewinntrend? & \multicolumn{4}{|c|}{ ja } \\
\hline Anzahl der Betriebe & 164 & 6 & 4 & 1 \\
\hline $\begin{array}{l}\text { Mittelwert der jährlichen } \\
\text { Betriebsgewinne in } €\end{array}$ & 26934 & 7831 & 23020 & 12319 \\
\hline $\begin{array}{c}\text { Mittlerer Quotient aus } \\
\text { Standardfehler und Mittelwert }\end{array}$ & 0,11 & 0,07 & 0,11 & 0,15 \\
\hline Gewinntrend? & \multicolumn{4}{|c|}{ nein } \\
\hline Anzahl der Betriebe & 1100 & 39 & 10 & 15 \\
\hline $\begin{array}{l}\text { Mittelwert der jährlichen } \\
\text { Betriebsgewinne in } €\end{array}$ & 27989 & 13913 & 26070 & 20283 \\
\hline Mittlerer Variationskoeffizient & 0,83 & 1,22 & 1,88 & 1,41 \\
\hline Betriebstyp & \multicolumn{4}{|c|}{ SONSTIGE } \\
\hline Gewinntrend? & \multicolumn{4}{|c|}{ ja } \\
\hline Anzahl der Betriebe & 166 & 3 & 2 & 1 \\
\hline $\begin{array}{l}\text { Mittelwert der jährlichen } \\
\text { Betriebsgewinne in } €\end{array}$ & 23699 & 11330 & -41503 & 27436 \\
\hline $\begin{array}{c}\text { Mittlerer Quotient aus } \\
\text { Standardfehler und Mittelwert }\end{array}$ & 0,21 & 0,10 & 0,15 & 0,05 \\
\hline Gewinntrend? & \multicolumn{4}{|c|}{ nein } \\
\hline Anzahl der Betriebe & 1276 & 19 & 7 & 12 \\
\hline $\begin{array}{l}\text { Mittelwert der jährlichen } \\
\text { Betriebsgewinne in } €\end{array}$ & 25828 & 6263 & 1781 & 1020 \\
\hline Mittlerer Variationskoeffizient & 1,17 & 2,60 & 2,41 & 1,41 \\
\hline
\end{tabular}




\section{A.3 ÖKO-BETRIEBE NACH RECHTSFORMEN}

Öko-Betriebe nach Rechtsformen (Stand: Mai 2005)

\begin{tabular}{|c|c|c|c|c|c|}
\hline \multirow[b]{2}{*}{ Land } & \multirow[b]{2}{*}{ Insgesamt } & \multicolumn{3}{|c|}{ Davon Betriebe, deren Inhaber sind } & \multirow[b]{2}{*}{$\begin{array}{l}\text { Juristische } \\
\text { Personen in \% } \\
\text { aller Betriebe }\end{array}$} \\
\hline & & $\begin{array}{c}\text { Einzel- } \\
\text { unternehmen }\end{array}$ & \begin{tabular}{|c|} 
Personen- \\
gesellschaften
\end{tabular} & $\begin{array}{l}\text { Juristische } \\
\text { Personen }\end{array}$ & \\
\hline \multicolumn{6}{|c|}{ Betriebe } \\
\hline Schleswig-Holstein & 380 & 331 & 26 & 23 & $6,05 \%$ \\
\hline Niedersachsen & 1083 & 935 & 114 & 34 & $3,14 \%$ \\
\hline Nordrhein-Westfalen & 1091 & 999 & 51 & 41 & $3,76 \%$ \\
\hline Hessen & 1340 & 1234 & 77 & 29 & $2,16 \%$ \\
\hline Rheinland-Pfalz & 500 & 433 & 51 & 16 & $3,20 \%$ \\
\hline Baden-Württemberg & 2988 & 2696 & 225 & 67 & $2,24 \%$ \\
\hline Bayern & 4171 & 3956 & 170 & 45 & $1,08 \%$ \\
\hline Saarland & 76 & 71 & k.A. & k.A. & k.A. \\
\hline Brandenburg & 581 & 410 & 73 & 98 & $16,87 \%$ \\
\hline Mecklenburg-Vorpommern & 611 & 443 & 75 & 93 & $15,22 \%$ \\
\hline Sachsen & 266 & 208 & 24 & 34 & $12,78 \%$ \\
\hline Sachsen-Anhalt & 254 & 188 & 39 & 27 & $10,63 \%$ \\
\hline Thüringen & 187 & 131 & 20 & 36 & $19,25 \%$ \\
\hline Stadstaaten & 35 & 28 & k.A. & k.A. & k.A. \\
\hline Deutschland & 13563 & 12063 & 949 & 551 & $4,06 \%$ \\
\hline Früheres Bundesgebiet & 11664 & 10683 & 718 & 263 & $2,25 \%$ \\
\hline Neue Länder & 1899 & 1380 & 231 & 288 & $15,17 \%$ \\
\hline \multicolumn{6}{|c|}{ Landwirtschaftlich genutzte Fläche in ha } \\
\hline Schleswig-Holstein & 28136 & 21932 & 3407 & 2797 & $9,94 \%$ \\
\hline Niedersachsen & 64108 & 50599 & 8100 & 5409 & $8,44 \%$ \\
\hline Nordrhein-Westfalen & 42430 & 36040 & 3939 & 2450 & $5,77 \%$ \\
\hline Hessen & 54302 & 45951 & 5341 & 3010 & $5,54 \%$ \\
\hline Rheinland-Pfalz & 19297 & 16104 & 2551 & 642 & $3,33 \%$ \\
\hline Baden-Württemberg & 92834 & 76945 & 12745 & 3144 & $3,39 \%$ \\
\hline Bayern & 127696 & 113479 & 10690 & 3527 & $2,76 \%$ \\
\hline Saarland & 6146 & 5773 & k.A. & k.A. & k.A. \\
\hline Brandenburg & 131431 & 43721 & 22552 & 65158 & $49,58 \%$ \\
\hline Mecklenburg-Vorpommern & 109879 & 45891 & 29773 & 34215 & $31,14 \%$ \\
\hline Sachsen & 31191 & 12864 & 6691 & 11636 & $37,31 \%$ \\
\hline Sachsen-Anhalt & 49344 & 22938 & 10757 & 15649 & $31,71 \%$ \\
\hline Thüringen & 24421 & 7152 & 7523 & 9746 & $39,91 \%$ \\
\hline Stadstaaten & 1259 & 771 & k.A. & k.A. & k.A. \\
\hline Deutschland & 782475 & 500160 & 124426 & 157888 & $20,18 \%$ \\
\hline Früheres Bundesgebiet & 436209 & 367595 & 47130 & 21484 & $4,93 \%$ \\
\hline Neue Länder & 346266 & 132566 & 77296 & 136404 & $39,39 \%$ \\
\hline
\end{tabular}

k.A. = keine Angabe

Quelle: STATISTISCHES BUNDESAMT (2005) 


\section{A.4 ÜBERBLICK ÜBER DIE PROGRAMMIERUNG IN Z-TREE}
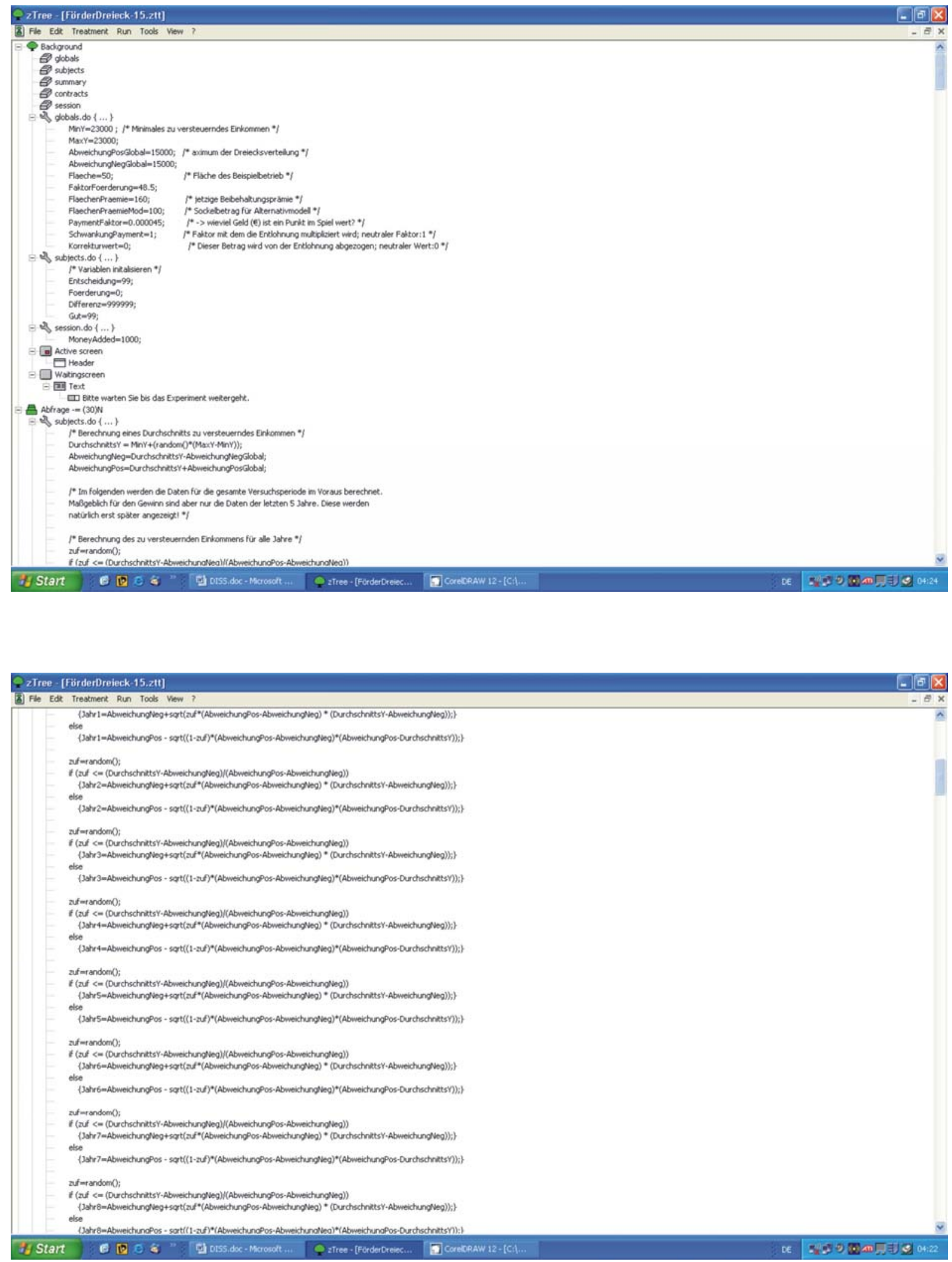

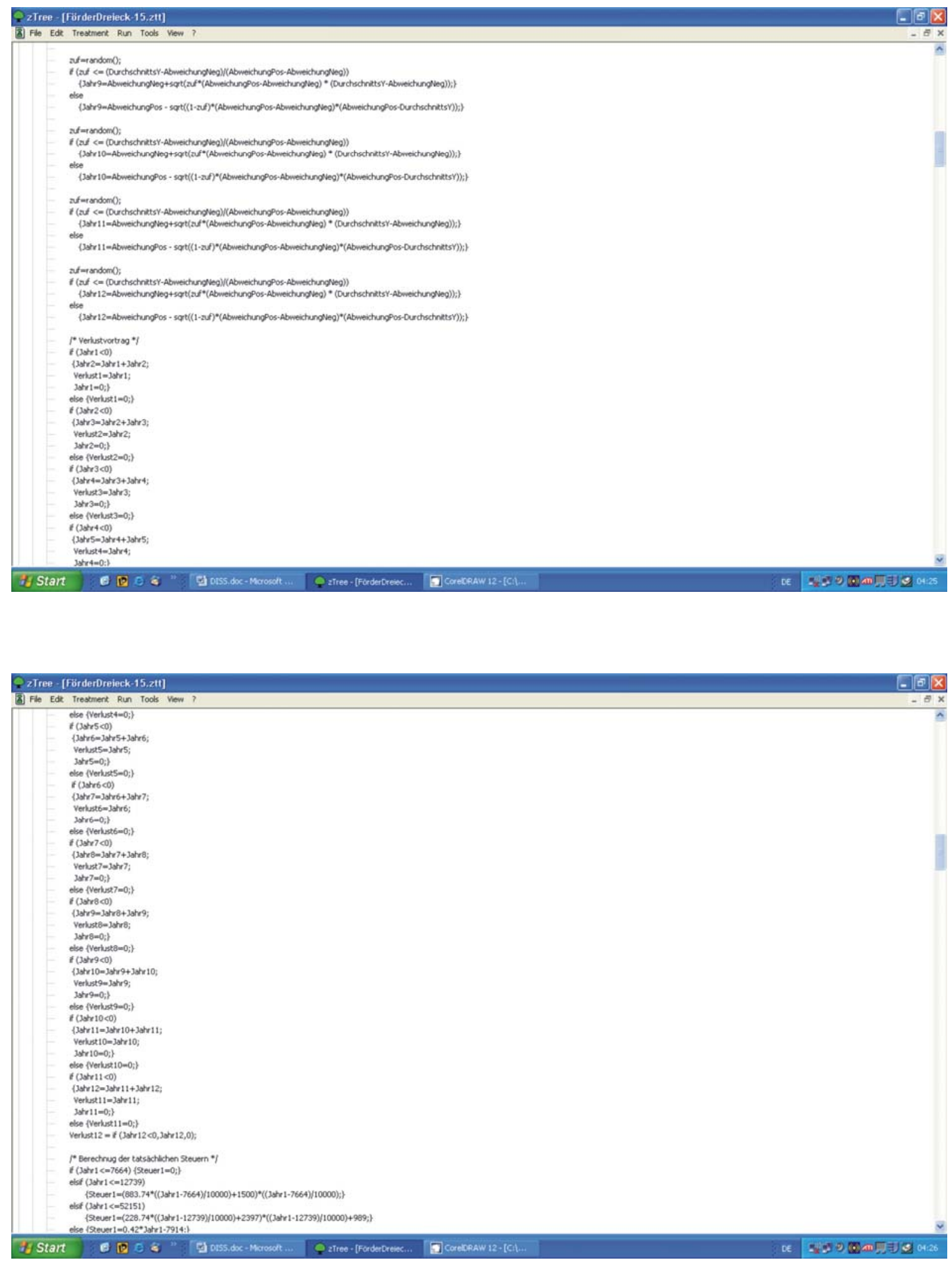

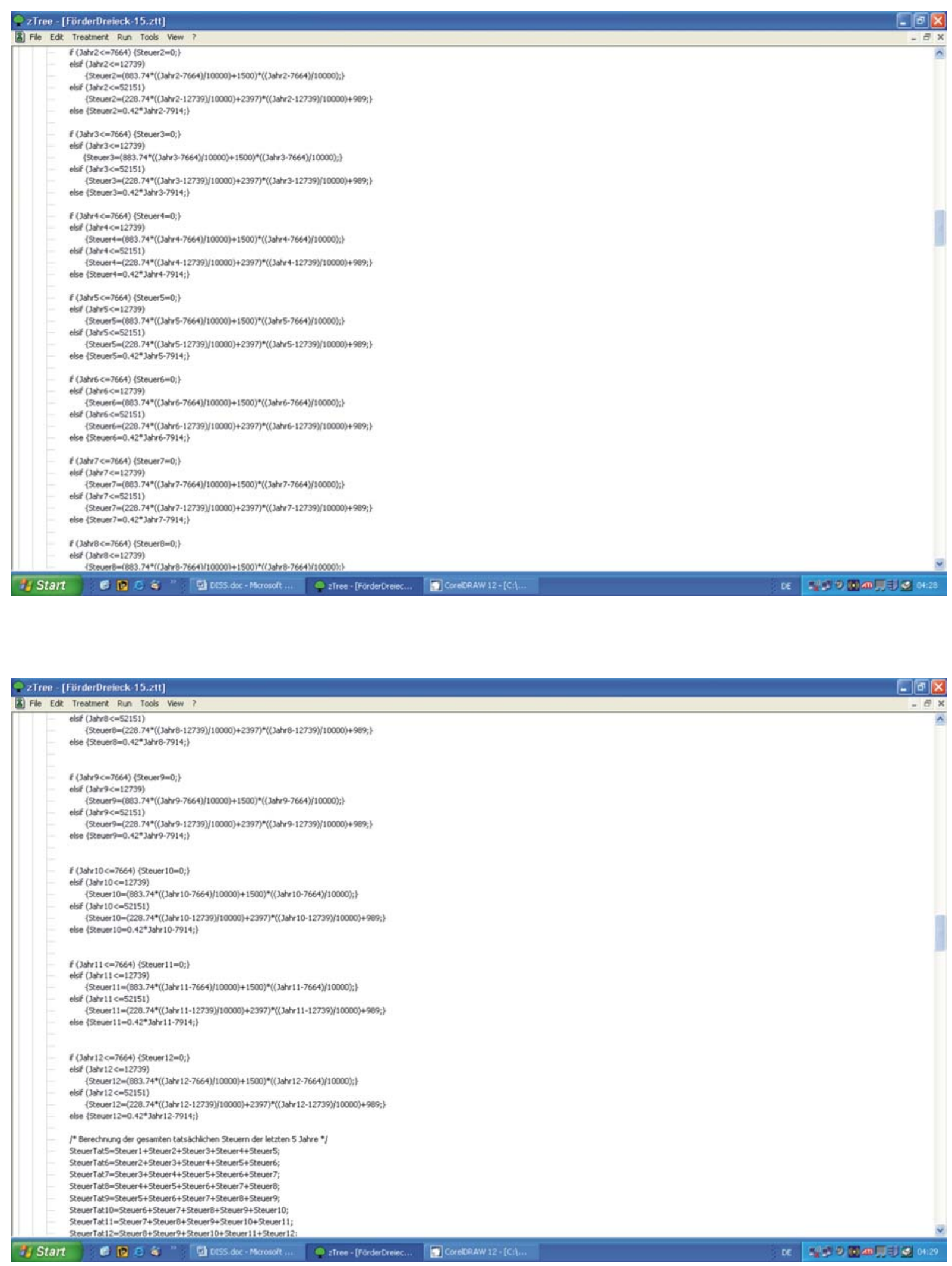

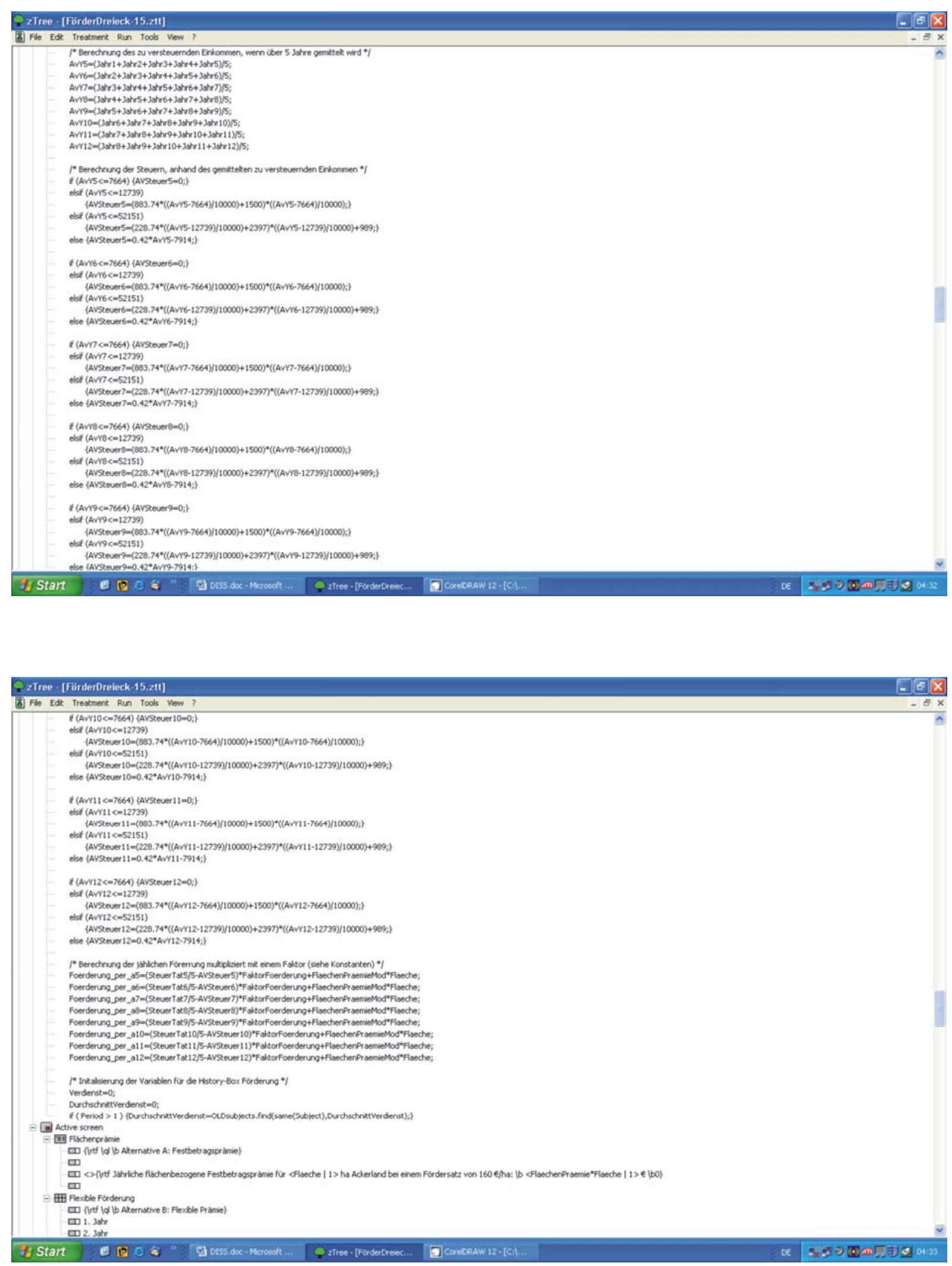

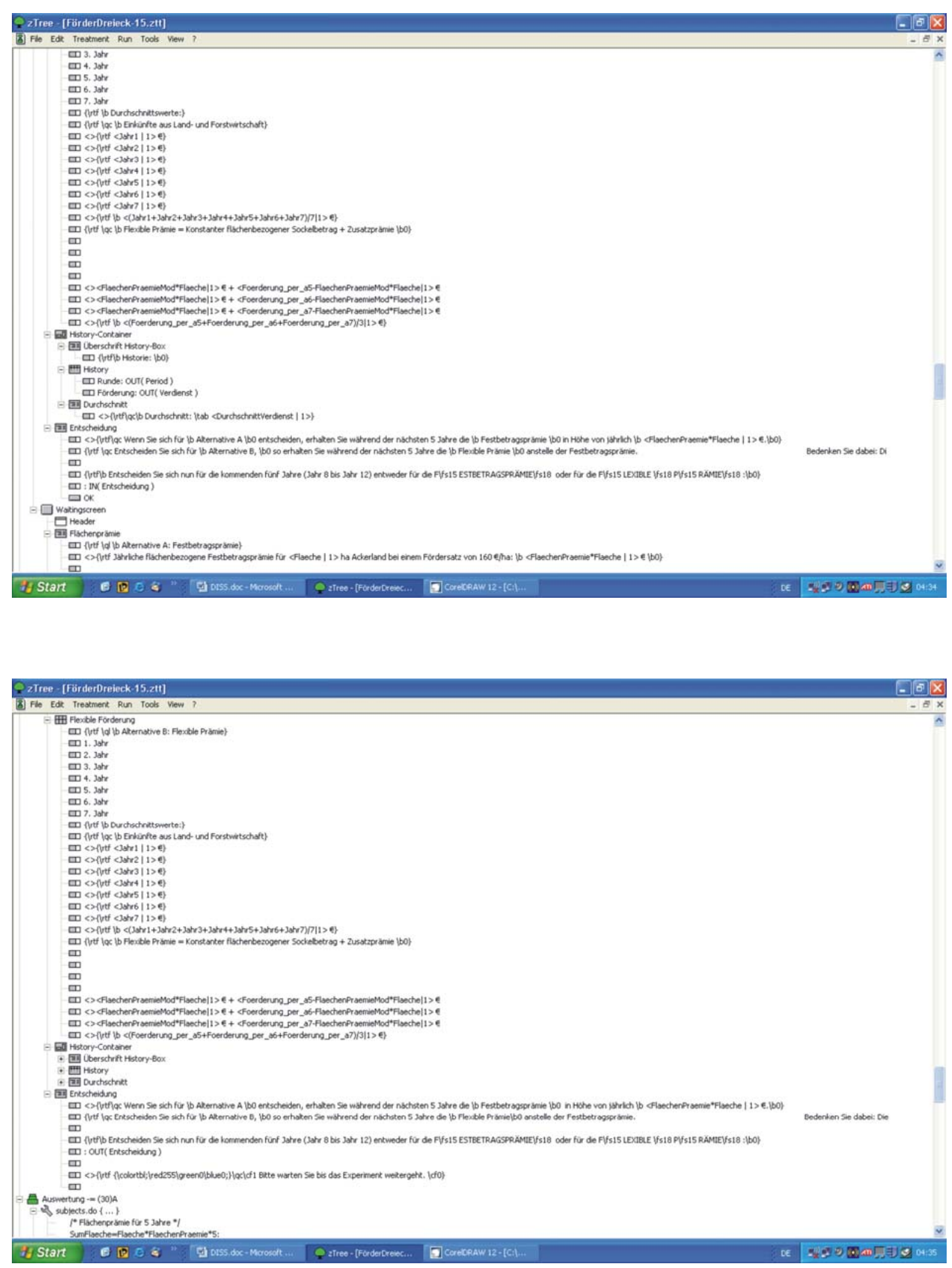

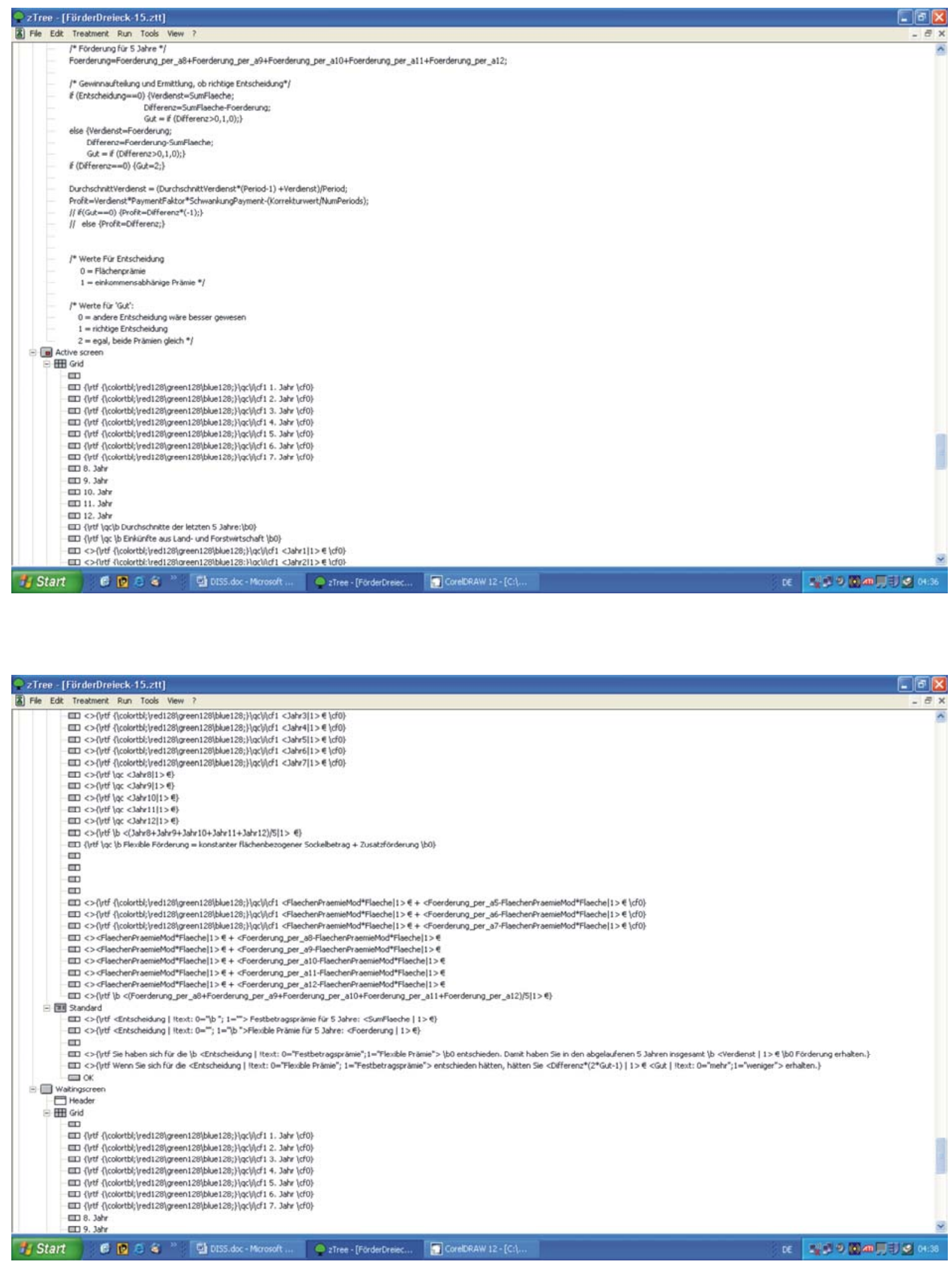

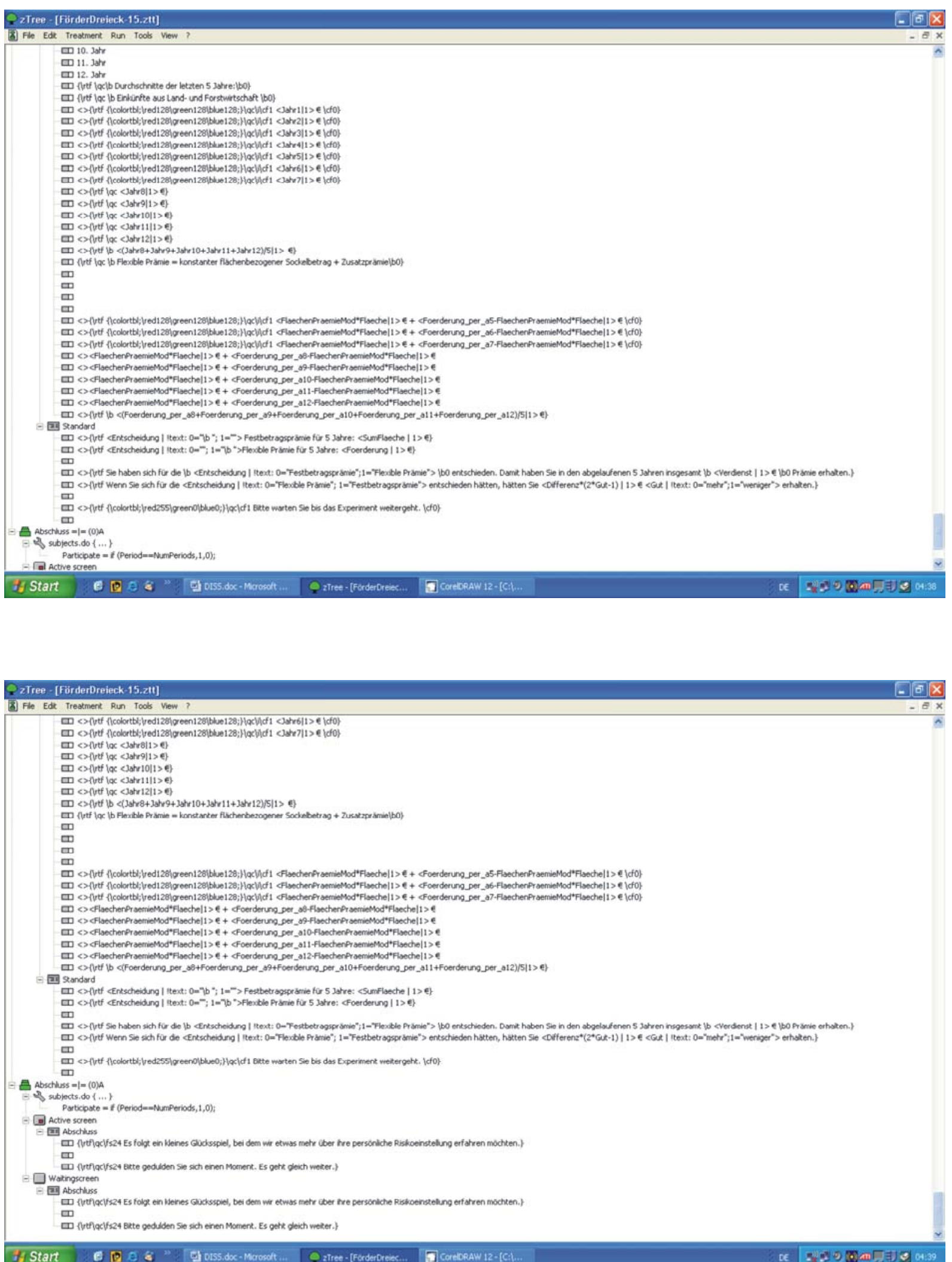

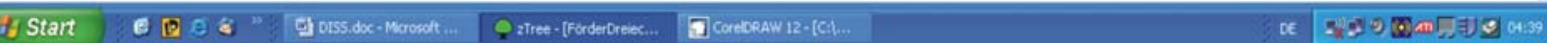




\section{A.5 EXPERIMENTINSTRUKTIONEN FÜR DIE TEILNEHMER}

Herzlich Willkommen zu einem kleinen EDV-Basierten Experiment zum Thema „Flexible Prämie vs. Festbetragsprämie zur Förderung des Ökologischen Landbaus“.

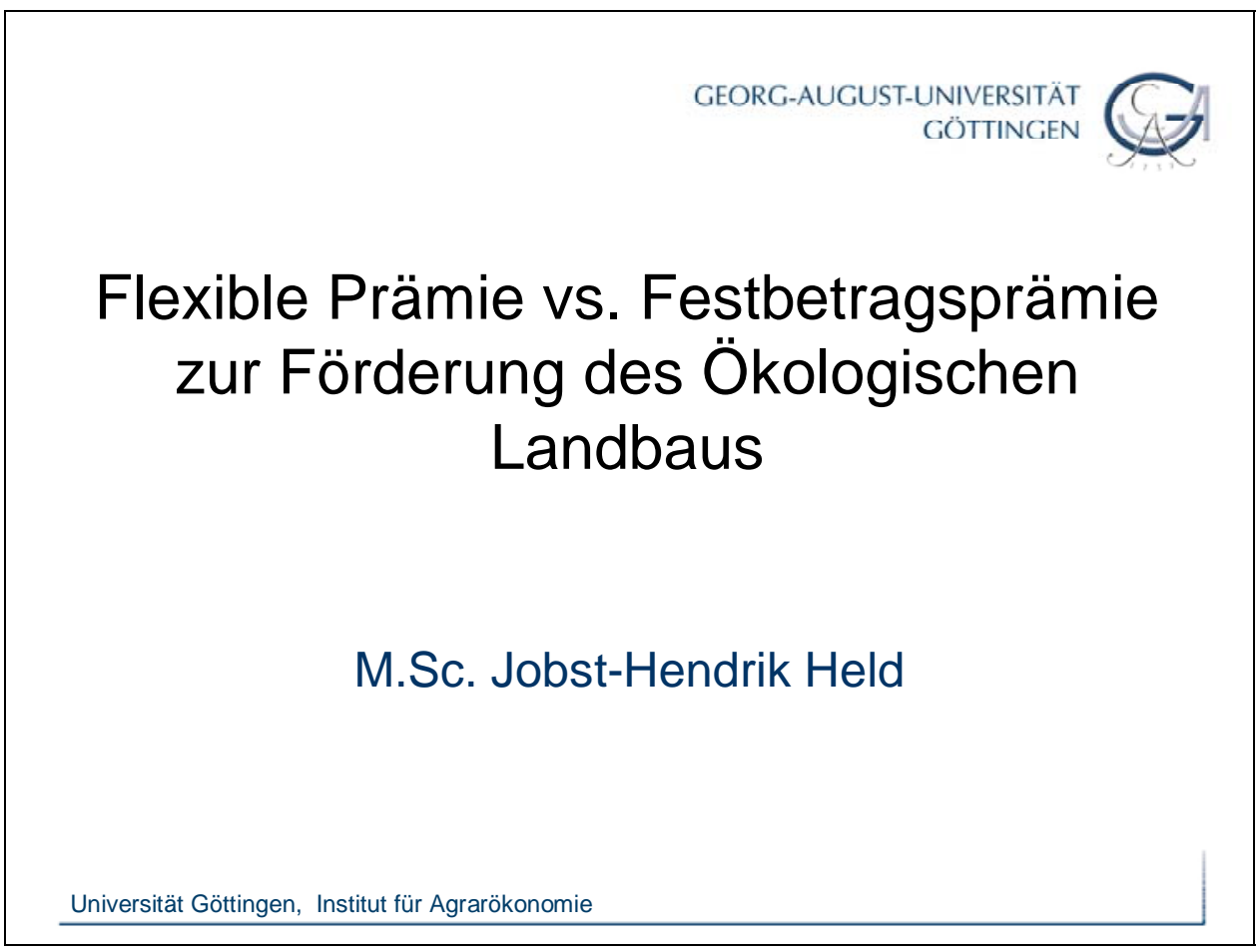

Unser Experiment beschäftigt sich mit der flächenbezogenen Förderung des ökologischen Landbaus. Die derzeit in Niedersachsen gültigen Prämiensätze sind auf der folgenden Folie dargestellt.

\section{Die flächenbezogene Förderung des ökologischen Landbaus}

- Umstellungs- und Beibehaltungsprämien im Rahmen der Agrarumweltprogramme der Bundesländer

$\Rightarrow$ Derzeitige Prämienausgestaltung in Niedersachsen:

\begin{tabular}{|c|c|c|c|c|c|}
\hline \multirow[b]{2}{*}{ Bundesland } & \multirow[b]{2}{*}{ Nutzungsart } & \multicolumn{3}{|c|}{ Prämienhöhe } & \multirow{2}{*}{$\begin{array}{c}\text { Zuschuss Zu } \\
\text { den } \\
\text { Kontroll- } \\
\text { kosten }\end{array}$} \\
\hline & & 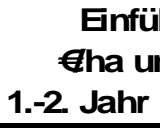 & $\begin{array}{l}\text { rung } \\
\text { d Jahr } \\
\text { 3.-5. Jahr }\end{array}$ & $\begin{array}{c}\text { Beibehaltung } \\
\text { €/ha und Jahr } \\
\text { ab 6.Jahr }\end{array}$ & \\
\hline \multirow{4}{*}{$\begin{array}{l}\text { Nieder- } \\
\text { sachsen }\end{array}$} & Ackerland & 285 & 160 & 160 & \multirow{4}{*}{$\begin{array}{c}35 € / h a, \\
\text { max. } 530 € \\
\text { /Unter- } \\
\text { nehmen }\end{array}$} \\
\hline & Gemüse & 750 & 300 & 300 & \\
\hline & Grünland & 285 & 160 & 160 & \\
\hline & Dauerkulturen & 1.220 & 770 & 770 & \\
\hline
\end{tabular}

Quelle: NIEBERG, H. (2005): Flächenbezogene Förderung bei

Einführung und Beibehaltung ökologischer Wirtschaftsweisen

Die Prämienbemessung erfolgt dabei ausschließlich analog zur bewirtschafteten Fläche mit differenzierten Prämiensätzen für verschiedene Flächennutzungsarten sowie für Umstellungsund Beibehaltungszeitraum. 
Wichtig für das folgende Experiment wird vor allem die Beibehaltungsprämie im Ackerbau sein, die in Niedersachsen derzeit $160 € /$ ha/Jahr beträgt.

Zur aktuellen Bedeutung der flächenbezogenen Öko-Prämien:

Nach einer im vergangenen Jahr von Frau Dr. Nieberg (Bundesforschungsanstalt für Landwitschaft, FAL) durchgeführten Untersuchung halten über $80 \%$ der ökologisch wirtschaftenden Betriebsleiter die gegenwärtigen flächenbezogenen Öko-Prämien für wichtig bis sehr wichtig für die Wirtschaftlichkeit ihres Betriebes.

Ein ähnliches Bild zeigt sich auch bei einem Blick in die Buchführungsergebnisse der ökologisch wirtschaftenden Testbetriebe.

Umso problematischer ist daher die aktuell höchst unsichere Finanzierungssituation mit Blick auf die zweite Säule der Agrarpolitik und damit natürlich auch mit Blick auf die ÖkoFlächenprämien im Rahmen der Agrarumweltprogramme einzuschätzen.

Einerseits führt die ansteigende ökologisch bewirtschaftete Fläche $\mathrm{zu}$ einem stetig wachsenden Finanzmittelbedarf, der in den vergangenen Jahren wiederholt dazu geführt hat, dass einzelne Bundesländer ihre Förderung vorübergehend begrenzt haben. So haben im Jahr 2005 bereits 5 Bundesländer (Baden-Württemberg, Brandenburg, Hessen, Sachsen und Schleswig-Holstein) die Förderung für Neuumsteller eingestellt. Für 2006 wird NordrheinWestfalen noch hinzukommen.

Andererseits bedeutet der im Dezember 2005 geschlossene EU-Finanzkompromiss für den kommenden Finanzplanungszeitraum 2007-2013 für die 2. Säule der Agrarpolitik deutliche Einschnitte. Daher sind zukünftig erhöhte Unsicherheiten bezüglich der weiteren flächenbezogenen Öko-Förderung wahrscheinlich.

\begin{tabular}{|l|}
\hline Die flächenbezogene Förderung des \\
ökologischen Landbaus \\
- Hohe Bedeutung für die Wirtschaftlichkeit der Betriebe \\
- Aber: \\
- Stetig ansteigender Finanzmittelbedarf bei zukünftig sinkenden \\
Finanzmittelbudgets zur Finanzierung der Öko- \\
Flächenprämien \\
- Konsequenz in vielen Bundesländern: \\
- Aufnahmestopp für Neu-Umsteller und Neuflächen in 2006 \\
- Umfangreiche Änderungen bei der Konzeption der Förderung in \\
2007 \\
Erhöhte Unsicherheiten bezüglich der Zukunft der Förderung \\
Fragestellung: \\
Gibt es alternative Förderinstrumente/Prämienausgestaltungen, \\
mit denen die Effektivität der Förderung verbessert und ein \\
Fortbestand der Förderung gesichert werden kann? \\
\hline
\end{tabular}

Aufgabe der Wissenschaft sollte es daher sein, Überlegungen hinsichtlich alternativer Förderinstrumente bzw. Prämienausgestaltungen anzustellen, die neben einer grundsätzlichen Sicherung der Förderung u.U. im Sinne der zukünftig reduzierten Finanzmittelbudgets auch zu einer effizienteren Förderung führen könnten. 
Im Folgenden soll daher mit der so genannten ,Flexiblen Förderung' ein Instrument vorgestellt werden, dass sich u.U. als Alternative zur derzeit geltenden Festbetragsprämie eignen könnte.

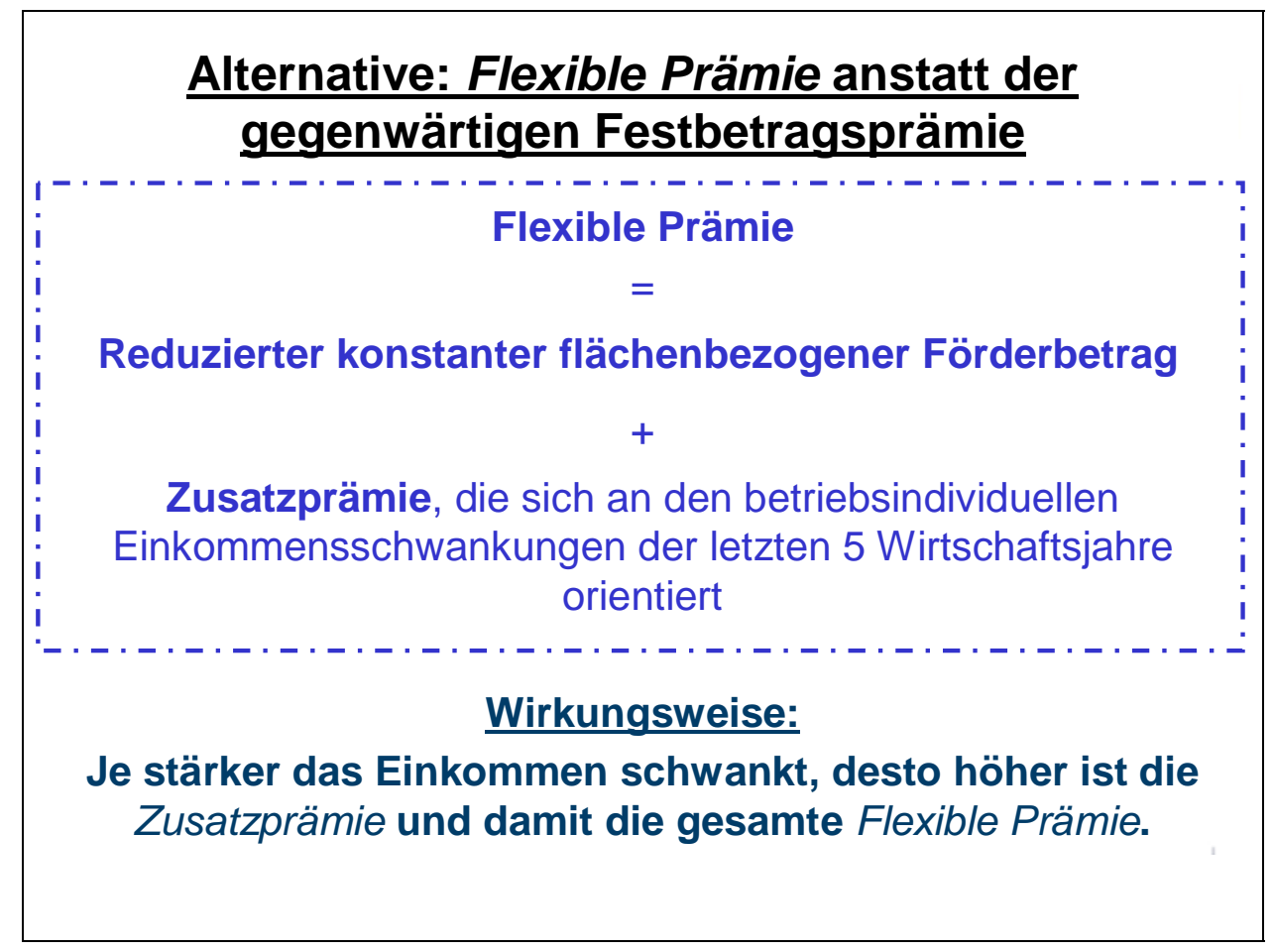

Die Flexible Förderung besteht aus zwei unterschiedlichen Bestandteilen:

Zum einen ist da der reduzierte konstante flächenbezogene Förderbetrag, der im Folgenden auch als konstanter Sockelbetrag bezeichnet wird. Er weist die gleichen Eigenschaften wie die derzeit geltende Festbetragsprämie auf, ist in seiner Höhe jedoch deutlich geringer. Die Tatsache, dass er lediglich an die bewirtschaftete Öko-Fläche gekoppelt ist und für eine vorgegebene Fläche daher konstant ist, unterscheidet ihn von der Zusatzprämie, also der zweiten Komponente der Flexiblen Förderung.

Die Zusatzprämie ist unabhängig von der bewirtschafteten Öko-Fläche und bemisst sich einzig an den einzelbetrieblichen Einkommensschwankungen der letzten 5 Jahre. Sie ist also in ihrer Höhe nicht standardisiert wie der Sockelbetrag sondern wird jedes Jahr betriebsindividuell festgesetzt

Es ergibt sich deshalb für die Flexible Prämie die folgende Wirkungsweise: Je stärker das Einkommen (Gemeint sind damit die steuerlichen Einkünfte aus Land- und Forstwirtschaft) schwankt, desto höher ist die Zusatzprämie und damit die gesamte Flexible Prämie. Schwanken die Einkünfte im historischen 5-Jahres-Zeitraum überhaupt nicht, so entfällt die Zusatzprämie für den jeweiligen Betrieb und es verbleibt lediglich der Sockelbetrag. Im umgekehrten Fall ergibt sich bei sehr hohen Einkommensschwankungen des Einzelbetriebes eine vergleichsweise hohe Zusatzprämie, die zusammen mit dem konstanten lediglich flächenabhängigen Sockelbetrag zu einer vergleichsweise hohen Flexiblen Prämie führt.

Mit dem folgenden Experiment soll nun zum einen diese Flexible Prämie mit ihrer spezifischen Wirkungsweise Öko-Landwirten, als potentielle Empfänger, vorgestellt werden und daraus folgend die Akzeptanz für eine solche Flexible Förderung gemessen werden. 
Dabei gestaltet sich der Ablauf des Experimentes wie folgt:

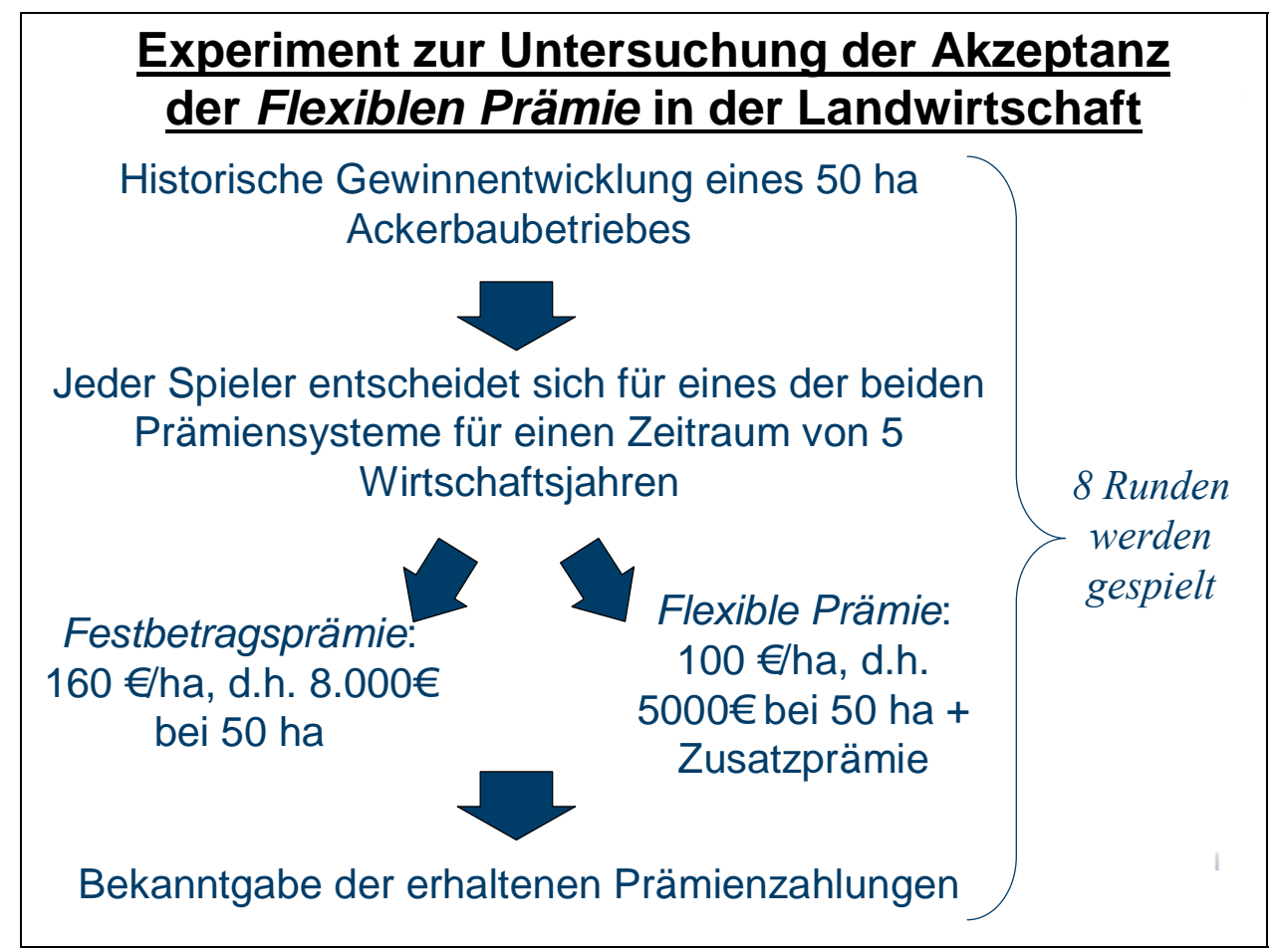

Der einzelne Teilnehmer wird in die Rolle eines Betriebsleiters eines 50ha Ackerbaubetriebes versetzt. Ihm wird auf dem ersten Bildschirm die historische Entwicklung seiner Einkünfte aus Land- und Forstwirtschaft als Grundlage für die Zusatzprämie dargestellt. Auf dieser Basis soll sich jeder Teilnehmer für eines von zwei zur Auswahl stehenden Prämiensystemen für die nächsten 5 Jahre entscheiden. Es wurde ein Zeitraum von 5 Jahren gewählt, da das dem geltenden Verpflichtungszeitraum für Agrarumweltprogramme entspricht.

Die beiden dem Teilnehmer zur Auswahl stehenden Fördersysteme sind:

1. eine Festbetragsprämie von $160 € /$ ha Ackerland pro Jahr (Diese Alternative entspricht damit genau der gegenwärtigen flächenbezogenen Förderung.). Bei der vorgegebenen Fläche von 50 ha ergäben sich bei Wahl dieser Alternative also konstante $8.000 €$ Förderung pro Jahr.

2. die bereits vorgestellte Flexible Prämie mit einem konstanten flächenbezogenen Sockelbetrag von $100 € /$ ha und Jahr und der Zusatzprämie, die sich an der Schwankung der Einkünfte orientiert. Im vorgegebenen Fall ergäbe sich für die 50 ha Ackerland daher ein jährlicher konstanter Sockelbetrag von $5.000 €$ zuzüglich der Zusatzprämie.

Der einzelne Teilnehmer befindet sich also in folgender Entscheidungssituation:

Er kann unabhängig von der historischen Entwicklung seiner Einkünfte die Festbetragsprämie als sichere Alternative wählen und bekommt damit in jedem Fall die jährliche Prämie von $8.000 €$. Für den Entscheidungszeitraum von 5 Jahren wäre das eine Summe von insgesamt $40.000 €$.

Andererseits kann er sich auch an der Schwankung seiner historischen Einkünfte orientieren. Unterlagen die Einkünfte in der Vergangenheit hohen Schwankungen, so ist die Wahrscheinlichkeit groß, dass die Flexible Förderung im Vergleich zur Festbetragsprämie zu höheren Prämienzahlungen für den Landwirt führt. Im umgekehrten Fall, also bei nur geringen Schwankungen, muss der Spieler damit rechnen, dass die Festbetragsprämie sich im 
Endeffekt als vorteilhaftere Wahl herausstellen könnte. Bei der Beurteilung der Schwankungen ist aber zu bedenken, dass sich die jährliche Zusatzprämie immer an der Schwankung der Einkünfte der letzten 5 Jahre bemisst. Würde sich der Spieler nur für das kommende Jahr entscheiden, so würden also die letzten 4 dem Spieler zum Entscheidungszeitpunkt bekannten historischen Einkünfte zuzüglich der zum Entscheidungszeitpunkt dem Spieler noch unbekannten Einkünfte aus dem kommenden Jahr in die Berechnung der Zusatzprämie eingehen. Der Spieler muss sich aber im Experiment für den kommenden vollständigen 5-Jahres-Verpflichtungszeitraum entscheiden. Deshalb nimmt der Einfluss hoher historischer Einkunftsschwankungen, der für die Zusatzprämie des ersten nachfolgenden Jahres noch außerordentlich hoch ist, in den folgenden weiteren Jahren sukzessive ab. Im Gegenzug nimmt der Einfluss der noch unbekannten zukünftigen Einkünfte auf die Zusatzprämie zu bis diese im letzten Jahr des Verpflichtungszeitrums nur noch auf fünf zum Entscheidungszeitpunkt unbekannten Einkunftshöhen basiert.

Wenn sich der Teilnehmer also aufgrund hoher historischer Einkunftsschwankungen für die Flexible Prämie entscheidet, so vertraut er auch darauf, dass sich diese hohen Schwankungen auch in der nahen Zukunft fortsetzen bzw. sich keine deutliche Glättung der Einkünfte einstellt.

Nach der Entscheidung des Spielers werden die Ergebnisse, d.h. die Auswirkungen der Entscheidung des Spielers, angezeigt. Der Spieler erfährt nun, ob seine Entscheidung im Nachhinein richtig war oder ob er bei einer anderen Entscheidung eine höhere Prämienzahlung für den abgelaufenen 5-Jahres-Zeitraum bekommen hätte.

Das Experiment geht über 8 Runden, d.h. dass sich jeder Teilnehmer nacheinander für insgesamt acht fünfjährige Verpflichtungszeiträume zwischen der Festbetragsprämie und der Flexiblen Prämie entscheiden muss.

Das Ziel eines jeden Spielers sollte in der Optimierung seines Prämienvolumens liegen, d.h. jeder Spieler sollte versuchen, über die 8 Spielrunden soviel Fördergelder wie möglich anzusammeln. Das gilt umso mehr, da sich die Auszahlung am Ende dieser Veranstaltung in ihrer Höhe direkt am erspielten Prämienvolumen orientiert.

Nach diesen theoretischen Erläuterungen zur anstehenden Entscheidungssituation werden auf den folgenden Folien nun zwei Fallbeispiele behandelt: 


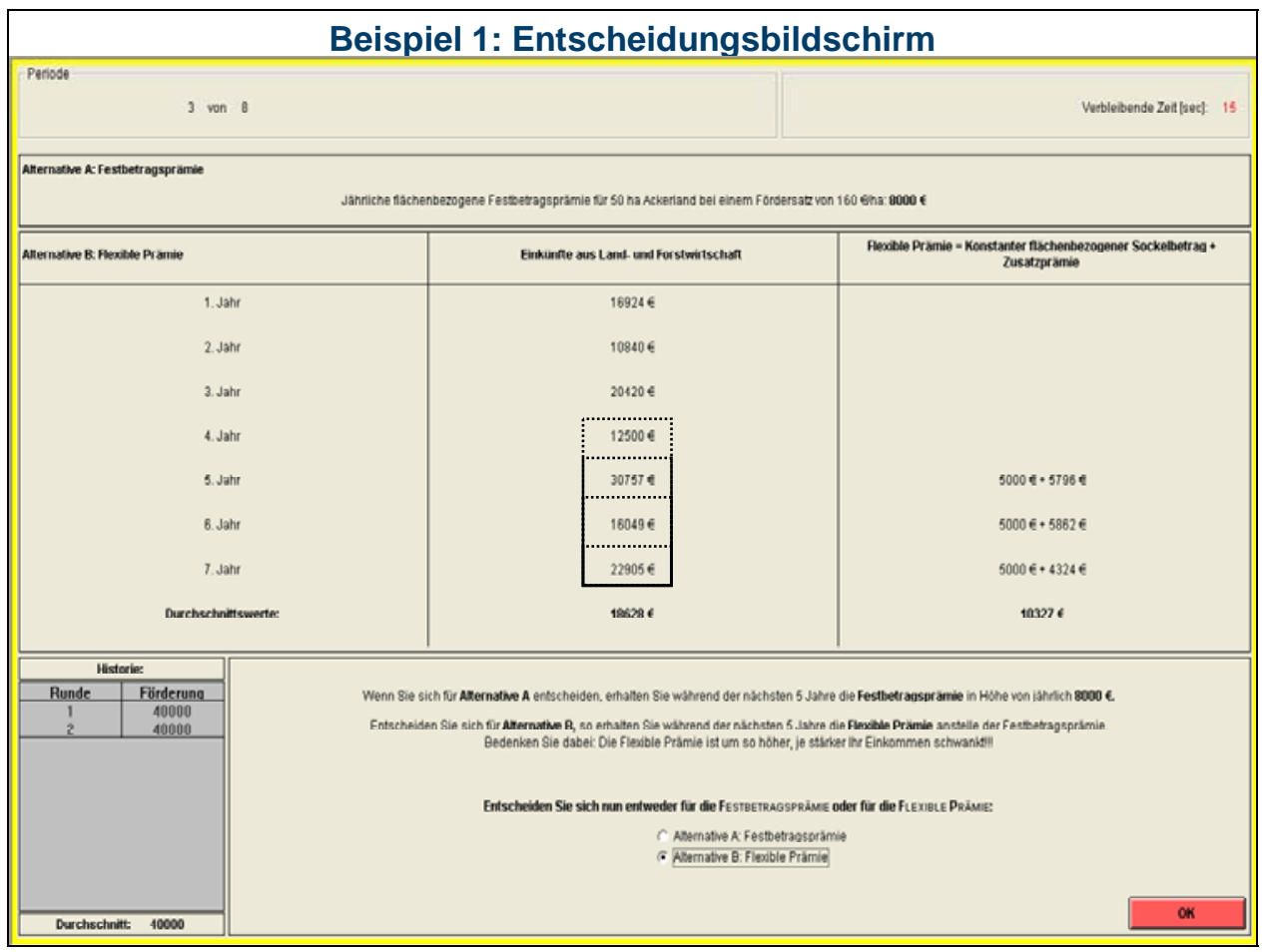

Die dargestellte Abbildung zeigt den Entscheidungsbildschirm, d.h. den Bildschirm, den jeder Spieler zu Beginn einer jeden Spielrunde angezeigt bekommt und der die Grundlage für die Entscheidung des Spielers bilden soll.

Der Bildschirm ist dabei so aufgebaut, dass unterhalb der Kopfzeile, in der die Spielrunde (hier Runde 3 von den insgesamt 8 Runden) und eine Zeitangabe angezeigt werden, der Bildschirm in folgende 4 Bereiche eingeteilt ist:

- Zwei Informationsbereiche, jeweils mit näheren Daten zu der jeweiligen Förderalternative. Zur Flexiblen Förderung werden dabei neben der historischen Entwicklung der Einkünfte aus LuF auch die imaginären historischen Flexiblen Förderbeträge angezeigt, die sich ergeben hätten, wenn es die Flexible Förderung in der Vergangenheit schon gegeben hätte.

- Informationsbereich mit der Historie der eigenen Entscheidungen

- Entscheidungsbereich, in dem durch einfaches Anklicken der gewählten Alternative und nachfolgendem einfachen Anklicken des OK-Feldes die Auswahl bestätigt wird.

Die Entscheidungssituation für den Teilnehmer stellt sich in der angezeigten Fallgestaltung für den Teilnehmer nun wie folgt dar:

Dazu erinnern wir uns zurück: Die Zusatzförderung als Teil der Flexiblen Förderung bemisst sich an der Schwankung der luf-Einkünfte der vergangenen 5 Jahre. Der Spieler soll sich nun für die kommenden 5 Jahre entscheiden. Die Zusatzförderung des kommenden Jahres bemisst sich damit an der Schwankung der Einkünfte der Jahre 4 bis 7 und des kommenden Jahres. Dementsprechend bemisst sich die Zusatzförderung des übernächsten Jahres an der Schwankung der Einkünfte der Jahre 5 bis 7 und der beiden kommenden Jahre (Die einzelnen gestrichelten Kästen werden zur Veranschaulichung nacheinander eingeblendet). Da die Einkünfte der kommenden Jahre für den Teilnehmer zum Zeitpunkt der Entscheidung noch nicht ersichtlich sind, trifft er eine Entscheidung unter Unsicherheit. Je weiter die Jahre in der Zukunft liegen, desto unsicherer ist für ihn die Höhe der Zusatzprämie. Während er für das 8. 
Jahr die Höhe der Zusatzförderung noch relativ gut vorhersehen kann, ist die Zusatzförderung des 12. Jahres für ihn überhaupt nicht mehr vorhersehbar, da sie sich vollständig an der Schwankung der zukünftigen Einkünfte bemisst.

Wir wollen uns den vorliegenden Fall ansehen: Die Einkünfte des Teilnehmers unterlagen in der Vergangenheit hohen Schwankungen. Das trifft insbesondere auf die letzten 4 Jahre zu; die Jahre also, die in die Berechnungen für die Flexible Förderung für die kommenden Jahre eingehen. Der Spieler könnte daher vermuten, dass die Flexible Prämie auch für den zukünftigen 5-Jahres-Zeitraum vorteilhaft sein könnte. Da der Teilnehmer aber naturgemäß keine Angaben zu seinen zukünftigen Einkünften hat, muss dies nicht zwangsläufig so sein. Es wäre genauso denkbar, dass sich die Einkünfte in den kommenden Jahren wieder soweit stabilisieren, dass sich am Ende des 5-Jahres-Zeitraums herausstellt, dass im Nachhinein die Festbetragsprämie vorteilhafter gewesen wäre. Wie bereits gesagt: Die Entscheidung des Spielers ist als eine Entscheidung unter Unsicherheit.

Aufgrund der im vorliegenden Fall deutlichen Schwankung der Einkünfte entscheidet sich der Spieler hier aber für die Flexible Prämie, indem er diese im Entscheidungsbereich markiert und das OK-Feld anklickt.

Durch das Anklicken des OK-Feldes bewegt sich der Spieler auf der Zeitachse genau 5 Jahre voran. Er befindet sich dann also zeitlich genau 5 Jahre nach seiner Entscheidung und kann nun neben seinen Einkünften dieser 5 Jahre auch die Auswirkungen seiner Entscheidung im dargestellten Ergebnisbildschirm einsehen.

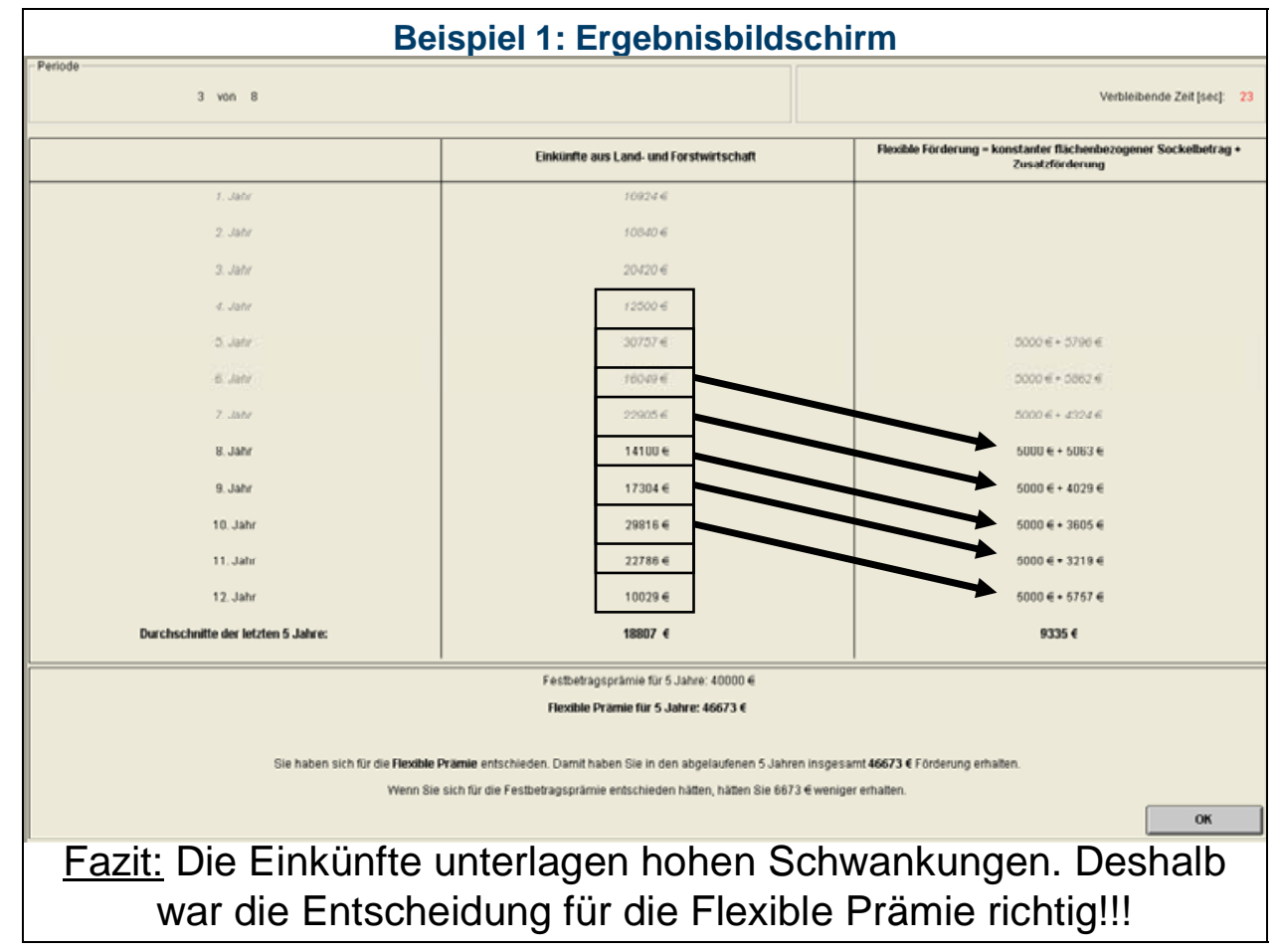

Der Ergebnisbildschirm ist hinsichtlich seines Aufbaus mit dem Informationsbereich zur Flexiblen Förderung auf dem Entscheidungsbildschirm zu vergleichen. Die Daten zu den Einkünften sowie zur Höhe der Flexiblen Förderung, die bereits zum Entscheidungszeitpunkt bekannt waren, sind auf diesem Ergebnisbildschirm grau dargestellt. Demgegenüber sind die Daten des abgelaufenen 5-Jahres-Zeitraumes schwarz dargestellt. Aus ihnen und aus der im Feld darunter angezeigten Ergebnisaufstellung kann der Teilnehmer nun ablesen, ob er mit seiner Entscheidung im Vergleich zur alternativen Förderung die höhere Auszahlung bekommen hat. 
An dieser Stelle möchte ich nochmals auf die Entstehungsweise der Zusatzprämie eingehen. Die im Folgenden hintereinander eingeblendeten (Die einzelnen Markierungen (Kasten mit Pfeil) erscheinen per Mausklick nacheinander auf dem Bildschirm) Markierungen zeigen die Zeiträume, die für die Berechnung der Zusatzprämie jeweils relevant sind. Wie bereits gesagt: Die Zusatzprämie bemisst sich an der Schwankung der Einkünfte aus luf der vergangenen 5 Jahre.

In diesem Fallbeispiel zeigt sich, dass sich der Spieler im Nachhinein mit der Flexiblen Prämie aus Sicht der letztendlichen Höhe des Förderbetrages für die richtige Alternative entschieden hat.

Seine Einkünfte aus dem 50ha-Ackerbaubetrieb unterlagen auch in der Folgezeit verhältnismäßig hohen Schwankungen. Im Vergleich zu den im Vorhinein feststehenden $40.000 €$ Förderung aus der Festbetragsprämie hat dieser Spieler über die Flexible Prämie ganze $46.673 €$ Förderung bekommen.

Zur weiteren Veranschaulichung noch ein zweites Fallbeispiel:

Wie der Entscheidungsbildschirm zeigt, unterlagen die Einkünfte in der Vergangenheit in diesem Beispiel insbesondere in den für die zukünftige Flexible Förderung relevanten letzten 4 Jahren nur verhältnismäßig geringen Schwankungen. Es wäre daher für den Spieler zu überlegen, in diesem Fall die Festbetragsprämie zu wählen, da mit der Flexiblen Prämie eine geringere Prämienzahlung verbunden sein könnte.

So entscheidet sich der Teilnehmer in diesem Fall für Alternative A: Festbetragsprämie.

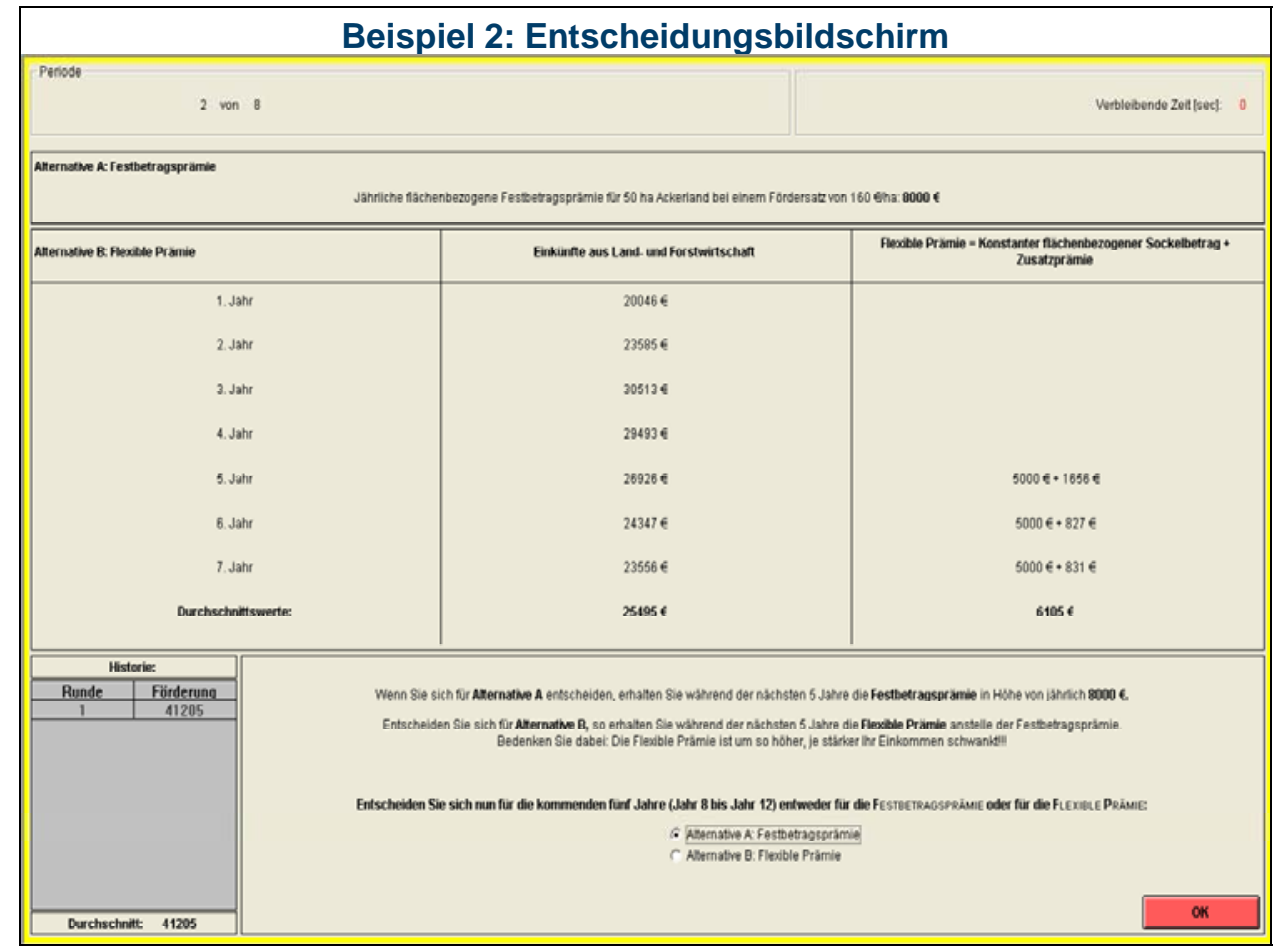

Der anschließende Ergebnisbildschirm zeigt nun wiederum, dass sich der Spieler aus Sicht einer möglichst hohen Gesamtförderung für die richtige Förderalternative entschieden hat. Hätte er sich für die Flexible Prämie entschieden, so hätte er im besagten 5-Jahres-Zeitraum anstatt der aus der Festbetragsförderung konstanten $40.000 €$ nur $30.762 €$ bekommen. 


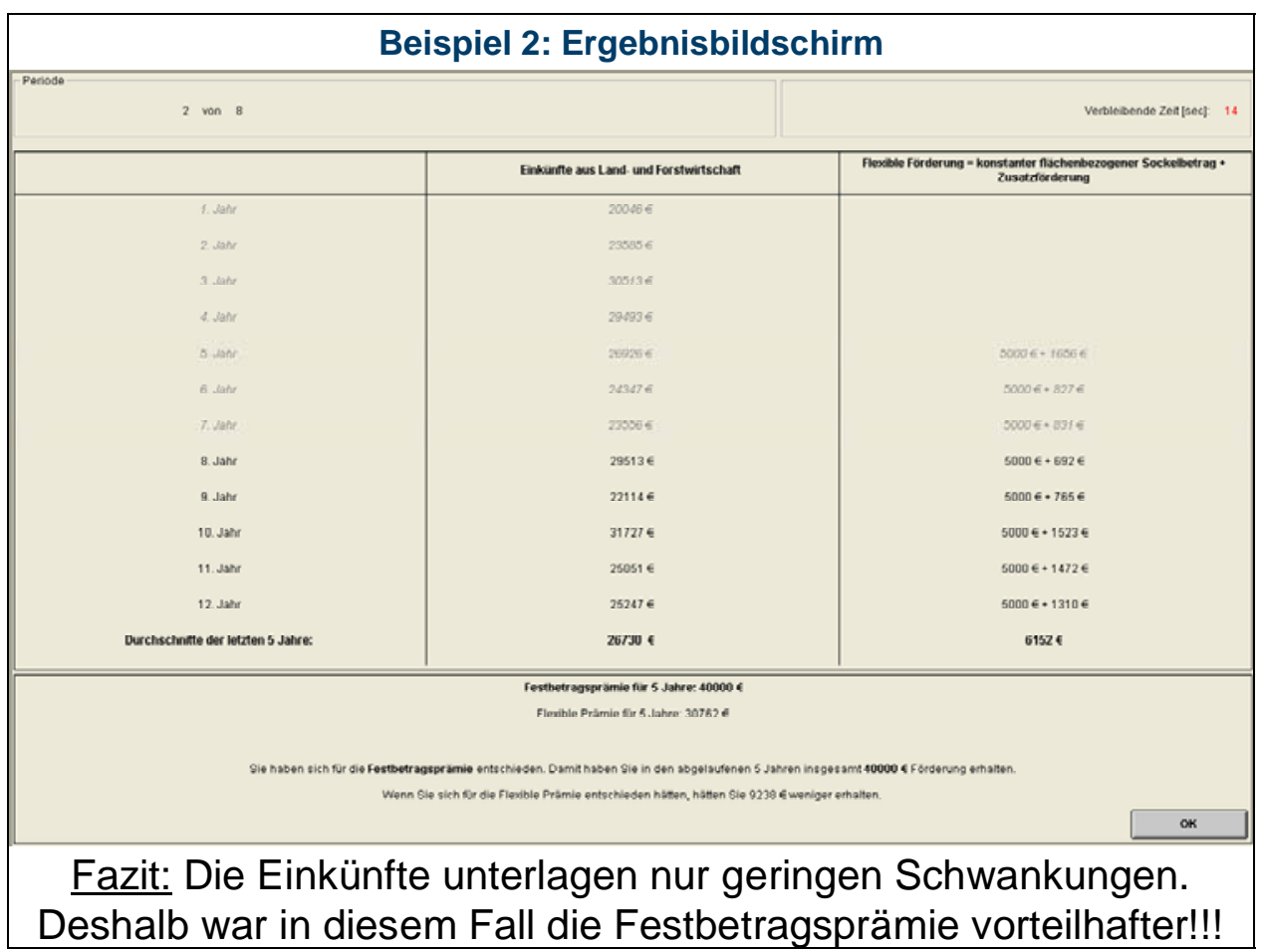

Bevor jetzt mit dem Experiment begonnen wird, sei im Vorfeld noch auf drei für den Ablauf des Experimentes und die Entscheidungsfindung wichtige Punkte hingewiesen:

1. Während des Experimentes gelten für die Teilnehmer wichtige Verhaltensregeln. Um die Aussagefähigkeit und damit die Verwertbarkeit der Ergebnisse des Experimentes nicht zu gefährden ist es den Teilnehmern nicht gestattet, sich zu unterhalten oder auf den Bildschirm eines anderen Spielers zu schauen. Durch die Anordnung der einzelnen Laptops sowie durch das Aufstellen der Zwischenwände ist bereits von mir als Veranstalter versucht worden, ein solches Verhalten weitestgehend zu erschweren. Ich bitte dieses zu respektieren und sich an diese Regeln zu halten.

2. Für Ihre Entscheidungsfindung ist die folgende grundsätzliche Herangehensweise elementar wichtig: Versuchen Sie sich so weit wie möglich in ihre Rolle als Betriebsleiter des dargestellten 50ha Ackerbaubetriebes hineinzuversetzen! Konkret bedeutet das für Sie: Überlegen Sie immer genau, für welche Förderung Sie sich in der gegebenen Situation wirklich entscheiden würden. Wenn Sie also im Extremfall schon nach dieser Einführung für sich selbst zu dem Schluss gekommen sind, dass die Flexible Förderung für Sie generell keine Alternative zur Festbetragsprämie darstellt, dann wählen Sie bitte auch nur die Festbetragsprämie im Experiment.

3. Bedenken Sie aber bei Ihrer Entscheidungsfindung auch: Die Auszahlung für jeden Teilnehmer am Ende dieser Veranstaltung bemisst sich an der Höhe des im Laufe der 8 Entscheidungsrunden angesammelten Fördervolumens. Analog zur Situation in der Wirklichkeit sollte es also auch hier das Ziel des Spielers sein, möglichst hohe Förderbeträge zu bekommen, um so auch den letztendlichen persönlichen Auszahlungsbetrag zu maximieren. 


\section{...Durchführung des Prämienexperimentes...}

Zum Abschluss des Experimentes folgt nun noch ein Glücksspiel.

\begin{tabular}{|l} 
Glückspiel: Simulierter Münzwurf \\
$>$ Auf dem Rechner wird der Wurf einer $1 €$ - Münze \\
simuliert \\
$>$ Zeigt die Münze "Kopf", so gewinnt der Spieler $50 €$ \\
$>$ Zeigt die Münze "Zahl", so gewinnt der Spieler $100 €$ \\
$>$ Vor Beginn des Spiels muss jeder Teilnehmer \\
allerdings ein Geldgebot (zwischen 50 und $100 €)$ \\
für die Teilnahme am Spiel abgeben, das sich \\
daran bemessen sollte, wie viel ihm die \\
Teilnahme am Spiel wert ist. \\
$>$ Dabei ist zu beachten, dass nur die $50 \%$ der \\
Teilnehmer mit den höchsten Geboten zum Spiel \\
zugelassen werden.
\end{tabular}

Dieses ist wie folgt konzipiert:

Auf den Rechnern wird der Wurf einer Münze simuliert. Zeigt die Münze „Kopf“, so gewinnt der Spieler $50 €$; zeigt die Münze „Zahl“, so gewinnt der Spieler $100 €$. Vor Beginn des Spieles, d.h. vor dem Münzwurf, muss der Spieler jedoch ein Gebot für die Teilnahme abgeben, das sich daran bemessen sollte, wie viel dem Spieler die Teilnahme am Spiel wert ist. Weil der Spieler in jedem Fall $50 €$ gewinnt, gleichzeitig aber auch nur maximal $100 €$ bei dem Spiel gewinnen kann, ist auch nur ein Gebot in dieser Bandbreite sinnvoll. Bei Bemessung des Gebotes ist allerdings zu beachten, dass nur die $50 \%$ der Teilnehmer mit den höchsten Geboten zum Spiel zugelassen werden. Die restlichen 50 \% der Teilnehmer werden vom Spielverlauf ausgeschlossen. Sie behalten damit ihren Einsatz; haben aber im Gegenzug auch keine Möglichkeit Geld zu gewinnen.

Die Überlegungen des Spielers hinsichtlich seines Geldgebotes sollten also von den folgenden Kerngedanken gelenkt sein:

1. Zum einen besteht für den Spieler bei einem zu niedrigen Gebot die Gefahr vom Spiel ausgeschlossen zu werden und damit die Chance auf einen Geldgewinn zu vergeben.

2. Zum anderen besteht für den Spieler bei einem zu hohen Gebot die Gefahr, dass die Münze nach dem Wurf lediglich „Kopf“ zeigt und somit der Spieler Geld verliert.

Insgesamt werden 4 Runden gespielt, d.h. die Münze wird viermal geworfen und jeder Spieler muss viermal ein Gebot abgeben. Wird ein Spieler in einer Runde aufgrund eines zu niedrigen Gebotes vom Spiel ausgeschlossen, so hat das keinen Einfluss auf die nächsten Spielrunden. Wurde er also in der ersten Runde ausgeschlossen, so kann er in der 2. Runde wieder ein Gebot abgeben. Die einzelnen Runden sind also vollkommen unabhängig voneinander. 
Wie der Spieler im gleich folgenden Beispiel erkennen wird, sind die Gewinnbeträge jedoch in den einzelnen Runden unterschiedlich. Während in der ersten Spielrunde für alle Spieler die Gewinnbeträge $50 €$ bei „Kopf“ und $100 €$ bei „Zahl“ gelten, differieren sie in den Folgerunden.

Der praktische Ablauf des Glücksspieles gestaltet sich dabei wie folgt:

Die Gebote für die einzelnen Spielrunden muss der Spieler bereits vor der Durchführung der 4 Münzwürfe abgeben. Der Spieler gibt also zu Beginn des Spieles nacheinander seine Gebote für die einzelnen Runden ab und erfährt erst danach die Ergebnisse der einzelnen Runden.

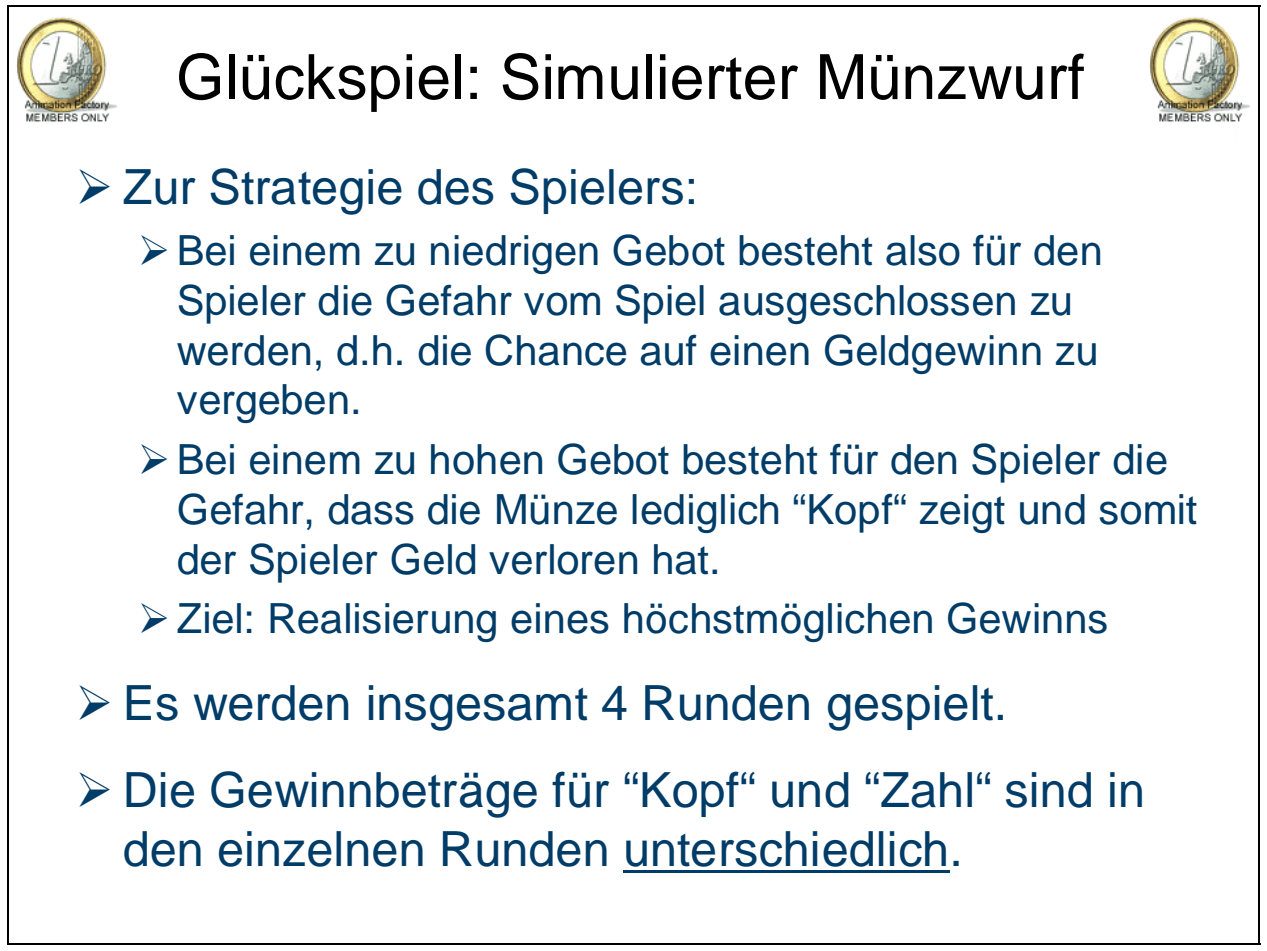

Ziel des Spielers sollte wiederum sein, möglichst viel Geld zu gewinnen. Denn das liegt auch in seinem persönlichen Interesse. Der Gewinn des Spielers hat nämlich wie das Gesamtprämienvolumen aus dem Prämienexperiment Einfluss auf die letztendliche Auszahlung an den Spieler am Ende der Veranstaltung.

Das folgende Fallbeispiel soll den Spielverlauf näher veranschaulichen: 


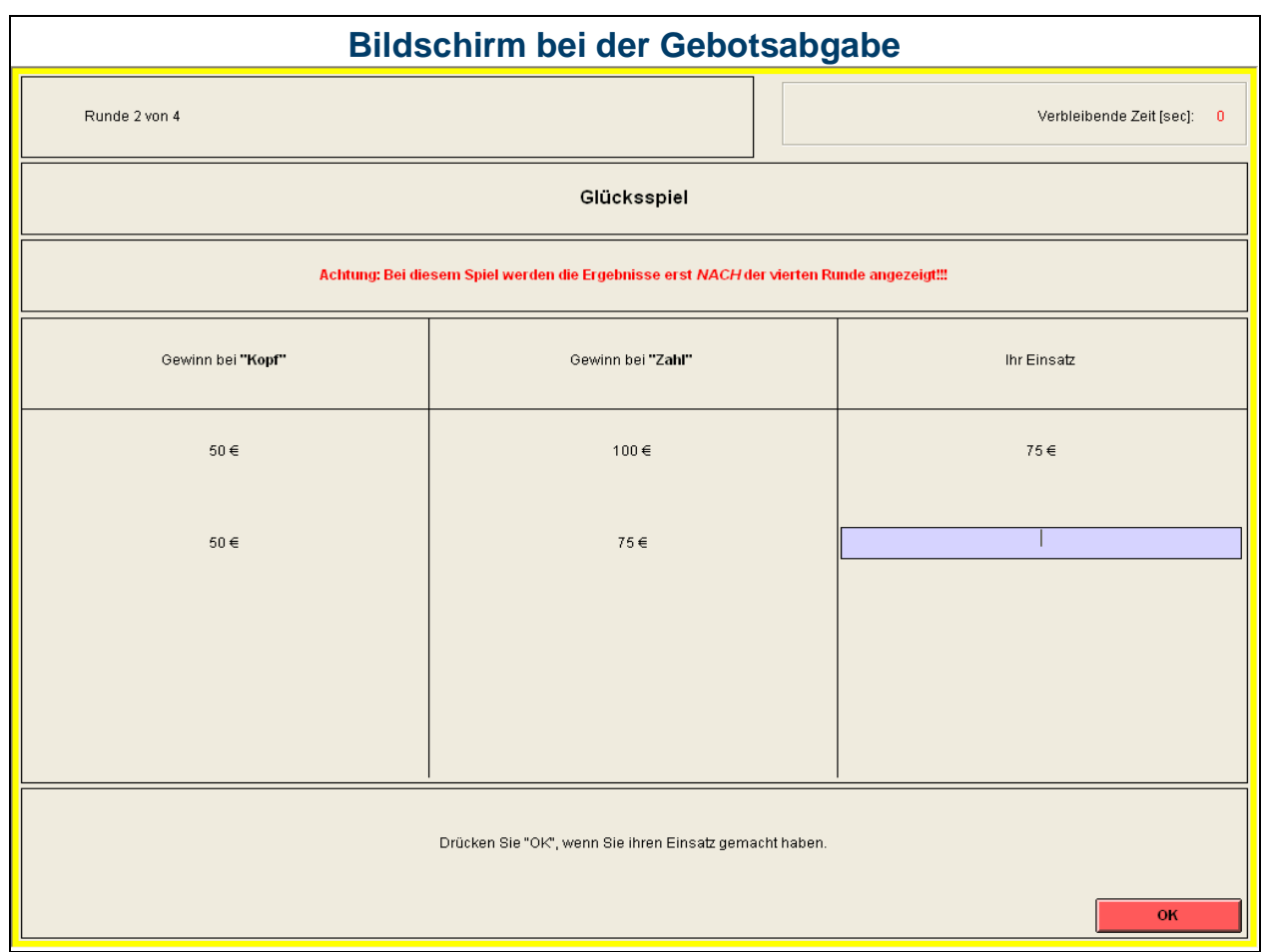

Die Abbildung zeigt den Bildschirm bei der Gebotsabgabe. Dieser Spieler hat sich in der ersten Runde für ein Gebot von $75 €$ entschieden. Ohne das Ergebnis des ersten Spieles zu kennen, wird er in der Abbildung jetzt zur Abgabe seines Gebotes für die zweite Spielrunde aufgefordert, in der der Gewinn bei „Kopf“ wiederum $50 €$ beträgt, während der Gewinn bei „Zahl“ jetzt nur $75 €$ beträgt.

Sobald alle Spieler für alle 4 Spielrunden ihre Gebote abgegeben haben, erfolgt ein automatischer Abgleich der Gebote über das Netzwerk und die Simulation der vier Münzwürfe auf den Computern. Anschließend werden den Spielern die Ergebnisse der einzelnen Runden mitgeteilt.

Das erfolgt im Auswertungsbildschirm, den die folgende Abbildung zeigt:

\begin{tabular}{|c|c|c|}
\hline \multicolumn{3}{|c|}{ Auswertungsbildschirm } \\
\hline Runde 4 von 4 & & Verbleibende Zeit [sec]: 25 \\
\hline \multicolumn{3}{|c|}{ Auswertung } \\
\hline Gewinn bei "Kopp" & Gewinn bei "Zaht" & Ihr Einsatz \\
\hline $50 €$ & $100 €$ & $\begin{array}{l}75 € \\
\text { nicht teilgenommen, Kopf, Gewinn: } 0\end{array}$ \\
\hline $50 €$ & $75 €$ & $\begin{array}{c}\quad 00 € \\
\text { teilgenommen, Kopf, Gewinn: }-10\end{array}$ \\
\hline $75 €$ & $100 €$ & $\begin{array}{l}90 € \\
\text { teilgenommen, Kopf, Gewinn: }-15\end{array}$ \\
\hline $60 €$ & $90 €$ & $\begin{array}{l}73 € \\
\text { teilgenommen, Zahl, Gewinn: } 17\end{array}$ \\
\hline \multicolumn{3}{|c|}{ Drưcken Sie" "OK", wenn Sie ihre Ergebnisse angeschaut haben. } \\
\hline & & ок \\
\hline
\end{tabular}


Der Spieler erfährt nun, wie er in den einzelnen Spielrunden abgeschnitten hat, d.h. ob er zur Teilnahme an der jeweiligen Spielrunde zugelassen wurde und welchen Ausgang der Münzwurf für ihn genommen hat. Daraus resultierend ergeben sich für ihn auch seine monetären Ergebnisse aus den einzelnen Spielrunden, die er $\mathrm{zu}$ seinem persönlichen Gesamtergebnis addieren kann.

Im dargestellten Fallbeispiel hat der Spieler insgesamt kein Glück gehabt:

In der ersten Runde hat sein Einsatz von $75 €$ zur Teilnahme am Spiel nicht ausgereicht, d.h. er gehörte in dieser Spielrunde nicht zu den 50\% der Teilnehmer mit den höchsten Geboten und wurde folglich für diese Runde vom Spiel ausgeschlossen. Sein Gewinn/Verlust aus dieser Runde betrug daher $0 €$.

Dem gegenüber genügten die Gebote des Spielers in den Runden 2 und 3 zur Teilnahme am Spiel. Bei den folgenden Münzwürfen hatte der Spieler jedoch kein Glück. In beiden Fällen zeigte die Münze nur „Kopf“, so dass der Spieler in beiden Runden Geld verlor.

In der letzten Runde, in der er wiederum mit seinem Gebot zur Teilnahme am Spiel zugelassen wurde, hatte der Spieler dagegen endlich Glück. Die Münze zeigte „Zahl“. Damit gewann der Spieler in dieser Runde Geld.

Dieser Gewinn konnte jedoch die Verluste aus den beiden vorigen Runden nicht mehr ausgleichen, so dass der Spieler das Glückspiel letztendlich mit einem negativen Ergebnis abgeschlossen hat. Genauso gut hätte das Glücksspiel für den Spieler bei den abgegebenen Geboten aber auch einen ganz anderen Ausgang nehmen können, wenn nämlich die Münzwürfe häufiger „Zahl“ ergeben hätten, der Spieler also mehr Glück gehabt hätte.

Bevor mit dem Spiel nun begonnen werden soll, sei wiederum auf die bereits vor Beginn des Prämienexperiments angesprochenen Verhaltensregeln verwiesen. Auch während dieses Spieles sind Gespräche unter den einzelnen Teilnehmern sowie Blicke (wenn überhaupt möglich) auf den Bildschirm eines anderen Teilnehmers nicht erlaubt. Nur so kann gewährleistet werden, dass die Ergebnisse keinen Verzerrungen unterliegen und folglich Aussagekraft besitzen.

Im Anschluss an dieses Spiel möchte ich jeden Spieler bitten, den vor ihm liegenden Fragebogen sorgfältig auszufüllen und bei mir abzugeben. Ich werde dann auch die Auszahlungen an die einzelnen Teilnehmer vornehmen.

\section{...Durchführung des Glücksspiels...}

\section{Ende der Veranstaltung}




\section{A.6 FRAGEBOGEN FÜR DIE TEILNEHMER AM EXPERIMENT}

1. In welchem Bundesland befindet sich Ihr Betrieb? $(n=72)$

SH: $17 \%$; HE: $15 \%$; NW: $15 \%$; MV: $21 \%$; RP: $14 \%$; BW: $17 \%$; BY: $1 \%$

2. Welche Rechtsform hat Ihr Betrieb? $(n=72)$

\begin{tabular}{|l|c|}
\hline Einzelunternehmen (Haupterwerb) & $41,7 \%$ \\
\hline Einzelunternehmen (Nebenerwerb) & $27,8 \%$ \\
\hline GbR & $15,3 \%$ \\
\hline OHG oder KG & $0 \%$ \\
\hline Genossenschaft & $4,2 \%$ \\
\hline Kapitalgesellschaft & $6,9 \%$ \\
\hline $\begin{array}{l}\text { Sonstige, } \\
\text { und zwar...offentl. Gebietskörperschaft; e.V......... }\end{array}$ & $4,2 \%$ \\
\hline
\end{tabular}

3. In welcher Bewirtschaftungsweise bewirtschaften Sie ihren Betrieb? $(n=72)$

\begin{tabular}{|l|c|}
\hline Konventionell & $3 \%$ \\
\hline Ökologisch & $97 \%$ \\
\hline
\end{tabular}

3.1 Wenn ökologisch: Seit wann? $(n=70)$

Kleinster Wert: 1972; größter Wert: 2006; $\mu=1993 ; \delta=7$

3.2 Wenn konventionell: Eine Umstellung meines Betriebes auf ökologischen Landbau in den nächsten Jahren halte ich für $(n=2)$

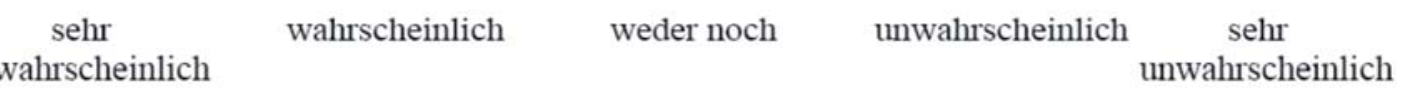

$-0 \%-10--0$

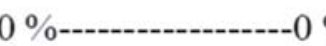

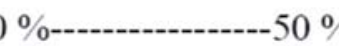
$-50 \%-----$

4. Über welche Flächenausstattung verfügt ihr Betrieb? $(n=72)$

\begin{tabular}{|l|c|c|}
\hline Ackerfläche & $\mu=126,74$ ha & $\delta=217,11$ ha \\
\hline Dauergrünland & $\mu=127,40$ ha & $\delta=374,96$ ha \\
\hline Sonstiges (Wald, Hoffläche, Unland, etc.) & $\mu=43,94$ ha & $\delta=180,26$ ha
\end{tabular}

5. Welcher betriebswirtschaftlichen Ausrichtung ist ihr Betrieb voraussichtlich zuzuordnen? (Richtet sich danach, welcher Betriebszweig mehr als $2 / 3$ des gesamtbetrieblichen Standarddeckungsbeitrags erzielt) $(n=72)$

\begin{tabular}{|l|c|}
\hline Ackerbau (inkl. Gemüsebau) & $23,6 \%$ \\
\hline Gartenbau & $0 \%$ \\
\hline Dauerkultur (Weinbau, Obstbau) & $0 \%$ \\
\hline Futterbau (Milchvieh, Rinder, Schafe) & $45,8 \%$ \\
\hline Veredelung (Schweine, Geflügel) & $1,4 \%$ \\
\hline Verbund (früher: Gemischt) & $29,2 \%$ \\
\hline
\end{tabular}

\section{Erstrecken sich Ihre Aktivitäten noch auf weitere Betriebszweige? $(n=72)$}

\begin{tabular}{|l|l|}
\hline Energieerzeugung (Biogas, Windkraft, Photovoltaik...) & Ja: $26,4 \%$ \\
\hline Direktvermarktung & Ja: $30,6 \%$ \\
\hline Tourismus & Ja: $8,3 \%$ \\
\hline Pensionspferdehaltung & Ja: $6,9 \%$ \\
\hline $\begin{array}{l}\text { Sonstiges, und zwar ....Behindertenbetreutung, Brennerei, Fleischerei, } \\
\text { Lohmumternehmer............................................................... }\end{array}$ & Ja: $6,9 \%$ \\
\hline
\end{tabular}


7. Welche Tierhaltungszweige befinden sich auf ihrem Betrieb und welche Kapazitäten bestehen in den einzelnen Bereichen? $(n=72)$

\begin{tabular}{|l|c|}
\hline Tierhaltungszweig & Vieh im Jahresdurchschnitt \\
\hline Milchkühe & $\mathrm{n}=24 ; \mu=65 ; \delta=86$ \\
\hline Mutterkühe & $\mathrm{n}=39 ; \mu=139 ; \delta=402$ \\
\hline Bullenmast & $\mathrm{n}=22 ; \mu=126 ; \delta=316$ \\
\hline Rinderaufzucht & $\mathrm{n}=39 ; \mu=63 ; \delta=96$ \\
\hline Schafhaltung & $\mathrm{n}=8 ; \mu=84 ; \delta=107$ \\
\hline Sauenhaltung & $\mathrm{n}=8 ; \mu=31 ; \delta=46$ \\
\hline Mastschweinehaltung & $\mathrm{n}=15 ; \mu=155 ; \delta=255$ \\
\hline Ferkelaufzucht & $\mathrm{n}=4 ; \mu=110 ; \delta=104$ \\
\hline Legehennenhaltung & $\mathrm{n}=7 ; \mu=82 ; \delta=107$ \\
\hline Putenmast & $\mathrm{n}=2 ; \mu=4010 ; \delta=5643$ \\
\hline Hähnchenmast & $\mathrm{n}=1 ; \mu=8500$ \\
\hline Sonstiges, und zwar $\ldots \ldots \ldots \ldots \ldots \ldots \ldots \ldots \ldots \ldots$ & $\mathrm{n}=0$ \\
\hline
\end{tabular}

8. Im Folgenden sind einige Statements zu den allgemeinen Rahmenbedingungen desökolandbaus aufgeführt. Inwieweit treffen folgende Aussagen auf Sie zu? Bitte geben Sie Ihre Zustimmung oder Ablehnung an. (Teilfragen 1 bis 5: $n=72$; Teilfrage 6: $n=70$ )

\begin{tabular}{|l|c|c|c|c|c|}
\hline & $\begin{array}{c}\text { Stimme } \\
\text { voll- } \\
\text { ständig } \\
\text { zul }\end{array}$ & $\begin{array}{c}\text { Stimme } \\
\text { zu }\end{array}$ & $\begin{array}{c}\text { Weder } \\
\text { noch }\end{array}$ & $\begin{array}{c}\text { Lehne } \\
\text { ab }\end{array}$ & $\begin{array}{c}\text { Lehne } \\
\text { voll- } \\
\text { ständig } \\
\text { ab }\end{array}$ \\
\hline $\begin{array}{l}\text { Ich bin davon überzeugt, dass die derzeitigen Öko- } \\
\text { Flächenprämien zukünttig auch bei knapperen } \\
\text { öfentlichen Haushalten nicht in Gefahr sind. }\end{array}$ & $8,3 \%$ & $25 \%$ & $23,6 \%$ & $26,4 \%$ & $16,7 \%$ \\
\hline $\begin{array}{l}\text { Was Fördergelder angeht, bin ich stets auf dem } \\
\text { neusten Stand. }\end{array}$ & $16,7 \%$ & $54,2 \%$ & $20,8 \%$ & $8,3 \%$ & $0 \%$ \\
\hline $\begin{array}{l}\text { Bezüglich der weiteren Entwicklung des } \\
\text { Ökolandbaus in Deutschland bin ich sehr } \\
\text { optimistisch. }\end{array}$ & $16,7 \%$ & $43,1 \%$ & $22,1 \%$ & $18,1 \%$ & $0 \%$ \\
\hline $\begin{array}{l}\text { Das gegenwärtige Fördersystem muss reformiert } \\
\text { werden. Ansonsten wird die Förderung in Zukunft in } \\
\text { Gefahr sein. }\end{array}$ & $25 \%$ & $47,2 \%$ & $18,1 \%$ & $6,9 \%$ & $2,8 \%$ \\
\hline $\begin{array}{l}\text { Bei der zukünftigen Ausrichtung meines Betriebes } \\
\text { spielen Fördermittel eine entscheidende Rolle. }\end{array}$ & $36,1 \%$ & $36,1 \%$ & $12,5 \%$ & $13,9 \%$ & $1,4 \%$ \\
\hline $\begin{array}{l}\text { Nur für bereits ökologisch wirtschaftende Landwirte: } \\
\begin{array}{l}\text { Wenn ich die Zeit noch einmal zurückdrehen könnte, } \\
\text { würde ich nicht wieder auf Ökolandbau umstellen. }\end{array}\end{array}$ & $7,1 \%$ & $4,3 \%$ & $4,3 \%$ & $22,9 \%$ & $61,4 \%$ \\
\hline
\end{tabular}

\section{Nur für bereits ökologisch wirtschaftende Landwirte:}

Welche Bedeutung messen Sie den gegenwärtigen flächenbezogenen Öko-Prämien für die Wirtschaftlichkeit Ihres Betriebes zu? $(n=72)$

\begin{tabular}{|c|c|c|c|c|}
\hline $\begin{array}{l}\text { völlig } \\
\text { unwichtig }\end{array}$ & $\begin{array}{c}\text { geringe } \\
\text { Bedeutung }\end{array}$ & $\begin{array}{l}\text { durchschnittliche } \\
\text { Bedeutung }\end{array}$ & $\begin{array}{l}\text { hohe } \\
\text { Bedeutung }\end{array}$ & $\begin{array}{l}\text { elementar } \\
\text { wichtig }\end{array}$ \\
\hline
\end{tabular}


10. Wie stehen Sie persönlich der Förderung des Ökolandbaus grundsätzlich gegenüber? $(n=71)$

\begin{tabular}{|l|l|l|}
\hline a.) Die Förderung sollte abgebaut werden. & $11,3 \%$ & $\rightarrow$ weiter nach Frage 10.1 \\
\hline b.) Die Förderung sollte verändert werden. & $40,8 \%$ & $\rightarrow$ weiter nach Frage 10.2 \\
\hline c.) Die gegenwärtige Förderung sollte beibehalten werden & $47,9 \%$ & $\rightarrow$ weiter nach Frage 10.3 \\
\hline
\end{tabular}

10.1 Bei Antwort a.): Warum sollte Ihrer Meinung nach die Förderung abgebaut werden? $(n=8)$ Antwortverhalten: 4 Teilnehmer erhoffen sich höhere Marktpreise; 2 Teilnehmer erhoffen sich einen Abbau von Fehlallokationen; 1 Teilnehmer erhofft sich einen deutlichen Bürokratieabbau; 1 Teilnehmer wünscht sich die Kopplung einer reduzierten Förderung an die AK im Betrieb.

10.2 Bei Antwort b.): Wenn Sie die Möglichkeit hätten, über die zukünftige Prämiengestaltung mitzubestimmen, welchen Vorschlag würden Sie der Politik unterbreiten? $(n=23)$

Antwortverhalten: 6 Teilnehmer schlagen eine Kopplung der Förderung an die AK im Betrieb vor; 5 Teilnehmer stimmen für eine grundsätzliche Erhöhung der Öko-Förderung; 3 Teilnehmer fordern ein Ende der Förderung von reinen Landschaftspflegemaßnahmen; 3 Teilnehmer stimmen für eine Kopplung der Förderung an Einkommenshöhe und Betriebsgröße; 3 Teilnehmer votieren für eine Kopplung der Förderung an die Umweltfreundlichkeit der landwirtschaftlichen Produktion; 3 Teilnehmer geben an, keine Ideen für eine Veränderung zu haben.

10.3 Bei Antwort b bzw. c.) Worin sehen Sie die Hauptziele der Förderung des ökologischen Landbaus? $(n=27)$

Antwortverhalten: 18 Teilnehmer sehen die Hauptziele der Förderung im Schutz der Natur und in der Erzeugung hochwertiger Nahrungsmittel; 7 Teilnehmer sehen als Hauptziel die Existenzsicherung von Öko-Betrieben sowie die weitere Ausdehnung der Öko-Fläche; für 2 Teilnehmer liegt das Hauptziel der Förderung in einer indirekten Verbraucherpreissubvention.

\section{Woran haben Sie Ihre Entscheidung im Experiment vorrangig festgemacht? (Bitte nur} maximal 2 Nennungen!!!) $(n=72)$

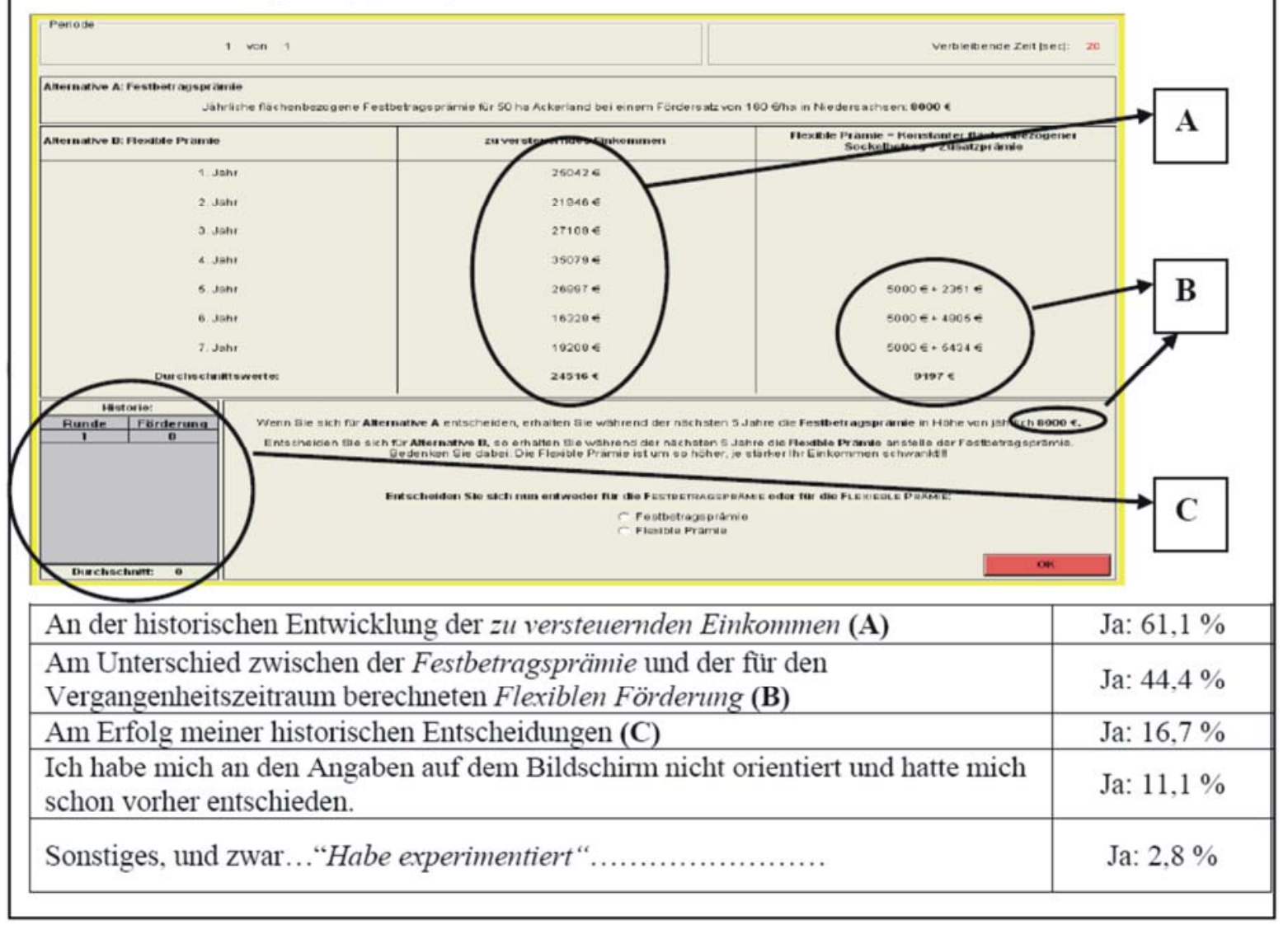


12.0 Angenommen die Flexible Prämie stände für Ihren eigenen Betrieb im nächsten Jahr wirklich für den folgenden 5-Jahres-Zeitraum zur Auswahl. Für welche Prämienart würden Sie sich dann entscheiden? $(n=72)$

\begin{tabular}{|l|l|l|}
\hline a.) Flexible Prämie & $45,8 \%$ & $\rightarrow$ weiter nach Frage $\mathbf{1 2 . 1}$ und 12.3 \\
\hline b.) Festbetragsprämie & $54,2 \%$ & $\rightarrow$ weiter nach Frage $\mathbf{1 2 . 2}$ und 12.3 \\
\hline
\end{tabular}

12.1 Bei a.): Warum käme die Flexible Prämie für Sie infrage? ( $n=33)$

\begin{tabular}{|c|c|c|c|c|c|}
\hline & $\begin{array}{l}\text { Stimme } \\
\text { voll- } \\
\text { ständig } \\
\text { zu }\end{array}$ & $\begin{array}{l}\text { Stimme } \\
\text { zu }\end{array}$ & $\begin{array}{l}\text { Weder } \\
\text { noch }\end{array}$ & $\begin{array}{l}\text { Lehne } \\
\text { ab }\end{array}$ & $\begin{array}{l}\text { Lehne } \\
\text { voll- } \\
\text { ständig } \\
\text { ab }\end{array}$ \\
\hline $\begin{array}{l}\text { Bei der Flexiblen Prämie habe ich die Chance auf } \\
\text { eine höhere Auszahlung. }\end{array}$ & $27,3 \%$ & $60,6 \%$ & $9,1 \%$ & $3 \%$ & $0 \%$ \\
\hline $\begin{array}{l}\text { Die Gewinne auf meinem Betrieb unterlagen in den } \\
\text { vergangenen Jahren starken Schwankungen. }\end{array}$ & $30,3 \%$ & $48,5 \%$ & $21,2 \%$ & $0 \%$ & $0 \%$ \\
\hline $\begin{array}{l}\text { Ich stehe neuen Sachen grundsätzlich immer } \\
\text { aufgeschlossen gegenüber. }\end{array}$ & $15,2 \%$ & $48,5 \%$ & $27,3 \%$ & $6,1 \%$ & $3 \%$ \\
\hline $\begin{array}{l}\text { Es wäre für mich sehr einfach, meine jährlichen } \\
\text { Gewinne stärker schwanken zu lassen, um so höhere } \\
\text { Prämienzahlungen zu realisieren. }\end{array}$ & $3 \%$ & $30,3 \%$ & $36,4 \%$ & $21,2 \%$ & $9,1 \%$ \\
\hline
\end{tabular}

12.2 Bei b.): Warum würden Sie sich für die Festbetragsprämie entscheiden? $(n=39)$

\begin{tabular}{|c|c|c|c|c|c|}
\hline & $\begin{array}{l}\text { Stimme } \\
\text { voll- } \\
\text { ständig } \\
\text { zu }\end{array}$ & $\begin{array}{l}\text { Stimme } \\
\text { zu }\end{array}$ & $\begin{array}{l}\text { Weder } \\
\text { noch }\end{array}$ & $\begin{array}{c}\text { Lehne } \\
\text { ab }\end{array}$ & $\begin{array}{l}\text { Lehne } \\
\text { voll- } \\
\text { ständig } \\
\text { ab }\end{array}$ \\
\hline Die Flexible Prämie habe ich nicht verstanden. & $2,6 \%$ & $2,6 \%$ & $10,3 \%$ & $33,3 \%$ & $51,3 \%$ \\
\hline Bei der Festbetragsprämie weiß ich was ich habe. & $46,2 \%$ & $28,2 \%$ & $15,4 \%$ & $7,7 \%$ & $2,6 \%$ \\
\hline $\begin{array}{l}\text { Die Gewinne auf meinem Betrieb unterlagen in den } \\
\text { vergangenen Jahren nur geringen Schwankungen. }\end{array}$ & $46,2 \%$ & $41 \%$ & $7,7 \%$ & $5,1 \%$ & $0 \%$ \\
\hline $\begin{array}{l}\text { Die Höhe der Auszahlung bei der Flexiblen Prämie } \\
\text { ist mir zu unsicher. }\end{array}$ & $28,2 \%$ & $38,5 \%$ & $17,9 \%$ & $12,8 \%$ & $2,6 \%$ \\
\hline $\begin{array}{l}\text { Die Idee einer Kopplung der Förderung an die } \\
\text { Einkommensschwankungen gefällt mir nicht. }\end{array}$ & $30,8 \%$ & $28,2 \%$ & $20,5 \%$ & $17,9 \%$ & $2,6 \%$ \\
\hline \multicolumn{6}{|c|}{ 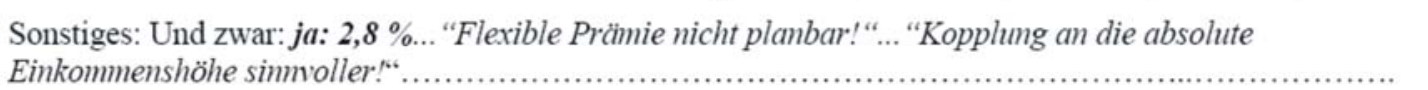 } \\
\hline
\end{tabular}

12.3 Für alle Teilnehmer!!! : Der Mittelwert der jährlichen Förderprämie für den vorgestellten 50ha Ackerbaubetrieb im Experiment betrug bei der Festbetragsprämie $8.000 €$ und bei der Flexiblen Prämie 8.400 $€$. Es gab also einen durchschnittl. Risikoaufschlag von $400 €$ oder $5 \%$.

Wie hoch hätte der Risikoaufschlag mindestens sein müssen, damit Sie sich für die Flexible Prämie entschieden hätten? (Beachten Sie dabei: Wenn Sie sich in Frage 12.0 für die Flexible Prämie entschieden haben, müsste dieser Wert jetzt kleiner oder gleich $400 €$ sein. Wenn Sie sich in Frage 12.0 für die Festbetragsprämie entschieden haben, müsste er größer oder gleich $400 €$ sein!)

$$
\ldots n=55 ; \ldots \mu=815 € ; \ldots \delta=606 € ; \ldots
$$


13. Wie gefällt Ihnen unabhängig von der Flexiblen Förderung grundsätzlich die Idee einer Förderprämie, die sich an den einzelbetrieblichen Einkommensschwankungen d.h. am Einkommensrisiko des jeweiligen Betriebes orientiert? $(n=72)$

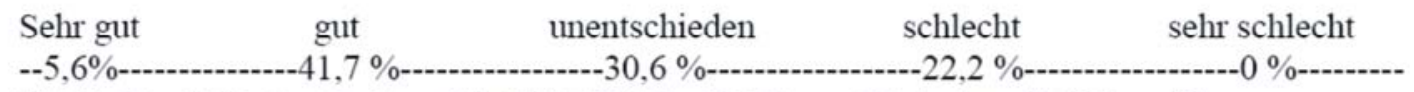

Bei "schlecht" oder "sehr schlecht": Welche Kritikpunkte haben Sie? $(n=13)$

Antwortverhalten: 5 Teilnehmer fürchten Fehlanreize und Fehlallokationen durch die Flexible Förderung; 3 Teilnehmer fürchten zusätzlichen administrativen Aufwand; 2 Teilnehmer wehren sich gegen eine Offenlegung ihrer Einkünfte aus LuF; 2 Teilnehmern fehlt die nötige Planungssicherheit; einem Teilnehmer gefällt die Idee nicht (ohne Angabe von Kritikpunkten).

14. Sehen Sie im Falle einer Einführung der Flexiblen Prämie für sich selbst einen zusätzlichen Anreiz zur Betriebsumstellung auf Ökolandbau bzw. einen zusätzlichen Anreiz zur Beibehaltung des Ökolandbaus? $(n=72)$

$\begin{array}{cccc}\text { ja } & \text { vielleicht } & \text { weiß nicht } & \text { eher nicht }\end{array}$

Wenn ..eher nicht" oder „nein“: Wie wichtig sind Prämienzahlungen bei Ihrer Entscheidung für eine Bewirtschaftungsweise (ökologisch vs. konventionell)? $(n=35)$

sehr wichtig hohe Bedeutung durchschnittlich geringe Bedeutung völlig unwichtig

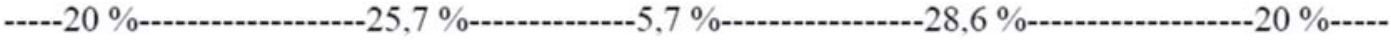

15. Worin lägen aus ihrer Sicht die größten Schwierigkeiten für eine Einführung der Flexiblen Prämie, die sich an den einzelbetrieblichen Einkommensschwankungen orientiert? $(n=44)$ Antwortverhalten: 28 Teilnehmer sehen die größten Schwierigkeiten im notwendigen Organisations- und Verwaltungsaufwand; 9 Teilnehmer glauben, dass infolge einer Einführung der Flexiblen Förderung Fehlanreize und Fehlallokationen entstünden, die einer erfolgreichen Realisation entgegenstehen; 7 Teilnehmer zweifeln an der notwendigen Akzeptanz bei Politik und Berufstand für eine Einführung der Flexiblen Förderung.

16. Welches waren für Sie die Hauptargumente, Ihren Betrieb auf Ökolandbau umzustellen? Bzw. sofern Sie eine Betriebsumstellung auf Ökolandbau planen: Welches sind die Hauptargumente für Sie? $(n=63)$

Antwortverhalten: 40 Teilnehmer gaben eher ideelle Gründe (bspw. ,eigene Weltanschauung“; „Naturschutz“; „Produktion gesünderer Lebensmittel“; „Wirtschaften ohne Einsatz chemischer Pflanzenschutzmittel") an. 13 Teilnehmer gaben eher betriebswirtschaftliche Gründe (bspw. „profitablere Nutzung schwächerer Standorte“; „höhere Prämien“) an. 10 Teilnehmer gaben sowohl ideelle als auch betriebswirtschaftliche Gründe an.

17. Welchem Geburtsjahrgang gehören Sie an? $(n=67)$

Kleinster Wert: 1932: größter Wert: 1977: $u=1961: \delta=9.1$

18. Welchen Ausbildungsabschluss haben Sie? $(n=72)$

\begin{tabular}{|l|l|}
\hline Landwirtschaftliche Lehre & $12,5 \%$ \\
\hline Fachschule / Staat. gepriffter Agrarbetriebswirt & $18,1 \%$ \\
\hline Landwirtschaftsmeister & $18,1 \%$ \\
\hline Landwirtschaftliches Studium (FH/Uni) & $33,3 \%$ \\
\hline Keine landwirtschaftliche Ausbildung & $18,1 \%$ \\
\hline $\begin{array}{l}\text { Sonstiges, } \\
\text { und zwar ......................... }\end{array}$ & $0 \%$ \\
\hline
\end{tabular}




\begin{tabular}{|c|c|c|c|c|c|}
\hline \multicolumn{6}{|c|}{ 19. Bitte geben Sie an, ob die folgenden Aussagen auf Sie persönlich zutreffen oder nicht. $(n=72)$} \\
\hline & $\begin{array}{c}\text { Stimme } \\
\text { voll- } \\
\text { ständig } \\
\text { zu }\end{array}$ & $\begin{array}{c}\text { Stimme } \\
\text { zu }\end{array}$ & $\begin{array}{l}\text { Weder } \\
\text { noch }\end{array}$ & $\begin{array}{l}\text { Lehne } \\
\text { ab }\end{array}$ & $\begin{array}{c}\text { Lehne } \\
\text { voll- } \\
\text { ständig } \\
\text { ab }\end{array}$ \\
\hline $\begin{array}{l}\text { Mir ist die Zeit am Schreibtisch genauso wichtig wie } \\
\text { die tägliche Arbeit im Betrieb. }\end{array}$ & $13,9 \%$ & $33,3 \%$ & $15,3 \%$ & $23,6 \%$ & $13,9 \%$ \\
\hline $\begin{array}{l}\text { Ich riskiere auch mal Dinge, die eventuell scheitern } \\
\text { können. }\end{array}$ & $11,1 \%$ & $62,5 \%$ & $18,1 \%$ & $8,3 \%$ & $0 \%$ \\
\hline Ich bin an technologischen Neuerungen interessiert. & $26,4 \%$ & $58,3 \%$ & $12,5 \%$ & $2,8 \%$ & $0 \%$ \\
\hline $\begin{array}{l}\text { Bei der Umsetzung geplanter gesetzlicher } \\
\text { Bestimmungen sehe ich mich als Vorreiter. }\end{array}$ & $1,4 \%$ & $11,1 \%$ & $58,3 \%$ & $19,4 \%$ & $9,7 \%$ \\
\hline $\begin{array}{l}\text { Der Betriebsleiter sollte seine } \\
\text { betriebswirtschaftlichen Kennzahlen aus dem } \\
\text { Stehgreif kennen. }\end{array}$ & $15,3 \%$ & $66,7 \%$ & $11,1 \%$ & $6,9 \%$ & $0 \%$ \\
\hline $\begin{array}{l}\text { Um meine Zukunft mache ich mir keine Sorgen, da } \\
\text { sich gute Unternehmen durchsetzen werden. }\end{array}$ & $9,7 \%$ & $43,1 \%$ & $34,7 \%$ & $6,9 \%$ & $5,6 \%$ \\
\hline $\begin{array}{l}\text { Ich verbringe sehr viel Zeit an der Planung meines } \\
\text { Betriebes. }\end{array}$ & $8,3 \%$ & $51,4 \%$ & $36,1 \%$ & $2,8 \%$ & $1,4 \%$ \\
\hline $\begin{array}{l}\text { Ich beobachte den Markt sehr genau und passe mich } \\
\text { frühzeitig an sich verändernde Marktbedingungen an }\end{array}$ & $8,3 \%$ & $56,9 \%$ & $25 \%$ & $9,7 \%$ & $0 \%$ \\
\hline
\end{tabular}

20. Kein Betrieb kann überall gleich gut sein. Jeder hat spezielle Stärken und Schwächen. Wo
liegen Ihre? Wie schätzen Sie sich im Vergleich zu Berufskollegen ein? (n = 72)
\begin{tabular}{|l|c|c|c|c|c|}
\hline \multicolumn{1}{|c|}{ Wir .... } & $\begin{array}{c}\text { Stimme } \\
\text { voll- } \\
\text { ständig } \\
\text { zu }\end{array}$ & $\begin{array}{c}\text { Stimme } \\
\text { zu }\end{array}$ & $\begin{array}{c}\text { Weder } \\
\text { noch }\end{array}$ & $\begin{array}{c}\text { Lehne } \\
\text { ab }\end{array}$ & $\begin{array}{c}\text { Lehne } \\
\text { voll- } \\
\text { ständig } \\
\text { ab }\end{array}$ \\
\hline $\begin{array}{l}\text { sind besonders gut in der Produktion (hohe } \\
\text { Ernteerträge etc.). }\end{array}$ & $8,3 \%$ & $45,8 \%$ & $37,5 \%$ & $8,3 \%$ & $0 \%$ \\
\hline $\begin{array}{l}\text { produzieren besonders gute Qualität (Geschmack, } \\
\text { besondere Sorten oder Ähnliches). }\end{array}$ & $15,3 \%$ & $58,3 \%$ & $22,2 \%$ & $4,2 \%$ & $0 \%$ \\
\hline produzieren zu besonders niedrigen Kosten. & $7 \%$ & $42,3 \%$ & $33,8 \%$ & $15,5 \%$ & $1,4 \%$ \\
\hline erzielen besonders gute Preise am Markt. & $11,1 \%$ & $41,7 \%$ & $41,7 \%$ & $5,6 \%$ & $0 \%$ \\
\hline nutzen besonders intensiv Fördergelder. & $9,7 \%$ & $41,7 \%$ & $37,5 \%$ & $8,3 \%$ & $2,8 \%$ \\
\hline haben besonders lukrative Marktnischen besetzt. & $5,6 \%$ & $25 \%$ & $55,6 \%$ & $8,3 \%$ & $5,6 \%$ \\
\hline $\begin{array}{l}\text { verfügen über eine optimale Betriebsorganisation } \\
\text { (Arbeitsverteilung, Maschinenauslastung etc.) }\end{array}$ & $12,5 \%$ & $37,5 \%$ & $41,7 \%$ & $5,6 \%$ & $2,8 \%$ \\
\hline
\end{tabular}




\section{A.7 ÜBERBLICK ÜBER DIE ERGEBNISSE DES PRÄMIENEXPERIMENTS}

\begin{tabular}{|c|c|c|c|c|}
\hline \multicolumn{5}{|c|}{ Experiment Nr. 1 in Schleswig-Holstein mit 12 Teilnehmern } \\
\hline $\begin{array}{c}\text { Teilnehmer- } \\
\text { Nr. }\end{array}$ & $\begin{array}{l}\text { Anzahl der im Sinne der } \\
\text { Funktionsweise der } \\
\text { Flexiblen Förderung } \\
\text { plausiblen Entscheidungen }\end{array}$ & $\begin{array}{c}\text { Anzahl der im Sinne der } \\
\text { Funktionsweise der Flexiblen } \\
\text { Förderung unplausiblen } \\
\text { Entscheidungen }\end{array}$ & $\begin{array}{l}\text { Anzahl der unplausiblen } \\
\text { Entscheidungen für die } \\
\text { Festbetragsförderung }\end{array}$ & $\begin{array}{l}\text { Ergebnis gemäß } \\
\text { Einteilungsschema }\end{array}$ \\
\hline 1 & 7 & 1 & 1 & teilweise Akzeptanz \\
\hline 2 & 5 & 3 & 1 & teilweise Akzeptanz \\
\hline 3 & 7 & 1 & 0 & volle Akzeptanz \\
\hline 4 & 5 & 3 & 1 & teilweise Akzeptanz \\
\hline 5 & 7 & 1 & 0 & volle Akzeptanz \\
\hline 6 & 4 & 4 & 1 & keine Aussage möglich \\
\hline 7 & 7 & 1 & 0 & volle Akzeptanz \\
\hline 8 & 6 & 2 & 1 & teilweise Akzeptanz \\
\hline 9 & 6 & 2 & 1 & teilweise Akzeptanz \\
\hline 10 & 6 & 2 & 2 & teilweise Akzeptanz \\
\hline 11 & 7 & 1 & 0 & volle Akzeptanz \\
\hline 12 & 7 & 1 & 1 & teilweise Akzeptanz \\
\hline
\end{tabular}

\begin{tabular}{|c|c|c|c|c|}
\hline \multicolumn{5}{|c|}{ Experiment Nr. 2 in Hessen mit 10 Teilnehmern } \\
\hline $\begin{array}{c}\text { Teilnehmer- } \\
\text { Nr. }\end{array}$ & $\begin{array}{c}\text { Anzahl der im Sinne der } \\
\text { Funktionsweise der } \\
\text { Flexiblen Förderung } \\
\text { plausiblen Entscheidungen }\end{array}$ & $\begin{array}{c}\text { Anzahl der im Sinne der } \\
\text { Funktionsweise der Flexiblen } \\
\text { Förderung unplausiblen } \\
\text { Entscheidungen }\end{array}$ & $\begin{array}{c}\text { Anzahl der unplausiblen } \\
\text { Entscheidungen für die } \\
\text { Festbetragsförderung }\end{array}$ & $\begin{array}{c}\text { Ergebnis gemäß } \\
\text { Einteilungsschema }\end{array}$ \\
\hline 1 & 2 & 6 & 2 & keine Aussage möglich \\
\hline 2 & 3 & 5 & 4 & keine Aussage möglich \\
\hline 3 & 7 & 1 & 1 & teilweise Akzeptanz \\
\hline 4 & 6 & 2 & 0 & volle Akzeptanz \\
\hline 5 & 8 & 0 & 0 & volle Akzeptanz \\
\hline 6 & 7 & 1 & 4 & volle Akzeptanz \\
\hline 7 & 4 & 4 & 1 & gar keine Akzeptanz \\
\hline 8 & 4 & 4 & 0 & keine Aussage möglich \\
\hline 9 & 8 & 0 & 0 & volle Akzeptanz \\
\hline 10 & 5 & 3 & & volle Akzeptanz \\
\hline
\end{tabular}

\begin{tabular}{|c|c|c|c|c|}
\hline \multicolumn{5}{|c|}{$\begin{array}{r}\text { Experiment Nr. 3 in Nordrhein-Westfalen mit 14 Teilnehmern (davon wurde 1 Teilnehmer } \\
\text { ausgeschlossen (konventionell und ohne Umstellungsinteresse)) }\end{array}$} \\
\hline $\begin{array}{c}\text { Teilnehmer- } \\
\text { Nr. }\end{array}$ & $\begin{array}{c}\text { Anzahl der im Sinne der } \\
\text { Funktionsweise der } \\
\text { Flexiblen Förderung } \\
\text { plausiblen Entscheidungen }\end{array}$ & $\begin{array}{c}\text { Anzahl der im Sinne der } \\
\text { Funktionsweise der Flexiblen } \\
\text { Förderung unplausiblen } \\
\text { Entscheidungen }\end{array}$ & $\begin{array}{c}\text { Anzahl der unplausiblen } \\
\text { Entscheidungen für die } \\
\text { Festbetragsförderung }\end{array}$ & $\begin{array}{c}\text { Ergebnis gemäß } \\
\text { Einteilungsschema }\end{array}$ \\
\hline 1 & 6 & 2 & 0 & volle Akzeptanz \\
\hline 2 & 6 & 2 & 0 & volle Akzeptanz \\
\hline 3 & 8 & 0 & 0 & volle Akzeptanz \\
\hline 4 & 6 & 2 & 0 & volle Akzeptanz \\
\hline 5 & 7 & 1 & 0 & volle Akzeptanz \\
\hline 6 & 7 & 1 & 2 & volle Akzeptanz \\
\hline 7 & 2 & 6 & 2 & keine Aussage möglich \\
\hline 8 & 3 & 5 & 2 & keine Aussage möglich \\
\hline 9 & 4 & 4 & 2 & gar keine Akzeptanz \\
\hline 10 & 5 & 3 & 1 & teilweise Akzeptanz \\
\hline 11 & 6 & 2 & 1 & teilweise Akzeptanz \\
\hline 12 & 7 & 1 & 2 & keilweise Akzeptanz Aussage möglich \\
\hline 13 & 4 & 4 & 2 & 2 \\
\hline
\end{tabular}




\begin{tabular}{|c|c|c|c|c|}
\hline $\begin{array}{c}\text { Teilnehmer- } \\
\text { Nr. }\end{array}$ & $\begin{array}{l}\text { Anzahl der im Sinne der } \\
\text { Funktionsweise der } \\
\text { Flexiblen Förderung } \\
\text { plausiblen Entscheidungen }\end{array}$ & $\begin{array}{l}\text { Anzahl der im Sinne der } \\
\text { Funktionsweise der Flexiblen } \\
\text { Förderung unplausiblen } \\
\text { Entscheidungen }\end{array}$ & $\begin{array}{l}\text { Anzahl der unplausiblen } \\
\text { Entscheidungen für die } \\
\text { Festbetragsförderung }\end{array}$ & $\begin{array}{l}\text { Ergebnis gemäß } \\
\text { Einteilungsschema }\end{array}$ \\
\hline 1 & 8 & 0 & 0 & volle Akzeptanz \\
\hline 2 & 8 & 0 & 0 & volle Akzeptanz \\
\hline 3 & 7 & 1 & 0 & volle Akzeptanz \\
\hline 4 & 7 & 1 & 0 & volle Akzeptanz \\
\hline 5 & 6 & 2 & 1 & teilweise Akzeptanz \\
\hline 6 & 7 & 1 & 0 & volle Akzeptanz \\
\hline 7 & 5 & 3 & 1 & teilweise Akzeptanz \\
\hline 8 & 6 & 2 & 2 & teilweise Akzeptanz \\
\hline 9 & 2 & 6 & 3 & keine Aussage möglich \\
\hline 10 & 2 & 6 & 2 & keine Aussage möglich \\
\hline 11 & 6 & 2 & 0 & volle Akzeptanz \\
\hline 12 & 6 & 2 & 1 & teilweise Akzeptanz \\
\hline 13 & 7 & 1 & 1 & teilweise Akzeptanz \\
\hline 14 & 4 & 4 & 0 & volle Akzeptanz \\
\hline 15 & 5 & 3 & 1 & teilweise Akzeptanz \\
\hline
\end{tabular}

\begin{tabular}{|c|c|c|c|c|}
\hline \multicolumn{5}{|c|}{ Experiment Nr. 5 in Rheinland-Pfalz mit 10 Teilnehmern } \\
\hline $\begin{array}{c}\text { Teilnehmer- } \\
\text { Nr. }\end{array}$ & $\begin{array}{c}\text { Anzahl der im Sinne der } \\
\text { Funktionsweise der } \\
\text { Flexiblen Förderung } \\
\text { plausiblen Entscheidungen }\end{array}$ & $\begin{array}{c}\text { Anzahl der im Sinne der } \\
\text { Funktionsweise der Flexiblen } \\
\text { Förderung unplausiblen } \\
\text { Entscheidungen }\end{array}$ & $\begin{array}{c}\text { Anzahl der unplausiblen } \\
\text { Entscheidungen für die } \\
\text { Festbetragsförderung }\end{array}$ & $\begin{array}{c}\text { Ergebnis gemäß } \\
\text { Einteilungsschema }\end{array}$ \\
\hline 1 & 7 & 1 & 0 & volle Akzeptanz \\
\hline 2 & 7 & 1 & 1 & teilweise Akzeptanz \\
\hline 3 & 5 & 3 & 0 & volle Akzeptanz \\
\hline 4 & 7 & 1 & 4 & volle Akzeptanz \\
\hline 5 & 4 & 4 & 0 & gar keine Akzeptanz \\
\hline 6 & 6 & 2 & 0 & volle Akzeptanz \\
\hline 7 & 7 & 1 & 0 & volle Akzeptanz \\
\hline 8 & 6 & 2 & 1 & volle Akzeptanz \\
\hline 9 & 5 & 3 & 0 & teilweise Akzeptanz \\
\hline 10 & 7 & 1 & volle Akzeptanz \\
\hline
\end{tabular}

\begin{tabular}{|c|c|c|c|c|}
\hline \multicolumn{5}{|c|}{ Experiment Nr. 6 in Baden-Württemberg mit 12 Teilnehmern } \\
\hline $\begin{array}{c}\text { Teilnehmer- } \\
\text { Nr. }\end{array}$ & $\begin{array}{c}\text { Anzahl der im Sinne der } \\
\text { Funktionsweise der } \\
\text { Flexiblen Förderung } \\
\text { plausiblen Entscheidungen }\end{array}$ & $\begin{array}{c}\text { Anzahl der im Sinne der } \\
\text { Funktionsweise der Flexiblen } \\
\text { Förderung unplausiblen } \\
\text { Entscheidungen }\end{array}$ & $\begin{array}{c}\text { Anzahl der unplausiblen } \\
\text { Entscheidungen für die } \\
\text { Festbetragsförderung }\end{array}$ & $\begin{array}{c}\text { Ergebnis gemäß } \\
\text { Einteilungsschema }\end{array}$ \\
\hline 1 & 8 & 0 & 0 & volle Akzeptanz \\
\hline 2 & 6 & 2 & 0 & volle Akzeptanz \\
\hline 3 & 5 & 3 & 0 & teilweise Akzeptanz \\
\hline 4 & 6 & 2 & 0 & volle Akzeptanz \\
\hline 5 & 8 & 0 & 1 & volle Akzeptanz \\
\hline 6 & 6 & 2 & 0 & teilweise Akzeptanz \\
\hline 7 & 7 & 1 & 0 & volle Akzeptanz \\
\hline 8 & 8 & 0 & 0 & volle Akzeptanz \\
\hline 9 & 7 & 1 & 2 & volle Akzeptanz \\
\hline 10 & 5 & 3 & 0 & teilweise Akzeptanz \\
\hline 11 & 5 & 3 & 0 & volle Akzeptanz \\
\hline 12 & 7 & 1 & volle Akzeptanz \\
\hline
\end{tabular}




\section{A.8 ÜBERBLICK ÜBER DIE ERGEBNISSE DER LOTTERIE}

\begin{tabular}{|c|c|c|c|c|c|c|c|c|c|}
\hline \multicolumn{10}{|c|}{ Experiment $\mathrm{Nr} .1$ in Schleswig-Holstein mit 12 Teilnehmern } \\
\hline \multirow{2}{*}{$\begin{array}{c}\text { Teilnehmer- } \\
\text { Nr. }\end{array}$} & \multicolumn{3}{|c|}{ Spielrunde 1} & \multicolumn{3}{|c|}{ Spielrunde 2} & \multicolumn{3}{|c|}{ Spielrunde 3} \\
\hline & $\begin{array}{c}\begin{array}{c}\text { Gewinn bei } \\
\text { "Kopf" }\end{array} \\
\end{array}$ & \begin{tabular}{|c|} 
Gewinn bei \\
"Zahl"
\end{tabular} & Einsatz & $\begin{array}{c}\text { Gewinn bei } \\
\text { "Kopf" }\end{array}$ & $\begin{array}{c}\text { Gewinn bei } \\
\text { "Zahl" }\end{array}$ & Einsatz & $\begin{array}{c}\begin{array}{c}\text { Gewinn bei } \\
\text { "Kopf" }\end{array} \\
\end{array}$ & $\begin{array}{c}\begin{array}{c}\text { Gewinn bei } \\
\text { "Zahl" }\end{array} \\
\end{array}$ & Einsatz \\
\hline 1 & 50 & 100 & 63 & 50 & 63 & 63 & 63 & 100 & 70 \\
\hline 2 & 50 & 100 & 50 & 50 & 50 & 50 & 50 & 100 & 66 \\
\hline 3 & 50 & 100 & 80 & 50 & 80 & 65 & 80 & 100 & 80 \\
\hline 4 & 50 & 100 & 77 & 50 & 77 & 64 & 77 & 100 & 89 \\
\hline 5 & 50 & 100 & 75 & 50 & 75 & 75 & 75 & 100 & 75 \\
\hline 6 & 50 & 100 & 60 & 50 & 60 & 60 & 60 & 100 & 65 \\
\hline 7 & 50 & 100 & 70 & 50 & 70 & 65 & 70 & 100 & 80 \\
\hline 8 & 50 & 100 & 78 & 50 & 78 & 68 & 78 & 100 & 82 \\
\hline 9 & 50 & 100 & 79 & 50 & 79 & 62 & 79 & 100 & 89 \\
\hline 10 & 50 & 100 & 50 & 50 & 50 & 50 & 50 & 100 & 50 \\
\hline 11 & 50 & 100 & 70 & 50 & 70 & 56 & 70 & 100 & 82 \\
\hline 12 & 50 & 100 & 77 & 50 & 77 & 65 & 77 & 100 & 89 \\
\hline \multirow[b]{2}{*}{$\begin{array}{c}\text { Teilnehmer- } \\
\text { Nr. }\end{array}$} & \multicolumn{3}{|c|}{ Spielrunde 4} & \multirow{2}{*}{\multicolumn{2}{|c|}{ RAC }} & \multirow{2}{*}{\multicolumn{4}{|c|}{ RAC-Einteilung }} \\
\hline & $\begin{array}{c}\text { Gewinn bei } \\
\text { "Kopf" }\end{array}$ & $\begin{array}{c}\text { Gewinn bei } \\
\text { "Zahl" }\end{array}$ & Einsatz & & & & & & \\
\hline 1 & 63 & 70 & 70 & \multicolumn{2}{|c|}{0,0080} & \multicolumn{4}{|c|}{ risikoavers } \\
\hline 2 & 50 & 66 & 50 & \multicolumn{2}{|c|}{0,0169} & \multicolumn{4}{|c|}{ risikoavers } \\
\hline 3 & 65 & 80 & 73 & \multicolumn{2}{|c|}{0,0013} & \multicolumn{4}{|c|}{ risikoneutral } \\
\hline 4 & 64 & 89 & 77 & \multicolumn{2}{|c|}{$-0,0065$} & \multicolumn{4}{|c|}{ risikofreudig } \\
\hline 5 & 75 & 75 & 75 & \multicolumn{2}{|c|}{$-0,0085$} & \multicolumn{4}{|c|}{ risikofreudig } \\
\hline 6 & 60 & 65 & 65 & \multicolumn{2}{|c|}{0,0029} & \multicolumn{4}{|c|}{ leicht risikoavers } \\
\hline 7 & 65 & 80 & 80 & \multicolumn{2}{|c|}{0,0044} & \multicolumn{4}{|c|}{ leicht risikoavers } \\
\hline 8 & 68 & 82 & 74 & \multicolumn{2}{|c|}{$-0,0005$} & \multicolumn{4}{|c|}{ risikoneutral } \\
\hline 9 & 62 & 89 & 83 & \multicolumn{2}{|c|}{$-0,0220$} & \multicolumn{4}{|c|}{ risikofreudig } \\
\hline 10 & 50 & 50 & 50 & \multicolumn{2}{|c|}{0,0073} & \multicolumn{4}{|c|}{ risikoavers } \\
\hline 11 & 56 & 82 & 67 & \multirow{2}{*}{\multicolumn{2}{|c|}{$\frac{0,0311}{-0,0076}$}} & & risiko & avers & \\
\hline 12 & 65 & 89 & 78 & & & & risikof & reudig & \\
\hline
\end{tabular}

\begin{tabular}{|c|c|c|c|c|c|c|c|c|c|}
\hline \multicolumn{10}{|c|}{ Experiment Nr. 2 in Hessen mit 10 Teilnehmern } \\
\hline \multirow{2}{*}{$\begin{array}{c}\text { Teilnehmer- } \\
\text { Nr. }\end{array}$} & \multicolumn{3}{|c|}{ Spielrunde 1} & \multicolumn{3}{|c|}{ Spielrunde 2} & \multicolumn{3}{|c|}{ Spielrunde 3} \\
\hline & \begin{tabular}{|c|}
$\begin{array}{c}\text { Gewinn bei } \\
\text { "Kopf" }\end{array}$ \\
\end{tabular} & $\begin{array}{c}\text { Gewinn bei } \\
\text { "Zahl" }\end{array}$ & Einsatz & $\begin{array}{c}\text { Gewinn bei } \\
\text { "Kopf" }\end{array}$ & $\begin{array}{c}\text { Gewinn bei } \\
\text { "Zahl" }\end{array}$ & Einsatz & $\begin{array}{c}\begin{array}{c}\text { Gewinn bei } \\
\text { "Kopf" }\end{array} \\
\end{array}$ & $\begin{array}{c}\text { Gewinn bei } \\
\text { "Zahl" }\end{array}$ & Einsatz \\
\hline 1 & 50 & 100 & 50 & 50 & 50 & 50 & 50 & 100 & 50 \\
\hline 2 & 50 & 100 & 75 & 50 & 75 & 67 & 75 & 100 & 87 \\
\hline 3 & 50 & 100 & 100 & 50 & 100 & 100 & 100 & 100 & 100 \\
\hline 4 & 50 & 100 & 70 & 50 & 70 & 61 & 70 & 100 & 88 \\
\hline 5 & 50 & 100 & 70 & 50 & 70 & 55 & 70 & 100 & 80 \\
\hline 6 & 50 & 100 & 77 & 50 & 77 & 72 & 77 & 100 & 84 \\
\hline 7 & 50 & 100 & 80 & 50 & 80 & 66 & 80 & 100 & 88 \\
\hline 8 & 50 & 100 & 76 & 50 & 76 & 62 & 76 & 100 & 89 \\
\hline 9 & 50 & 100 & 79 & 50 & 79 & 67 & 79 & 100 & 92 \\
\hline 10 & 50 & 100 & 80 & 50 & 80 & 55 & 80 & 100 & 90 \\
\hline \multirow[b]{2}{*}{$\begin{array}{c}\text { Teilnehmer- } \\
\text { Nr. }\end{array}$} & \multicolumn{3}{|c|}{ Spielrunde 4} & \multirow{2}{*}{\multicolumn{2}{|c|}{ RAC }} & \multirow{2}{*}{\multicolumn{4}{|c|}{ RAC-Einteilung }} \\
\hline & \begin{tabular}{|c|}
$\begin{array}{c}\text { Gewinn bei } \\
\text { "Kopf" }\end{array}$ \\
\end{tabular} & $\begin{array}{c}\text { Gewinn bei } \\
\text { "Zahl" }\end{array}$ & Einsatz & & & & & & \\
\hline 1 & 50 & 50 & 50 & \multicolumn{2}{|c|}{0,0073} & \multicolumn{4}{|c|}{ risikoavers } \\
\hline 2 & 67 & 87 & 77 & \multicolumn{2}{|c|}{$-0,0024$} & \multicolumn{4}{|c|}{ leicht risikofreudig } \\
\hline 3 & 100 & 100 & 100 & \multicolumn{2}{|c|}{0,0013} & \multicolumn{4}{|c|}{ risikoneutral } \\
\hline 4 & 61 & 88 & 80 & \multicolumn{2}{|c|}{0,0017} & \multicolumn{4}{|c|}{ risikoneutral } \\
\hline 5 & 55 & 80 & 65 & \multicolumn{2}{|c|}{0,0397} & \multicolumn{4}{|c|}{ risikoavers } \\
\hline 6 & 72 & 84 & 81 & \multicolumn{2}{|c|}{$-0,0010$} & \multicolumn{4}{|c|}{ risikoneutral } \\
\hline 7 & 66 & 88 & 79 & \multicolumn{2}{|c|}{$-0,0107$} & \multicolumn{4}{|c|}{ risikofreudig } \\
\hline 8 & 62 & 89 & 75 & \multicolumn{2}{|c|}{$-0,0022$} & \multicolumn{4}{|c|}{ leicht risikofreudig } \\
\hline 9 & 67 & 92 & 79 & \multicolumn{2}{|c|}{$-0,0155$} & \multicolumn{4}{|c|}{ risikofreudig } \\
\hline 10 & 55 & 90 & 75 & \multicolumn{2}{|c|}{$-0,0071$} & \multicolumn{4}{|c|}{ risikofreudig } \\
\hline
\end{tabular}




\begin{tabular}{|c|c|c|c|c|c|c|c|c|c|}
\hline \multicolumn{10}{|c|}{$\begin{array}{c}\text { Experiment Nr. } 3 \text { in Nordrhein-Westfalen mit } 14 \text { Teilnehmern (davon wurde } 1 \text { Teilnehmer } \\
\text { ausgeschlossen (konventionell und ohne Umstellungsinteresse)) }\end{array}$} \\
\hline \multirow{2}{*}{$\begin{array}{l}\text { Teilnehmer- } \\
\text { Nr. }\end{array}$} & \multicolumn{3}{|c|}{ Spielrunde 1} & \multicolumn{3}{|c|}{ Spielrunde 2} & \multicolumn{3}{|c|}{ Spielrunde 3} \\
\hline & \begin{tabular}{|c|}
$\begin{array}{c}\text { Gewinn bei } \\
\text { "Kopf" }\end{array}$ \\
\end{tabular} & \begin{tabular}{|c|}
$\begin{array}{c}\text { Gewinn bei } \\
\text { "Zahl" }\end{array}$ \\
\end{tabular} & Einsatz & $\begin{array}{c}\begin{array}{c}\text { Gewinn bei } \\
\text { "Kopf" }\end{array} \\
\end{array}$ & \begin{tabular}{|c|}
$\begin{array}{c}\text { Gewinn bei } \\
\text { "Zahl" }\end{array}$ \\
\end{tabular} & Einsatz & \begin{tabular}{|c|} 
Gewinn bei \\
"Kopf"
\end{tabular} & \begin{tabular}{|c|}
$\begin{array}{c}\text { Gewinn bei } \\
\text { "Zahl" }\end{array}$ \\
\end{tabular} & Einsatz \\
\hline 1 & 50 & 100 & 90 & 50 & \begin{tabular}{|l|}
90 \\
\end{tabular} & 75 & 90 & \begin{tabular}{|l|}
100 \\
\end{tabular} & 100 \\
\hline 2 & 50 & 100 & 72 & 50 & 72 & 72 & 72 & 100 & 81 \\
\hline 3 & 50 & 100 & 90 & 50 & 90 & 85 & 90 & 100 & 90 \\
\hline 4 & 50 & 100 & 85 & 50 & 85 & 73 & 85 & 100 & 94 \\
\hline 5 & 50 & 100 & 78 & 50 & 78 & 62 & 78 & 100 & 81 \\
\hline 6 & 50 & 100 & 65 & 50 & 65 & 57 & 65 & 100 & 75 \\
\hline 7 & 50 & 100 & 79 & 50 & 79 & 75 & 79 & 100 & 79 \\
\hline 8 & 50 & 100 & 58 & 50 & 58 & 58 & 58 & 100 & 88 \\
\hline 9 & 50 & 100 & 80 & 50 & 80 & 70 & 80 & 100 & 90 \\
\hline 10 & 50 & 100 & 100 & 50 & 100 & 100 & 100 & 100 & 100 \\
\hline 11 & 50 & 100 & 100 & 50 & 100 & 100 & 100 & 100 & 100 \\
\hline 12 & 50 & 100 & 65 & 50 & 65 & 50 & 65 & 100 & 65 \\
\hline 13 & 50 & 100 & 100 & 50 & 100 & 90 & 100 & 100 & 100 \\
\hline \multirow{2}{*}{$\begin{array}{l}\text { Teilnehmer- } \\
\mathrm{Nr} \text {. }\end{array}$} & \multicolumn{3}{|c|}{ Spielrunde 4} & \multirow{2}{*}{\multicolumn{2}{|c|}{ RAC }} & \multirow{2}{*}{\multicolumn{4}{|c|}{ RAC-Einteilung }} \\
\hline & \begin{tabular}{|c|}
$\begin{array}{c}\text { Gewinn bei } \\
\text { "Kopf" }\end{array}$ \\
\end{tabular} & $\begin{array}{c}\text { Gewinn bei } \\
\text { "Zahl" }\end{array}$ & Einsatz & & & & & & \\
\hline 1 & 75 & 100 & 100 & \multicolumn{2}{|c|}{$-0,0335$} & \multicolumn{4}{|c|}{ risikofreudig } \\
\hline 2 & 72 & 81 & 79 & \multicolumn{2}{|c|}{$-0,0007$} & \multicolumn{4}{|c|}{ risikoneutral } \\
\hline 3 & 85 & 90 & 85 & \multicolumn{2}{|c|}{$-0,0029$} & \multicolumn{4}{|c|}{ leicht risikofreudig } \\
\hline 4 & 73 & 94 & 87 & \multicolumn{2}{|c|}{$-0,0490$} & \multicolumn{4}{|c|}{ risikofreudig } \\
\hline 5 & 62 & 81 & 78 & \multicolumn{2}{|c|}{$-0,0010$} & \multicolumn{4}{|c|}{ risikoneutral } \\
\hline 6 & 57 & 75 & 66 & \multicolumn{2}{|c|}{0,0251} & \multicolumn{4}{|c|}{ risikoavers } \\
\hline 7 & 75 & 79 & 75 & \multicolumn{2}{|c|}{0,0023} & \multicolumn{4}{|c|}{ leicht risikoavers } \\
\hline 8 & 58 & 88 & 78 & \multicolumn{2}{|c|}{0,1012} & \multicolumn{4}{|c|}{ sehr risikoavers } \\
\hline 9 & 70 & 90 & 85 & \multicolumn{2}{|c|}{$-0,0217$} & \multicolumn{4}{|c|}{ risikofreudig } \\
\hline 10 & 100 & 100 & 100 & \multicolumn{2}{|c|}{0,0013} & \multicolumn{4}{|c|}{ risikoneutral } \\
\hline 11 & 100 & 100 & 100 & 0,00 & 013 & & risikor & heutral & \\
\hline 12 & 50 & 65 & 56 & $-0,8$ & 3500 & & sehr risik & cofreudig & \\
\hline 13 & 90 & 100 & 100 & $-0,0$ & 507 & & sehr risik & kofreudig & \\
\hline
\end{tabular}




\section{Experiment Nr. 4 in Mecklenburg-Vorpommern mit 18 Teilnehmern (davon wurden 3 Teilnehmer ausgeschlossen (konventionell und ohne Umstellungsinteresse))}

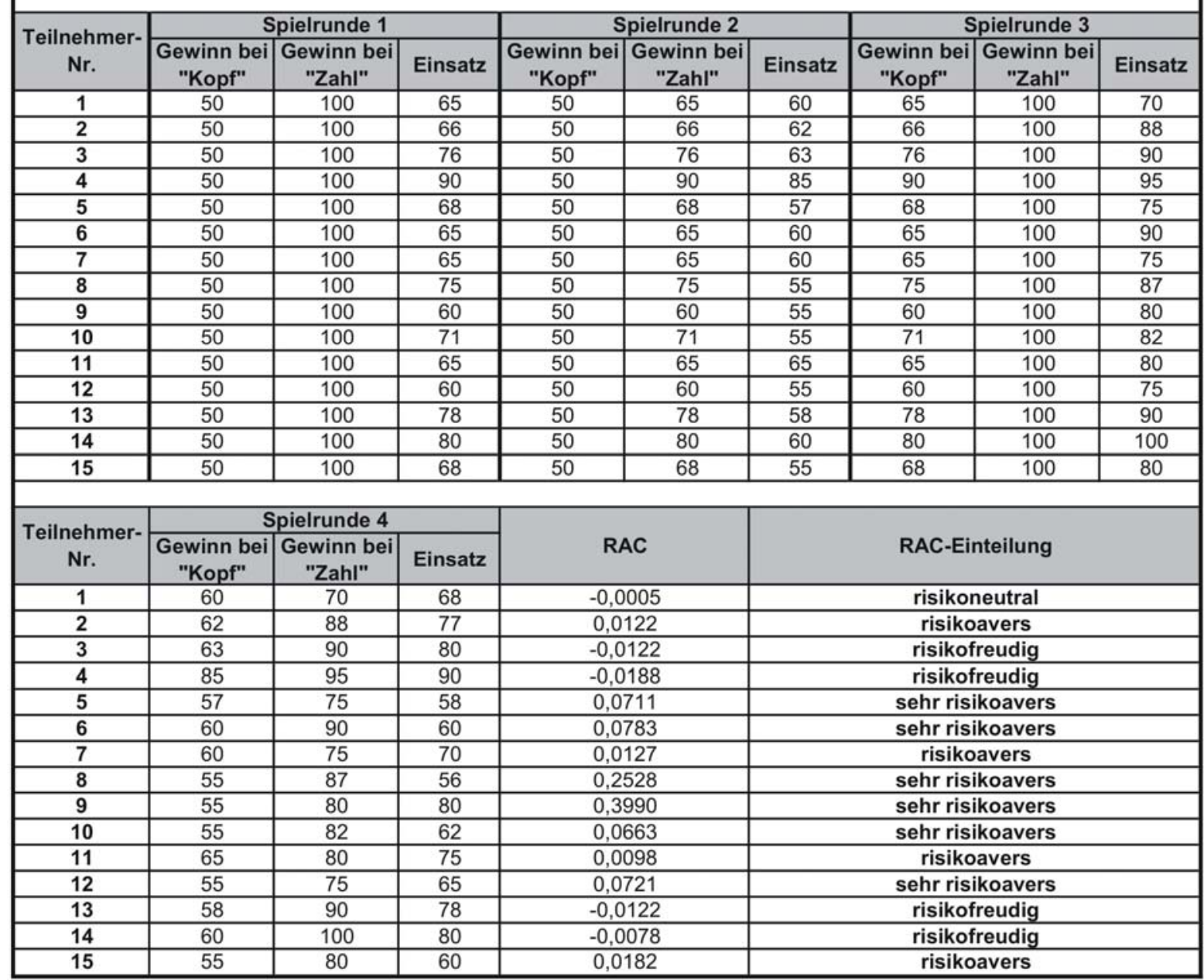




\section{Experiment $\mathrm{Nr} . \mathbf{5}$ in Rheinland-Pfalz mit 10 Teilnehmern}

\begin{tabular}{|c|c|c|c|c|c|c|c|c|c|}
\hline \multirow{2}{*}{$\begin{array}{c}\text { Teilnehmer- } \\
\text { Nr. }\end{array}$} & \multicolumn{3}{|c|}{ Spielrunde 1} & \multicolumn{3}{|c|}{ Spielrunde 2} & \multicolumn{3}{|c|}{ Spielrunde 3} \\
\hline & $\begin{array}{c}\text { Gewinn bei } \\
\text { "Kopf" }\end{array}$ & $\begin{array}{c}\text { Gewinn bei } \\
\text { "Zahl" }\end{array}$ & Einsatz & $\begin{array}{c}\text { Gewinn bei } \\
\text { "Kopf" }\end{array}$ & $\begin{array}{c}\text { Gewinn bei } \\
\text { "Zahl" }\end{array}$ & Einsatz & $\begin{array}{c}\text { Gewinn bei } \\
\text { "Kopf" }\end{array}$ & $\begin{array}{c}\text { Gewinn bei } \\
\text { "Zahl" }\end{array}$ & Einsatz \\
\hline 1 & 50 & 100 & 78 & 50 & \begin{tabular}{|l|}
78 \\
\end{tabular} & 50 & 78 & 100 & 78 \\
\hline 3 & 50 & 100 & 76 & 50 & 76 & 64 & 76 & 100 & 89 \\
\hline 4 & 50 & 100 & 70 & 50 & 70 & 60 & 70 & 100 & 80 \\
\hline 5 & 50 & 100 & 70 & 50 & 70 & 65 & 70 & 100 & 79 \\
\hline 7 & 50 & 100 & 51 & 50 & 51 & 51 & 51 & 100 & 51 \\
\hline 8 & 50 & 100 & 88 & 50 & 88 & 79 & 88 & 100 & 98 \\
\hline 9 & 50 & 100 & 75 & 50 & 75 & 75 & 75 & 100 & 80 \\
\hline 10 & 50 & 100 & 60 & 50 & 60 & 55 & 60 & 100 & 70 \\
\hline \multicolumn{4}{|c|}{ Spielrunde 4} & \multicolumn{2}{|c|}{ RAC } & \multicolumn{4}{|c|}{ RAC-Einteilung } \\
\hline 2 & 62 & 81 & 71 & \multicolumn{2}{|c|}{0,0064} & \multicolumn{4}{|c|}{ risikoavers } \\
\hline 3 & 64 & 89 & 77 & \multicolumn{2}{|c|}{$-0,0053$} & \multicolumn{4}{|c|}{ risikofreudig } \\
\hline 4 & 60 & 80 & 65 & \multicolumn{2}{|c|}{0,0217} & \multicolumn{4}{|c|}{ risikoavers } \\
\hline 5 & 65 & 79 & 69 & \multicolumn{2}{|c|}{0,0036} & \multicolumn{4}{|c|}{ leicht risikoavers } \\
\hline 6 & 70 & 92 & 81 & \multicolumn{2}{|c|}{$-0,0297$} & \multicolumn{4}{|c|}{ risikofreudig } \\
\hline 7 & 51 & 51 & 51 & \multicolumn{2}{|c|}{0,0292} & \multicolumn{4}{|c|}{ risikoavers } \\
\hline 8 & 79 & 98 & 97 & \multicolumn{2}{|c|}{$-0,1932$} & \multicolumn{4}{|c|}{ sehr risikofreudig } \\
\hline 9 & 75 & 80 & 80 & \multicolumn{2}{|c|}{0,0017} & \multicolumn{4}{|c|}{ risikoneutral } \\
\hline 10 & 55 & 70 & 65 & \multicolumn{2}{|c|}{0,0544} & \multicolumn{4}{|c|}{ sehr risikoavers } \\
\hline
\end{tabular}

\begin{tabular}{|c|c|c|c|c|c|c|c|c|c|}
\hline \multicolumn{10}{|c|}{ Experiment Nr. 6 in Baden-Württemberg mit 12 Teilnehmern } \\
\hline \multirow[b]{2}{*}{$\begin{array}{c}\text { Teilnehmer- } \\
\text { Nr. }\end{array}$} & \multicolumn{3}{|c|}{ Spielrunde 1} & \multicolumn{3}{|c|}{ Spielrunde 2} & \multicolumn{3}{|c|}{ Spielrunde 3} \\
\hline & $\begin{array}{c}\text { Gewinn bei } \\
\text { "Kopf" }\end{array}$ & $\begin{array}{c}\text { Gewinn bei } \\
\text { "Zahl" }\end{array}$ & Einsatz & $\begin{array}{c}\text { Gewinn bei } \\
\text { "Kopf" }\end{array}$ & $\begin{array}{c}\text { Gewinn bei } \\
\text { "Zahl" }\end{array}$ & Einsatz & $\begin{array}{c}\text { Gewinn bei } \\
\text { "Kopf" }\end{array}$ & $\begin{array}{c}\text { Gewinn bei } \\
\text { "Zahl" }\end{array}$ & Einsatz \\
\hline 1 & 50 & 100 & 55 & 50 & 55 & 51 & \begin{tabular}{|l|}
55 \\
\end{tabular} & 100 & 67 \\
\hline 2 & 50 & 100 & 75 & 50 & 75 & 65 & 75 & 100 & 80 \\
\hline 5 & 50 & 100 & 77 & 50 & 77 & 76 & 77 & 100 & 79 \\
\hline 6 & 50 & 100 & 75 & 50 & 75 & 60 & 75 & 100 & 80 \\
\hline 7 & 50 & 100 & 76 & 50 & 76 & 64 & 76 & 100 & 87 \\
\hline 8 & 50 & 100 & 78 & 50 & 78 & 68 & 78 & 100 & 90 \\
\hline 9 & 50 & 100 & 65 & 50 & 65 & 53 & 65 & 100 & 78 \\
\hline \multirow{2}{*}{$\begin{array}{c}\text { Teilnehmer- } \\
\text { Nr. }\end{array}$} & \multicolumn{3}{|c|}{ Spielrunde 4} & \multirow{2}{*}{\multicolumn{2}{|c|}{ RAC }} & \multirow{2}{*}{\multicolumn{4}{|c|}{ RAC-Einteilung }} \\
\hline & \begin{tabular}{|c|}
$\begin{array}{c}\text { Gewinn bei } \\
\text { "Kopf" }\end{array}$ \\
\end{tabular} & \begin{tabular}{|c|}
$\begin{array}{c}\text { Gewinn bei } \\
\text { "Zahl" }\end{array}$ \\
\end{tabular} & Einsatz & & & & & & \\
\hline 1 & 51 & 67 & 55 & \multicolumn{2}{|c|}{1,2487} & \multicolumn{4}{|c|}{ sehr risikoavers } \\
\hline 2 & 65 & 80 & 70 & \multicolumn{2}{|c|}{0,0026} & \multicolumn{4}{|c|}{ leicht risikoavers } \\
\hline 3 & 53 & 70 & 60 & \multicolumn{2}{|c|}{0,0713} & \multicolumn{4}{|c|}{ sehr risikoavers } \\
\hline 4 & 62 & 86 & 74 & \multicolumn{2}{|c|}{0,0031} & \multicolumn{4}{|c|}{ leicht risikoavers } \\
\hline 5 & 76 & 79 & 78 & \multicolumn{2}{|c|}{0,0059} & \multicolumn{4}{|c|}{ risikoavers } \\
\hline 6 & 60 & 80 & 70 & \multicolumn{2}{|c|}{0,0092} & \multicolumn{4}{|c|}{ risikoavers } \\
\hline 7 & 64 & 87 & 77 & \multicolumn{2}{|c|}{$-0,0034$} & \multicolumn{4}{|c|}{ leicht risikofreudig } \\
\hline 8 & 68 & 90 & 85 & \multicolumn{2}{|c|}{$-0,0233$} & \multicolumn{4}{|c|}{ risikofreudig } \\
\hline
\end{tabular}


